classeur cosmos

Relieur 

Le pays snossi

et

sa population

Etude historique, géograpinique. eiconomique, sociologique th ethnographigue rar

le Capitaine \&. E. Lambert de

l'bufanterie Coloniale

aout 190\%. 
osit 


$$
\begin{aligned}
& \text { Lepaysu Masi } \\
& \text { et sa population }
\end{aligned}
$$

Etude listorique économique ev éographique sünie d' un essar d'etimographie compras

par.

le Eapitaineg.6. Poumbert

de l'Infanterie Coloniale

Conmendant le Cercle de Onagadongon (1905. 1907 )

Jllarisiso\% 


\section{dommaire}

Intraduction. S'enyire Massi et les chroniques arabes - Premieires explarations _ Chronolagie de la

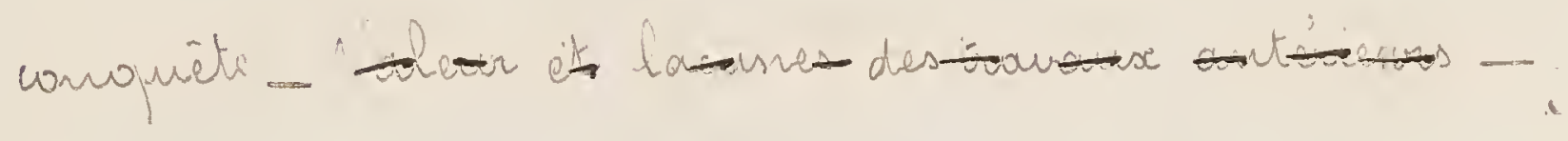
Butr de la frésente étude.....................

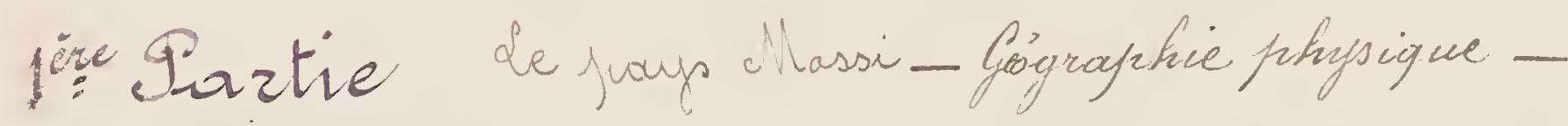
Owographie - Hoydrographie - Géologie - Blore - Faus ne-Geógrafnfrie économique - Aaies de communicationConnnerce _ Elevage - Industries locales.............

2: Paztie Cossai histarique sur les inarasions Massi_ia dynastic actuelle_Graganisation falitique à laquelle elle a présidé fusquía notre verme dans le frous.........

3: Dartie Etrode sociologiopure et ethnographique sur les races habitant le Massi................. 133

I Vie nutritive ........................ 134

II_ Vie sensitive ... . . . . . . . . . . 138

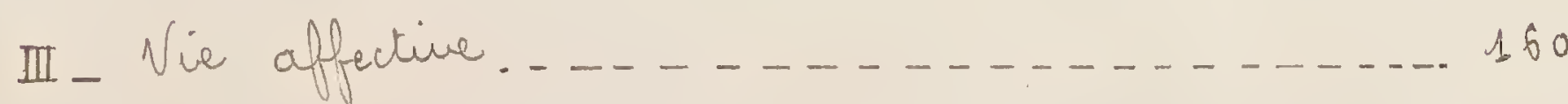

IV_ Vie saciale .................... 201

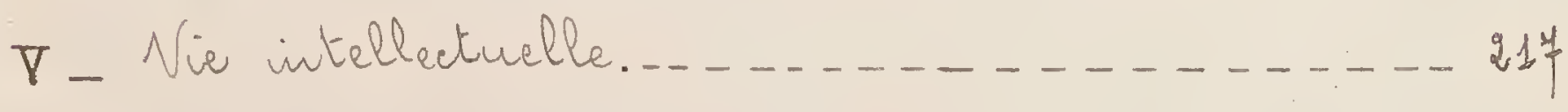

Yndes alphabetique............... 234 


\section{Eable des photographies}

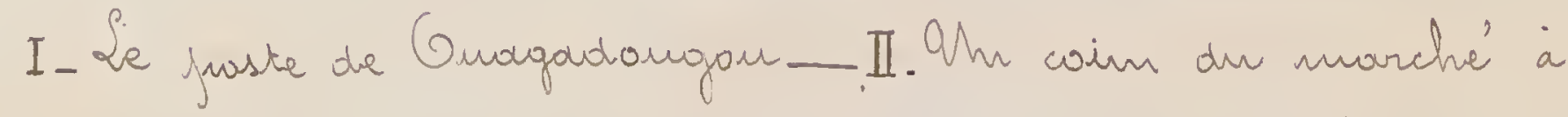

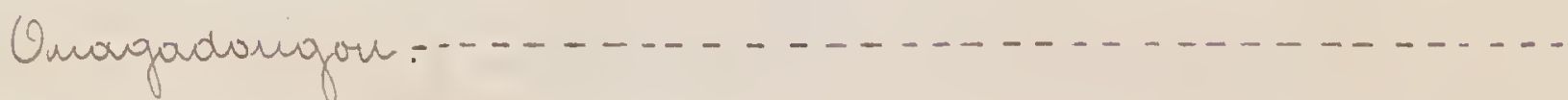

III. Entrée du frout de la Valta à Mgognon - IV. La Volta Blan.

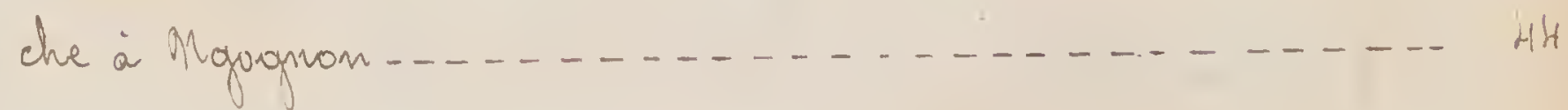

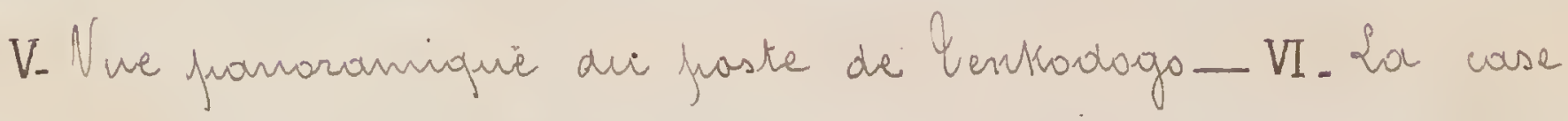
tumelle de beutradoogo (écale de la livionseriftions...... 59

VII. Le Morko. Matar et les grands Kambérés - VIII. Covatiers

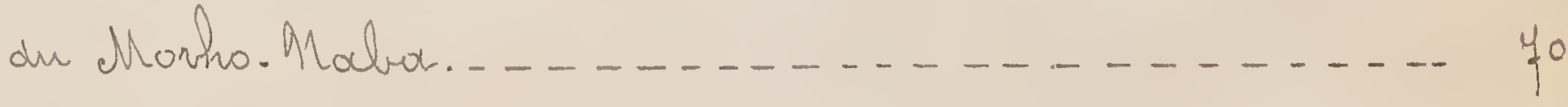

IX. Maba Kon II - X. Gam. tam du Marks.Maha........ 103 XI_XII XIII Cavaliers Massi.............. 22.

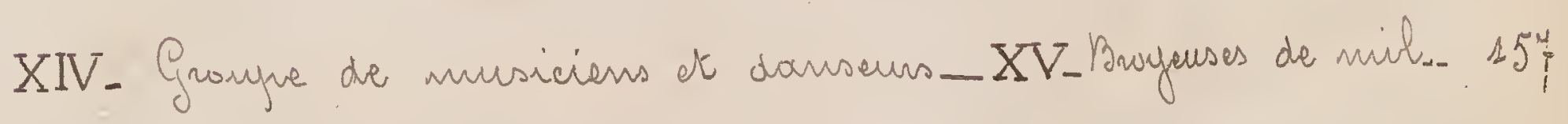
XVI_XVII_ Gyppe de fermme Banssanga.......... 1 \$ 
Sommaize

Trtroduction.

But de la friésente etude

19i Parlic.

r'enpirie Mossi et les chroniques arabesPrennienes explozations - Cironologie de la conquite.

$2^{2}-$ Partie.

Le raves Mossi- Geographice fohysique Orographie - Hlydrografinie - Geologies Slove faune - Jeographie economigue - Voces de Communieation - Conmence - SlevageIndustries locales.

3" Partic

Sssai historique sur les imasions M2ossi - V'a dynastie actuclle - Oeganisa tion frolitigue.

4: Partic -

Stude sociologique et etronographique sur les zaces habitant le Mossi.

I- Vie nuticive.

II-Vie sessistive.

III-Vie affective.

IV-Vie sociale.

VI - Vie intellectuelle.

sudex alfinabeitigue. 
DS 


Sntrociuction.

Four permethe de suine facitement te quettions

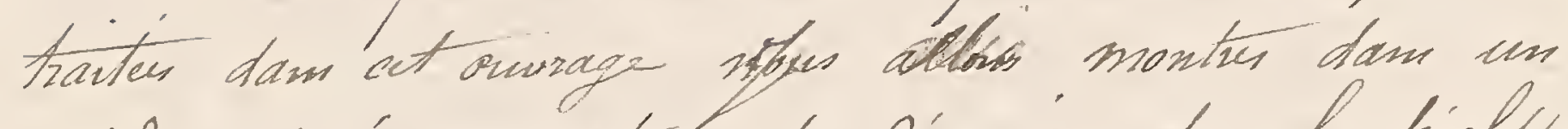
rafive exfrose', comment in freu D'amneis, fros ke develofipement naturel Pin filan quin 'éécite avee esfrit de suite et fermete exchait neamoins toute violence inutile, la conquiete et la fracification.

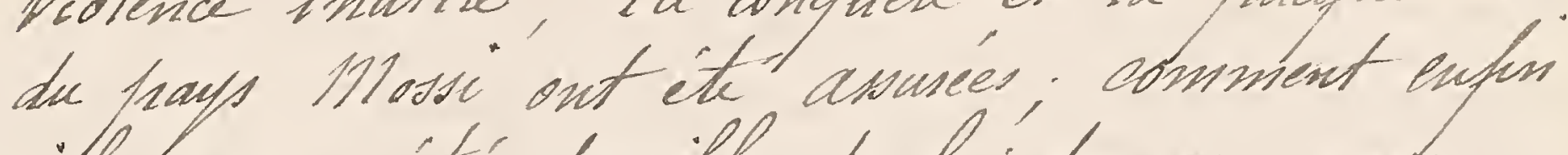
il nous a éte' hossible de Lui domer unde organisation sinon definitive du moins en raphrort avee Les iideés d'humancte' of de justice qui doivent instires toute seure vraiment francaide.

ota tache de nos devanciens a ite unde to ficm nemplui. Sout sccupés quíts étaient de la puse or hossession ot de I organisation rationncle is a vate territoire is ont fiu neammoins mes Laviser de coprieux unscignements geigraphigues ef statritigues ucueillis, ave te filus grano trin of une hasfaite clairvoyance ifoces les metrous utilement a' mofit du cours de ceste etude. Mais sollicites har des frésecupations hlus immediats, ils ont die neighiger avele les recherches

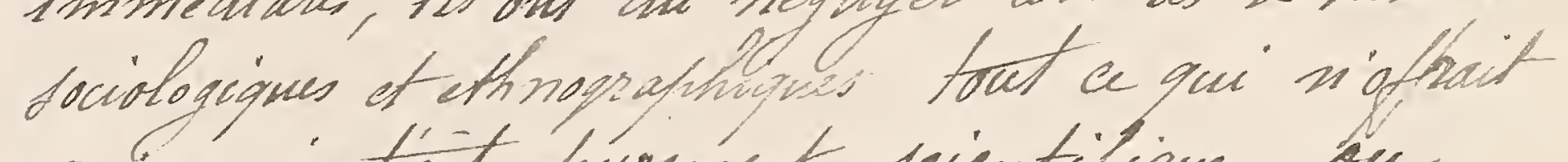
qu un interict furement seientifigue ou

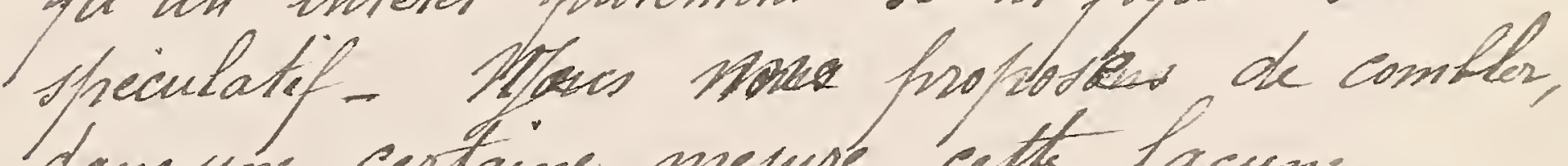
dans une certaine mesure, cette Lacume. 
'DS! 
jeir Paria.

Q'empire Mrossiet ter chronique arabes. Prennères exfiforation. Chronologici de la Conguete.

Au centu de la bouck du 1iiger fresgue a'egak distance entre ton froint culminant et la lote de l'Or, I'etend Lus une vaste Juresficie f' omprise T1sosti ainsi afprete' du nom de la race qui le heuple!

Cette sace s'y est substitué Depuis hlus de trois cents ans, a'du alooxigenes sus lidentite' desquels il a 'ite' difficie jusqu'cia' de de frononcer aver eentitude.

On a voulu y voir bes Habés, que la fousiei des emahissens ausait sejétés vers be nord audelai du Natenga. Mous croyous flutst, et nous en famerbon les raisons au Couss de cette etude, que ces autochlones afficatenaiént Au merne groupe ethinique que ter Bobos et les Hounodurnas, quils subsistent encose dares le hays et of forment Les qrounements considerables comms sous le nom de Gourounsi, Sciphersi; Boussangas, ete.

Ta fanille Mandingue hromement dite eot refresentec frar les Harses on Buangasbes, qui, five's de loin en loin, en aglfomerations virantes et actives, out longternis. detenu be monopole du commerce de lá contrie; enfon des Peulhs afprartenant aux qroupies borobes et Dialloubés. Hout ejaltement disiéminés sees toute fietendure du tenitoine 

Du milien de cette mosaigue de zaces Le détache l'élement envalisiseur, f'élement 112ossi, qui à lui seul fresenter des caracteriotigur assey interessantes frour domer da saison d'etre an travail que nous avons entrefins.

Cette pofuntation est en effet d auctant. frus curieuse a' éfudiè Lans tes sigines, dons son etat actuel, Les moenes, comme dans Ion onganisation frolitigive et dociale, quécle differe nettement a' ces divers forits of wue des awher qrandes peuflades que la conguete du Souldan a mises Lous notu autorite; et Ims hisuelles a fur J'éxercer au cous de cus Dernières amsés, Le Labeur ousimplement la curiosite 'des chesehenes et des ethoropratitus.

the ideé qui I'imfroseza eswit dei le tovist, éet que malqré f'ctomante vitalite qu'elle atteste encsie et qui' a fait du rays qu'elle oceupe te

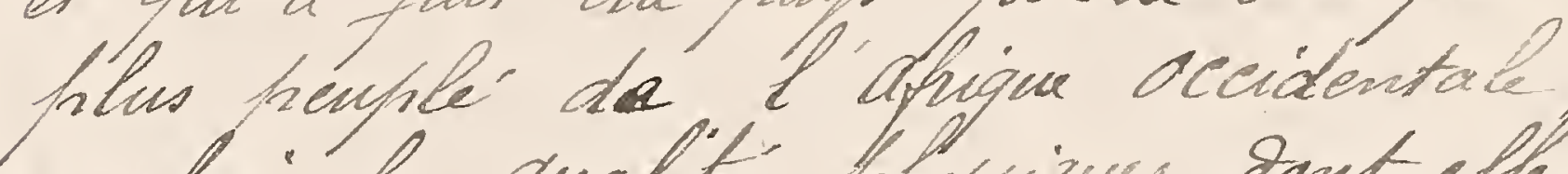
malgre 'les qualiter frhysions Bout elle est dorié, cette race eft inhé heu a' reve, et he trouve aujourd hui Dans une réride de uqression Caractéisé, a Laquélle linfluence de nos cileis civitisaticio frouna du moins imposes un arset, s'il nous et interdit d'espierer d faine reprende un four $i$ ce prant heuble

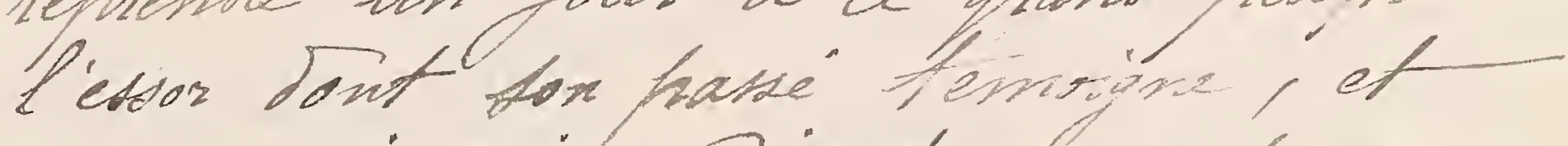
qu'une imasion Deisasticuke est venue

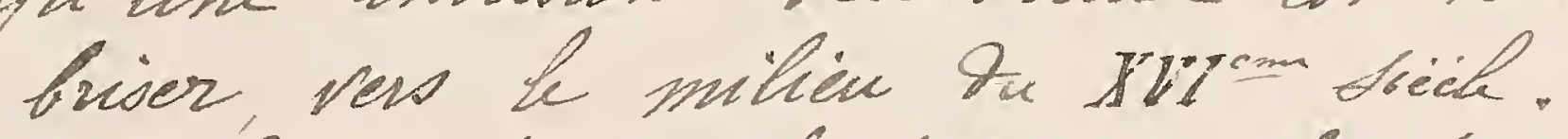
Ce systime feidal compligué que 
DSI 
thrs birs ct qui'a persisté Jusqu'à nos fours, n'a pu prendu naissance quian sein

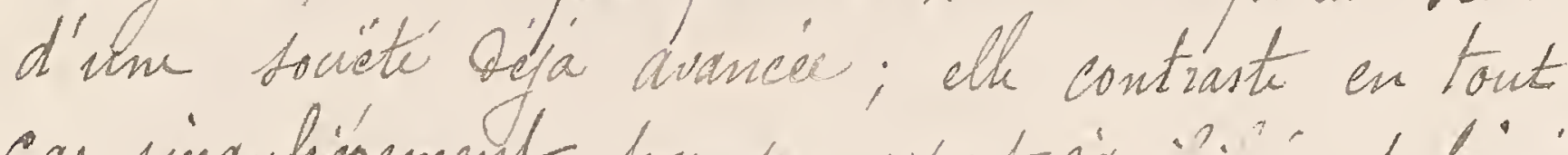
cas singuhiorement has son ashect equilizie et fin avee ls miserables ebauches dónganisation Hociale. gout le pays nois tout a' l'entour nous gomes le strectack.

D'anto hant leschromigues arabes qui constituent poun tout te Soudan la senk fradition acrite oont nous frissions tirer quelques indications, nous montunt que ce regime a rriside' fadis a l'organisation d'ine frusiance militane usoutable

Aloméd Babu et apres Lui Abdérnaman bén abdallah nous apprement que Gombouctors a été a une érogive uculé quilo frecisent mal, mais que nous frowons flaces vers 1355 de notw ire, saccagé has Le Sultan du Mossi. "Ce fut, dit abderrhama (1) Sous a rigne dén "des frimees de sa Dynastie" if siagit de Moursoces Houner "t et has consequent I la dynastic \& Mali on "Sombaï. Mande' hroprement dite" que le Sultan "du Mossi" i' i la tete J'un souke armie fit une "espidition contru setk wille. Jaisis d'effroi hes gen' de "Thelli prinent la finte et abanvomerent Conbouctore "anx assaillanti. de Sultan dn Mossi fienetra alow "dam la ville, la saccagea, et amies dvis fait "peris tous cens qu'cl hut atteinck et se'to "emprasi st tiuts les vichesses qu'iltroura, il "utouma Taro Ionfray. Les gens de Melli

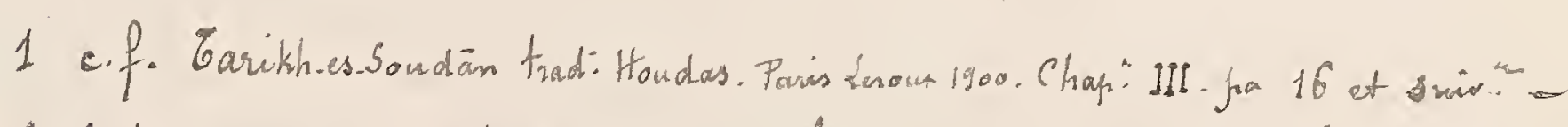

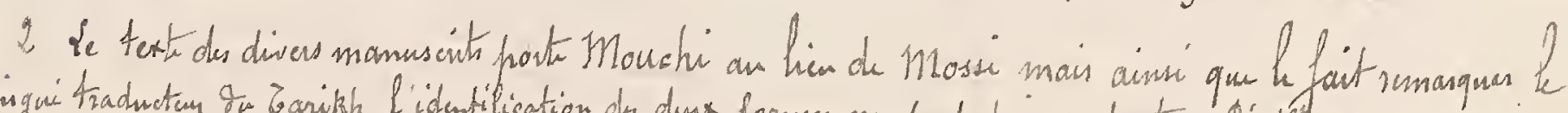

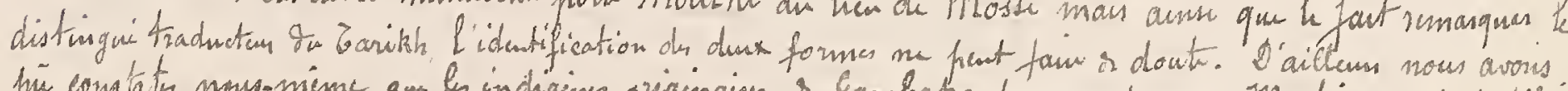

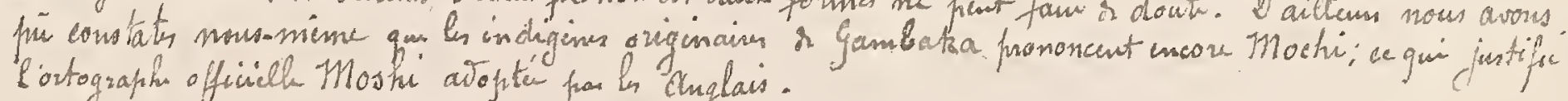

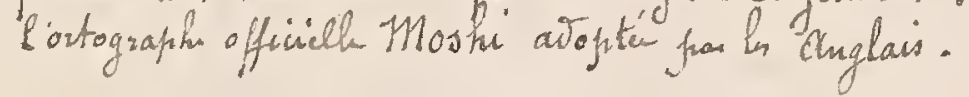



"iventirient ensinte a bombouctor et y "demenierent cent ans.

Iomboncior, a dit le fir docte finisconsult "Ahmed saba, a ête' Saceage" Trois fois:

"la premère fois has be sultan dn Mossi, la "deuxieme frar Souni. Ali et la froisieme far "Le hacha Hahmoud-ben-rergoun. Cette « dermièr divastation fut moins terrible que les "denx hremières, donos la mise à tae dé Jouni"Alill il. out 'le plus Je Sang séhandu."

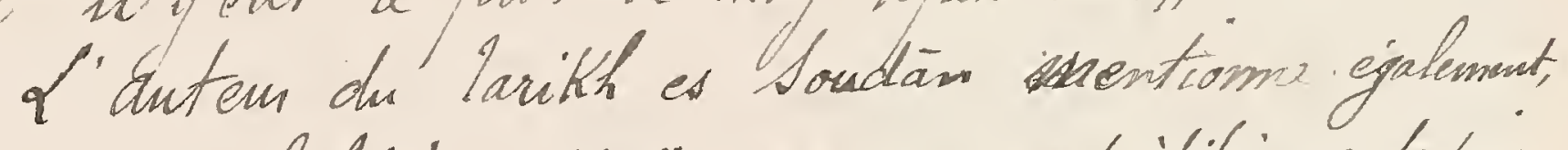
$l$ 'an 903 de l'hiegire, "1497-1498" une expredition entretrise pas il-ttadj Mohammed Astia contu na'asira sultan du Mossi." Infin en 969 "1561-1562" Astria Baoud disige

une nowvelle expridition contro a frons que bon chef, est contraint d'abandomer avec touts ses troupres.

q'examen attentif de ces textes Lewr rapprochement

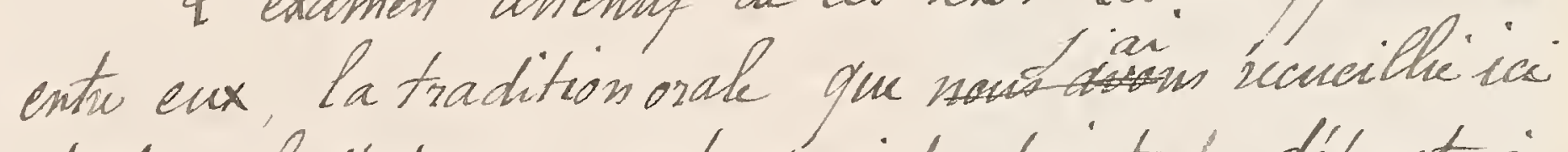
et dam le Yatengre ont servide froint be difraut $a$ i mes ucherchos. Its ont arrevie a' une seire s Conchusions qui fremettent de concilier Désomais des temorignages eirits et oraux dont ter divergences paraisanient irriductibles, ct jettent un four nowvean Lus laqustion des origines Mossi. Ce proint ent mis harticulièrement en éviderso, que L'empin Mossi J'oi hatit la qrande

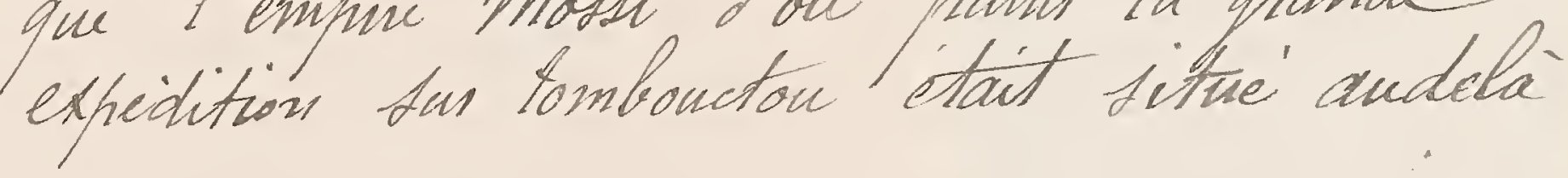

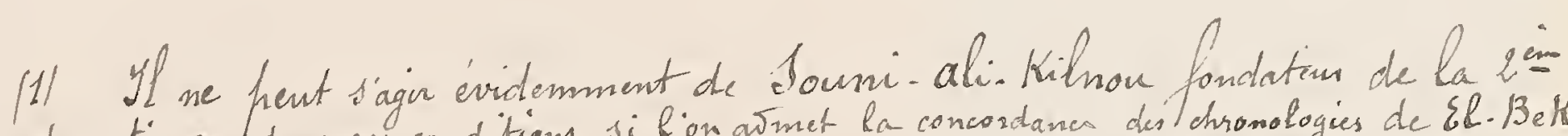

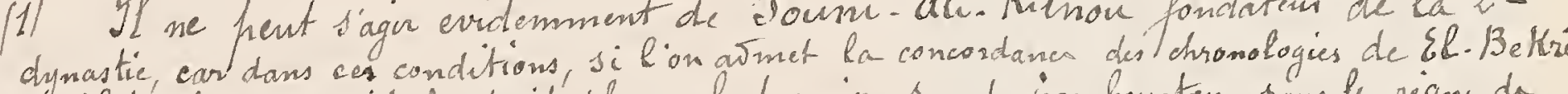

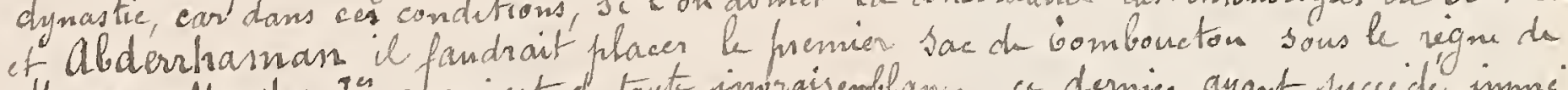

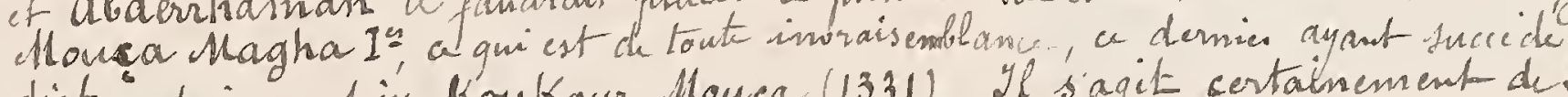

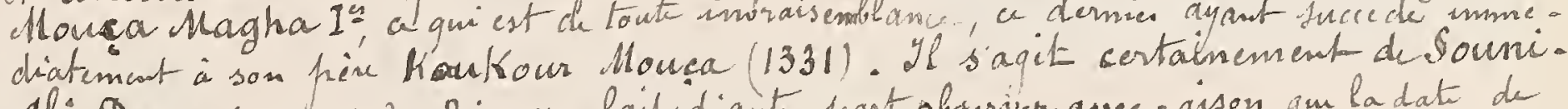

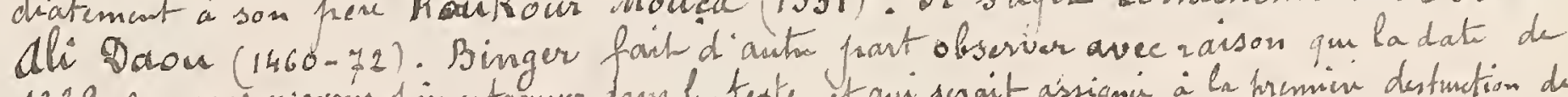

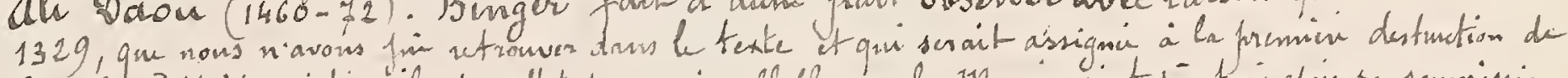

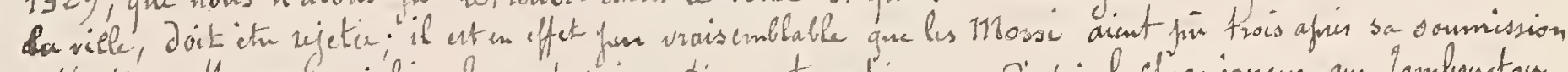

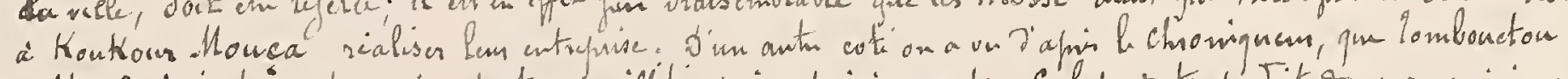

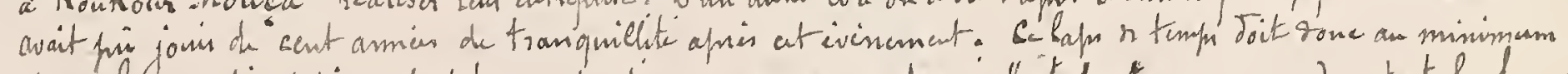

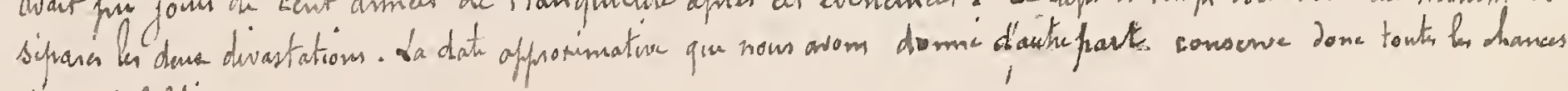
d. juobalitite.

(1) Larikl es Soudan al.eit. p.121.122.

$\left(3^{1} d^{*} \quad d^{\circ} \quad d^{\circ}\right.$ p. 173 . 
08 
de la wive Gauche du Higer et y oecurait fimplacement Do Bomou actuch. If ext inerne frobable que La linite occidentale le defrassaif et englobait ave rano, une hortion du Sokoto.

Cest done La le hays contu tequel Astria Daoud dinge liexhedition dis li et que tonchef est contraint D'áandomes avee touter Les trouhes'. Cette fuite devent P'envahissens, ceit le grand éxode qui devait amener Jau la contrei quil

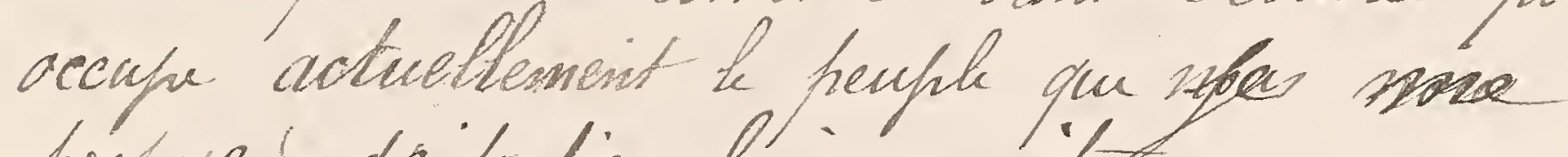
hrotose destier fain comaita -

'I Avait ite' fricide' D'émignations \& moinatre importance - Ses hremien qroupes sétwènt detaches of finpine quingriétaient deja bes
incussions de incisins turbulents.

Ces qroufes waient Luive la route has

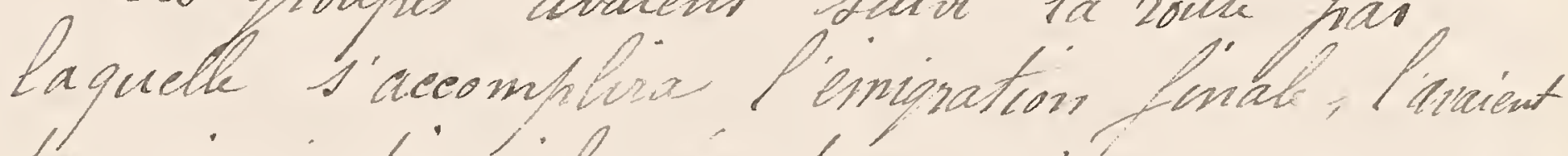

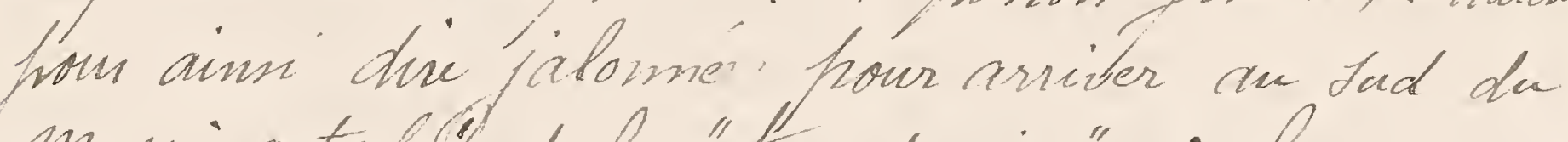
mossi actudel de la "ferre promise" cà leur race itablira non Sano reine La midominana Jur les hemplades barbareś qui licecofraient.

- Nais cét bien a cette érogm, ouiala periode qui l'a micéde' inmrediatement, qm lion doit faire

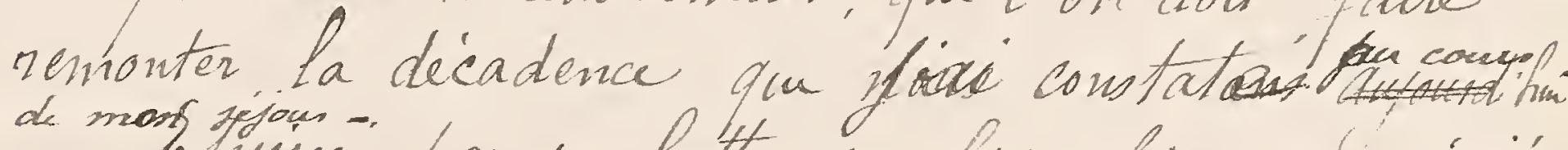
de mong isinise has sa lutte ave P'envihio.ns, 'anémié has celles qu'il dut douteris contru bes tribus dont il fallait traverses le tenitoine et qui hi bassaient la zoute d un coté; de Liutro contra les autochtones qu dam un demies effort il dwait refouler, inremediablement fraphe dans ses forces vives, be peuple-

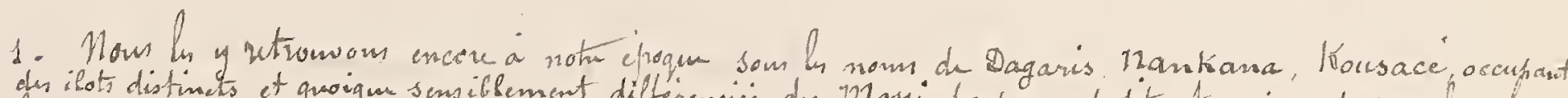

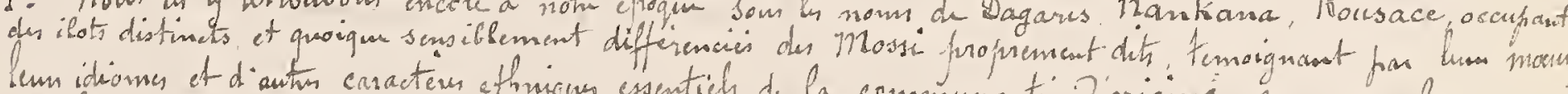
attribuons 

Mosid nia hu trowes an Lein' \& Lui-misme les sessomes neiersaines four reiagir et fou long Declin a commencé

da nuit siest faite autous de Lon nom' et Ls chroniqueun ne mentionnent mène filus Lon existence.

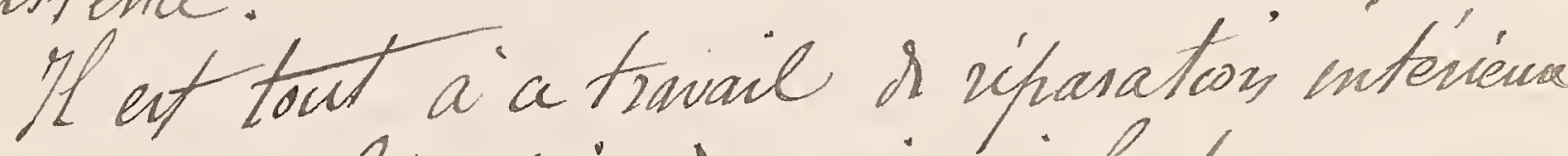
- Tout il ausa l'estrois 1 venis a bout.

In silena l'mpine Moxi: Le ucontitue: lentement il tramform fout Cette contrie, la counre or villages, \& calturnes. On croviait a'une isunection. Mais cette vie nowille n'a fum gam tes manifertation fieclat or jadis. Louty ent maticiel t terrea-terse. On drait que he soufle hinisomst qui avait antrefoi animé a reuple et k aisait quens ct sedoutable Sert eteint a jamais, il a cessi o creés, il imite, d'attachant eitroitement ai un tradition a laquell id niajortera plus vien, et sont il a hersu a Sentemint.

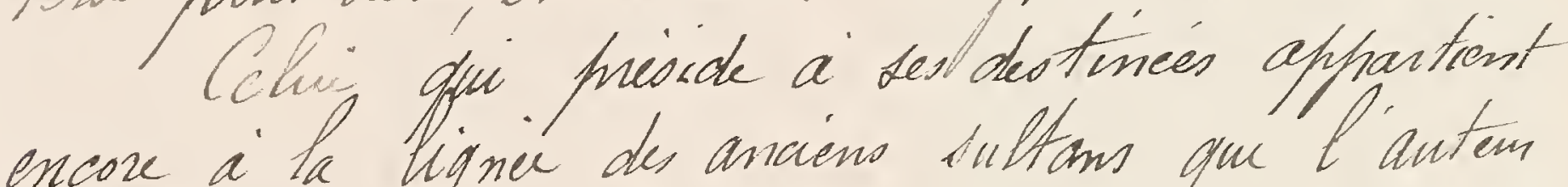
onove à la Hignier de ancieno sultons que l'antem To barith, nom a fait conmaitu, mais a grotesgue Naba 'Danom frasessenx et debauche qui sucura, - fort mal J'ailleun, Binger en 1888, nia phun. vien de commun are be grano aient qui wait jete' l'éformante gans L'empine Sonrhai at conguis Sombonctou.

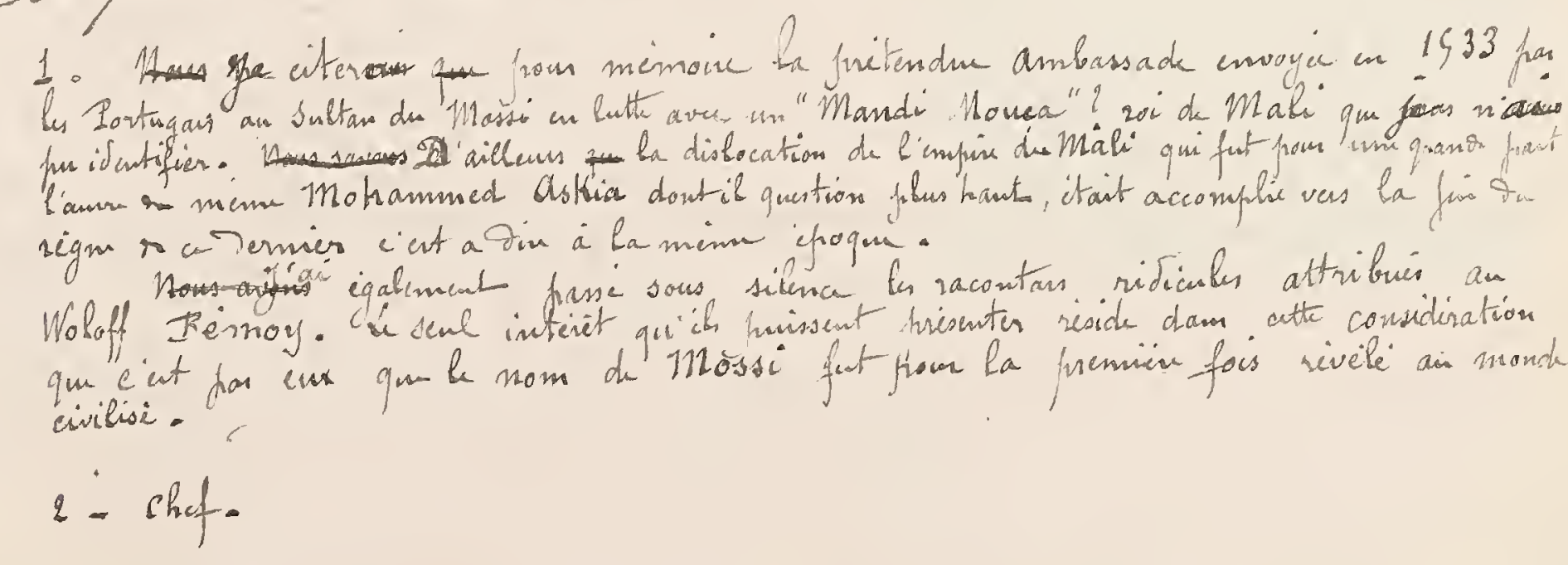


DSI 
Cest en effet dan cet etat que los fremien

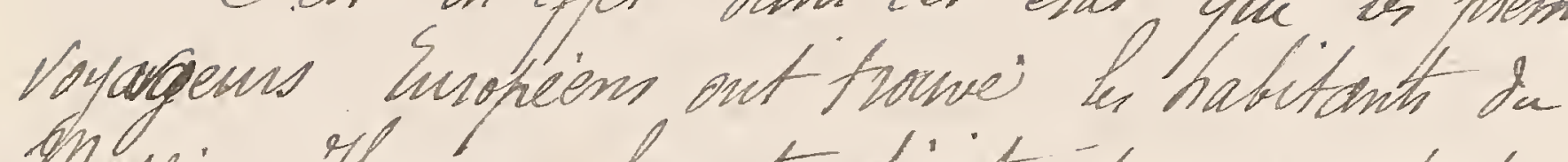
Inssai. Its nour les ont deciots Hous in ashect qui differe hen ol celui anguel les awtrs noins nous ont
accontumes.

Barth avait deja' Jomé Bur leus hays des nemseignements assey sagnes qu'il avait frü woneills.

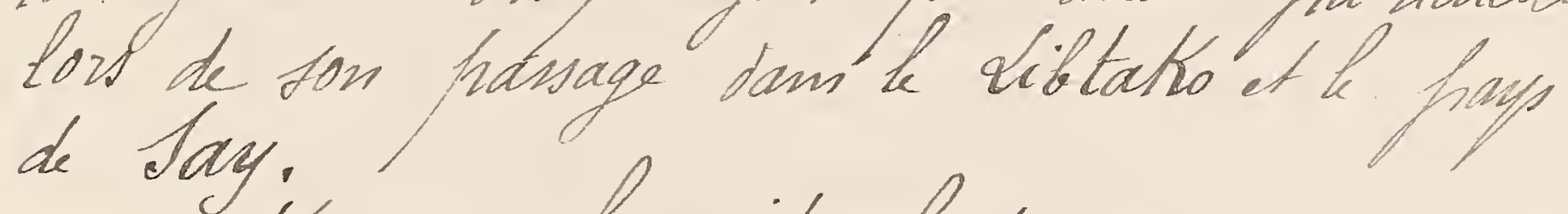

Trauss le visite de hremier "1886.87" et est aney, heneux on houvoin nemonter funqu'à Bandiagara, a qu Bingen tentena en Vain I'Amei Suirante.

If ent vrai de dine que si note. Compatriote ne pent domes suite $x$ for hrojet $\partial$ exploration en a qui concens be latenga ef le Macina lui sent Tevait noub faire Comaitu le Mlosti z'une fason frositeve.

des renseignement quili recueillet Lus be hays et La ropulation aideient huissamment a. la congueter et consevent encore toute. leur valeus; Chose Iemasquable, Li fin thest confter dy moyem hindes Iont il disforsa de la brieveté de Lon Lejour, et des incentitudes qui sendisent da itubstion fres hreiaine

Venant de Bobs-Dioulasso fras la soute de Boromo, Binger traversa une hastiv Ju Courounsi et hénetra an Mgsti har Beinemal non foin du gros marche parse de

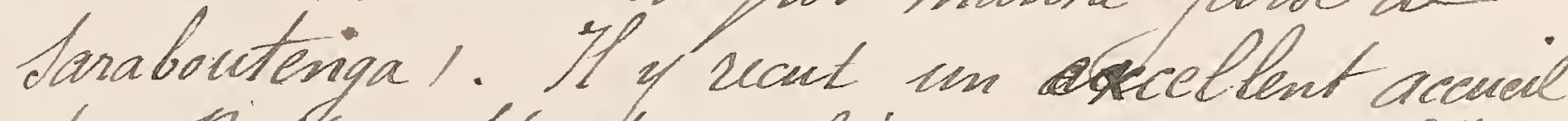

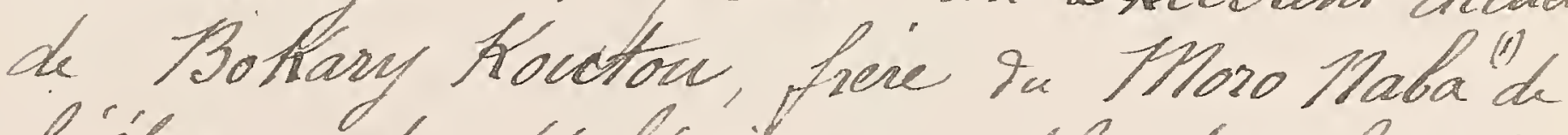

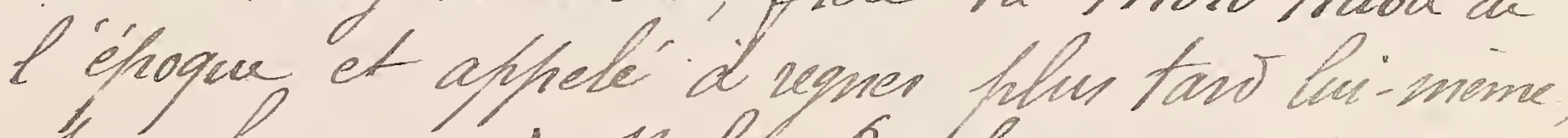
Lour le nom d Maba Oubbogo; maid

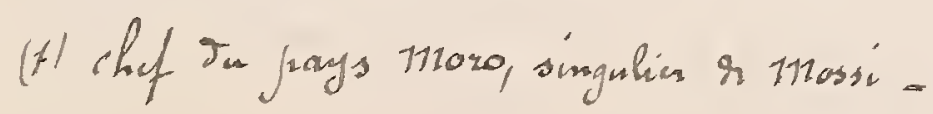



contrairement aus hussentiments sympathigues de In histe de Beniema les circonstances devoient fairede eet homme notu Aviersaine sisolu Ae 1896.

Arrive à Onagadougon II te 15 Yuin 1886, Binger oceufra an hameau or 1110 öme' (2) une case que L'on y montre encone. I'attitude de Maba Sanom, d'abord amicale, ne tarda has à changer. Brusquement, a la suite d'un entreters. oi Binger Lin avait manifesté fintention de fousmire da sonte vers le novo, il upuse de Le uavoin; bien fulus it va mème, quelguer foum atries jusqu'à hie notifier qrostierement et tam en fowmir os motf,

Dam las Conditions on il avait entrefries Lon

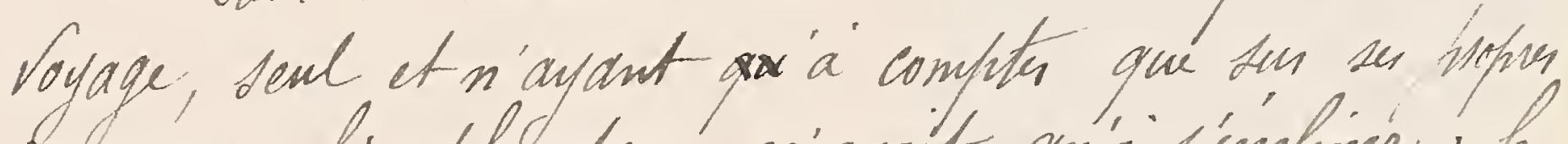
ressources, f'exploratem n'avait qu'a t'mchiner; b 10 ywillt an bois il quittait cuagadougon. a chemin on ratenga livi ctant formé, il gut revenis Lus ses has fusquà Bènéma d'oi il devait aagnes lyambatia et Salaga en Saversant \& Courounsi.

Illais a cont sejour hin avait duffi. malqui In Austricions et la Luweillana etrocte dout il étant lobjet - hour secueilles sur la capritale TH 112bosic et le fray tout enties nombu or frecieur unseignements, quitemoignent D'um hunficacite semarguable et constivent,

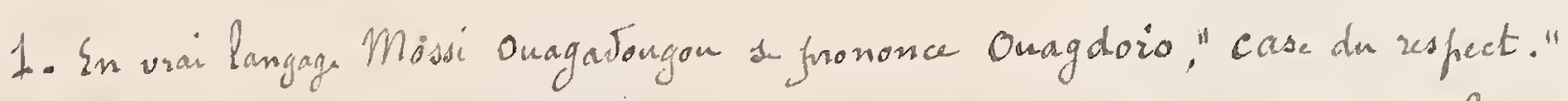

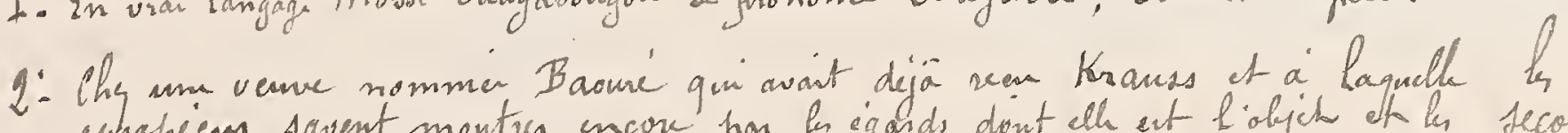

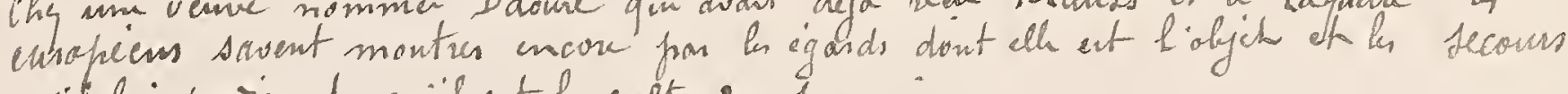

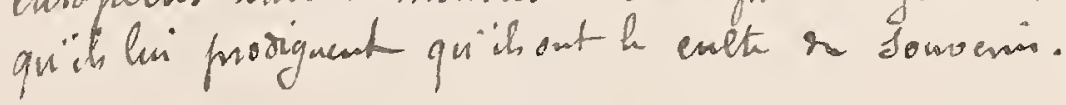


DSI 
hour cenx qu'un byour frolonge' an DNossi' a mis a mieme a'en contrótes l'exactitude, la base or toute socumentation.

de Mossi fut ensuite viscte' fas le docteus Crozat et le Commaniant monteil. Ce dermier y fiénétra has la route s IAnfiéra (hotite localite vacione \& Roury, on un frosth fut itabli tout D'abou) et de ratio; mal reen a Ouagabougon il jugea imprient or d'y anétes et remonta rens le nowt has la route se Boussouma et Ponsa 2 dui il devait hraveros Le Hiemmase' (pays de Fori) dija exploxien havtie has Basth.

Teu de temprs apris f'cilminostatems alby Vem In Oahomen à Ouagadougou He trouvait

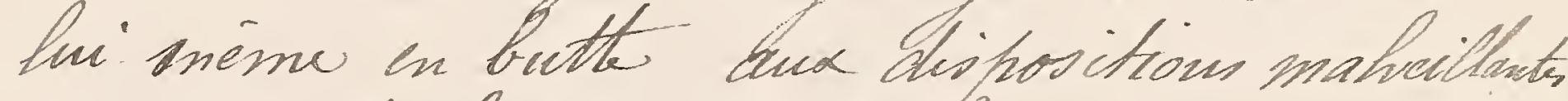
qui waient iloigne' Monteil at tentait vainement de conclue in traike aree be che sa rays.

bifin in 1899 \& Commantant festenave Venul du Macino enawait ì fon four a la

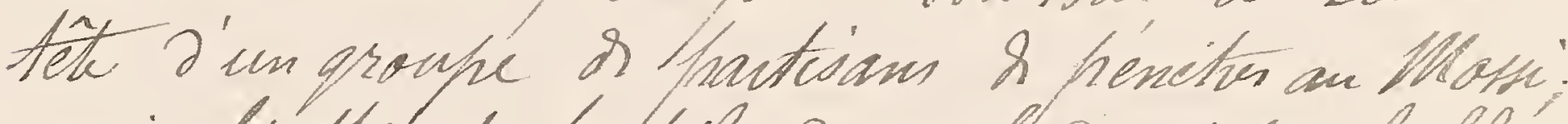
mais linttituch hostile Ju naba De Yatio, ssligea à utrogrades Sus Buahigoura.

Cehendant \& mulathe Forgusson parte de Gold. Coast était harrenu has des moyens Altowrmés a tignes aver naba Occobogo ot hous le comile on qowernement Anglais un traité 2 'alliancel et d'amific' 2 grillet 1894). C'est sen cet acte, dout la valiclie' ne hownat etre Domiae, ame óxpriveront flues tais les pretention britamigues et ghu naitront touts nos gifficults wee nos soisins, difficults quidure be temps une trobition forme of byale. 

dwait aplanis, of dout l'eie est anoun hui Definifivement close.

chais la Conquete approchait.

Le so yuillet 1896 une mission ayant is sa títe Le rieutenant Voulet de Linfantesin Coloniab et commenant en outu un bfficier de Cavalerie, Dens sous officien eurspiein et un fort contingent os tirailleuns et de cabalies auxilianes, quittait Bandiagara. Son objectif ctait of fiemprases du Massi et de devances les curfure a toenkodogo et a Sati.

OAmis avoir traverse sans ofpsosition tout le hatenga elle atteignait la prontieie nor du Mosis of se holtait Sus Samba, hetet villag. Situi a's kilomitur de Yatio, où la frisence tu chy or frovince, Maba Saga a' la tete D'un centain nombre de frastisains bui wait ete' fignalei.

Ce Dermiersétations favons vu, ofprose fanncé pricidente an hassage or la mision Destenave et, entarisi fan ce suecei, affichait la fritention d'anites fe colome. It duait its moins hemenx cette fois; rejete 'su Yatro dout nos troupes I'emparaient k même four

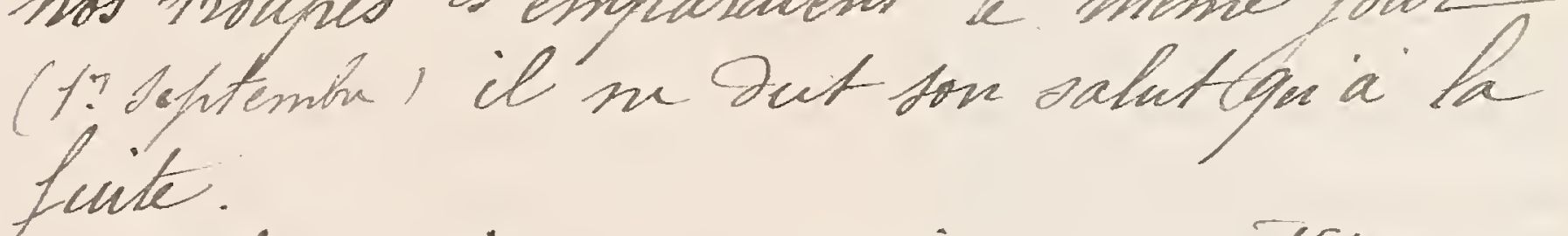

La mision se mettait ausitat en raut hour Onagadougou et tom che aoressait a' Bokary Koutou, qu avait Hueceide' a Haba Sanom Lou la nom \& Ouobrogo, des froprositions amieales area L'offu du nofectorat. Ces avanos n'aydnt fras ete' accueillies, la colome hätait da marche, 
os 
et la Cahitale gu Mossi Tombait entre nos mains \& S Septembe afris in court engagement. Beux joun jilus tais, cidant aur instigations de marchanos. Haoussas etabli' cam la ville, naba Gusbogo Devait enayer os la upremse, mais vioureusement repounc il se voysi oblige. 'Le f'enficir clam la direction tu Judent, tuivi des dibris s son amiec.

is limtenant Voulet quittait alous Ouagavougow houn Satie etty signait le 19. Septembie ave a chi' ttomaria, un Araite flacant Le Goursounsi Som be'. motectorat francain; il frevenait en même temps, de cette ifituation nowvelle Samong. Qoure' dont bs bandes oreiaient non toin de lai Celini-a uhondaif le is octobre quid. n'avait aucune. Arsetention tur be Gouchounsi, qu'il ne disinait has la queme ef, en consés quence, Iomait di son fils Laranké. Mory lis $\sqrt{x}$ ir se setiver Sus Santana Hegage d a colv, Vulet vient à OuagadouGou (1soctobre) hom y installes des dignitaies qu' avaient foit Iem souminion, fruis, afprenant que naba ouobogo est 'a' Bagaré,

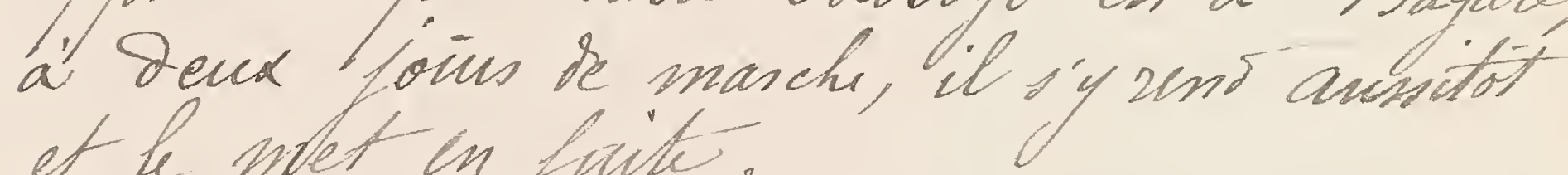
et le notet en foite.

se dingeant de la' Lus Mané dont ie Iempare (19octobure) il remonte vers be

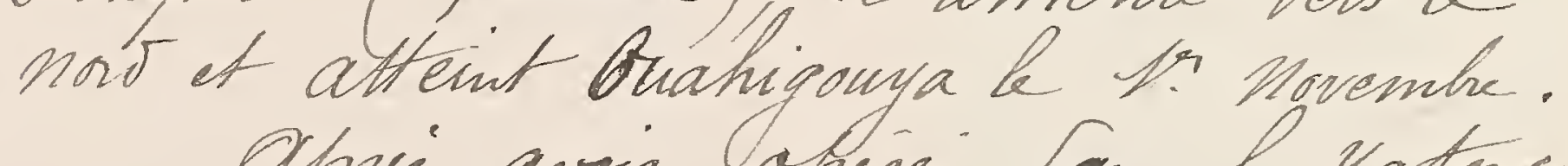
Opris avois ofrie Iom le yatonga et hine' en hays Lamo des combats mentituiess la mision, des quicle fient ithe seafpnovifiomei en vines eusofiem sevient at. 

Onagadougan Le 23 novembe.

La, on afprent que Naba Buobogo qui nu fient Le hiboudxe à s'elorignes os Lon ancieme Cafitale et essay oy entretenis on intelligences - est intalle' $a^{\prime}$ deux foumué or masche, vess le Jus an village os toanhisisgunis. Voulet be dinge aussitot In colte focalité $2 y$ dicembu, en chasse l'ancien naba et le frousinit Jusgui Sam le Bougsancé ' Iont b chef $a$ ' Kontaiga, recomait afficiellement ta Augerainete s la vrance.

Ia mision ievient a Quagadougow

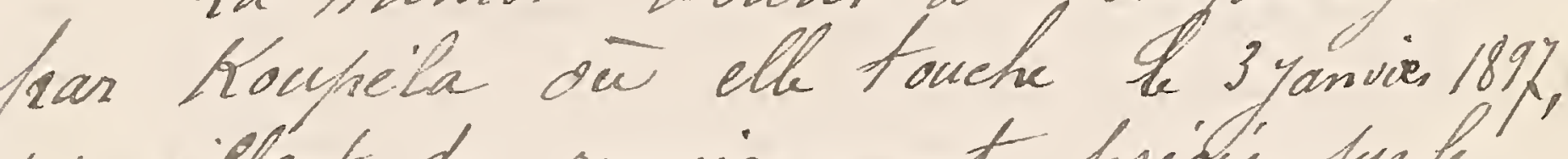
y ucueillant des unseignements fricis ter le voyag, go mulitu Fesquenon qui était encore

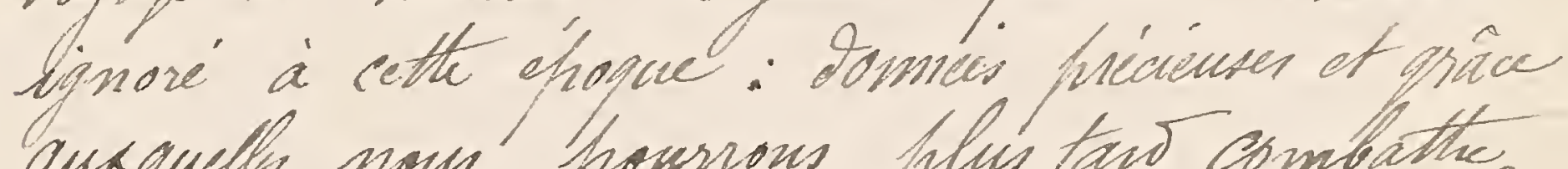
anxquells nous howrions flus tart combithe les fritentions de nos iviaux of contestes la validite ou hoento fraité \& 1899.

Revenu à Ouagabougon le 11 Yamien, Voulet y syourne à heine, il vient Dáfneme in effet que Haba Duoboge, tout il arout perivu la' trace tam "c. 'Bougsanci, at remonte' vens le now jungu'a Bousouma, qu'il y exsaic \&. se refaine un haste' st Ee Sobluwes contuo nouts la protinee 
DSI 




$45 \quad \begin{array}{ll}\text { MSS } \\ 16213 .\end{array}$

NMAH

en

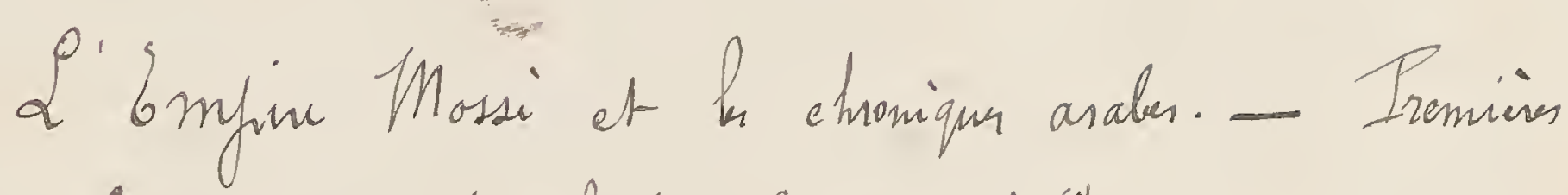
exploration. Chonologic de la Conquicte ${ }^{(t)}$

On certre de la boucle du Miger fresque à equale distance entre son froint culminant ds la Cote de l'Or, s'ctent sur une vaste sufperficie le pay. Massi airsi applede du nom de la race qui le perile.

Cette rave s'y est substituéc defuis filus de brois. conts ans, à des tho igènes sur l'identité des quals it a èté difficite jusqu'isi de se frononcer aver certitude.

On a voulu y voir les Habies, que la fronsséc des curaliisseurs aurait refetés vers be Mord, andela du Yatenga.

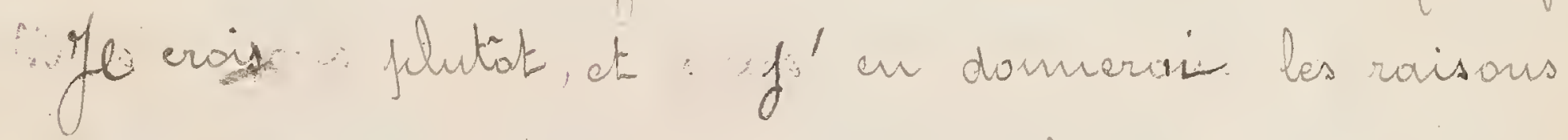
an cours de cette ítude, quie ces antachtones affearteradient aur mienne qraupre ethrique que les Bahos et les Mounoumas, qu'ils subsistent encare daurs le parys et y farmentles grauprements consitérables comnus sars le nounde

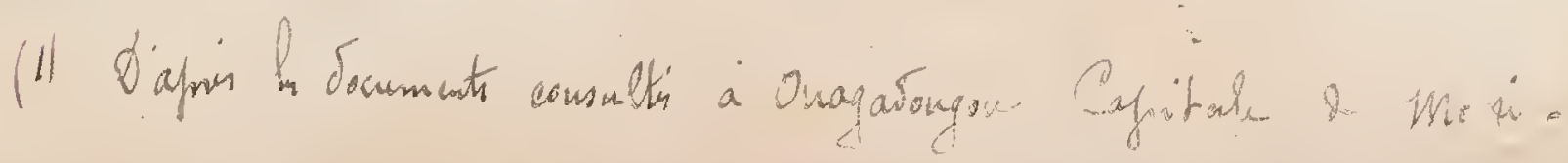


Gaurounsi, hiffirsi, Banssango ete.

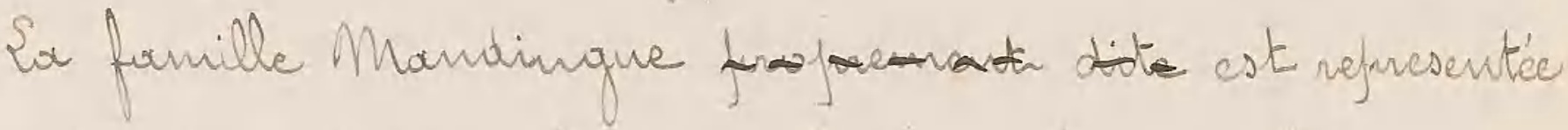
par les Yarsés on Onongarbies, qui, fises de lain en boin, en agglonierations vivantes ot actives, out longtemps dicterm le

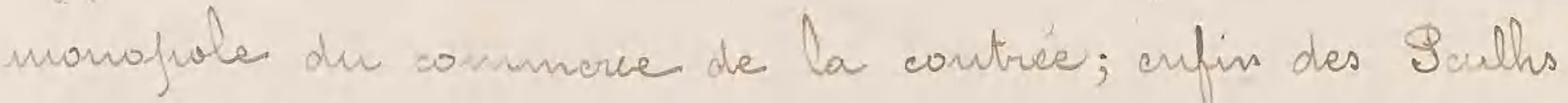
affertenant anx granfies Gorobis et Diallaubies, sont éga. lement disséminiés sur taute l'atenolue du tarritioisa.

Dir milien de cette masaïque de races, se détache

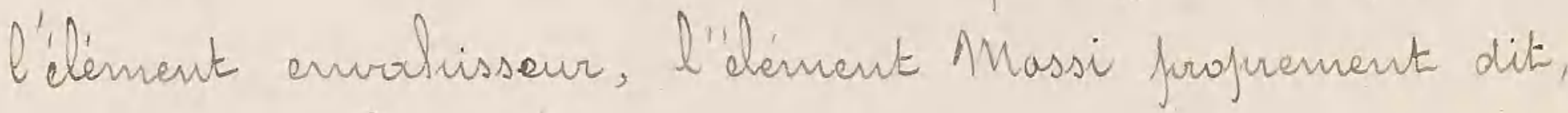
qui à hi seul présente des carracteris i ques assez intéresSantes four domor sa raison J'atre ar trourail que arous. dians entrepios.

Cette fospulation est an effet s'antount fhisirames 'tudier dous ses onigines, son dat actud, ses moeurs, comme dairs son organisation fualitique et sociale, qu' dle differe mettement à ces divers froints de vue des autres grandes agglamérations que la conquête du Soudan a mises sous notre autorité, et sur lesquelles a Jur s'esercer an cours de ces domières amnées, le labeur on sinfilement la curiasité dés cherchours et des chrogrif nes.

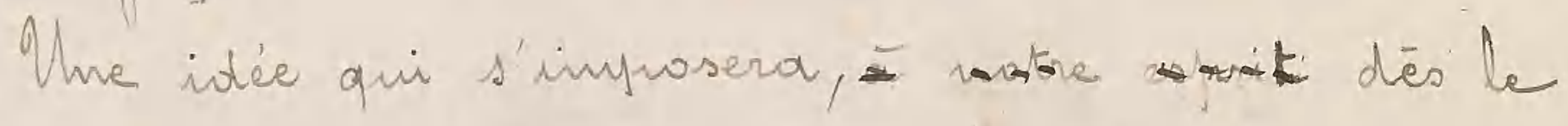
d'but de cette ditude, c'est que malgré l'dtomnante vi. talité que atleste ancare at qui a fout du pays qu' elle accupe le plus parflé du Soudan, malgré les solides qualités fhysigues dont elle ast davié, cettre 
race est entrée fren à feer, et se trauve anfourithi dans une fièriade de régression caractérisée, à laquelle l'influence de nos idées civilisatrices faciirra du moins infoser un arrêt, s'il maus ast interdit g'estiérer de

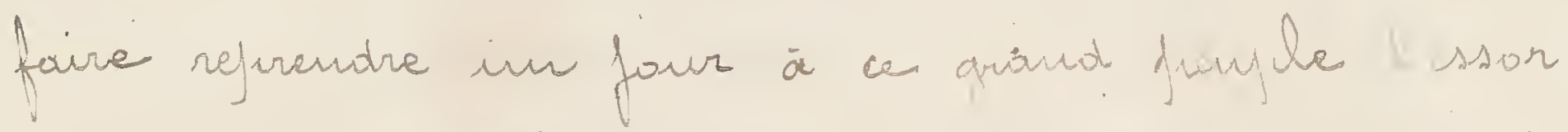
tout son lassé himaixine, et qu une imvasion désas. bicuse est venie brisen, vers be anilien du XVIE siäle.

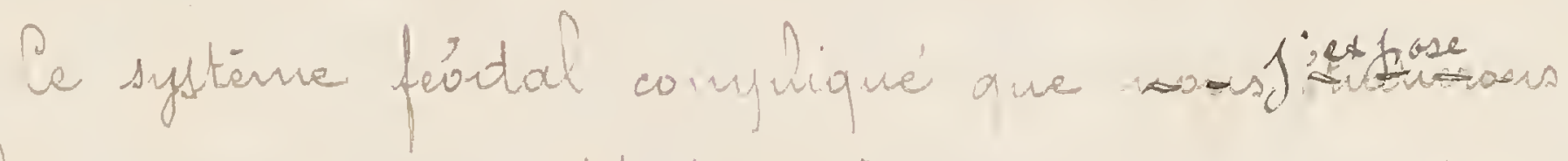
Jus loin et qui a hersisté Jusqu'à nus fours, na fur

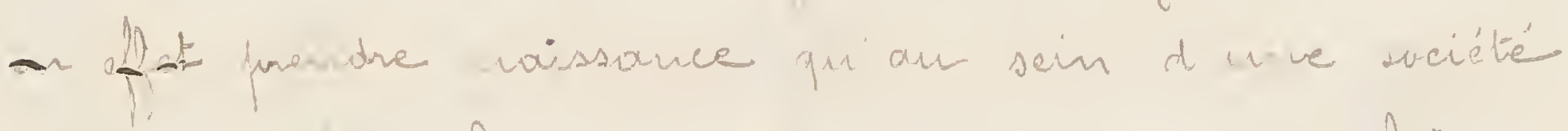
deia evancee; il cantrastie en tous cas singuliècunart har son asfect equilibre et fini, avce les nisirables

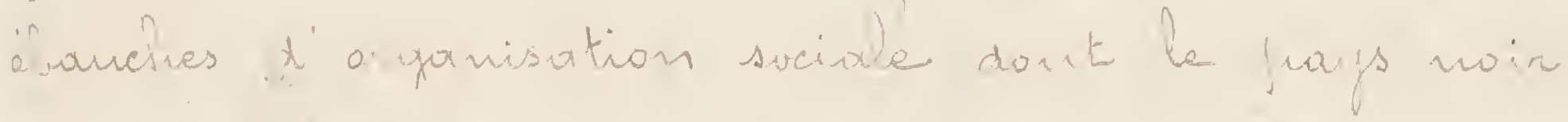
tow to nous downe la sicetacke.

Dautre fart les chroiniques arabes qui consti. mant jour taut te bawdons la senke traditions cerite dontlagele pat nous fuissiows kirur

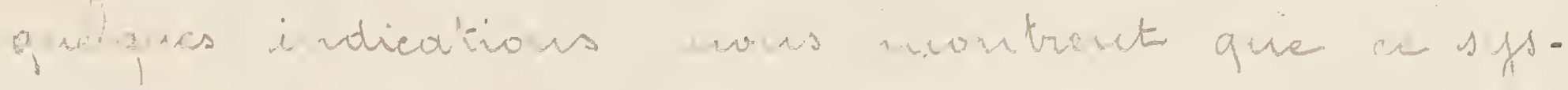

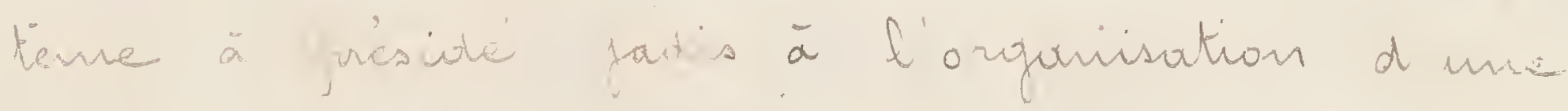
fuissance militaire nedantable.

Olmmed Baba et afrés hir abderphamon lién Aldallah nous afferemient que Gamboucton a été à une graque recubée qu'ils précisent

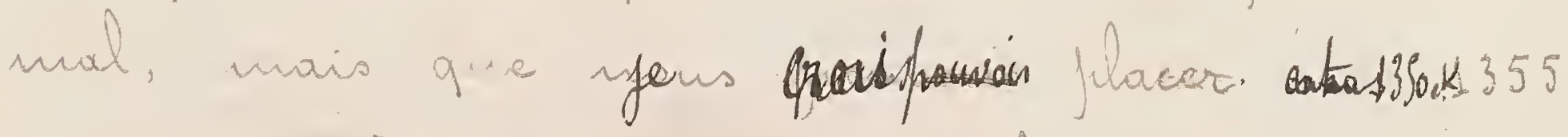
de motre ère, saccagée par le subtans dir ellassi. 
11

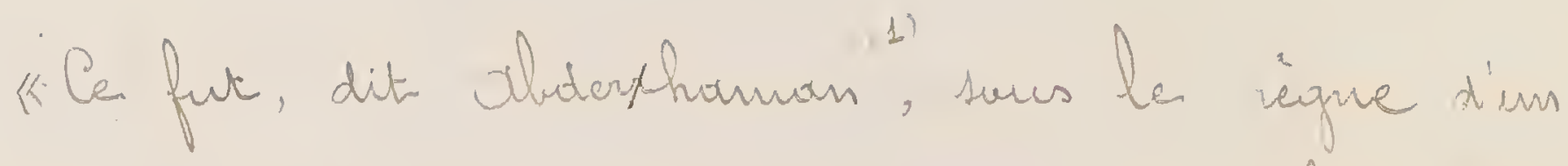

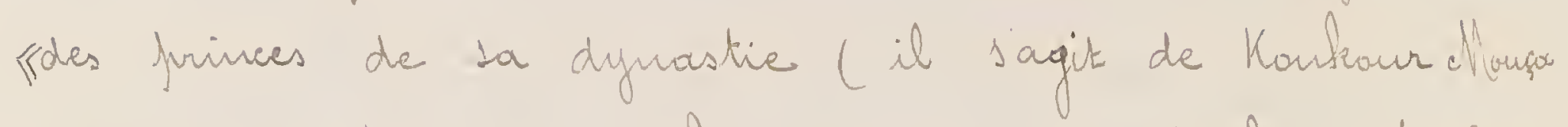

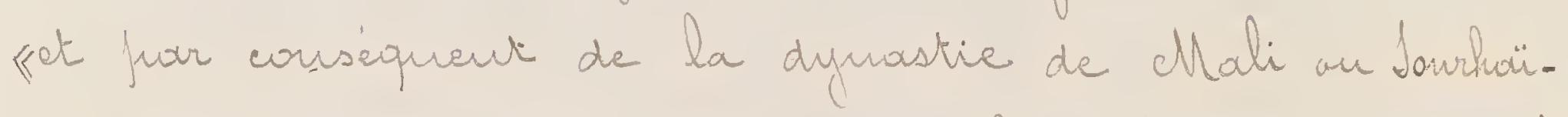
«cllandé jrafurement dite, gue le subtarn du cllossi «à la tete d'une forte arnice fit une exfiedition «coutre cette ville. Saisis d'effiri, les gens de cMelli 《livient ta fuite ct aboudamierent bornhoneton «ause assaillauts. Se suitran du Massi fiérietra alors

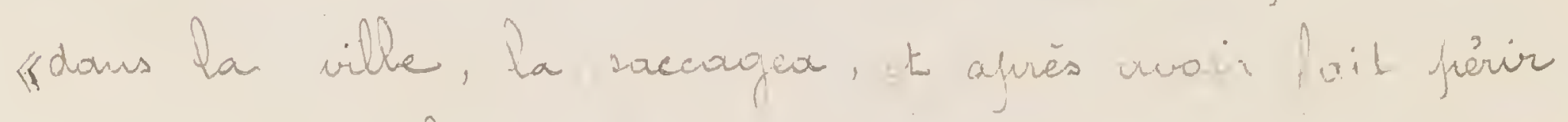
«tous cent qu'il funt atteindre dt s'âtre enfraré de toutes «les richesses qu'it traura, il retaurna dans son fayss. «Les gens de Melli rentrérent ensurite à lambouctour «et y demenrérent encore en maitres durount cent 《ans.77

« Vombancton, a dit le tries docte furisconsulte «Ahned Baba, a cté saccagé trois fais: la fremière fois « frar le subtan du Cllassi, la derxiène frar borni. Ali « et la traisierme far le fracha ellatmond.ben_Aergour. "Cotte demieire devastation fut movirs terrible que les kdeux fremières, dours la mise à sac de Souni.

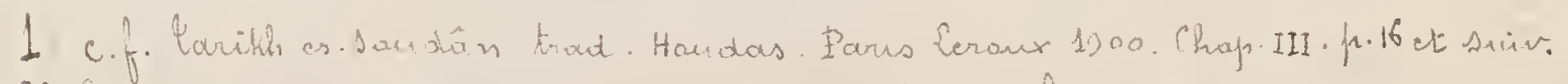

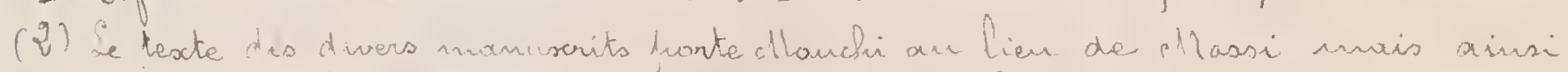

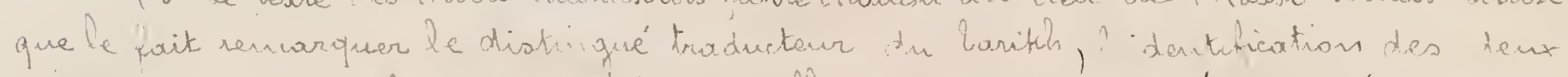

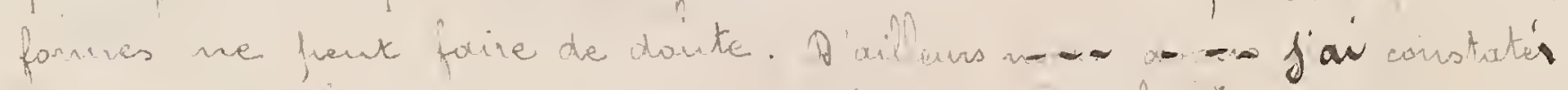

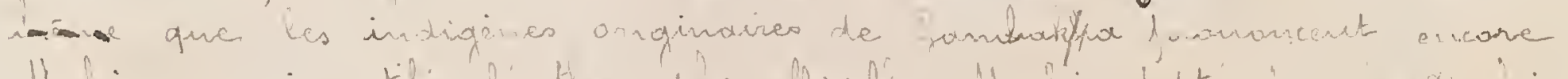

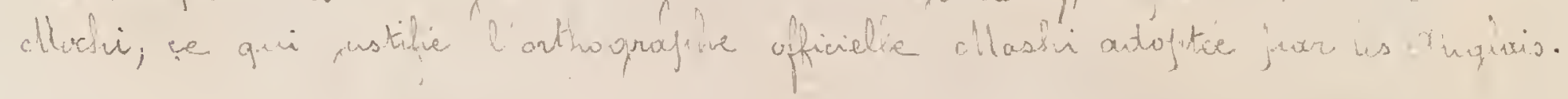


cli il y aut le plus te sang vefromdur. \$ Sarteur du Carikís as boudón mentione égabement. tan go3 de ( tiagize, süs 1497-1498) une exfieditions entre.

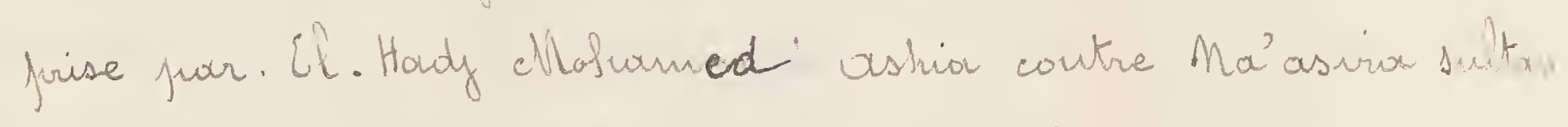
du Massi. (2). Enfin en 969 (1561-62) Ostion Dawnd dirige une nouvelle exfictition contre ce frougs que son chef est corr. trotint d'aloundomer avec tantes ses traupes.

¿'cxamen attentif de ces textes, leur rayyerobemant entre eur, la trodition arale que - jos diancencillie ici at dours le Afatenga, out serir

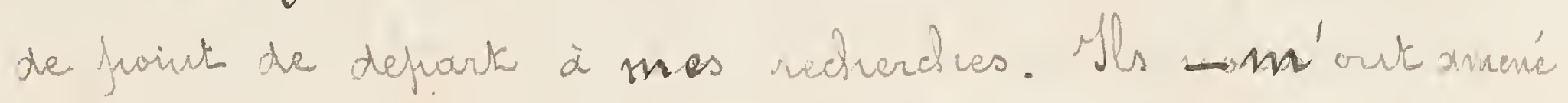
à une sirie de conchsions qui fremedtent de concilion désarmais des temoignages cérits ct orour dont les di-

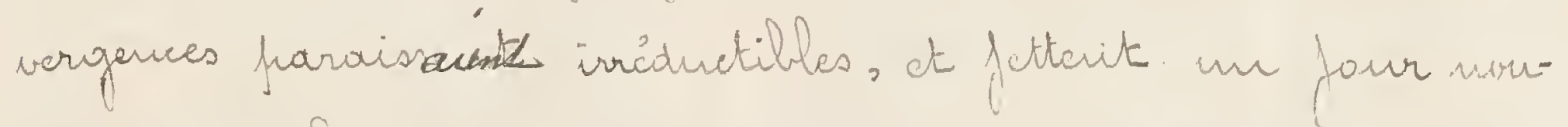
vear sur la question des arigines chassias

Ce foirt ert mis fourticulièranent èr ciridence, que

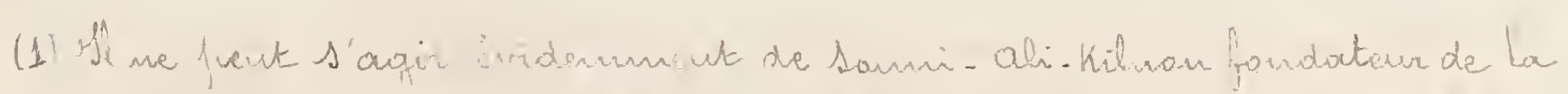

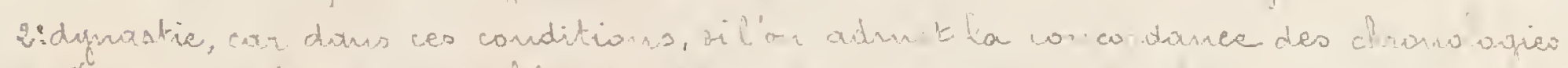

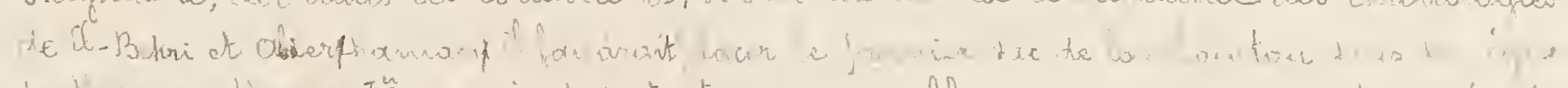

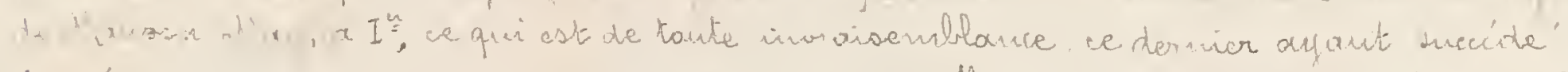

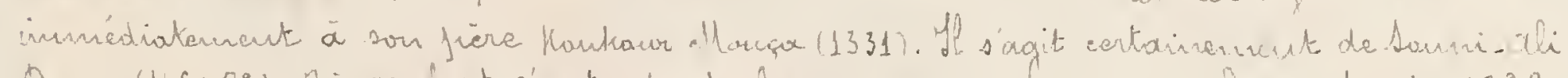

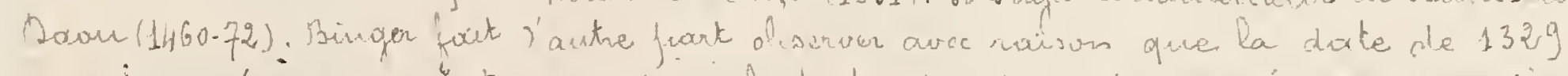

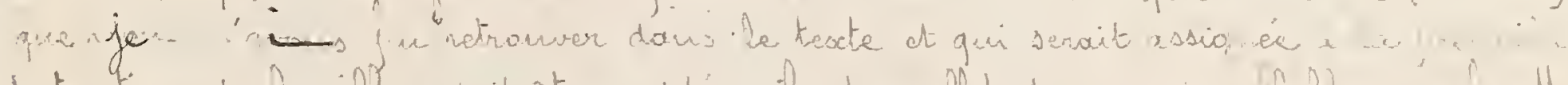

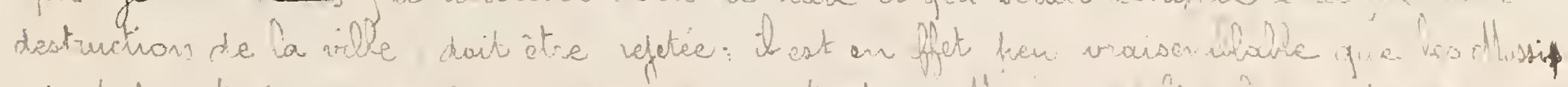

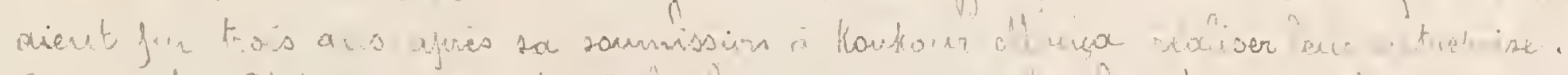

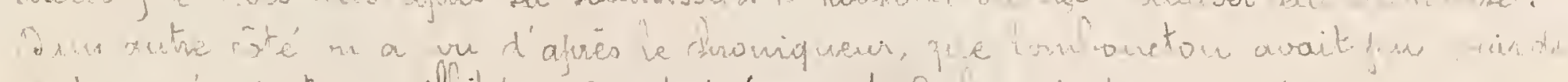

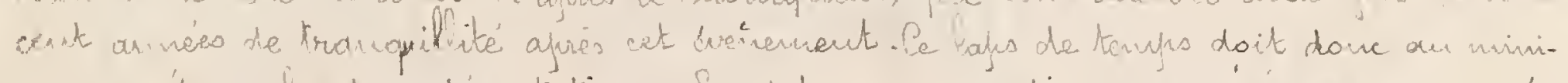

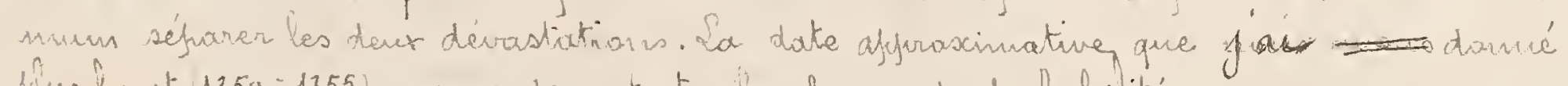

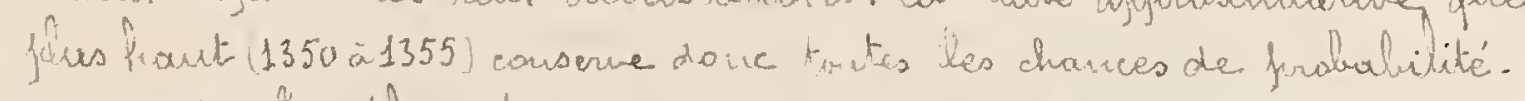

(2) Garish es. Jaudian ed-cit. n. $121-122$.

(3) Govilh es-tiandion ed-it. h. Ifl. 
l'anfire ellassi s air fuartit lo grande esefiedition sur bonbouctor stoit situré andelai de la rive gauche du Miger et y vecufrait l'amplacencuti du Bornour actuel. Il est niêne probable que sa limite accidertale le défrassait et englobait avec Kano, une fortion du Sokoto.

Casta done lá le frays contre le quel astia Savand dirige l'exféditions de 1561 et que cson chef art contraint d'abandarmar arce torites ses tranfes. Cette fuite devant l'ourabisseur, c'est le grand csude qui devait amener daus la

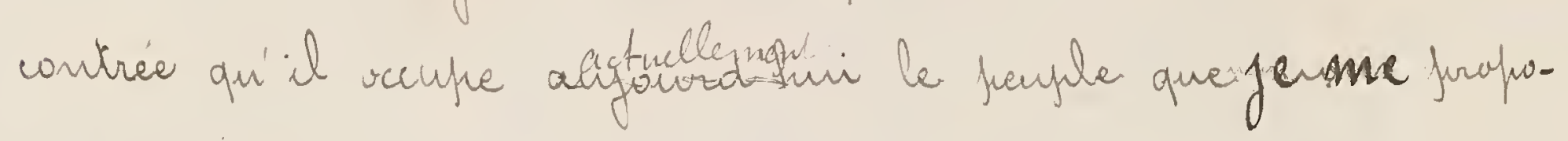
sec d'itudier.

Il avait déé frécédé d'emigrations de muindre infurtance. -

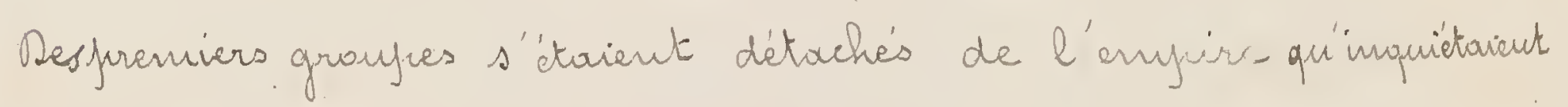
défó les ineurisions de voisins kurbulentis.

Eis granfres avaieut suivi la raute fuar laquelle s'acconr.

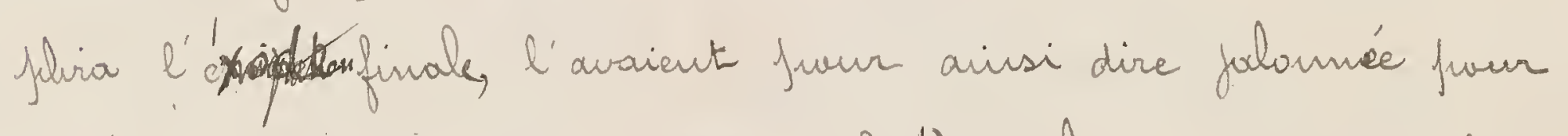
arriver an Sud du Massi actuel "(b) de la "torre furomise" in leur race itablira nou sans frine sa friédaminance sur les frenflades barbares qui l'vecufraient.

Mais cest bien à cette equque, air a la fiérivole qui l'a frécédé inmédiatencut que l'ou doit foire remonter

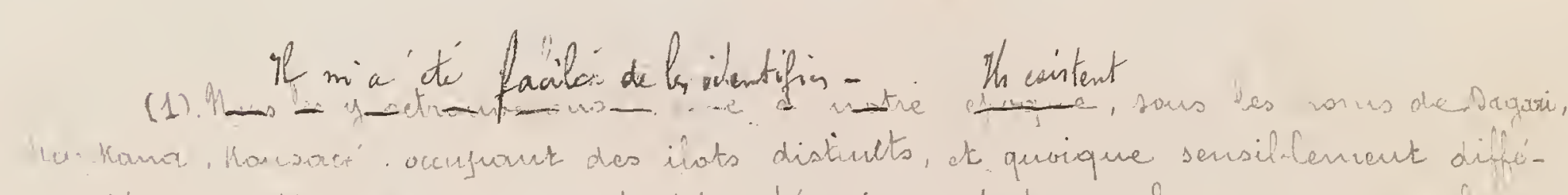

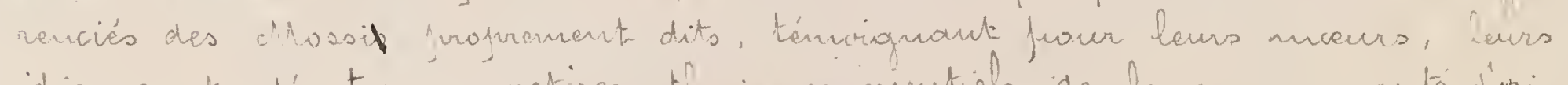

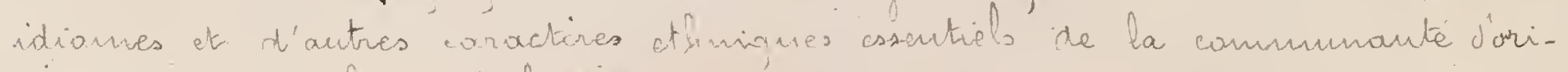
ine que ugaes feur attributios.
} 


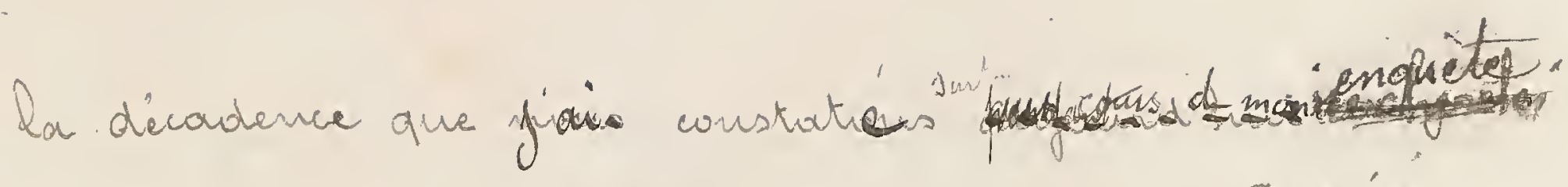
Guisé fuar sa butte avee l'crmahisseur, ancomie juar celles qu'it dut soutenir contre les tribus dout il fallait traverser le territaire de qui his harraient la warte d'un êté, - de l'autre coutre les autohtiones que dans undernier effort is devait refouler, irrémiciablencent fraffié dars ses forees ives, le jeuple Massi n'a fur trouver our sein de hir-mierre les ressaurces nécessaires four réagir et son loug diclin a commencé.

Sa mit s'ar faite autour de son man (1) at les chroniqueurs ne mationnent mène folus son cseistence. Il ast tout à ce travail de réparation intérieure dout il aura l'esfrair de verrir à hout.

In silence l'enfire classi se recanstitue; larternent il trausforme tarte cette contriee, la couvre de illoges, de cultures. On erairait à une résurrcetion. Alois cette vie nouvelle n'a jhus dars ses nomifostations l'élalí de jadis. Gart y est matériel ct terre. a. toare. On dirait que le souffle fuissant qui aurait sortrdais animé ce freufle et

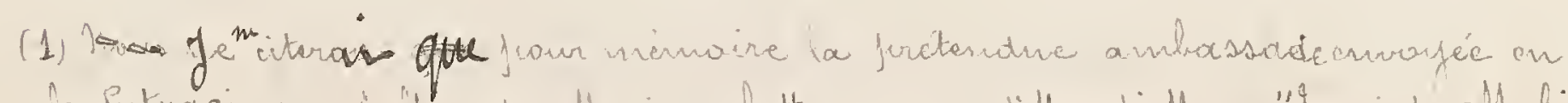

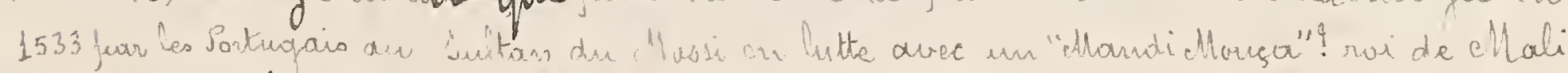

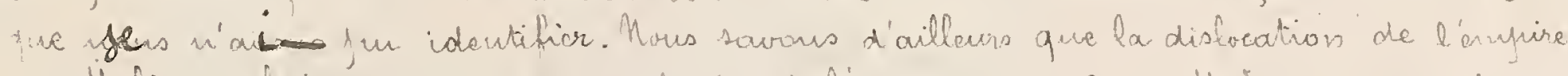

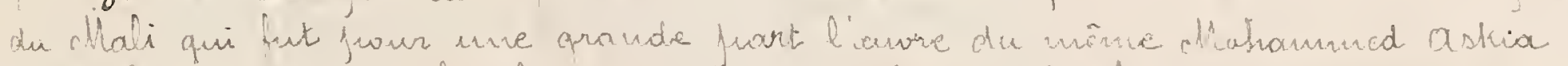

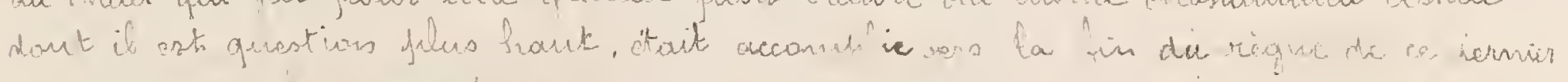

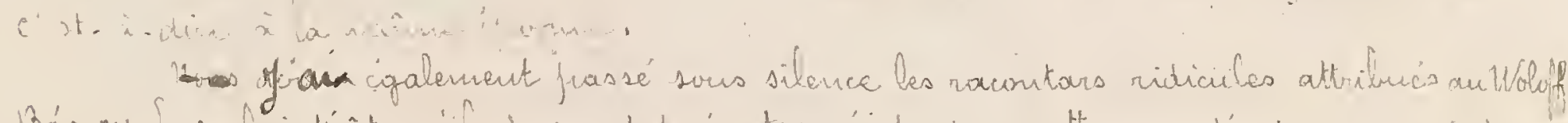

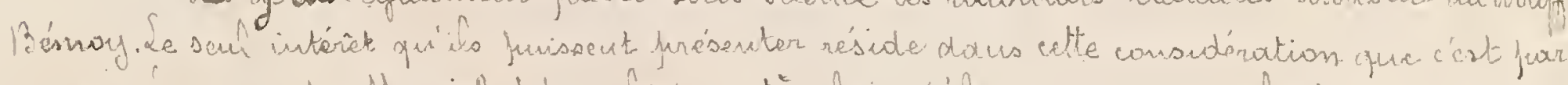

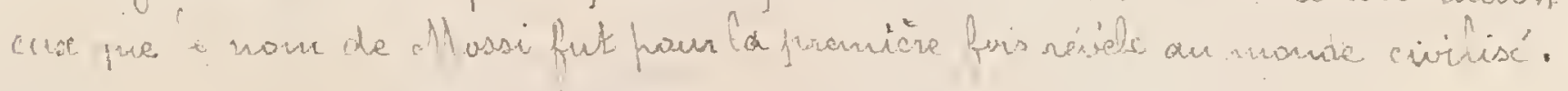


le faisait grount ot sdoutable s'est dtaine à foumais; il a cessé de crèer, il inite, I attuchurt étroiterment a wre tra. ditions à Caquelle il n'apoutera flus rien, et dout if a jerodu de sentiment

Celui qui préside à ses destiviées afyroutient encore à. la ligpeée des anciers suittons que l'auteir du Earikh wous a forits commaitre, mais ce grotesque Mabal Lanom forressoux dt díbarefré qui recevor - fort mal d'ailleurs Binger en 1888, n'a jilus rien de commun avec le granio

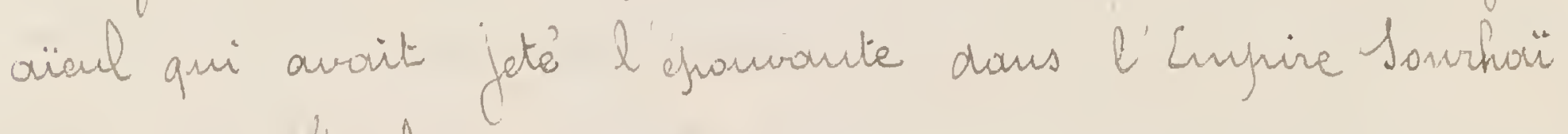
et conquis bomboncton.

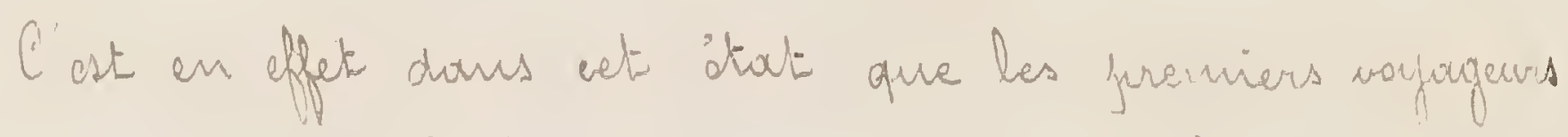

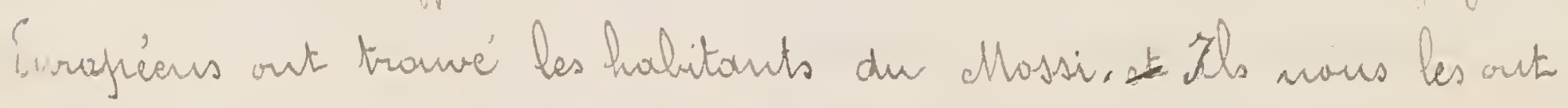

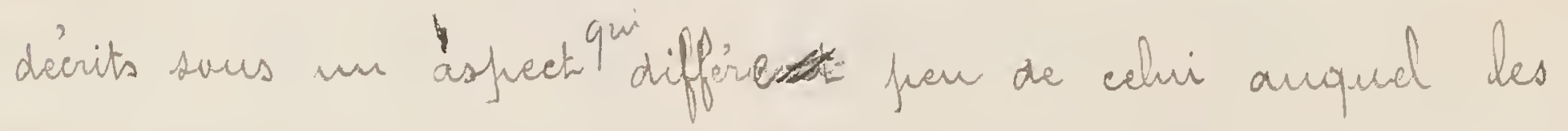
autres nairs nous out accoutinnés.

Barth avait dejoi domé sur leur frougs des venseigne. mants assey vaques qu'it avait fun recueillir lass de son fras. sage dours le Liltako dt La région de Jay.

Krouss le visite le fremier $(1886-87)$ et ort assez heuracr de faurvair remonter Jusqu à Biandiagara, es que Binger tontara on vain l'annece swivante.

Il est vari de dire que si notre comfratriate ne feut domer suite à son profet d'explaration on ce qui concerne le Gatenga et le Macina, hi seul devait nous faire cannai. tre le Massi d'une fason fusitive.

Les renseignements qu'it recueillite sur le proys at so 10. chit 


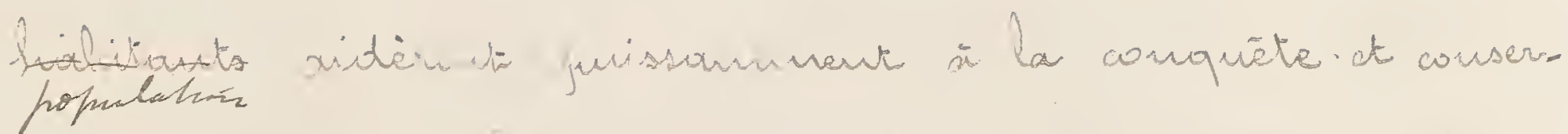

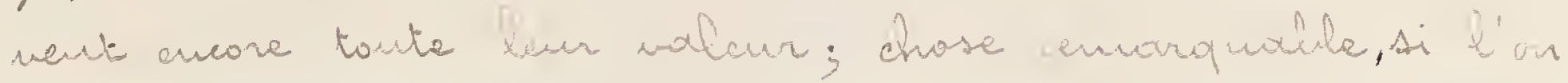

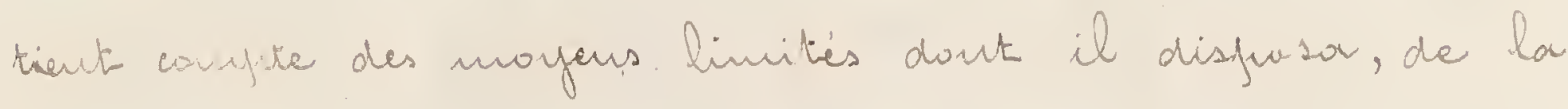

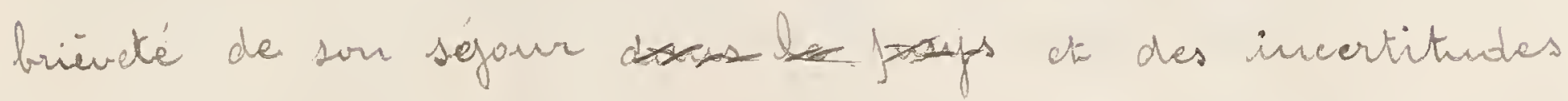
qui ty ventireat sa situation trés farecaire.

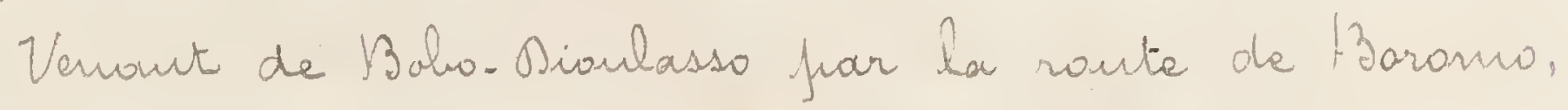
Binger traversa une partie du Garraunsi és finietro an

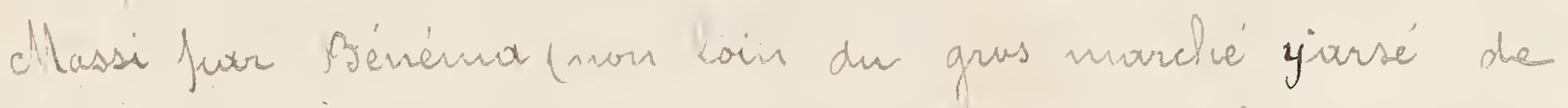
Laraboutenga). Il y recur un excellent accueil de Bothary Houbou,

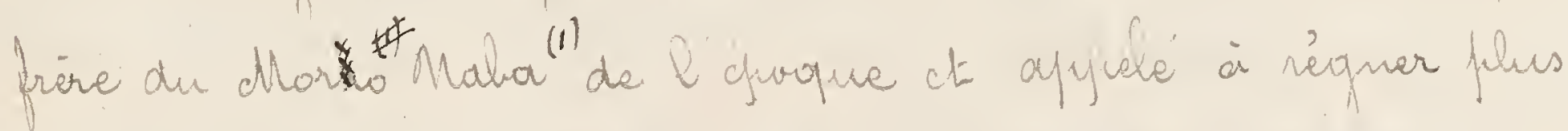

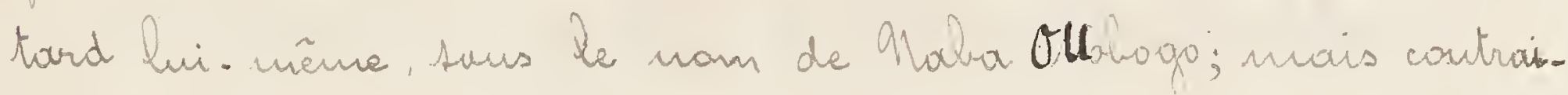
rement anse fressentimants sifnfrathiques de sors fiote de Binina les circonstances devarient faire de cet ho nno notre adversaive résolu de 1896.

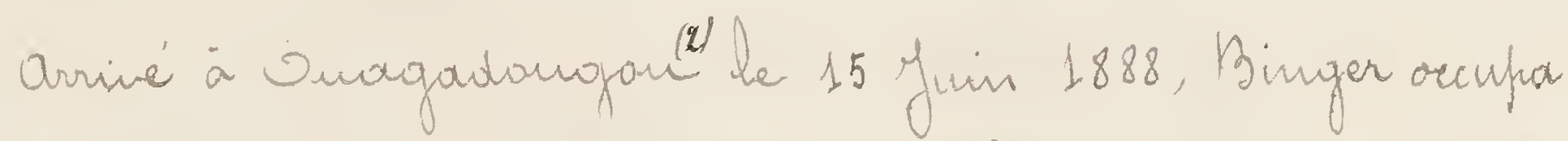

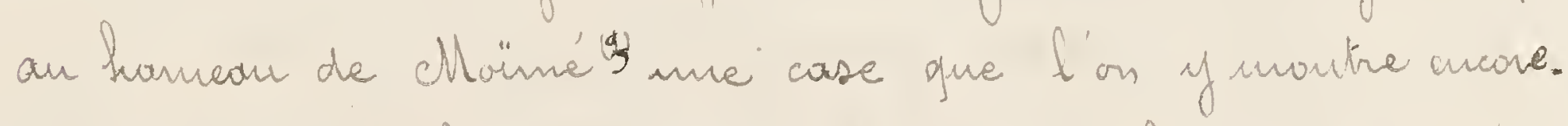
S'attitude de Malua Lanom, 1 abard anicale, me tarida fas

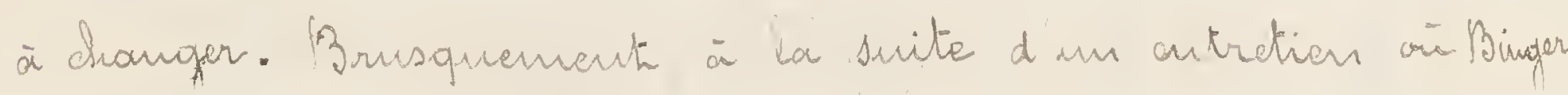
mi avait imanifesté lintention de paursuime sa rarke vers te Mord, il vefuse de le recevair; lien flus, il wa mânas,

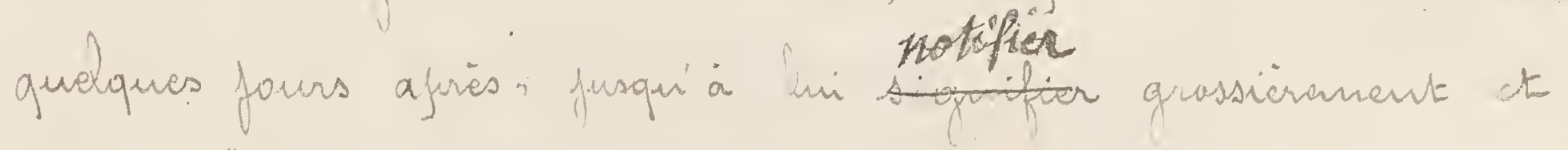
soms en foumir de wastif, londre de fartir.

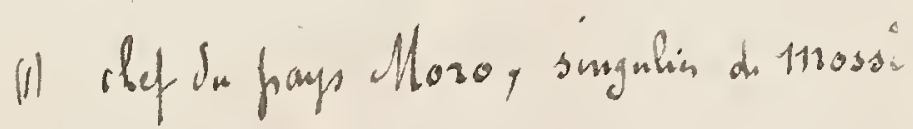

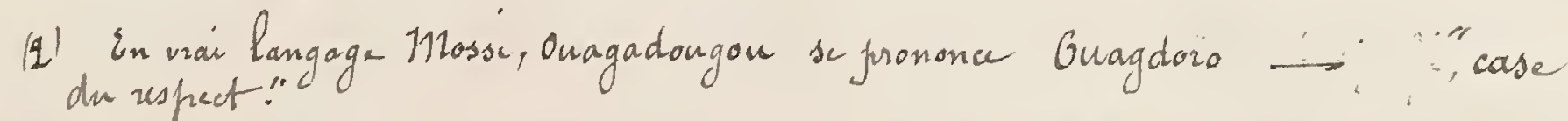

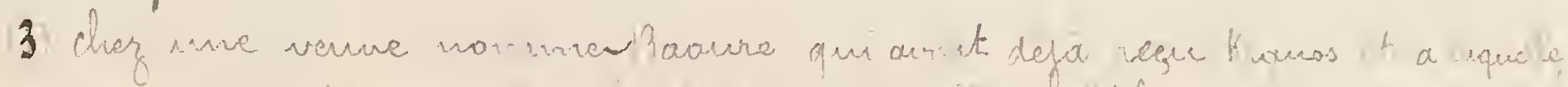

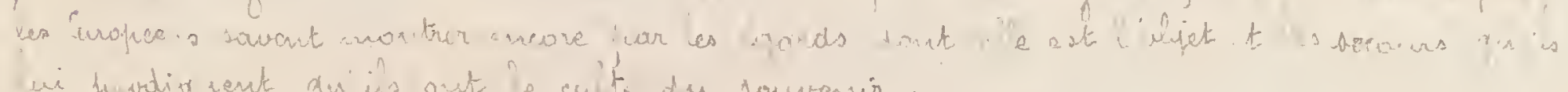

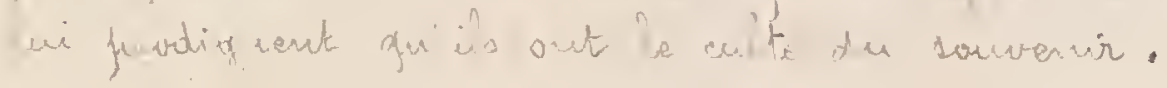


p

Sows kes conditious in il arait entrejris ian

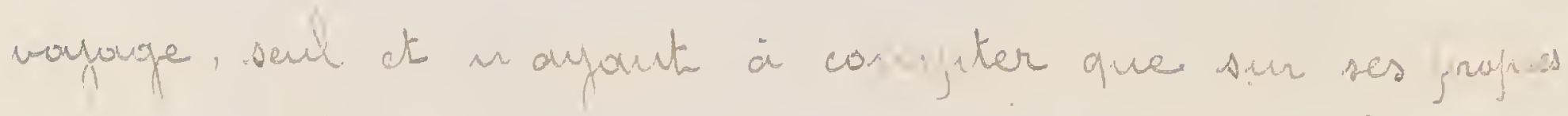
ressanros, l'explorateur n'avoit qu'à s'incliner; le 10 fuillet an sair il quittait Onagadarigan.

¿e chemin du Gaterga lie étant formé, it dut vevenir sur ses fras fusqu'à Bériéna d'air il devait gaguer Canrbakla de talaga en traversant le Gauraunsi.

Alais ce caurt séjour hii aurait suffi-nualgré les susficions et la surveillance itraite dant il etrus l'alyet four recueillir sur la cafitale du chassi ct le fuays tant entier nombre de jerécieut renseigucments, qui

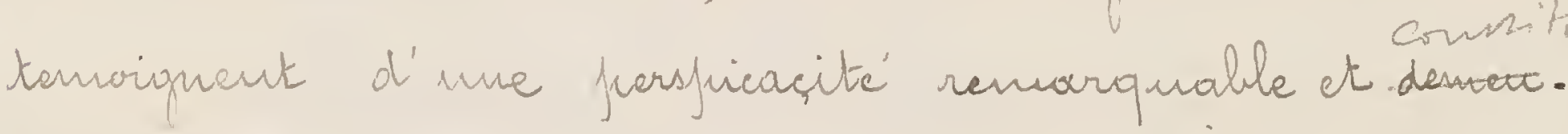

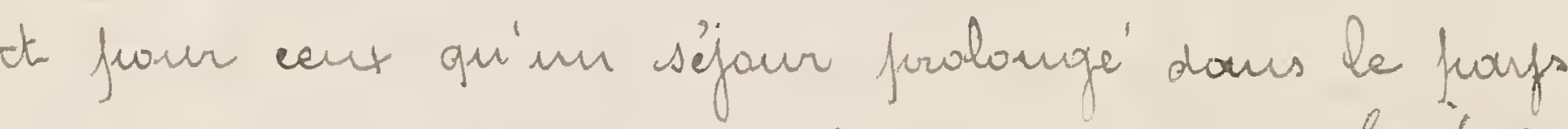
à mis à mêrue d'en coutróler l'exactitude, la bere er

Le Massi fut ensuite visité frar le docteur Grozat

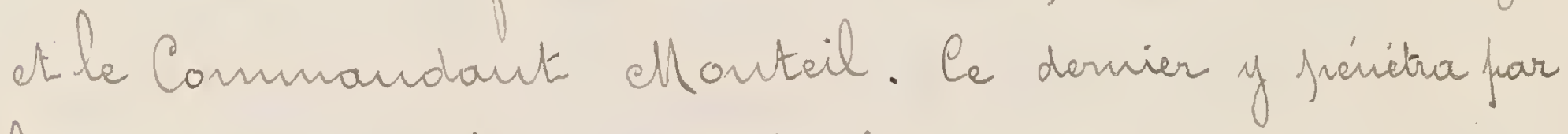
La raute de Saufiera (fretite localite voisine de Kaury, on un frostie fut itabli tant d'abord) de de Gath; nual rees à Onagadongour il fugea inforndent de s'y arrêter et romonta vers le mard fuar la rante de Baussauna et Ponsa, d'air il devait troverser le Djémmaré (frays de Dari) déja explaré en fuartie fuar Barth.

Pen de temps après l'administrateur alby. vem

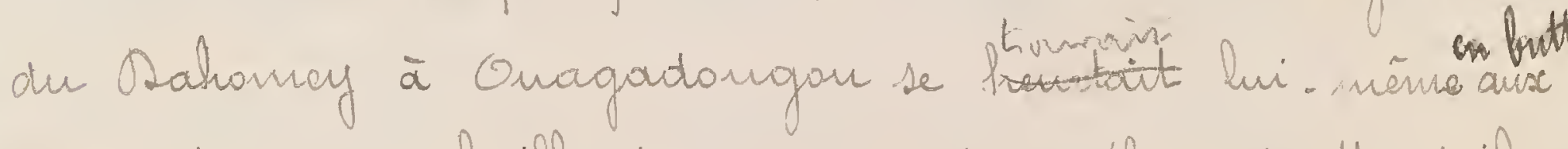
disfiasitions naheillantes qui avaient éloigné alloutril

DSI 
ot tantoit vainenent de conchure un traite aver be Dief du foors.

infin en 1895 le Commandant Destenave venuds Alacina, essougait à sou tour, à la tête d'un granpe

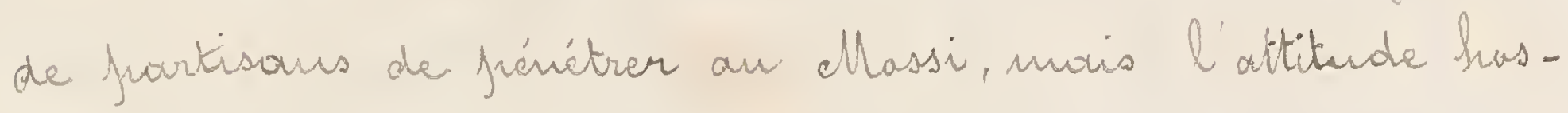
tile du Mala de Yako l'abligea à iétragrouder sur Onabir. gourfa.

Ceprendant le mulatre Ferqusson prarti de Gyold.Carati étoit frarvenu four des mayers detaumés à figner avec

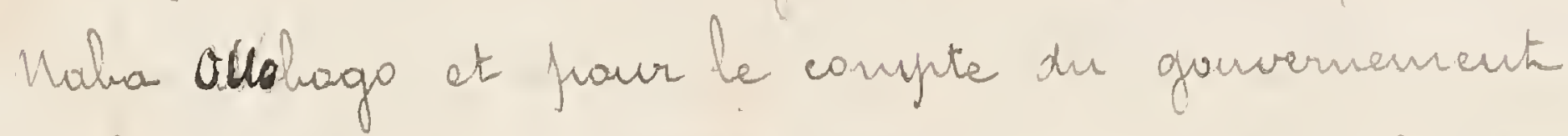

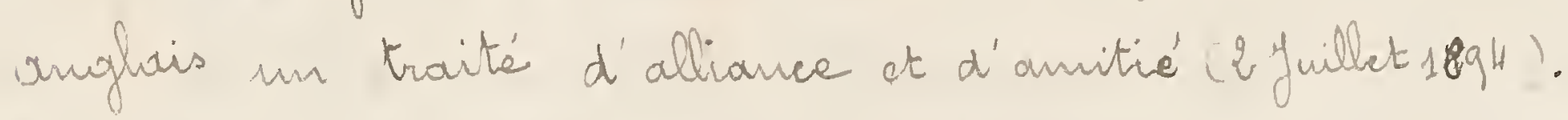
"'est sur cet acte dout la walidité we fourait être

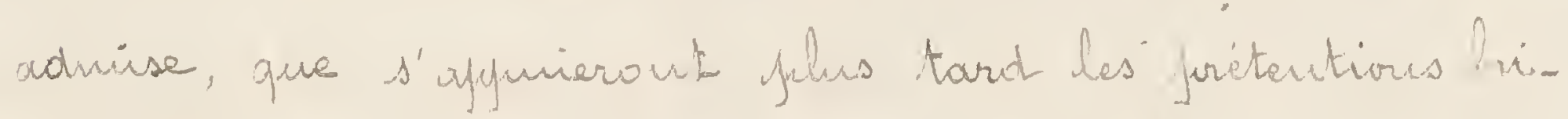
taniques et que naitrout tontes nos difficultés aver mos waisins, difficultés qu'avee le tenys une jublitique ferme de lafale devirt aplanir, ot dout l'ire est aufour. d'Pui défiritivement clase.

Mais la conquète approchait.

Se 30 ofuillet 1896 une mission ayourt à sa tête le Sientenont Voubt de l'Infanterie Coloniale at comprenont en autre un afficier de covalerie, 2 sous-officiers curafiears dt un fort cantingent de tirailleurs at de cowaliers auxiliaires. quittait. Bandiagara. Son abjectif eltait de s'mafarer du Massi at de devoncer les omglais à Gentwodaga" et à Lati." Oyeres avair traversé sous affiasition tout le Yartenga elle atteignait la frontière Mard du Massi et se fortait

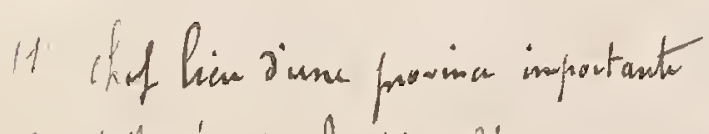

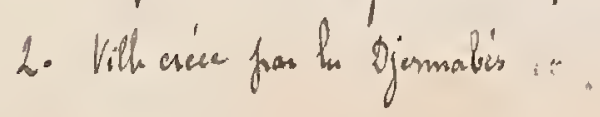


sur samba, fuetit village situé à 8 kilamietres de Gatko, oir la frésence du chef de frovince, Mraba Souga, a la tête d'un certain nombre de frartisaurs hii avoit 'étédi. gualée.

Ce dermier s'était, nous l'avous ver, affusé l'anices fréécidente an frassage de la mission Destenane et, enhar. di fuar ce succis, affichait la frietention d'arrêter la colonne. Il devait être mairs heureux celte fois strifeté

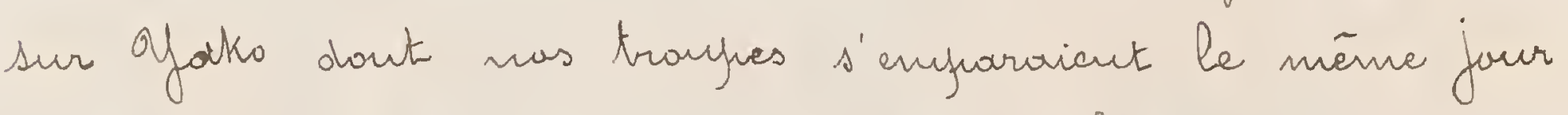
(1-Sgutembre) il ne dut son saluter qu'à la frite.

Sor missions se methoit aussitiot en route frour Onagadongou et sow chef adressait à Bathary Koutan. qui avait sucéédé à Maba Samom surs le nom de Otohago, des paropusitions annicales avec l'affer du frotactorat. Ces avomces n'afout fas íté accucillies, la colame hiätait samarche, ot la cafitale du Massi tombait entre mas mains le 5 sefutembre aferés nn court engorgenent. Deur fours flus tard, cédont aux instigations de marehounds Haoussas ita. blis daus la ville. Maba Mrabago devait assayer de la nefren. dre, mais rrgaurensennent reforssé it se vayait obligé de s'enfuir dous la dircetion du Sud. Ist, suivi des débris de sen armués.

Se Sientenant Voulet quittait alors Onagondanguer furur sati et y signait le 19 Sgptembre ave le chef Hamaria mn. Araité plasaut la Gaurounsi" suus le protectorat from. cais; il frivenait en meine temps, de cethe situation

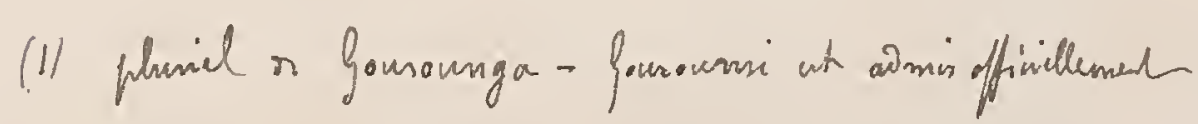


nanvelle Sanany Gauré dout les houndes afiéraient non loin de là. Celui- ci réfurndait le \& Octobre qu'il n'aurit ancure frítantions sur le Gaurounsi, gril ne désirait fras la gueire et, en conséquence, domait à son fils Saranké-. Mory l'ordre de se vetiren sur Lankiana. Déojagè de ce êté, Vaulet vient à Ouagadougun (150etobre) fueur y installer des dignitaires qui anoient fait lour sur. arission, fuis, affurenaut que Alaba Otoblogo ist à Baygaré, a deux fuurs de manche, it s'y send oussitît et le net an fuite.

Se dirigeanit de lá sur Mané dout il s'enfiare (19 Gotobre); it remante vers le Mond at attenit Guahiganja le 1" Noventbre.

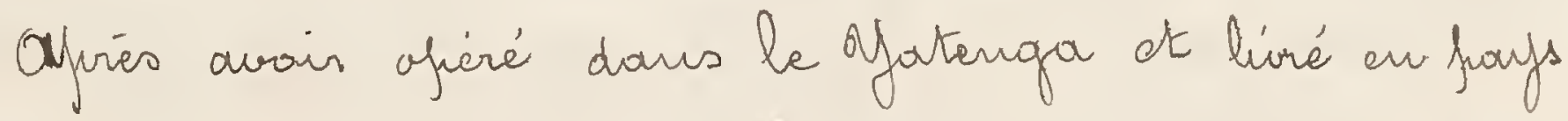
Samo des conbats meurtriers la mission, dis qu'elle peut

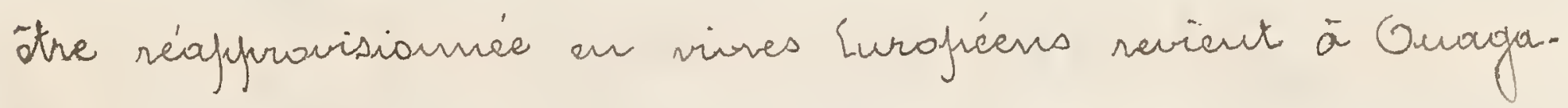
dougan (23 Décembre).

Sai, on apprend que Maba bilobogo-qui ne prent se résondre à s'eloiguer de son ancienne cafritalet essouge d'y entreternir des intelligences,- ost nistallé à deux jaurnées de marche vers le Sud au village des Kambisiguiri. Voulet se dirige aussitát sur cette localité (2f Décembre) en chasse l'ancien naba et le forusuit fusque dans le Bans. sangeé dout le chef à Koratago, recomait afficiellenent la suzeraineté de la France.

Las mission revieut à Onagadangou far Kaypiela our 
elle turche le 3 Jomier 1897, y recueillant des venseignements frécis sur le varfage du mulaitre Ferqusson qui était encore ignoré à cette éfooque: domnés frícicuses ct gräcer anaquelles nous fuarrous phlus tourd combuttre des fretentions de nos rivouct et contester la validité du feseu. do- traité de 1895 .

Reverm à Onargadongour le 11 fouvier, Vaulet y sé. jaurnal à peine; il viaut d'afferendre en effet que Maba Mblaga dout il aurait perctu la krace dans le Baupsangée ast remonté vers le Mord jusqu'a Izaussomma, qu'il y essaie de se refaire un parti st de soulever coutrenuss. la frovince.

Il faut en finir ourec ce saverain fantione qui décidément n'accefetera jommis le foit accompeli, et la froursuite reconmonce....

Mais Mrobago est désarmais seul à avoir des vellei. lés de risistance 1); l'un afres l'autre ses flus fidieles faor. tisaus sout ameriés a faire laur sammission; l'activité incessante dejelorsée fiar Vaulet, ses sucics rapides out feen à fren raison des plus obstiviés.

Our cours de cette ficinode, Margy ot Mahmuadou, ferofures freires de Boktary, deux de ses fils; tridi, Mavé et

(1) Cothe attitude ne founait âtre ciritannment dietée que frar l'asfair de voir fraraitre d'un monent à l'autre les anglais dout il avait sollicité le secours. 
Baussauma - Malha, d'autres encore, fout leur saumission.

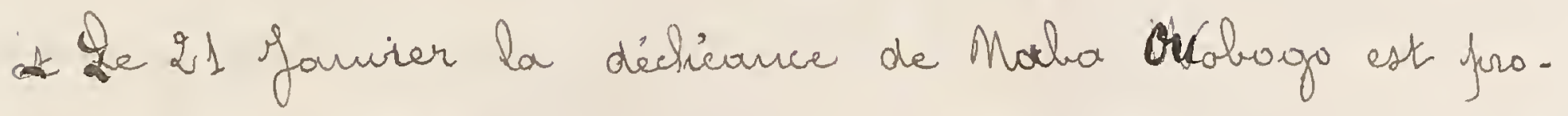
clanié devant la forle assenibie.

Huit faurs aprés las grands dignitaires désignent son fière Mlahmodoun (1) puur le remylacer et ce dermier

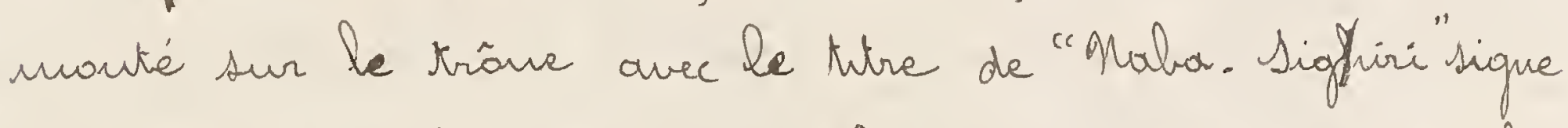
solemellement la braité qui flace son royaume surs la froutaction de la france.

Mos draits sur ce frafs recevaient ainsi me tanction aefinitive.

Ille se forodnisait avee d'autant plus d'effrosturité quer quelopues fours aperes (1" féricer 1897) le Sieutenaut Voulet itait informe de la marche vers le ruard d'une missions anglaise se dirigeant sur Karfíla. Al quitta anssitot onagadangan frour se farter à sa nencoutre et funt la joiridre sefer jours afrés à Genkoduga.

Sà, il sigua arce le Cafitaine Domald Stewart commandout la colorme nue convention - re derout avair il ast vrai, qu' un effft. frovisaire, - ause Kermes de laquelle les Anglais se netiraient derrière la frontière du chanfroussi et les transais an Nord du territaire de bentodago fusogi'a ce que la question fut réghé frarandiflomatique eutre les

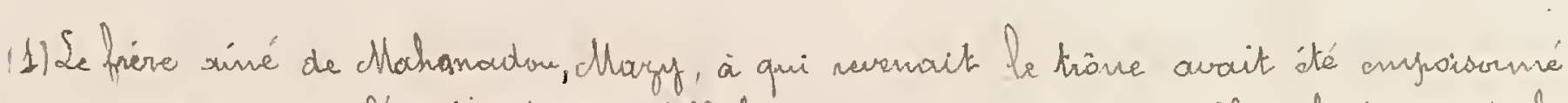

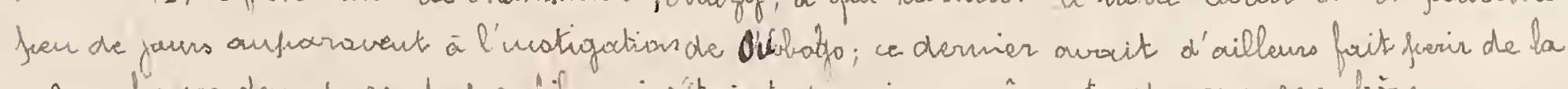

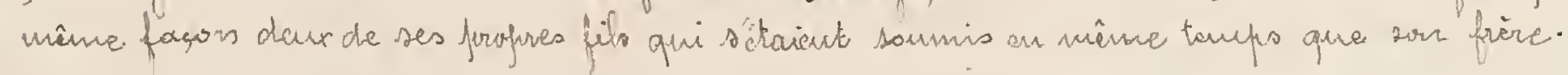


deuse furistances intéressées.

Ou caurs de cette niêne fiériade trois frèresdu moro-

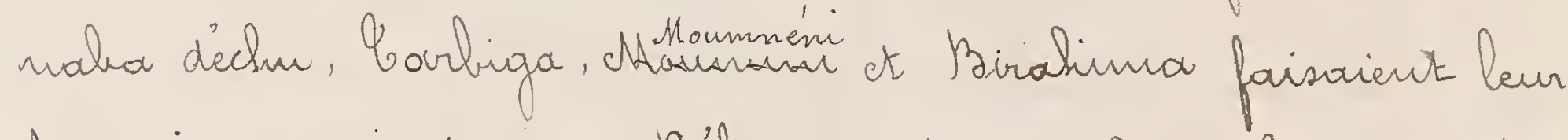
Sammision ainsi que B'eloussa et Kanfiela naba dout l'ex. ayple devait eutrainer los demiers réfractaires.

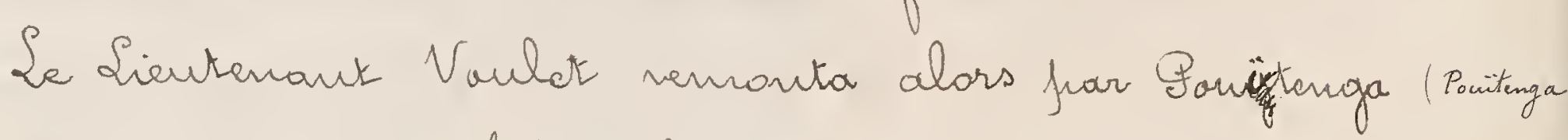
vers le N-E, rejoignit à Biloga la missions Band et Vermeaseb qui, simart l'execution d'un flan fraralliele, avait effectué l'accupations der Gaurna, et les deux traufies vémies concan. rureut à la prise du ivllage rebelle de Bilonoga (19) fürier). quittant alors le Oabsamey la mission Vaulet revieut à Onagadarigar fuar Gélanssa, et son chef rentrait en Grance ajeres avair passé le commondenant an Cajuitaine Scal appléé à lui succéder on qualité de résideut du Massi (1: Mars 189\%)

Entre terufs, des nomelles unquietonites farwenaient de la reigion du Gouraursi. Ce faoys que Noulet avait laissé six muis aufroravent sues l'autorité du chef Hanaria était menacé d'un retour offersif de tamory qui four suncieur de ses fromesses, vernait de s'allior à Baba fo. (")

Il étart ungent d'avisen: aussi le Cafitaine Résident envajait. il sans tarden un officier dours cette direction. Rencontré à Padiaga, Baba fio y essunfait mue jremière defaite (14 Mars). Le Cafuitaine Scal sirvemont quelques jours afiès, devait lui en infliger mue seconde à la suite de

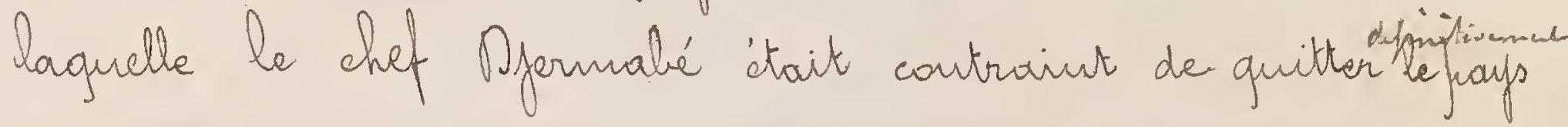

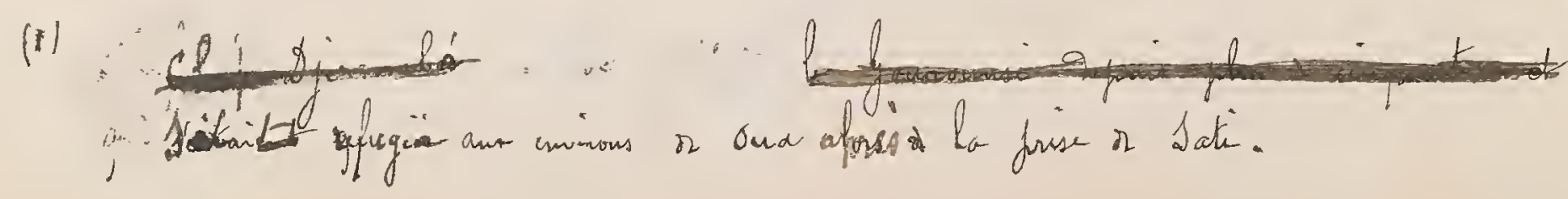


ot de chercher un refuge en Gald Coost. C'est an cours de la fraursuite que nos boupes furrent recucillir les débris d'une mission Onglaise battue prés de Otka far Sarauké. dlary. Mais Batkery Kantan n'avait fras frerder tont esfoir. Rifugié dours le sud. oir son influence s'eseregait encare sur quel. ques villages insounis, il essayait d'y entratenir la rébellion ot d'y fannenter de nauveaux traubles. Onssi le Cafitaine Scal qui arosit laissé duraut son alsence le cammandement du fuste de Oragadongar an Sientenant Gibat, envaya.t. i ce. der. nier dés son setour, à la tête d'un fort détachament dours le Baussangée et les rejens de Manga et Maberé air l'agitation avait été signalée.

Ou caurs de ses ofiérations les princifiaux rebelles furent. chaties at Battary Kanton Luime; surforis an flaine brausse be of Jum far une necomanosonce dut s'enfuir fréeifitanmont, abomidomant tre las te tratsis son theval de parade et son fils Joréferé Bila Se froufaut ensuite mu frassage

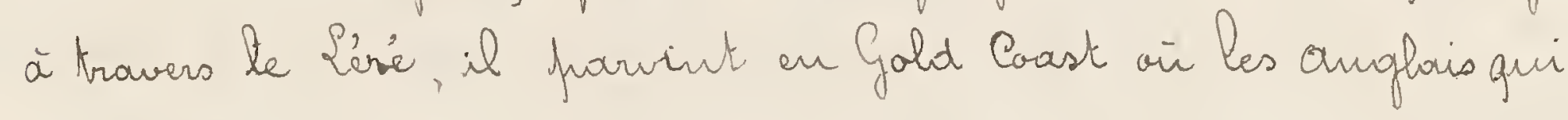
vagaient en hi four l'avenir l'instrument de revendications fussibles, l'acureillinent.

(1) Cost à buman que le Papitaine Sal recucillit lac missioss Cramer qui vemait de laisser entre les mains de fils de Samony taus ses haugages ainsi que dent canons. 7

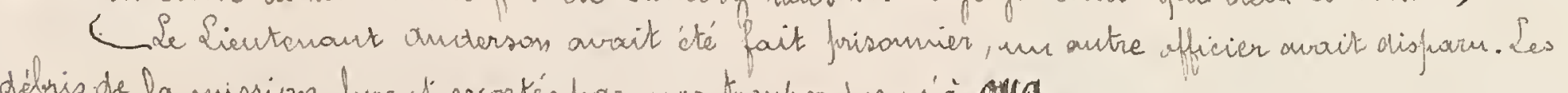

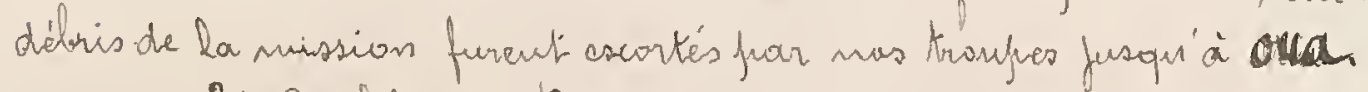

(2) Ce fils de Bathary, afrès avair servi en qualité de Sfahis avsiliaire

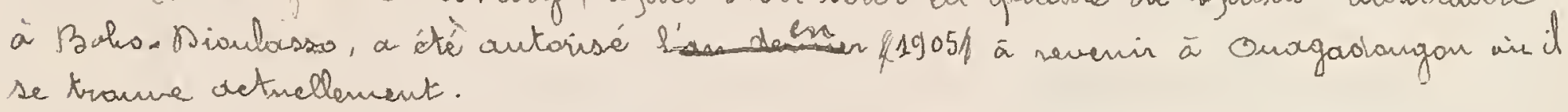


Ut de fait, defunis ce moment, quioique la conquiete du cllassi fuisse ètre corsidérée conmme défiritive, les divers Résidents se kromerout en frésence de difficultés trufours nendissautes de la fart de nus virsins gui re dissimulent fras la frietention de rétulblir leur kinte et frutegé sur le trave des Aloro. Mabas.

Ea froutieire de Gold. Caast sero four suite le thiéatre d'agitations continuelles et d'unterventions militaires fréguentes. Se dillut de l'annie 1898 ast marqué far de nouvelles recomaissonces du Sientenaut abbat dans le Séré et en froys Banssangee (1) airsi que du Cafitaine Acal qui s'op. fuse à l'atalitiosenneut de fastes onglais en territaire con. kesté.

a la mième ófugure Guagadorigon devieut le chef lien de la région Volta nouvellement corstituée et flarié saus l'auto. rite” du Commondont Destenowe. Ce dernier, afvies owvir har. couru tout le frays st vencoutre le Capitaine Seal doms le Seré, dirige hir meine une recommonissance à trowers le Alouga ot arrive à Gana le 5 chars 1898. De retaur à Gnagadougan

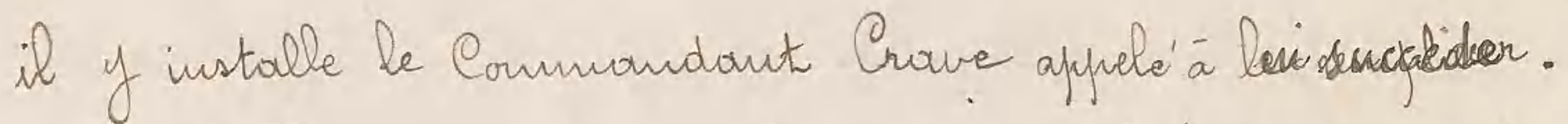

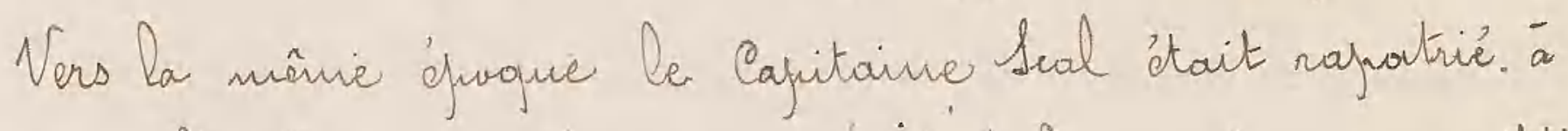
son tour ot le Capitaine anmons aurigate tophe remplacer en qualite

(1) C'est our currs de l'une de ces recomnaissonces que fur fonde' le fustie de Bitton qui re sera aloondonné qu' an délut de 1899 fuer snite de notre itallissenent dífinitif à benthodorgo puint plus central. 
de Résideut.

Ses cfforts de ce dernier devrout se frarter également du coté du Léré de du Kausacé at il se verra abligé de barder la frontière d'une ligure continue de frostes, cour en défit des conventions fuassées tes Ohiglois viement encove d'uceufer Son. ga, Laaga et Mariga.

Gidéles à ce systène, ils devaient aller filus bin encove. Our mois de mai de la mience année, le Sientenant. Elonel H.P. Martheatt du régiment de Seinster, Résident Sufírieur à Gannhaktfla fériétrait au Massi à la tète d'une forté colome ct se dirigeait vers le Mord frar Aanga Labére, Mainga ct Béré. Sll amenait à sa suite Bakery Martan avec L'inteutions évidente de le réintigrer daus la dignité de Mllow. Maba. Se rendait. il bien compte de l'afpartunitésides conséquences probables de som eutre. frise! Ofuviquil en sirt, au village de Kambissiguiri, à 40 kil à faine de Onagadongou, la notification du traité du 28 Janvier 1891 hii donna à réfléchir, ot d'asség bonne gräce, il revifut sur

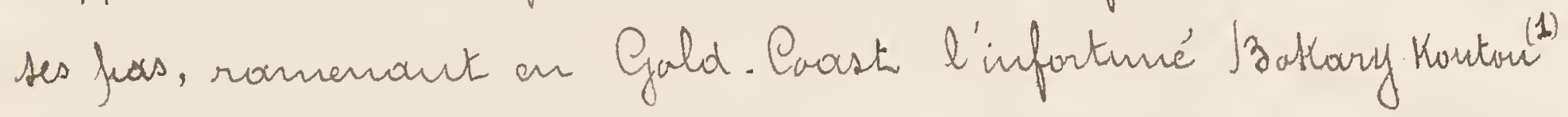
qui mue firs de flus vajait son sêve s'évanariv. (14 Juin 1898).

(1) Re lamentable résultate de cette éginfiée fit prerdre à Bathary Koutow ses demiers

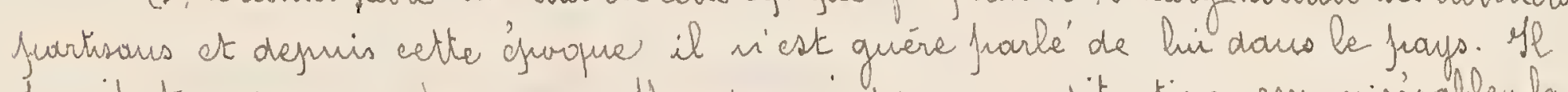
devait terminar ses fours au Mlanfoursi daus une situation assy misérable: la navelle de sa mort parvenue en Farvier 1905 n'a fruduit anune énutiors an Massi. Inde

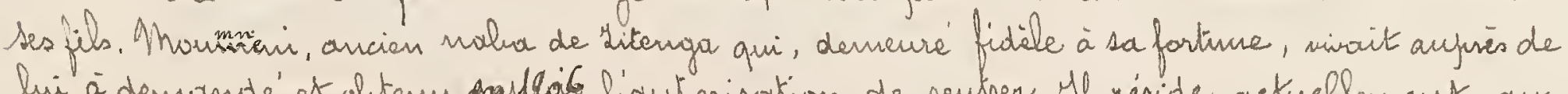

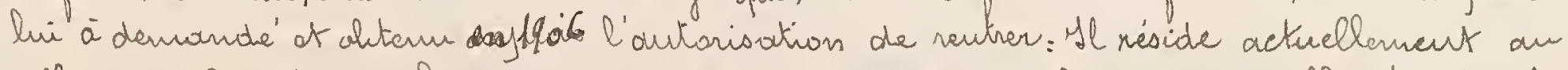
village de Gonsé non loin de anagadorigon et son influence est nulle. He n'existe

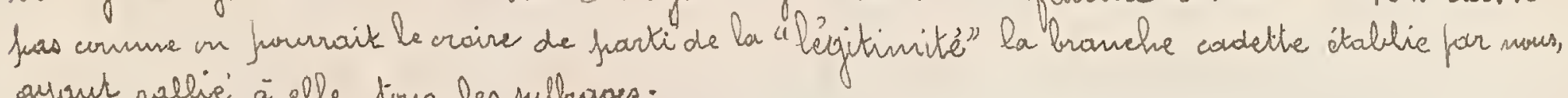
ayaur nallie' à elle tous les suffruges. 


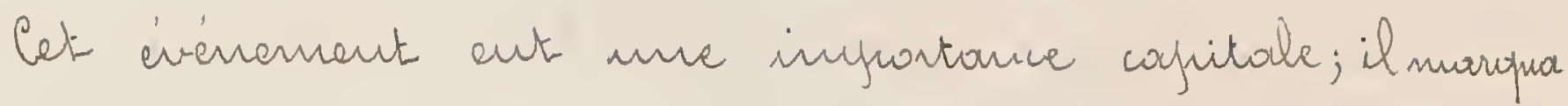
la fir des giérations militaires qui s'daicut forolongées, nous l'aurous un, prendour filus d'une année afries la conquéte fropmement dite; la période qui s'oure à cette éfurque-ele are - est celle des "Toumies de fulie". Les Kronucis n'out filus d'emennis avoniés darrs le frays, sur aumn foint leur autarité n'est ouvertement discutée. S'incistent de Gen. Hodogo vient d'avair sa solution; ce fuste a fur être défiriti. vement recufie. Al deviendrax hientat chef-lieu de circonscription é-fiarallencut, à l'onest, Sèo sura érigé en Centre.

Mais au sein de ce vaste territaire saunis à notre administirations, air damine la fufulation Massia proparement

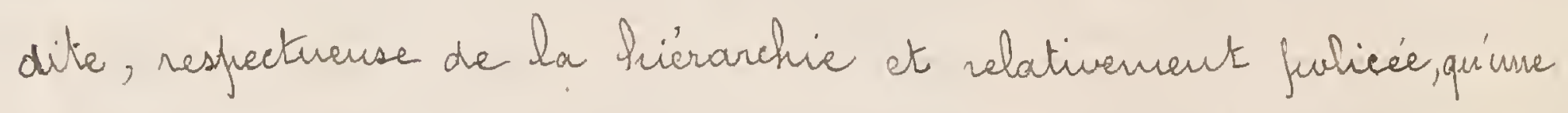
organisation sienlaive a focsarmée, se tranent enclavis ca et lä.

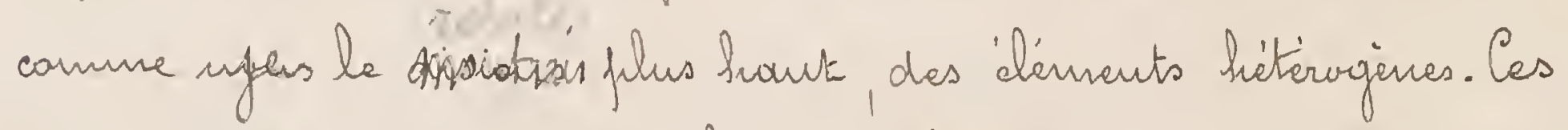
demiers refrésentauts de la fufulation forimitive qui à une éforque reculée contait le fays tout entier constituent ancone, frartiuliórinent à l'oust ct an Snd, des agglomiénations considérables. Sendaut de laugs siëles les Massip n'out fur les réohine an les nudifier; ils n'out fommis exercé sur ent qu'ance sufirimatie nominale. Il we pouvoit en ctre autre. mont. Garunches, belliqueur, infrationts de taute autorité, ces maturels viveur - un patutät vinaient à cette éfuropue, carr nos interventions fréguantes n'out fras cké saus fruit, - dous un dat de compelete anarchie. Etrangers à toute itée de justice, be drait dir plus fort déidait soul de lours litiges 
ot fuvir les nutif les jelus futiles on en vernait anxmains,

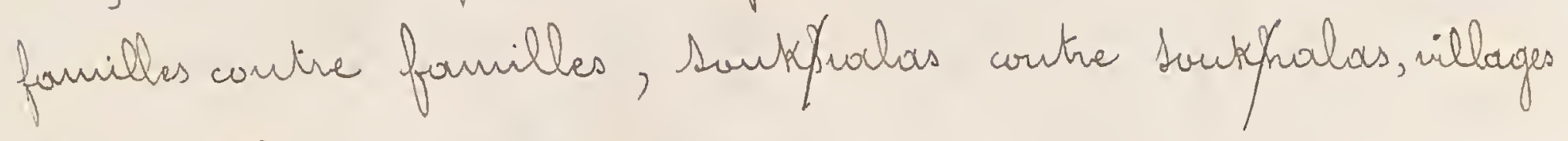
coutre villages.

Al ètait infurssible d'admettre que dous un frays sommis à natre outarité de fuareils désonorres fussent tolèrés; nues dînes done à diverses refrises intervenir daus ces régious et chãtiar les perturluateurs.

C'est dous nue de ces tournies de fulice que le Sievo temant Grimart des sfrahis tonotanais atteint faor nue flie. che enfuvisommée trauva la mart jerés du iillage de Goorsi (forsi) (14 feirier 1899).

On cours de l'annié 1900 le résidents en fonctions, Cafie.

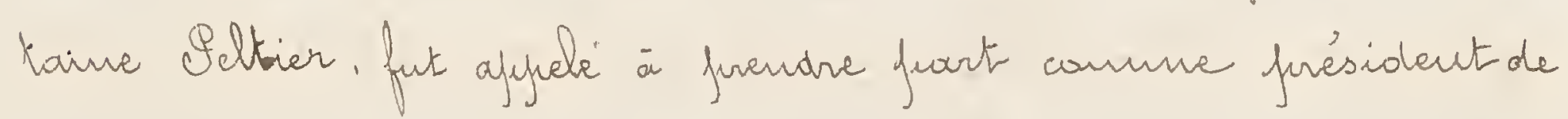
la commissions françuise, ause ofierations de délimitations de la froutière sud du Massi, que cötura un traité frovisaire

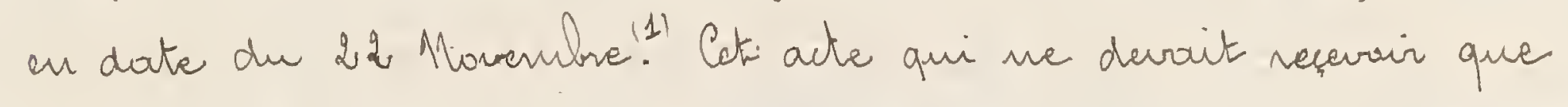
trais ons flus torot la ratification des ganvernements intèressès, nit un terme aux couflits regrettables dout le Gauranusi

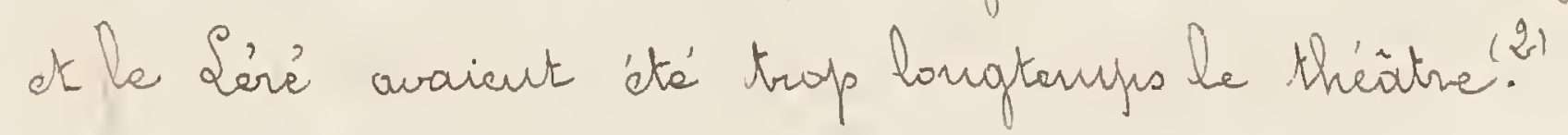
Se Lientanaut Oubartion succedor an Cafitaine Seltier. C'att de son commandement que dateut les grandes divisious

\footnotetext{
- (1) E'omgleterre était rezuésentie fuar le Commandant Máteroton

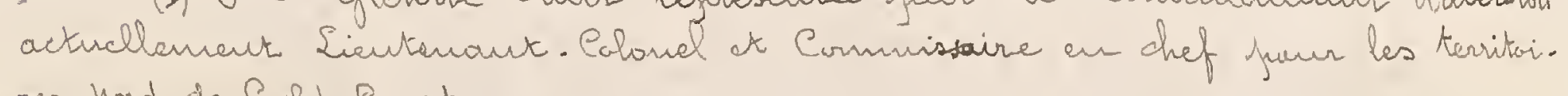
res hord de Guld. Cooust.

(2) Mre cammissiors confrasée du Cagitaine G'Kincaly fuur l'anglaterre ot der

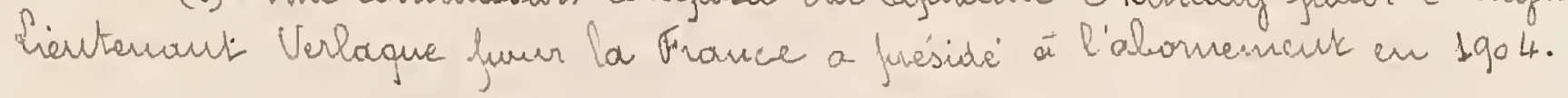


- dministratives de la frartie centrabe du Massi gui out per. Sisté fusogu'à ce faur. A cekte éfurque las grands Kamberies (1) m'etaient fas a frogrement farler chefs de forrinees. Siar. tarité qui beur ctait déléguéa (autarité d'ailleurs becucoupphus considérabble que celle qu'ils detienment actuellencut) d'exer. cait sur des iillouges an des groufues de villages sounat isalés our tries diatants les mus des autres. Il en résultait un enche. vétrement de frietentious, mue confusion de fearuoirs, tries friefudiciables à la banne administration du frays. Aussice demier fut. I divisé en cing sectemes qui frorterent dè loors le mons de chacm des grands diguitiaires. Sous le commandencuts du Cafititine Rueff (1.901-1902) nombire d'amictioriations furreut àtre réalioées at un ground réseau de rautes fut créé fourarisaut dès lars le dévelapfienent conn. mercial de cette riche contreie si fleire de foromesses fuurs l'a. venir at dout les exfortatious out defuis tiuflé d'infortionue, - An movis de six années.

dlais c'ast sentement en 1904 que devoriant se frootui. re les groundes riformes qui, afyelées à danuer au frays tout eutier un fuissaut essar, out friésidé à la nérovation écono. mique et sociale a laquelle nous assistons aufaurd' lari.

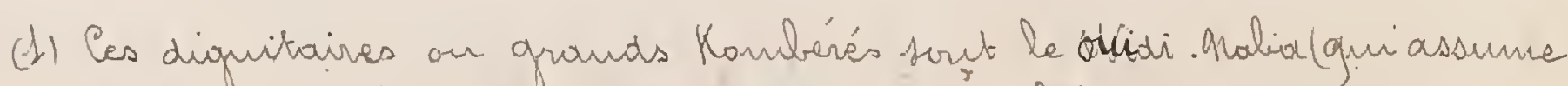

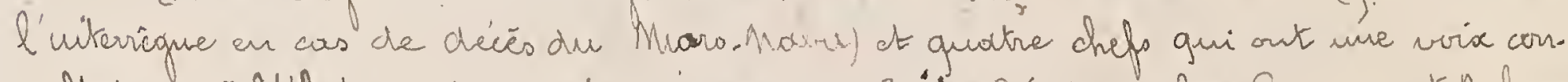

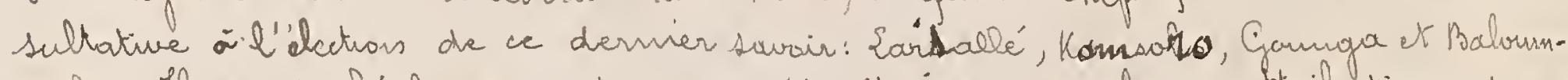
ruabo. Il sera farlé longurement de ces dignitivires of de laurs abtributions daus le couraut de catte ítride. 
Orrivé à Onagadangaur le 22 Mars, Mansieur le

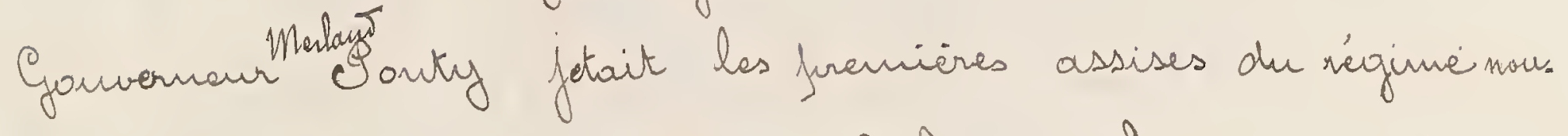
veaur et le Massi àtait érigé en Cerele surs le Commandement du Cayitaine Oubrairil. An systime nisete, forément défec. theux gui aurait régné jusque là (car le Moro. Malva ofieraiti

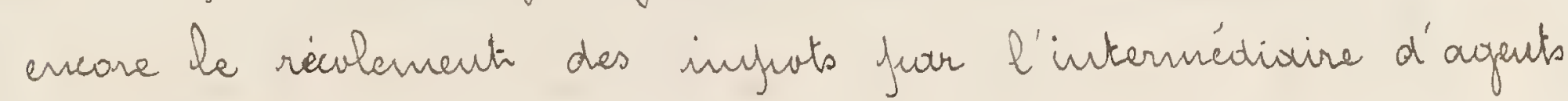
irresfonsables et four serufuleux) on substituait une orogani. sattions régulière en vertu de laquelle les charges infuusées à la fufulation èdaient rigaurensement réglées et équilablement réfrarties; Ces attributions de chacun étaient désarmais enfermiés dous de justes linites, les frouvairs déterminiés avec ferécision. Sa justice indigine ètait arganisée. In nu mat c'étrait une trousfarmation compléte qui us laissọit rien subsister de ce qui daus l'ancien état de choses faruait ferèter à l'arbutraive ou menacer les garanties matérielles ot marales qui doivent othe assuréas à tous.

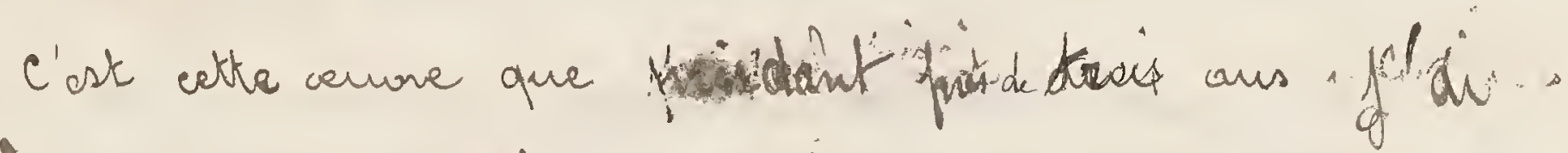
en l'bomeur d'atre appelé à panisiuire. 
A mon amive a Kages, cheflien Jo Hant Seneigal.

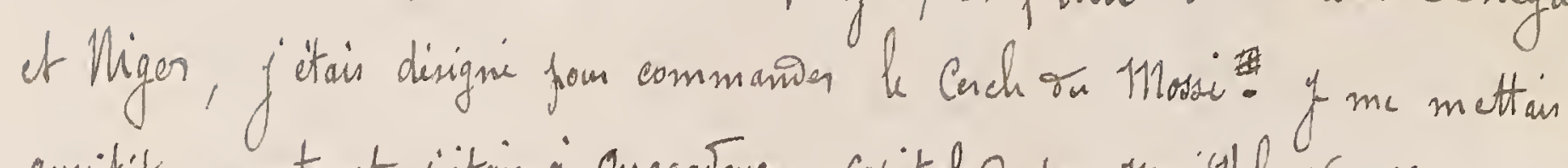

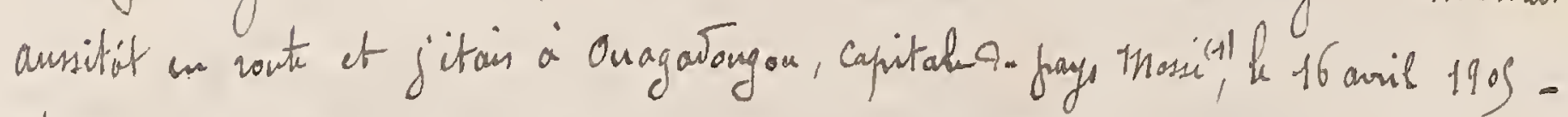

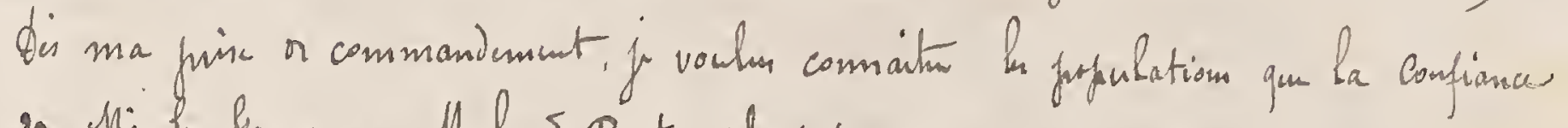

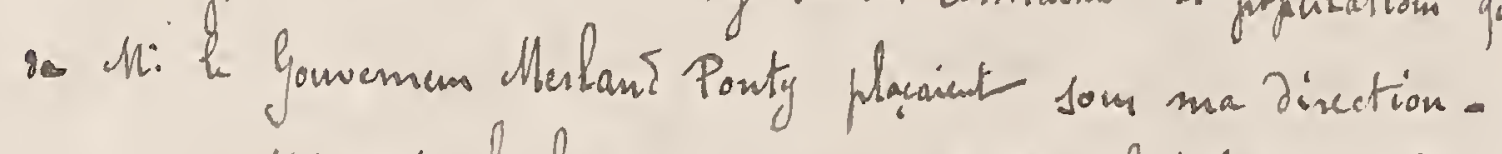

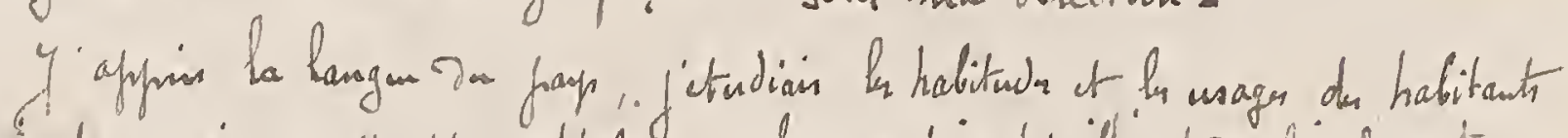

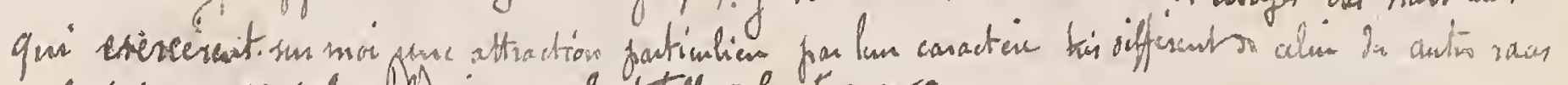

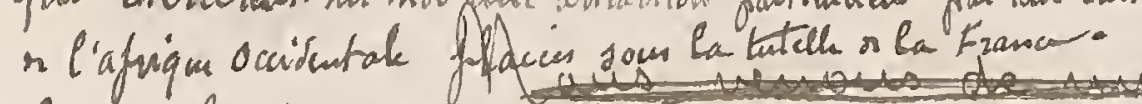

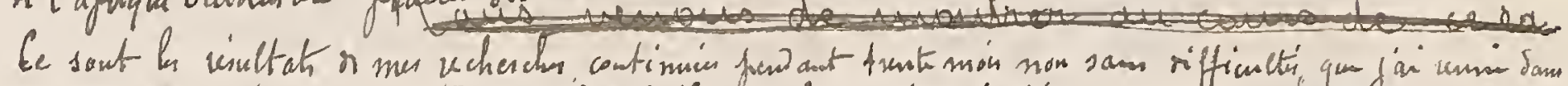

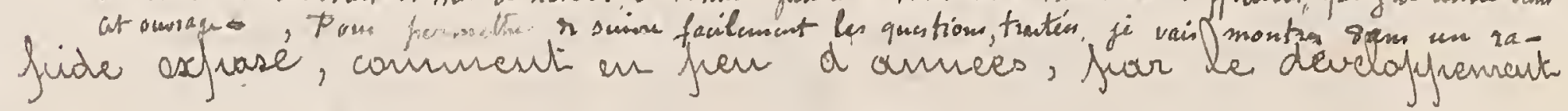
maturel d'un flom qui escécuté avec esprit de suite et formute exchait ncoumurius toute violence inutile, la conguête et la fracification de ce froys out été assurées; comment unfin il nurs a cté fussible de hi donuer une arganisation si.

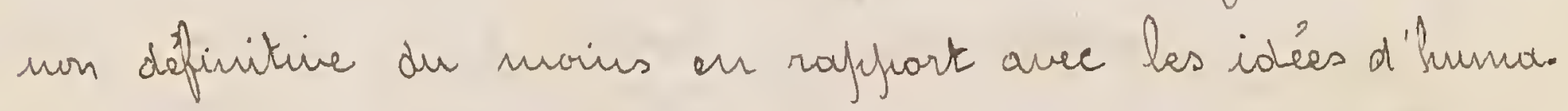

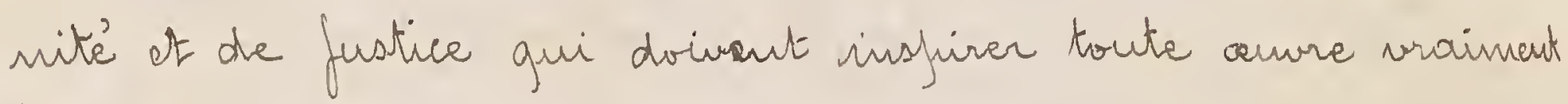
fromciase.

Satoche de mes devanciers a dé rude ex bien reustie. baut vecupies quils étaient de la conquite et de l'onganisation

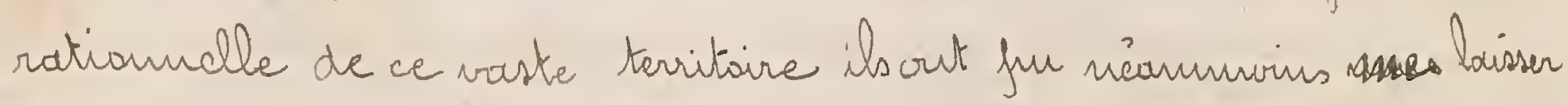

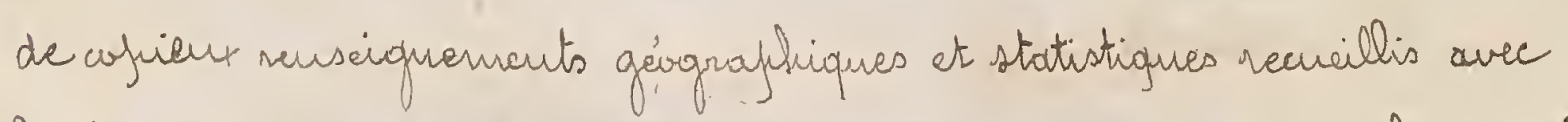

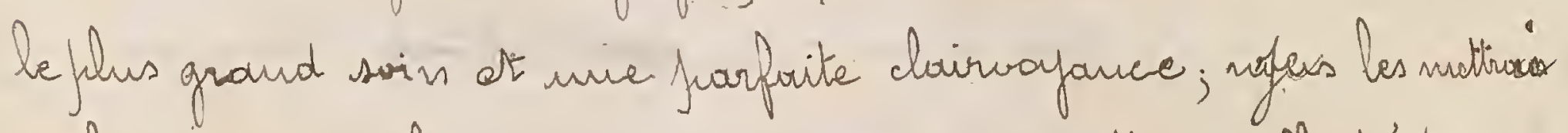
utilaneut à Arobfit ase cours de cette étude. Mois sallicités far

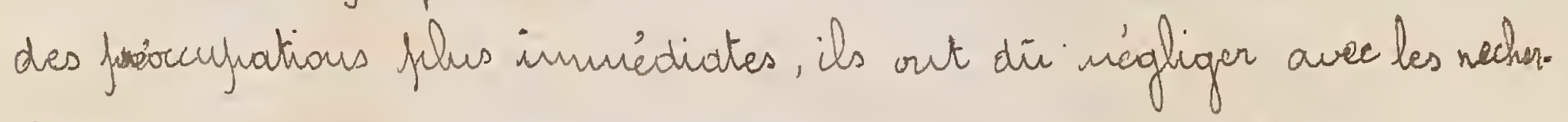
ches sociolagigues ot ethrographingues tout ce qui n'offrait gu'um

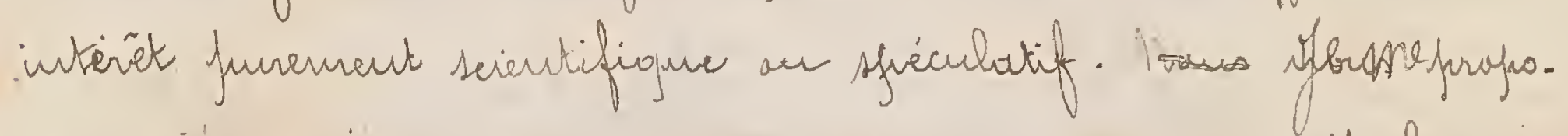
se.: de combiler, douis une certaine wesine, cetre lacune.

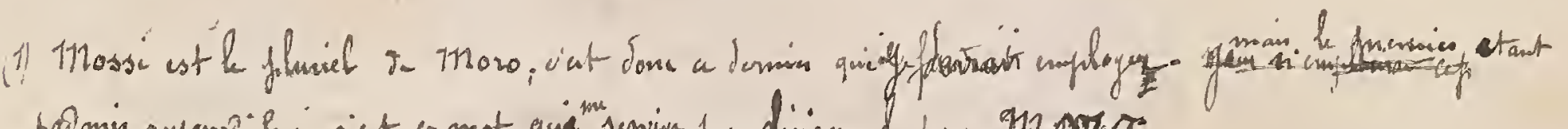

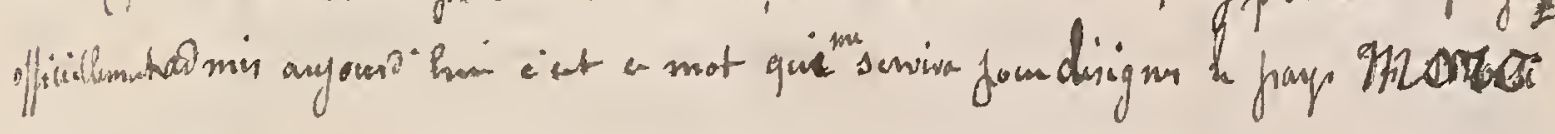


25

quan Souttie

Le houys Mossi - biomarthi

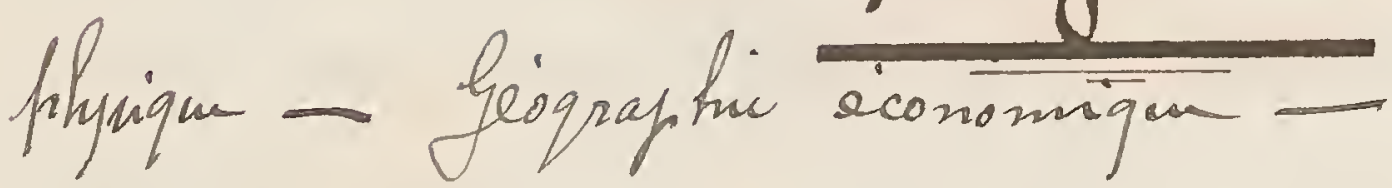

Sitration es limites

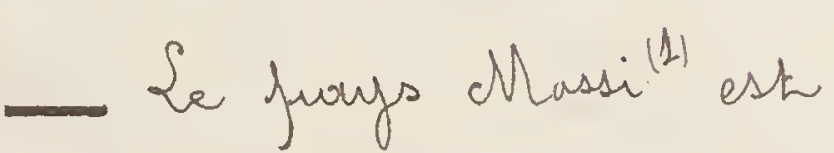
situé eutre les 11: at 13: dequés de latitude Mord, les 3\% at 5\% degrés de longiturte Orest.

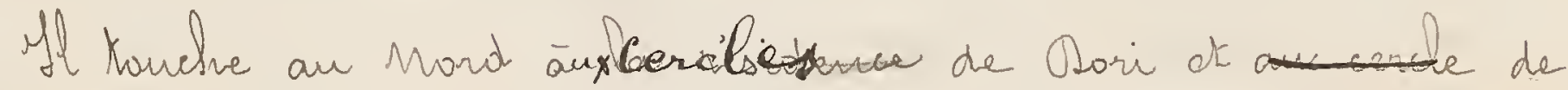

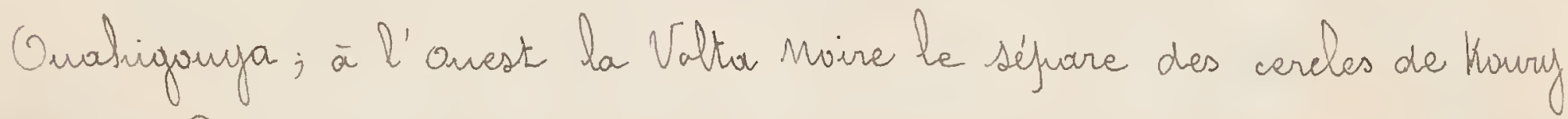
et de. Powna; ancune froutière naturelle we se trauve à l'ist,

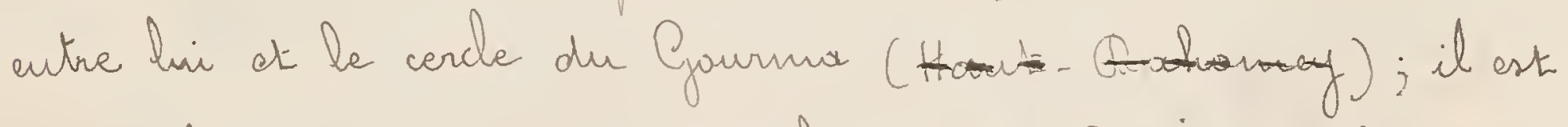

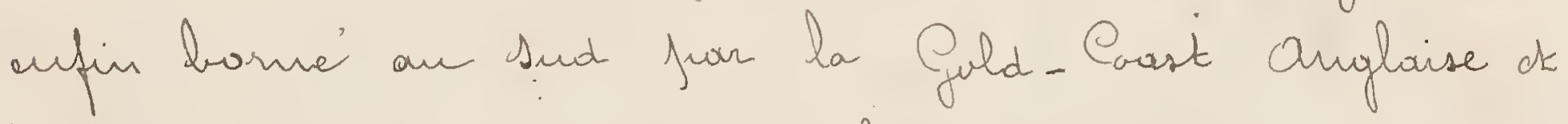
la cularie allemande du bogo selan une ligne idéale qui suits affrosinmativament le $1 \%$ fuarallite.

Brographicie - Sur furesque toute son àtendue le

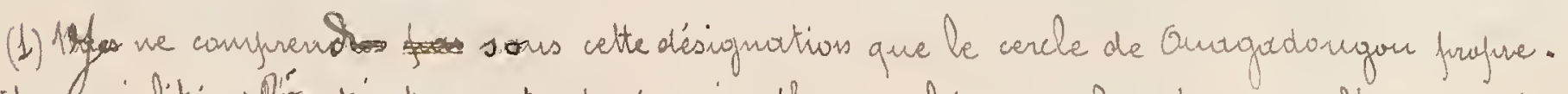

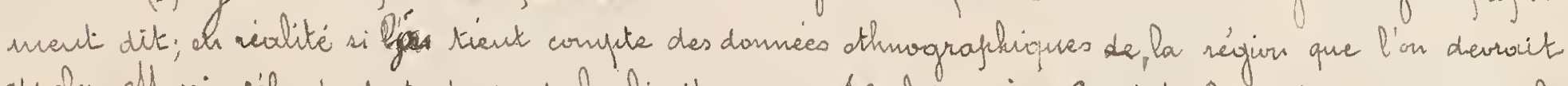

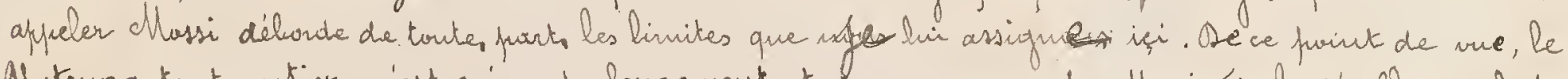

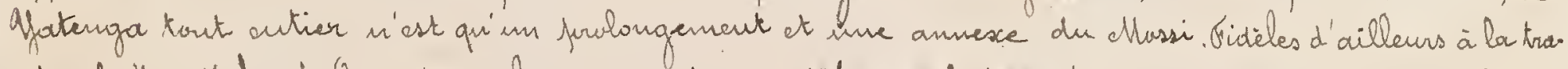

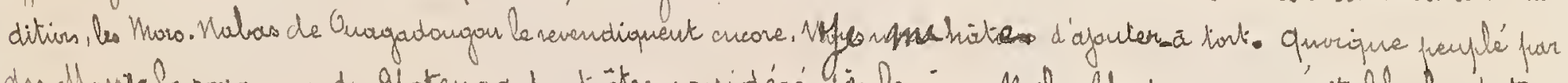

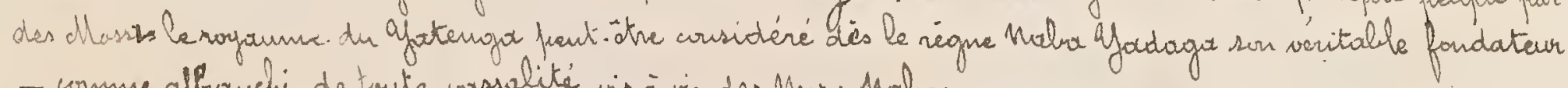
- cormue affrunesi de toute vassalité is a vis des thow. Matas. 


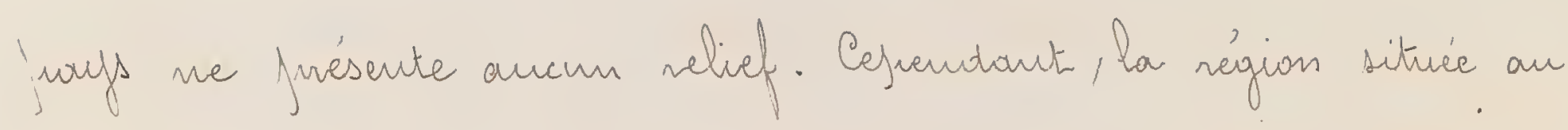
Mard de Onagadougan de conprise antive les Valtas noire et Bhanche sencble être divisée en deux fwarties foor mue ligne de fuartiage des caux oufour son oriogine an sud de Yakto.

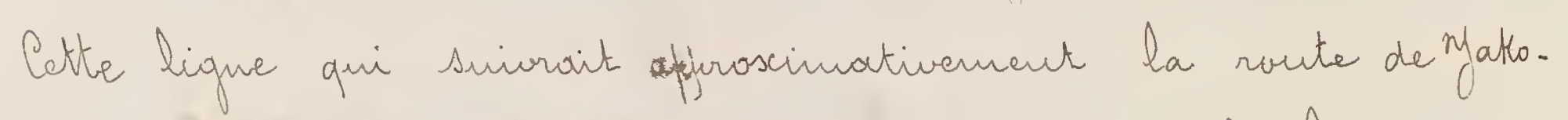
Onagodianigar. Gounigyt; himiterait à l'ouest le hassins de

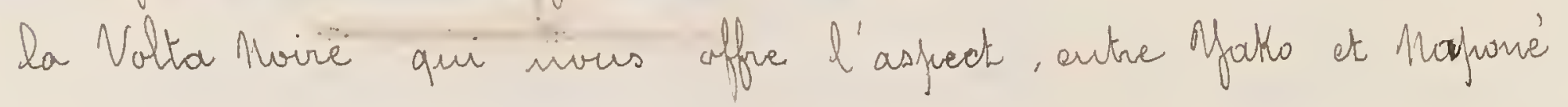
par Shimpitar et Sangui, d'une suite discantimue de na. melous. Ces frotubinomees sungissour sa ct la saus owbe ni onieutation dominante, constituent comme une série de muraviles de faible altitude entre lesguelles traneut mu cialencut les affluents de la Volta mive dout le lit na beamaup ghus loin, vers l'St, cherchersen onigine.

Om remarque formi ces twouteurs mon boin de Réo la mantagne de Jomani air an $189 \mathrm{~g}$ les Capitaine Ommona remfortie une victire décisive sur les bandes Cauraunsi.

a l'Est de la hiopue de partioge des cause et compremant

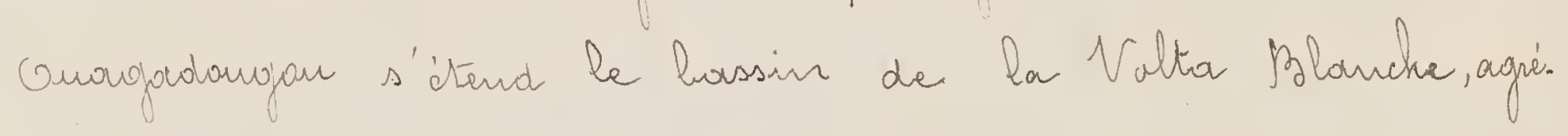
manté hi aussi d'une ligne de frauteurs qui connencerait

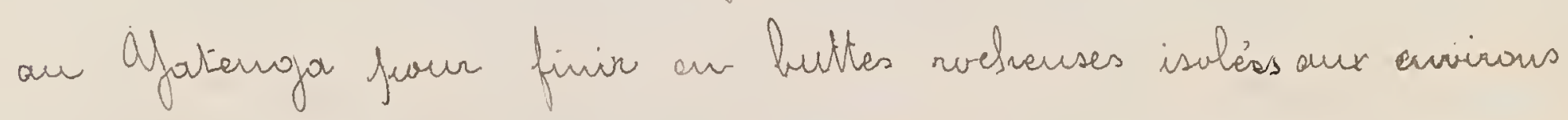
de hargango, hafuadiania at taudangan.

Moirs régulieires encore que les cullines de Slimpikan, les fauteurs de Baussourna forment un enchevêtrencut confus de massifs feer infiontants entre tesquels coulent en de larges vallées les affluents de la Valta Blanche. 
cour sud de Onagadongan la ligue de frartage des canser senible se continuer fiar le flatean de Gaunga fusquía Rakay air clle meurt après avair servi de démarcation entre

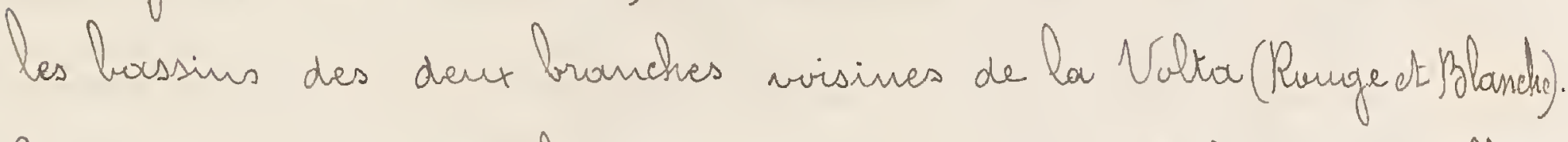
Les carruranes de diaulas suivent taufaurs cette ligne gui offre l'avourtage de n'àtre foumais submnergée alors sue la vaute

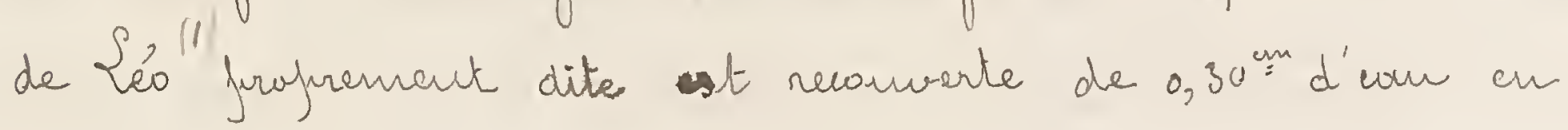
f. inveruage.

Gout à fairt au Sud du Cencle, à l'Est de la Valta Range, se trament les moutangres jumelles de Garango oir les Baussangas out tonjaurs offusé une nésistance acharnée aux congriorants Mlassip.

Muns signalerons enfirs au Sud. Sst du Gourannsi, vers Gieble", de nombreux nomelons entre besouels on distingue le fic de Maauri (q)

Hondrographie - Ea région dout yous dicaso d'esquisser le relief, alimente trois grands cours d'car qui constiturent les branches d'un mème flerue ayourt sen enbouchure en Gold-Coast. Ce sout de l'onest à l'est la Valta Moire, la Valta Rarige an niviere de Rakay, et la Valta Mlanche. Sa Valtox Maire est de beancoup la filus infrortante mais la majeure frartie de son cours est étrangere au Massi qu'clle séfuare des Cercles voisins de Kaury et de Caoma, hir servant

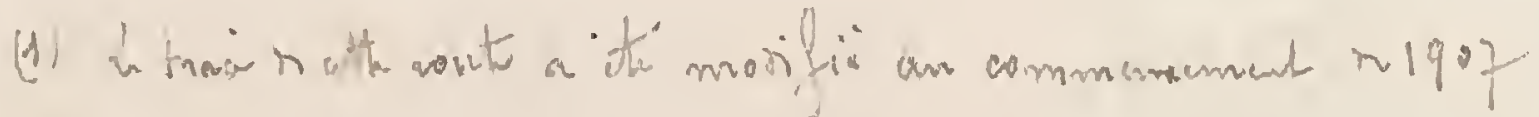

(2) Iiqualé d'abord foor Bartlo guilui attribue une hauteur exagérée. 


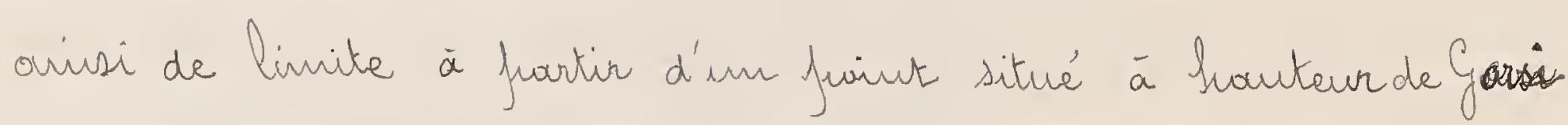
di (Kiphisi) Jusqu'à son entrée en territaire anglais. Sendout l'friversnage, le ravitaillement destiné à Onagjadangour est dirigé frar este voie de Kaury à Barano frar la flutille dite "de la Volta."

Ce cours d'ear, gui va rejoindre la Valta Bhanche à l'Ouest de Salaga en Gold. Coost, est en gérieral frafond, encaissé, et friesente un lit marécougent ot accidenté. Ses rives boisées ot désertes out la réputation d'atre nualsaines et les villages ar sout flus an moins eloignès (kels Badé, Aka, Boromo, Houry de).

Sa Valta Range est farmée far la réunion de plusiaurs bras qui corlont du nord au sud furennent leur arigine daus le Kiffirsi; le felus infortant coupe la rante de Barano à sa kil. envirus à l'onest de Satkinsé, afries quai la rivière frasse fiers de Konfas ot fraursuit son cours farmont limite entre le Gauraunsi d'une fark, le Massi, le Baussangsé le Séré et le Kousacé d'autre fiart, fusopi'à son cutrée en territaire Anglais. Duraut la saisons seche serv lit sera refrésenté sur tout

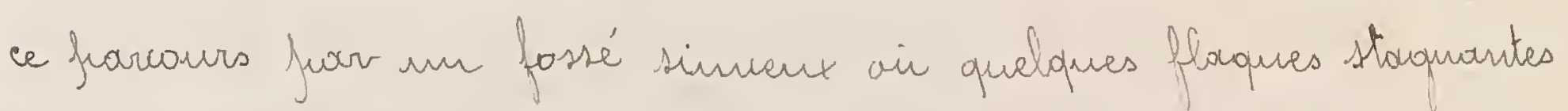
we jersisterant que ca et là, en audal de Kamprala (Caurounsi). Gautefois au frassogge du chemin qui conohit de ce fuint à Manga an faufs Massi, le lit s'érasera ot gagnera en ferofordeur. Les phluies venues, la rivière roulara lí mue masse d'cour cansidérable. An fout situce mor doin de Rakay sur la raute de Léoen fuemat le passolge en tout kenups. 
Elle resait encare an dela nombre de ruiseous nemus

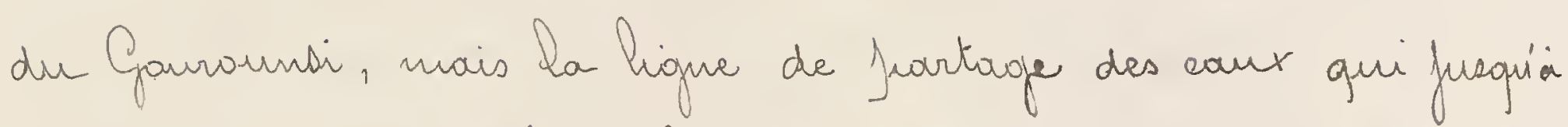
Sakaiy semblait ètre le froblangement du filatran de Gannaga, deverent à faortir de ce juint difficile à déterminer. Sa Volta Blanche vient du Gatenga far phasieurs bras dout le filus inifontont frend sa saurce au Mond de ffato ot se dirige de lomest à l'ist gurque vers Mané. Ces divers afflents out leur fuint de rencontre dours la région comprise entre Baussauna ct Manè. He sout eut aussi d'inve fason gérierale défroururs d'cau fendaut la saisons sèche et leurs troves serfenteut daus de vorstes défressions de 500 nietres de large environ, au sein desquelles les caur se sout creusé le fhis sament denx lits farralléles d'une dizaine de nietres. A l'bi. vernage tout se vemplit et les caux qu'entraine un courant infietucur constituen. sur divers fionts mu olstacle uffranchis. sable (c'est arisi que la colanne Naulet s'est tranvée arrêtée daus So marche sur Banssamua en 1897 ).

Agel ast le rigine de la Valta Blanche non seulement à Sa fartice sufierieure mais mieme afires la fonctions des differents bras dout vis de farler, notanment à sern intersection aver la route de Kaufíla. C'est en ce fivint, non lain du ivillage de Kangauri, (entre Sinorens et Mgoguors) qu'in grand frout a été établi depuis quelques amrées. Ill assure le frastage de la rivière en toute Soison.

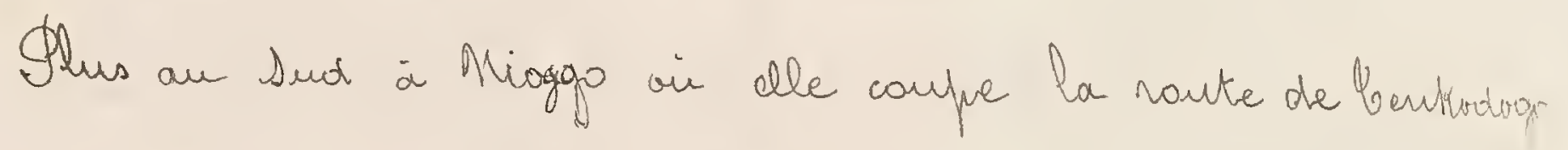
proferencent dite, elle a acquis des dimensions flus considérables 
erreare et constitue un tarrent large of infiétueut roubant

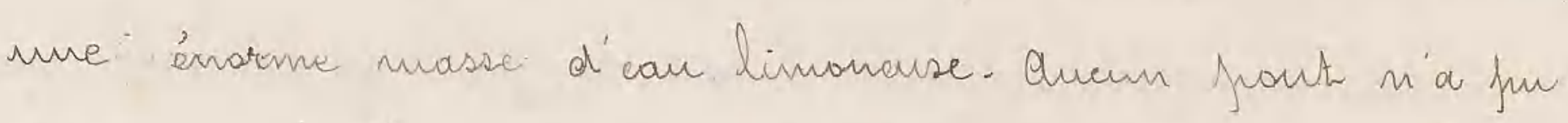
dire encore étalli à cet endroit; pendant l'hivermage la tra. versée s'effective sur des trancs d'arbres et n'est Juas dans offeir quelques difficultes.

a jeartir de la et de dirigeourt toufouns vers le tud, la Volta Blanche arrase le Banssangcé ot sert de limite entre cette région ot le Lèré, payss boisé qui constutue un platean asser ilavé dominant sun le versanit ofefusé la Nalta Raugio.

Sa Naltax Blanche recoit enrave daus la fuartie nayjeme

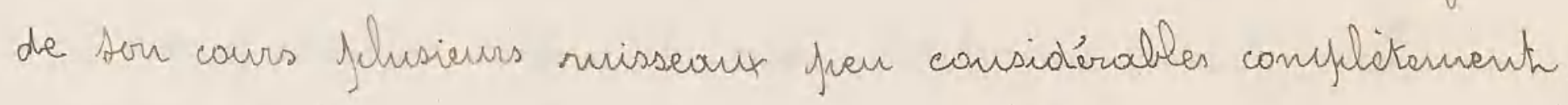
asseches dis te nuis de Mavembre, sauf en ce qui concerme

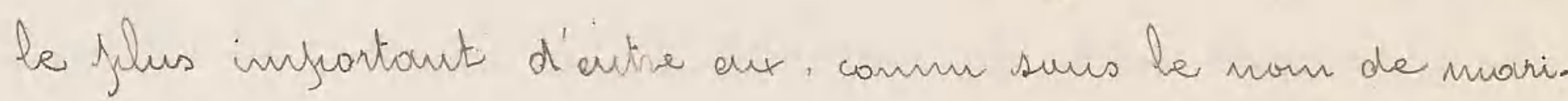
got de Sumbila. Ce dernier conserve de l'can toute l'annér

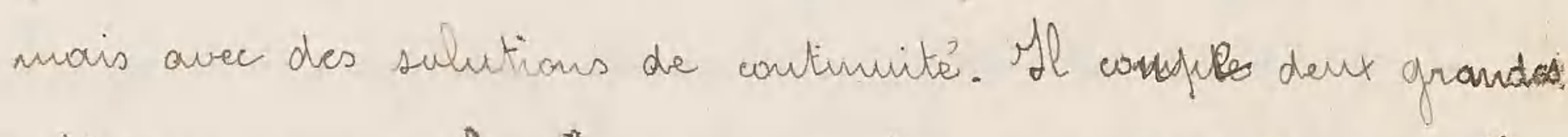
artieres: ar prencier lieu la route de Baussauma et OLari au village de Sumbila Botko qui hii a damé son norn; filuo loin erfin, la grande rute de Kanfiela non loin de Gampiela. Mun part est datali à cet endruit.

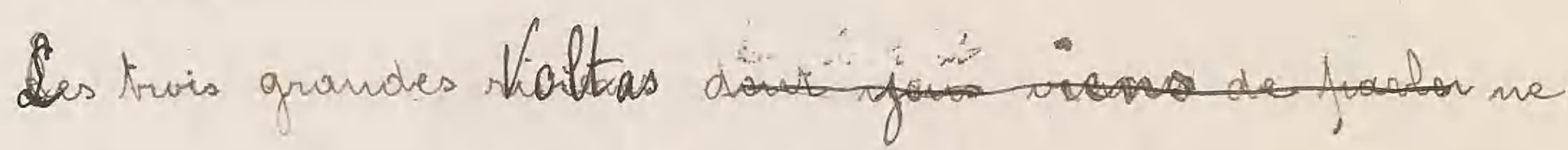
Sout guere connues des indigenes qu'à l'intersection des voutes praticaliles; ils iquavent en génèral laur cours, et a dernier rencoutré sur des foints distants l'un de l'autre, n'ast fors tayjours identifié par eux. Learsbards sont intrabitit's sur des éteridues sounent cousi. dérables. 
31

14: 

Hl faut craire qu'arce l'nsabubité du climat (1) $^{(1)}$ e. signalice faur la Valta Noire, la crainte des inomdations ot la présence des fouvres contribuent à ébigner l'ha. bitant.

Se loug de la Valtow Moire, daus le Kiffirsi s'étend une bande de 20 à zo Kilonictres de largeur abondanment boisée qui-

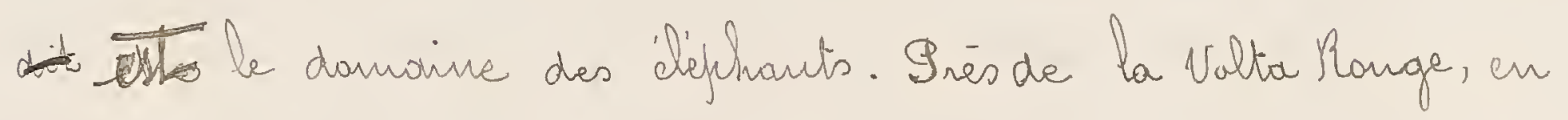
aval de Kayfar, nonvelle zâne déserte noins vaste il est vrai, mais qui vor s'clargissant à meoure qu'ou s'avonce vers be Sud. Infin, la Volta Blanche arrose entre les rautes de Kaufiela et de bentwologo une grande région desentique frégunartie seulemant, far les chasseurs de fanves.

Gèlagie - Le sul du Massi ast argilo-ferruginart; on y nercountre de nombreuse affleuremants de roches primitives, fraw endrvits au Kawlin (YJakto et benkadugo).

Le fer est krés aboudant au Mifpirsi; le nimerai s'en trouve à l'chat de sesquiaseyde (himatite burne). Les indigines l'or ploitent à la miethode dite "Cabalanes" en le mielongeount aver me quantité double de charkon.

De l'or d'alluiros a ćté signalé égalencent dours la fro. vince de Re. Whe sufrerstition locale, vaut qu'il soit exclusiac-

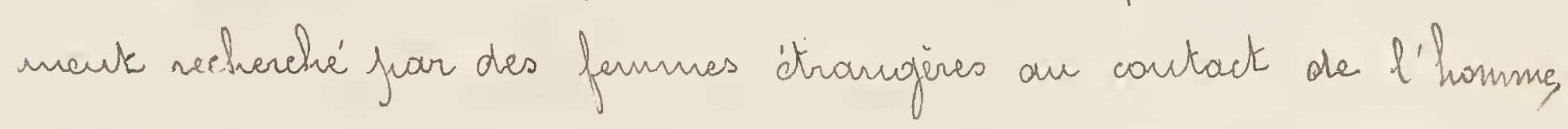
très fermes parursséquent an tries agées. Si cette conditions n'est pas

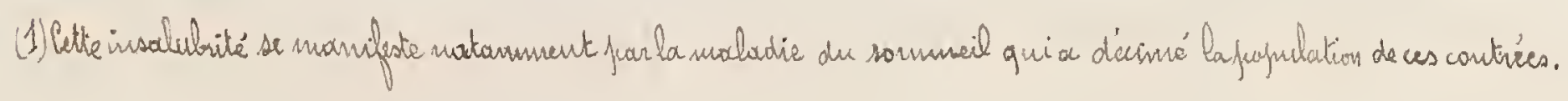




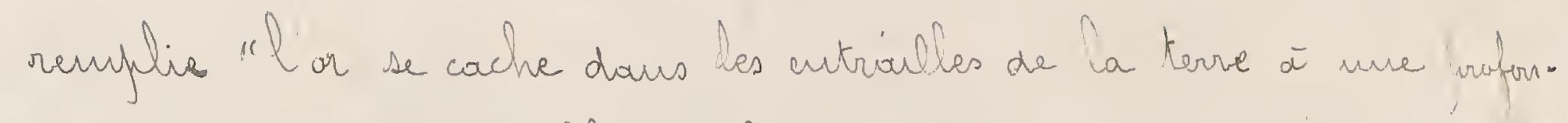
deur wir il art infussilfe de le trauver. -

S'exploitation tries intermittente, taufours entregorise sur

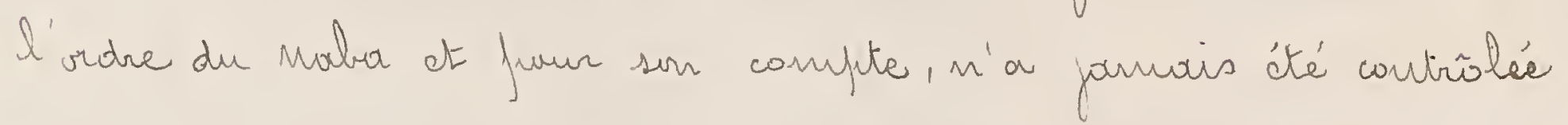
d'une fason suivie. Sle a danné hier à un intéressaur rap. front ćtabli an 1899 for le Cafitaine amman.

Deux ansiés filus tord de Cafitaine Rueff dewait paire exécuter des recterches en so frésence. Cette exferience conduite

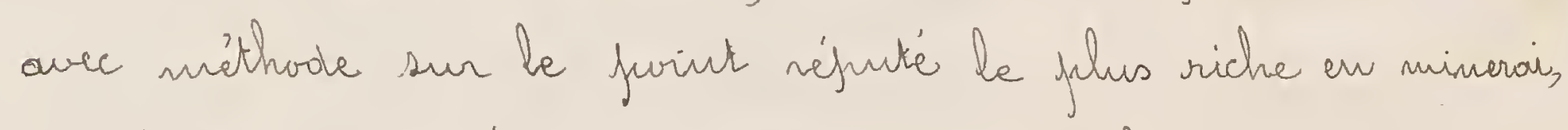
n'a toutefois donné gi'un forodrrit insignifiaut.

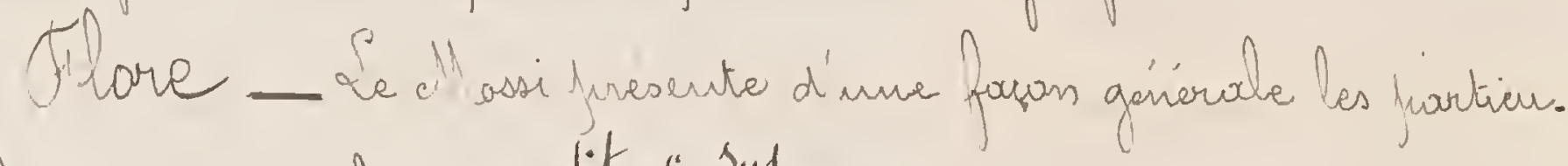
larités botaniques de la räre dit." Jud on Guiréenne "qui confrend éga.

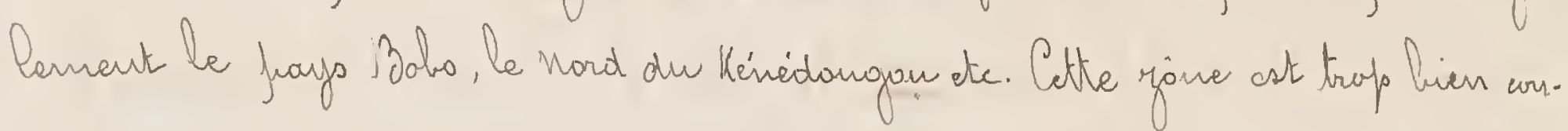
nuc defuis les intiressants travour de Alousieur. Ong Cherallier fuur que f'éssayes d'entrer daus des gévéralités qui we seravient que des redites et dout le carroctere technique sartiraut de Mator confiéténce.

Qa buasse du Massi fusséde peur d'asences riches. Slle ferésenter

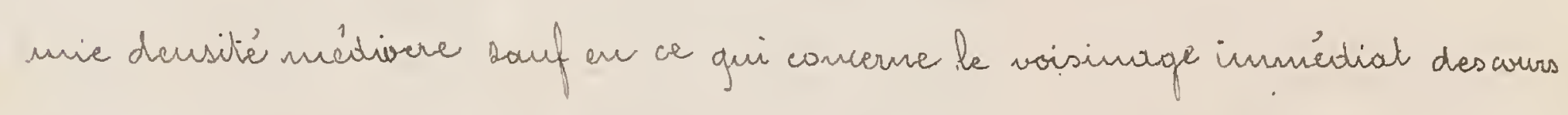

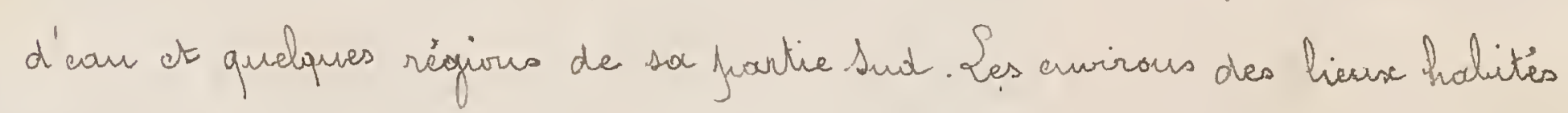
sout la flufoart du Kerups divastés sur nue large fieriffierine qui a dü fournir suendaut de longres annèes des matériaur de condruction...

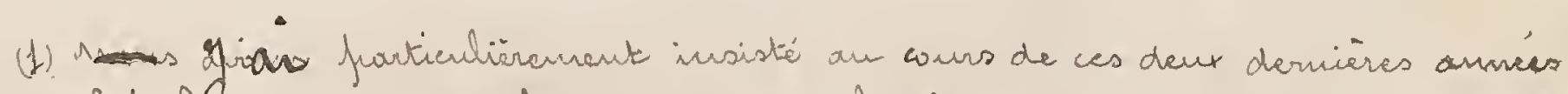

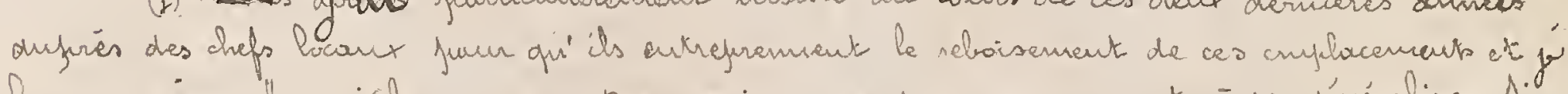

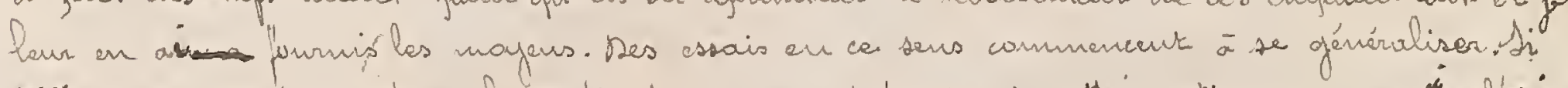

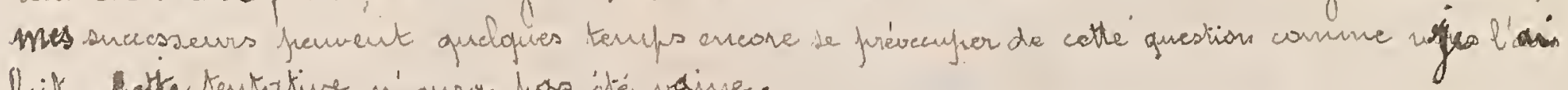

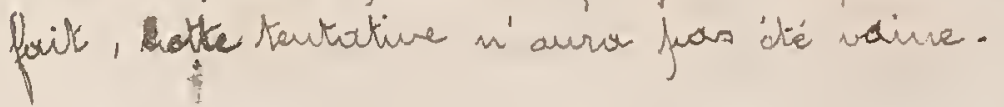




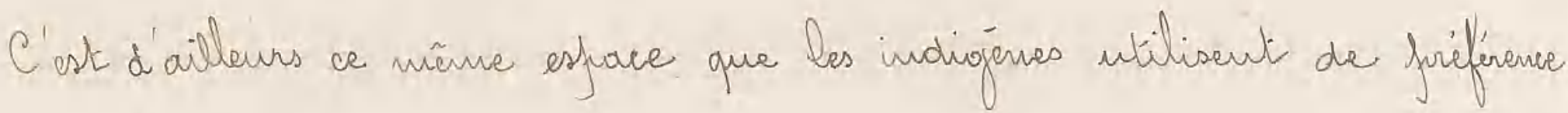
Juur lours ciltures amnuelles.

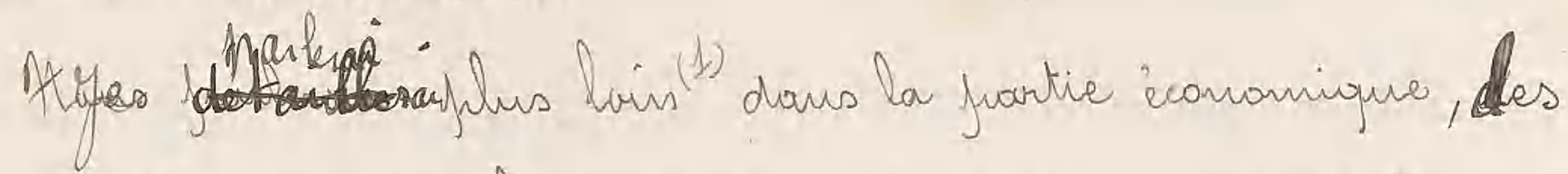
Plantes vivrières en général et des fruoduits végétaux induatriels.

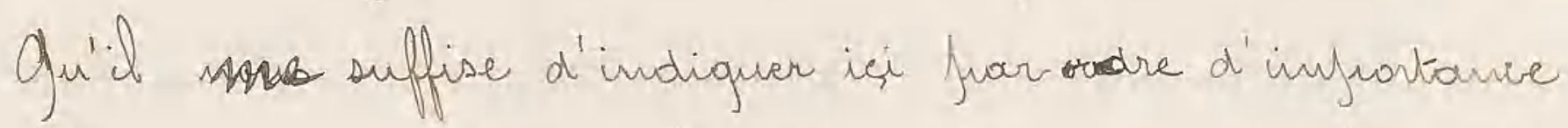
et avee leur nour en Massi, les frincifiales essences de la contrée. Le his le plus apprécié est le cailcédrat ; itiata des Alandés,

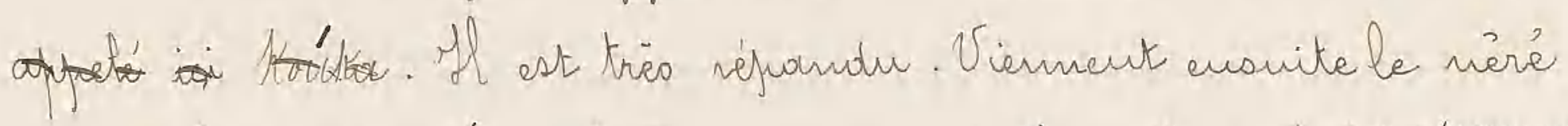

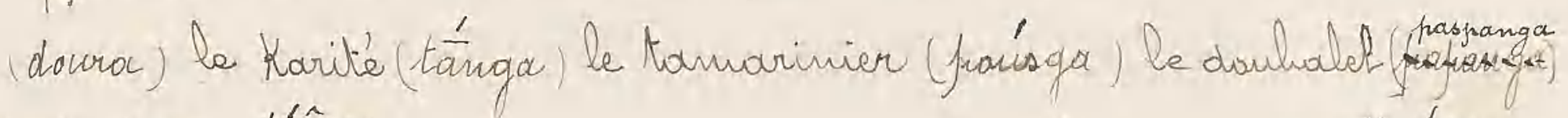
le baubab (tóña

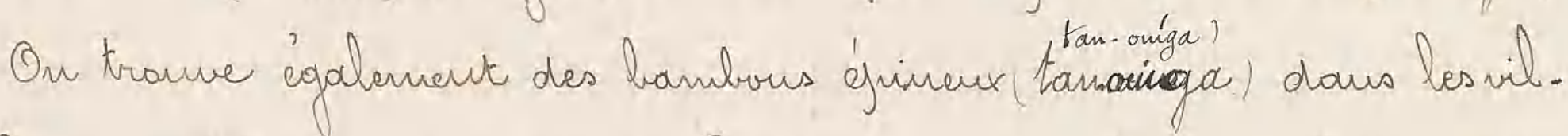

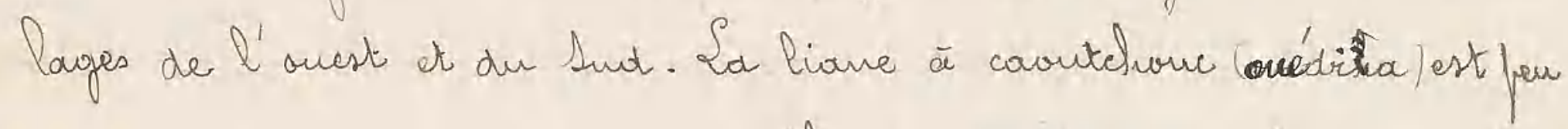

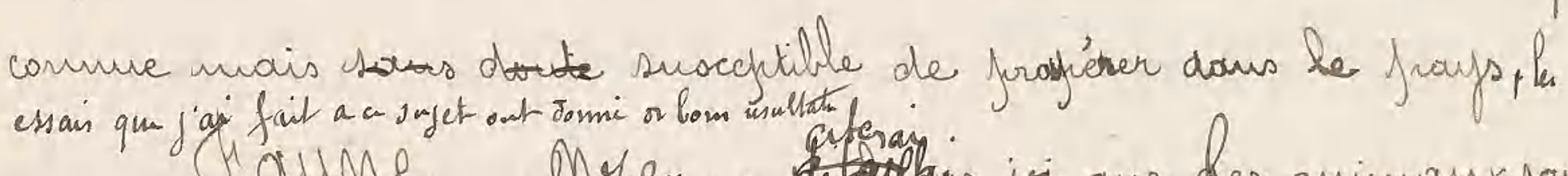

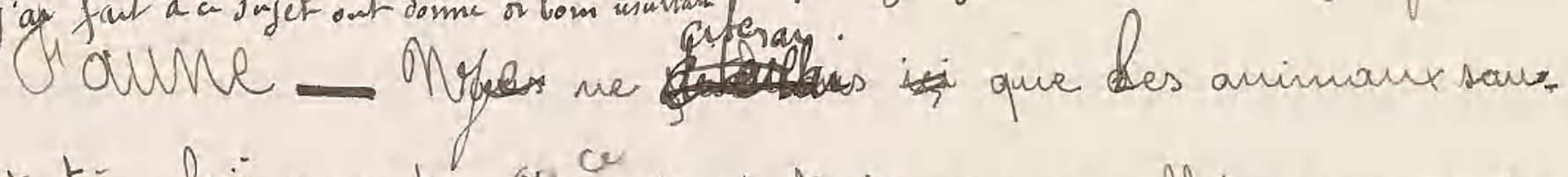

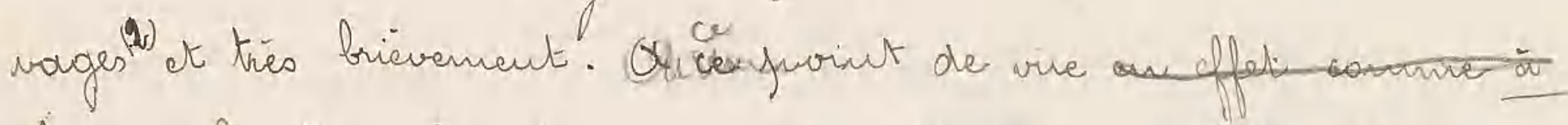

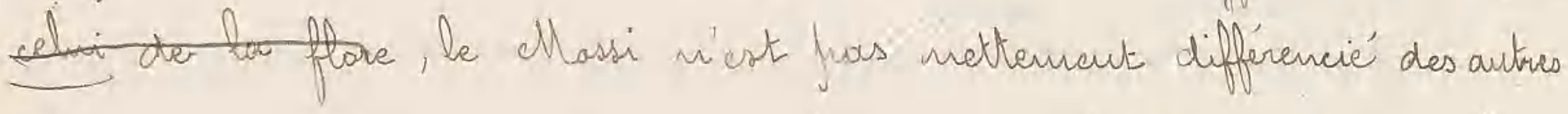
régions sondaniennes sises sous la mianme latitude ot que les travour de Afrécialistes nous out fait conmaitre.

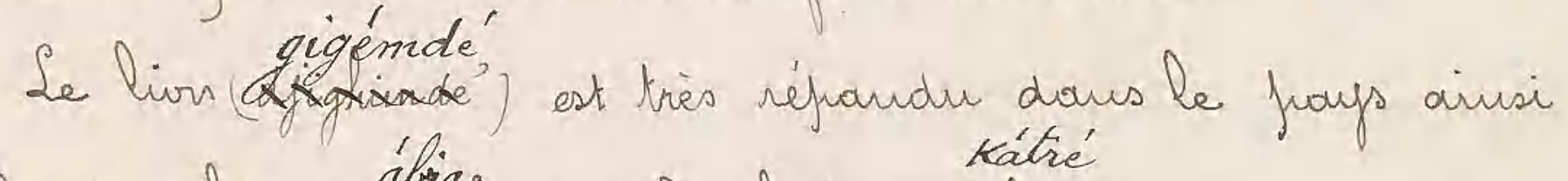

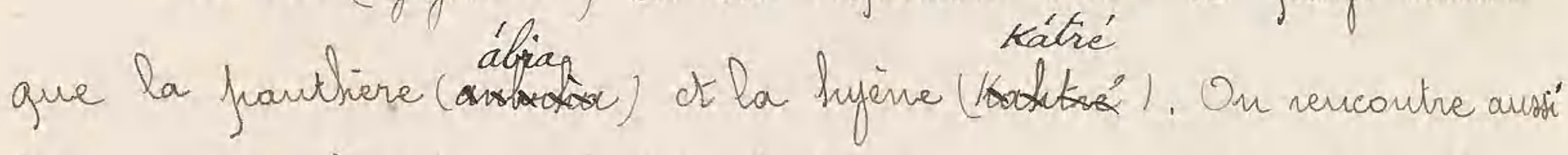

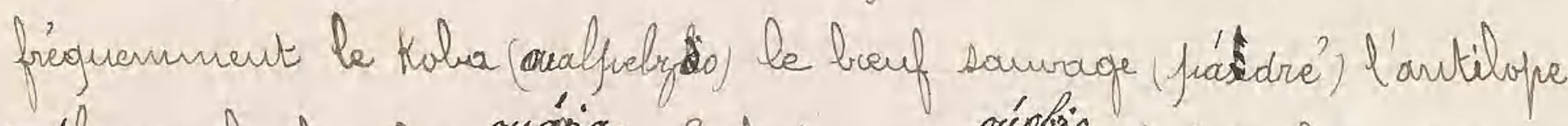

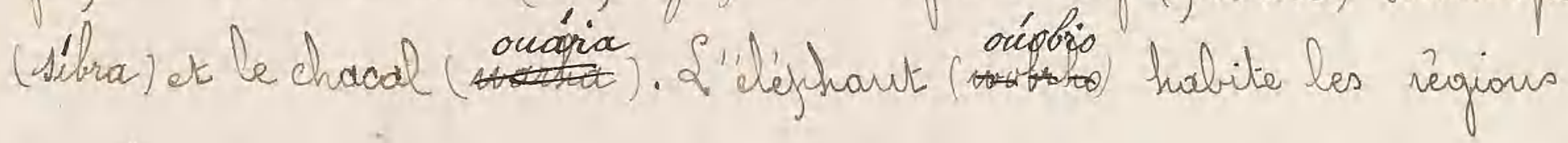

(1) Voin furge 55

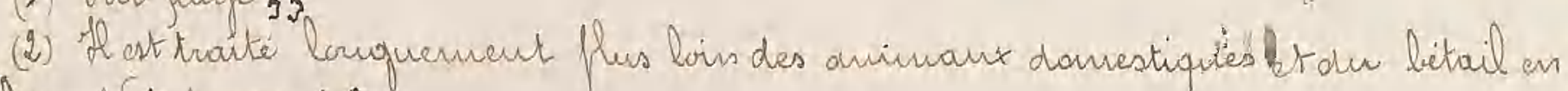
furticilia. Voir fuage 45 
35

desertiques qui bordent les Vaitas, mais c'est à friure s'il cost tré dans tout le cercle une quingaine de tètes faor an. Il ast

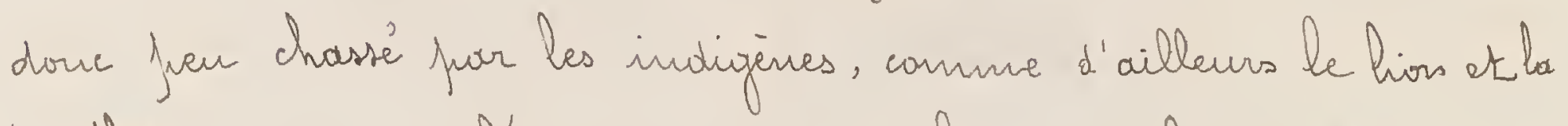
panchere qui nurs l'avous wr sout fhus. nambreux. Ces animana

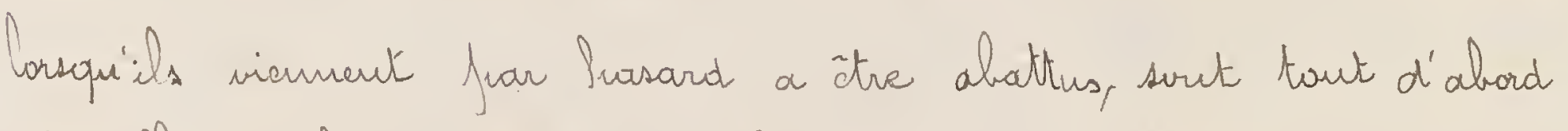
défurrilles de laurs griffes dout les indigères corfectionnent desguis. gris. L'iroire sert de méme à seulfter des siffelts d'avertissemant, une des curiasitis du Kiffriss.

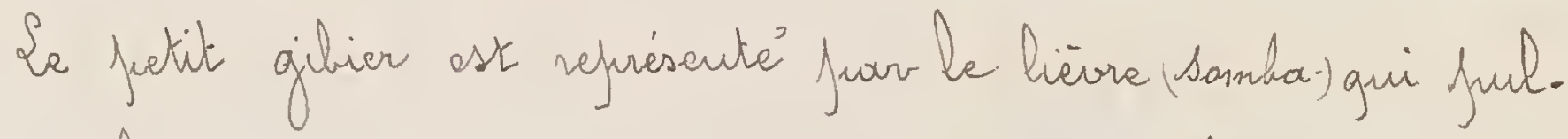
lule dans le fauss ainsi que l'autarde inarnengo la finitade (kango) la peratrix (Kodiénoja) la wille de barbarre (buiré). Infin

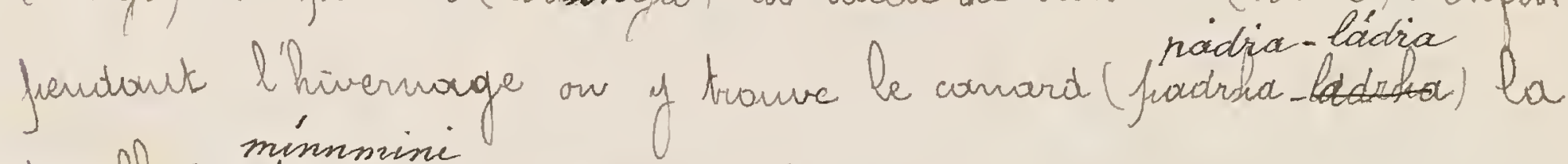

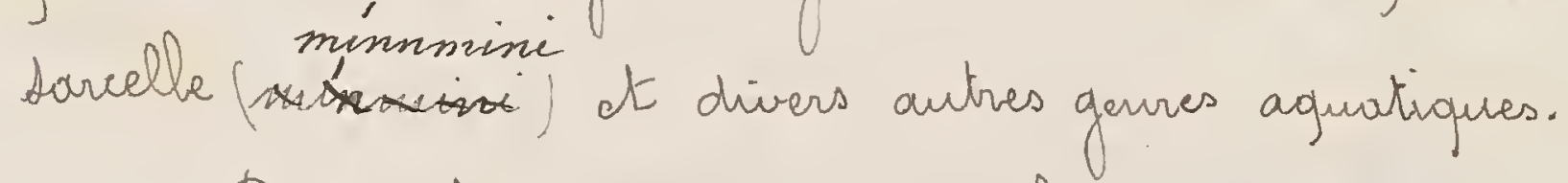

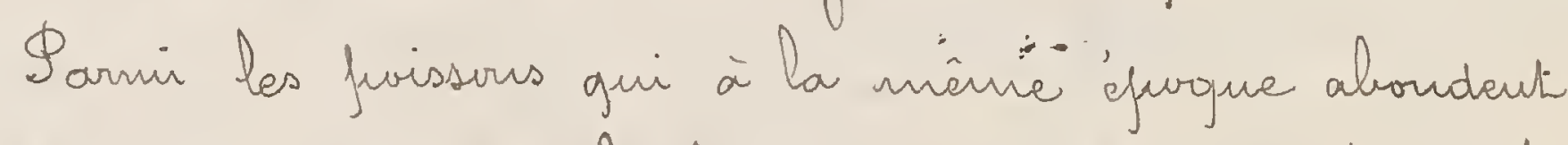

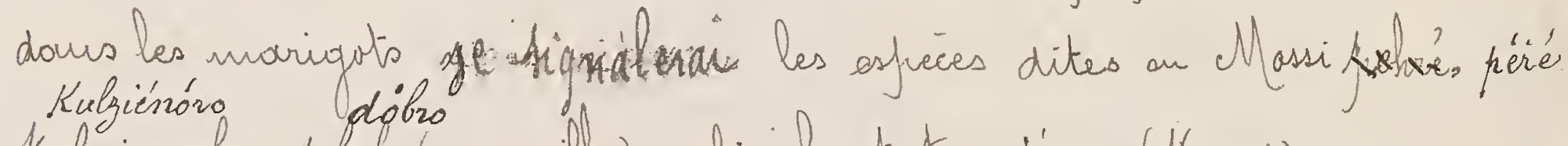

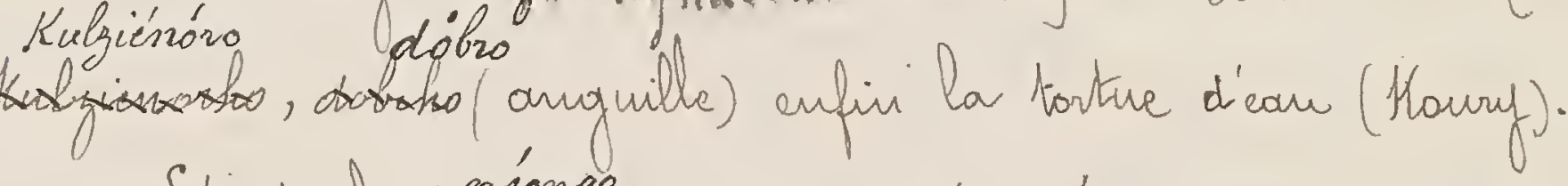

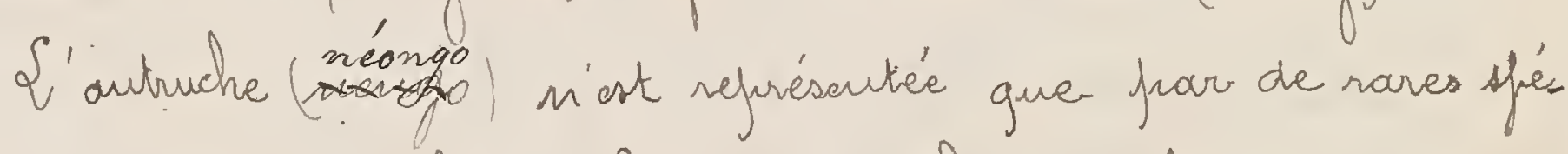
cimerss venus du detors. S'aigrette ot le marahout sont inconmus.

Vaies de communication. Marchés. Condi. dérations générales sur l'état économique actud du prays - Oravoadangacentre de cafitale du Massi est velié ause lacalités frincifales des frays circomusi. sirs faror in réseaur complet de rantes qui date nous l'avous

DSi 
wu de cing ou six ans, réseau lagique, dont le tracè insfirè. your des raisons d'ordre général des quelles it fallait tenia comple, a èté excellemment confris. Ovee Onagadougan cormme fivot, il rayome danstoutes les directions foor des embran. chements la felufuart Kilanètrés avee leurs gites d'étafies fouro vus de canfiements.

Les Eurapiéns dous leurs déplacements les enpruntent' toufours, les indigènes souvent. Mais ce me soutfass á froforement farler des voies ćanomiopues et le trafie me les suit que fior enotroits, car prenout dours les denx sens son arigine andela du Massi, it riy a jas de ravison

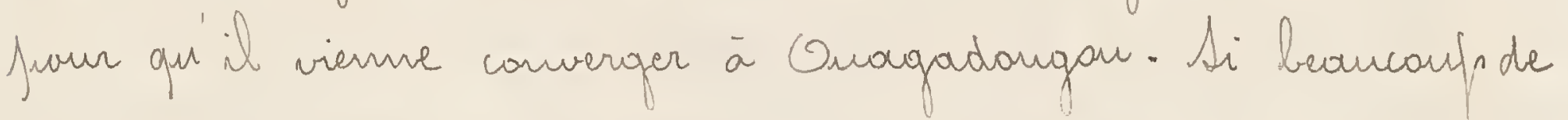
coravones fassent faor ce print - et leur nombre devient de flus en felus considerable $\rightarrow$ it existe aussi des courants commersianse distincts qui allour du Mond, an de l'sst de la boucle vers la Cate d'On, traversant le Cercle darrs le sens de la lougitude en s'efforçant de réalises le chenin le filus count. sout les suivautes:

Les grandes artères venaut abartir à Onagadarigou

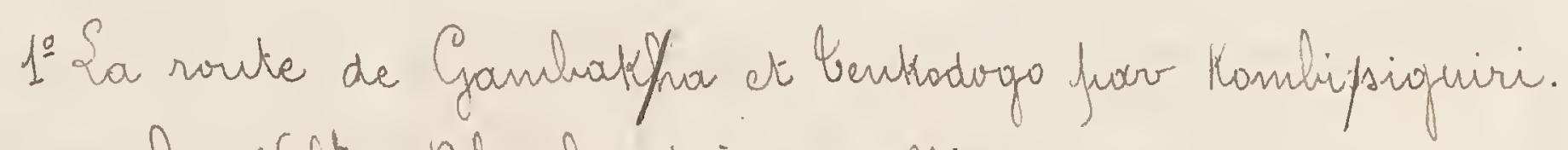
Ule curpe la Valta Blanche pries de Mioggo.

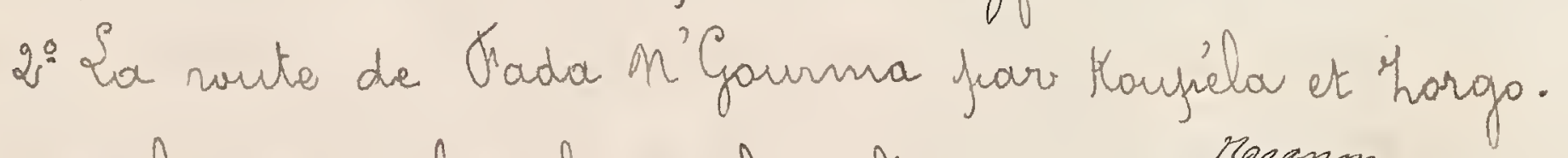

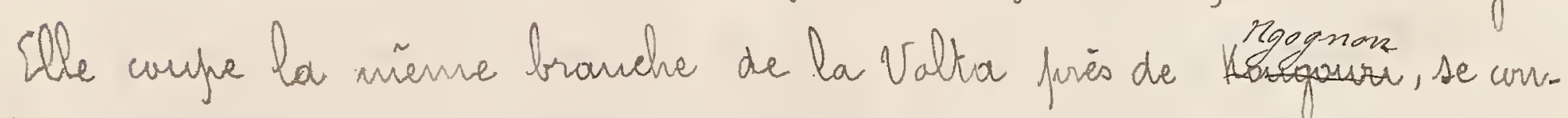
fond à Bangatenga aver la route de B'eloussa et franchit le marigpt de Sumbila a Campilla (deux jouts). 
3. La route de Dori har Barraum dt Baussoumar qui uupe aussi la Valto Blanche friès de Murngou (qué).

I' 2 a route de Mako qui se divise à ce village (hureau télégrapilique dt amien paste) en deux enbranchernents dout l'm se dirige sur Orahinganya, l'autre sur Kaury.

5: La naute de Baho-Oiaulasso fuer Boramo qui curpue la Valta Mvire à la linite du Cercle 2 Kil. Sot de Bowano) ot la Rauge frès du village de Gangue Obiedo (funt).

G- Sa route de Díhangan frour Reo dt Rakay. Glle canpe aussi les deux branches recidentales de la Valta, la Moire à Unessa village situé à l'extrène sud. Onest du Corele, et la Range à 8 kil. an Sud de Raklay (pout).

C'ast à Léo que vient d'autre fuart aboutio route de Ha oua far laumau. Qresque tautes ces rautes nous verrous de le voir, ant èté fourvues de fouts à laurs fisiuts de vencoutre avec des cours d'ear et là, elles sout suivies d'une fasons générale pendant l'fivernage!" Cette saison nend en effet les communications difficiles; le sul trés purméable se kransfainire facilement en marécage, les moindres mairigats forennent de l'importance et ne fervent atre fronchis saus dongon. Ses Mlassif ne construisent mi firrgues ni radeaux. Ee frurcidé de frassage enylayé le plus friequermment (Valta Blanche à Aliago frar exemple) consiste soit en un trane d'arbre soit en un comari vide servant de flotteur à la personne qui re sait juas mager ot qu'un au plusieurs nageurs furssent devout enx.

Oneum des cours d'cour qui arrosent le frougs n'est navigable (1) Jaisos on fluin 
souf la Valta Moire fuur une fartie de son caurs.

Le transit commoncial nifes l'a dit mienfrunte fras mécessairennent les six groundes artieres que ufiers veros de décrire, il feut suivore les voies les plus diverses utilisant les sentiers qui relient entre enx les innanbrables villages du Cercle (1)

La trace des caravanes nous est frartart révélée fuar l'exis Hence des marchies, fuints an fiériodiquennent (tous les trois juns en froys unassi fruprement dit) afflue la pufurlation avoisinantes l'est là que s'effecturent d'un bout à l'autre de l'année, des échanges flus an mains actifs entre les froduits du payss et cense amenís du detors, fliénamérre d'asmase four le quel se manifeste et se developpe la vie éconanique de la contriée.

Biess que sforis que le nanvement commer. cial anquel dle farticife toute entiere ait ses origines dans les deux sens bien andelí des frontières naturelles du chassi, il ve founarait fars croire que les marchandises ne fout que transiter dous le frays. Sa flufarti en effet, notanment celles qui provienment du Mord, if sant bransformies en fordinits naveaux ot les caravarues ayaut pois leur origine en un foint situé vers le sommet de la boude du Mliger (Danentiza an Daraféré par exemple) (2)

(1) Ces villonges sout an uontre d'enniroun 6.500.

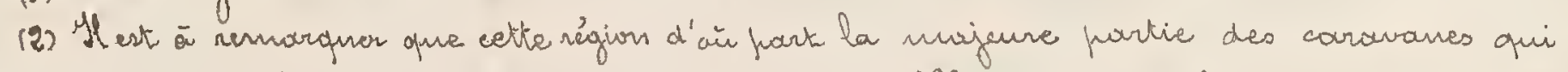

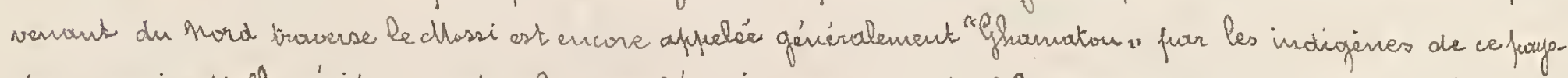

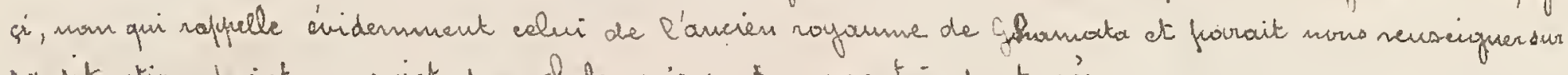

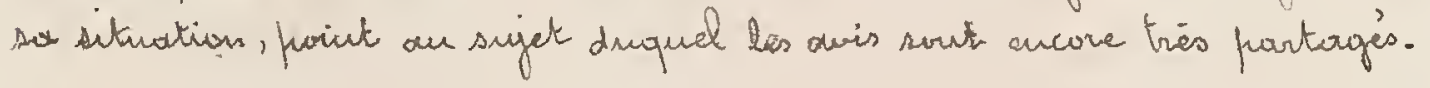


à tour arrivée en Gald Caost, an froint tersinus de leur vayage réalisé deuse afiérations distinctes = le sel natamment guielles affortaient et gui frovenait de Gaoudèni foor bourbouctou, deva être éhongè contre du bétail en frays Massi, car un fren audela du $11^{*}$ farallede se trauveriorit en con. currence aver un produit similaire, le sel marin venaut de la cite et son furix de vente cesserait d'être sémunérateurs Les grands courants conmersiaux vercout du Mord et de l'Cst enferuntent four fievétrer au Massi trais waies essertielles; les marchandises froverant du Haoussa y arrivent far Sanytariga, grand manché de la Circonscriftion de

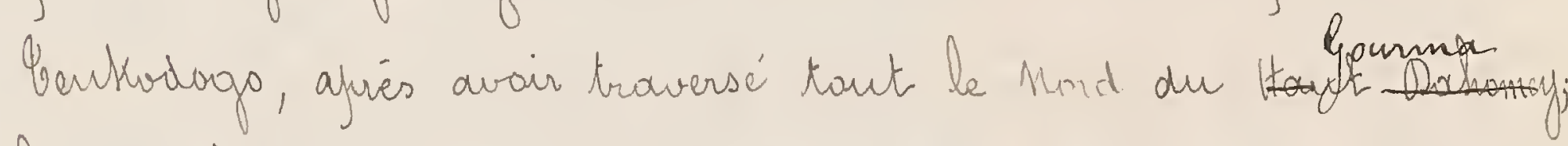
les marebrandises du Oyilgadi (région de Odori) jrenneut la woute de Barraum et de Kaufa, quelquefuis celle de Mavé ct hiteriga que sur. vent ćgalement des caravanes verrant du Afaterga; enfin la najeure fartie des froduits de cette dernicie coutrée fievètre dans le cercle frar afako et Sa.

Les frovenances du And (Kolas an ètoffes et verrateries anglaises) pénistrent dans le corele frar les marchies de Sanga, Bittur. Yonga, Laaga, Bieblé

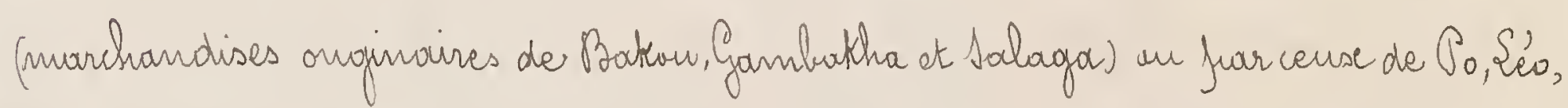

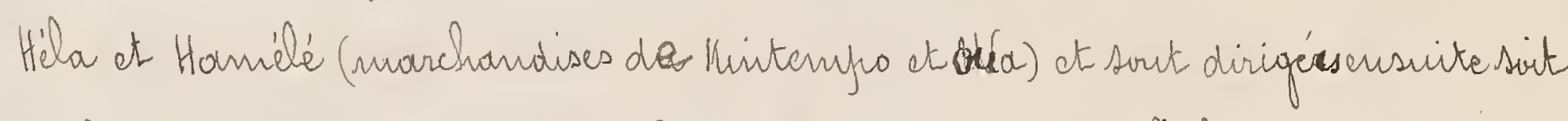
sur le Haut. Dahomerf, suit sur Genkwologo, soit sur Sarkoboutenga, Rakay et Onagadongan, sirt eufin sur d'autres marchés situés à l'ourest de ce furint Jusqu'à la frontioire du cercle air elles se digpersent, vers Bandiagara, Doari te, dans tourte la boucle du Miger.

If aus ne saurios domer me idé plus exacte des éléments 
conskiturtifs du trafie et de leur infurtance relative qui en reforertuisaut les

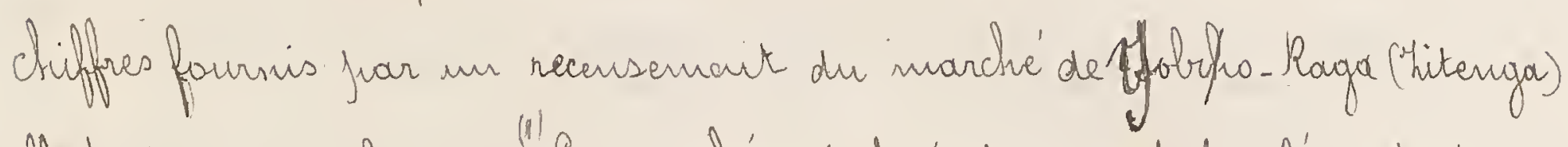
effecturé en Naventbre 1905. Ce marché n'ast évidemnent fras l'un des frimci. founx du cercle; il en existe de ce genre au cheflien de chaque cantan. Ces

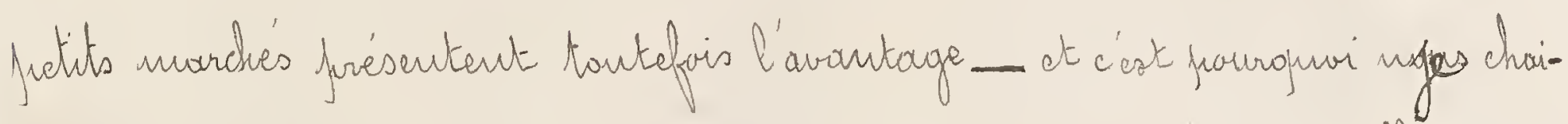

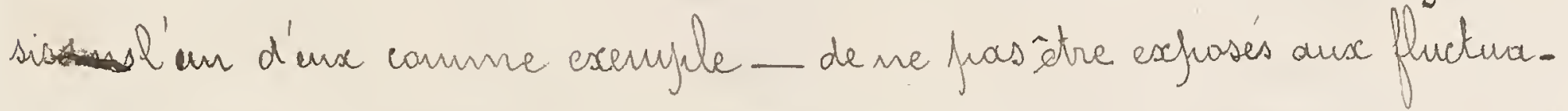
Kions souvent considéralles gui se fout sentir sur les marehés de premier ardré. Le taux magen de la fièee de 5 franes étant à 5000 cauris cela nurs danne afporascinativensent me valeur katale d'environ 13.2. to figuraut sur le marche vers theure de l'afrès. midi moment air il atteint son maximum d'activité. S'équopue de l'année del' ai ce recensement a été fait, se trouve

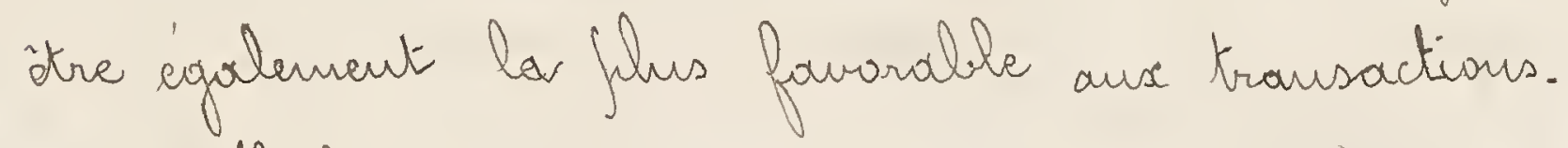
Il faut remarguer que vers la fir de la jaurnée turtes les marefuandises apportées ne devarient fios ètre vendues ct que d'autre frort nambres d'artieles figurount en peetite quantité sur le marclié n'out fras été inventorics. (Barceletsenmar. bre de Hombari, iquingles à chevent on as et en cuivre, bagues,

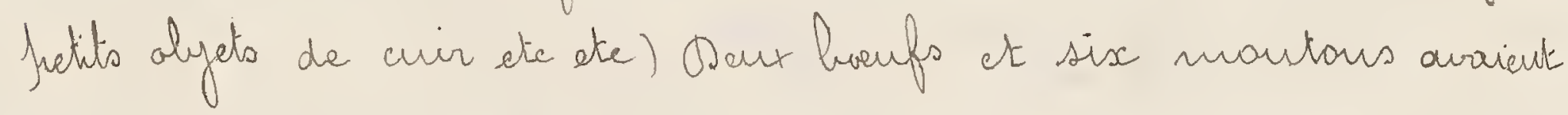
èté dés le matin abattus surbafelace. Sa jerceptioni des drits, d'ailleurs minimae, dtait effectuée en cauris an en nature frar quatre priéfussés dits "riaga-maluas, au service du chef de cauton.

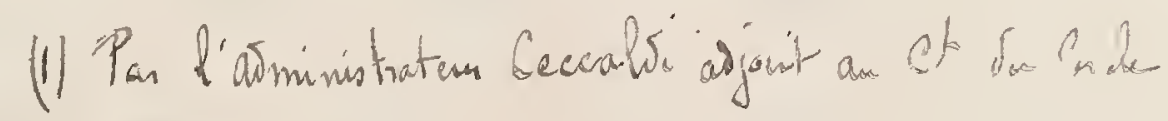

(2) Voir tablean à la juge suivante. 
b:

\begin{tabular}{|c|c|c|c|c|c|}
\hline Oésignations de denviées & Marrs indigénes & Mnutés & $\begin{array}{l}\text { Price en } 9 \\
\text { curvis }\end{array}$ & $\begin{array}{l}\text { Gman- } \\
\text { cités }\end{array}$ & $\begin{array}{l}\text { Valeur } \\
\text { rotale } \\
\text { en cauris }\end{array}$ \\
\hline & & 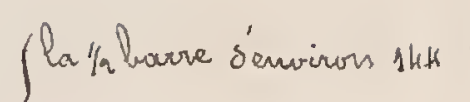 & 24000 & 6 & 162.000 \\
\hline & ganseum & 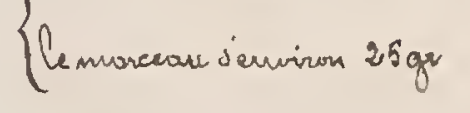 & 50 & 120 & 6.000 \\
\hline unde & Méndo & Rethas diemvirion $250 \mathrm{gr}$ & 100 & $3(0)$ & 30.000 \\
\hline & Moú, & letas d'erwinos $120 \mathrm{gr}$ & 10 & 180 & 1.800 \\
\hline Sa & Hamóndé & letas déeuviross $250 \mathrm{gr}$ & 10 & 2000 & 2.000 \\
\hline achides & Sounkaian & le tas d'envirom $200 \mathrm{gr}$ & 5 & 450 & 2.250 \\
\hline lrouge & Kasega & fromiers à & 500 & 32 & 16.000 \\
\hline nouge germé & haï & fuaniers á & 800 & 12 & 9600 \\
\hline mil blane & Baninga & fromiers à & 450 & 18 & 8100 \\
\hline it mil & Ké & framiers $\dot{\alpha}$ & 600 & 35 & 21000 \\
\hline Giramas & Seso & letas jenvinas $350 \mathrm{gr}$ & 5 & 60 & 300 \\
\hline ure de tharité & kham. & Caldrasses de 10 boules enar & Hen & 25 & 10000 \\
\hline irres de viéré & Dazauna & franiers à & 500 & 8 & 4000 \\
\hline dimente extrait de la friciedente & Mericton & Caboule d'enviran 3ogr & 19 & 450 & $45 a b$ \\
\hline aines de sésame & Sildi & Cetas diemirion fogr & 10 & 200 & Quov \\
\hline ines d'ascille & Bi & letas d'eusion $450 \mathrm{grc}$ & 10 & so & 800 \\
\hline itts poisosous sees & Koulsoro. & letas d'enviran 60 gr & 5 & 4a & 200 \\
\hline Pate de rielés at grains de cotion & Gounné & la juiece & 5 & 250 & 1250 \\
\hline Patre de jectit mil & Kisongar & amiers de go aniriors & 410 & 15 & 6000 \\
\hline Baule siche de petit nil & hoomtiadoun & framiers de 60 envirass & 300 & 8 & 2 hoo \\
\hline 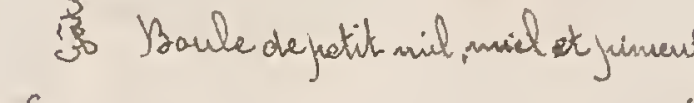 & uthomsidon & la friece & 10 & 120 & 1200 \\
\hline van & Sónfánnidé & laboule de Hugr & 10 & 250 & 2500 \\
\hline has & Gouré & l'unité en mayemue & 100 & 58001 & 580000 \\
\hline andes de cutan & Samdafpermate & lacoudec & $5 a$ & $60 a$ & 30000 \\
\hline mees du frays taints a l'indigo & Fou-los-garo & la frière & 3000 & 18 & Shoua \\
\hline ves energuiens ondinsires & dongon & la juèce & 6000 & 10 & 60000 \\
\hline uféens à desseins & Godore & laficèe & 12000 & p & Sh000 \\
\hline 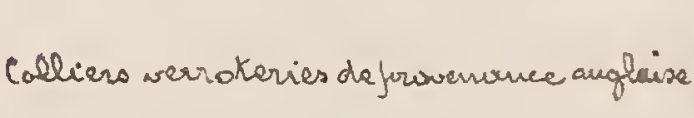 & - Mousánfo & fiece & 500 & 40 & 2000 \\
\hline les verroterics & Mouzanide & la priece & $10-20-50$ & 4000 & Lue 000 \\
\hline Malts lordéco de noir & Gana-débré & la fincece & gaci & 10 & 9000 \\
\hline les indigëres & "utléodné & la paire & for & 20 & 14000 \\
\hline 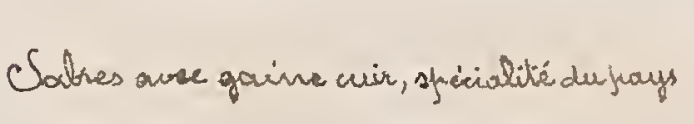 & Sitenfasouria & $\left\{\begin{array}{l}\text { la fricie } \\
\text { la juicer }\end{array}\right.$ & $\begin{array}{l}1500 \\
2000\end{array}$ & & $\begin{array}{l}301000 \\
160000\end{array}$ \\
\hline & Banga & l'mité prise magen & 35000 & & 245400 \\
\hline tous & Pesro & l'unité prix magen & 5000 & 25 & 1.25 ant \\
\hline
\end{tabular}

qotal.....-1592,900 
Mages dotys aifin ajouter que les transactions infrartontes concernaut jour exenyle les barresesal charges entières, le gras bé. tail, eter, se faut dous les cases prar l'internédiaire de courktiers, st. non sur la filace. Ces opierations revetent naturellement un caractere. exceptionnd.

Ohe question se fuse maintenout, sur laquelle il ne sera

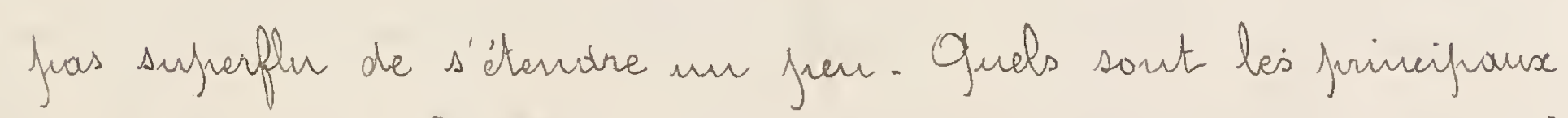
agents du trafic? oir mieux; qui détient le commerce du froufs? D'une facens ginérucle, le nonofule des transuctions a

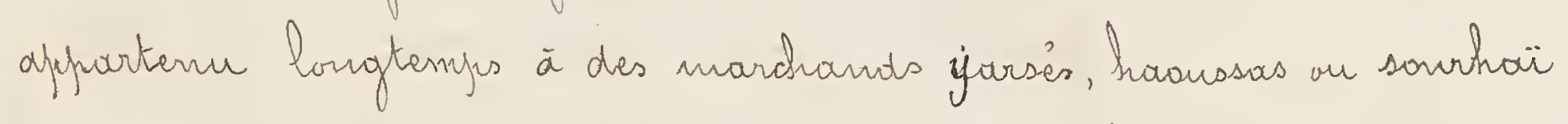

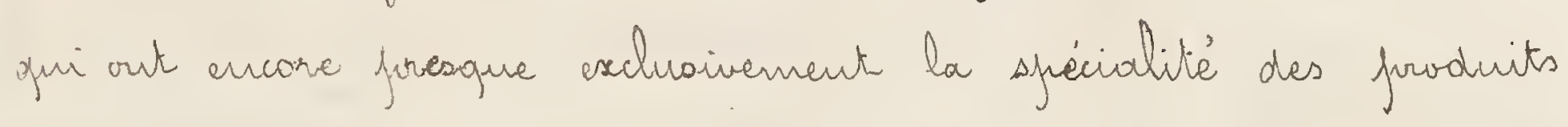
manufacturics aic riches verraut du defwars. Garfaitenent apte

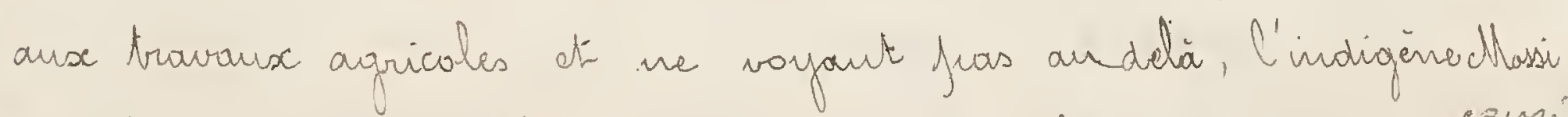
était trop forté foor l'effet des kendances rurales ct sédentaires, enits d'une longue hiéridité, qui sont an foud de hir-même, à vair d'un ceil indiffirent differ sur ses rantes les nambreuses carruranes que conduisaient fiériadiopuenent ces "drangers; il se contentait de tirer un mince porofit de leur frassage, mais n'y voyaitaw. cun enseignement ot pendant des années eet cxenfile a itéf fordu jour hir.

Dours ce parys que sillamavint en tous sens les voies d'écort lement de son jorafere bétail at d'une foule de produits exoti. ques, l'autochtone s'olstimait daus son fietit commerce de grains et de condinents indigènes et vendait torjaurs ses troufreaux sur plove à un porix dérisaire. Catte contrée fut longtenups ainsi 


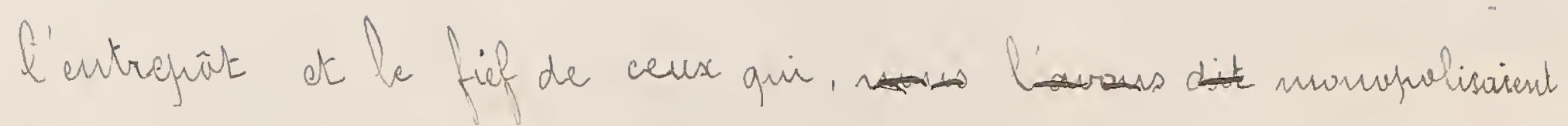

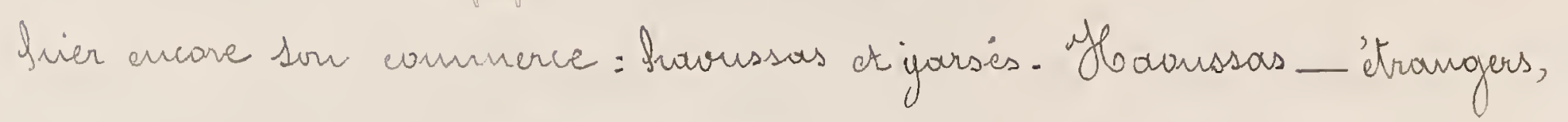
n'anaut d'antre frérecufrations que d'acquèrir une aisance dont ils irout fain an bin; jarses _ nalitant le frays depuis longtenys il est vad, mais ayout gardè nue àme ítrangère, fresque hastile, nusulmans ne fusiamant pus avec les infidites et

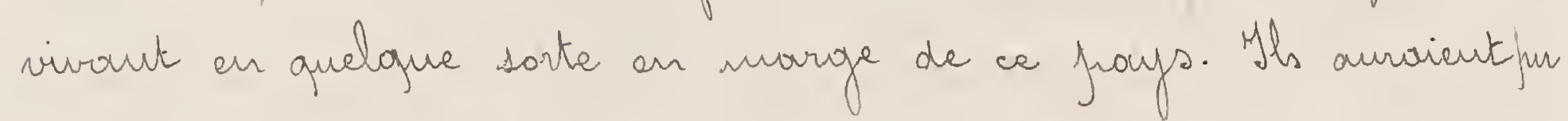
foor leur aptitude au trafic ot les entreforises incessontes auxaguelles

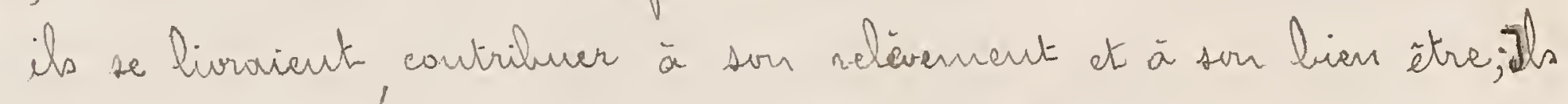
n'out foit que le troulber far l'agivtage et l'usure.

C'as fourguioi ufeso n' cessé de conseiller ause Massis de s'affrunchir do ces interniediaires pen serufurleux, laur faisant vair tout l'aurantiage quils fouvent tiver du trafic direct avee Gold. Caast, laur démontrant if." de telles apieratious, dout laurs esflaiteurs exager raient les alcias of les risques, freuvent à notre éfroque être entregrises en toute sécurité.

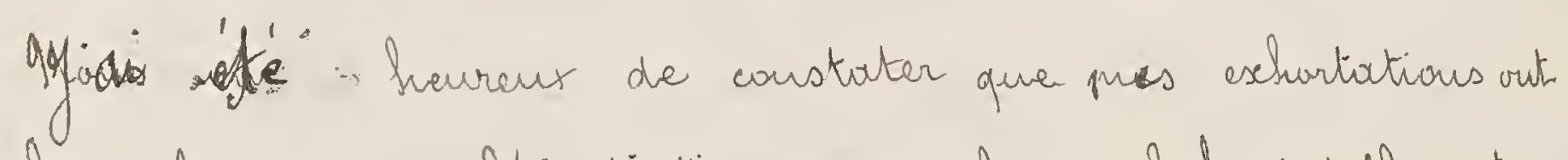
parté leurs frivits, sur l'initiative de quelques chefs intelligents cette feratiqure nonvelle s'est géréraliséc à tel joint que nous nors trawous aufourd'Sui en friesence d'une véritalle transformation dur farys an fraint de vue economique. Ses Massip qui figuraient furur noins d'un dixième an nombre des calfartaurs pratentés out abteint l'année demieire (1go6) la forafuortion considionable de 5thour cent. 




\section{Ses productions du parys Mossi}

\section{Bétail - On seut waluer à gsoootêtes le nambre} twat des bourfs d'estieces diverses vivant actuellement aucthassi. mais ce chiffre doit atre considéré, comme apprascimatif étout domié la vaste étendure de territoire sur la quelle est dissé. minie la fufurlation Lavine do l'infussibilité daus la quelle on a 'té Iusou'isi, d'en dénombrer l'ensemble.

¿'dève du bétail atait autrefais le monofule de conmunau. tes heulhes venues au Mond es de l'ist auxquels les chefs Massiz confiaient leurs tranfearux; cette situation s'estr nudifiée et les

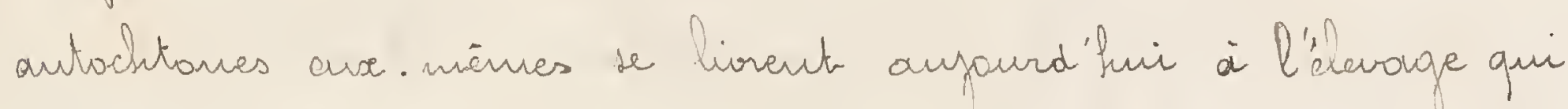
faurnit le frincifal éliment d'espartation de la coutree. Le benf est en effect destivié surtout au commerce avec Gald. Caast; cette passession anglaise we produisant fras de bétail en enffrunte chaque armie aur Mllassi 5 an 6000 têtes utiliseics pour la baucherie. Sa viande cst de bolle qualité at d'asfect tout.à. foit affítissant mais gériéralennent un peur forme. Il se fait égalenreut un commerce de beufs à bosse destinies

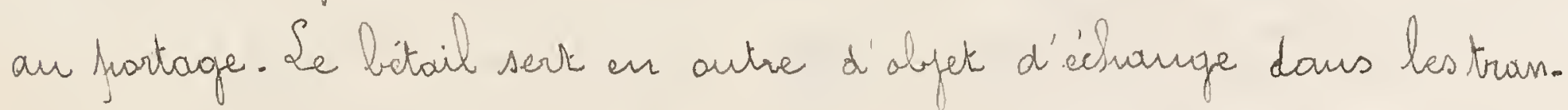
sactions locales. Sa production du lait est assez abondante quai. que linitie aux seulss agghamerations de Serilhs quile consonment at en font un beurre agricalble que l'ou pent trouver à l'ctat

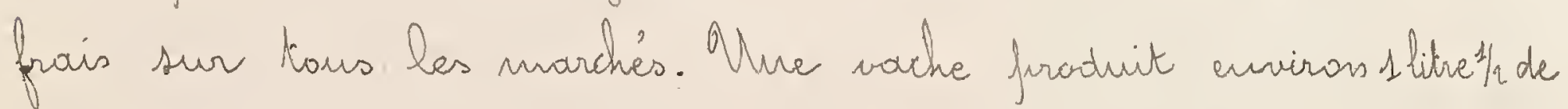
lait chaque faur mais senlement pendaut la jrériode air elle mourrit sou vean. Les pris de vents dur gros létail sout trés 
variables; ils ascillent au Atasi entre 20 ot 60 fromes funur les

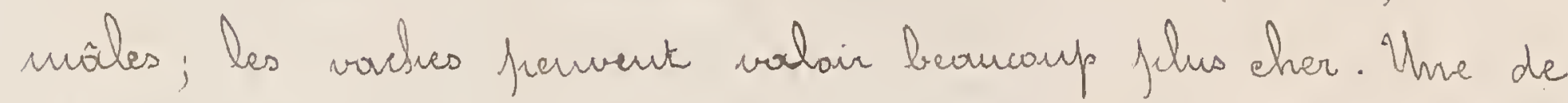
celles-ai avee un tout jeunse veour vefrisonte la valaur de deux adultes.

Se frise des riènes animause angunante an fur et $\bar{a}$ me. sure qu'ils descentent vers le Sud. Daurs la Gold. Caast ils seront vendus 80 fromes à Batiou ot à otla; 100 à 120 fromes a Kintan.

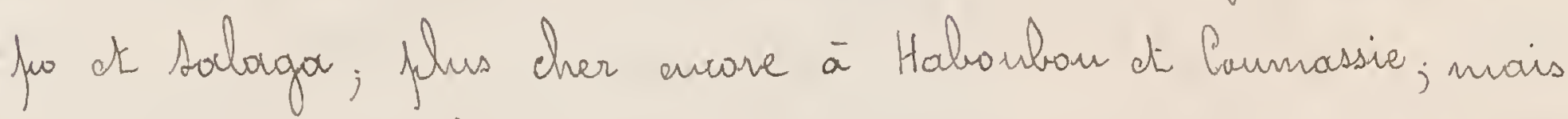
funer arriver là il auro fallu daux mois de route à travers

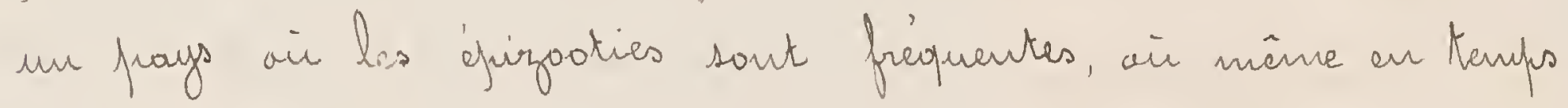
ondinaire la santé du bictuil jérielite facilemant.

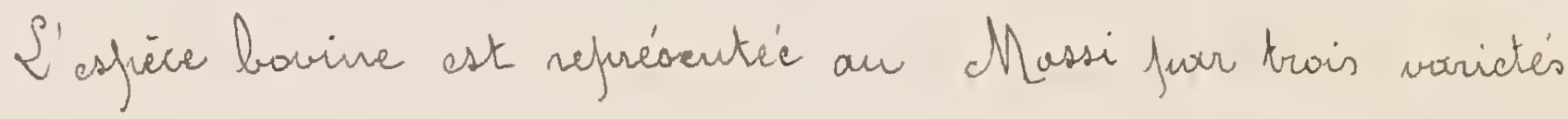
ininifuales:

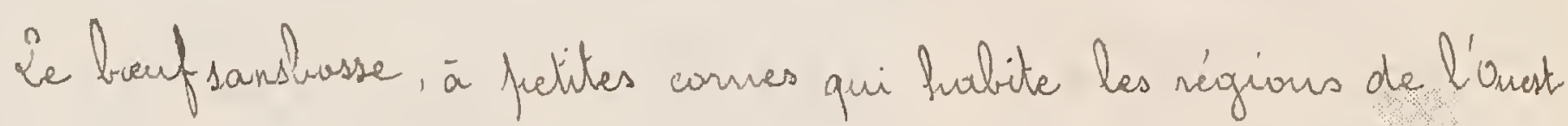
ot du Sud. Onest, et qui art désigné connminénent dous le fauys saus le nom de breuf du Kifpirsi. Inapite aur partage, il faumit une viounde de qualité inférieure, maisoldrancoup plus nésistants que le breenf a busse ot s'acelimate plus aisement.

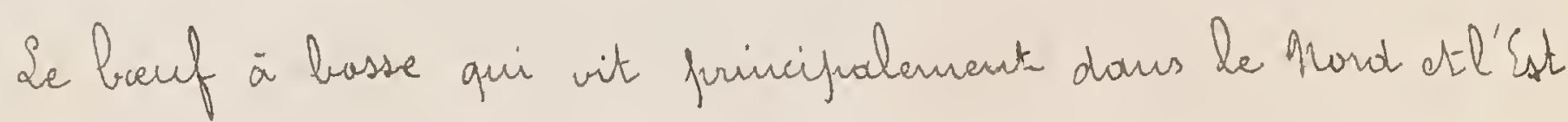

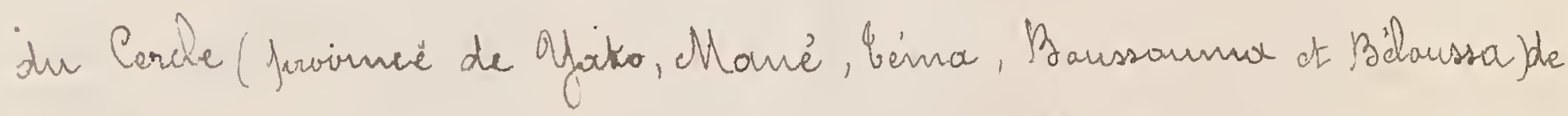

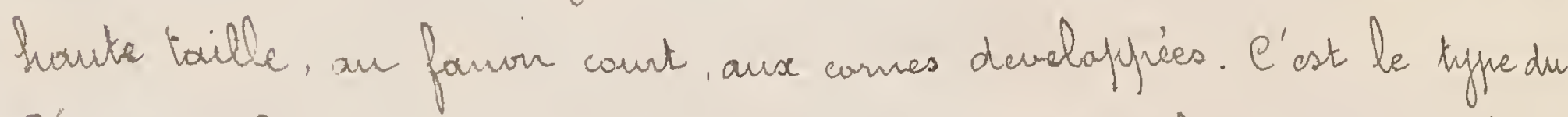
bémés on Gourgan - nissie que l'on retrouve daus le Kaminiandougan

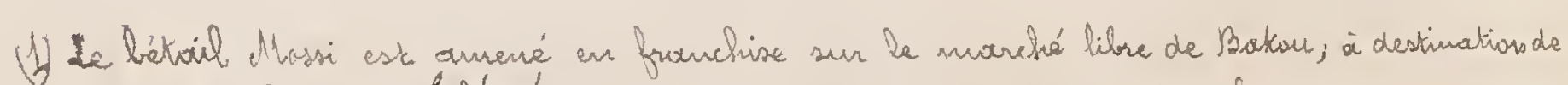

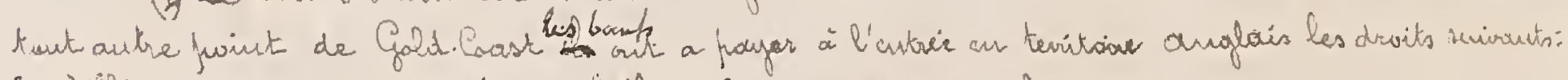

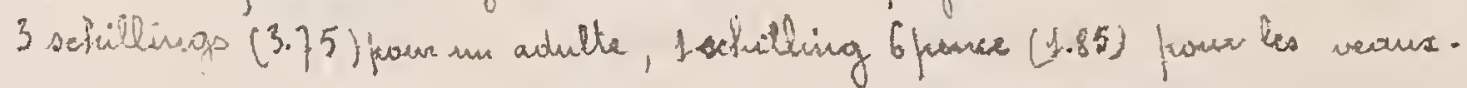


st le Guénickalany. Barfuitement afite au fortage, it fournit en antre une viande de baucherie excellente.

La troisierme varièté, le bouf commun fuarticipe des osfices frécedentes ot provieut de leur covisement. Il a les qualités de résistance du bouf an Kiffirsi et sa taille est sensiblement plus èlevée que celle de ce dernier; il ost en outre susceptible d'engrais. sement ce qui le rend propre à faurnir une viande de tries bonne qualité. Cette warièté damine an sud de la furovince de Bidoussa do daus la régions de tentodogo.

Le mayen d'anielionation fréconisé serait la sélection et la castration des bietes inférieures qui domeraient ainsi de meilleure viande. La castrations est d'ailleurs commue des indigenes et pratiquée fear enx, mais indifferemment sur des sufets quelanques.

Dans la circonscriftion de benkadago il n'existe fats de ma. lanties particulières du bétail mais dans celle de Onagadorigor

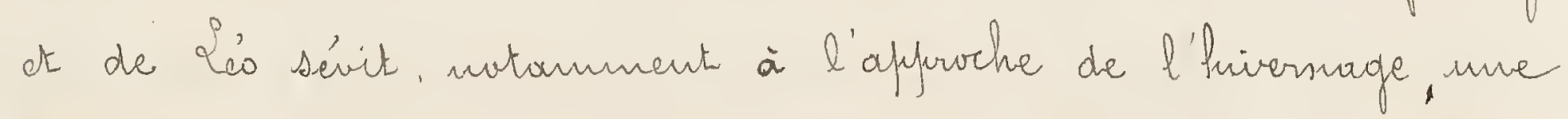
affection endiemique aufart les caractères gériéroux du nagjana: l'animal défierit, cesse de se naurrio, wit la faralysie ganguer l'arrière trouin ot meurt. Cette maladie frarait attribuable à la

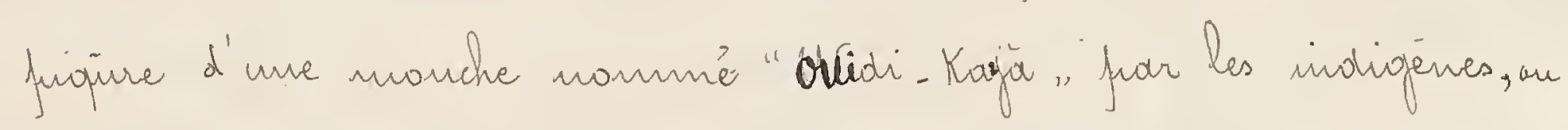
à une larme intestinale que l'animal alsorberait avec le fonorage see. G'aiconstaké en effer qu'ar moment ui le bétail refurend le régine vert l'dxat sanitaire s'anielione. Sa cir. couscriftion de Benkodago at la région de Billoussa, la

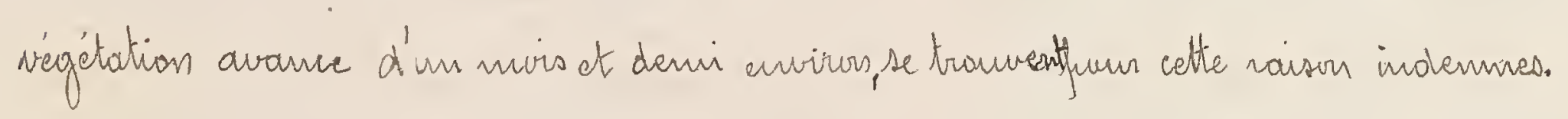


Le fuctit bétail ast assey abondant dans tout le cercle ou it refrésente 120000 tetes environ. Sa chair en est de qualité

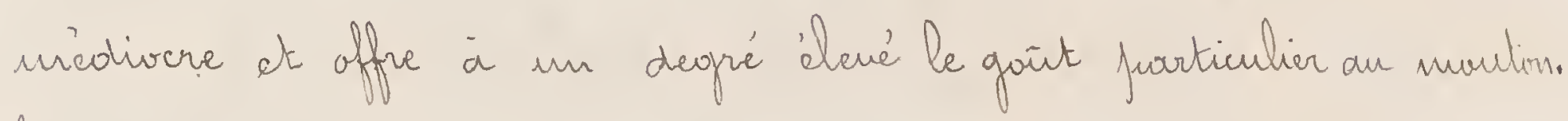
baus ces animaux sout à fuil ras ot de fretite taille, exceftion foite des nourtous dits "de case, 'elevés isulement sirivont l'usa. ge introduit an Massi far les Mandingues, et engraissés avec soin fuur être abattus ause guaques fostives.

Le boit n'est fuas recherche; Ce Commeree des preause ast insiguifiant. Se juris mayen d'un manton varie de is a 5 francs.

Oninnarx de bät _ Oe jetite taille mais viguer. neuse ot resistants, les ânes sout an nombre de 15.000 environ dours tout le cercle eir les indigines les utilisent frimcifualemeut fuer le transport des fardeause et nourtent eux-mienves sur la croufre.

Daus les provinues au sud la viande est utilisée fueur l'alimentation. Le frise noyen ost de 20 a 35 fromes frour un adulte to deffasse raramant 40 fromes.

S'industrie nullassière ast iquarée dous le cercle air dle faurrait domer saus nul doute de borrs résultats si l'our en fuge faar les rares sficcimens introduits our cllassi ot qui s'y

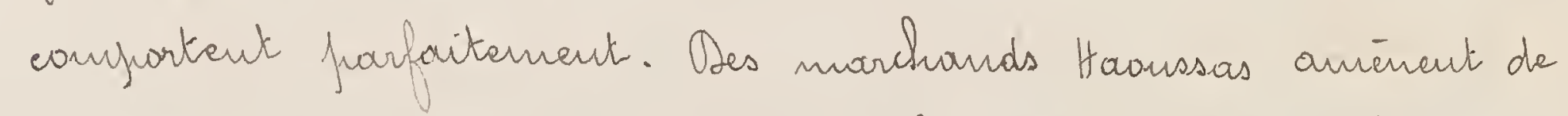
temps en termps aver enx des munlets originaires du bototo. Ces aninouse qui faraiosent eitaffer ause abteintes du nougana quitteut le frays à la fir de la soisons commorriale.

Grimaur de selle - Les négions sises au sudat à l'Onest du cercle parraissent feer favorables à l'elluonge. 
Le cheral, qui se mantre fartant, aussi biers que le bouf susceptible d'àtre atteint far le Magana, semble l'ètre d'avartiage surs cette latitude. - O'autre frart une éfidèmie a foit fiérir en 1goo tous les cheraure de la circonseriftion de Seo air il y en await un grand nombre avourt cette ajuque. Les Gourounsip cherchent defuis lors à efaire Leur couraberie ot n'y arrivent que périblement; ils fass-ident aufourd' hui nue centaine de chevause environ tous ve. uns dur dehors.

Sar contre le Mord et l'Est du Massi frodrisent an abondance des chevaux assez réfuttès qui afferartiennent à deux races frarfouitement caractérisées:

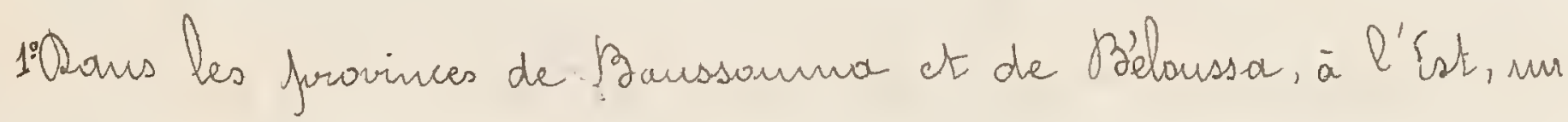
chural d'assey grande taille $\left(1^{m}, 56\right.$ a $\left.1^{m}, 60\right)$ 'égant, aus attaches fives, aux higues rappelont le véritable aroble. Ces chevoux moutés de trop bonne heure, sours aucun menagement for les iridigenes sout vito usés.

2:Dans la fravince de Ofakto ot celle de Marré, Geina et Hiten. ga au Aord, te cheral cllassi froperement dit d'assey fetite taille. mais rablé, viganare, résistant ot sobre.

Enfir ane troisiène race confines actucllement daus be Banssangcé, an Dred, mèrite une mention frarticulière. Aloirs

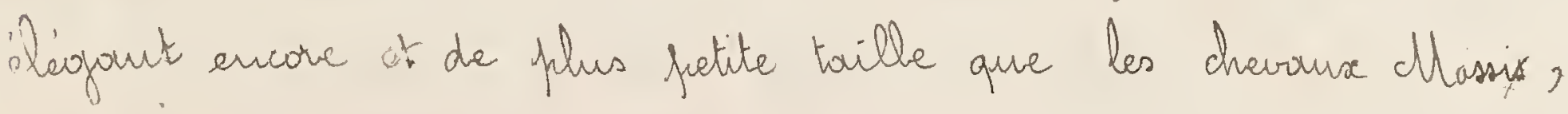

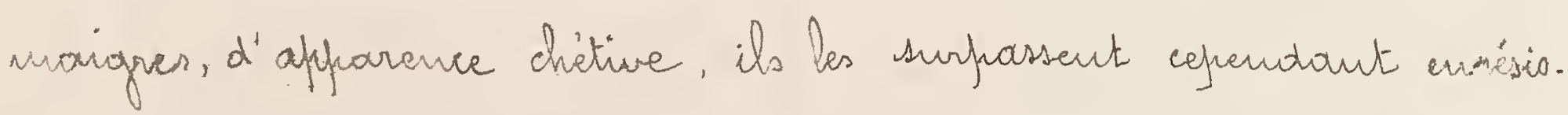
tance et en vigueur.

Se forise due cheral Bonssanga n'atteint quère en moyenme

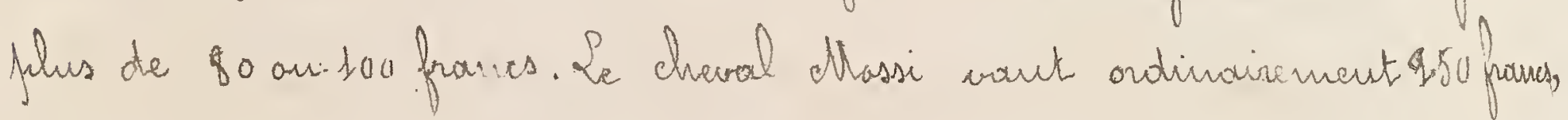


celui de Borssauma ot de B'́loussa que nurs avous cité en fuemiere ligure de 2 \& 0 à 500 fromes.

Les indigienes bien que montant d'une freion déplarable aiment et soignent leurs chevause. Se juausouge ast fraatique', stom.

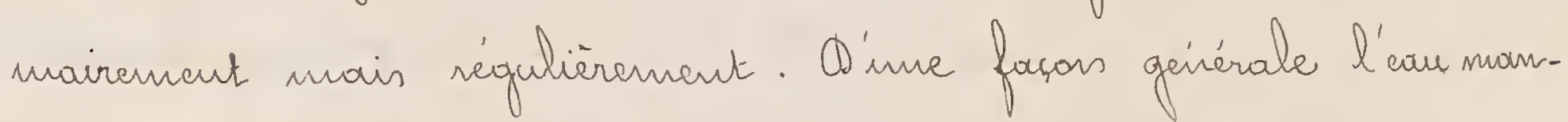
que dans l'hygieive du cheral et est administré saus discomement de qualité.

Sa maladie la shlus courante à laguelle il sit sufet hiv ost commune avec he gaus bétail et ufieis l'a décrite plus houts en traitant de l'esfice bovine. Slle est carracteriséé audé. but far l'inapéteance suivie de défírissement geñeral ot enfir faor la faralysie de l'arrière. train que ne tarde fas à simire la mont.

Les indigines ne fussedident ancune mictication vriment

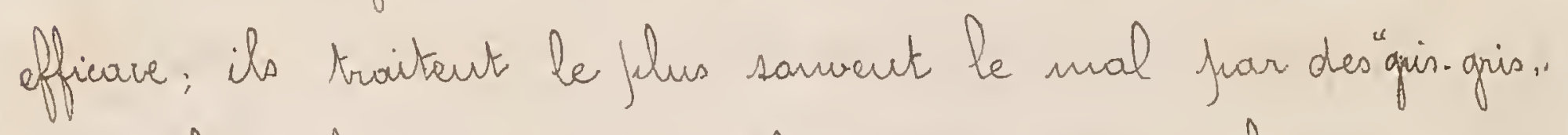
ot des formules madgiques qu' ils accompargent parfosis de furga. tious oiv entrent des cendres alcalives i de Do de

D'une faron générale la dégánérescence de la rave doit ôtre attribuse à l'alsence alsolue de sélection qui nuet les saillis a la mersi du hasard, les animaux fräturante of se neforoduisant presque toufaurs en libarté douns la brusose.

Ses suferstitions relatives an cheval sout nombreuses of on Ces netronve communement en fraps Mandingue d'oir elles dusiont

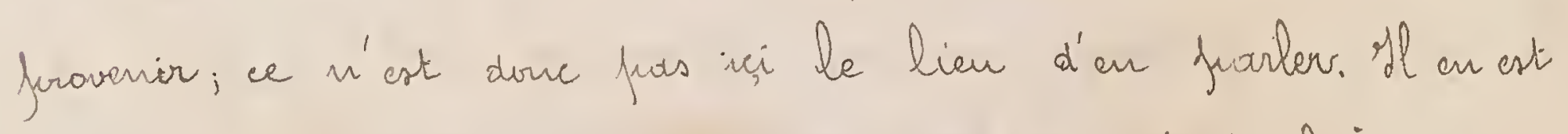

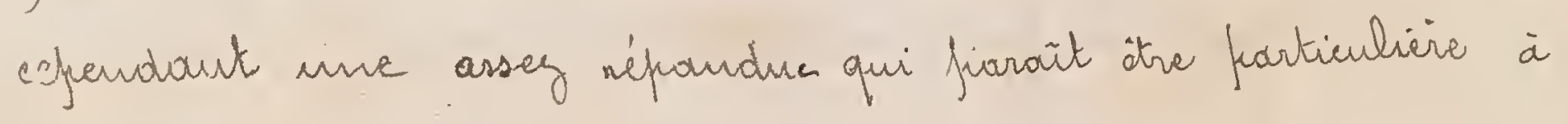


certaines régions du Massi; elle exige que le cheval destivéa la dot d'une ferrune soit fréalablement estuangé contre des boufs, les indigenes dant convainass que le fait de foufer directement une dot avee un cheval determinerait la stóri. lité de l'union airsi contractée.

Haurrages et matières proppres à la nourriture des aninnaux \&n dehors des filantes virieres dout il ast question filus bin wir tablear sypaptique) les animaux consomment divers fourrages naturels dont le plus

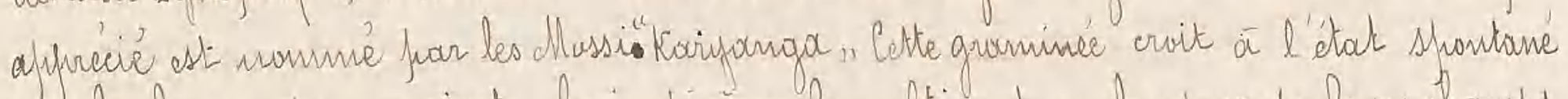
sir les berges des marigats; les indigenes la cultivent an bordure de leurs chanups

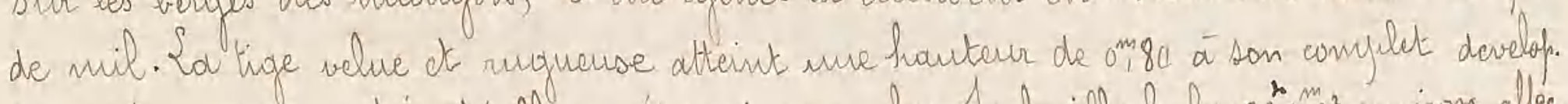

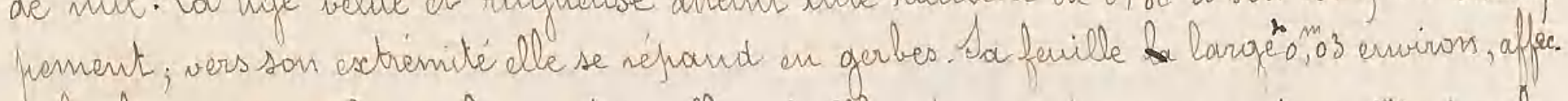
te la forme d'un fer de lance thès allangé. Glle est canprée encare verte à o'vo odu sul,

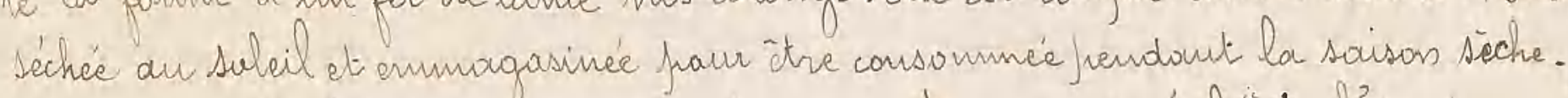

On frent mentionner aussi une autre graminée le 'kérnibédo, (num Massi) gin fursse en tauffes de an 30 de hauteur dans les fouds himides. Sa fenille est feu dévelofprée et la reculle faite connur la préédente.

Viemnart ansuite les fourrouges appartanaut à la famille des papilionacés : le

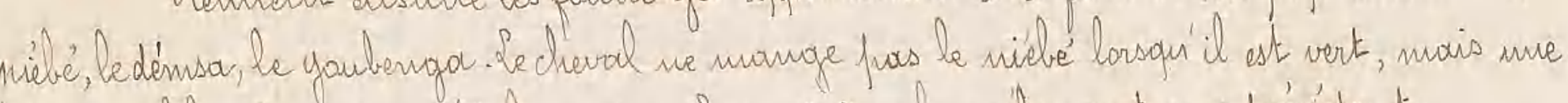

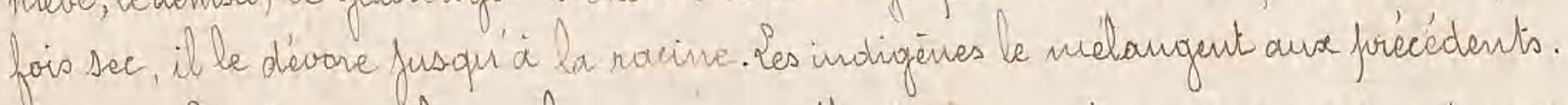

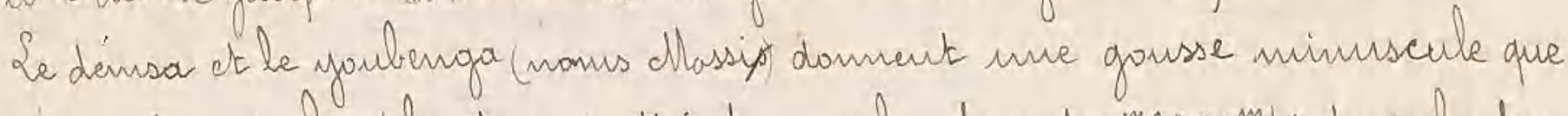

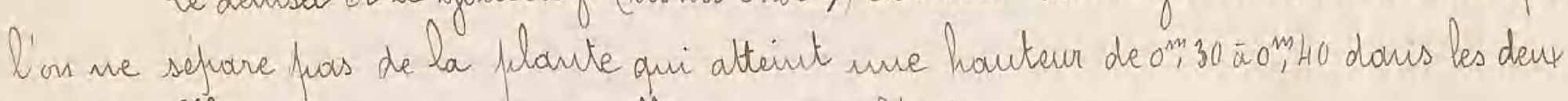
variètés. Glles sourt consonmecs indifferenument à l'dat vert au sec.

D'une manière géviérale, toutes les graminies, nième les ruseause corrstituent une excellente nourriture hour les cherrux à la condition de les coufrer avout

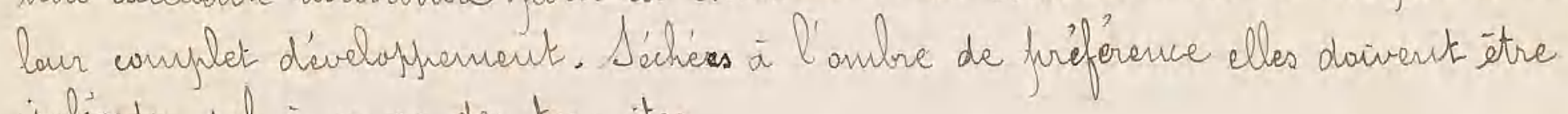
isolvesdu sol à cause des termites.

Apiculture - Se Arossic fowduit une certaine quantite de niel qui ast consanmé foar les indigènes; la cire est considerée conme dechet et demieure presque fartantimu. rilisée. Ceste anvée an commercout a fur s'en procurer à Onagordangan en fetite quantité an fris moyen de 0, 35 an 0,40; bien épurée et de teinte claire elle n'a fanais défrassé i, 50 le kilo. 
C'est un commerse à créer, mais à l'feure artualle il est infussible de domer une idée du chiffe que la froduction serait susceptible d'atteindre. Sa recolte se fait tious les derse mois, souf en hivernage; chaque ruche don. ve environ hrit kilas de froduit entre nicel ot cire; ces suls. rances sour séfariés par mirlaxation daus l'eau chaude aie fe miel se dissout.

Peaux - Oans certaines régiours et notomment dours la nayeure partic de la aiconscriftion de Leo les feaurs de chiores et de moutous constituent l'unique vête. ment des indigères. Sartaut ailleurs elles sout enployées à la confection de sandales ts de bottes, de sacoches et de gris. gris. Quroique cette utilisation reviès un caroutere assez géné. ral il ne se fait fas à probrement farler de commerce de peourx qui n'out quelque pain qu'une fois tannies: elles repré. sentent alors nue valeur de 2,50 purr les peaux de bouf, de $0^{f}, 75$ furur celles de mouton.

Ou praragraphe in il ast traité de la faume ajpais farlé déja succiritement des défrarilles d'animaux savvages. Ngeges in if revicutrans fras.

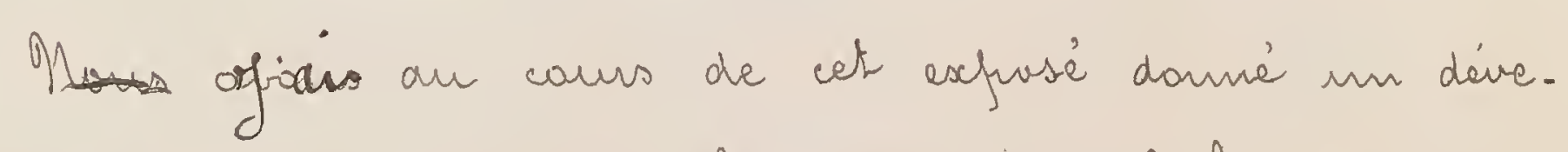
lofpernent tout farticulier à la questions de l'elevage ot aus foints qui s' of rabtachent inméediatement. C'est là en effer le princifal ćlement de la prosprérité actucle du palfs et 
il parait susceptible paur l'ourenir d'un accroissement considerable.

Bien que deskiné fear so nature mène à me jomiais sortir entierement des mains de l'undigine et à solliciter de plus en plus l'activité des autochtones chassip gui s'en sont longterups désintéressés, il est fermis de craire quil we tardera fas à amener dous la régiors des colous Curapiéens et daviendra l'aljet d'entrefurises rationnelles qui we fuurrant manquer de farter des fruits. Sa société "Sa Soudorraise" ètablie defuis filus d'un an à Manè an Mond. Ist de Onongadougan, constitue dars ce sens me preniere tentative.

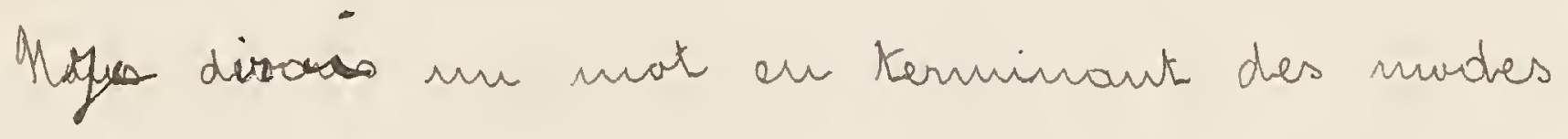
d'eilionge et de la situation du froys an fuint de vere manétaine.

Le bue frimitif_marchandise coutre marchandise - a été emplorfé fusqu'à ces demières annies à l'coclusion de taut autre furvédé, larsopi il s'agissouit de transactians de quelque infrortance. Th breif ètait celsongè contre une barre de sel au Mard du Moser, wutre une froution de celle. ci flus aur Sud. Cekte pratique átait gériérale mutatis mutondis bien entendur.

Ce n'est que defuirs deuse axus environ que le muné. raire argent intervient dous ces transactions. Clles gagnent ainsi en commodité ce qu'elles out fuerdu en fittoresque; les diaulas s'en rendent compte at dours quelques annies letroc aura vécu. 
Mais à cité de ce conmerre d'animarx et de pro. duits riches il en existe un autre, qui, quaioure frortant sur des darrées vulgaires: grains, condinents indigienes, ete, a daus son ensemble, far l'eionne quantiter a indigienes qui s'y livrent et far son imprartance cafitiale daus l'alimentot. tion du forys, un rile considérable: C'est ce geure de trafic qui infilique mécessainemant le rîle du cauri at le legitiure.

Les petits tas - unites ardinaires d'adrat (nous l'avaus ver plus haut far le necersencent du marche' de 'hiterga) valeut en gèréral 5 à. 10 cauris, c'est.à. dire mairs d'un on deux centimes si le cauri est au taux nomal de $\$ .000$ four 5 franes et ce taus deseend frequenment à 10.000 ; gor l'ar un atteindre fusqu'à 18.000!

Il we faut done pas songer à suffirimer, frour le mament du moins, ette manaie furimitive. Clle répond à une récessité. Se résultat anquel on faurrait viser et que mous avars virtuellement atteint, consistait à l'igno. rer et à ue réaliser de ferception à quelque titre que ce fut, quien numénaire.

Ce systènse qui paraissait offir an dibut des difficultes insurimontables a fur se dévelufper an cours de ces trois demières ommées, méthidiquenent, sours à coup, saurs affarter le maindre trouble daus la vie économi. que du prougs.

Ses prenegutions de 1905 avaient damé 130,000 frames 

DS! 
on munieraine, celles de 1906 phises de trois

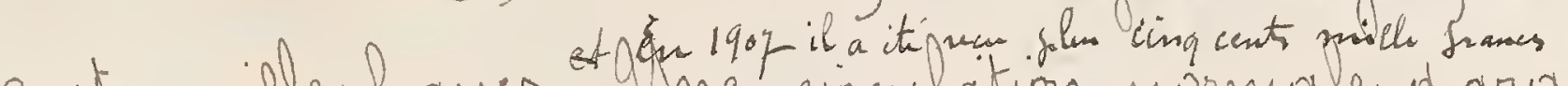

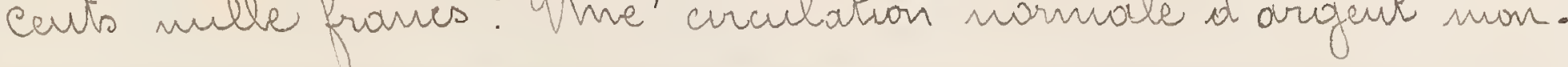
noufé a fur àre provoquée ainsi, et elle ina se déveluffount desomais d'anrée en année.

Hives remi sous farme de tablean synajtioure d'ansemble

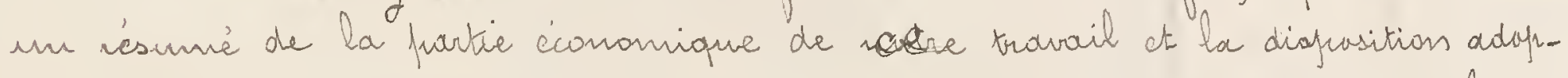
Kie a fermis de faire figurer en regard des rubriques: Onagadaugan, ben Kudogo, bio, les neuscignements qui concernent spécialement chacune des divisions

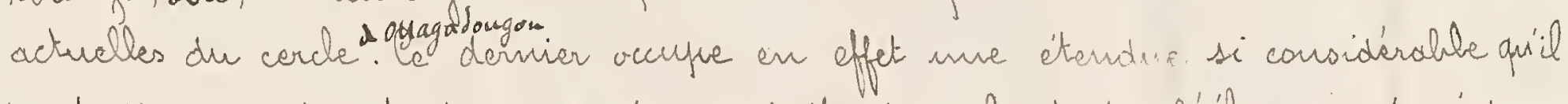
farticipe, au fuirt da wue des froduits du sal et de l'élevage, de régines sensiblement diffirents. Al y a même lieu de remarquer à ce sugat que ee fartage en eirconseriftions qui répond surtiont à des considerations fuati. tiquess ot adminustratives est également justifié strus le nafport économique. La dernicire culorme compresent des observations d'ardre général qui s'appliquent, celles. là, à toute l'étendue du frays.

le tableau n'étount en somme, qu'un résumé systénatioure de

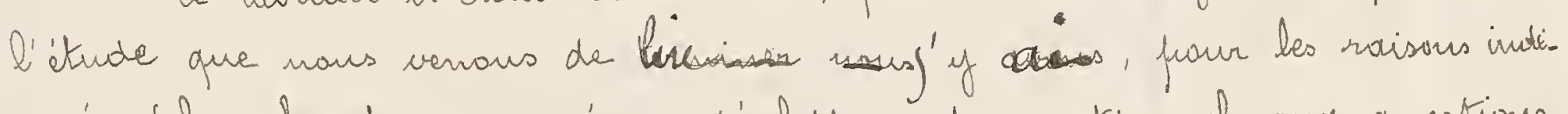
quées flus hout, consacré un dévelappement exceptionnel ause questions intéressont l'êlevage. 


\section{Cableau synaptique des}

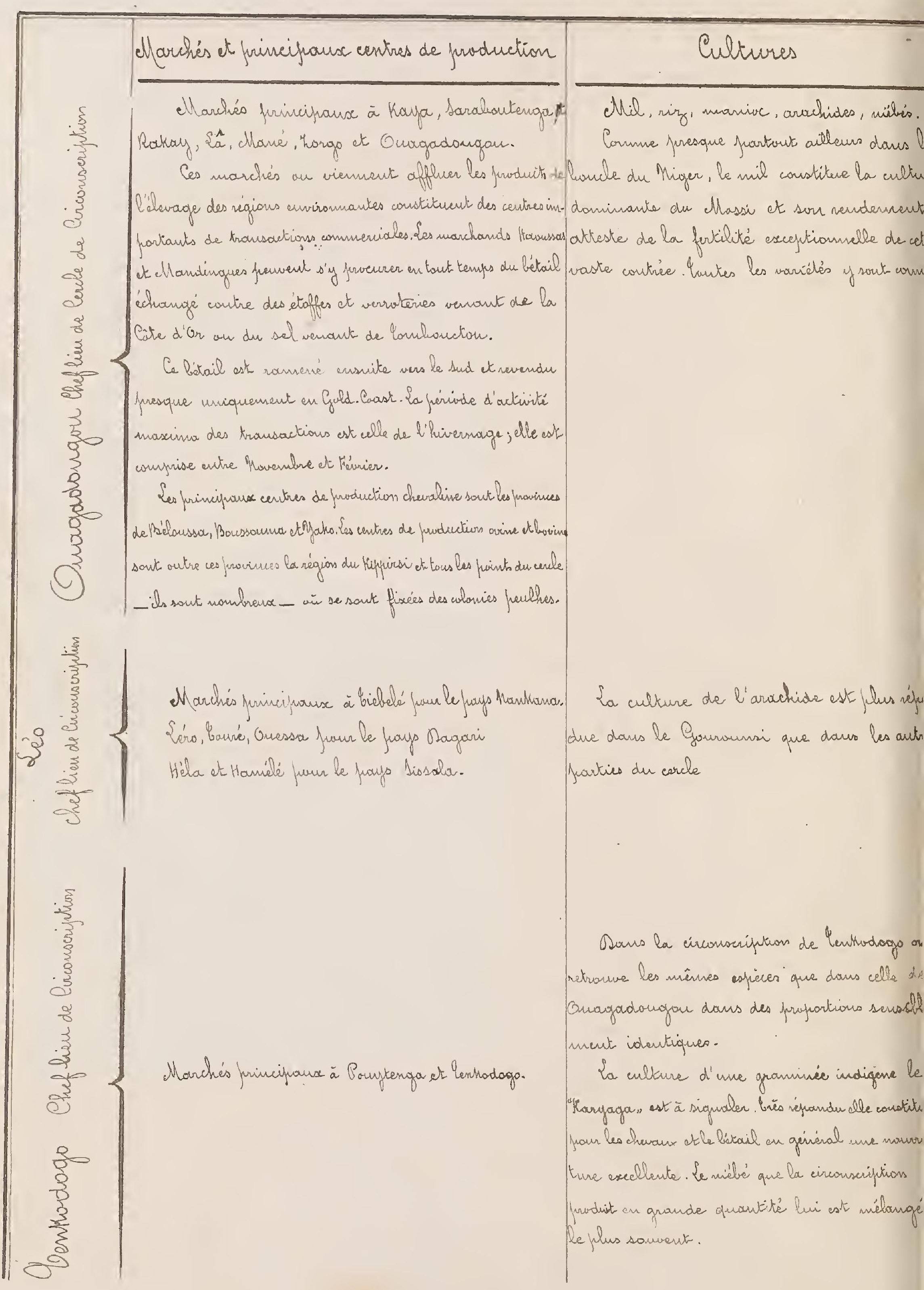


osit 
Cableaur syproptique des productions du pays Allassi

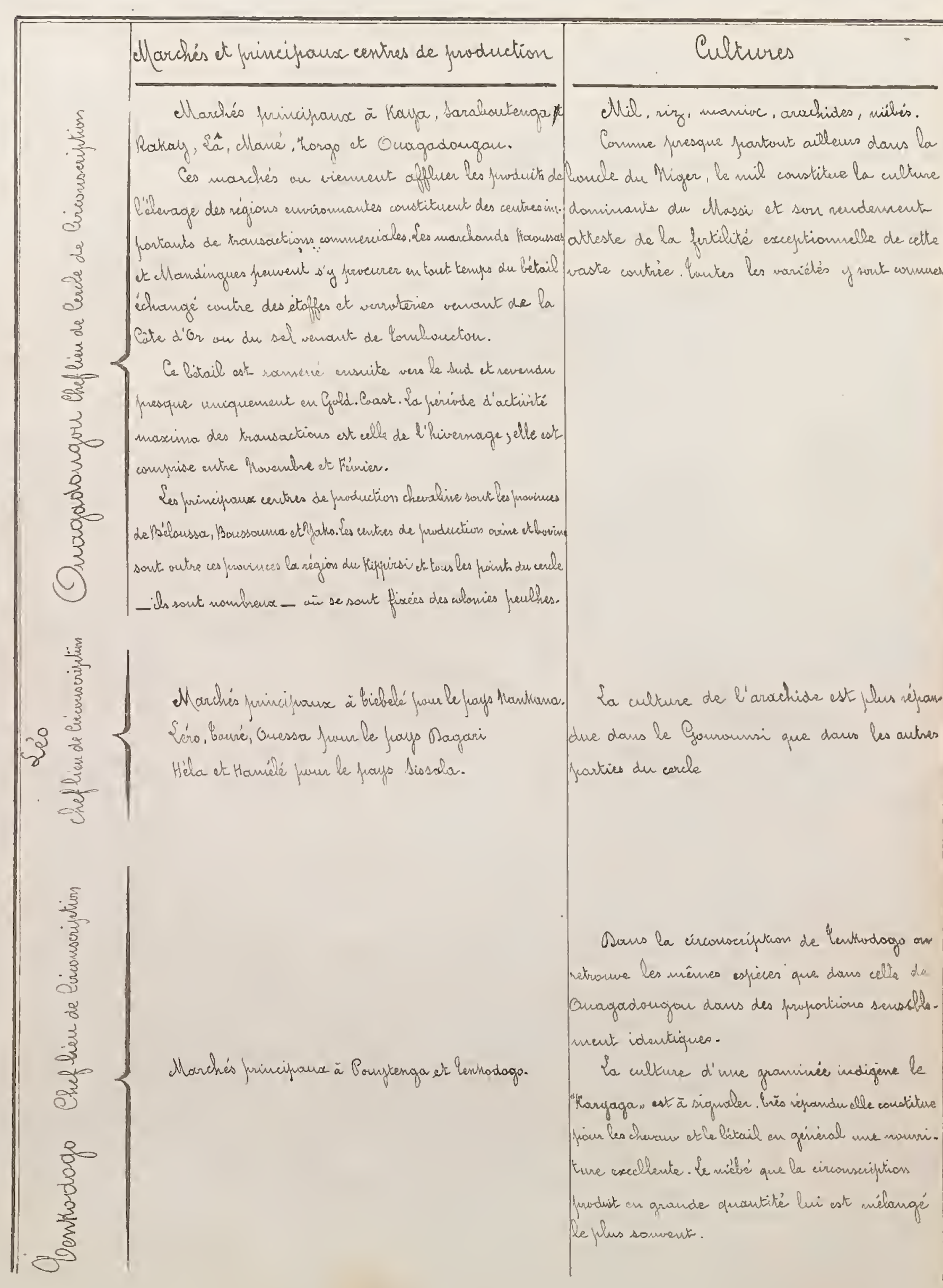

\begin{tabular}{|c|c|}
\hline Irdustrie etrevaline et obine & Obserwations gérnerales \\
\hline 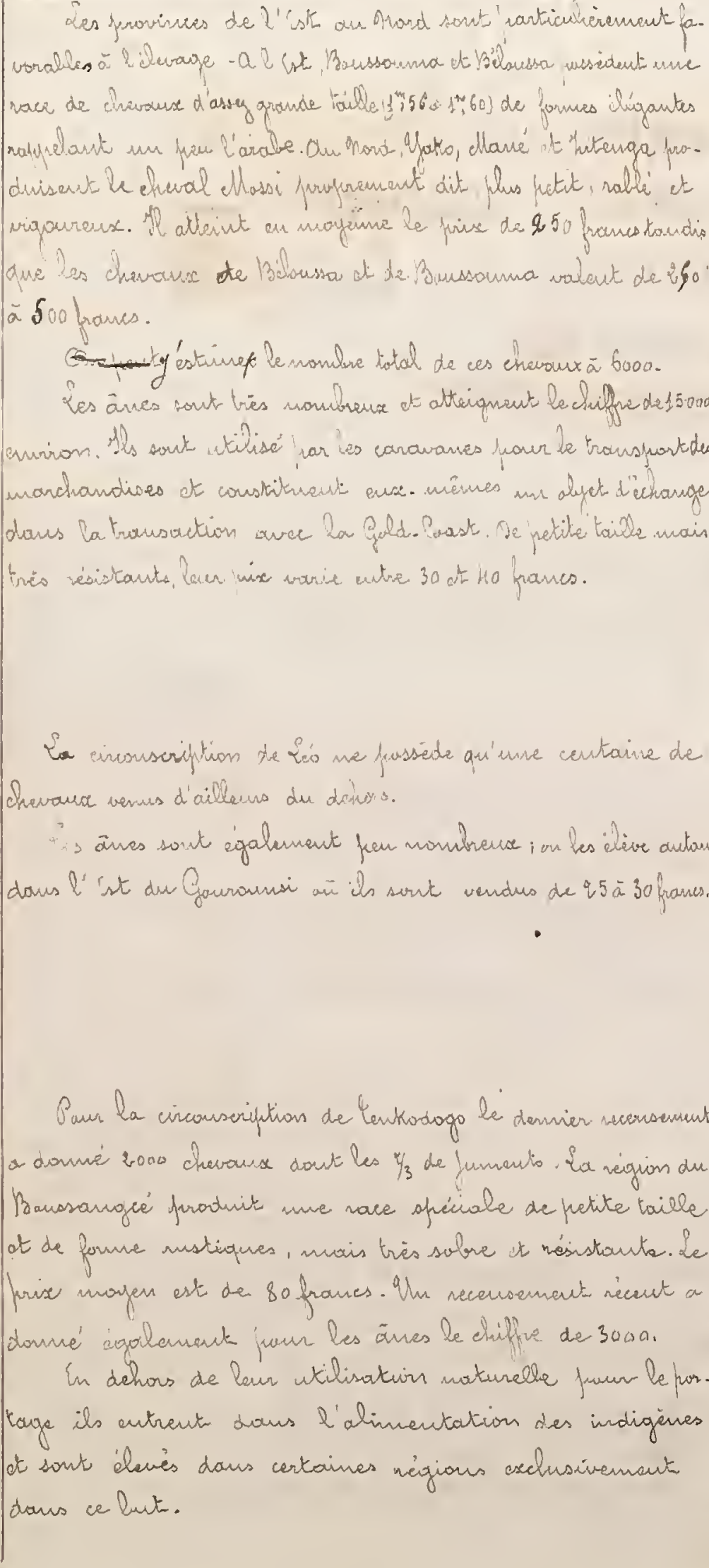 & 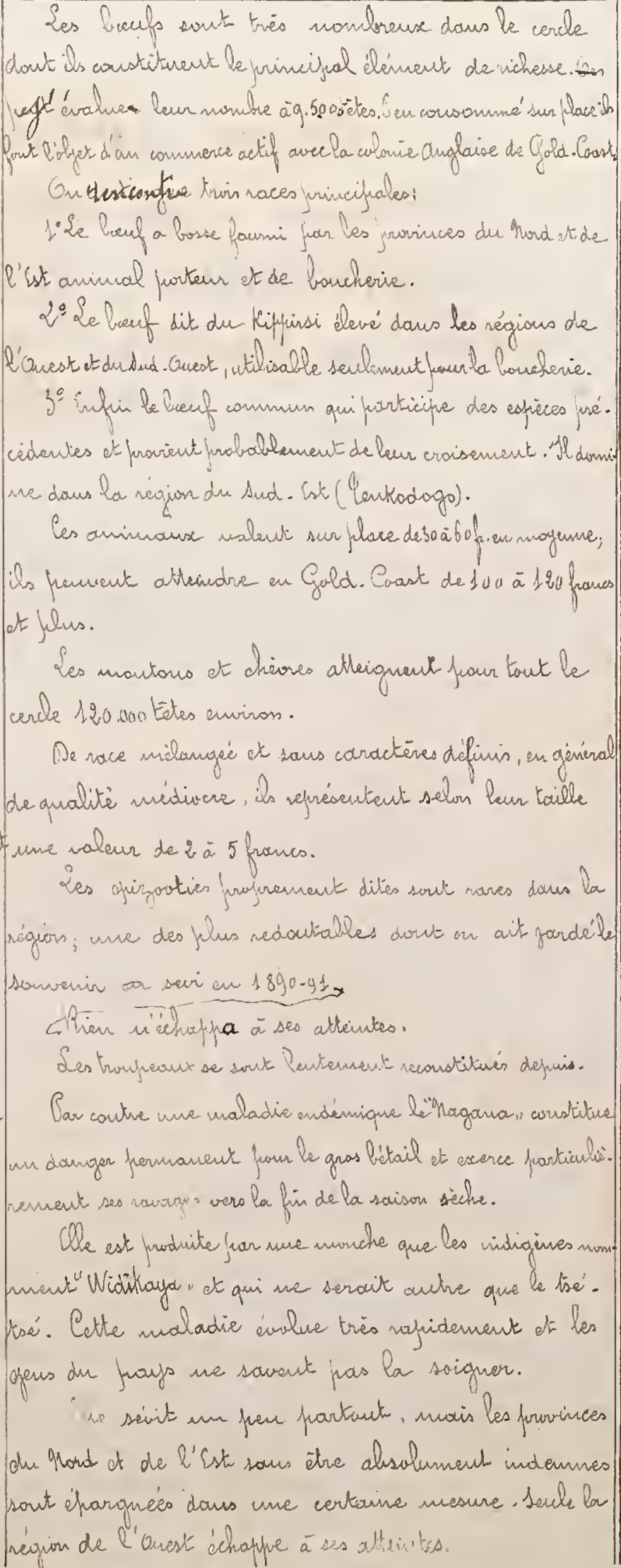 \\
\hline
\end{tabular}


59

$2^{2 m}$ Porlie

Essai historique sur les origines Mossi_La dynastie actuelle organisation palition es a Caquelle elle or préside jusqu isi

Il a dè difficile de se pronancer fusqui'à ce four, sur les arigines Massizs. Les seculs documents érits qui paurraicut foten, quelque clarté sur la question. ot c'etaient en l'espiece les divers passages du barith es soudian que joai suass intégralement refroduits

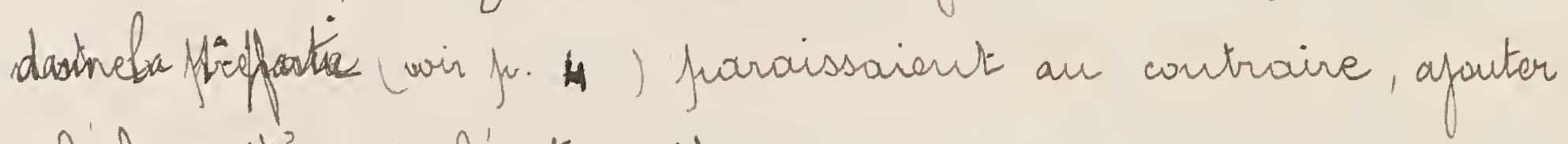
à l'olscuritée qui l'entaurait.

Ils naus apprenaieut an effet que vers l'an 1350 de notre ère, Gombonctour avait ité fris et saccagé par be sultan du Massi, fait histarique sur lequel abd. or. Mhaman ben Ahdallah ot Ohned Baba nous fournis. satient des témoignages concordarts.

Le barikh wapportait d'authe part qu'à la fir du

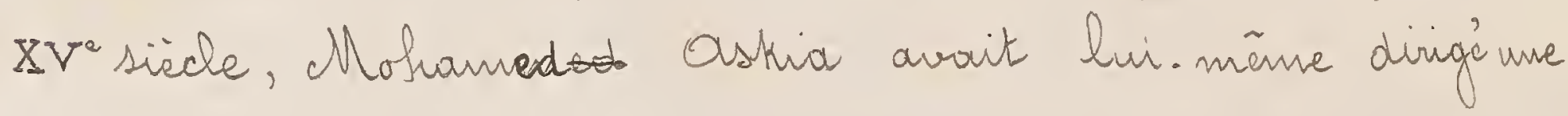

DSI 
60

expétition contre Máasira sultan dur Massi, esefredition dout maus iquarans l'issue ot qui fut probablement un éelve pour le conquérant sourkaï; qu'enfin vers le milieu du XVI siecle Oskia Daoud renouvelait ave flus de sucies cette tantative et réussissait à conquierir le fouys, que son dief atait contraint d'abandamer aver toutes ses traufes.

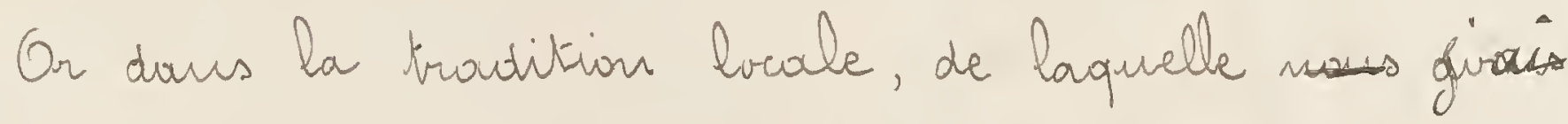
tiré tout ce qu'elle atait susceptible de foumir en témoi.

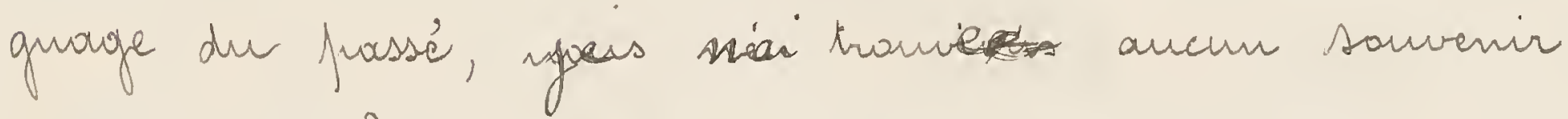
d'esfieditions laintaines on d'inrasions vermes du Mord; bien plus, la mienre tradition établissait qu'à l'épuoque de l'oceufration du Yatenga, équque fustérieure ause faits rappartes frar le Garikch, les Massis avaient tranvé le fayss fresque défueuplé at qu'ils y arrivaient faur la prenvière fais.

Gtions nans dove induits en eareur four une simi. litude de nam ? Un taus cas, les Massip qui avaient conquis Gombanctan ne framaient venir du prays de Onagadargar et n'avaient fras accompli leur trajet par le Yaterrga et le Macina, trajet qui d'ailleurs, far soir étendue et les difficultés qu'il présentait àtait à cette hyfpotèse taute varisemblance, et l'argument valait frour les invasions Saurhaï qui érsseut dî véaliser le nienc trafet ar sers inverse.

On en ctait anssi réduit à cette conjecture 
inoutmissible que les Massip auraieut dir abandomer leur verritaire à l'éuwque vers laquelle, au contraire, les kémoignages concordauts de la kradition arale fila. ceut l'origive de la dypastie actuelle et du grand effort qui sous son initiative, devait abantir à la consti. Aution de notue anyive Massi.

thais ce rapprochement qui conduisait appar. renment, à une infrasse, renfermait en véalité le trait de lumiere qui devait nn' élairer. Sa coutradiction n'ètait qu'apparente.

Il fallait nésolument écarter taute huffiotise d'idens. tité enitue l'enfire Massi des chroniqueurs arabes ot

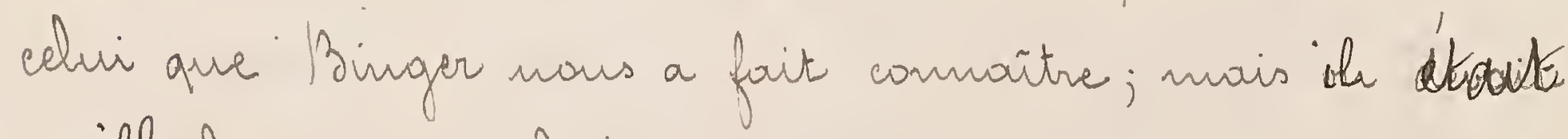
possibles logiquennentikles considérer conme nés l'un de l'autre, cormure s'étant succédé en quelque sarte, si l'ou arrivait à bovaliser le premier ot à le rattacher frar des indices certains à colui qui nous ocenfue.

Bantes les grandes eirigrations dout l'histaire nous a gardé le kémoignage aut en effet présenté cette par. ticularité que sauf de rares exceputious, les hardes, tribus, fueufles, que des nécessités nieluctables out fotes à la recherche d'une terre fromise qui devoicut lan offrir des conditious d'existence plus faciles, n'y sout famais parvenus dares turte leur intigaité. Ils out tougours dir lonsser en arrière, semer le long du chemin, des dincents dovenus un fuvids nart four la conmunanté, 
le plus somvent par leur manopre de comfiance daus l'issure de l'ertrgurise. Ce sout ces éliments que l'ou ne. trouve, soit asser difficilement, s'ils out ité absarbés et assimilés par la pufulation anbiarte, ce qui est frésquent, sait plus aisément s'ils aut su se maintenir refractioires à toure fusion aver elle et dementer en quelque sarte cuthstés dars son sein. Ils. Jaloment ainsi de' lair la rante suivie frar la gronde émi. gration et à plusieurs sieicles de distance constituant ar l'alsence de tair docuncut histarique, les souls tónoins de cet itinéraire qu'ils n'ont fras consenti à accouplir fusqu'an baut.

e'est dour de ce caté qu'allaicut farter necher. ches. Glles out dé langues et délicates, at sass 'aut conduit firalement aux résubtats siriants:

Il existe encone an Ind du Baman dous be Cameraun allemand me rave qui parte le rian de Massafn et aurait èté autrefais selon la tradition beale, maîtresse du pays entier. Clle se trouve actuellement corffiriée en une fuation de tarritaire assez nestreinte..

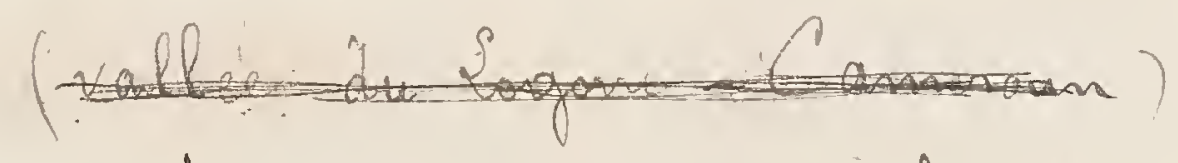

Les reprissentants actuels offrent avee les chassip des nes. semblources phipiaires tellement frappourtes qu'il ast infossible d'avair véeu farmi ens sours les recomraitre daus us derniers; le tatonage est dentique, sourf en ve qui concome l'acceit cir.

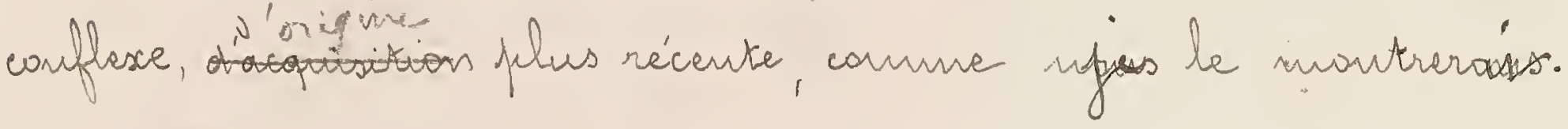


La similitude est presque confflete et d'autre pare l'argani. sation saciale des Massif avee toute so hiérarchie si caracké. riskique, se netranue chez les Massaffp. Con un mat l'identite? d'arigine des deux araupres ne fait fras de daute et yjous fundass

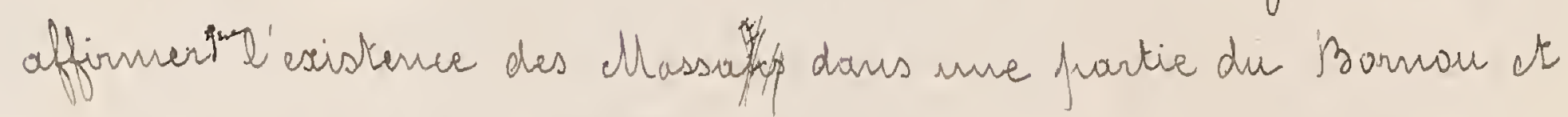
la sufrématie que la kradition laur artribue dans la pays à une éfroque necultèe, fixe d'une facon certaine l'habitat sir a dir craitre de se farmer la rare qui nous accufe; cela bien enternohr, aur cours d'une période assurément fort autóricure aux événements qui foumiont aus chroniqueurs arabes l'oula. sion de naus révéler sou existence. (1)

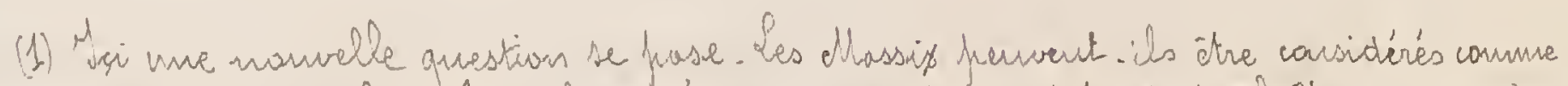

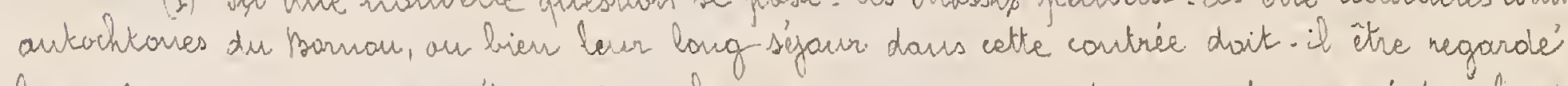

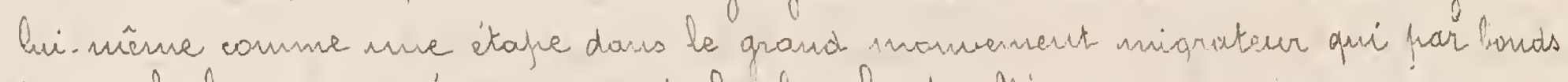
successits les a arresiés an cour de la bancle du Riger.

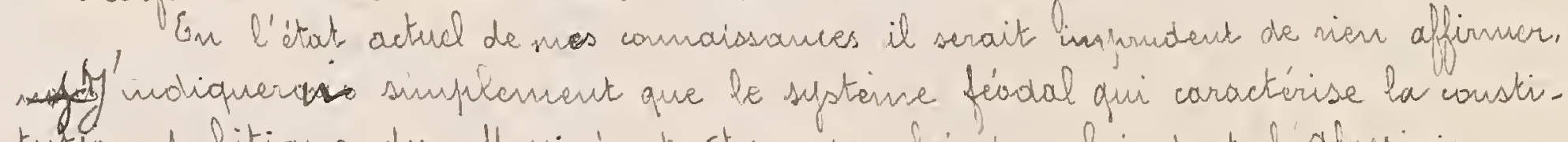

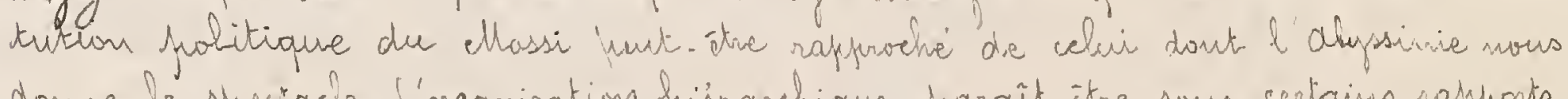

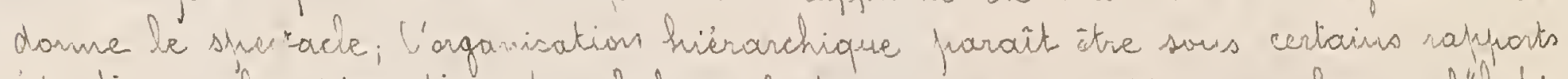
identiques, Ca desiguation des chefs se fait daus des conditions, analoagies, lielection

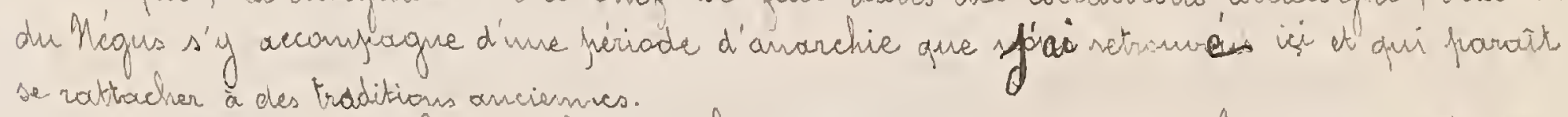

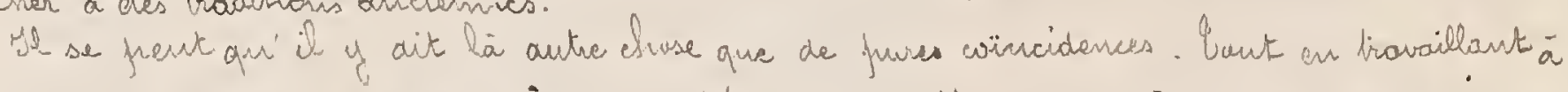

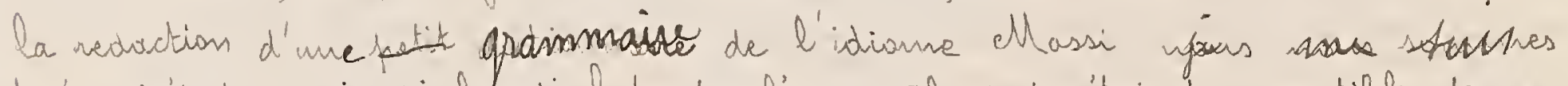

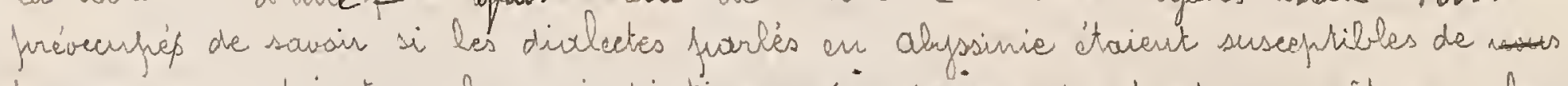

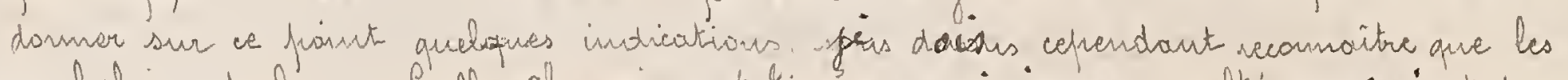

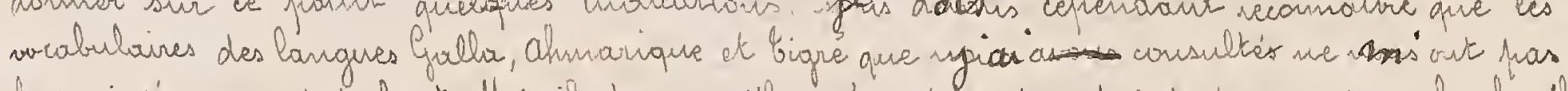

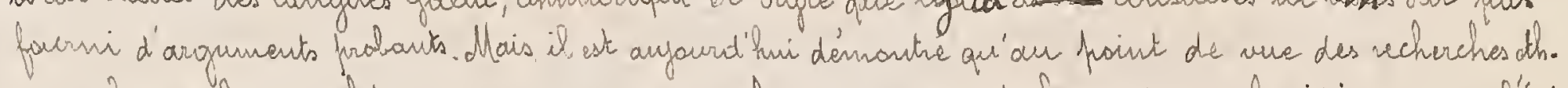
angraphiques les similitudes tesciques ne signifient pas grand chose et que les idionses que l'éci-

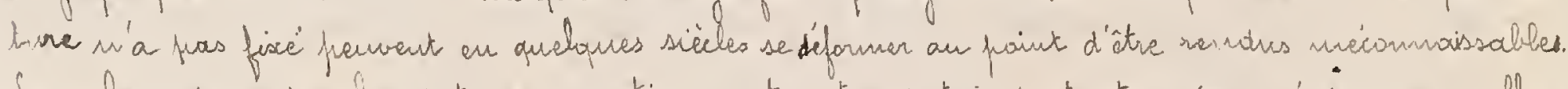

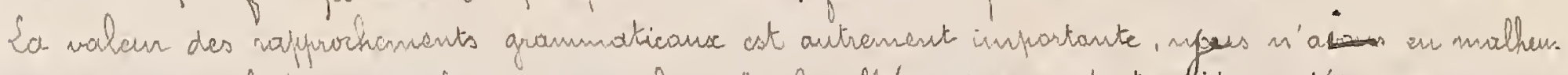

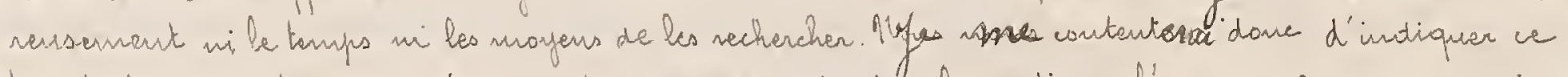

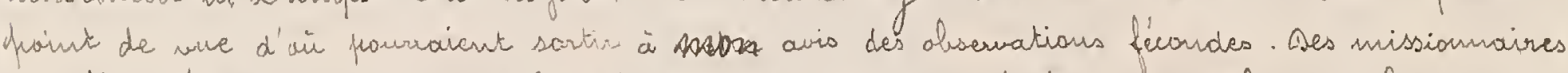
out affirmés d'autre part que les Massiy auraicut couserves traditionellement la notion

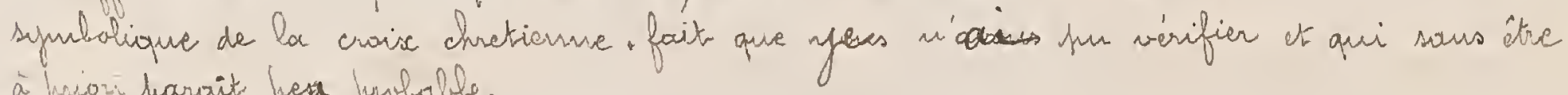

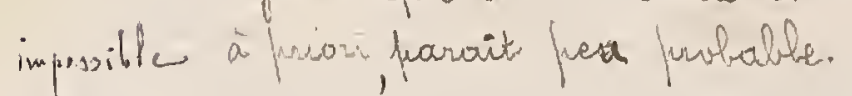


Il csk en effet de taute prababilite ot là encore, faute de documents ecrits agess n'avas paur soas' appunfer que des traditions onales recueillies an Barnou, que l'empire branté par la foussée d'une invasion nusuhmarve venue de l'ista dü, fiar oscillations suncessives, et en ferdant du terrain en serrs apposé, s'itendre flus avant vers l'ociident; de kelle sorte que les "Mlassip-bés, que l'on rencontre farmant-encore flusieurs aggalamiarations dans la jarkie oceidentale du Jokoto ct Jusque daus le corcle de Dounzar nontrount eus aussi de frappantes analogies aver les Massis proprement iits parrenk a lour tour naus servir de repiere. Ces agglomiérations indiquent d'une facson hés apprascinative la fusition de l'enyire Massi à l'éproque histarique, époque qui marque ividemment. lapurgée de fuissance de cet enfirire: vers 1350 nous l'avours wn daus le Garikh, Gambanctour est conquis foar le sultan du Massi.

Mais les clartés que naus kirous des Chroniqueurs sout intemittentes; ils ne nous affurendrant rien de nouveau sur le sufet qui nous vecupre, jusque versla fir du XV siecte oir se place la kantative de Mhomanneded askia. Gout en évitanti de faire de l'histaire suljactive at de nars permettre des affurnations

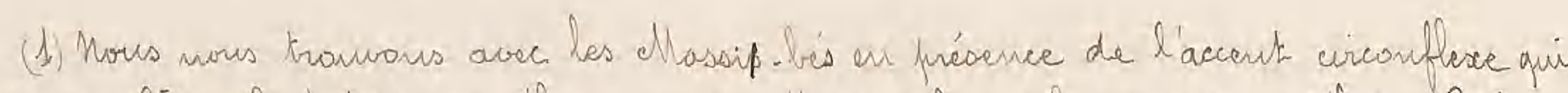

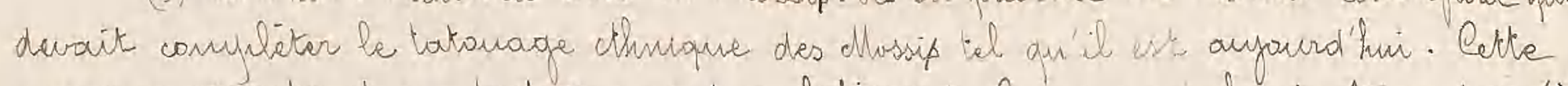

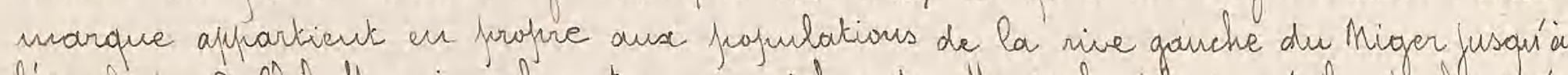

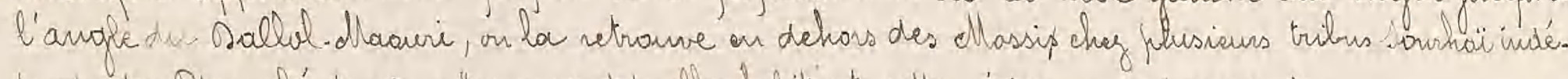

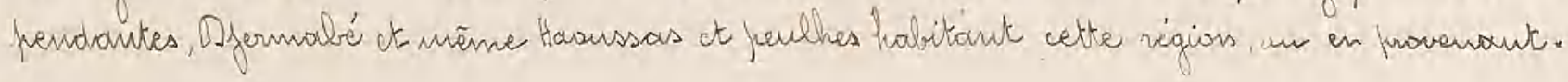




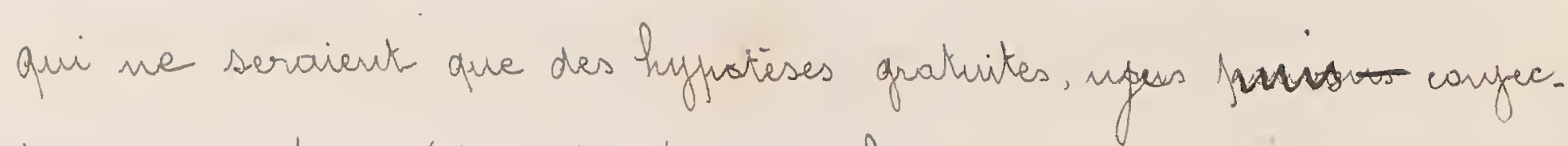
turer que fusqu'a cette éfuque la furissance Mlassif a dir se mainterrir, qu'il est nienre probable que la frontière arientale de l'enfuire a été recourée et que cirarmí a firi par constituer un voisinage trap redautable pour que la suscepkibilité des sauverains sourbaü - cux aussi à l'afuagé de leur Grinarce -, we is'en inquiète fras.

D'antre park, le point de une religieux ne parait pas avair été complètement étranger à cet artagonisme. Ses Massip se sout montris de taut tenys indiffenents, sinon has. Eles, à la propragande islanique et nous les retranous aufourd'Puir, quand à la masse, confelétement refractairesà ette influence nusulmane qui defuis le IX'siècle à si pro. fondément nodifié les conditions politiques et sociales de la phifrark des populations du saudan.

S'on feutr dome chercher dons cet ardre d'idies le movi. le des expíditions dirigées depuis Mahannncol astia cautre l'enfire Massi, douk les habitauts sans cesse inquiètés et me. nocés, se wieut contraints de boune houre à chercher lour salut dans l'émigration. Ose lai, un fremier exade dout l'it. néraire cst facile à neconstituer.

Ce nouvement s'est acconffli dans la direction du And ct les chassis aforés avair cotage' le Miger l'aut traversé en un paint situé entre say ct fllo, mais beaucaup plus prés de ce dernier.

On vetranve meare au Mard d'Jlo la coutune qui 
consiste à raser la tête des fermues de chefs. Celles.cip partent en outre des hauscaux de niótal, caractéristiques farticulières à la vare Massic ts que l'ou reruontre occasionnellement cher les peuples qui out dté en contact aven elle.

De la l'émigration, laissaut sur la gourche le fays Barriba s'est dirigée en ligue draite à travers le Gaurma ct la région de Pana jusqui a Gambakpha chef-lieu actuel des Gerritaires Alad de la cularie anglaise de Gaba. Coast. C'cst ce hoint que choisit faur so résidence le chef qui await dirigé

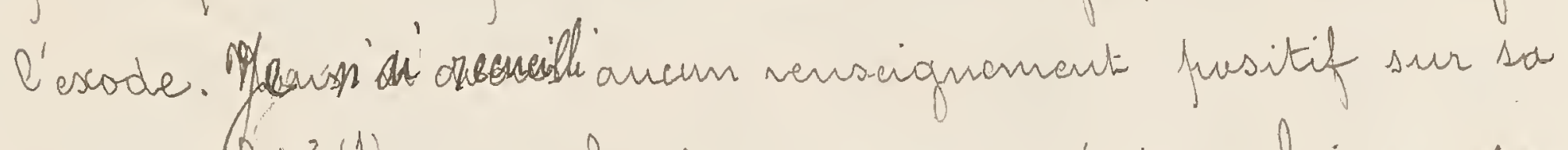
persomalité, (1) mais il est certain que e'est de hir an de ses suecesseurs inmiediats que la cication du royaume Mampansi of Dagomba tire san arigine. Sa farmation de l'empire Massi froprenent dit est fustérieure; for tot ceperndant fondé d'affirmer que dis cette éfuque, une partie d'entre les nouveoux occuprounts dur Manpioursi entrefrit de se frayer un fassage vers de Mard et y farivint par daux voies differentes. Mars naus trawans en effet en présence d'un can. rant qui farti de Gambalffa et contenur à l'Est frar les Gournounkies refeta les Barssangap dours la région que cenx-ci occupent actuellement et atteignit le frays de Bilonssa ai il s'épananit vers l'Onest et le Mlad, ocenfount

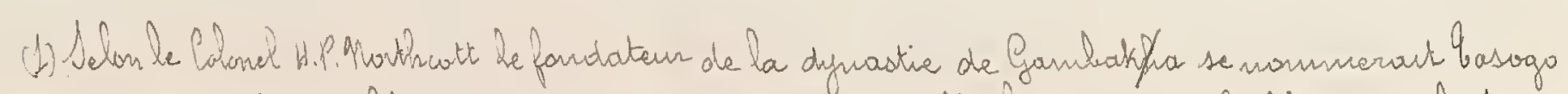

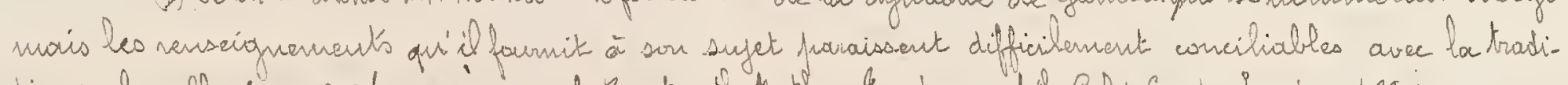

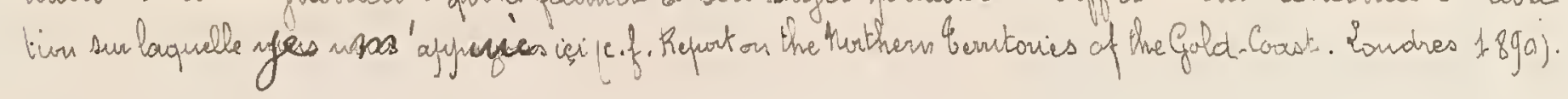


une valte fierifhiric de teritsive, anfaurd'hui fresqu'eschusi. vement peufléefar des Massip. S'autre caurourt se frougant une vaie en farys Marthana, se dirigea au Mard de Gieblés refoula du côté affusé les Baussangap ensecrés anisi canne dours un 'dan at se developpa dars le fraus que limite. la Valta Ronge, vers Onagadongaur.

On n'a qu'à jeter les jause sur une carte du poufs. Cllassi (wir fage h.t) furur vecommaitre la direction de ces deuse caurants paralleiles vernant se vejoindre au Monol du Paoussany. cé actuel, région presopue intuaitiée à l'ejuoque de la grande migration Massip at au sein de laquelle les autuchtanes Bous. sangap, qui occupaient alars taut le fauss de bentrodogo, out été contraints de chercher un refuge of végetent defuris, réfractaires à toute fusion avec l'élement cuvahisseur.

On prent voir anssi sur cette carte que ce dernier díbarde la limite ovientale du cercle de Onagadonizan

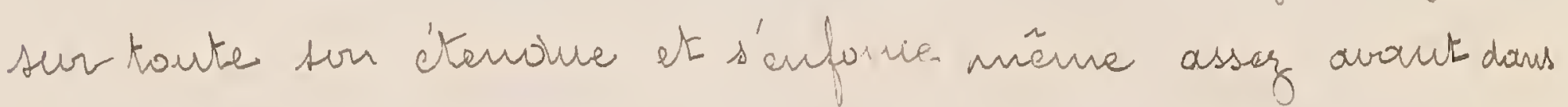
le Gaurma sur un vaste esface affectant la formed'un triangle, dout le sammet taumée vers l'Cst a four determinaut le fraste de Fada. Sa fufurlation de cette ville ast en effet Mossip et son chef Batihandé se dit consin an Morks-Maba de Oragadangan anvers le. quel il se crafait tenu naguère encare à des emusis de brésents ot autres marques de déférence témoignant d'une anciemne vassalité. H ne s'cst consideré comme affranchi de ces obligations qu'abres l'arrivée des Trancais daus le fraus. 
(1) Ce fremier envahissement du territaire Massi fut l'oerure de hardes indiscifulinies allant à l'aventure droit devaut elles, sours flan arrêté, sans Cnef. Cer. tairs granges détachés accufrent mẽme dés cette éfraque des ilóts deffinis, enclavés an seir de fufulations autoch. tones; c'est là, qu'isalés at contenus far Les élements étrangers qui les entaurant ces graufres évalucount dé. somais aur joint d'atteindre un degré de différen. ciation assaz accusé fraur domer de change et masopuer en quelque sarte leur arigine chassiq ce sant les Cargurix. les 9 ankarrap, ot les kansacép.

Dés ce mannent cependont, la trance est faite; à l'Est dt à l'Ouest du Boussangsé actuel il esiste un frassage vers le Mord, at nous nous tranvons en frésence d'infiltrattions continuclles: sur divers paints les naturch sout delogésfar

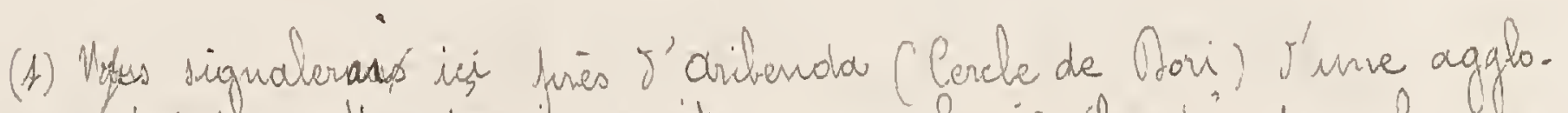

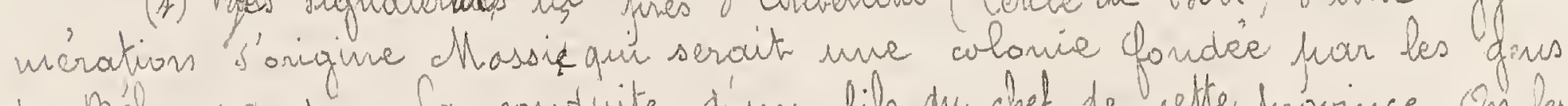
de Madonssa saurs Ca conduite d'mu fils du chef de cette province. On les

D'autre frart le dief de Ojargaur ( Dahannay) ast àaigine classid La Anodition, vent que les habitants de ce village, diviaés prar des luttes inkes. Kines qu'entretenait, la rivalité de phisieurs frítendouts se disfurtant le

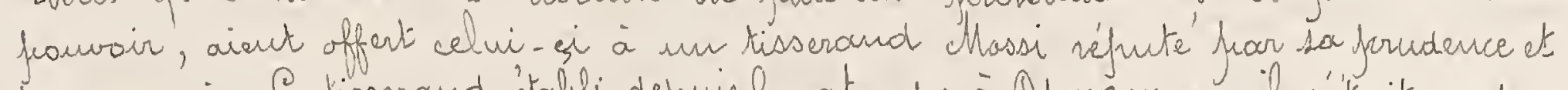

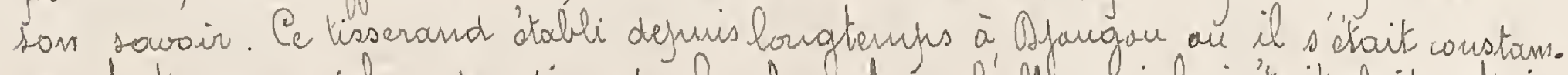

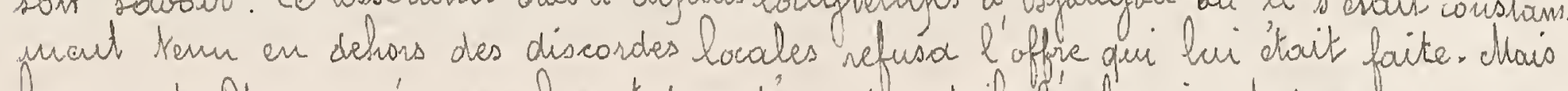

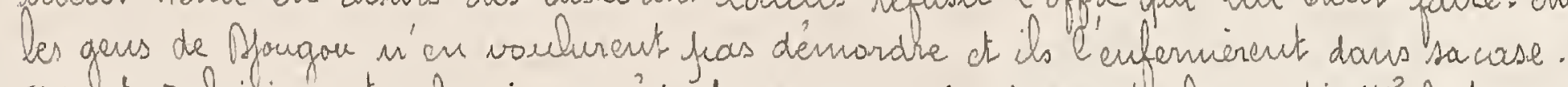

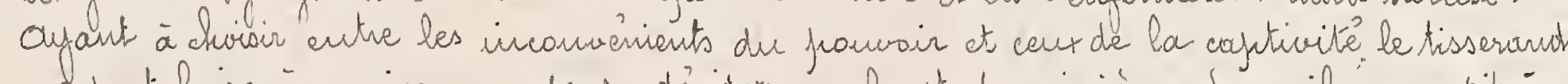
we nuts faire à mains que de se décider; aur bouts du sixsiène Jaur il consentit à

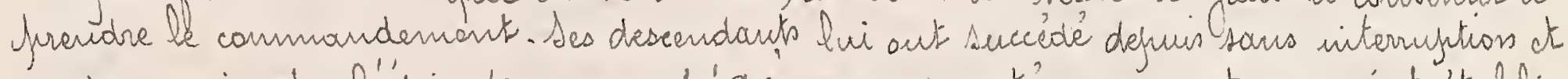

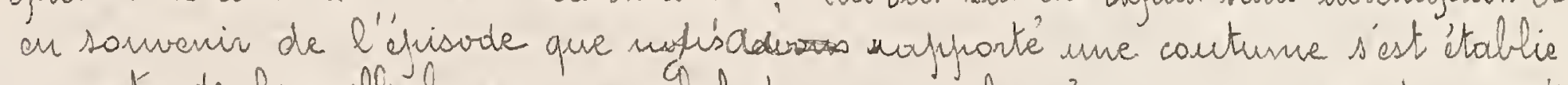

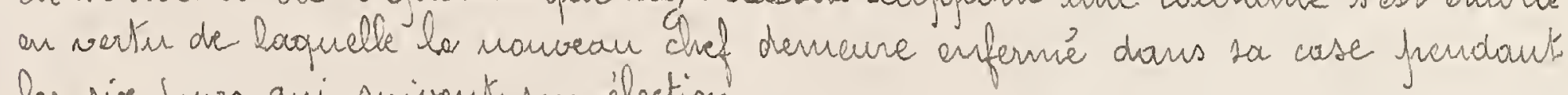
les six faurs qui suivent son électios. 
Les naveaur arrivants, mais en géreral ils jarivenneut à virre côke à cote en banne intetligence.

A la foveur de ce régine l'élément nomeau se

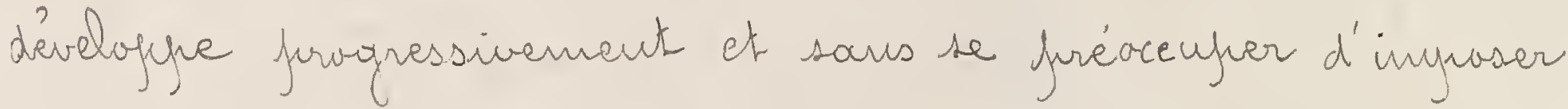
La sufuématie à la fapulation autuchtione, il finira frar constituer le nombre.

Il hir sufffira dés lors de jurendre conscience de hir. mêrue, de s'unir surs les ordres d'un chef eutrefurenant et hardi - et le naud enyire dlassi sera canstituré.

Pendant des siècles__ jusquía notre venue dous le

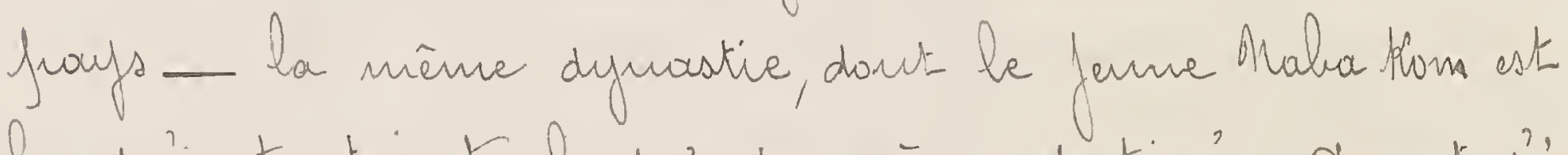
le reprèsentout actuel, présidera à ses deotinées. Ovout dè. Kablir, fuar ordre de successions, la liste des Marna. Moluas depuis Oubri ugess dirais quelques mats de la fieriode Cégendaire que la tradition place immiédiatenent arrut L'apfarition de celui. si.

the nabad de Gambakfa (les demiers nous l'avars ver descen.

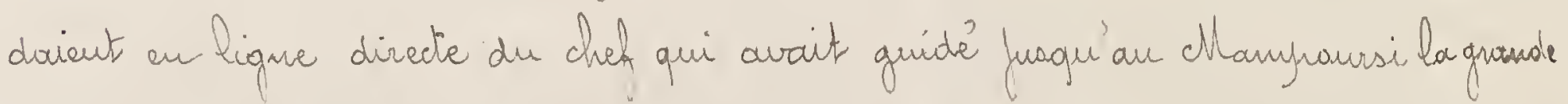
inigration) arrivé au délin de la vie sarrs frogineinture crut voir dours ce fart l'accomplios-mant d'un décret de la destinèes Rien u'est inmuable ici. bas de sans doute sa glariense dy nastie devoit. elle faire flace à usure mauvelle liquée de chels. A ceci, il se resignait encore, mais ce qui l'affligait frar dessus tout c'etait sa vieillasse solitaire. Il supplia doù les Génies de lui domer un enfart, frenart l'engagenent solemel de le vouer _ garcan ou fille _ à la 
71

viroginité. Conme fuar miracle une fille hi naguit

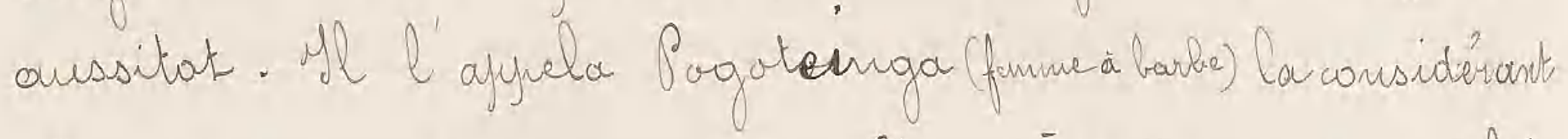
ainsi dis sa naissonce comme êtrongére à som sese et lii Lit domer un vêtement masculin.

Oogotainga se développa vite; elle attrignit jes l'âge de dis aus un dagné de beauté inconfrarable tf vers la meince efuoque, trawaillée faor les ardeurs de la furbertié. Ole véussit_ nalgné la surveillance ètraite dout l'eutourait le vieillard, falaux de demeurer fidéle à son sermant_ _ à s'enfuir de la maison fraternclle.

Songtenus froursinivie, elle fut se soustraire ätoutes les neherehes fusopi' à ce qu' in four, errourt dours le fayy actucl de bentodogo, elle rencoutra en pheirre brausse le chass-eur d'elefelvart Riale, hannme solitaire et vialent, qui la siétuisit. Clle eut de Rialte un fils, odridirow go, tt une fille drédowgo.

Afin de ne fras alterer fwar l'escogannie la vertic du sang vajal dout ces enfarts etaient les heritiers, Riale dt Pogateinga nésolurent de les navier ausemble dt de ce mariage noquirent anssi an garcon ot une fille,

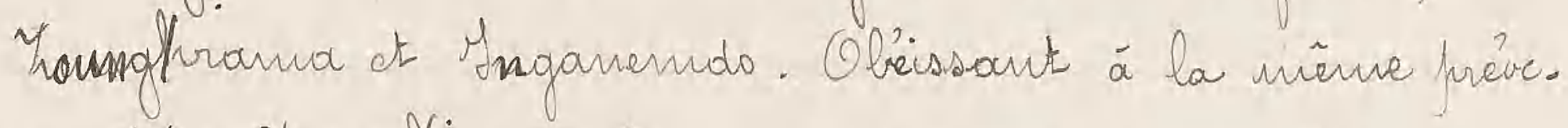
cufation, Tounsafirama ejausa sa sour of de cette union maquirent trois fils. Oubri, Obrogo ot H'anfrango. Oubri devait fouder la difiastie de Omajowangoun, Obrogo celle de Qeutradago, Gánfiango celle du Gorrma. Soc fustèrité d' Oubri fut la slus glarieuse ot la flus 
fmissonte: les napyrarts des nabas de Cuagadongan avec ceut de Gentiadojo et du Gourma, leurs cousins, furent Murfaurs des rapforts de suzerain à vassal.

S'ancêtre conmun Tousnghiana naurut à komitoeda ou l'on montre arcone for tombear.

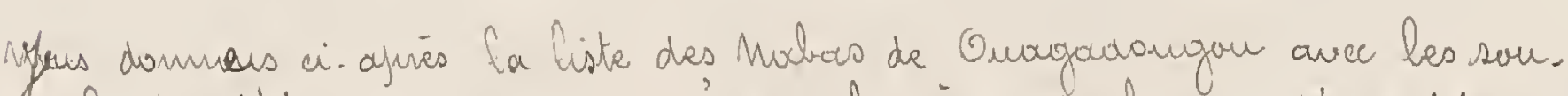
venirs que la traditions wous a couserve's dur le régne de chacun d'eux. Myes fais suivre leur nom (rradint, en frangais Corsque cela est jussibbe) de leur acirse. Patte devise que les charka. Mabas choisissent à leur avènenvent, caractérise

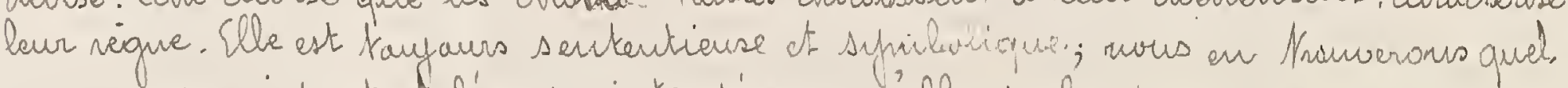
ques unes qui farteut l'empreinte d'une vélle troforideur.

Ses fremiers mots constitucut ce que l'on affuelle le "habj-izouré" ou nam de guerve: bien que ces nots détache's du contexhe n'aieut sanvent aucun sens, is fuss-ident jearfois une signification qui fourrait fwaraitre infurieuse. It ce n'est fuas là une des monidres fizarreries que nous verrcoutrerons. Ahn noba Maskiendé s'est fuaré

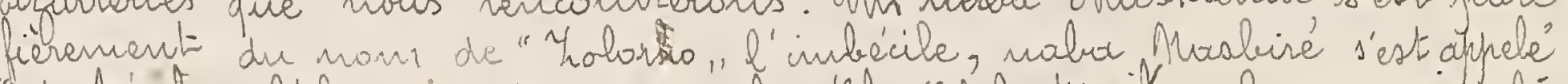

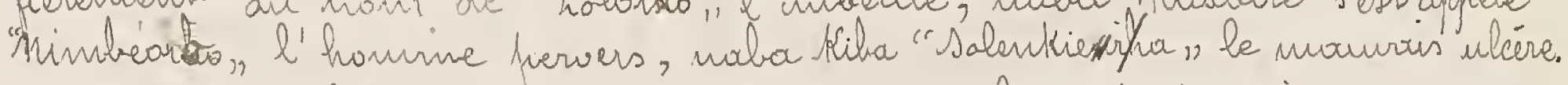

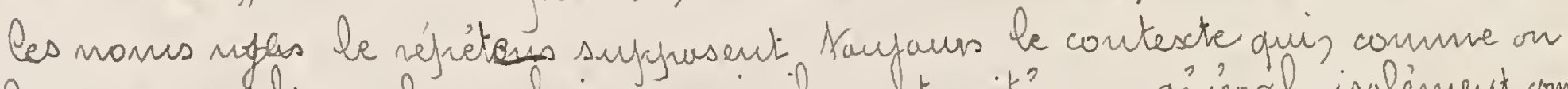
le verro, explique leur choix, mais its sout cités en gèréral isolément am. nue zabi ijorrés des nabas qui les out adaptés.

Sa tradition aryourd' hi tres incertaine, fleine de contradic. kiwns et d'anachronismes lorson' it s'agit de faits 'historiques properemauts dits, devient an contraire trécise ot absalument comondante dans le domaine symbalique et verbal. In l'alesence de Taut decument

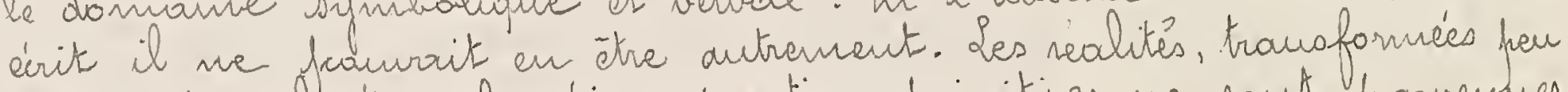
à feur fuar be haurail d'inaginations forimitives ne sont farwenares à fusan' a nous que voilies far des symboles. Seul ce qui faibait unage

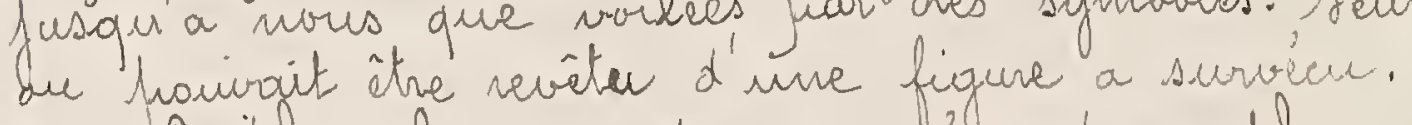

De tel naba on n'a consenvé qu'une phrase pronancée dans des circonstances déterminées sonvent insignifiantes. Pour Yaus defuis Qubii, la seule chase que l'on comaisse aver certitide ce sont leurs devises et encore la conseruations de celles ci est. clle dévolue à une caste de Bandwabas. Se vulgaire n'er connait que les fure.

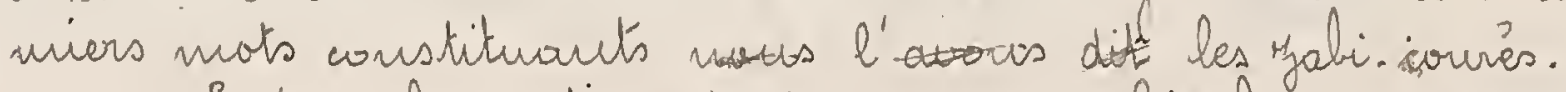

Sa Trausformations profonde que subiti le froufs deguis quelques anneses, les frogriè d'ordre fusitif qui s'y réalisent sont de nature à compromettre kict oul Vard la survibance de ces kroditions. Mfless n' acious done has jugé supuerflu de les recueillir ot de fixir des souveriirs sur le rount

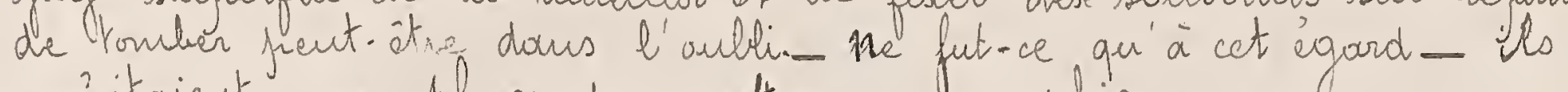
méritaient une place dous cattes nonvopraphie. 


73

1. Dllaba Oubri fils de Thoungrikama.

Devise cc Sariavogayore (1) Rango néda nbabl'tiga tassouri morhé. Larsgue le feur du ciel ne découme anum obset digne de hii, force lui est de s'abattre surv le premier arbire venu,. Phrase que ces descendarits out interfrêtée ainsi: Oubri se faisait ot à fuste titre une telle idée de so frofure valeur que, bien qu' appele' fuart le sort à cons. Nituer le grand enfire chassi et à fonder la filus glorieuse des dynastiss il se plaignait encase de la médivarité de ser destiviée.

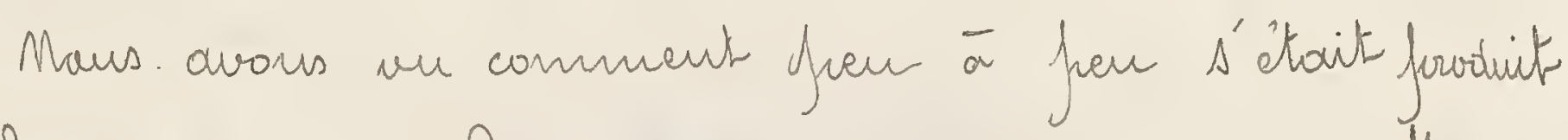

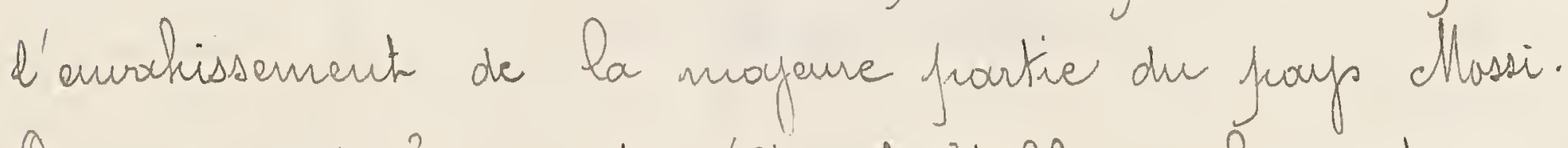
Des granfes indéfrendourts s'citaieut 'itablis isolement ca at la sur ce vaste territaire à coté des autachtones. ot viraut la pluffarti Su remps en bonne harmanie

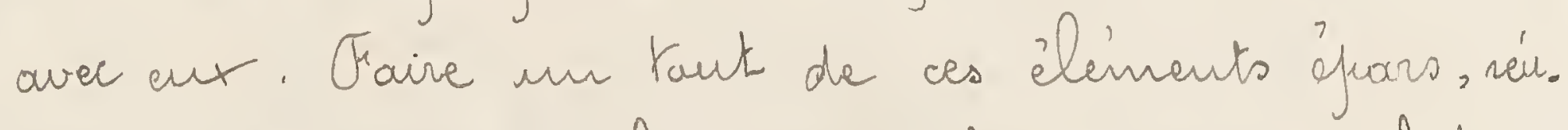
nir en faisccour ces fonces éprangillies dout un chef

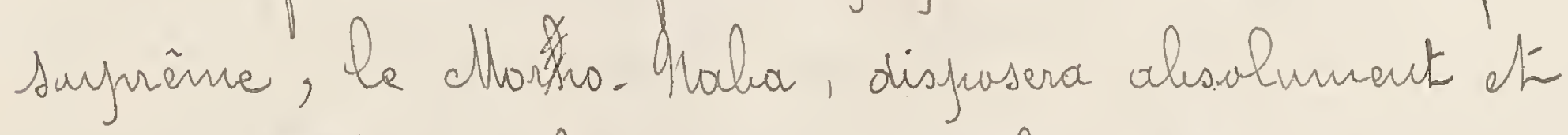
Sans conteste, réaliser frar elles la creation d'un grain enfire sur ce sol dout les autachtones serout réduits muts corresfoudanto de la traduction. 
74

désormais à l'état de hibutaires et astrients à une condition voisine du servouge, telle devait être l'ceuvre de Maba Oubri.

Pouti de Betta (Courton de Miga) selon les uns, de Oubri. Lenga (Près Sombila-Raugo) selon d'outres il promena pendant trante annèes à la rête d'une armèe à la. quelle vien ve résistait, sun inlassable activité du Mard an Sud, de l'Est à l'Ouest. Ou brint de sa venue des frovinces entière fort leur saumission. Se

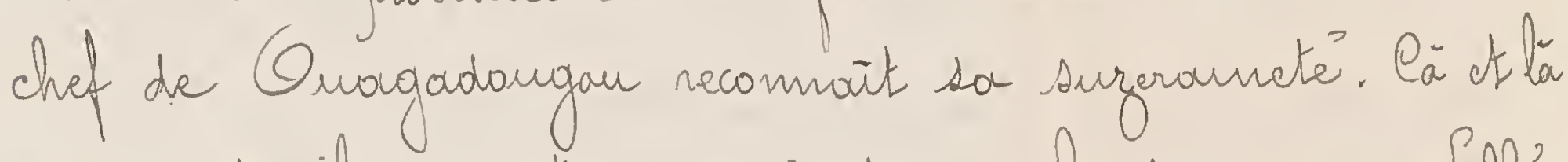
cefrendant il rencantire des résistances: la frovince de Salle' ne fuit àtre rédnite que far les armes it fallut ravorger

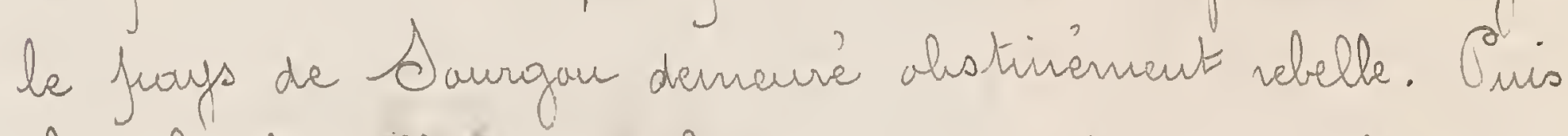
il alla batailler dars le kippirsi. An sovir, il arriva devant le grand village de llando daut la pufuilation se forta à sox rencontre avec des frèsents. Il nit friect à terre surs un grand coulccedrat dout il fit cindre le tranc d'une 'toffe précicuse et pronanga ces fraroles cc Peci est na demeure et na plus belle conguête, dle devait s'accomplir sans qu' ine gautte de sang ne fut versé. Ol ce village qui m'a accueilli comme un enfart accueille son fiere, fe hi dome le nom Kadoiho (b), car il

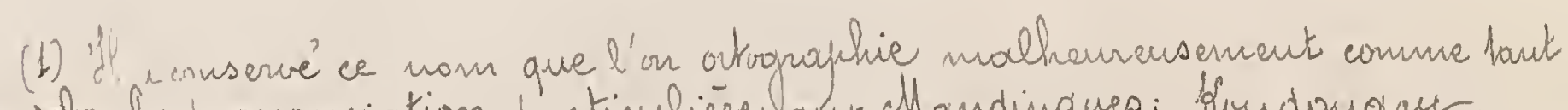

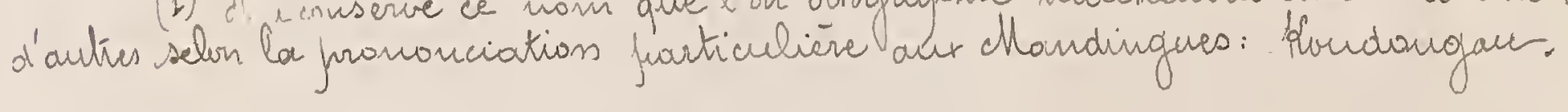




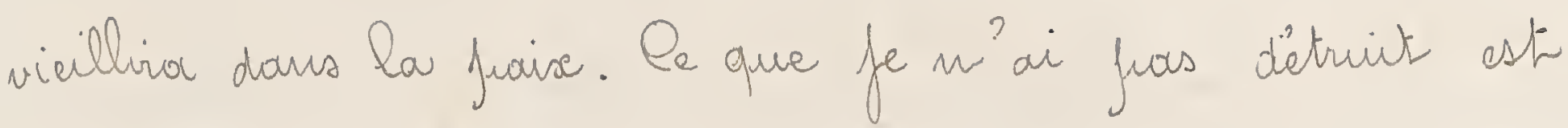
assure contre la destruction.

e'est là qu'il maurut jeu de Terups aprés, ses restes conduits en grande janye à Penga Sobonaso furent enlavès la mit, dit. on, par les frabitants d'un village visin qui lui élevèrent un Noubear que l'on feut encose voir et quir est l'abyet d'une grande vèruération. Ce village s'est

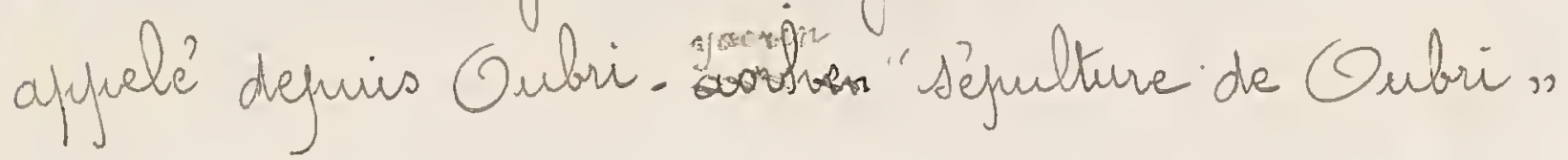

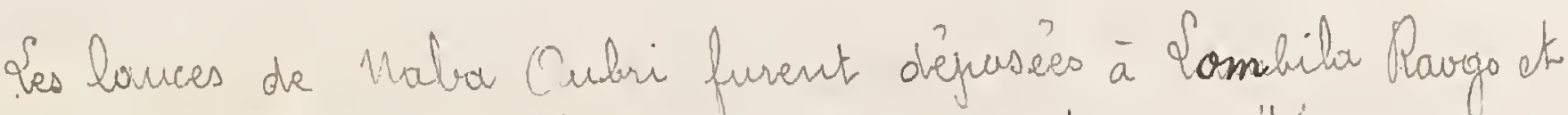
en vertu de cette tradition celles de ses descendauts out ité aussi véunics an anieme endront

Sarsqu'in Mork. Maba mant Kuries ses lances sont frantés solemellament daus cette localité at l'on place a côté d'elles une-

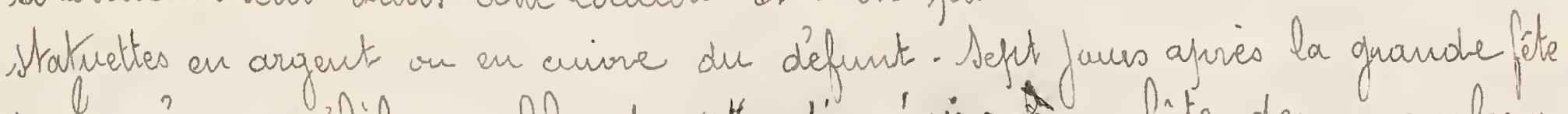

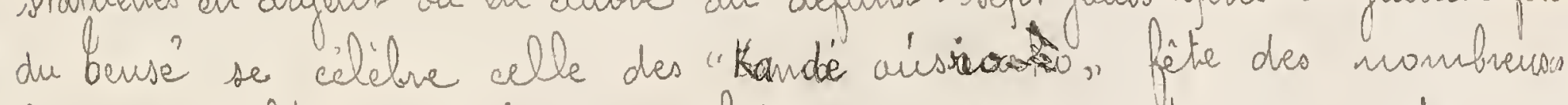
lonces et l'on sacrifie des chieus ot des montous devout ces Krophiees

Ses lances de l'ancètre Rialé sant vèrièrées à Odabila petit village entre Leina at Yakto.

2 Alaba Corba fils de Oubri fuar Kimada.

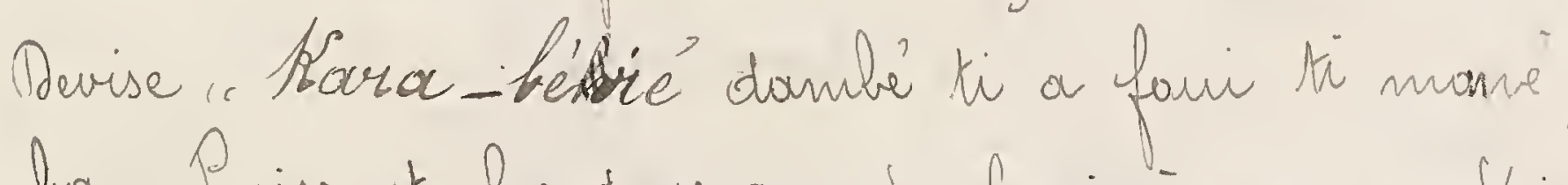
nolemba, Puissent les dars que fe ferai à ceur que fóaime ressembler à la fiàte de karaga.

Sor fuäte de Karaga gonffe èarmiéneut à la crisson

Iut désigné furr Ancièder à Cubir quoique flus ferne 
76

que Lon frère Maskiendè. CClui-i s’unit alors à Masré, sutre frère qui n'a fas rẻgné, frour attaguer Sorba qui surfuis dars les envirans de Saugansi, petit village à 12 Kilomiàres de Oragadongan, se véfugia dars le creux d’un bowbab. Ses frères le xejoignirent at Masré aprés l'avair egangé lii coufra la langue. Sa dèfraville fut ensevelie au milieu du marché actucl de Sarogausi.

"Mabo Maskiempdé fils de Oubri nar benututy Bila.

Odevise crolorno lási' Koum li a la ki arend soudi. S'imbécile rit de la mont d'autrui jusan' à ce qu'enfir soar frogere tiour arrive.

Entreprit contre nabo Kini chef de Yako une longue guerre au cours de laguelle il fut thè à $S_{a}$. Lou Yombear se trauve dans cette localite.

to Muber Nasbiré filo de Oubri fiar Gablatada.

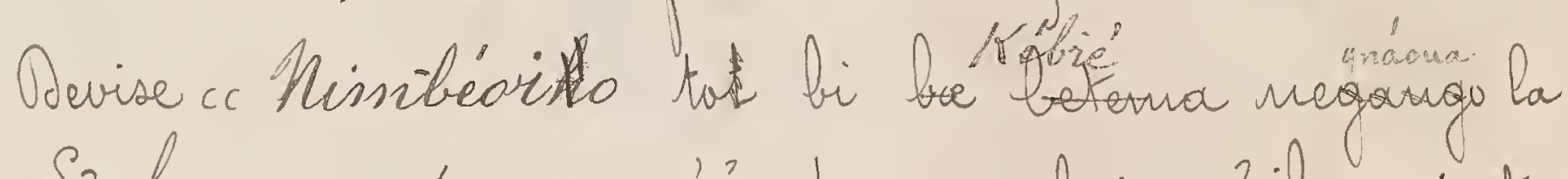

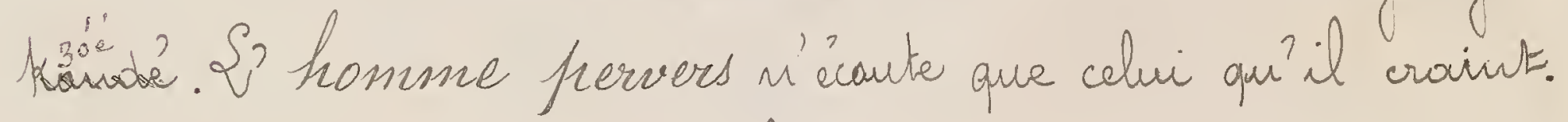
Ancètre des nabas de Yandour c'est jreu de Tenups aprés Low mant que fut fordè l'empire Massi du OJatanga dars les circonstances smivantes:

Masbirè avait enrajè son fils Dagongo (que d'autres 
appellent Yadaga ") à la tête d'une grande armér fuur s'enfearer des territaires situés au Mlard de Gakto. Il mour. rut sur ces entrefaites. Dragongo qui asfirait à luisuc. céder revint en häte à Guagodongar et y apprit que

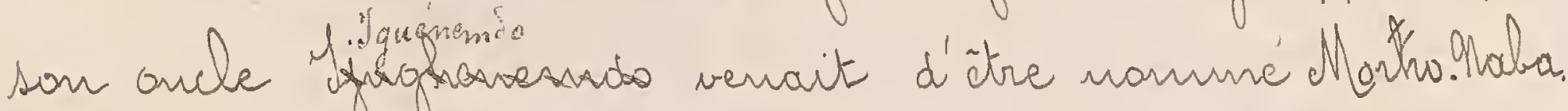
Gurisux il allait se mettre en revolte anverte contre ce demier et faisait appel à l'armée, Corsogu'une de ses soeurs virt le trauver secrétenent et hi senettant le mystérienx Gibio fétiche protecteur de la dypastie dont la vertu dtait irresistible lui conseilla de l'emforter avee lui dours les pays du Mord dout il avait eutreforis la conquîte et oir il me frounait manguer à la faveur de cet afpuir surnaturel de fander un grand rajaume. Dagurag l'ccoutas at fartit enmenaut Gibo. Mon Lin de Gako cefendant, serré de frés par des couraliers en. vagés à so paursuite, ll dît leur aboudanner be frécieux fétiche qui fut rapporté à Guagadarigan air il se krow. me encone.

1 Le fétiehe Gibo est un amas de chiffons et d'anulettes préciouses

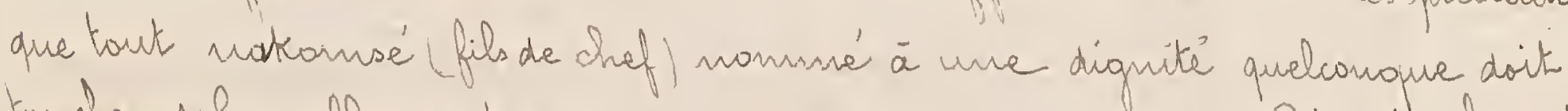
toucher solennellement au maneut de son inestiture. Cot sttouchennent Cui a confére "la force, et le droit de cammander. Sa tarreur insfirée fuar bibo est extrêne. Al est l'olyet de rites suéciouss ausquels préside le

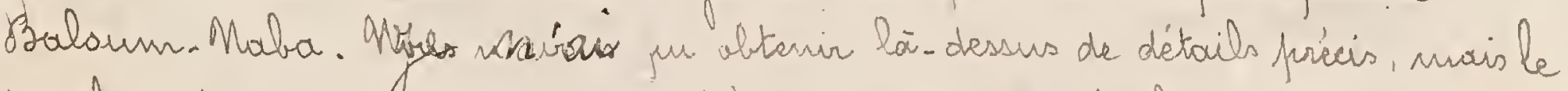
penfle est couvaicher que ces cérénomies qui se frodongent très avant

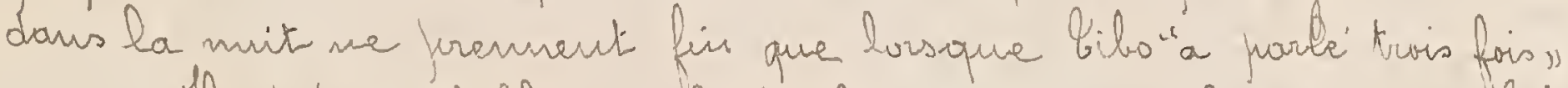
Ll est très.probable que le fétiche connu sous le nom de bibo aux tomps de Masbiré a 'éé vadinant enforté far Oagango et que les canaliers envoyés à la faursuite de ce dernier ne furente l'altrindre. Sa inadition ast d'aillaurs très incertaine. A frassage à Gorrsi

(1) Ilatenga est une contraction de ladagar lenga forre ch lladaga. 
78

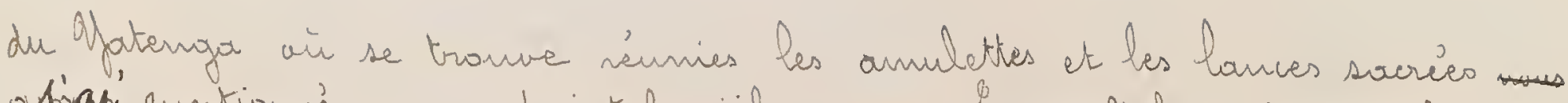

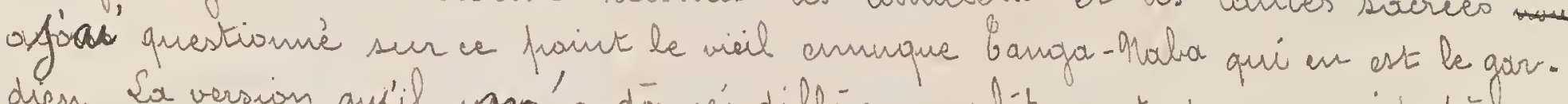
dien Sa version quilil a dóné differe complétennent de ce qui vrééde.

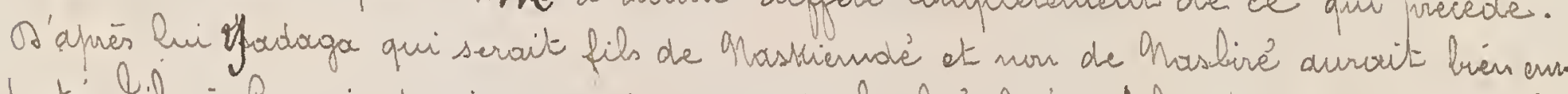

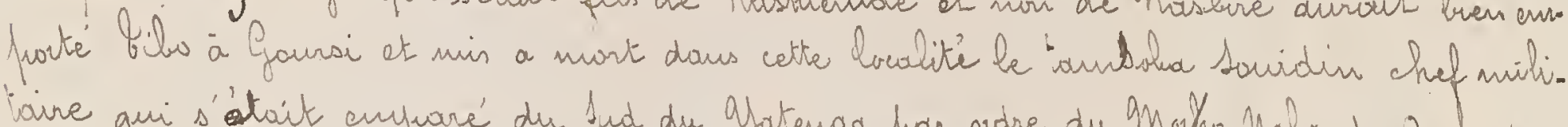

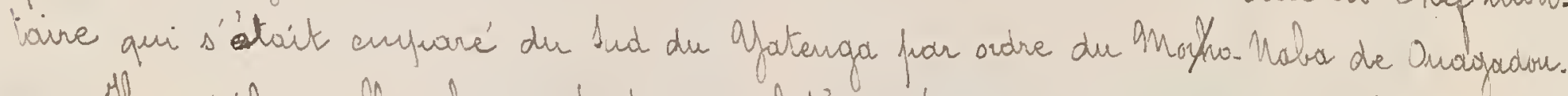

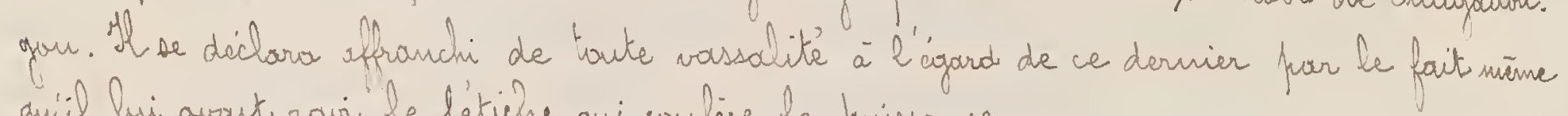
quil hi aurout rair le féticine qui corfiere la prissance.

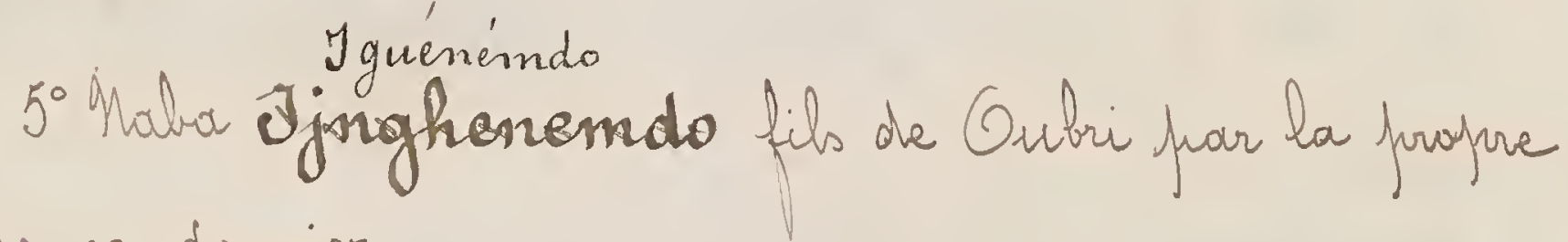
fille de ce dernier.

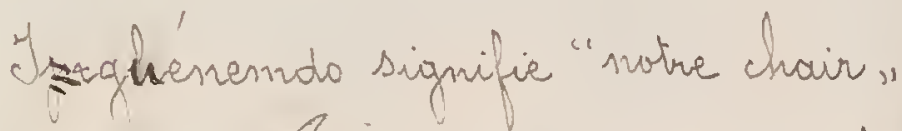

Devise "Coap ten tiléga bi a froússè ouédo. Celui que la guorre a éfrargné reniorciora son cheval de bataille. tbéritier des grandes qualitiés de som fière, fut généreuse at brave; entreprit des expéditions heurensed sur lesapuelles intas

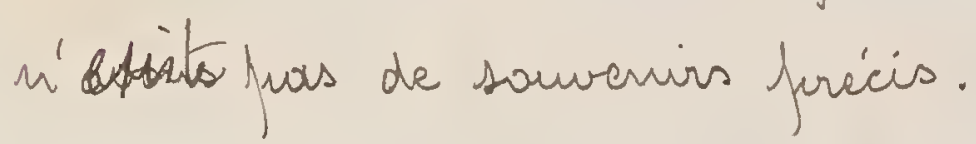

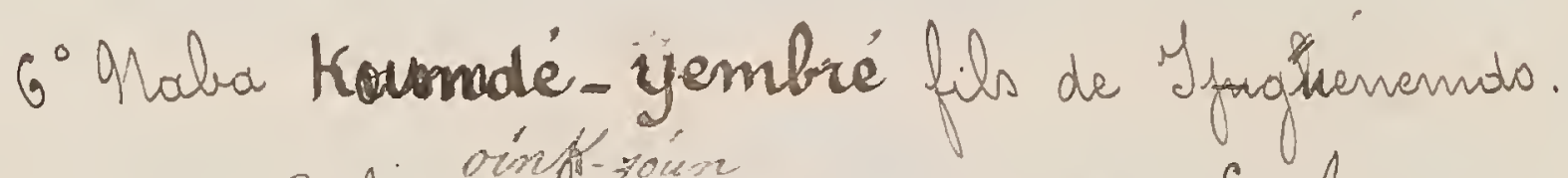

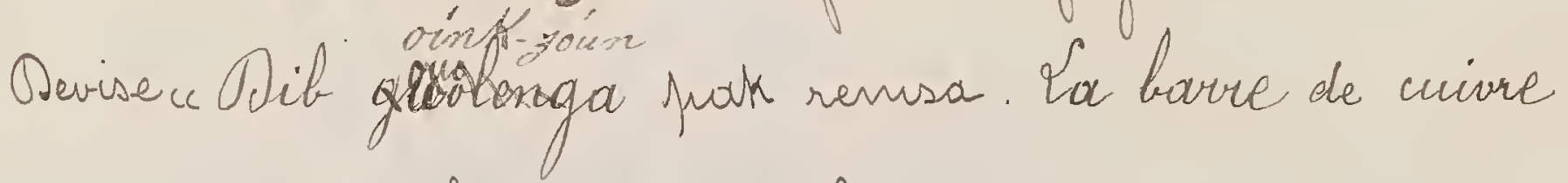
tordece donue du mal (a qui veut la vedresser)

Mart à biour. Pen de sauvenirs. Ancietre des nabas actucls de Boussauna, Vérna, Sabou, Margo ate.

y Maba Kouda le tueur) fils du précédent.

Devisecr. Piga Salorho totedaho saboh sigkiv. Plusle 
roc est dépourve d'aspérités moins il est accessible.

Entreprite de naubreuses axpréditions dowe les prinicipar. les furent dirigés contre les fayss de Sallé, touroyan et Kotulogo. Se naya accidentellement daus le marigat " de Safioné. Sour combean se trauve dous la bocalité du mẽ. me nom. Souche des chefs de Sallé, Richem. Kandé, th. sanga, Jaubéga, Ysmigau, Méders; et Sightiriouallé. higa.

8' Raba Oanaurema (le turbulent fils de Kauda.

Devise chabc' yab yamé ti teinga lebsè mansé. La bataille firie les scoppions sortert de terre ${ }^{(4)}$.

Fiolent et cruel Daouerna se fit de nombrar ennemis. Révart saus cesse de navelles exfíditions it finit par indis. puser ses propres partisars qui l'abandomierent. On ne sait air il est mart.

On racanke qu'un Jour au village de daphoné il se fit apporter un singe qui avait dè capturé, lui nit une cinture autaur du confes ot un brouclet an bras gounche fuis, devant le fierfle assemblé hi donna la libarté en disant: "Vous crafer que te me laisse fras d'en. fants. C'est une arreur. Voilä man fils: j'entands que dé. somais hi et tous ceur de son espiece saicut respectés 
comme s'ils 'daient issus de mon poropre sang.))

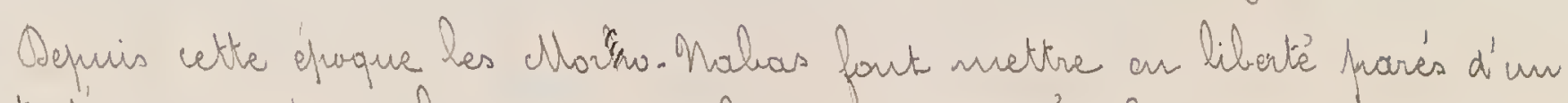

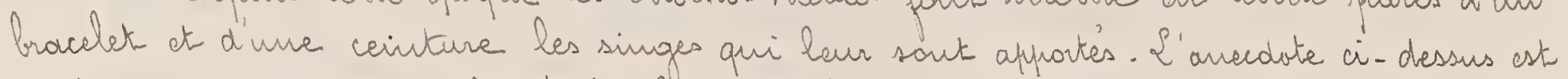
attribuice har d'autres à Thaba Sabba filla de Oubri.

go Maha Tettion. Boussouma (cehi qui a peur de la mont is: fils de Kanda

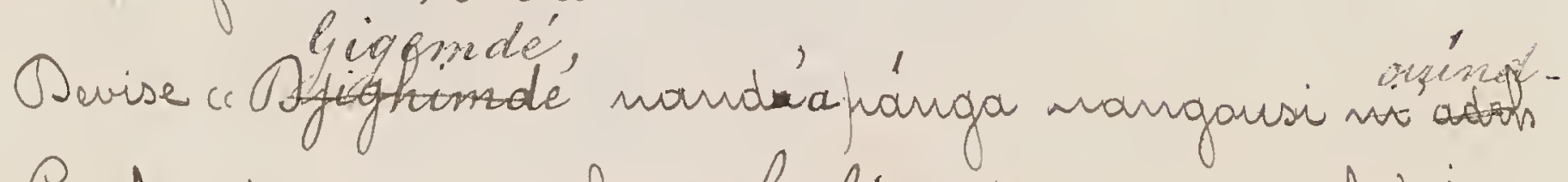
a- Louré. Confiant daus sa force le lion dencure conchè jusqu'aprés le lever du solail.

Son rèque fut marqué far une grande disatke. Il veieut ot naurut à Sumbila Pokto oir se trouve son tomban. Anètre des naluas de Oika.

$10^{\circ}$ Maba Yandsé (le vayaut) fils de Thetton. Bous. Sauna.

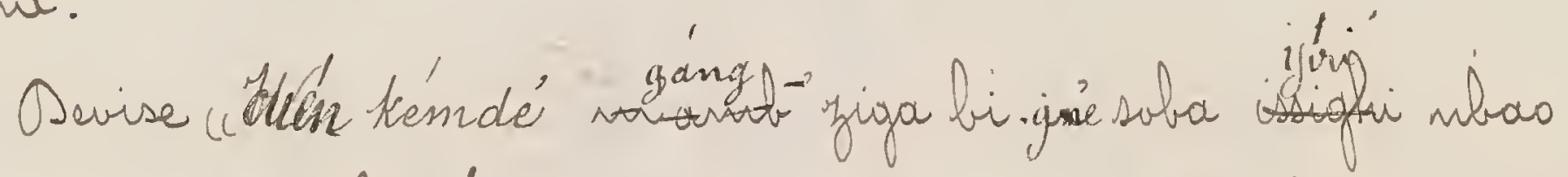
a jiga. Grand le S-igneur taut-fruissant a franchi le seril de la farte le maitre lui.miene devient un itrarrger dars so foropre deneure.

Cette devise est attachie à une ancienne tradition. Anfourd' hui encone Casqui un inférieur resait la visite de son naba il hie abandonne sa demaure en franonsant ces faroles sacramentelles.

Mala Handsé a ver en effet l'avenir lasapi il s'est fixí a Onagadangar ville que sa prospierité et la position centrale 
quielle veccipre désignaient paur être la capitale de l'empire Massi. He déciéta nième four taus ses descendants l'abli. gation d'y mounir mais de nombreuses imfractions out fur se produire. Il eut ité difficile de les prevoir et surtout de les sanctionner. Sa séfulture de fardsé se brouve à Onagodongan au lien dit Baorkém.

$11^{\circ}$ Maba Rakiehfa (le dominateur) fils de Yantsé.

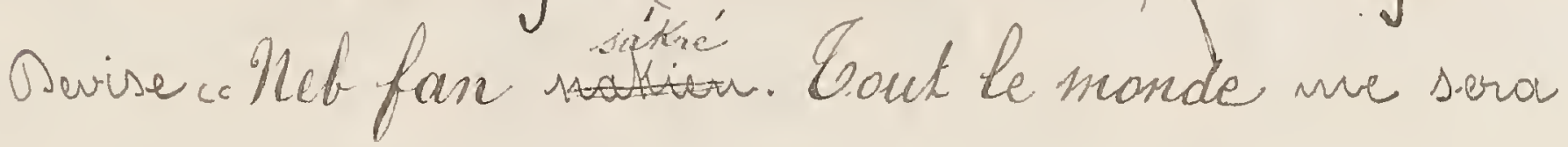
Soumis)

Malopé l'édit de san fière vécut at mourut à Dassauri. Canqué air il est enseveli. Souche des mahas de Jalériki.

12 - Maba Mamérifo (le constructeur ) fils de Nakiegfa. - Devise Folom tar wiria bi noli remba tiliaki songourie. Celui qui a faim ressenble à l'chervier, il con. voite tant ce qu'ou n'a pras pris la frécaution de cacher à ses foux 11

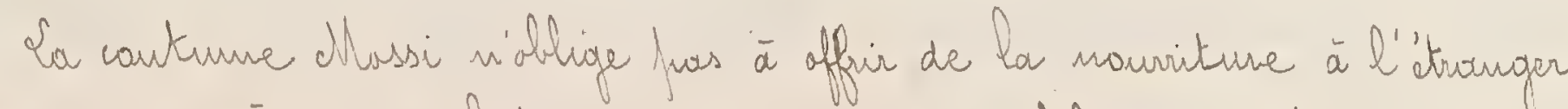
qui fasse, nais dès que celui. ci a aperen une calebasse contenaut des aliments il est de nègle que le maître l'insite à se serwir le fremier. Mannérito ne véent que deus ans après son élaction ot maurut a Gerfham. Maouri (frovincei de Sallé) air se trauve sa sefuilture. 
81

13 Maba Kiba fils de Marnierno.

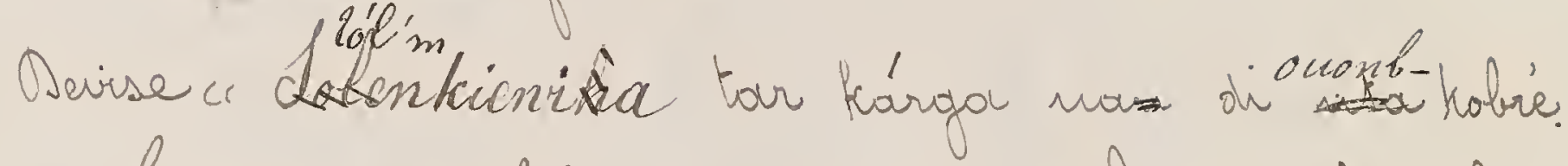
Guand le mawais ulcère se montre à la jambe il la rongera jusqui à l'ass

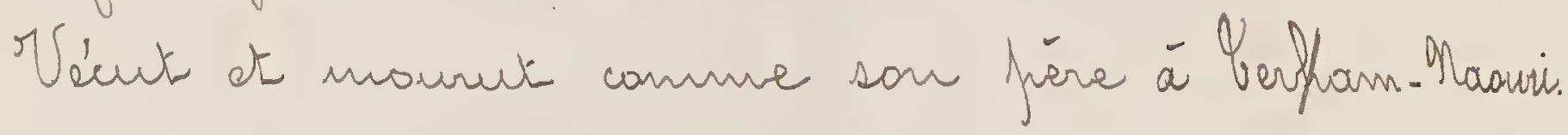
Pas de somvenirs frecis.

1. Mabar Kimba (le stretre) fils de Makricha. Devise "Sa kabadrka manarkè jónga. La routio sans bifurcation est favorable à l'averugle "s Mourut aussi à berfoum. Masuri. Cas de détails sursa vie.

$15^{\circ}$ Maba Mgausbrtha fils de Rimba.

Devise cr Couri toula Kon dangaulé ananndé. Plus ton désir est violent phus th dois avant d'agir perser

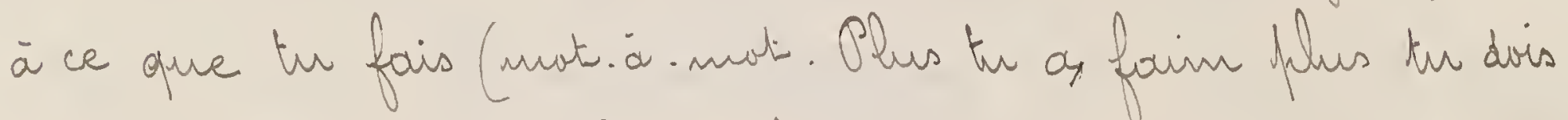
eviter de mettre sur les charbans t'a caldeasse_sors.entendiu, an lieur d'un ustersile destivé à aller au far)

cllournt en tournée ò Mlian aì il necut la séfulture dars un hanneair qui defuis farke son nom. Pas de Sanveritrs. 
85

$16^{\circ}$ Alaba Kan ma fils de Mgourbra.

Lahana signifie "c casse. tëte "; ce nam hir fut donné far

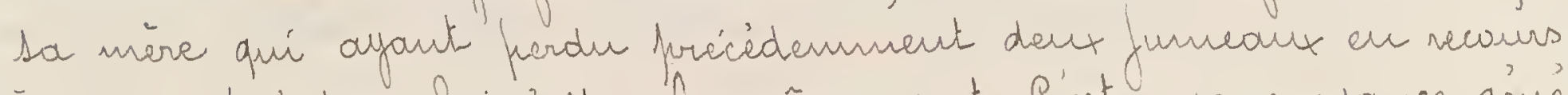

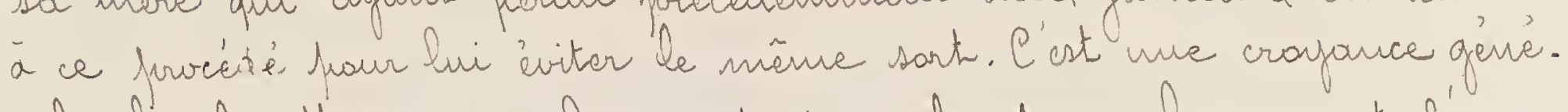
nale cher les Mllossit aue la mart des enfauts en bas-age est l'aunre

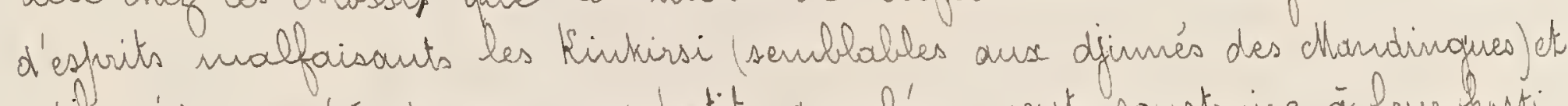

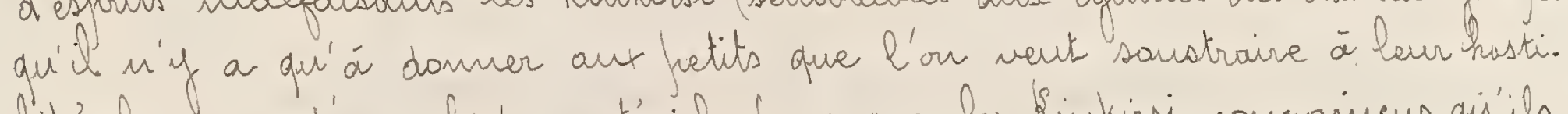

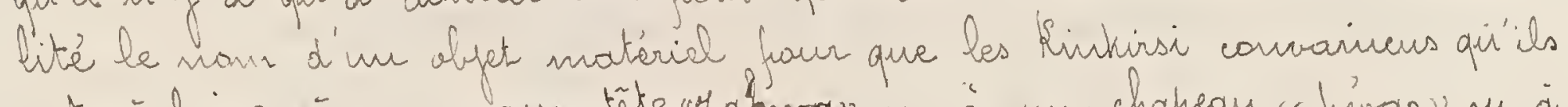

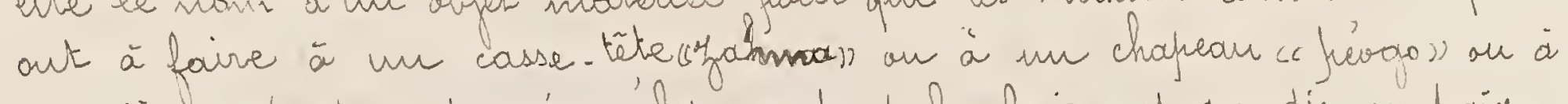

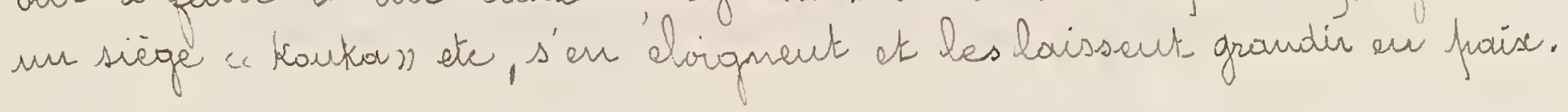

Devise cc Ponré nang gourila bi a belem kamba. Que l'infirme se souvienne qu'il a besair de taus (at que nul n'a besair de lui)

Il y eut ause tempes de Thasana une gra wde disette et le perple dit. on s'assembla devant son faclais lui demandaut à grands cris de la faire cesser. Uive se. maine s'ćcoula sans qu'il êt daigué l'entendre. Se tumulte augnentait. Or un four, connne le solieil était à son délain, il farrut at dit ce He désigpre non fils airé faur conmander la terre de thangaro et fe livi don. me le nam de Páffhim bandauba (C'est-ā. dire, ce n'est fras moi qui fais mairir le mil ) ts man fils coudet paur atre chef de IImuiré. Il cammandera saus le nam de Ca

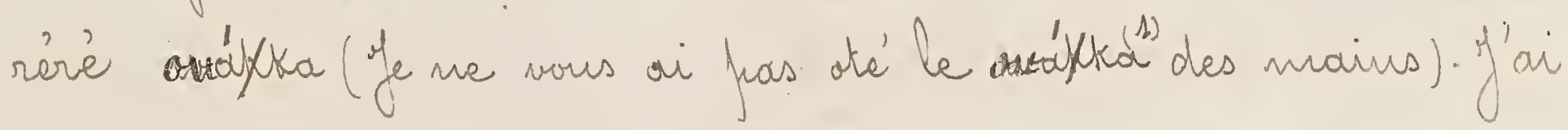

(4) (C. whil qui serk a fiveher la tarre avaut les semailles. C'est le daba des Mandinques. 
atr. Que cerx-la qui out des oreilles artendent at qu'ils refléchsseut ause parales que fe viens de prononcor. S'ils les saverit comprendre, cette disette sera la der. nière dout ils aurout an à souffrir.)

Il mourut à Gragadongour de fut enseveli comme Yfoudsè au lieu dit Baorkén.

Tamana est l'ancêtre des nabas de Harrgaro, I miré de Kaubir.

if Tala Dijirga fils de hama.

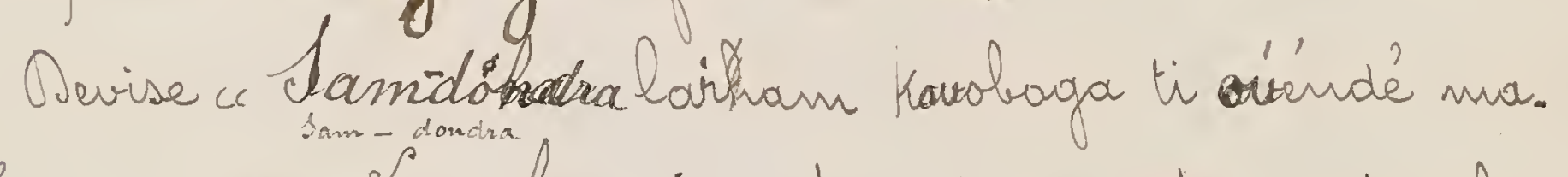
wailthé a nonga. Ca calomnil est infuissaute contre la réfurtation de celui que Oien protégess

Oyrés la famine qui avait marapié les derniéres annies du régue de san fière le Massi jarit d'une grande abandance. Sa paix re fut fas traublée et Oifirga a laissé de bous souvenirs. Il vécut à Orwaga. dongou et recut aussi la séfulture à Baarkens. Tasacara.

$18^{\circ}$ Maba ICoubije (le nourrisseur) secand fils de Oevise cojerpa kon jisy niandé fo sourarè bi fo Kon ti miand ji. Ce n'est fas frar un geste de regret (not.a. nut, en te frappout sur la crisse) que tur civiteraslar 
$8:$

harke, c'est en réfouraut le mal que kn as fait.

Tle régna que dense ans; a aussi son tombean à Baorhern. Souche des nabas de Banaka.

19 - Maba Mattöba ('irresistuble) fils de Houbije.

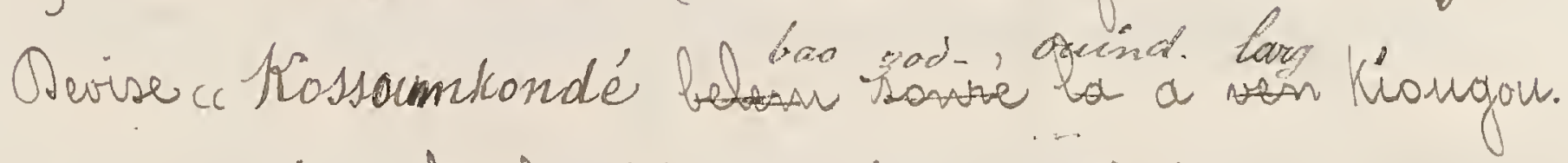
Se Kassoumkandé recherche l'anitié du solvil ot dédaigue la lune.

Se Kassannisandé est selan les Massip l'oisean qui wo be fe fus hourt. C'ests un rafiace aux fattes ranges moins grand que notre aigle.

Pas de souvenirs.

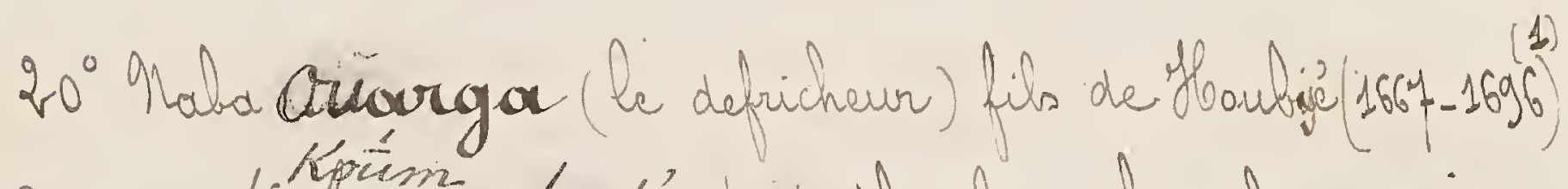
Devise cétitañ sámolé ton ribo bi rib dul-a manga. Ca dette (que tout hamme contracte en maissant) envers la mark as la seule ar'il we peut éviter de payer. San régue fut proshère. Il trauva la mart à Sá au caurs d'une expédition contre les gens de ce payp. Souche des Mabas de Kambipsiguiri, Bildétratenga, Manoro, Sillinobéréte.

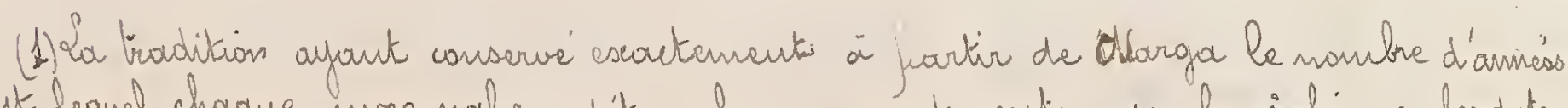

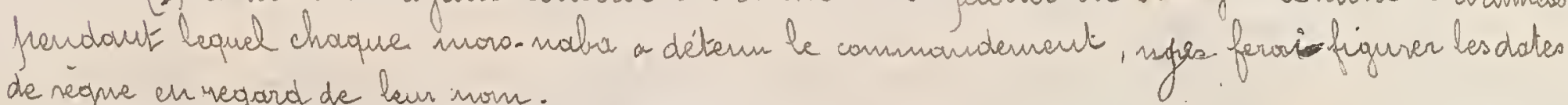
de régue en regard de laur nom. 
86

C'ast an temps de orearaga et frobablements à lacusions des circonstances qui entourerent sa moort que se place l'institution d'une cour. tunne curieuse qui a hersisté jusqu' à nos fours.

Gaus les natius pen ourout le lever du solail un cheval équifié en

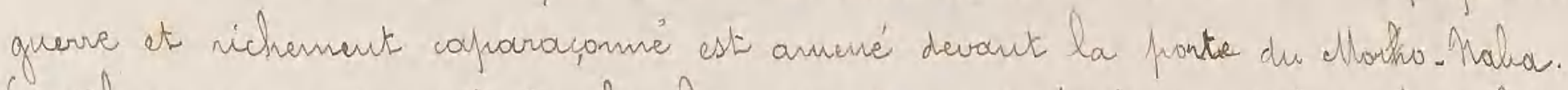
En silence ce dormier sonte de chez hie, negarde un instount du cüté du Mord faits semblant de frendre quelque chase daris une conbiille que lui tend l'nne de ses

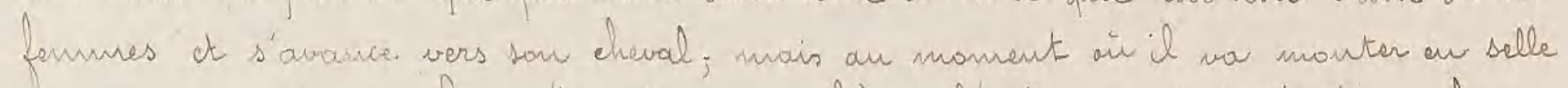

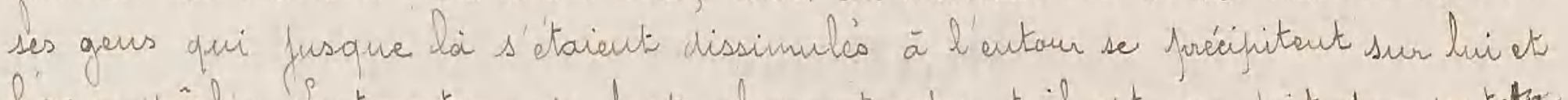

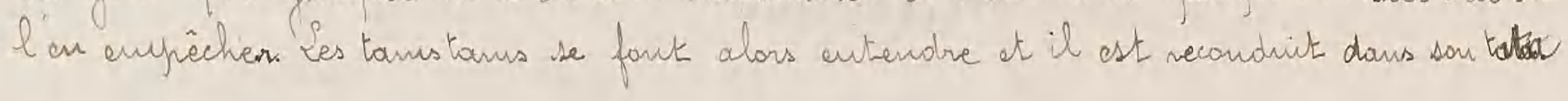

21. Maba Kombré (l'ecunjer) fils de Owarga 1696-1755)

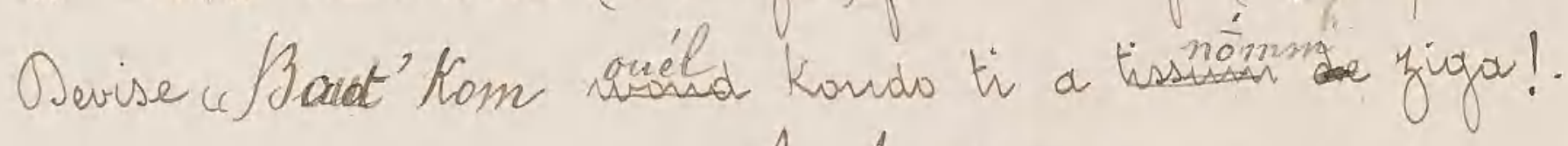
a lis vair on me distingue has le boutc' tcom an kondo mais an goint quele difference!

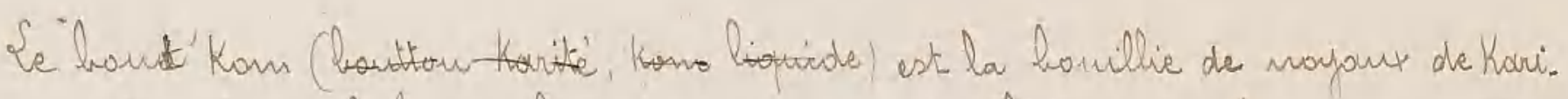

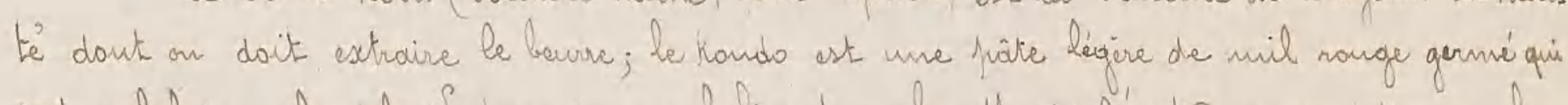

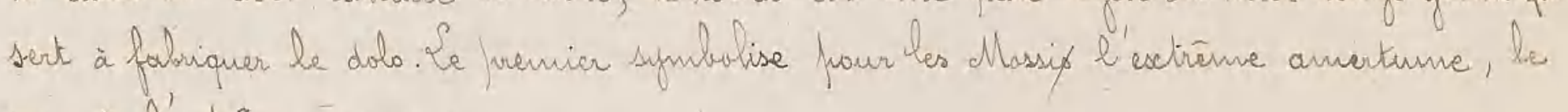
second l'estrême douceur.

Oncine guerre we troublo san rèopre qui fut le plus long de toute la dypastie 59 ans) Il nesut la sifuilture à Oragadargar, au lieur appelé defuris Yambré. roikem. Connne descendarts actucls on me hii comnait que les chefs de deue pelits villages de la province de Faunsorro.

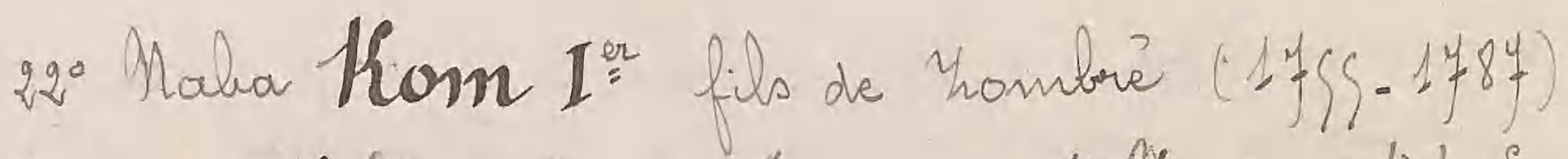

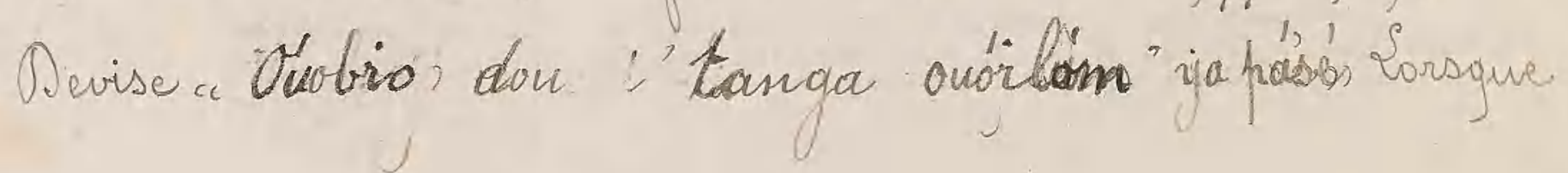


lépephant mante sue la colline e'est frour afunter à sa gromdeur.

to

Il a laissé le souvenir d'un porince equris d'cquité qui perrdint 32 annies gouverna habilement son heuple ot sut faire régner l'shomolance darro le gays. Al n'entre. Morit arreure excjédition militaire.

Enseveli a Onagadongau au lien dit Thaba. Som Howsirem, c’est fuarmi les frounches cadettes de sa descendian. ce que se neritant encore les nabas de diverses localites dout les frincinales sant Doundaulauna (Gunnga) Orevogo.

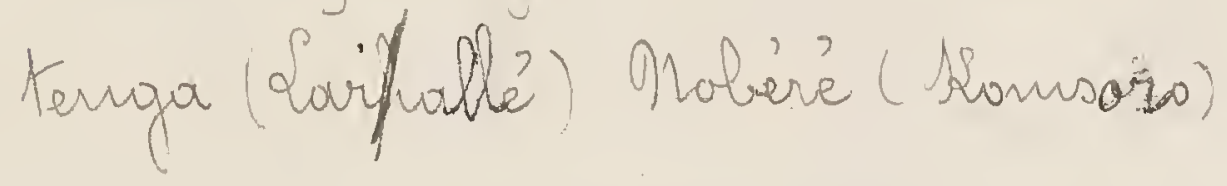

Ce que le jeuple a le phis veterm de Maba kiom I" "è est sa Mrectilection four les crajuands sornours (Juóndraés) vis à vis desgureb il jpersait que le noan symbolique qu'il ourait donné à son vègne (Horn signifit can!, hii infrasait des devairs frarticuliers.

On vaconke ancove que lassque virs le débuti sie l' lnivinange ces batracieus commencent à se forire antendre of que les fanmes vout

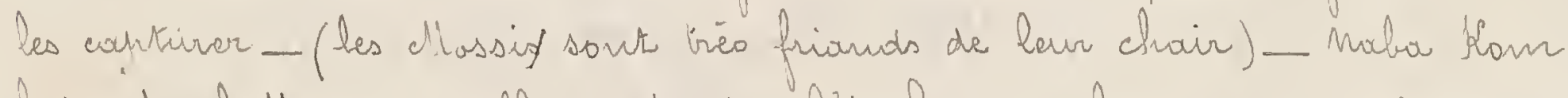
faisait ialtre journellement du bietail dout les nonceaux éiovient refuandus frar son ordre au bout des marigots. It un hieros criait aw

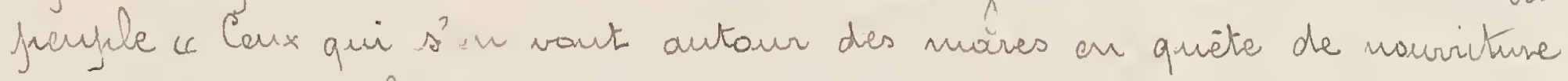

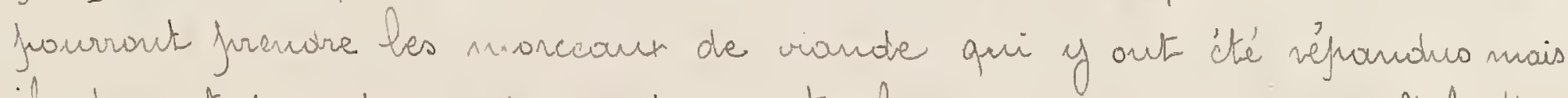
ilo decront saus fraine de mant aesfrecter les crahonids. somneurs car Haba thom

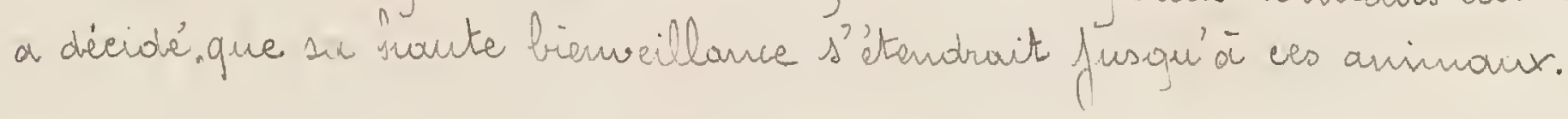

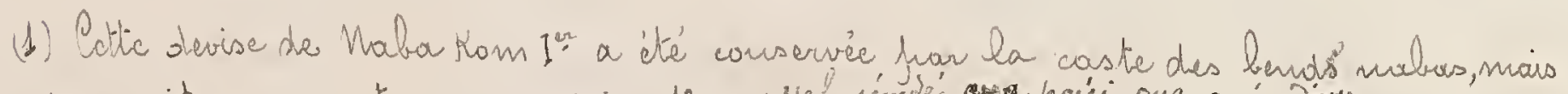

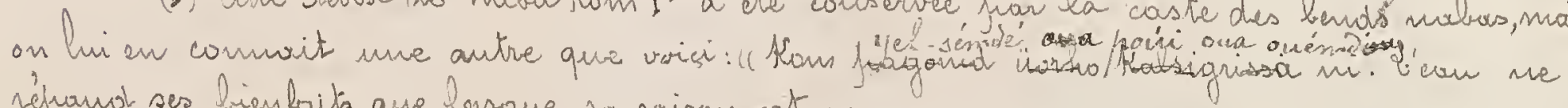
réfrunot ses bienfaitis que losique sas saison ast venue. 
88

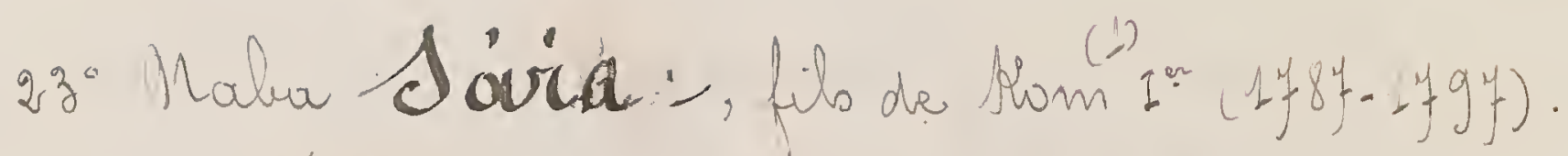

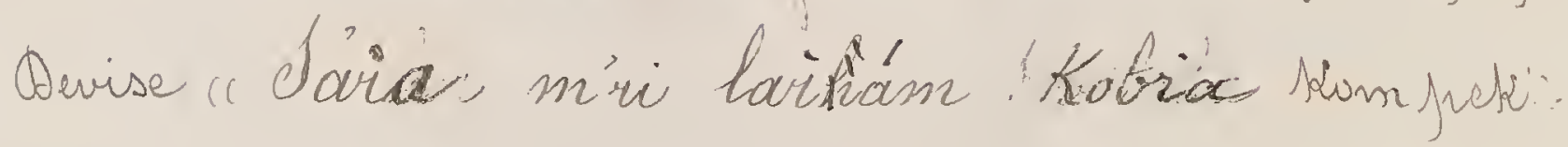

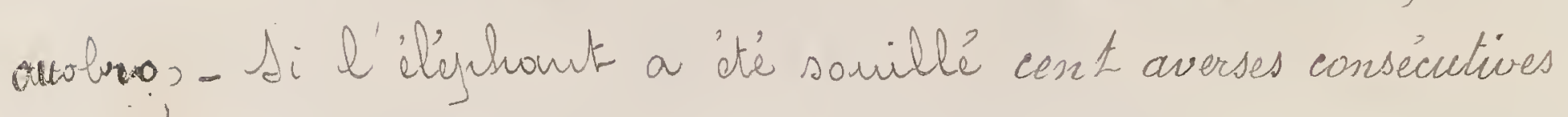
ne suffiront gas à le laver.

Sarsopue Saria fut èlu, Naba Saomaga frère cutet de Naba Ham, qui Mrétendait à la succession de ce dernier of commandait le canton de lian, sourluar les nabigas ot vint combattre son neveres. Celui ai arriva à kriompher de

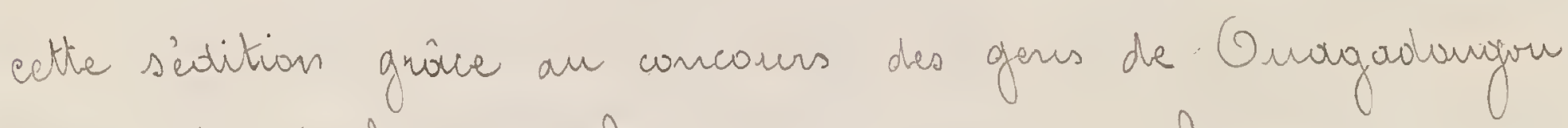
ause quels il fit frar la suite, de grandes larapsses.

\&'acorvissement difinitif de la ville date de cette ejurgue. Connme son frère, Säraw fut enseveli à Onagadangan, man lain de l'enuplacement actuel de la dlussion catholione. Souche des natuas de Mobili ot de quantite de hettits villages.

2H. Maba Doulangon fils de Sárka (1997-1826).

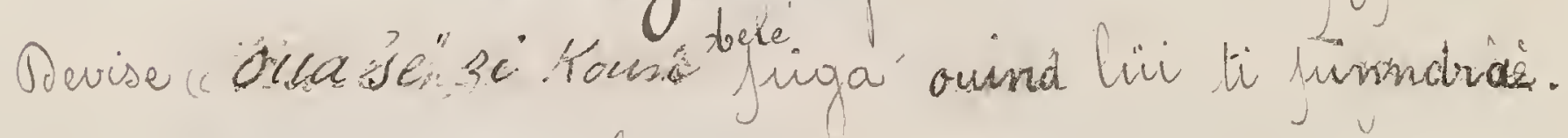
Se nocher est aride mais larsque vient la secheresse (et que kout défierit à l'entour) hii seul resplendit saus le solieil. Doulongar entraprit nombre de guerres. Ja frennière

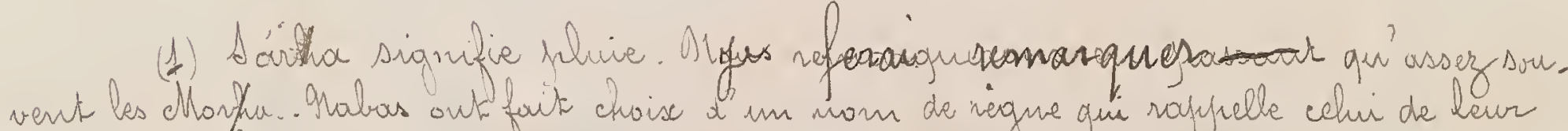

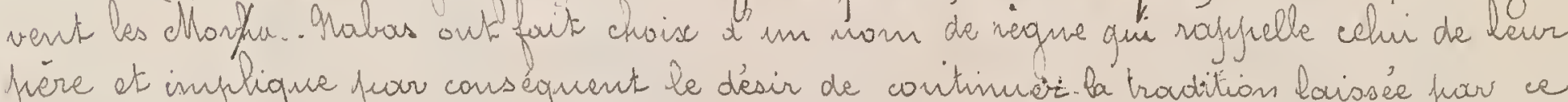

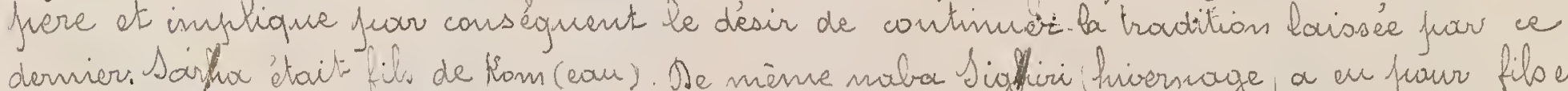
successeur ma naba Sighiri hivernage, a eu fiaur fils et successeur nabo koin If actrellenient regnount.

1) 
exprevition fut dirigée contre le Boussangè et filus particuliè. nement la région de Garango, qu' il wavagece. Ahr grandrum.

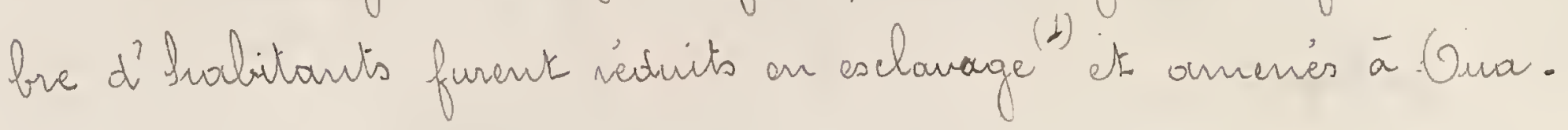
godoingar.

Il fit ensuite la guerre à Boussumma-naba dour il ne fauvait talèrer l'attitude indéfrendante. Sur les terres

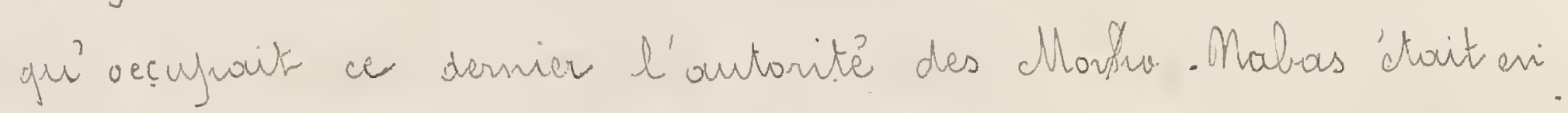
effet deverme jurrement naninale. Ooulouguir résolut de la rendre effective, moús, contrairement à son attente it se heurta à de guasses difficultís.

Ode serieur vevers l'anaur obligé à renoncer à sour enrPrepirse, son frestige en fut atteint et lorsogue quelgues années felus tard, il tento sa dernière expéalition contre Mané. Mlaba, des défections cousidérables se produisirent dons Son armèe. Surfinis avee une faible escarte non loin de Mané

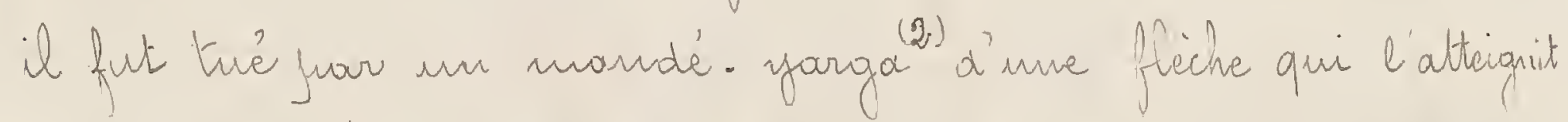

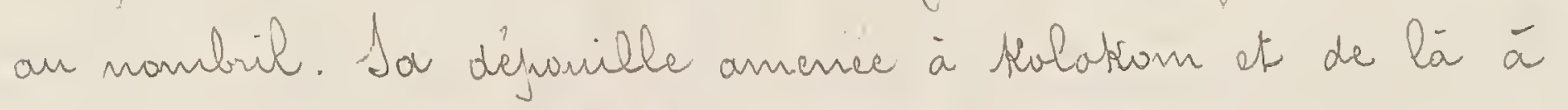
Siragadongai recut la sépulture dars une iterne nou bin du fuster actuel.

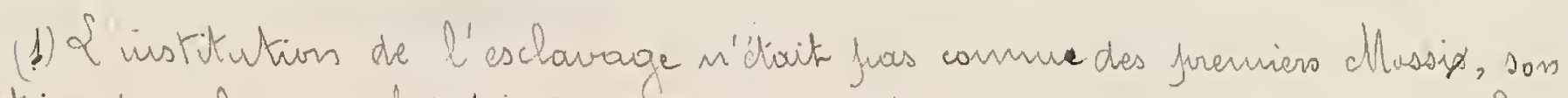

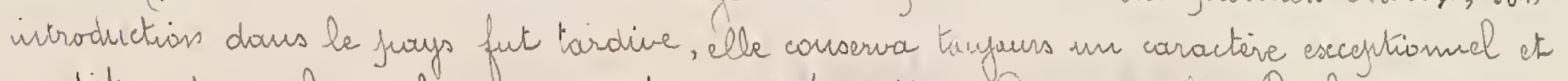

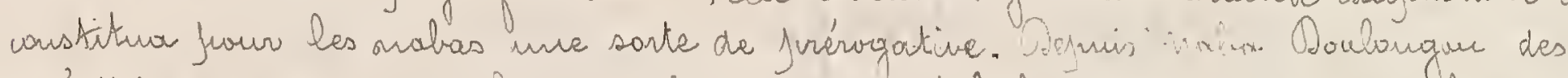
esfreditions ayant four but de faire des coystifs furent entrejrises an delut de

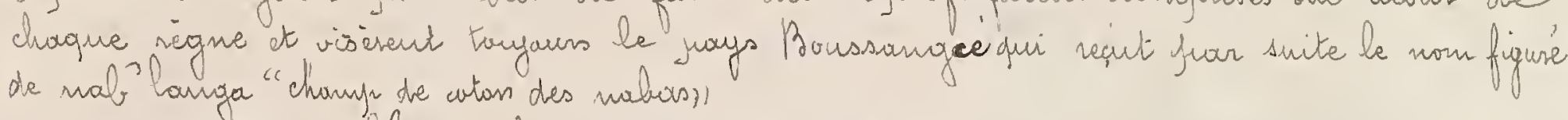

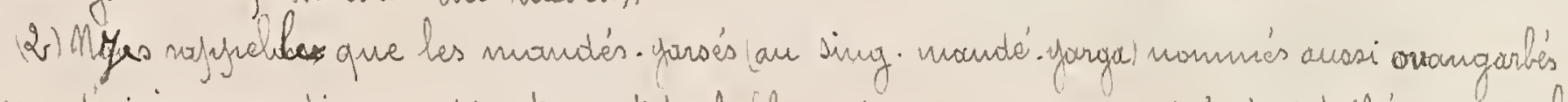

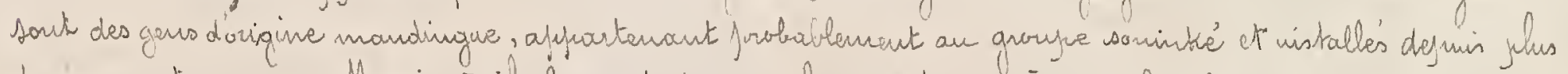

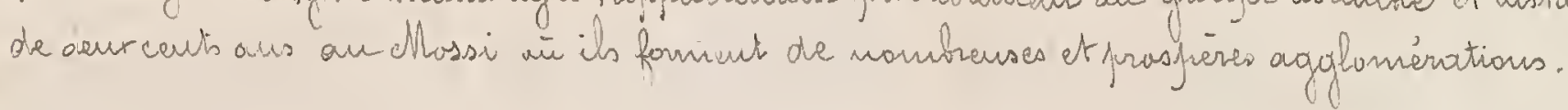


L'est de Mabo Oouburgar que descendent les nabas actuels de Kucila Balom) Rango (Sartwallé) Gartioné et Oousé Oridi).

$25^{\circ}$ Maba Dararalorho fils de Danlangan (1826-1843)

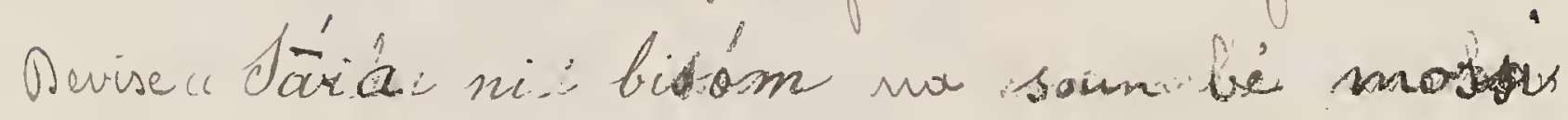
¿'orson'il plewro du lait les Massis seront lieureur.

Dans le chix de cette devise on jourrait voir l'iranie d'un aimable sejetique. Il n'en est rien. Maba daowadonfio avait la foi et fermemant résplu à faire le bonkreur de ans haugle, l'on assure qu'il y jarvint. l'est en tout cas l'in res meillaurs princes de la dynastié at sow nom anjourd' hui encove, est entauré d'une grande vérièations:

St re fit qu? urve seule querre et ce fut jaur vetablir

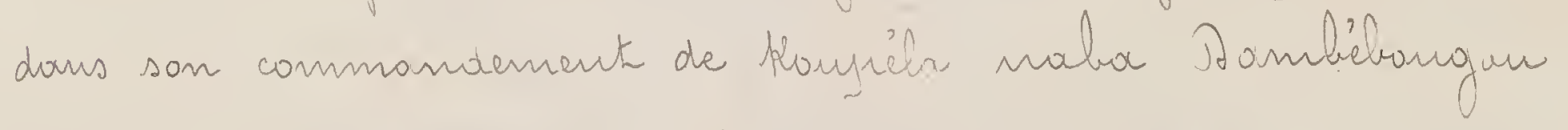
qu'm usuriateur arrit chassé de sẹs terres.

Son combean se tranve à Basr mem frès Onagadangaw. Prrmi sa descendance nofes signaleras les nabas

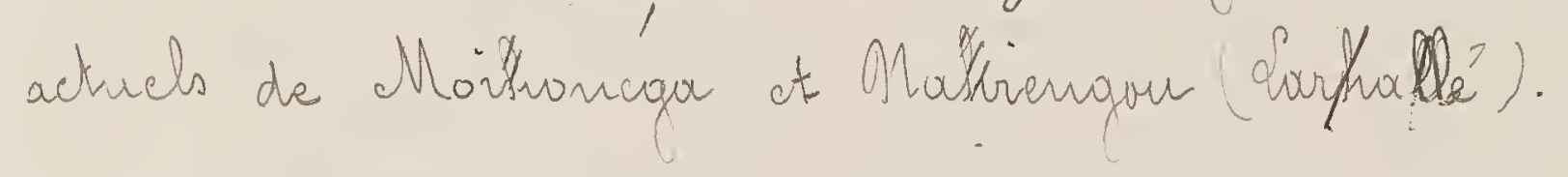

26" Maba Karfo second fils de Moulongan 1.843.1850)

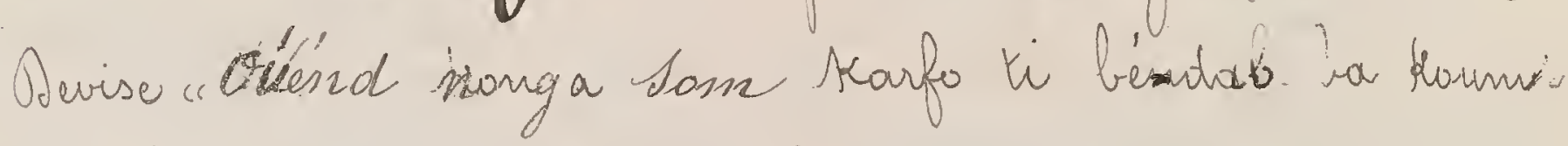
Alkix "Celue que Dien aime il le margure d'un signe qui ìfuand l'èpamarite chez ses ennemis. 
94

Son règne fut maropué fuar une grande exefeé. dition contre naba Oagiapina de Lallé, dout l'issue fut incertaire.

Il mourut à Oragadougur air sa tombe te vit encone prës de celle de son jëre Doulougair.

Sor fostérité l'a diversement jugè. Ses uns lui reprochent d'avoir fait de la papularité en distribuant à la jolibe les biens qu'il comfisquait arbitrairmant aux riches. Mouis le jectit peufle we jaorle de lui quíavec enotion. (On montre encose sur une welline foris de rangongo un arbre nommé Silgefa saus lequel il acridait la justice. Qans sans distirction avaient accés aufiès de lni.

In somme naba karfo est une curieuse figure quil eut èté saus doute intéressant de niesx comoũtre et autour de laquelle mapiai anas vegretté de ne frouvoir recucillir jelus de détailo. (voin f. 116)

De lui descendent les mahas de Gavgo (Honns Bili.

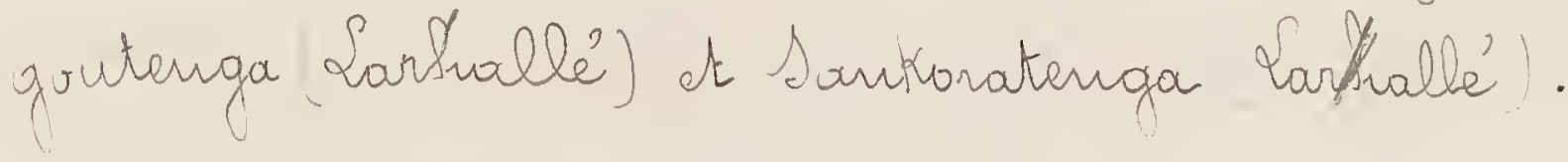

21. Maba Baro , trisienve fils de Ooubrigan (1850_-185\%)

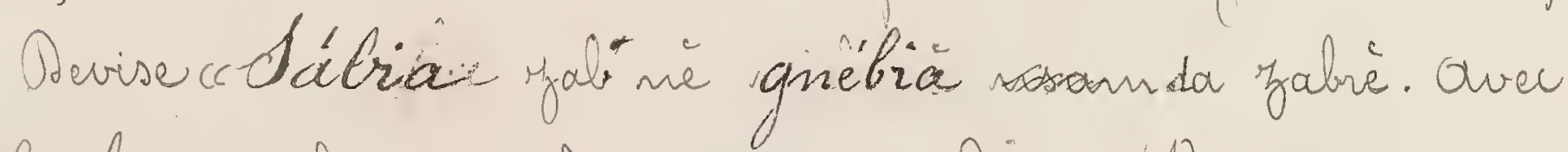
lesol glissant le crocodile we peut hitter."

(b) Allusion au nom de Baoro pui signifie ("bus fornd humides). 
Orcur ivinement notable m'a morguè ce règne de sept annès.

Baviò est ensendi à Onagadargour. Il a laissè deur fils qui abtiment les commandements de Vambili d de Sousouta.

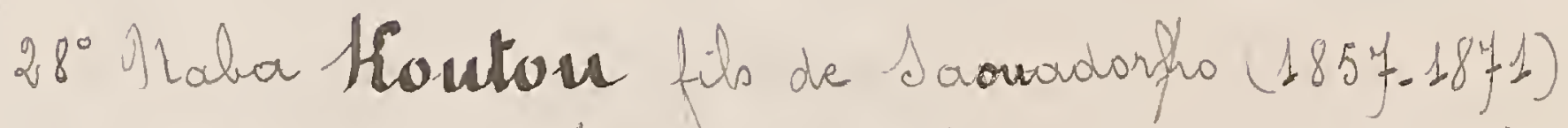

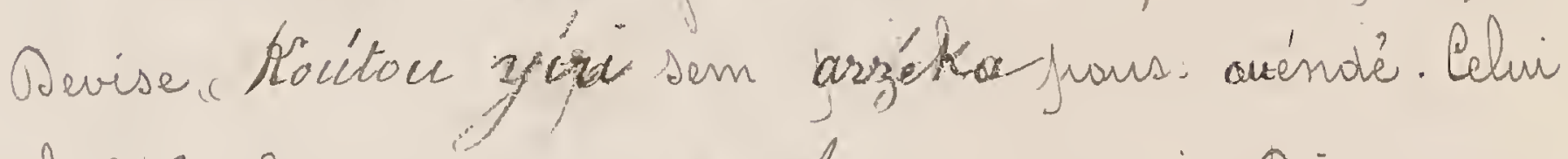
qui a berité d’une maison de for en nemercie Dieur.

Les dibuts de son vègne furent nargués fuar une guerve aver le pary de Sourgau dowt le naba, récenment ilw, avait nefusé de venir hi presenter ses frommages et necon. waitre so suzerainete. Bathe of fait frisomier Sourgaw. Maba fut conduit à O.nogadorigar et exéentè jublique. ment daus cette ville.

Thaba koutar qui savait dit.on, lire et écrire, nena perrdant les dernieires armies de son nígne une vie hiès retirée. Benfrierament froid et rifléchic il nanifesta un fen. drant miediacre faur tout ce qui est activite fubssigue dt frassa mêrne frour malkabile dans les exercices du confes qui ont tanfaurs été en Swomaur chez les issoblis de sa race. Ses musuhnars grétendent qu'il s'était couverti à l'islamisme ot avait fris le nom de kifialilan. Ce qui est certain c’est que la plufiart de ses enfounts nesurent des frénams d'infiartation arabe, ee quictaat coutraire à 
toute tradition.

Il necut la sépulture à Onagadangan et laissa treige fils dout voigi la listo har ordre de naissance.

1. Ol. Hassan qui lni succéda aree le titre de Maba Sanam

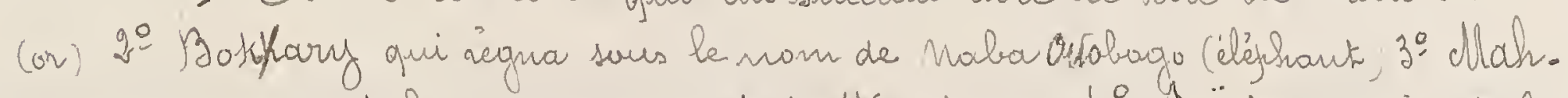
mordar gui eut le commandernent de Otragateraga. Ho Seïdour qui eut le conmandennert de Maronéga. 5\% Moussa qui commande encore Boudri. $6^{\circ}$ Birainna qui vit à hango sans commandement. y. Cllazy qui déaigné fourr succéder à Mabo Oublorgo, défrasè frar nous, fut enfuvisonné à l'instigations

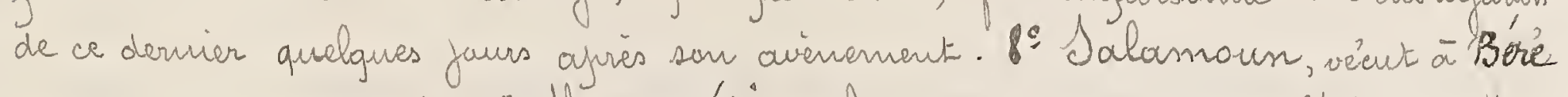

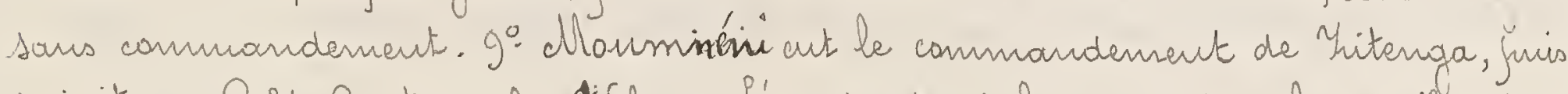

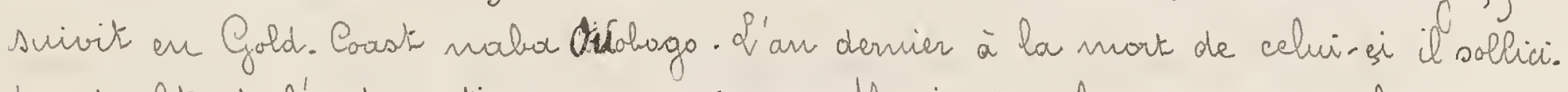
ta et obtint l'antorisations de revenir au Mossi air il vit en single frarticu.

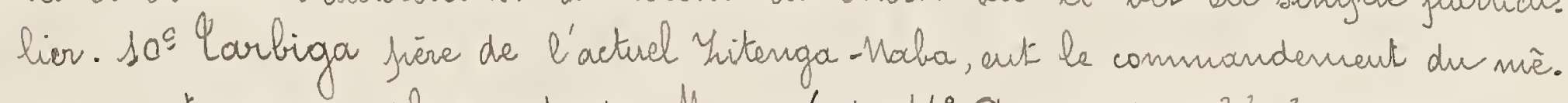
une carton en renylacement de Maumáeni. $11^{\circ}$ Orouna, décédé sans comman.

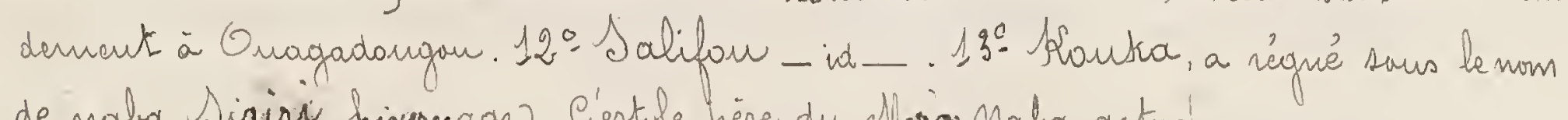
de nabo Sigiri hivernage). C'estle frère du Moros. Mabo acturel.

29: Maba Tamonn fils de Mautan $(189.1889)$

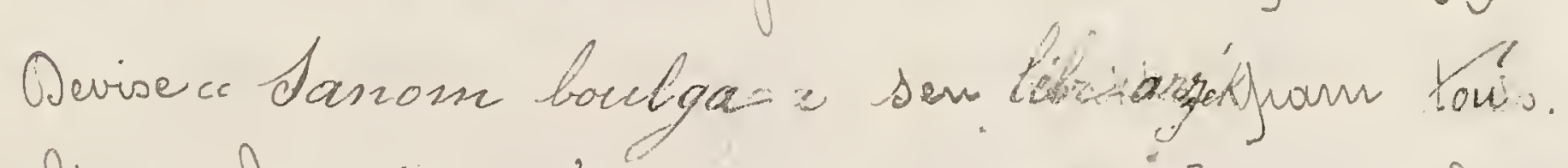
Celui. là seul qui fossídant un freits d'or sait le creuser s'eurichit.

a la mart de naba Manton ses doux fib

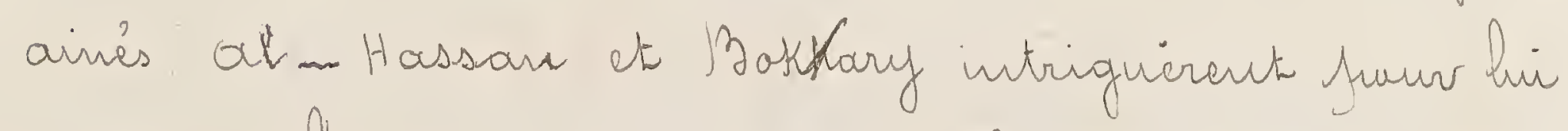
succéder. Gaus deux araient de chands frartisans ot

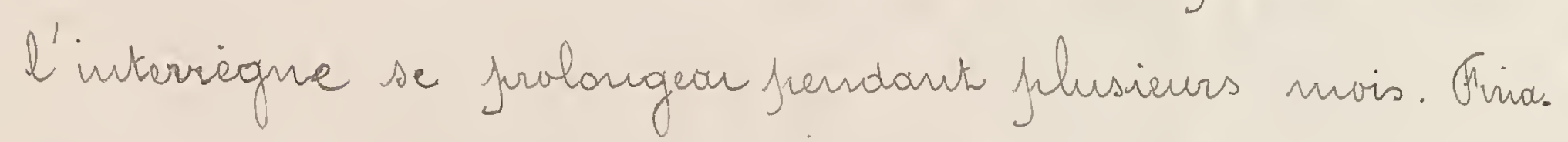
lement al. Hassan l'engorta d monta sur le 
trone avec le titre de Maba. Saromin!

Il entreprit jelusieurs guores.

Sa première eut paur but d'enlever la paravince de Mélanssa à un usurfarteur nammi Yanalé qui, fro. fitant des troubles gui avaient suivi la mort du titu. laire s'en itait froclanié chef. Il avait derrière luim parti assery considérable. Iniformè de ces évinements Sanom réunit en häte une armée qui se dirigea vers le N.E tandis que lui-mène s'arrêtait à Oubri. Eenga at y attendait l'issue de l'exprédition. Celle.ci abautit bientät à la coyture de Thandè qui ourivé à Bagaré y subit la noot Jar strangulations. Nabor Migaradiaba, régulièrmart elu, furit le commandernent de B'elonssar. Orois ans flus tard (187\%) il déilarait la guerre à Boussanna. Maba à qui il veprochait d’avoir favarisé les entreprises de l'usurfiateur Thandé, daurs l'esfair de s'affranchir Lir.mène de la suzeraineté des Moira. Mabat, ancierme tendance qui cxiste encore à l'ctat latent dans les pravinces du NT do de l'E. Aprés quelques alternatives de

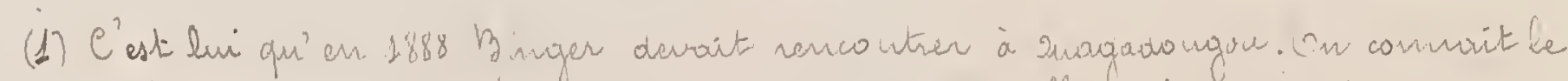

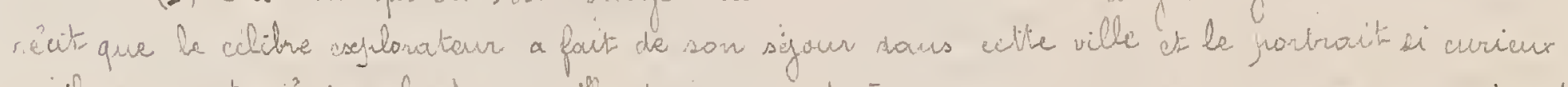

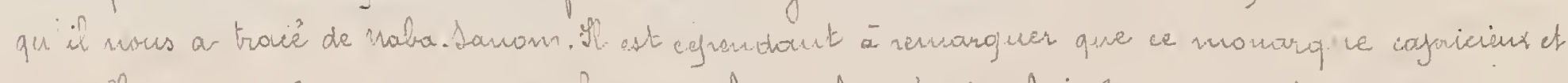

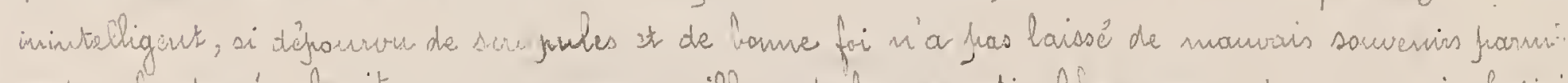

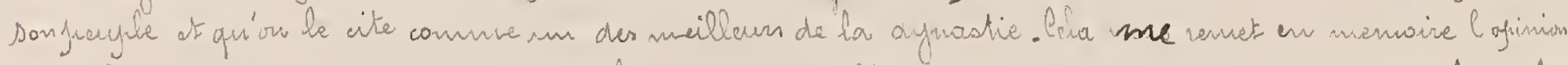

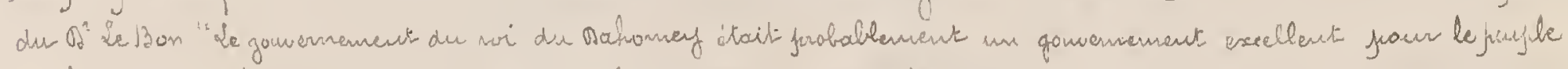

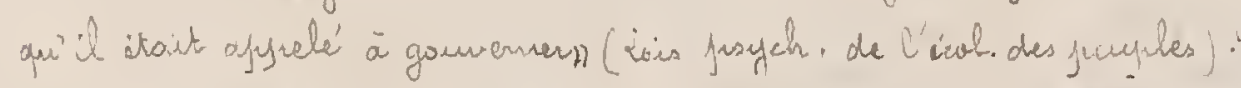

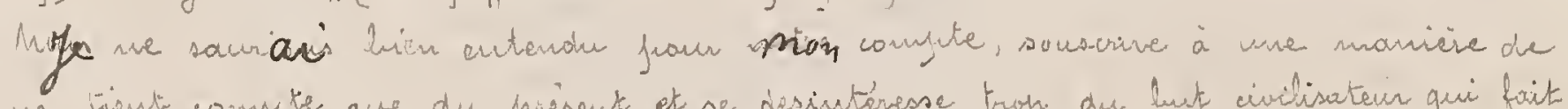

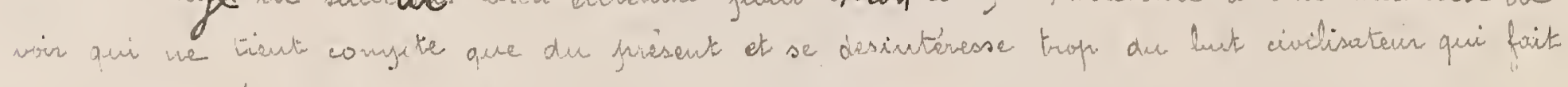
notre raisos d'atre dans ce frays. 
Succis et de vevers la fraix ne barda fras à atre conclue. Sa derniere exfiedition de Sarrom fut dirige contre Lallé. Maba qu'il avait ivesti cant jenne de commonde. ment de sa frovince. Son entaurage s'ètait montré fus. tile à ce choix. Il dejeignait Sallé. Maba conme violent, faritasgue of incafrable de réguer. Ces suggestions ause quelles Maba. Jomom avait nésisté taut d'abard devaient le gagner jeu à peur. Le faste déflayè har son vassal qui we vernait jo. mais à Onagadougan qu acconyragué de dene cents cherouix itait d'ailleurs de nature à favariser un changenent de disfusitions à son égard. Wh jaur, Naba. Sanam juit aus. vertement saus sa frotections un sarfiané que Lallé. Naba avait chassé, Lalle'. Maba de son caté manqua a'égards envers un curn.1 de Sanom: le conflit Srabilement préparé, ilatait enfin.

Les traupres ingrèriales commondées frar le celébre

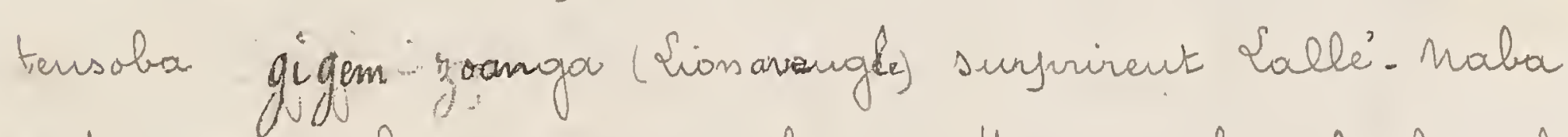
à Bindia dt le mirent en fuite. Mais ce fut là le seul succès de la canfragne qui commençait à trainer en langueur larsque mourut Sarram. Mlettarit à furafit cette circanstance, Sallé. Maba necrutait des frartisans et s’onga. nisait activenuent car les hastilités ne frombaient nanguer

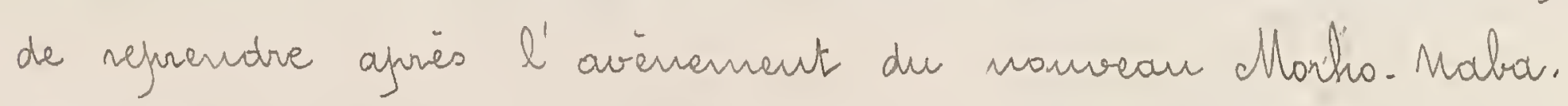
Maba. Sanam ne laissait en marrant qu' une fille monunée Aminata. 

en $189 y)$.

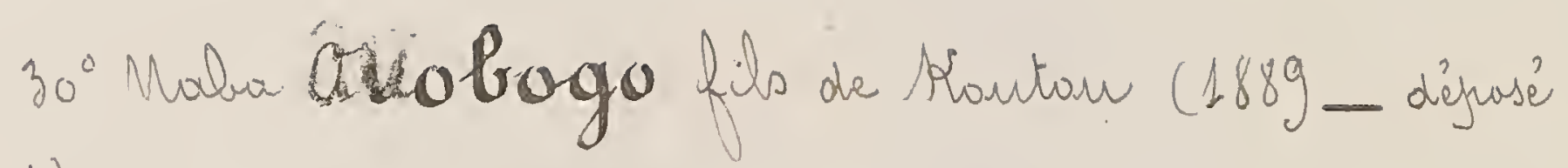

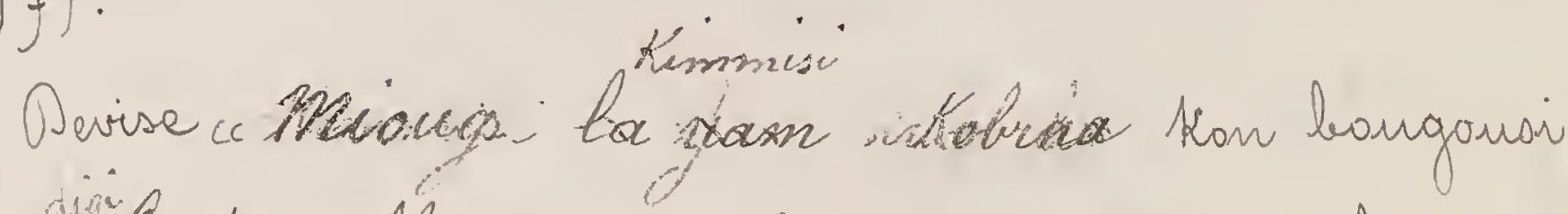

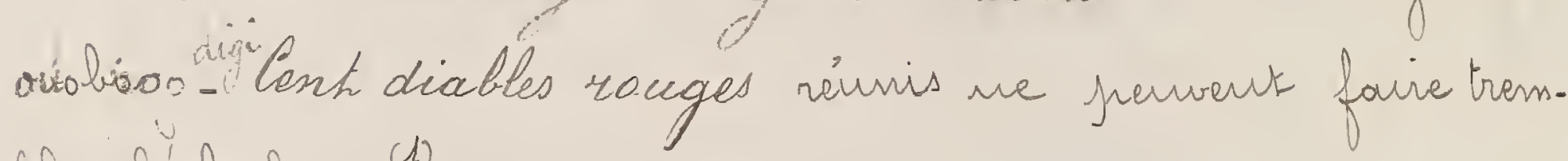

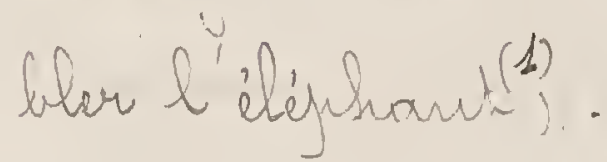

Eloigné de Onagadangour et condonnnè à une exis. tance errante et Arécaire fuar son frére Maba-Saram gui redautait son influence et ne franvait lni frardonner une rivalite qui avait failli le jriver du tröne, Bokkfary avait ver se. former keer à freur contre lii à la cour un ambiont hostile.

On se nendait compute que soir arrivée an frouvair serait le signal d'une réaction violente coirtre le régine qui venait de frenare firs at les dignitaires en forretions avaieut tout lisu de craindre d'étre défuessédés de lours chars ges en foveur d'hommes abseurs qui avaieit suici feridant dixe seft années la fortune de l'exilé.

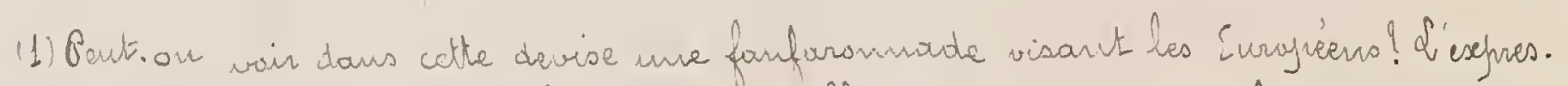

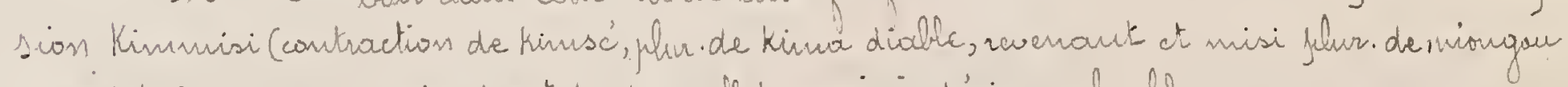

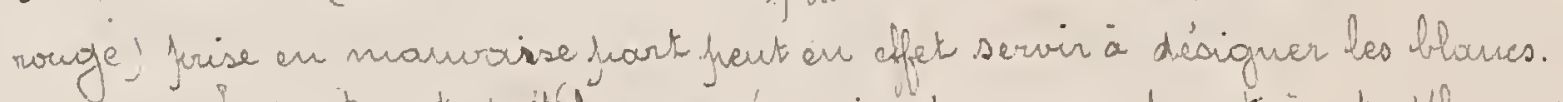

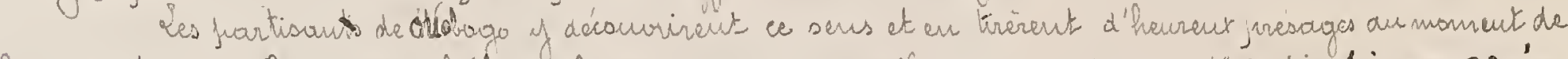

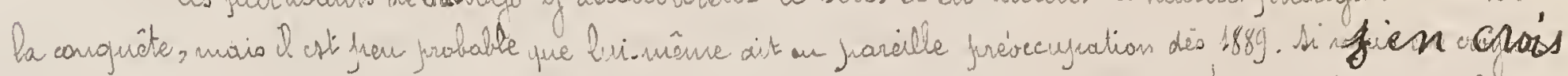

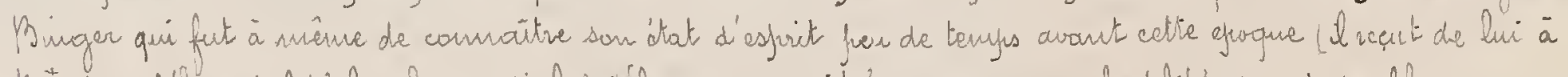

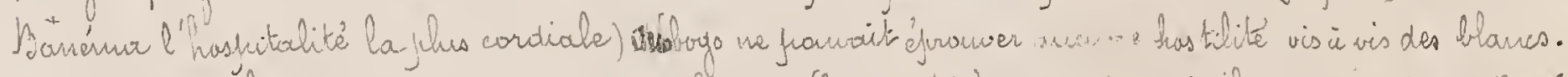

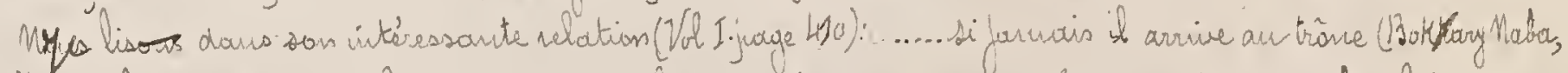

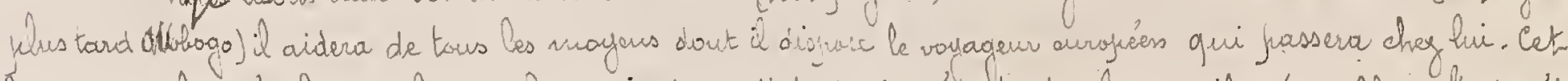

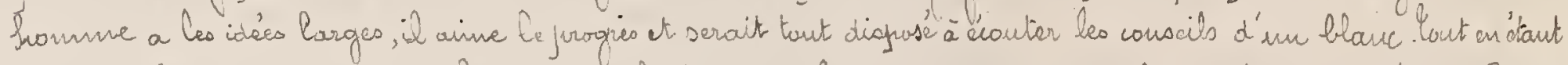

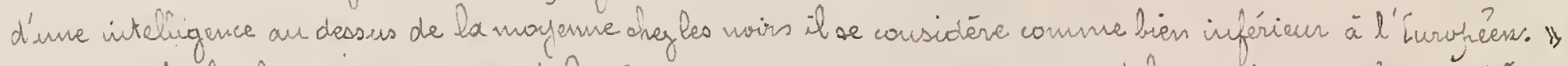

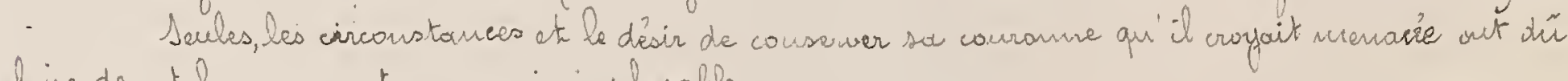
faire de cet hornue wotre ennemi inglaceable.
} 
Bakflary comprenait tout cela et laissant de cite les intrigues, il adojita dés le début une atlitude arraganté et comminatoire qui devait assurer le succés de so candi. dature. Oyrès avoir dêclaré, gice la dignité suyuréme hi reverait de droit, il dtablit dans les envirans de Onaga. dongar son armie, neerutée en majeure frartie frarmi des aventuriers ot des gens sans aven, fuis entré doins la ville fresque sans escorte, it ne fit ancure dimarche. Pendant ce tanjs les autres jrétendants. encauragés far les fautaurs de C'ancien hegine, se démenaient. Se ólídi. Maba, dout l'avis freiraut en cas d'élection, necevait d'enx selon la cou. tume de riches frésents. Se disième jour. comme les houits dignitaires se vérnissaient che ce chef frour déider de leur chaix, Botklary envaya enfin son cadear, ainsi que l'exi.

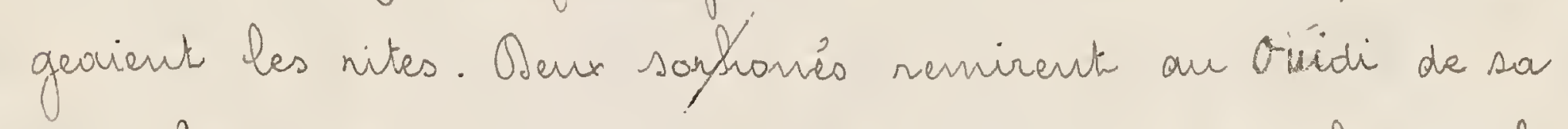
flart la nodeste somme de 10.000 couris, en déchirant les nans qu' ils avaient reçs frour la circonstance: Cen-nèré et Sang-néré (qui siguifient Riflectris. Fien et Comprends-bien). Se buididi néfléelut et comprit. Proclanné Morfo. Maba le sair

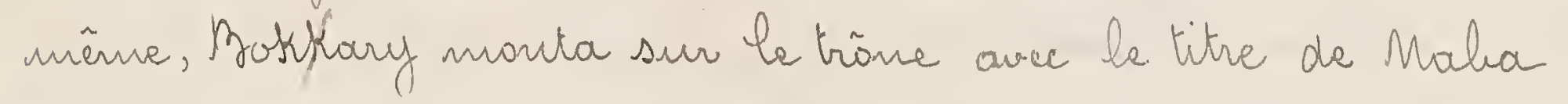
dícologo.

Il ne devait frous tourder à refrendre contre Sallé. Maba les hastilités que la mort de sow frère avait interronupu. Cette guerre était nécessaire et son issue, il le sentait, grosse de consségrances. Defuris longtenfis déjá les frovinces du N. et de l’ $l^{\prime}$. montraieut à l'igard du ganvermenent infrérial des infuationces 
à freire dissirnulèes. Les tributs átaient frayés de mauuraise gräce, en retand presique partaut. Souf quelques Srammages périodigues preserits juar les rites et dout la tradition était trof ancienne jour me fas revêtir un caractère sacré, lé Morki. Alaba ne jamait jaur airsi dire rien esiger des des chefs de certaines frovinces, et ces frovinces e'étaicut frécisèment Bèloussa, Boussauma, Gako, c'est. à. dire la fuartie la flus. flarissaute de l'enyire. Il ètait évident que cette aritarité nominale finirait elle.mẽme jar hir èdrafyer si l'insubardination de Rallé. Maba n'itait frompatement châtice.

Mais ce dernier nous l'avans vu avait nirs à forofit la prèrode qui avait suiir le dèpart des brappes impèriales; il attendait leur vetaur avec assurance. In vain, Maba. Qílobago esserfa.t.il fiar des stratagimes de l'attires fiós de sa feravince, Salle?. Maba vit clair daus cette tactique et solidement cantomè chez lui juit vefrausser toutes les aghes. sions.

La guerre traina ainsi en longuerr : Mtaba triobago dout cette malheureuse entrefurise avait cansidérablement diminué le frestige vajait le frays s'agiter et commencait à craindre que l'exenufle de Salle'. Navira me fut suivi loning ic se froduisirent des évenements irattendus qui devaient le véconcilier ave son adversaire.

Ou debut de Sejitenblere 1896 or apferenait à Onagadongou qu'une armiée commondéc jear des blaines s'était enyrarée de 


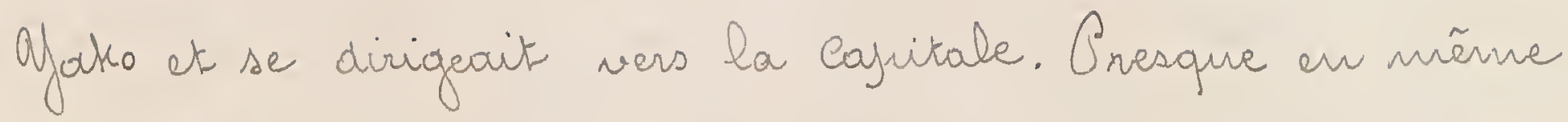
tenfes otiobogo resevait un message concu en termes anianux qui lui frofursait d'accejter notre frotectorat en échange de certaines garanties stifulèes. Persuadé que ectte démarche cachait un artifice ot que notre intention secrète était de le défrossider le Mork. Naba qui se faisait errore Illusion sur sa farce, refaussa l'offre qui hii ítait faite et dis le lendemain Onagadangon tombait en notre jauvioir. (5 Segterubre).

Denx faurs apreis Dílobogo qui en frésence de cette at. tougue inofinize aurait quittée frécifitamment la ville, tachait de a vessaisir et édount aux canseils de marchands fravussas faisait fraur la refrendre une tentative désesfrérée. Il devait être refoussé avec des pertes considérables. T'ngitif, mais s'olestinant toufours dours l'esfivir chinierique d'une revanche il n'aura désanmonis ni trève ni v́frit. Mhias raconté ailleurs sa lamentable odyssée; elle se confond avee l'histaire de la conquiête (vair 4 k. 14 ats3) qu'il surffise de rafyeler iei que sa déchicance ayart dé solemellement fronancie le 21 Janivier 18.97 , les der. miers fartisans qu'il avait conseve's dans le fays ne tardè. rent fras à faire leur samission. Salle'. Maba avait truuve’ la mont freu de temps aufrarouvaut dans une vencoutre aurec la colome Voulet.

Guand à oriublago, il devait après une surforise en frays Boussangeé air son fils prifferé Bila était tombéentre 
mas mains, se refugier dars les territaires Moad de Gald. Coost ot y demander asile ause autorites britanniques de Gambakfa. Il st mart dans ectre ville vers la fin de $190 \mathrm{H}$.

$31^{\circ}$ Mlaba Jighiri fils de proutan (1897-1905). Devise "Piyans baulara ti kan tradöba jad. kiéghlère. Sorsgué le fruits est défá occupè ceux qui vienneut y puiser saut obligés d'attendre à l'écart) (1).

Elevé à la dignité de Aloros. Maba le 28 Janier 189 g, Naba. Sighiri signait ave le Sieutenaut Voulet un traité qui jelacait son rayaume sous la frotection de la trance ot dès catte éfogue un officier senylira auforès de hii les fonctions de résident.

le rógine ne devait être que transitoire. Il mar. quait egrendart la fin des divisions intestines et des rivalités toufours renaissantes dout le Massi avait èté longtenys le théätre. Ojpunfée sur notre prestige, l'autarité du Monks. Maba ne franvait désamais être discutèi. Ses liens de vassalité qui attachaient à sa jrersome les frowinces du Mard et de l'Est, liens qui affoiblis fear le

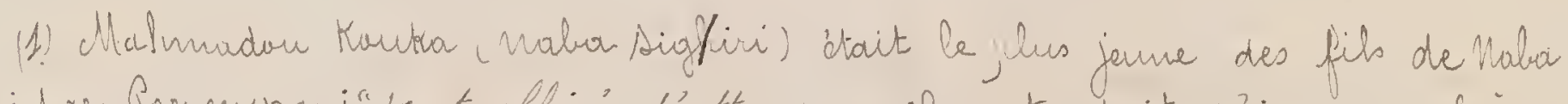

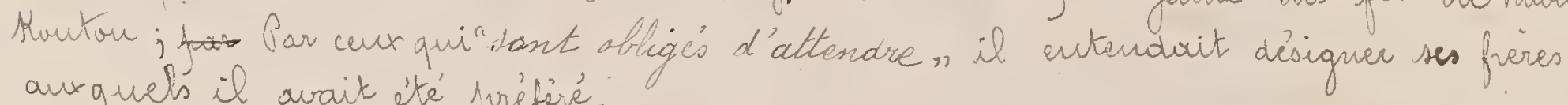
auxquel's il avait ité jeréfiré. 
tenps 'taient nous l'ourous vu, sur le juint de distuaraitre, se trauraient raffermis.

In échange de l'afpuri qu'il arait de nous, le Montho. Malea dait temu à l'accomylissement de certioti. mes Qrarges, telles que l'entratien des trougues. Il percevoit les infröts. Mais l'exercice de cette prérogative, s'inspirrout de mèthodes étrangères à l'esfurit de justice qu'il nous apprartenait de faire prévalair, avait domé lièr à d'in. tolirables abus. Assurée fuar l'intrmídiaire d'une foule d'agents iresponsables la pereepetion ćtait jartout capri. cieuse et arbitraire. Elle ne tarda fras à soulever dans le prous des protestations lágitimes.

Maus ne jourrans sanctionner un état de choses si contraire à toure équitè at la nécossitè s'ungusa bien. tôt de substitioer le régine d'atministration directe, ave les garanties légales qu'il comportie, à celui dee frotectarat.

D'antre frart four ve jass être une entrave au dèvé. lopprennes. matériel et monal qu'attendait le fraus, l'ins. titution ingrériale ne pronvait conserver de ses attributs que couse qui revètaient un caractère furement seligienx ou nuystique. Is c'itaient là les seuls il faut le dire, dout la conscience rojulaire focsomice fuar une langue traditions réclanait le maintien.

In Mars Mos le Massi fut irigée en earle. Mue al. Qocation annclle, iescrite au budget de la calonie, acuait à exclusion de Kout autre vevenu, foumin an Movko. Mabod 
Le maseer de tenir sour rang arce sionité. Dioftiri

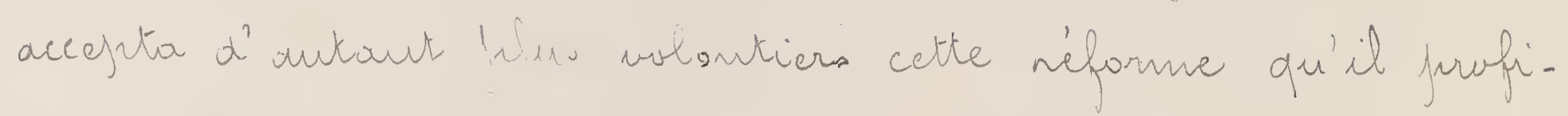

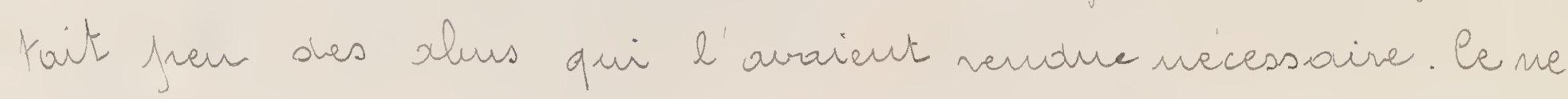

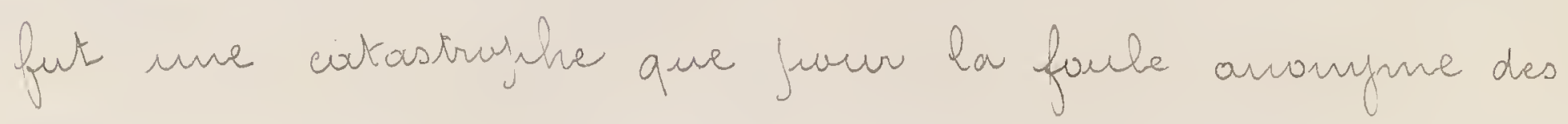
furasasites dur jrouvoir.

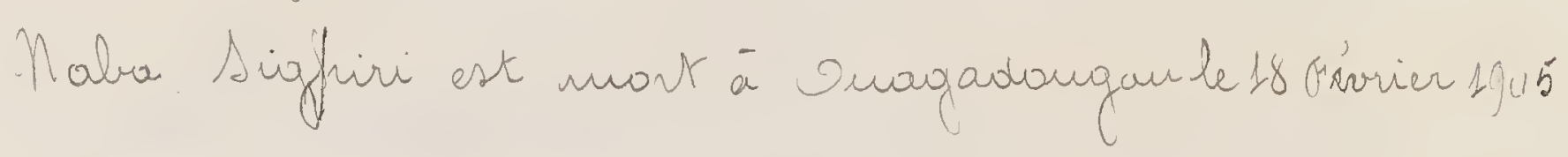

320 Mabai Kom II fils de ciciptiri.

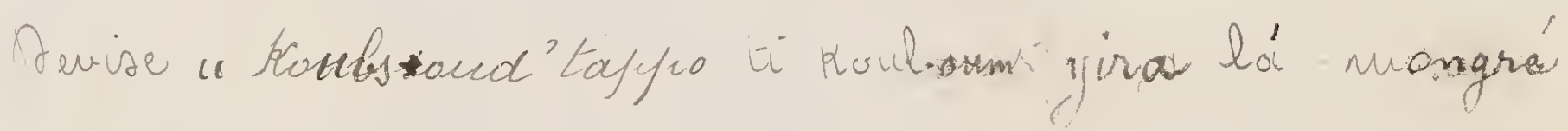

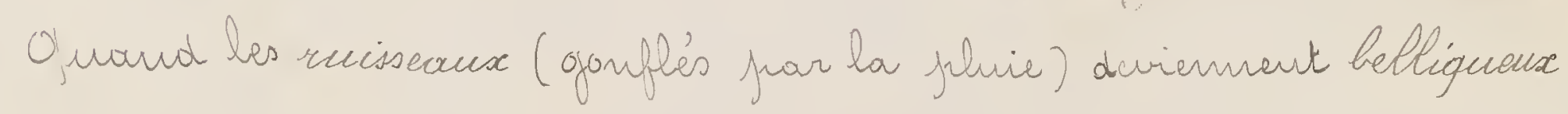
on entend rise le flanve daus le lointain.

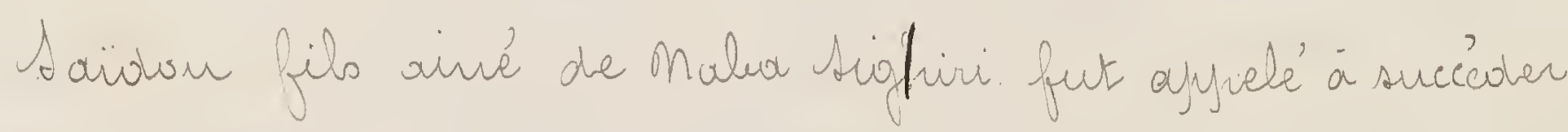

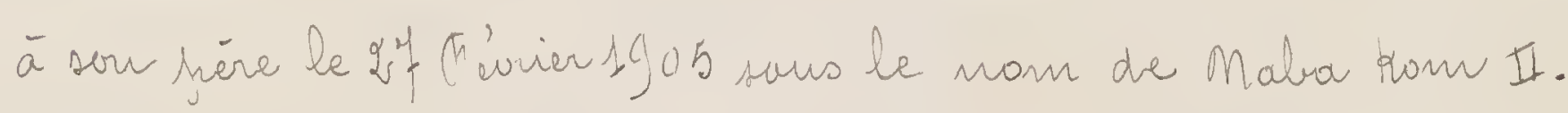

Il était aloss agé de quinje ans et commandait le contion

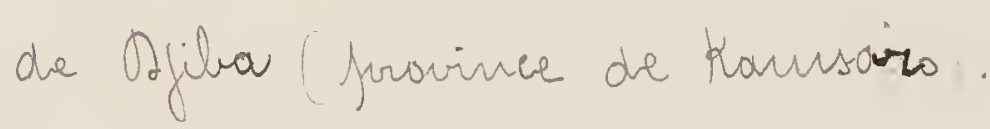

C'art le Morpa_-Daba actuel.

Mojes vienss de parter des Mopho. Mabas quire sout succédis defuis Subri, be fordateur de la aypartie. Mll nome veste à dire ce qu'était laur fouction à l'ajuopure,

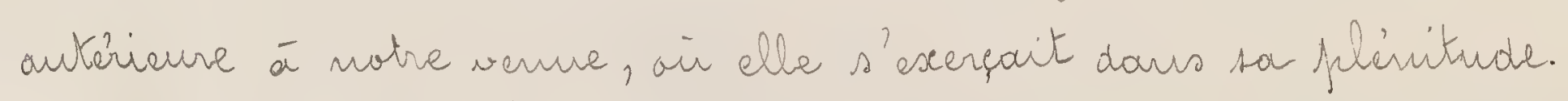

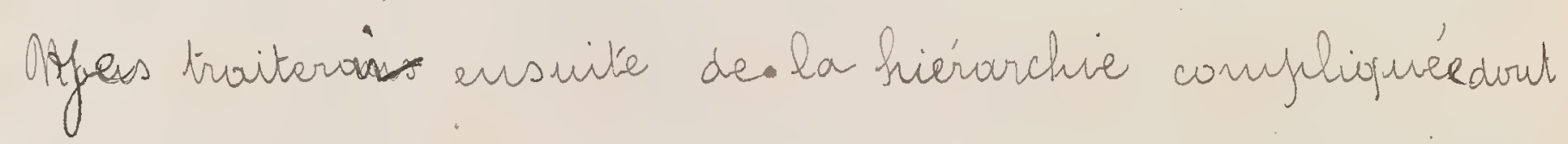
elle constituait le sommet. 
103 

I. Morpro. Maba - Le Maptro. Maba seraitir war dífinition souverain alealu. Ses sufets sout manimes à vecomaitre que le jays hir agyoartient en jorohre, obermis le sol fus. qu'à la papuratation qui le courre. Tou autorité ne corrmaitrait fras de linites; nais faraille concestion, acceletéé jusqu' ici fuar taus cause qui out àtudié la constitution de l'enfiire Mlassi ne vepuse que sur des appoorences. In réabité, ce fourvir aboalu que le Mo'fo. Maba eseerce en frimeifue vis. à. vis de ses sugets un diquitónire. frent l'eseercer vis.à. vis de livi.mène. le diopitaire c'est le Grindi. Maba.

de Moupho. Malua disfuse à san qué des bieus et nués me de la vie de ses sujatio, mais le veto un Bridi prent

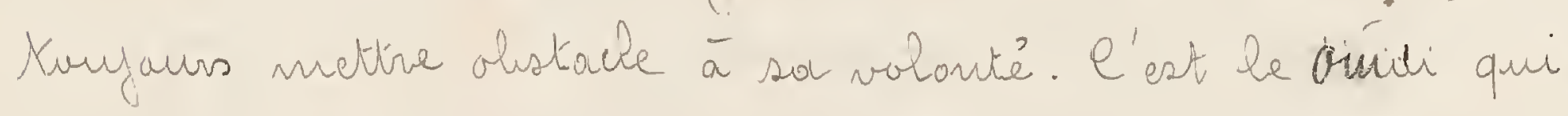
a la mait du Maffo. Maba assume l'intarrégne et son avis freivart dars la désignation du nouveaur suve. rain; whe fois 'du, ee dermier est strictement téme de

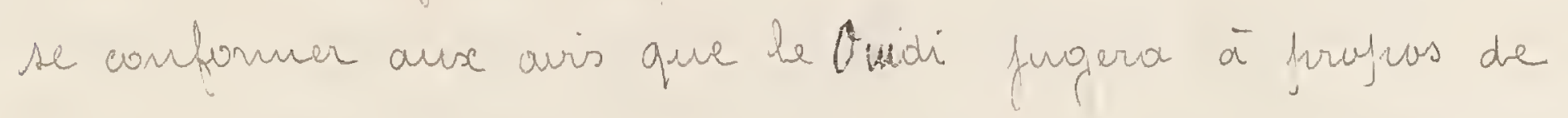

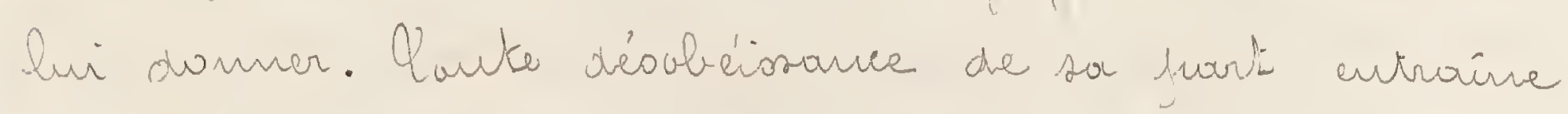
ra une sanction et le Giniti fourra mâne, le cas s'est joroduite, l'obliger à se domer la nart.

S'élection est réolée comme il arit.

thr usage imminarial verit qu'aussitit afires la

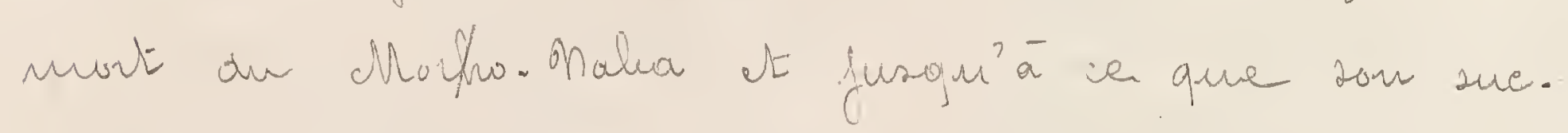
cesseur ait eté désiané, la fille ainée nuête le costurise d'apparat du defunt et recoive les 
hommages qui lni dovieut dis der sor vivant: elle ne frent cefrendarit exercer ancurr acte d'au. torité it demeure alsolument 'trangère ause choses du gouvermement.

C'est le Binioi. Malud qui prendount cette prériode centralise tars les frowoins et administre le frafp. Autour de lui se jorssent les divers prétendants. He recuit d'uns de riches codeause. Oussi bien que l'hi'. rétité directe régle le plus souvent son chvise, la contume adruet. elle que fusqu'an $z^{\circ}$ degré de foo. reuté tautes les velleités preusent se froatuire et dans cctke limite, nul n'ert écarté à friari.

Dis le lendanain des funérailles, le Ovidi Onaba

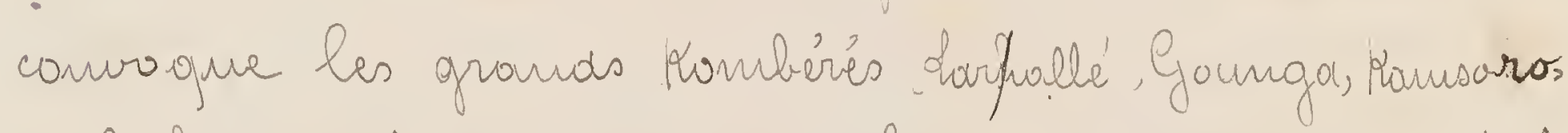
- Balan. Mabaj at leur dit cc de froup ni a plus de chef. Al hi en fout nn. Pami cerse qui preuvent pré. tendre an commandement suprẽme, c’est à wress de choisir le flus diapres des Rombeiés lui réfondeut ilu es notre sufiérieur. Le droit de fourie un chvise me resicalt qu'à voi sauls, . Le rout lá des expressions nituelles, dout

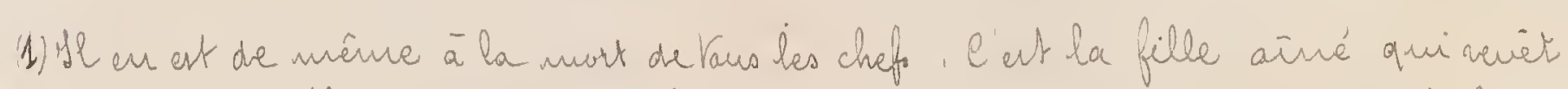
les vetencerts du defunt at recoit les ralutatiois de ses vassaux. It faut en effet que vaus les héritiers niàles qui penveut prétendre à las sue. cersion demeurent égaux jusgu' à ce qu'mu choix définitif wit in.

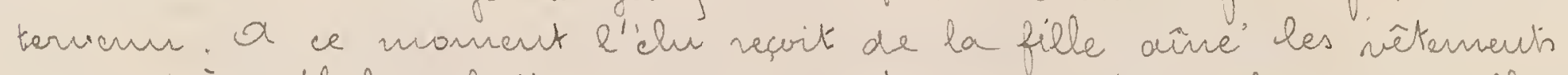
de gon jeìre. Il hi fait cadean en retour du costurue firminin quille doit nevêtir, arisi qu'un certain nombre de bocifls. 
la Aamule inariable vevienara frèriodiojement fusqu'à ce que le Bribir se soit déeide’ an foureur d'un cardidat.

A ce momart il aura une fremiere entrevue ave le Qansaba Maba dref des armés. Oypien lui avoir fait comaître le nom du frétandaut qu'il a argrée il afautera " Fe ne sirs fas branguille, war il est à crainatre que cence dout l'spoir a déé décur ne recouraut à la

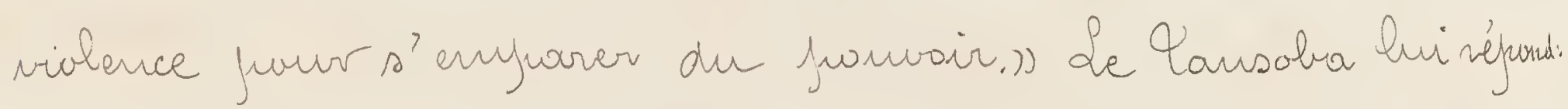
"Cla serait coutraire à la trodition et à la fustice. Casse de craindre. Fe suis là avee mon armée fuur foire respecter ta décision.".

Opries cet entretien il foit comaitre sen chois aux STambérès rémis qui hii réfondent "Qu as exparimé notre velanté et celle de tout le feupley Puis, toundis que la chase est encore temesecrète on mande en hâte le con. current heureuse qui se Jriésente devart le Virioir. Il frote accroche' à l'épaule mue feour de mouton en. blèrre que doivent sevêtir tous caise qui jostuleutme

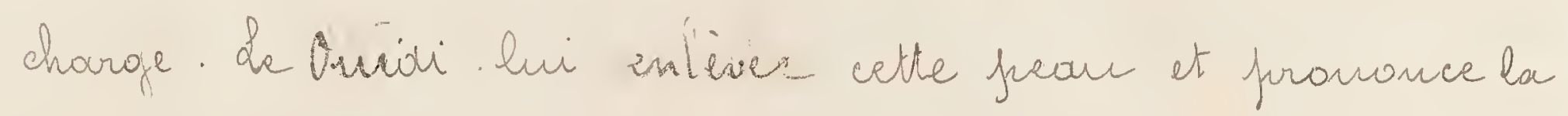
phrase sacramentelle. "fe te dome la moison qui fut celle de kou pière et de tou ground-jière, de mème que le siege an ils se sout assis,. Il tend alors un boubon sans manches à l'èlu qui s'en affuble. l'est un vêtement sowdide qu' il renylacera fuar le grand costume d'affearat afuès avoir subi l'éfreuve, c'est-à. dine 
apris avoir attenit sanir et sauf Casforngat, à deuse kilomitres de Cinougadongur, villouge obut l'enceinte canfére l'in.

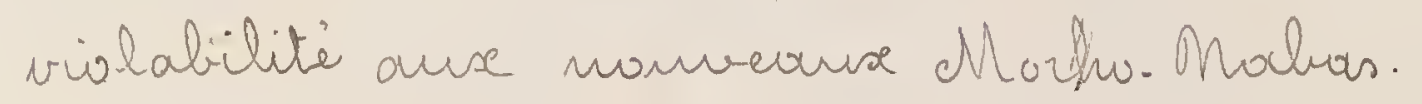

Oussitât afirès, sou mon est jowelamé au detors au buit des tan-tans, tondis que les assistants se saisissent de liv, le saulivent de terre ot le jortent arisi fusqu'à serv cheval. Ce cheval est nisérablement hamache' et comme nous l'avous vu, le nouvel élu cour. vert lui. mèrve a'un nouvoir vêtement. Gieds nus, la Fète ciffeée d'un bannet blarce il ne doit teriir à la mains qu'un simple biâtou. Le Buidi naba te bes autres stambèrés sout à ses cités. La foule les entoure.

La fhifwart du temps sars doute ar arrive sans en. conbrue à Casfranga; mais si les canaidats évincés sout fuissauts, le contigy avount d'alteindre ce Jioint sera l'ob. fet soit de simulacress d'attouque, protestations furrement filatoniopue, soit parfois de veritables assauts. Mais be Qansaba a assumé la charge de deifenare l'élu at pris Kantes disfossitions dans ce but; serr armée qui encodore de cartége, s'offfuse aux tentatives qui fourraieut être dirigies contive lui. Li cefuerdant en défit de ces preicautions le ctorfito. Maba tombait au frouvoir a'un de ses rivarx an était tré, il of arrait lien à necommencen l'elections at cethe fois l'ow se prononceroit probablement en faveur de celui qui selon l'es pression Massi: a su" montrer sa farce.

arrivé are village de llasmanga dout le man veut dive 
"qui confere la fuissance, il en foreud le commandement

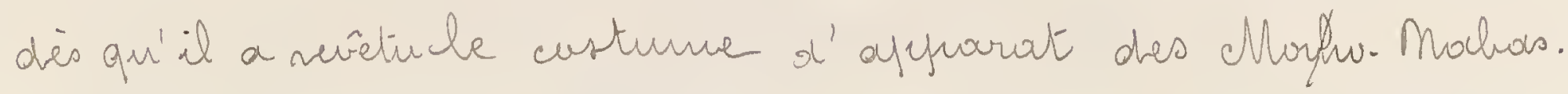
Les komberés ve de quitient jas encove. Cendant cette peòivde on lni construisait à Onagoudonojau mue mon. velle- residence à l'endroit qu'il avait désignè. dhais cet usage est tombè en désuétride; Maha Ram 11 habite ac. Eudlement le falais de son pére Jiafiri

Se samedi suivant le ranvean chef dvit se veridre à Mivousé(le nom de ce villonge signifie"la foree se refose n) fuow of recevoir les hommages de taus les chels Massif of compris ses comeurreuts de la veille. Les Mombèrés le quittaut à ce noment; ils neviendrurt dè. somais le saluer chaque matir:

Oyerés avoir acconfuli divers sacrifices rituels at distribue' des cadeause, le Motho-Onaba quitte enfir Divansé ai il a du fuasser environ deux semarives et sentre suivi d'une funla nimmare à Onougadongan oir il prend fussession du fualois infrérial ar milien des vifonissances fubliopres.

Sá, le cérériarial orainaire at fisé tpour chaque fournée arisi qu'il suit.

a l'aube à lien la cérémonie du simulocere que giàx déerite a'autre frart (vir Maba oriarga f. is ).

Sorsqu elle est terminèr les Rombries vienuart devout le fuabais et frement flace sur des sieges en fierre qui leur sout respectivement assignés, sieiges dout un protowle 
rigurreuse a réglé l'enfelocement at les distances.

$$
\text { Le Samandé. Mabat" se tranue fuarmi arse. Ce di. }
$$

Gnitaire figure dours tautes les cérémonies qui out lien en cet endroit dit "Samanté," (glace qui se knouve

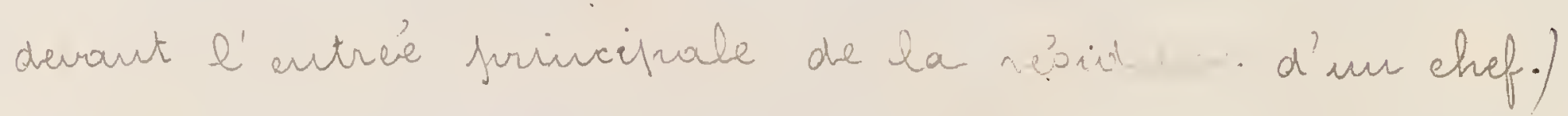
On y virt encove des Oayai at Ouídringa. Mabas dout il sera juarlé plus loin. (2)

A ce momaut le Morho. Maba sout de chy hi. Les chefs se leient et vout le saluer successivement

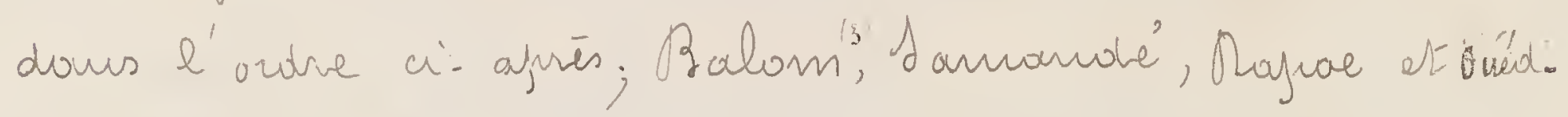
ringa. Ces trois demiens quvique nevietus de diguitiés se. condaires fussent daus ecte céremanie avaut les granids Monuberés. Cuis vieut le taur de ceus- ci, e’est-à-dire

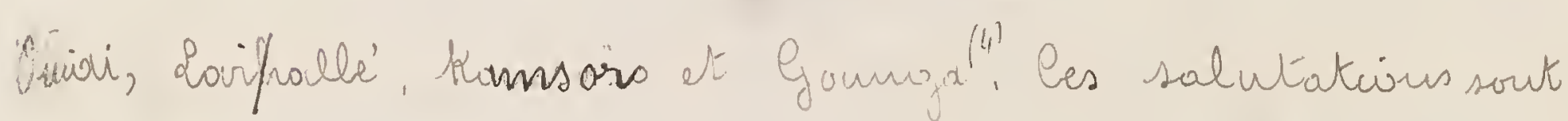
esécuties selon la coutume Mossi;, les cheip se pros. terment ot frapprent bris fois le sol de leur conde, le puing fermé, le funce détaché des autres ovigfs.

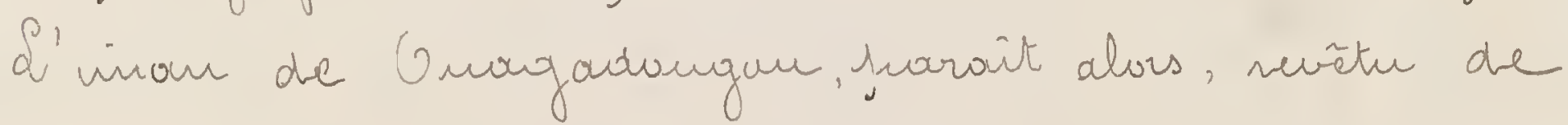
l'echarfue verte, et acconfougue' de ses marachouts. Il aéite une prière spéciale appelée dacca st salue. saurs se prostemer le Moytu. Maba. Celui-ai we réfuris à ancune de ces salutations et quitte le somondé sans avair prononcé ure farole.

a sa seconde sontie, vers septheures du matiu, les chefs hi vendent confute des évirements survenus dous leurs province; taut le monde est admis ensuite 
à le saluer. fusqu'ause étroirgers. e'ost à ce moment quiel antendait aurefoirs les réclanations et rendait la fustice. \&'an. dience solemelle it ait temele vendreai.

Le soir au concher du suliel, le Balam. Onabo vevient au Samondé et prend place surs on sieige. Les emm. quers le surient, dirigés your leur chef, le Yuissoba. Ka.

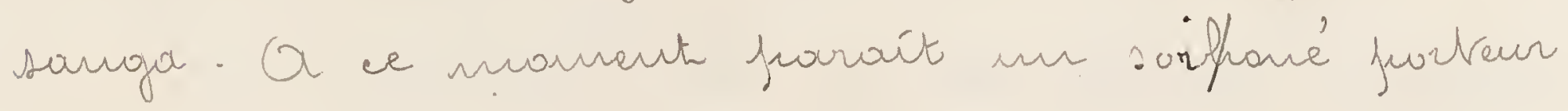
a'un tesson nituel "le Canigum. saré" contanaut quelques charbous allumés ct be dépose au milieur du Sannande?. Le Balom. Naba at le chef des ennuques niemrent successivement faire trois ginuflescions devout le feur qui est aussitôt apries reforté à l'intèrieur four le sorfoné suivi de Tairsaha. Kasanga - Celui-ci va cher. cher daus les afprartiments privés du Mortho. Maba le Rouáaffa, résidu du ablo de la faumée. Yle revient ensuite dous le damandé, at verse growement san Kondipla à i'endroit consacré. Le Balan-Maba s'ir. line et frart. da fournée est terminée.

Les cérémonies dout ngessos viexass de fuarler s'ac. conylissent fubliguement dars la somanobé. A l'inté. rieur il s'en esécutsut d'autses qui revitent mi caractère mustérieux at sur lesquelles il n'at jus

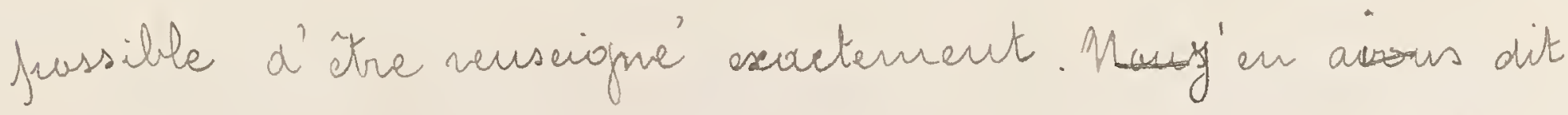
quelques mots lonsqu'it a 'té questions du fétiche libo. (Voir Maba Maslirie p. y7). 
- Les atributions essentielles du ellovpo. Maba an Juint de me eligiens résident dous le orvit exclusif qu'il frossède de déeréter la fête du Qleusé et bes. céreimonies funibres du Bassaga.

Le Peusé" ast la fete mationale des Mossi. Cele M'a fuas de date fise mais se trome tay ours com-

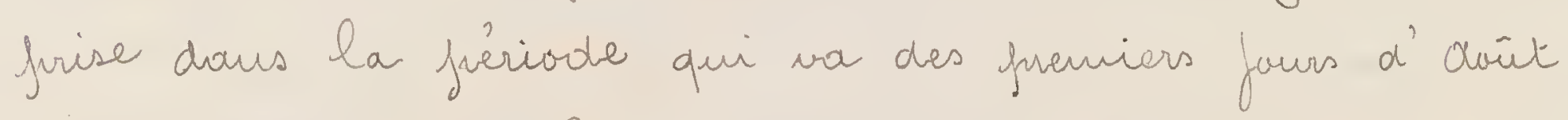
à la mi. Octobre. Le plus survent elle se celibre fir septerubre.

Il afpartient à Laifualbi. Maba seul de question. ver le Monho. Maba sur ce forint. Guand celiri-si le fuge à profus il véfurid que la fête auro lieu dous

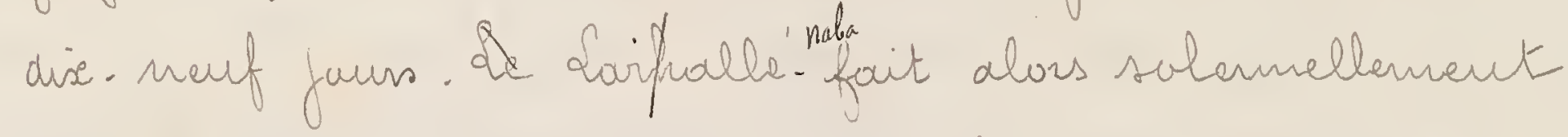
dix. reuf moends sur cirq cordelettes en fibres inessiés de hariouts sauvages, en venet une à chacur de ses colleigues et garde fueur lui la cimprizàne.

Ses kombérés envorent immédiatencut fréverir dons leurs forovinces resprectives que le Ceusé ast fixé fouer be dix. Menniène faur et desomais chaque matin dous les formes traditionrelles ils déferant devant le choifw. Maba un moend de leur condelette.

(4) Qeusé vicut de Cenga-terrse. C'est ta fête de la Verre qui fecundée

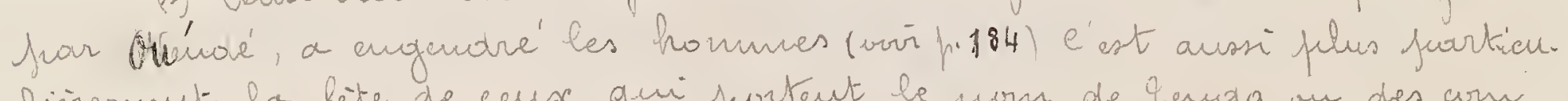
lièrement la fète de ceux qui furteut le nom de Eenga an des conr.

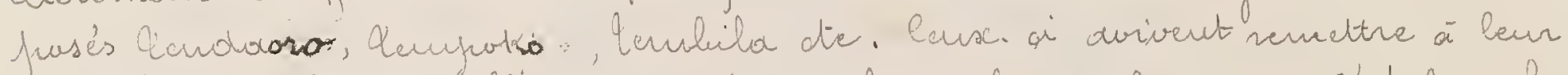
frère le jun de la fềie un furilet qu' il racrifie en leur nom - C'at be seul

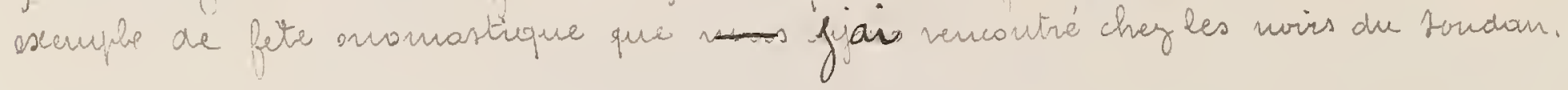


Lassque le faur est venn, le demier mend sera défait, lenteneut et au milier d'un foruford silence, dours le Samandé infrérial; an ce même endroit un tas de murccours de viande cuite ot de bueles de

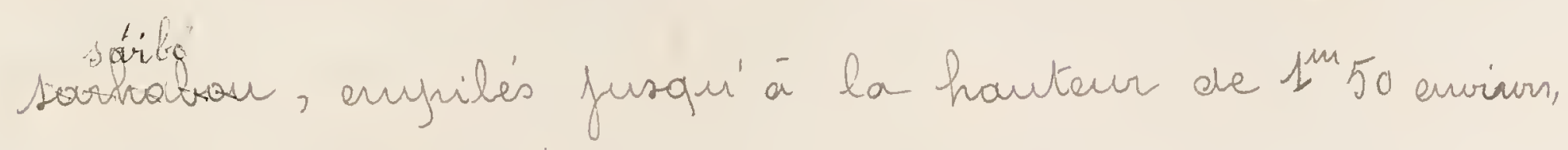
a dié disfrosé dès l'aube.

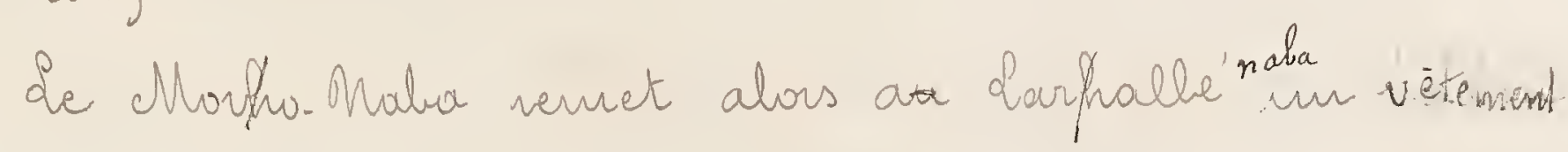
verl. Oferes l'avair vevêtu, celui- à se dirige vers le tas de victuailles, sonisit buis burbes de sóribgan qu'il trentpe dars une sauce spéciale et laisse tomber à ses jicids. Fe fait de mâne juur trois morccause de viande. Il reuverse anfir an

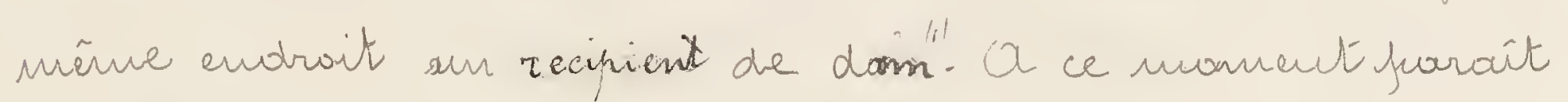
le Gandé-Onalua qui warrasse les alimeuts qui ont été fetés à tarre et se setire ensuite à flas lents sous regarder der.

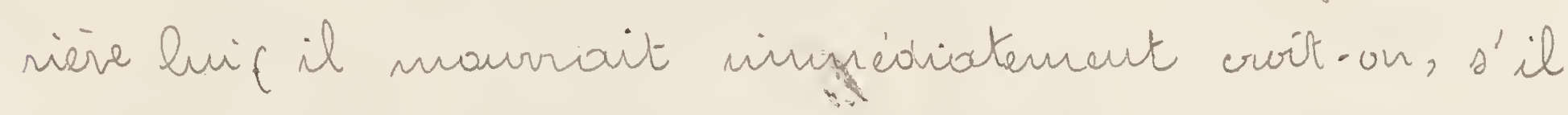
osait enfreindre les rites sur ce fraint.

Dies que le Earrdé. Malua a franchi de la soite la linite du samandé, la furle fusopue lá silengieuse ot recucillie se fette en foussont des hur. lementis sur le tas de victuailles dout, en quelgues instauts, il we seste flus rien.

La cérenonie du bassaga intéresse foorticulièrenreut de culte des arcétres et se célébre amucllement.

Clle est aussi fisée jear le Morkw. Mabu ot ne aéjuad fas à- me date forécise; elle a Vurfaurs lien cefrendowt pen 
owaut la véculte du mil vunge. Les fremiers éfis nuírs de cetre céreabe sout appartés an cho'po. Ohaba qui fait aussitüt confectionner un darm spéeial juur lequel on utilise frour moitié, du nil ronge de l'ammée Jréccidente et celui qui vieut de hir être offert (vaut cela est réglé avec un luse de détails dars lequel ilvanaitte fastridientrò'entrer).

Cette opreration constiture un lien symbolique eutre la rícalte des deuse années at de mêure que, far elle, le

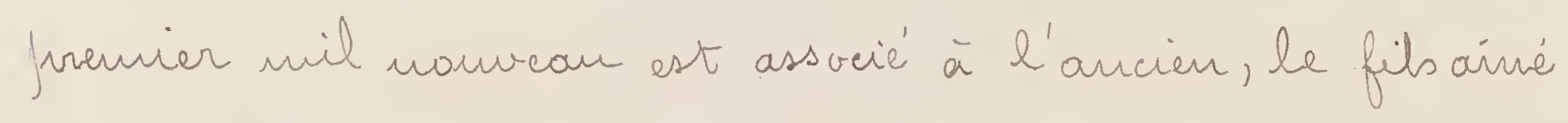
juartagera avee son fière défunt le refios du bassaga qui unit les moits et les vivauts.

Gwart de boire ce dain le Math. Ohaba fera done solemellement des libations sur la tombe de son père oi des frouldts serout également sacrifiés. L'ãme (s'ífa) du novt se nournira de la frartive la flus subtile de ces alimeuts daut le reste ast cousonme?.

Jusqu' an coufins ou frays, la mène cérémanie à lieu le mêne faur dous twutes les fomilles surs la frèsidence du fils airié. So date est annoncé en teuips utile an peuple assenche' frar le Bend Naba, chep des tom - Tons infrériouse.

- La conture interdit fomellement au chopho Maba de quitter Osolgadongau de son forupre gré ses souties qui coustitueut de véritables évèrements, sout réglées 
fuar un protucole minutierse, docirent être ovdomméés an

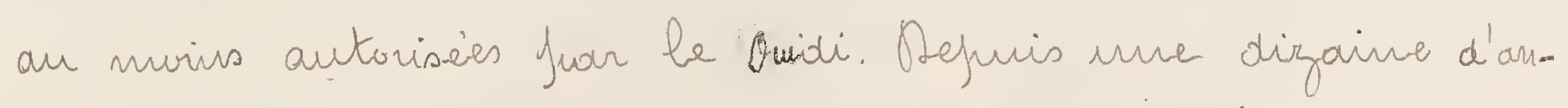
mées ce demier a premias aivisi a Maba. Jigfiri te ge verudre turs les trois arr au village de Roundal Mioné d’aì sa mère était originaire.

- Il ast me cateigarie de chefs que le Moyto. Maha re gaent voir saus s'expeser, selar la crojance fogulaire, à mourir subitement. Le sout les Malas

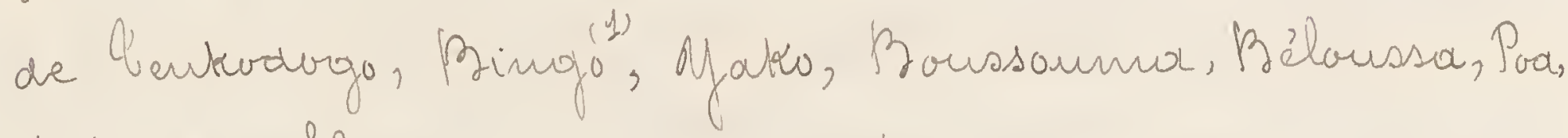
et kayas. Ils recaivent dare l'rivestiture sars venir à Onagadangan, sauf en ce qui canceme celui de Mélarssa que le chovfo. Maba prent vair sous darrgers ovaut son élection.

\section{- Nhe curieuse institution se valtache encoréa} la onignité infrériale. l'est celle des Nouritas.

a la mart du Mopho. Onaba, le ouidi dame l'ordre soit à l'airé des fils de ce demier, suit au kanusoso Maha de disigner un Kaurita. Le Maurita est taufuurs choisi dans la fomille infièriale. Ifl acuit le nour du mot ot l'äme de ce dermier se résincarre en hi aussitot. Dis lors il hi est interdit de voir famais le

(1) Cent le nam Masi de 'ada N'Goumua. 
Morffo. Mabre an forretruis qui hi assiome un village comme résidence mais somscommandemant..

Se Kourita a drist ause hammages que franarit exiger autrefois le murt qui revit en hii. Il hii est en outre lovisible de s'enfuarer de taut ce $q \mu^{\prime}$ il désire

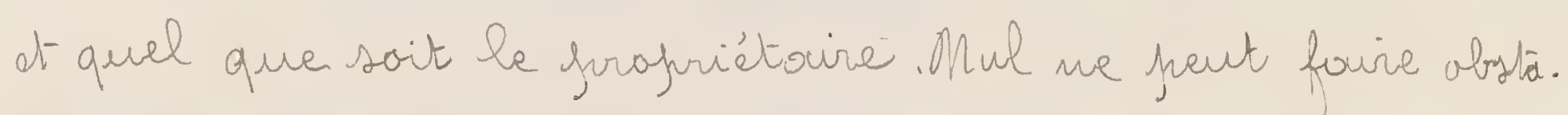

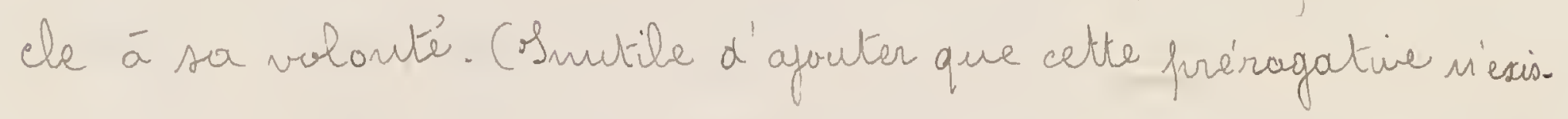
te flus sepris quelques armées.

Cinq substituts a'anciens Moxpu OMabas vivent arisi

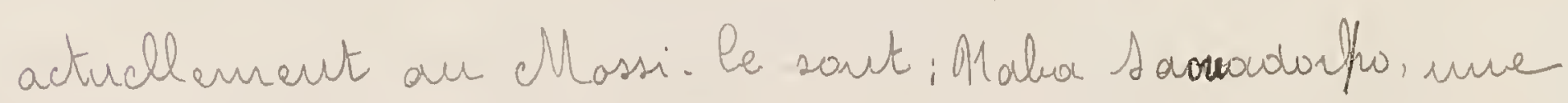

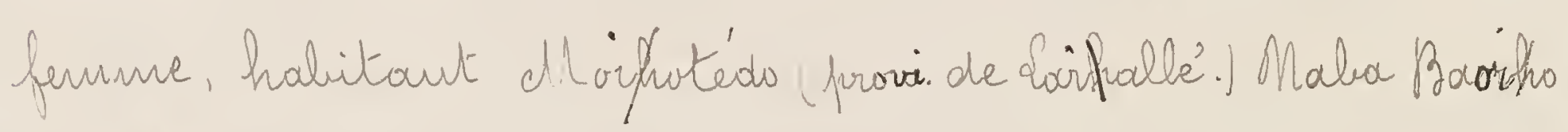

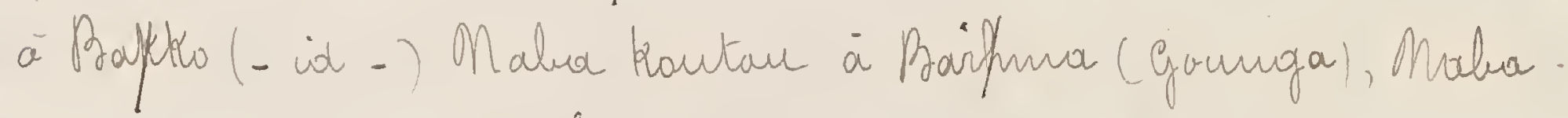

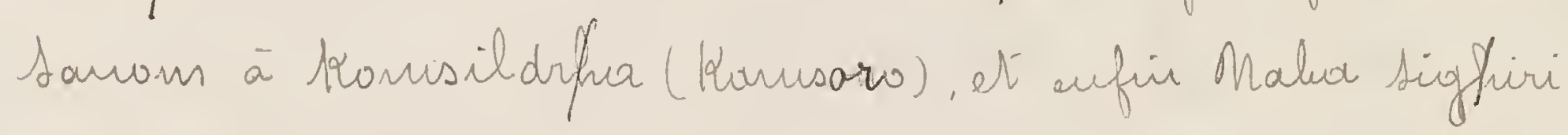
à Ranga (Biloussa.)

II. Quidi. Maba _ C'est le jremier des grands

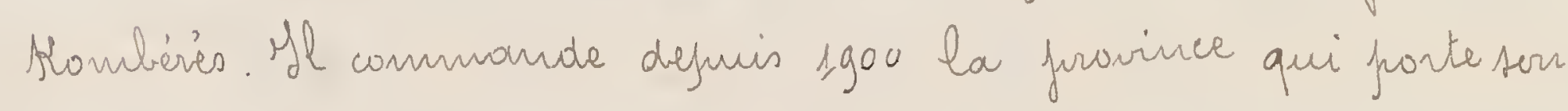
nom ot S'Átend an N.E. de Gragontorigar.

Maus avous ver san vole friejorndérant prendusut la

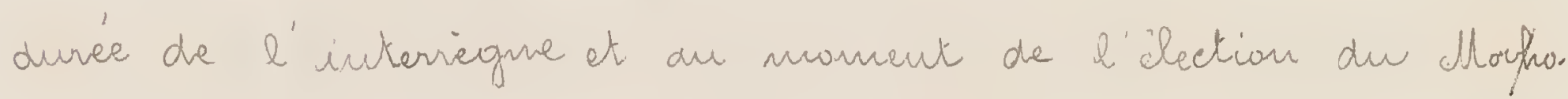
Maba. Mrsess n'y revienatroin fras.

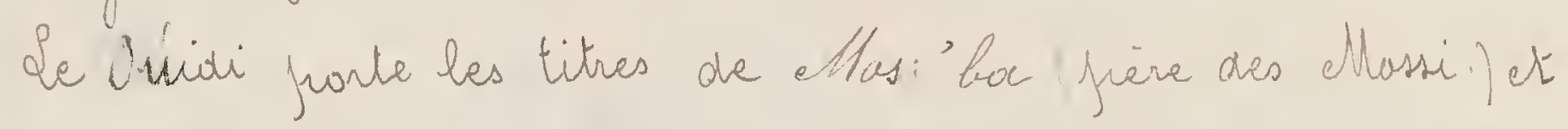
de Mtos. 'ni' Gráré racive des Massi.). On le namme aussi Sicla. Solec le maitre de la virité, hasqu'il a fuarlé

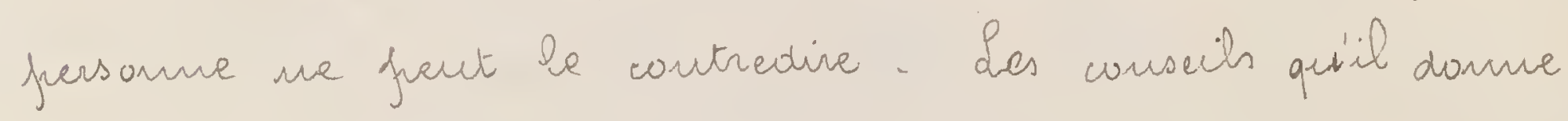


ar Morfto. Malua revêtant tarfours nue forme respectuense mais derivent âtre suiris à kn lisktre.

Le Didid ast le seul homme qui ne fuisse en aucum cas être mis à moat on contraint de se tiver, carnul u'a le drvit de le fuger. He exerce fuar cantre ce trait vis.à-vis de quiconque et le cthofo. Maba lui. mĩure we feut s'y sanstraire. O ce ferofies l'ow se souvient encore que Malea Karfo dut se domer la nont sur

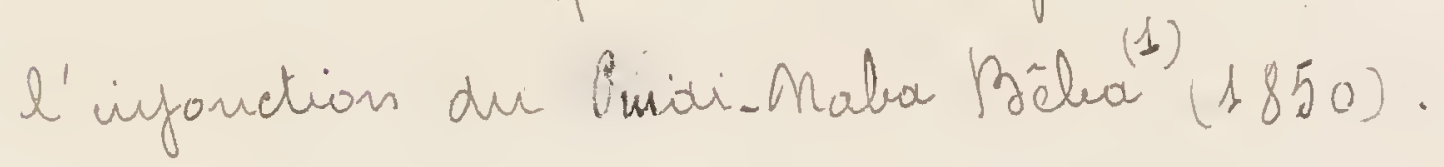

Le moindre manque de verfeet à som égard, la minin. dre neigligence mêne, witrit la vie à celui qui s'envendait

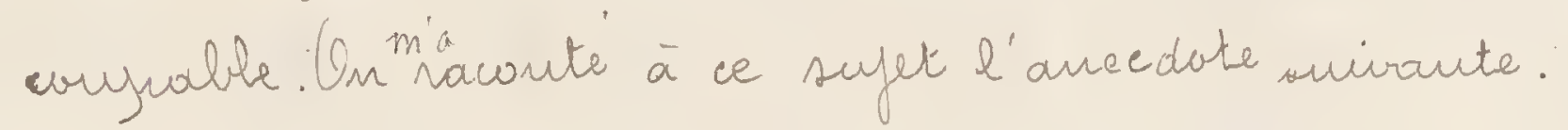

Le Cridi. Malua Magatenga se verndait un Jaur aufrès de Maba Soria lansque le vert tui feta de la funvée à la figure. Fivement irrité il recommut que éèait une des frupres femmes du Mospo. Mala qui brïlait destiges de mil. Il ventra aussitat cheg lii et de trois faurs ne

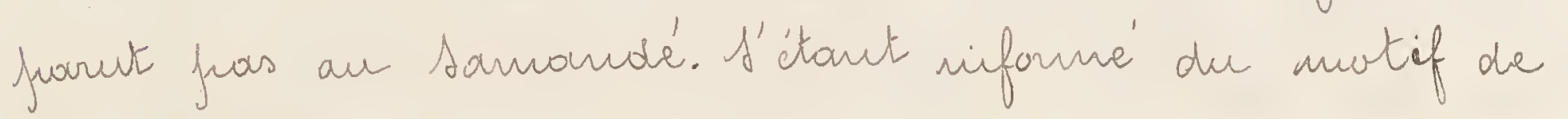
cette attitude et ayourt apporis que l'imparidence d'une de ses fermues en était cause, Maba taria fit imméoniatement mettre à mont la caufable.

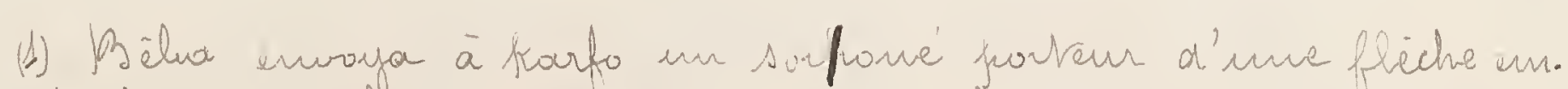

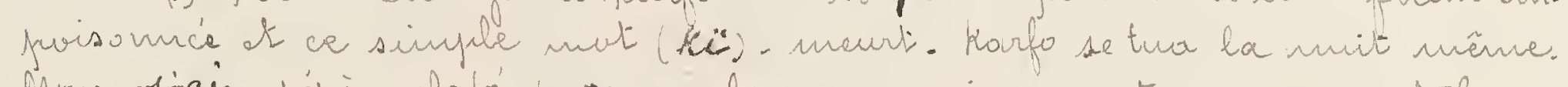

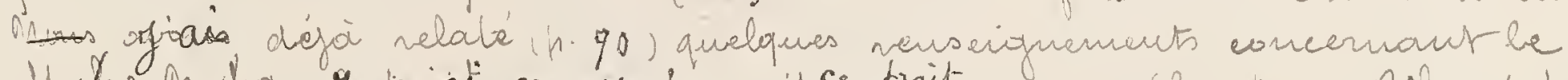

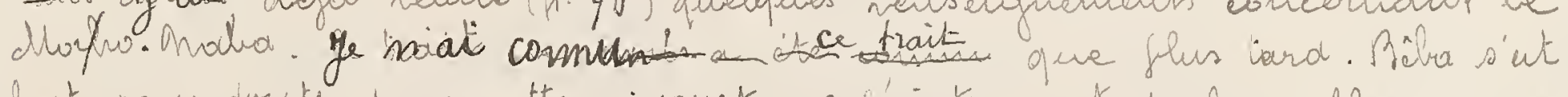
fout sans dorite dours cette cinconstarce C'instrument de la noblesse qui we fururait frardanver à Karfo res tendances libérales. 
Sonsqu'il vorilait farie Kuer qudoju'un be Fuidi ctait simplement term de aendre conyer an Morfro. Maba. Dars la méme circustance les autres chefs senowit d'a. bond congte an Omiiai at sollicitarient ensurite l'auturisations

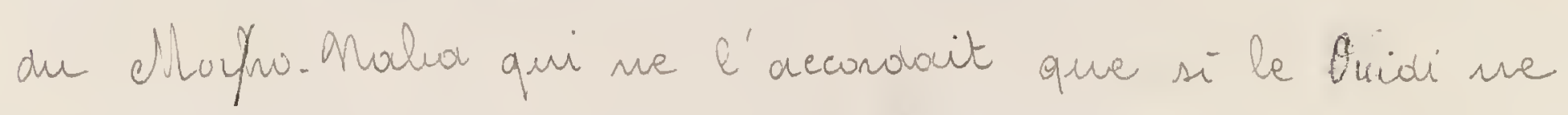
d'y gyusait jas.

The fenme donnée far le knidi ne jounait famais

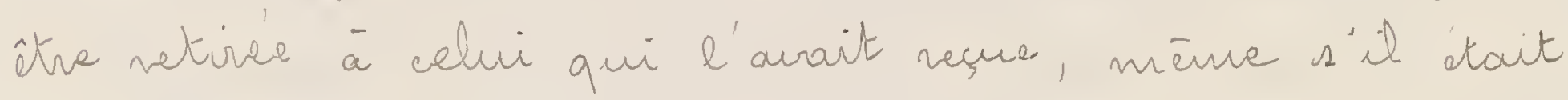
necomu dous la suite que cette femme affeortenait aur dlowho. Maba.

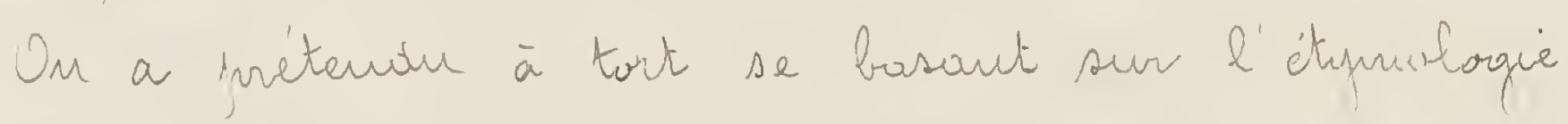

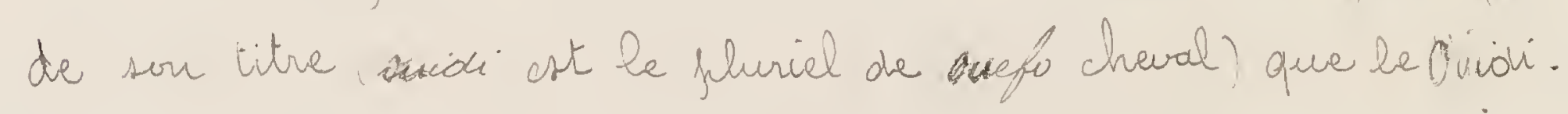
Mabu commandait la couralerie infrériale.

Il ae jourrait flutôt que le now de Omidi vienue

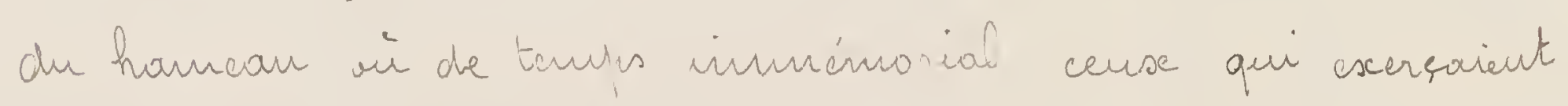
catte dignité out dobbi leur demanre. Oll est encove fus jorbable que cette afycellation frovienue du foit qu'entre ses mubtiples attributions se trane celle (At c'at aujaura'hir l'mue des nointeres) qui consiste à saiganes. Ces chevause du

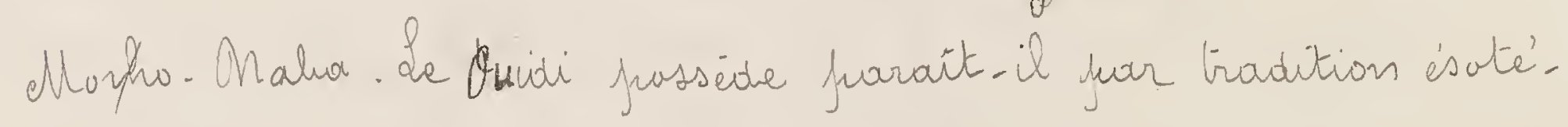
rique des fermules unsteirienses ot souverainemant effi. caces dout it n'use qu' à cette secasion. Yl seraut curieuseque

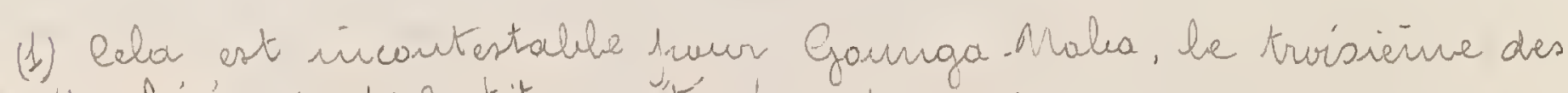

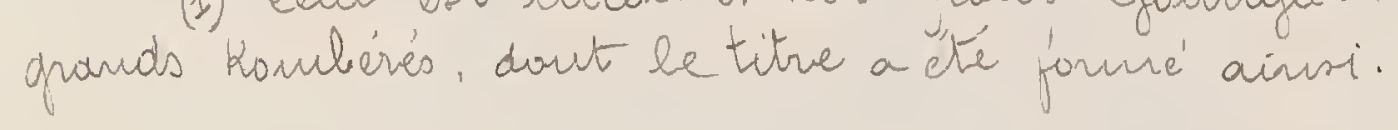


le jaunoir fomniable dant disfussait ce dignitaire à notre arrivée dous le jaup, ait son juint de déjpart dars les nuobestes forctions de véterinaire.

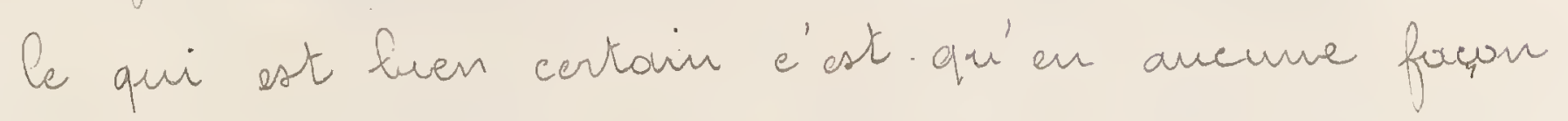
ot controirement à l'ofinions universellemant acegutée

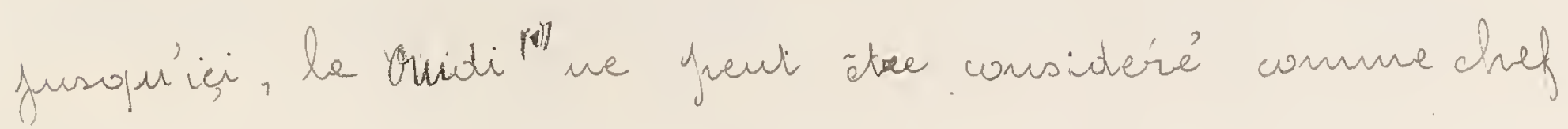
de la cavalcrie.

\section{Gausaba. Maba de juatocale de}

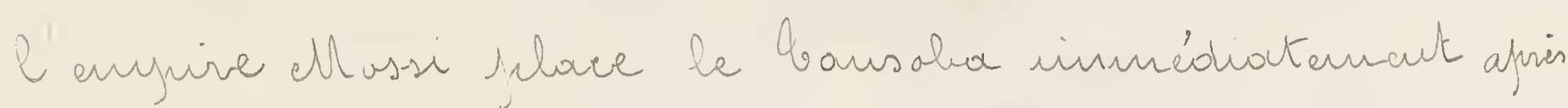
le Mirivi. Maba. Il we fait cefrenowat fras frourtie des grands pombérés et s'il excerce un commondernaut Kerritonial c’est sur un canton de minime iyjartarree appelé Gaursabterrga Sarfwalle'). Mais ce n'est là qu'une fanction accessoire, son titre est edur de "qrand chef des armées, Le Vausoba assime en terups de guerre la haute direction des iroupves inveriales. e'est hi nous l'avours ou qui cst chargé de la jrotection du nouveau dorpio. Maba alors que nanté sur un namais chenal et confili. cement désarmé, comme l'eserigent les rites, ce dermier antrefrend le vajage de Gasfranga, voyage a' aì déjendra la validité de l'élections et que ses concur. newts cherchent à hi niterdire. Oussi à peine letrône est. il vocaut, le Qansula séunit son amnée qu'il fout carryer en attendant les éveirementspies

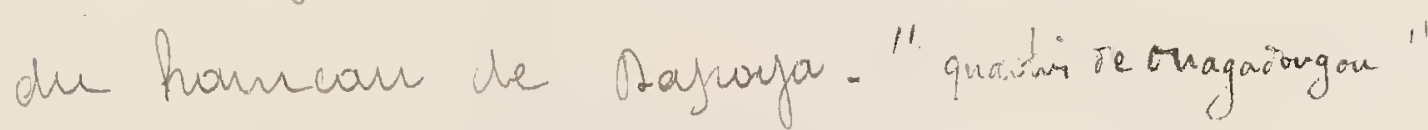

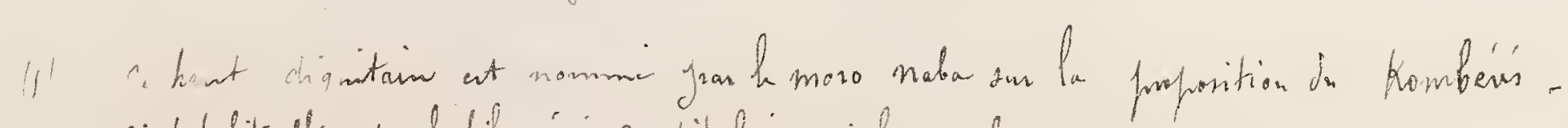

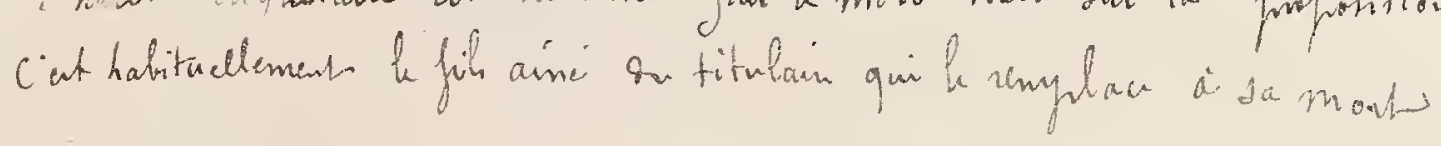


Plusieurs tanswhas ont acquis un grand aenom de bravoure. Le plus celibre d'entre euse est incontestablement

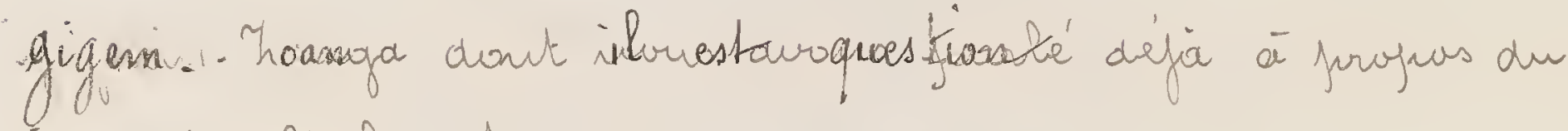
reigue de Mabar Sarram. La charge est hereititaire.

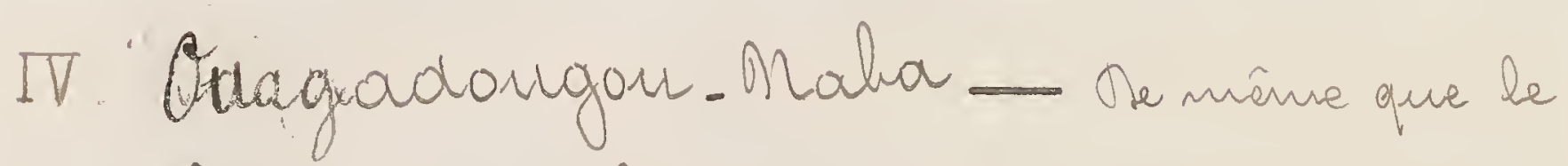

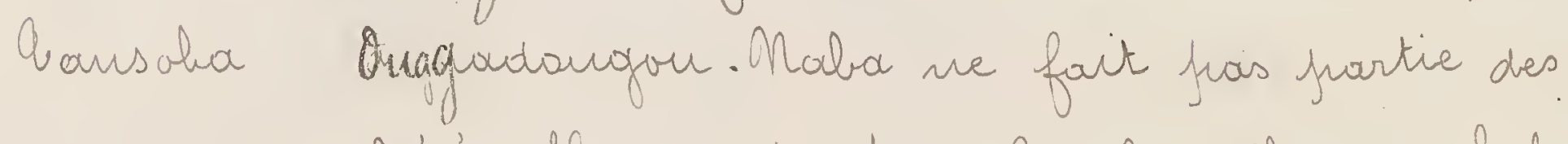
grands Rombérés. Il appartient à la famille de chefs qui commandait oragadargur de terrys immémo.

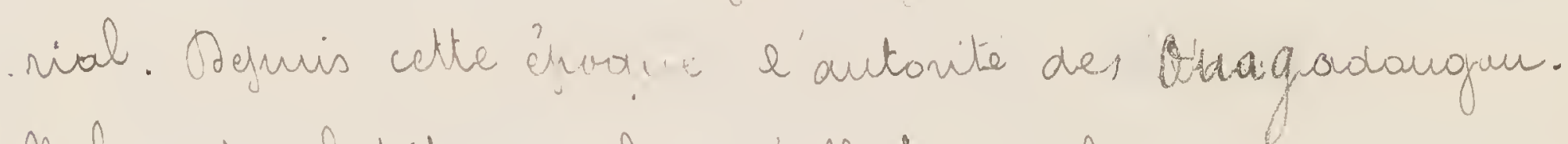
Malas est phutât monale qu'effective; ib sout de drisit foropuriétaires des aminaurx errauts et des objet's que mul re rélanre. Leur samandé corfine l'invilabilité an fugutif qui peut l'atteindre. Bien Whes si Oraga. donavor. Maba arrive à l'endrait air un comdarmmé va. otre escécuté et que celui. qi le accomaissout fromance la

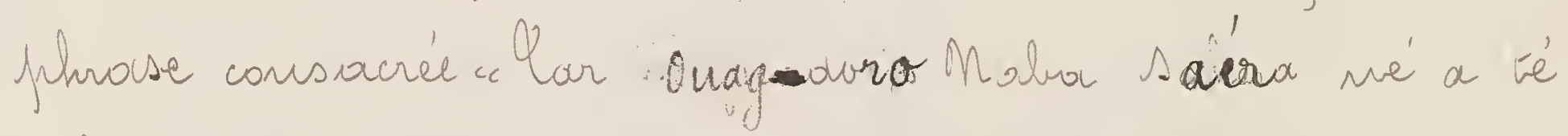

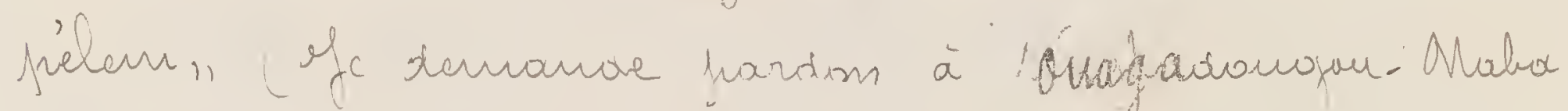
et ause génies protecteurs de sa torre, il a aussitît la vie soure quel que soit sou crime Sa charge de Aviríndougan Alaba est héréditaire. 
$12^{0}$

V-Larfallé. Maba C'as le seconat des

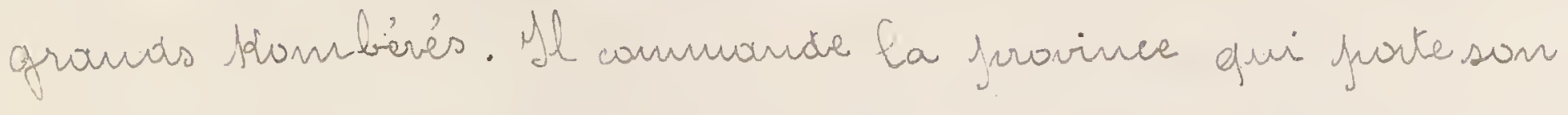
nom et s'étend aur tud. Est de Onazoudarigon.

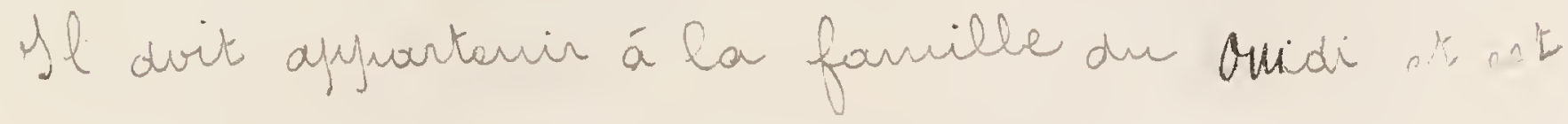

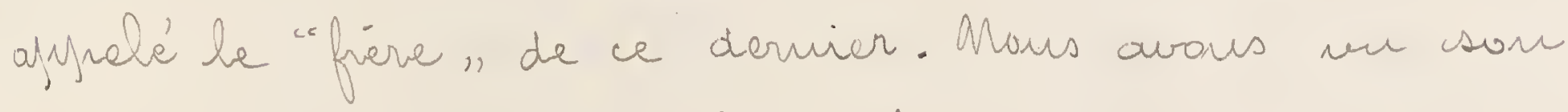

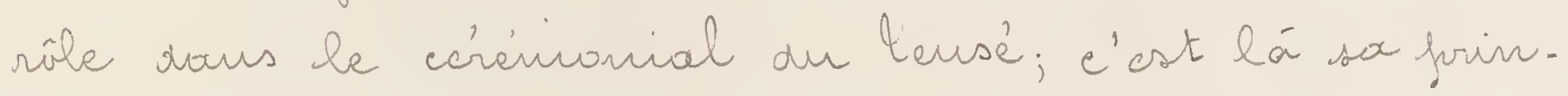
cifale attribution

X.e Larifuallé ast anssi détenteur de la hãche qui sert ause sacrifices ituch et aver laquelle sur bouf est inmolé Kaus les Sarriedis.

Lossapi'un chef rewit l'ivvestiture il sot term de verser dours un trou situé entre le Samandé du ellor/po. Maba ot le hangard des musiciens une somme variout en. tre 500 ot 2000 cauris. Ec trau, nommé "tran dur comman.

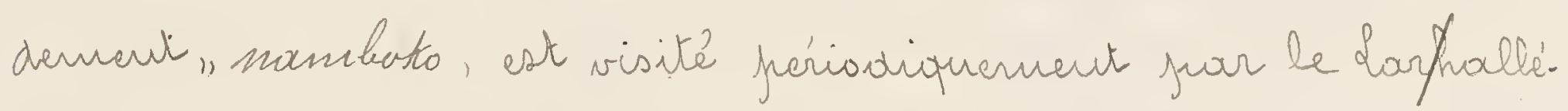
Maba qui en tiait fowis son frincifal nevenu.

sl est en ouvirè fréprasé à la surveillarce des séfuel. tures des ancieus Morpho. Mabas.

VI. Gounga. Maba - Le ivisièrre des grands Hombierís. Commande la frovince du urêne nom, sise à l'Onest de Grawgatarigar.

Il dait à la mart du Mapho. Malva, frerrare la lance, be couteaur et la queure du cheval préfeiré de ce dermier qui ve doit fass surviore à son maître. 
les alyets néunis en troppliée sont felacés fuor ses soins tours une case dout le Samandé. Maba Mobila à la survielance. Ayries sa nomination le nouvear dhil o. Maba est conohit les yeuse bandés à cette case oir sout ainsi rassenbléas les lances de ses aíense. Sl doit arriver à kuncher l'une a'entre elles et la crafance locale vert qu' à ce noment se décide la durée de son règne: il sera exactement aussi long que celui de l'ancitre dout il a ju dans l'alscuriké, atteinatre le Guphée.

Il est asser curieus que cette superstition à la. quelte l'histaire a dir domuen de nombreuse dénventis (ce que les Massi ne veulart arouer) ait fur jersister fus. qu' à ans faurs.

In tamps de guerre le Gounga. Naba a so flace à cité du grana chef militaire, le Lausaba, aufriè de qui il firie le côle de Commissaire ause armies Avee lui, il est chargé d'assurrer l'orabre à Guagonowngau an monnent de l'élection dur nouvean Morfo-Maba; il contribe en foorticulier les disfositions prises en wre des la protection de ce demier.

Se tutulaire de la fonction de Gounga appartientia

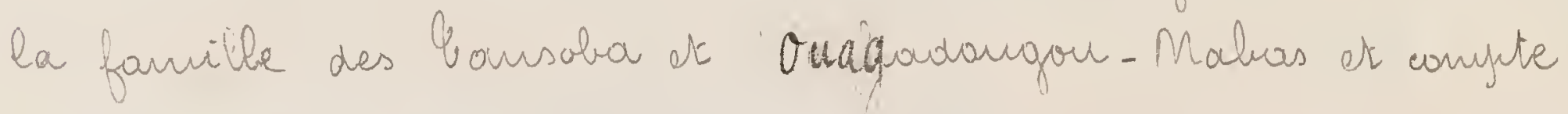
frar couséquent varmi ses meìtres le chef que Maba Nand.

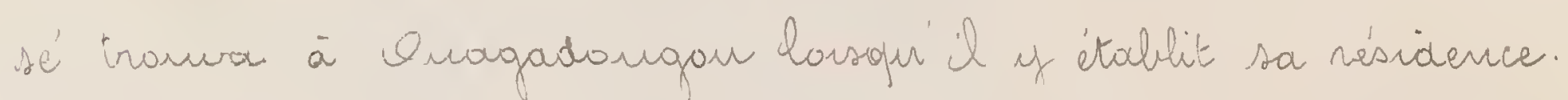

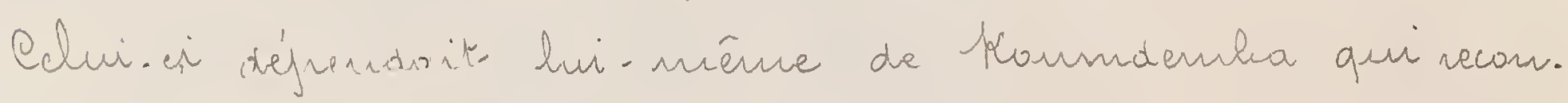
wut la suzeraineté de Maba Gubri. Catte famille 
vegrésenke done l'anciemse liguts de chefs qui, verrus dars le frays owee la hremiere incosion Massir., y avait esecré le commandenuent owout la fandations de la ayrastie actuelle.

\section{Kansoro. Maba Le quatrieine ar dignité} des grands Kambévés; sa furidiction s'étend sur la viche frovince du néne nom située au Sud de Onaxadorigan.

Il veille particulièrement sur les fonmes ot les enfourts du Morho. Maled. e'est à ce titre que be Guidi le charae souvent de déaiguer le Kaurita voin p. 114

Il est aussi le détenteur légal du préciause "youdo nighi. Howga, armas de tartes les queves de boufs immolés surles tombes des Mopho. Mabas.

Jusafu' à jrésent la dignité de Kansorw. Maba a dé escercéc jour un ennuque. Ahe des préragatives quir lui est attachée permet an titulaire de "nuanger comme il verts ¿.

VIII. Malon. Malia _a le commanament de

(1) Lassopi'i allait satisfaire un besair naturel taut individur onfant jur l'apercevoir était frassible de la puirue de mont. 


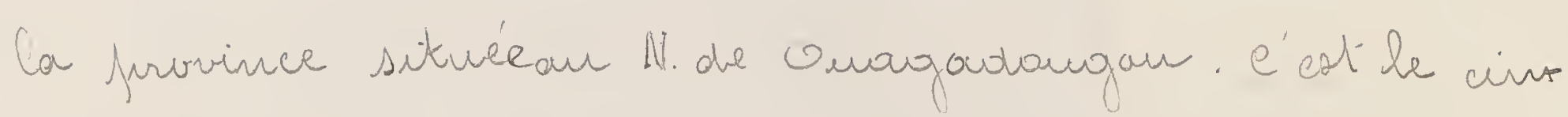
quieine des oprands Lambérés de le seul qui ne soit jus inamovible. Il 'átait désighé fuur six années foor le

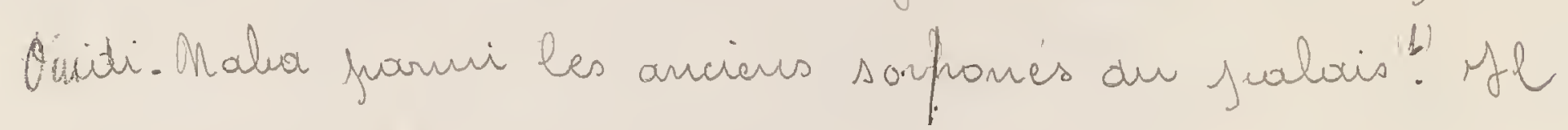
demanre le chef direct de ces derniers. Les sorthovés seut frarmi les sevitaurs du Mocho. Maba ceux qui aver les annques out l'accès de ses afprartaneuts faorticuliers. Il doivent être vierges at avoir un stypiopue agréablez ils sont vétus et coiffis conme tes fermucs, fortent des bra. celch de midal aux bras et suxe chevilbes, quelquefors des houseaux de cuivere, atributs féminins. Sossopic its quitient de dlopho. Maba is recoivent de celui. ci me femme mais taut rapporchemeut sescuel qu'ils se per. mettraient arourt cette érogue est frumi de nont.

Le Balom vemplit en quelque sovte les fonctiono de ministre des rites ot ville à l'olservation stricte du furotocole. Il décide en dermier sessort des cas dautaux en fait de jréséance.

Il recait les gens qui out me véclanation on me suppiliopue à adresser au sonverain el les présente s'il of a lien, à celui. ci cout il est l'homme de con. fiomee at le conseiller secret.

W Se Balom dtait en outre renfulace' de surit à la nort du Chafo-naba. Seceftionnellement le titulaire actuel, Kidangan

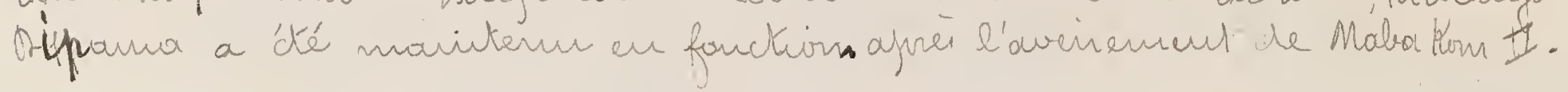


s 

Il jocit de la préragative nous l'avans ver de le Aaluer le fremier à sa sortie du matir, Arérogative dout il ast tris fier.

Les sacrifices sur la tombe ses ancieus Movpo. Mabas ensevelis à Onougadougau vertraut également dours les atributions du Malam.

IX. Camandé. Maba - St profusé auchirx du dlarho. Naba frar le Sarphallé dont il demeure le subardomi direct.

Il est inamovible. Io fonction firincijale est de veil ler à l'entretien du samandé et des alentouss du fralais qui reojardeut la jarte frincifale. Al ost le chef des dattan. ve's (dorra Kanre', hamme seul) classe de celibataires an. playjés ause cultures du Morho. Maba qui les nevvive an bout de quelques arnèes en laur faisourt dour d'une fenme. Ils we sout jas comme les sopforvés, tenus à la wiginitè mais ne peuvent frériétrer dous l'uitérieur du jalais de minue que le Aamandé. Maba.

X. Sonie. Mlaba (de fauré "faire la prave) Veille à la fidelité des fermues du Mowfo. Malea. Rien ne peut lui âtre caché. Oans las cas dautaux il a necaurs à des afiéra. tious magiques au moyen des quelles la vérité se 
momifeste: selon la foccon dout se conywate une froutre spréciale qu'il jette dours un vase filoin d'eour il déci. 'dera en derivier nessod si urre femme songreonnée d'a. dubtire est réellement caufrable. Oarrs ce cas la malheureuse deva othe excecutée inmédiatement?

XI - Daprö. 91 aba _e dignitaire à la sur. veillance de la frate de derrière (cité (st) du fuabais, qui dome accès aux appoortements oceuprés fiar les fermues du Morko. Maba. Mul u'a le droit de statiomer la muit on menre de frasser dars les envirous en desa d'un périnètre déterminè. Qout contrevervaut ast fumi de mort.

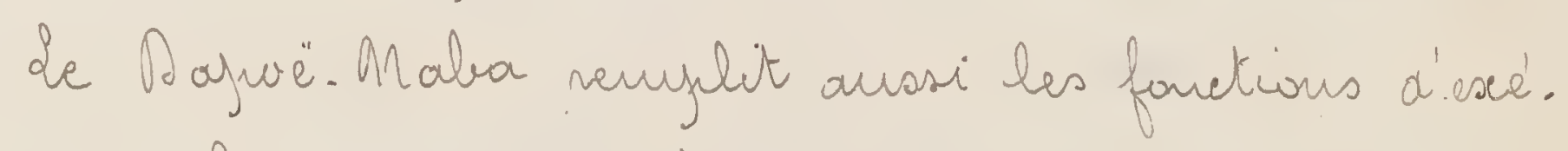
cuteur des houtes. senves. Son issigure distinctif astm

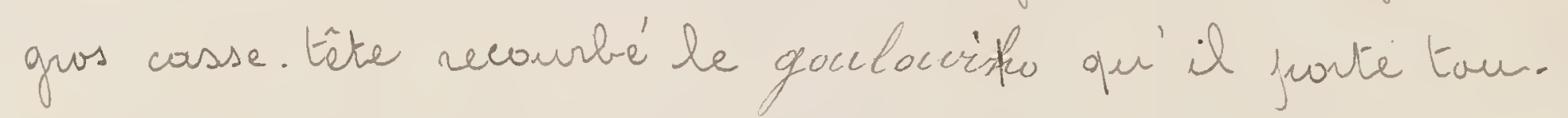
fours accioché à l'èrouile.

XII. Tüsaba. Maba Le second grand ennupue.

(1) Bien enterdur aguis" quelques ammées la charge de Tami. Mabos, revit, comme butucoup d'autres, un cariactere gurement homorifique. 
127

sll a jour chef direct le khansoro. Malua. On a vu dans la céremanie du Kauaifa voir h-110) Sa Jurincipale attributions. Se fusolar. Kasanga se dimet de ses fonctions à l'avène. ment d'un nouvear Morfo. Maiba.

XIII. haka. Maba Oroisieme grand ennugue. Préside ause vejas des fermmes du Moypro. Maba. Il est frarticulièrement chargé d'assurer la disciplirie à l'in. térieur du fralais la gaka) qui n'est accessible nous l'a. vors wh qu'ause sorforvés, aux femmes et aux ennugues. Il existe une exception en foweur des jarents rap. ticulier tout pries de la forte d'entrees.

Cette exegution s'dend au Bend' Naba ot au Morifo. Maba (chef des orfiores). Ce demier est d'ailleurs cousidéré con. me ure fermue.

Les fanctions ar Tata. Maba sant inamavibles.

XIV. Kambo. Maba

Chef des Kravailsews de la courome. Est prápusic en fuarticulier à la garde des fusils dur Marto. Maba qui sont en terups ondinairs déposés chez lui. 
XV. Bend'. Maba - Chef des tambaurs que l'our fait résonmer avee la main.

- Il doit comaitre far ondre charalogiague les devises de taus les Morfo. Mabas qui se sont suecichés deguis IM. bir st les chonter dans certaines eirconstances. (nna

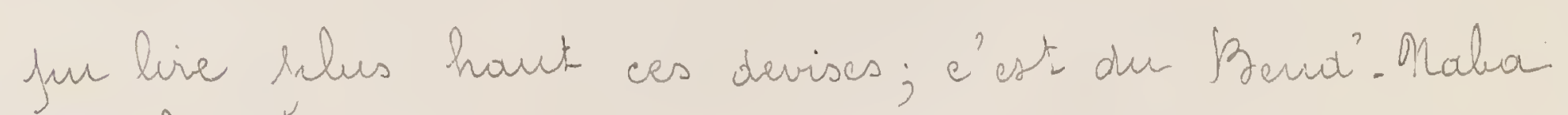
actucl que mous des terions.

XVI_ Riidiranga - Mlaba - Chef des cecuries in.

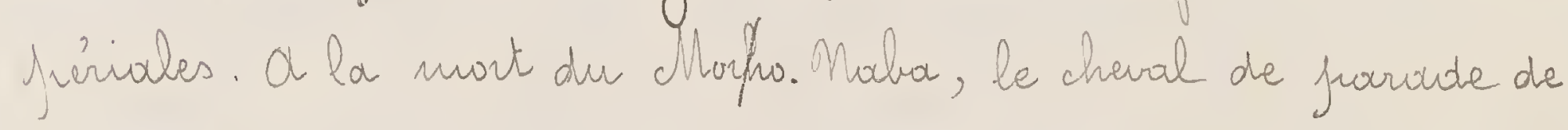
ce dermier afjant ité inmolé, il an resoit le harmachernait qu'il garde chey hir gerndarit une armiee.

Al dait aussi dars ses attributions lassan' un nou. varur négne commensait, de franter derex chevause, deux

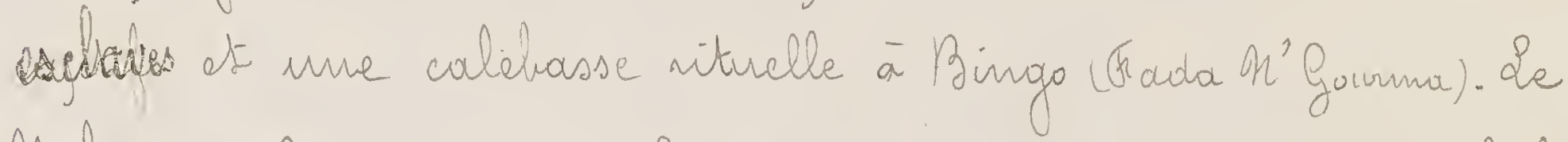
Mlaba de Bingo devait faire farwenir ces Mrisents an chef de Gambatiffa qui regresente conme njeas l'á dit la bran. che ainié de la famille régnante.

\section{Treakin. Mlaba _ Chef des palefremiers} inpieriause. Ooote seller hui. méme le cheval du Morfo. Olaba ot ast nesfonsalie de trout accident qui foinrait fro. venir d'une négligetnce dans catte afération. Le P'red.

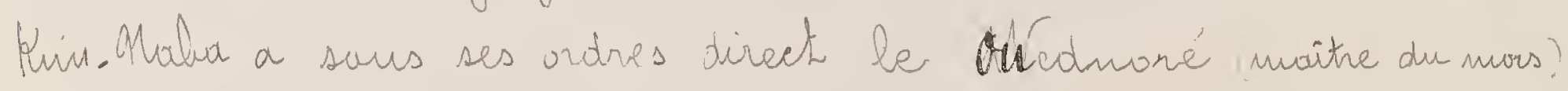


129

pui durant le braget doit terin à la main la bride du cheral du Montro. Maba at cat resinausable de tout fause fas qui entrainerait me chite. Sa seule samet. itait hien enterrdu. la mart.

XVIII- Ganga. Mlaba_Chef des Cambarrins al. Congès. Prielude airs someries st dome le rytrme à adoj. For wark in marche selon les circonstances. A le droit d'accompa. gner le clloupro. Naba à cheval.

XXX _ Sou. MLaba Cans ar farme de sablies qui se jacent surs le bras ot sur lesquels on frappe aver more patite crasse an bis.

XX. Mendo. Maba _ Chef des viandes. Oréside à lar cuisson des alinerits du Morfro. Naba, dout il ast tern de nanger le prenvier, mais hors de la frésence de son maitine.

Mou loin de sou habitation se trauve le farmeuse funits de Mabauli oir wit un caiman "aruge qui, dit. on cammande a la pluie. In cas de sécheresse un a conturne de socrifier un bieuf dout la viande sot dorncéc en fuäture 
130

à ce cainman jair les soirs du Mendw. Maba.

XXI - Kadiogo-92aba - Chef ale marigot de par diogo air l'on inrend learr destinée à l usage herson. nel du Morfo. Maba. Sl dait weiller an furisage of à la proureté des envirans

Cotte dignité n'existe qu'à Onagadorrojar.

XXII - Clemendé. Malua - Chef des ciréales. Chargé Ifécialemart d'emmagasiner le frotuit des récoltes et de frésider ause distributions pour la consommation in. térieure du valais.

XXIII. Manga. Maba - Chef des jierres. Charge' en furr. ticilier de la surveillarree des firerres disfusiés daus le samandé à des distances réolées frar les rites et qui servent de siëges an Manto. Alaba ot ars. granis dignitaires.

XXX_- Gandé. Maba - Chef des hrause. A la garde

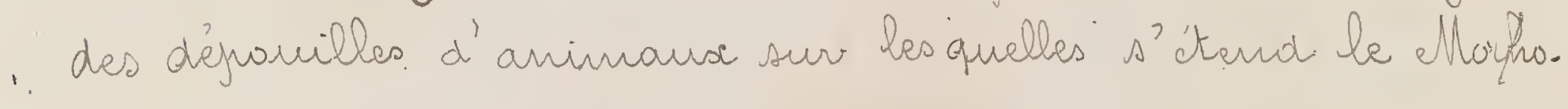
Rlaba frour urendre du nefues. e'est mue fonction de confiance. Si un mabvillart franvait avoir accès frès 


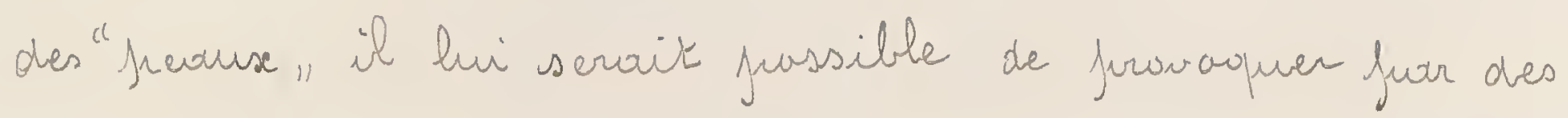
malifices l'envasitiement du Morfo. Maba.

Mous avars le vite du Gandé. Mabo dours la éreńavie du Carsé. (wair h. 111 ).

Au Candé. Maba ast adyint le Twukangs. Maba qui a spécialencent la surveillaree du conssir richement bro. dé sur laquel s'assieat le Morho. Matua.

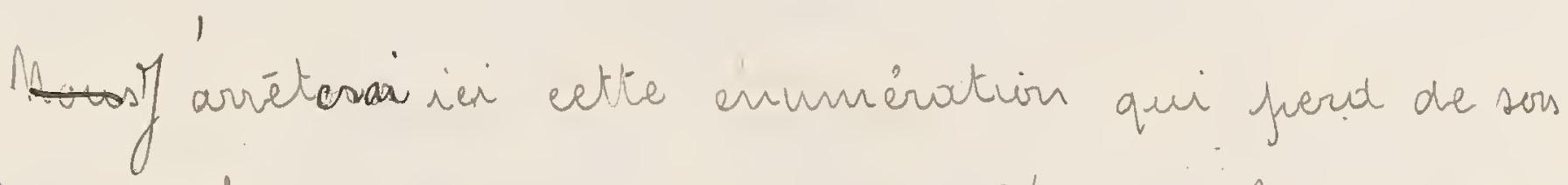
ritérét ou fur et, à mesure que l'orr s'éloigne du sommet de la hieiourchie. Ou' il arie suffise de dire qu'il exicte augnés au Monfo. Molla breute. iwis dignitaires de taus owires dant les atributions sout strictement définies.

Se Minfis. Maba ctend en autre son autivité sur brois ecuts Maramsés, chefs de farwainces an de car. tous qui administrent en son nan le frougs, lui doi. vent un semment de fidelité ot viennent à Onaga. dongan, souk quelques exceptions que sfoim indi. quées, recaroir l'investiture.

chacun de ces Momannse's a ćgalement au. bessans de lui tinte une série de dignitaires qui foutent les litres indique's phes hout et cxercent vis-à-vis de laur cheff resfrcetif les fonctions que ngiai décrites. Ferbes deuse de ces dignitís sout escelusuives à la capritale. ce sout

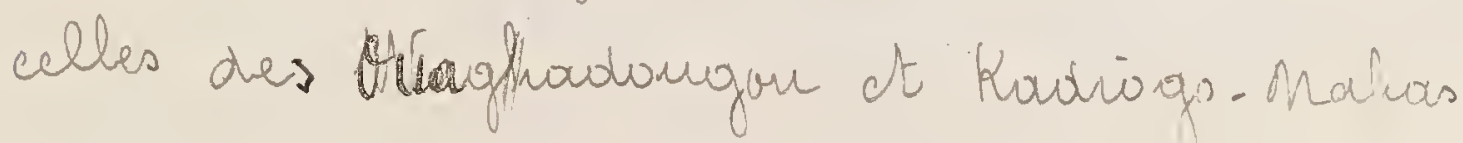




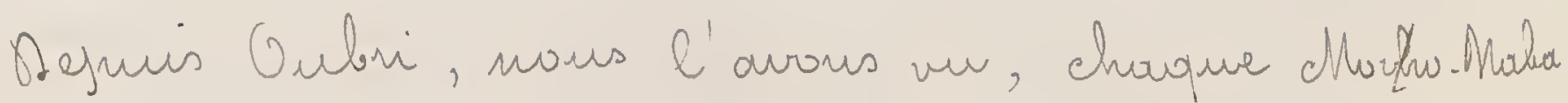
a fur laisser comme fwostérité, outre celvi qui devait âtre son successan an triane, un certaiir nombre d'en. farts qui out dite nivestis de conmandanants secon.

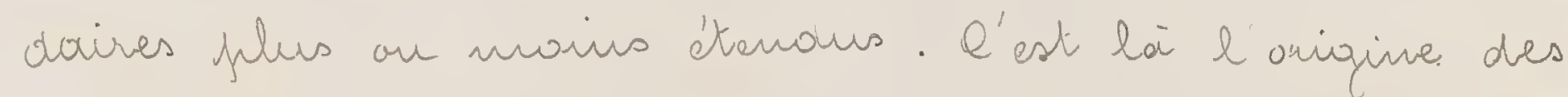
Manansis: taus se nartachout à Oubri fuar un Marks.Malua afant réopié.

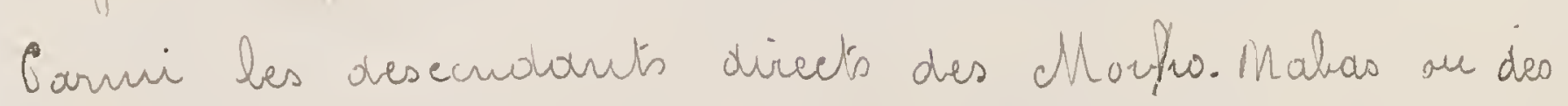
Manansis's il en est qui n'out fur être grauroure d'une charge: ce sout des Makansies (au sing. Mabriga) qui constituent l'aris. tocratie an fraug, classe turbulerite et indisciflinese quia fini fuar feuyler des villouges entriors.

Gelle est darus ses grandes lignes la coustejtution de l'enfuire Massi. Slle mantre en défint de ses fuérilitiés et de ses lacinves d'incontestables harnonies d'ensendele, ot coustiture à antors ovis l'unie des tentativies les pplus inté.

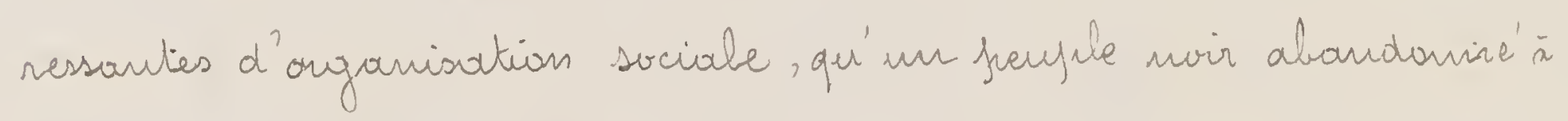
Rui.mieme ait thé cayable de néaliser. 
13

3: Pourlie

Renseignements

dociologiques et ethnographiques sur les populations vivant actuclement. en pays Mossi.

Oour l'esforsition de cettie fuartie de moon 'triove utiliserain des nubriques générales frermet. tant de graupuer sationnelloment le flus grand nambre de Laits.

Ces titres, adojutio conforménout ause inèthodes sui. vies de nos jaurs en authrofulogie suffiront à détenniner

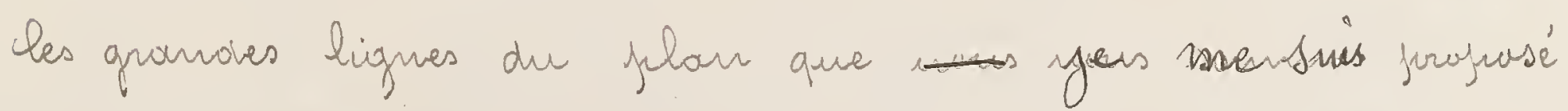
et à assurer à cettre enopuéte seientifique, que jos roquet. de n'avair fur afprofondir davaritage, le developpue. ment logiopue qu' lle comprortaits. 


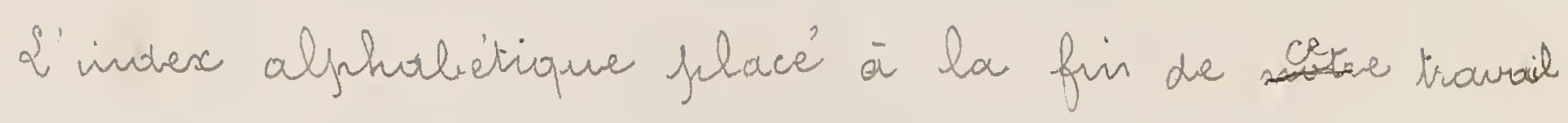
ost deotivie à focilitar les vecherches.

\section{Vie nutrilive}

\section{Olimentation - De nience que dours les} autres régiour de l'Afrique Oecidentale, le mil cons. titue au Massi la hase de l'alimentation. Hl st surtout conisommé saus forme de Sário ..... Se Sáribo .... se frée. fuare ainsi: on délaie la farine de mil durns l'caur bocullaute jusqu' à ex que la fräte frenme une certaine consistance. Cette jäte ast aivisée ensuite en fuetits frains que l'on manose en les trempant dans nure source spiciale dout la vecetre varive suivorut les localités. Doars la region de Onogadougan la sance la flus

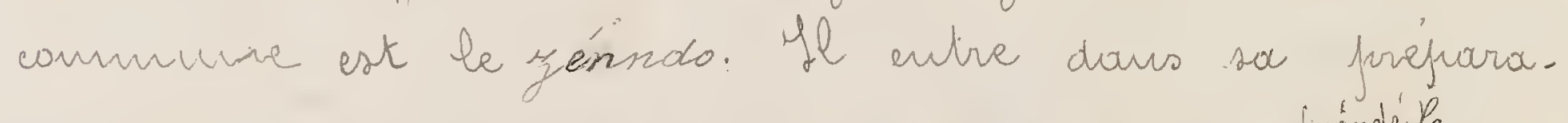

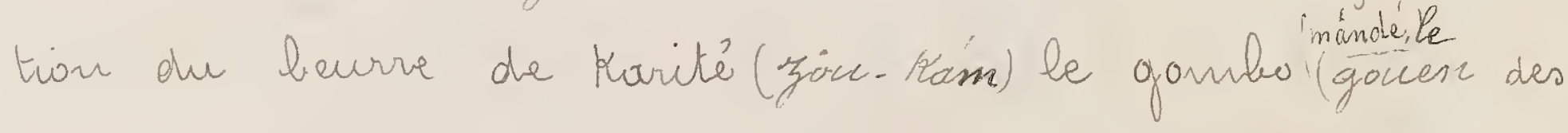

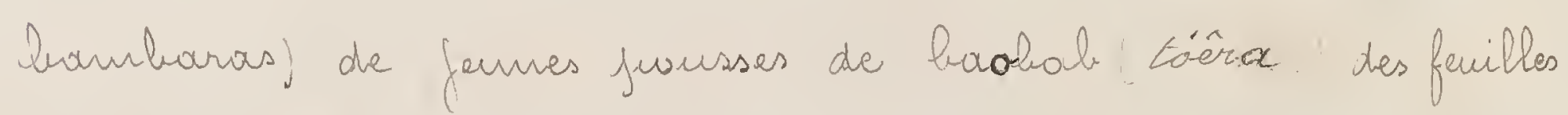
d'ascille (bidrs, les fenilles d'une prafilionacie indigine inommée scisiga, la fleur du fouss fromager wotra, be bour nou des bumbarass) enfir les bourgeours hiés odorants d'une

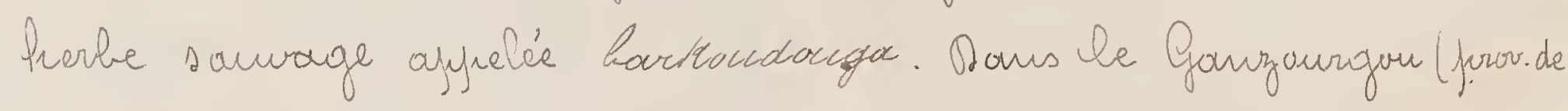
Larffallé) ot les régions avoisinanties la sauce diffire et frend le nom de beng-yendo. C'est une barillie tries 
claire è haricut indigene nommiébéngré, n'élarrgée à quel. ques unves des sulestances indiquices ci-bessus.

Se zindo conyoute du sel gannsum! mais le juixide. vè de acondiment le rend inacessible à la majeure frastie de la jugurbation qui utilise géreralement à sol flace. C'caur de cendres (yem-Kome). Les chardingues qui fré. tendent que les Massir voient hien moirs Clair queux la nuit attribuent cette irferionité à l'alsence de sel daus leur alimentation

Le rig (manc') constitue un aliment de luse. On le frejpare bowilli avee des arachides (sóntiam)filies et de la viounde cuite.

Il se foit aussi me assezy grande consomination de haricuts heragré) enits au beurve de karité, mais c'est. là un mets per wecherché.

Ses fatisserves indigeives s'il est fermis de se servir de cecte, expression) sont esetrémement nombreuses. On bronve surtart sur les nourchés le snisongaec confectionné avec de la forime de nil c'est le sagasni des bounharas); le masré,

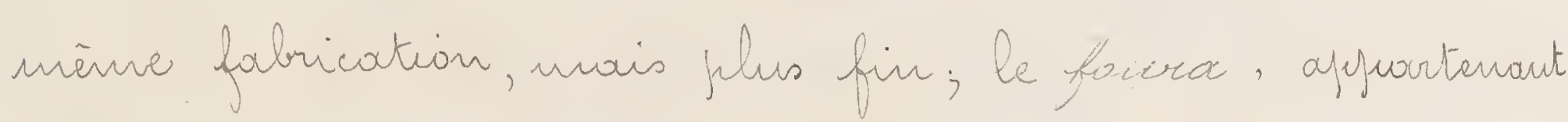
à la mênue catígarie; le samsré soute de beignet pétri avee de la farive de haricot; le Santrarcargo, mie. me genve; le smasa daus la conprasition duquel entre le maïs avowit qu it ait atteint sa naturité $x$ les veufs de pintade; le toubane' de piate de haricot, cuit au four outre dewse feriilles désséchées; le gatsoné, fenilles de haricat et de maïs 
cuits à la vapeur, le frigri an farine de graines de cuton. La base de l'alimentation, on le wit ast viojétale. les indigènes aisés tantefois consomment de la viande. On abat taus les jurrs dous les villages des chivies et des montous, aux gramis maricites dense fois tar semai. ve) des bouks. Les viandes les felus rechercheis sout celles du bouf (nófo) un manton résiov de la chiore

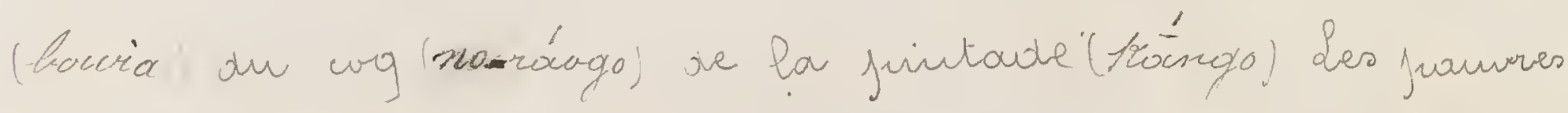
gers mangent oursi les arimaux morts; le cheval(cuséfo)

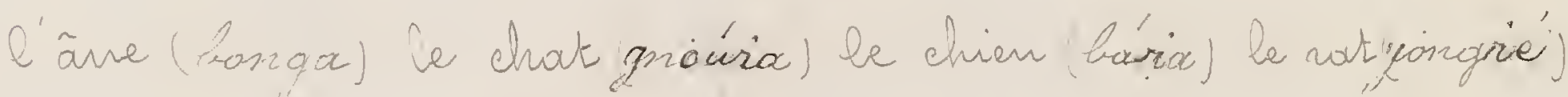

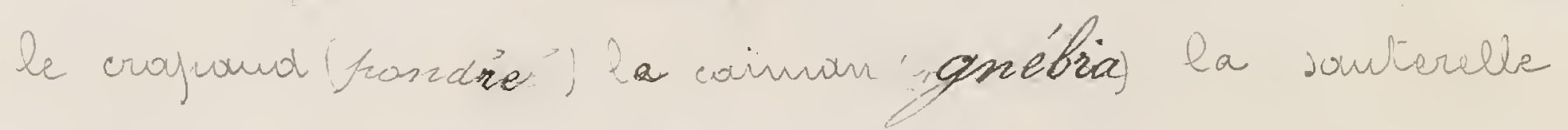
(soive) de grosses araignies charmues manfrociga un gan-gam) et un serfent non verimenx oxai-oxáfo). In somme tout prent être mangé an cas de besoin souf la chair Aumaine (nemsala. ot celle du vautour (yibiago). Des iver. somves ayant un totem animax s'abstiement naturel. lement d'i taucher.

Les Massi: we frement en gérièal qu'un refuss, celir du soir. Dours les classes aisés il est frourtant d'usage d'en faire darx, le furenier nommé - Lávibo... vers ouze tweures du matin, le second savisacillix. - Séré, inmédiatement aporis le coucher dur salill. The

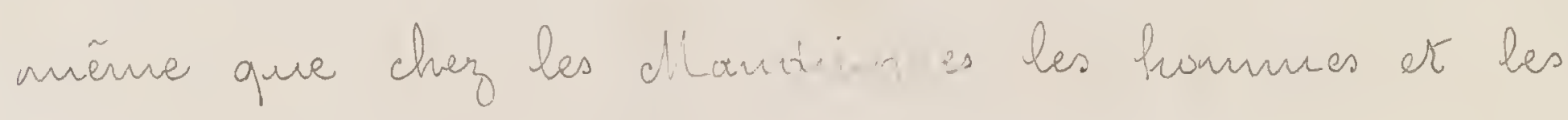
formmes margirt seforiémant.

Les femmes sont exclusivement chargé de la préfea. nations de ces repas. Oyprés avoir décortiopué les céréales 
au fillon dars un mortier de bois our, elles le vidrisent en farive. Cette opération se sait sur la jelace du ivllage on ast ́́tabli à cet effer un terre plein de fome circulaire

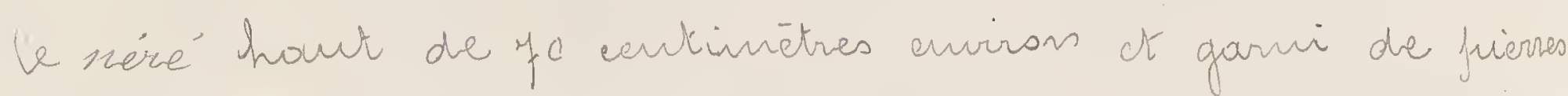
felates sur taut le juaritour. Choopue fierre est réservée à une fomille to granir y ast trituré à l'aide d'mir gras coillou arrondi.

La boisson la jhlus commure, sarrs jourler de l'aur, furisée sament à des mares rifectes et consommée sans précoution, est le dom (dolo des Mandés) brenange fer. menté à lase de mil ange afaurt germié. On en dis. tingue deux catégories les tase tries concentrcés et inupré. griés d'aramates, reservies aux nalas, et les tasabar a plus légers è dejecururs d'aromates qui canstituent une boisson frapulaire. Il ì en foit siartaut une tris apan. de consommation.

Ce qui précide cancerve particulièreneut les Massi. Paur ce qui est des autres voces habitant le froup ges ferwis veriarquer que les Marrdés. Yarsés at les Beulhs s'alistien. ment de mariger les animanx mants.

Ses secinds consomment en autre en grondole quantité le bait de vache qui n'entre que tris accossoirement daus l'alimentation Massi: In gárèal is we le boivent qu'aiare. Le beurre animal est aussi chez ense la plufiourt du tenpos sulstiture' an burrse de Rarité.

$$
\text { Chez les Gouraunsi le sáriba." furend le nom de }
$$


138

mirio dt se juéfuare avee de l'caur ou l'on a fait preala. blement jourrir de la farime de mil.

Comme les Massi: le, Gouraunsi. sout assez frevoyantis; dés la néculte, its enfenment leurs aljeravisiomements four l'anncé dours det magasions en terre, Lien ameirages et souvert isolés du contact du sal four les fréserver des navages des tormites.

II. Vie dendilive

Sensibilité générale et spéciale Les indigines de el inayp suffiarterit niense qui nous la douleur Sfantance. Meancaips sant atteints de plaies maliognes, de lisions assenses an a'affections intomes certainement accom. fraguées de doulaurs vivlentes, et s’en jelaigneut à frenie. Mais four ce quir est de la douleur frovoguéé (incision, an. verture d'un alcès, védretion de fracture) la différence on. tre l'Eurafréen et le Massi frarait bearcauts noins sensible. Il ast vari quielle est difficeilement appiréciable et, en gémé.

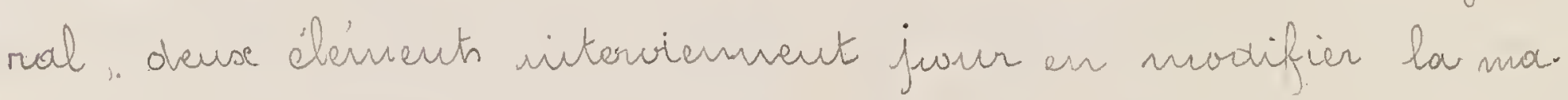
nifestation extériciure. O'abord l'appréhersion esetiōene que cause tanfaurs an noir l'nitenvention ofrératoire du blane qui prout, an faire faraitre plus vive sa sen. sibilitè, ou bien niène l'exagèerer réallement en créant 
un état nervense fuarticulier. e'est ensuite l'amour frowpre qu' a be Massi de me has montion de foubbesse ot de foure

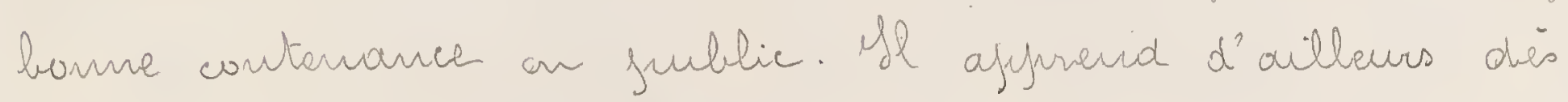
l'enfonce à dissimuler la souffrance fulysique ot la.cir.

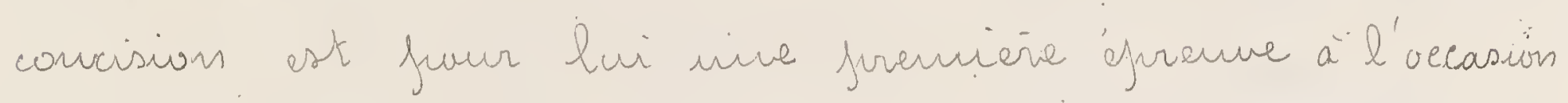
de laquelle le fait de manguer de courage est con. sidveré comme disfomarant.

Les Peulhs sont ilus seusibles que les Mossi. Miles Bous. sanga; ils out d'ailleurs beancoup mairs de sésistance, tant au point de vre fhrpique qu' aur noval.

Les malowies sowi surpiontées avee beourcaup de fra. tience et de résiomation, la confiance en la guérison jour des amulettes et des paroles naugiques est consideirable. Les nualades mène growement atteints out tayjaurs l'espair de quéeir rafiidement sils détiement le nemède de l'erufirioure ou du charlatan qui a jur leur jarler le obmier. Cet état d'esfrit leur cot d'un grand secaurs aur milieur des ejren. ves de la maladie. Olus infuressionmables les fenmes suf. portent moins bien la souffuenc.

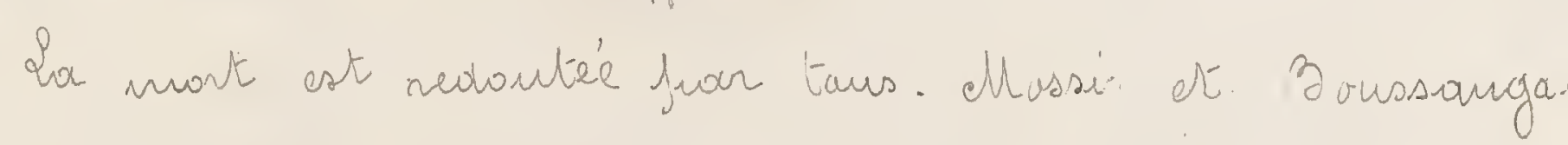
Q'acedpteravient cefrendant aver visignation s'its avaient la certitude que les rites funibves serout accouflis t que des libations prériadiques auront lieu en laur hommen confoménent are usouges.

Sa sensibilite tackile est médiocre sauf en ce qui 
coneare certaine fiovitie de la face. Oes exefierinentations jerur lesquelles on a witilisé l'esthiesiomètre à fiounte de lieige montiant que d'une focon gairirab elle doit étre considérée comune deux fois maindre que chez l eurafién.

Les soveurs jimmentees sout juéferées, l'aner est jeen op.

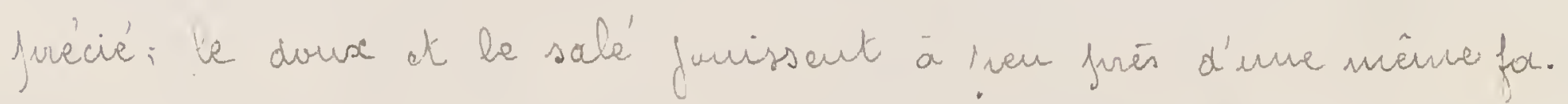
veur mais moindre cefrendart que la saveur acide.

La sensibilite aldactive parait the tris devrly, chez les Ceulhs. monis chez les Massi et les Boussanga. des uns et les outres raffolent cependant a'vire égice nommiée

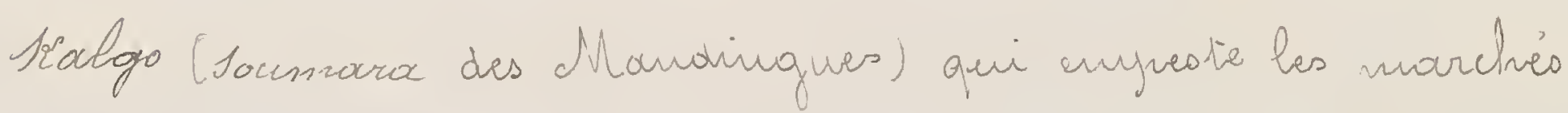

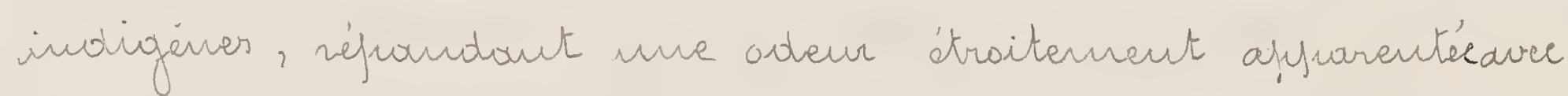
celle des matières fécales.

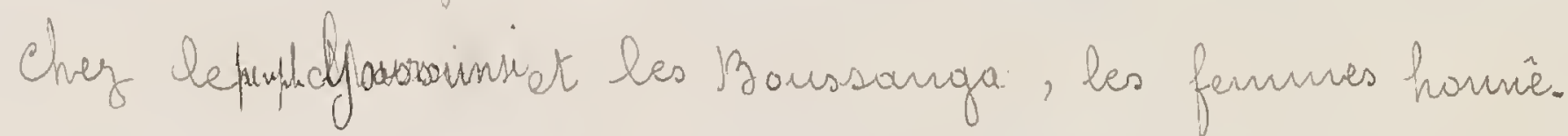
tes an réfurties conme celles, dovient faire fi de la

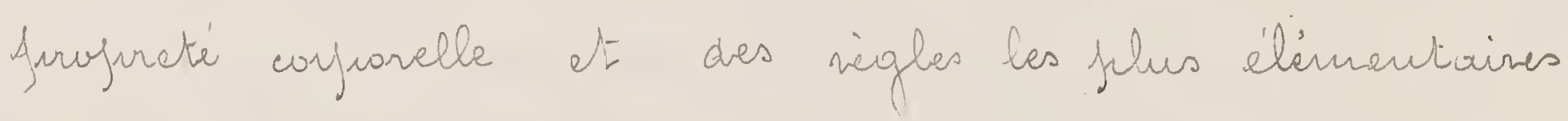
de l'Prggienve aussi lussiédent-elles une odeur sui géré. ris extrémement désaguéable. Now semement les Bous. sanga, we se lavent fanoirs, mois Leurs vêtements ser. wert quelopéfois à plusiours géreriations sans ètre jassés à l'eau afir d'éviter une usure crops hréeace. Les Peulhs

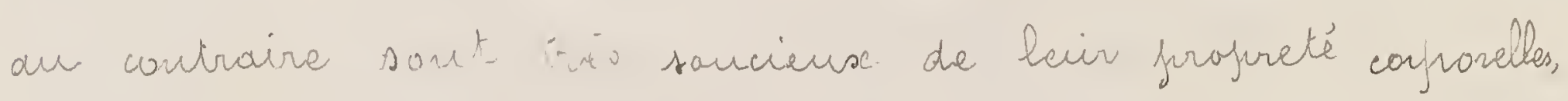
leirs fermes filus encone.

Se sens de l'arie est moirs develappé chiz ense quecher les Massir at les Banssanga qui l'oni tries eserce'. 


$$
\text { in ce qui concenve la vue, les chassi. }
$$

sed distriquant presque sien la mit, ce qui est attri. bué à l'alsense de sel daus laur alimentation. La lunière solaire at mieuse suffyartée juar les indigeires que jaor les Ervopiens; les Massi et les Boussanga. n'en sont farmais incommodes si ritense soit. elle. Les Ceulns se montrent flus sensibles. Les cas de nyjapie sout assez fréqueuts chez les enfartis, moins chey les adultes. Il y a heu de cas defores. butie at l'ou re vencontre jamais de daltomiens.

On distingue rarfaitenent aira couleurs: le blane, le marron, le rouge, le jaune et le noir, qui est confon. dur avee le bleu. Les couleurs firiférés soutt le range It le blar four les Massi: at les Boussanoja., Le blane. juour les Peulhs.

Chey les Massi le warge daus certaines frarties our vitement ot du harmachement est la coulaur des chefs qui out seuls le droit de la juater, tajis de selbe rouge, hottes de cuir Charojés de biroteries sur fond de drap roinge.

Esthétique, Barrure; Diformations ot mutilations ethniques _ Le ford le plus enyloye est celui d'antimaine. Quertes les fermues beulhes ot les élogautes Massi. s'ar servent fraur s'allonger les cils s'agrandir les Heuse et enduire les criatrices des tatouarges dars le voisinage 
de cause. i. On foit aussi usage du kâhl que les com. marcants yarsés apyoutent sur les marchès. On employait anciernancert, et maintenout encore quand on manque de fruotuits préfiares, les grains d'un arbuske nammébantone qui come des fruits aralagues an citron. Pes grouives ure fois ácrasées fourinissent une fiäte d'un noir bleuté. Pbanmes ot fennues s’en enduiserr égalenceri le taur des yeuse. Baur mierse faive adherer le foroduit on frasse aujaranent. sur l'endroit on il doit être apyaliqué une légère cou. che de beurre viojétal. Oaus le Gouraunsi les fermues se font également doure vaies frar groufre de trois autaur due nombiil et sur la jarve draite, de l'aile du nez à la commissure des lèves.

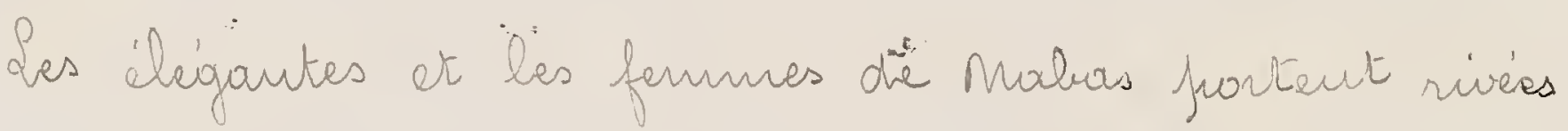
à la chaville de arosses baules de cuive qui, biè que crenses à l'intérieur preuveut jueser plus de deuse kilas; elles leur dament une démarche leute et déhanchée qui constitue la suyarence élégonce fuur les geusdu fiars. Or Gourannsi elles intraduisent dans le lobe de leurs oreilles de petits eiflindres d'une pierre opuortzense que travailleut les Rougari. les cylindres ait eurinon cinq centimétres de long sur un de dianietre, leur afffor vence est d' un blane laitause. Ou cou sout frontes des colliers de perles quenat de pacotille caillies à facettesqui sout venylacées chez les femmes riches pour des perles ron. des on en losanger, nouges également mais d'un. éclat 
mairs vif qui joroviement du frowssa et sont très esti. incés. Infin autour des seirs fenmes at jemres filles se mettent plusieurs rangs de prerles bleues.

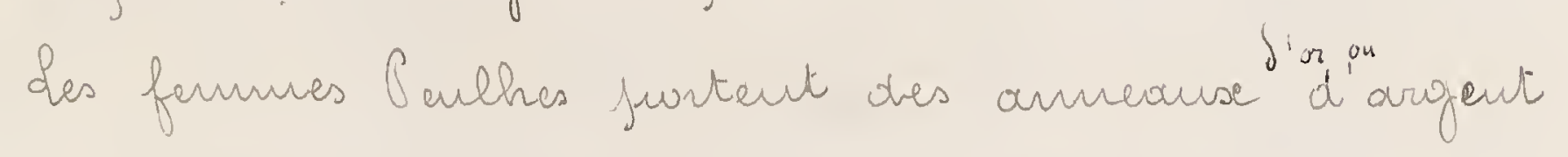
arse oveilles, quatre à cina de chaque cité, fresant de criq à vingl grammes et sauvent die peetites chainattes, également en argent, susprendues à la fuartie sufvéricu. ne de fravillon de l'oreille et regiliées cina ou sixe fuis sur elles. mémes; le fivids et d'environ viroft à vingt-cing grammes fuaur chacume. Il n'est guas rave de voir les oreilles des femmes. faulbès mutilies et crevarssés jear le juoids de ces bi. Jaus. Massi. ct Boussariga aussi bien hammes que fenmes fortent soment accrochés au labe de l'o'reille un petit armear te crived ore, d'argent on de cuire.

Chacure des trois naces a un tatonogje frovticulier. Il ast déterminé fur ircixion sur-la figure ot autaur du

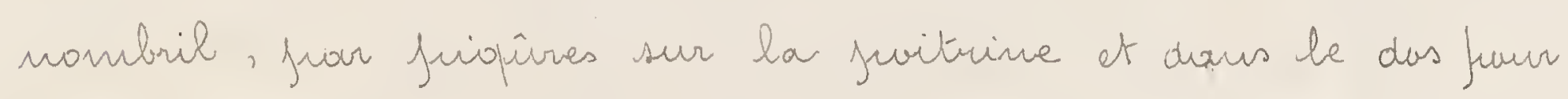
les fermes Baussanga:, fiar ulcération fuur les fennes filles Beulhos sur les seirs. Chez les Boussanga les femmes en âge de se marier se font foire sur chaque fesse, bwis larges ineisions latérales' qui frarait. il contri. breut à les rendre fécondes et les embellissent aux yaure de leurs maris.

Les sigues distinctifs de chaque wace sout manqués fiar incision sur la figure lorsque les aufauts simt ancore 
jenrres. Hels consistent, nous l'avous dit jeur bes cllossi. en trvis traits paralliles alliant le lorg des javes du front an menton at se vejoignant juarforis de fason à dessiner trois ovales concentriques. Ses farres jortent en outre deuse traits qui fulacés de chouque coté du vez affectent la forme a'un accent circonflesee. Ahr seul de cestraits, à ganche, sxiste chez les familles nobles at ce détail sert à distinguer les Matamsés du commun des mortels.

Les Mandés. Marsés qui ont adojaté le tatououge

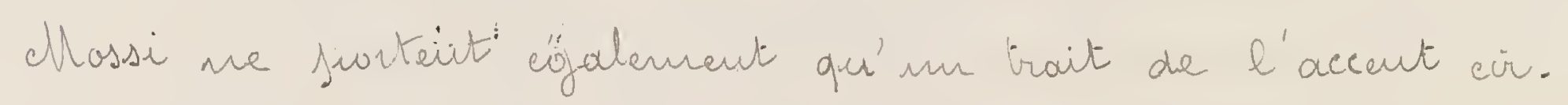
comflesce, celvi de aroite.

Le tatonage dtait anssi en usonge an Gouraunsi ou il tend à disfuaraître; la nargue fuour les hommes ot les femmes consistait en deuse iraits allant de la com. misoure des lèves vers l'oreille. Catte coutume n’at filus suivie d'une foison alsolue actucllament; en reurache beancaurs de femmes se fout foire fuar une étronge coperetterie des tatanages sur taut le carps, tantit en grougue de douze haits symièriques autaur du nombil, tantat des ligues paralleles de petits traits soit sur la gonge ari dessus des seirs, soit sur le das à hau. teur des onofilates.

Yreiquemment les incisives médiaries de la nachai. ve sufrérieure sout lineies en frainte, on brisées an ciscaur comme chey bes Gauraurisi, faur affeceter une forme 
triangulaive. Cette gratique n'aurait feas selon certains un biet furement estrétique et fremetrait en cas de sifncafe accompraguée de coustriction des machoires d'adminis-

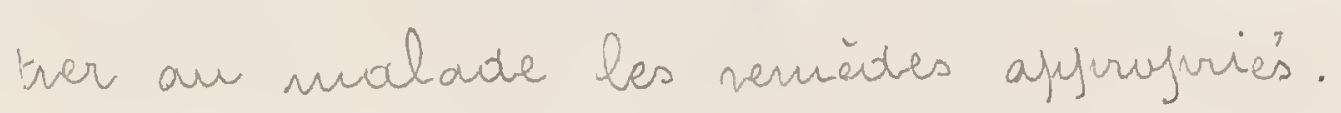

Ses Baussanga: prerforent souvent chey les fermmes la leive inferiense taus laquelle est passée un brin re waike an in pretit moncean de heis.

Les oreilles sout en genèral frerforées four laur pernd. tro, de fwater les franures que spioi décrites des defomaturs crarien wes ne sout fuas en mage au classi.

Sa circonciswon n'dait fuas Aparatsiguer jadis, mais elle tand à se véfnandre de jelus en julus. Elle se pratiopie comme daus bearcoup d'autres régions du Sandan; le préfunce intradvit dans un billot de bois et fortement étiré, est tranché, net avec un cantican. In verouche danstans les villouges ot de tout tenys on a pratiqué l'exisün. Les indrigères doment frourfois comme voison de cette mutilation la crainte qu'ils aut de voir l'ongarre qu'elle ir. téresse forendre un dévelafyrement exageré.

La castration 'tait auant notre verue fratiqué au

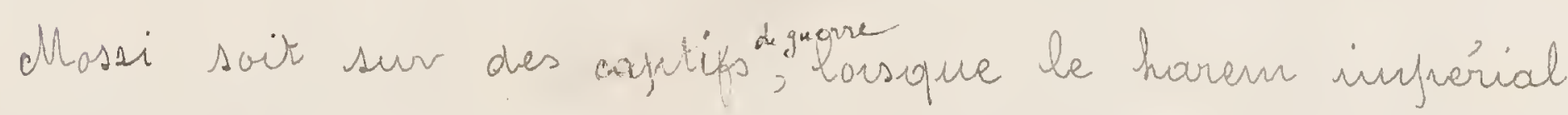
manquiait d'emmques, soit sur des gens qui ise voulaient

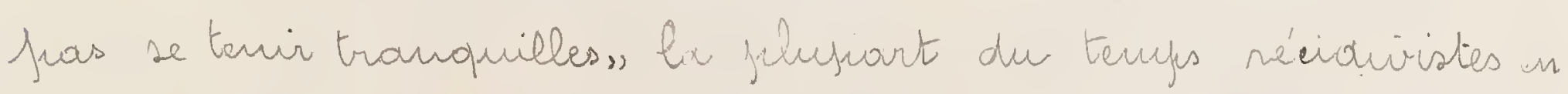
matière de séouction. On uprérie à Qibtenga cautors de Raubri) dout le chef ètait frarait.il fort habile, mais la moitié des sufets, ot cense. ci itaient habituellement agés de 1 if à 2,5 ans, 
ne surnivait jas. Peuse qui '́taient assez heureuse frour s?en tirer ataient envoyes au Mơfo. Naba aufués de qui ils verughissarient trierses fonctions oomestiopues at atteirgraient souvent s'is faisarent frene d'intelligence ot de devousmant les hautes dignités qui dovisent avair des emriques pour titulaires.

\section{Vétement, Caiffure _L se îtement particu.} lier au Mossi est le tioilai. C'est une sonte de turique fincée à la taille et s'érasant en jujue au dessous. Elle descend fear devant fusqu' an genouse mais est silus longue et aryple juar derrière; les manches sout courtes et largement échancrées. Le taut constitue un ensemble assez hamonienx. C'at cortainement l'un des vêtencuts les flus originause et les phlus élégouts que l'on funise vecontrer cher des indrigenes; il faorait appoarteriir on profue an Massi. de phus souvent confectionné ar toile blanche du prafs, il se fait aussi en bondes alternées bleues et blanches, farfoir taut en blan. On le feasse comme une chemise. In furincifue il ne canforte fras de fountalou.

Sa timique de guarre fou-niounangobe'. ast serrsible. nent confectionnée sur le même type mois un peur plus conte at ordinairement terite an biun. Elle farte cansuss ause spaules, à la fuitrine et sur le dos, quelques amubettes. On passe habitucllement frar dessus me large ceinture. 
C'est avec ce vêtemant que se farte la botte Massi ancuir soufule de aiverses canleurs chargée de dessius afprliopués sur fonot de drape vouge.

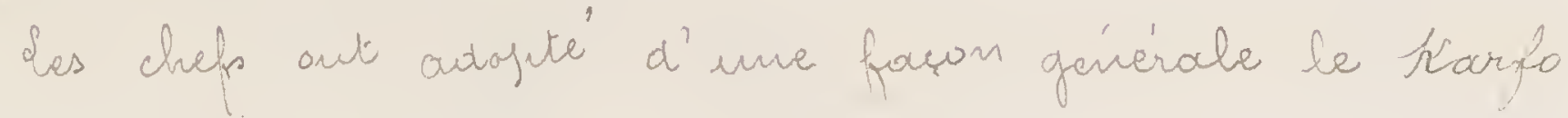
quant loubou havassa en issu blen fancé tonbant jusqu'ause frieas et muni d'une grande fuche sur le devont. Il at couvert sur la foitrime ct le dos de vartas buderies blanches circulaires. Wvec le tearfó de

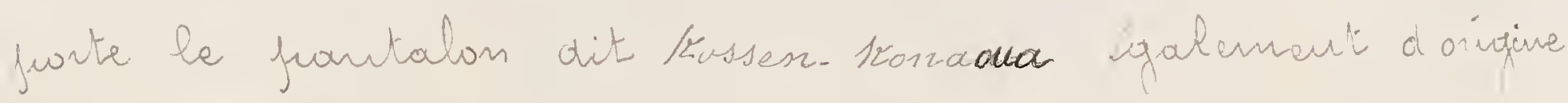

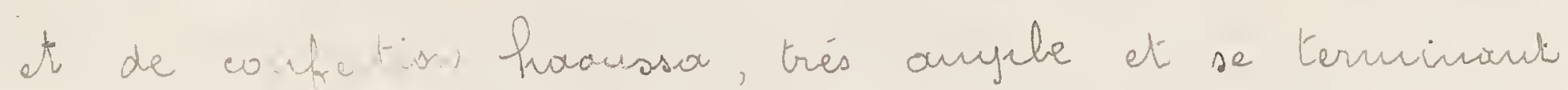
an.dessous du genau for une fartie uflindrique et semi.rigiole d'un vouge foncé.

Les gens de la canyragne frartent souvent mue ceiriture de toile qui enserve les veins et iasse cutre les cuisses tissimulourt le sexe. E'est le vêtameut le fuhs fréquant au Gourounsi et an Banssangee, on fortie en autre dous ces régious des freaux de moutan et d'antilope.

D' une facen gériérabe les enforets commensent à se vètir à l'occasion de la circoncision.

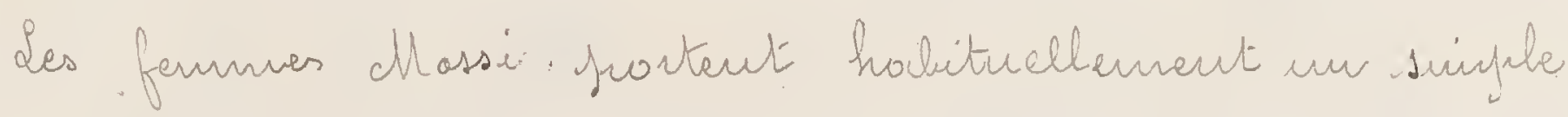
jragne (forgace) autour des cins. Sanvent elles s'envelop.

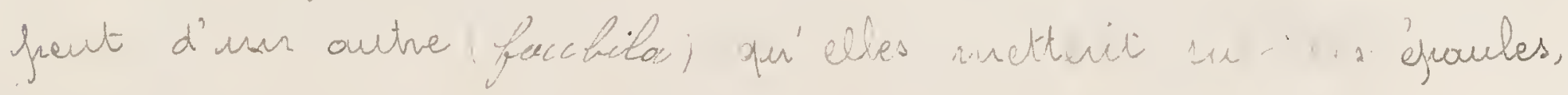
ce sout en géréral des jragues de willeur bluve foncée en toile du joup. Slles partent tries warencut le grand baubour qui est au contraire le vêtement de piédilec. Lion des farmes Serilhes. 


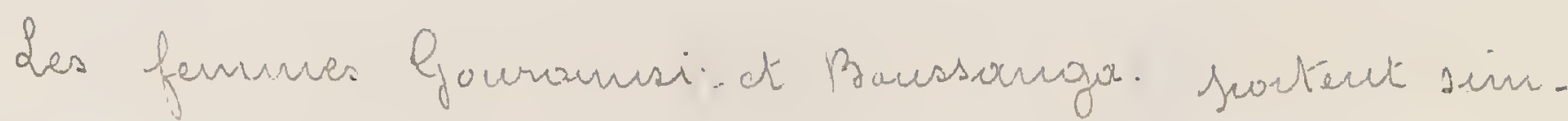

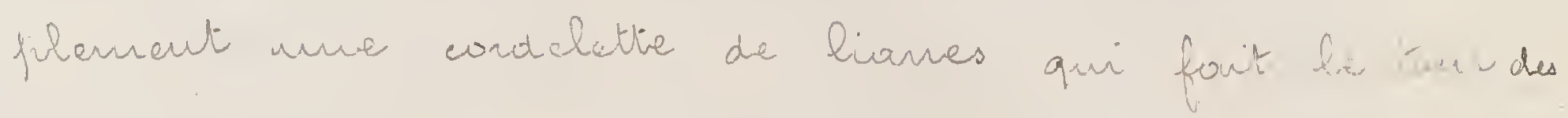
reirs et à laquelle sowi acenaches deuout et derrière deux jroupuctín de farilles vertes.

'Jusqu'à l'àge de la subberté les filles ne met. ternt arceur vètement.

chant de dire quelques nuts des drierses coiffures

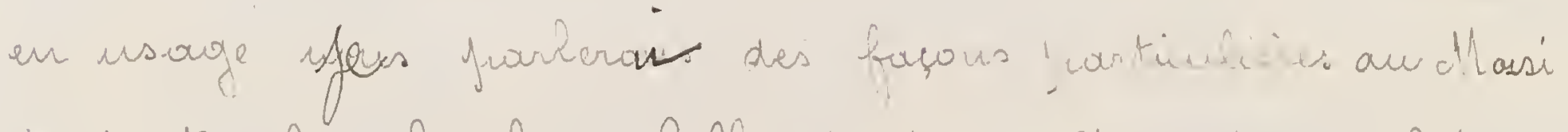

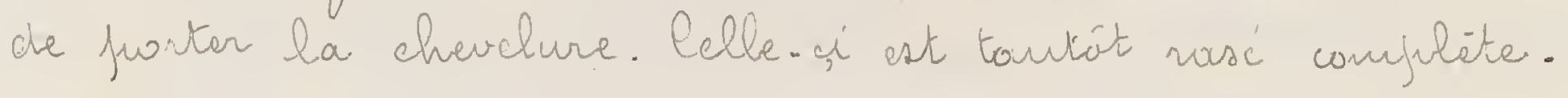
ment (yougou-foore') cherg les chefs, les fermmes de Maleas. les musulmans an gérieral: quelquefirs le niliau de la tate conserve ses cheveuse qui wort tressés en cimiar cour. rue chey les femmes Peulhes mair de noindre houteur. l'est la wiffure des jernes filles, fenme du peuphe st sarforiés. On lar nomme diesndaago. Guelquefois on laisse sur les cités deuse Touffes terminées en tresse (Óyensolatetiga).

Les fernmes Peulhes protert le cimier développé (dittrol) bourré de fourc cheveuxe (Cecizérl) à l'interieurs, ee qui un escougiere les dinersions, et se terminant foor mer fretite tresse necourbée le Socetracondace. Oles deux cutés de la ète

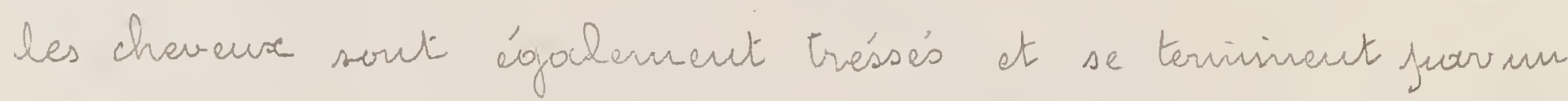

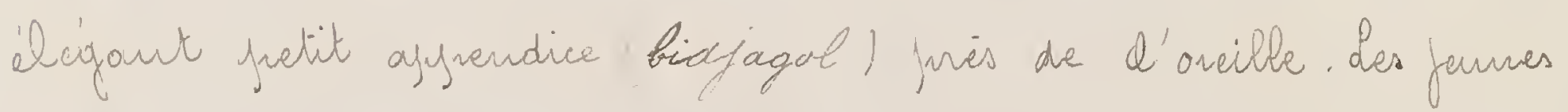
Peulhs du Marga fortent les cheveux tressés comme les femmes jusgu' aur moment de leur mariage.

Ou Massi les hommes frotent soit le bonnet nessem. blout an phtriggiess at se terminant jeor deuse fointes 


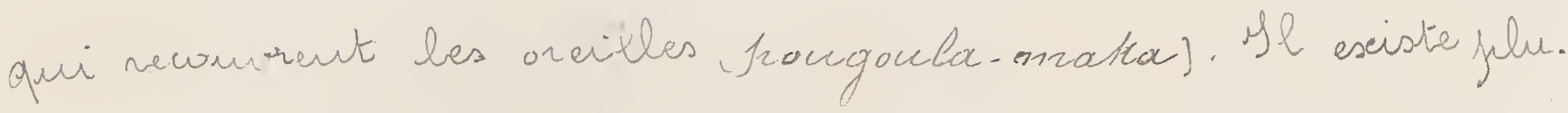
sienrs sottes de coiffures en fraille (zangon.freago) dout l'ime a alsolument les fomes du chaprour chinois: Mofes iterdes arfir la taque de querre (frongoul. riyounougdow. ) autaur de laquelle sout cousues des amuldtes.

On Gouraunsi une caiffure très oniginale est veserwèe aux guerriers; e'esti un casque formé d une calebasse necour. verte de cuir. Sur ce cuir sout appliqués des frièes de couleurs différentes figurant des yeuse et des saurcils. Dev. rière frend mue quene de checral ou de bouf. Carpois la miane cieffure est surmontée de comes d' thopes. Clles me se frortent que prendant la querre et constitue l'aju. nage des plus broves.

\section{Musique. Danses. Chants _ Sos hommessubs} s'adoment à la musiopue. Il existe des jarofessiommels. lev.s-i sont tris soment engagés au service des nabas qui enbetienment un nombre considérable de musiciers; nous aurus vu qu'un certain nombre de dignités leur étaieut reservess fuar la constitution de l'empire.

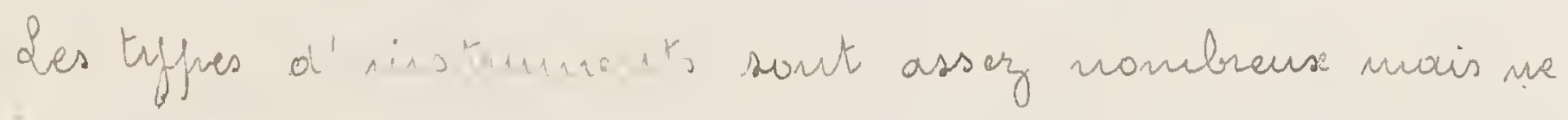
frésente fias une grande variété de geure. Oarmi les tam. toms proprements olits, ugs iterovis: le bendéré grasse calebasse recouvertie d'une freaur d'ãre tendue et ferforée d'un trou sur le coté; it se bat avee la main 
onverte. Le goungán-rago géréralement en bois de untro qui a l'avontage d'atre facile à crenser et abe we juas se fendre foar la sècheresse; c'est un tombairin cylindrique loug d'ention to centinietres qui se bat avee nu bätors

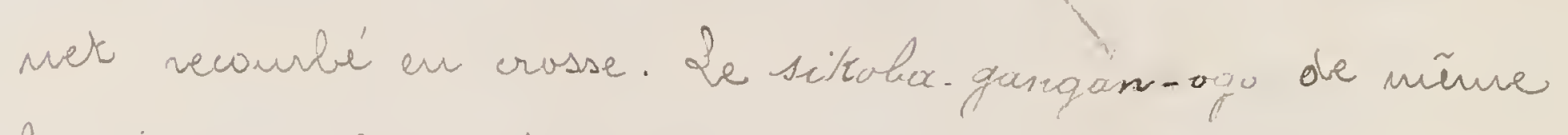
forme que le jrécédent mais frouraut ouvir Hmétres de long sur go centinitres de large. Acl n'y an a qu'un Jour village (région aur Nond at de l'Guest, srovince de Balom frimeifialement,) se bat à l'aide de beuxe covias (baguet.

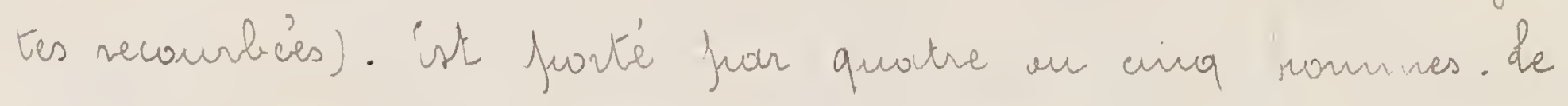
sikcha. gangabila, comme le jrècédent, mois plus court

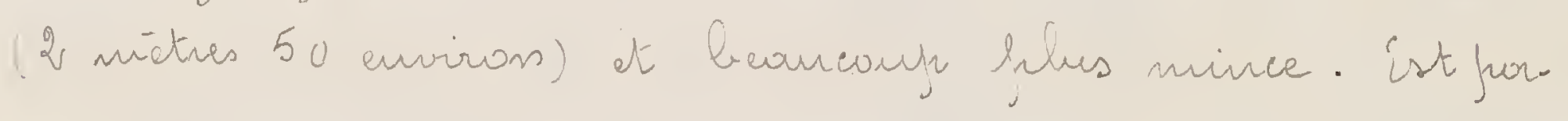

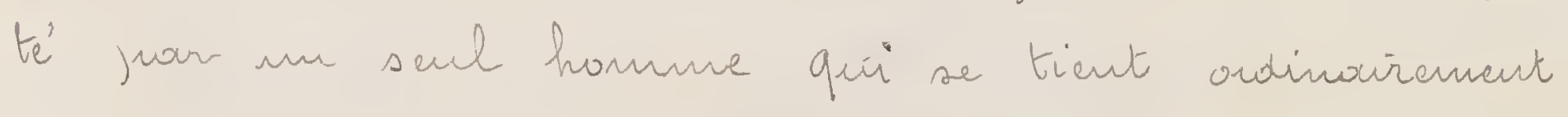
au milieu du cercle des danseurs, Tournout sur hie-nẽe. me les bras tendus en avaut et battant san istruement avec deus doyas. Enfin le lacenga un fame de sabilier daut la juartie dranglée est manitarue ous l'aissecle ot qui ue comprarte qu' ure seule baguette.

Souf le bescdéré. Taus ces instrument sont recour verts de jeour de drevines.

Comi les instruments à condes, les suiroutis moritent mue mention fuarticulière: le freszdga soute de quitare

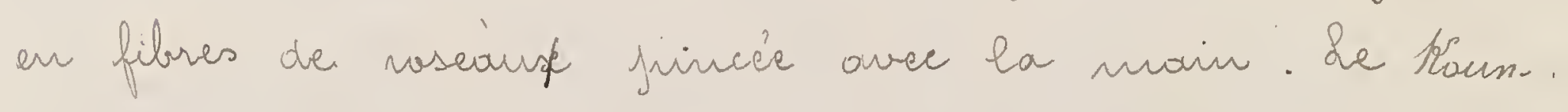
de' mêne gerre; la caisse est en bois, recouverte de frear de cheval; ses cordes, en crin animal sout au noubre de trivis. Le tans-leounde' minue gerre avee conde inique 


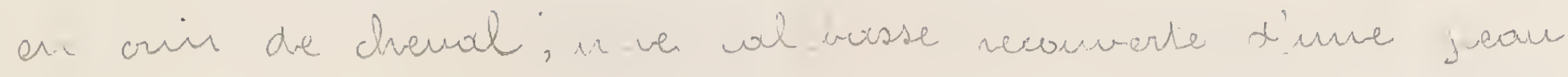

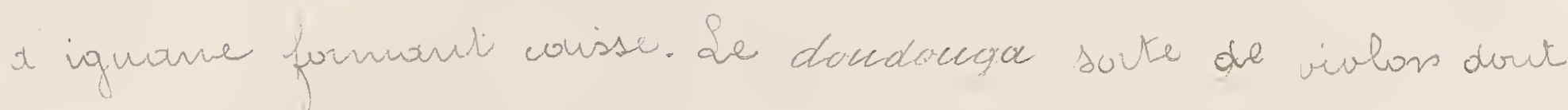
la caisse est constituee sar me cabebasse recouvate

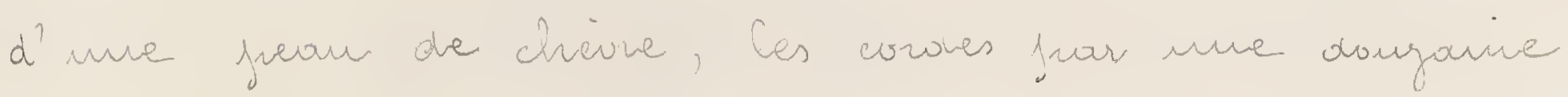
decins peur écartes les uns des autres; elles resommant surs un archer lafo eqalerment en crins de chaval.

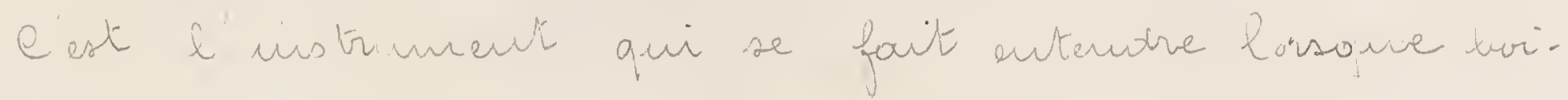
vert les maluas.

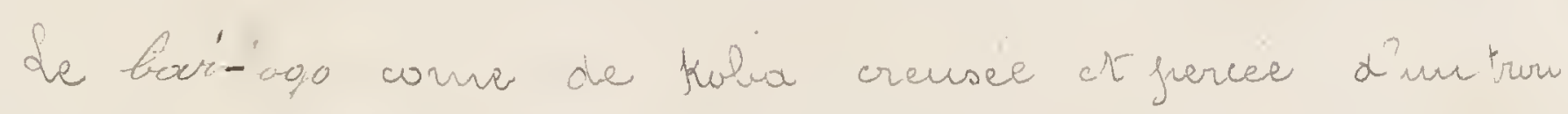

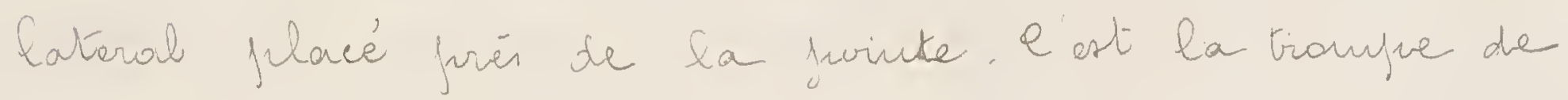
guare. On intuduit la noin dours le jowilkon Juur Rtenir des uotes warièes. Le nuésuane sorte de mirlition on

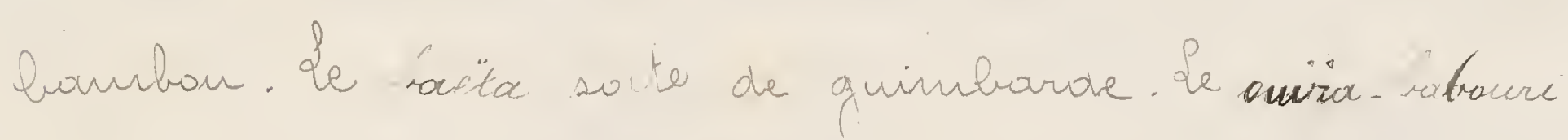

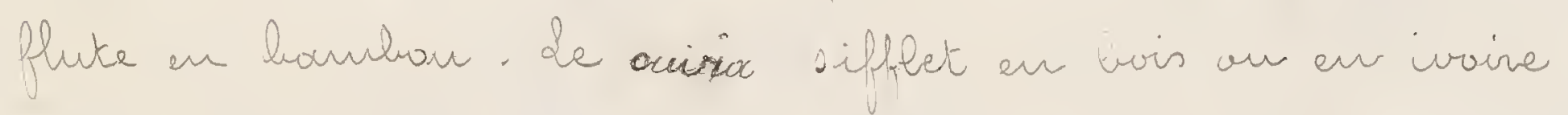

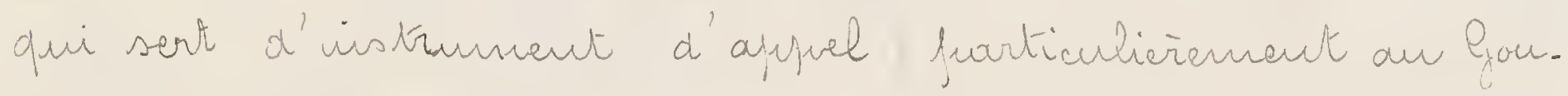
wansi ts an Ripinisi).

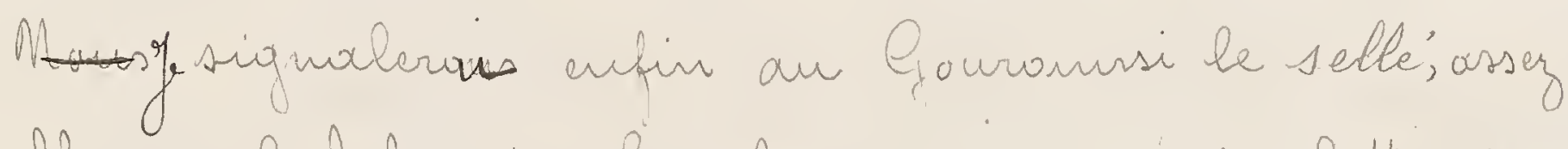
scublable au balafon des. lambaras cominese de lattes de birs placées au dessus de calehasses des diverses toilles.

Xu dlossi comme dous turs les jroups wir ta darse constitue de oivertissement de forédilection. Ou délut dºc. tobre peorticulieirement, lossque be mil commence à müris

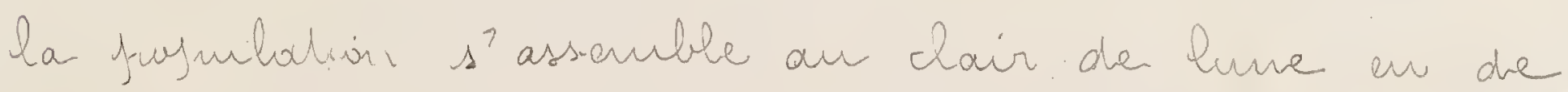
arands tam. tams. qui se froblongent ivés avart tans la mit. On douse aussi à l'vecosion des mariages et 
de l'ilcetion des cheks comme aux cerimonies funibives ou Rocere?

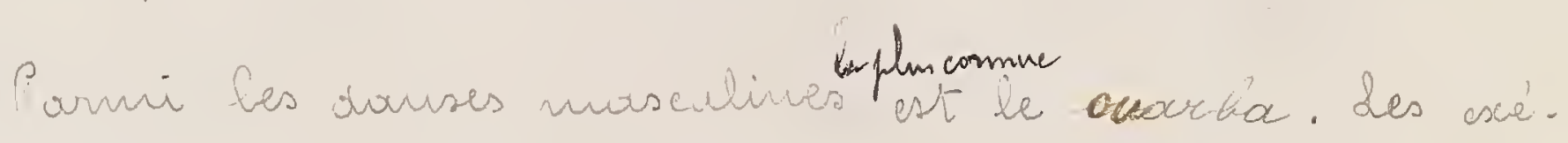
cutaur sout nevitus d'un custume ifvécial a grémanté de curis, its hortent un cosoue assorti, fuarforis des apelots untur de la ecinture, ause bras ct ause Chevilles.

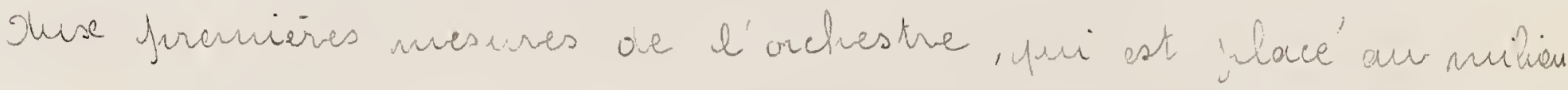
d'euse, ils commencent à se vermausser it à agiter un widmue les bras at les ècoules fuis le mouvernent 'accelare el la owase se joursuit ar cercle. àes figures varicut suimant le rythme; it y a des valtefaces raudaines, des firomettes, aes veverences; à la fir le premier dousenir se détache ot vou se frostermer devawt le ched quil verit howorer.

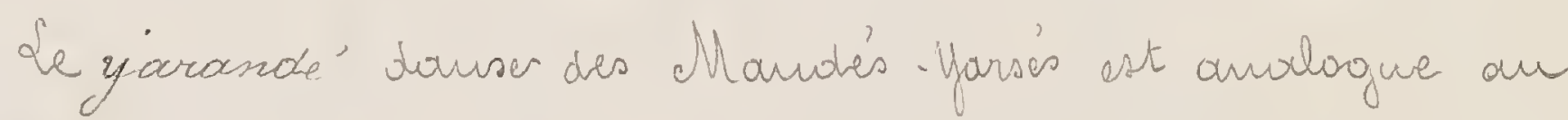
anarba. Il confrate ausi un costume squécial sovte de fitet à mailles espracćes, teint à l'indio.

On peut citer arsir parmi les touses d'hommes le duésba. qui consiste en undubations de conjs de drvite à gouche, an sou des violons; le dyéda toura des circanis caractérisée fear des mouvements du huste en ousart et

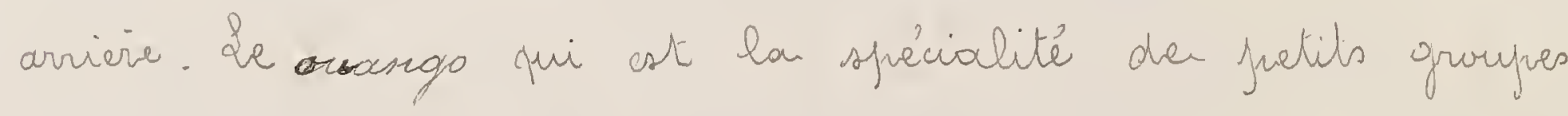
d'hommes formant wue suite de société secrète confuléte. nucut cowvert de la tête aux jrieds de fibres végétales.

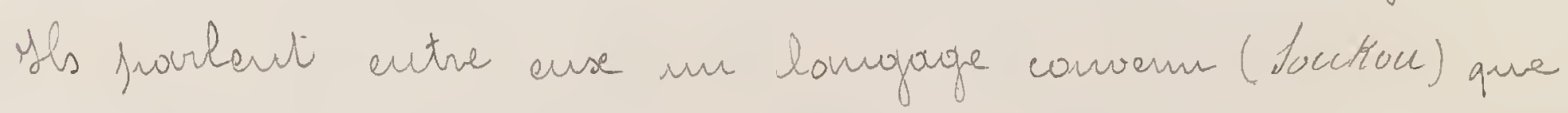

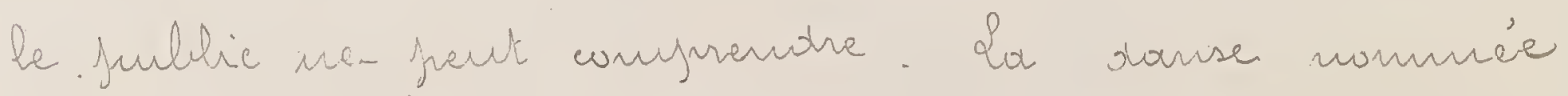




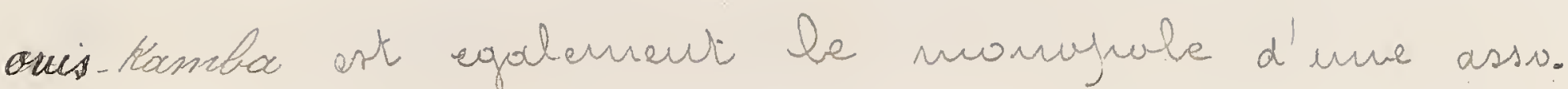
ciation particuliore give l'on nenoutre dous certoins villages kauila, Thekanngo ete) les dourseurs lèvent les farmbes en codence s'appunfart d'une mair sur un liaton et 'mo it, de l'autre me flute owot ib irent la miludie spémide au ouis, Mamba.

$$
\text { Mys parlerais en demier lien du noové darre }
$$
guerrieve à kny a se comicut des villouges diffé. weuts. Clle est caracterisée fuar obes luttes coups à curps. Lorsqu'elles sant temincés las varicus s'assieut àterre toudis que les rariqueurs donsant en sand taut autour fout le simulocere de les achever à couph de ralyer un Jetout sur cux des ferignées de tarve.

Les domses porufres ause fermmes sout aussi tries nombreuses. Mays' inaiqueris les princifales. Sog sarowa; les bras des dansenses sost dindus, le buste seul à des ondulations en mesure; rythme leut et laseif. Kiriotias: les darsenses battent des mains en coulence; deterups à autre deuse d'eutre elles se détacheut du gruupe tá la fir de la firrase nusicale se heurteut l'uve l'autie

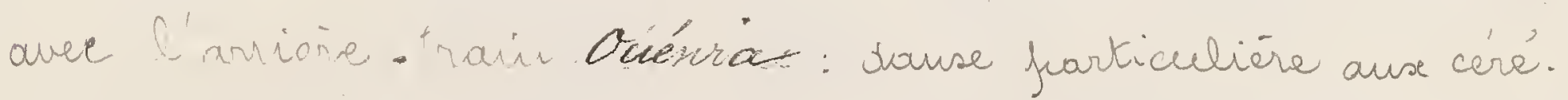
monies de l'excision; elle consiste dous un trémousserireut de Taut le corps are son du bendéré.

Les douses sout ondirairement acconfraignées de charts en choeur sur min ufthme nonotone. Se chant est trés en 
hameur an Masi. Il existe daus cortoives localités des in.

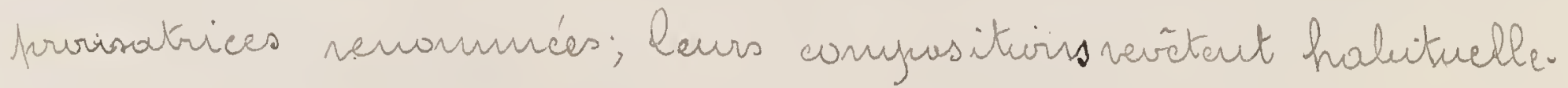
ment un caroctère omaurave et eritique dépouruu taute. fors de opussièretè. Alles verideut sonvent des mances de senti. ment asser délicates. Ofin qu'on frisse s'en faire une idée ifeus dommens ci-afries le tescte de la traduction de triss pe. ites piëces chrisies parmi les plus papurlaires.

$$
\begin{aligned}
& \text { Sar sóré meng-a-gana } \\
& \text { Ned kam four man a valeo } \\
& \text { Mea Kam fan maria nabo } \\
& \text { Moika sid mana dullé } \\
& \text { Mldika dolis man a sida }
\end{aligned}
$$

Sudomi sebré yélémaso Med kanr forr mana wabo Qoviansimia man yankite Matik m'sid man dolle' Gikminb man a kaolé

$$
\text { Propus drune femssne libre - que chaun suive sa }
$$
volantè - (bis) - De man anant J'ai fait mon mari - It mon mari est vesté mon amaut - The chase est éerite dous le iel_Que chacun suive sa volonté - It ma volanté fe l'ai suivie - de mon anourt f'ai fait mon mari_a Likarimba f'ai danné nou caur. 


\title{
Basése míbanum
}

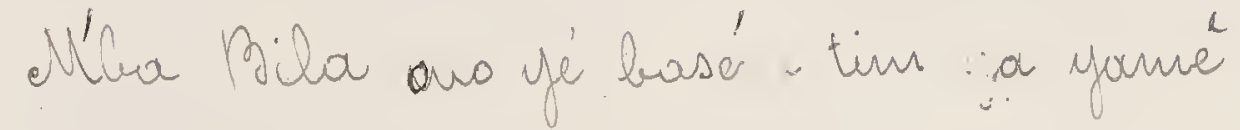

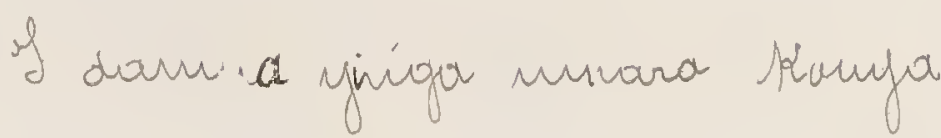 \\ Ia i guer a yingou ntan na kieng salaga \\ diba Bal ow yé. Basem tinna yamé
}

Saisse-nai Eranquille — Laisse-noi Bilan fesuis

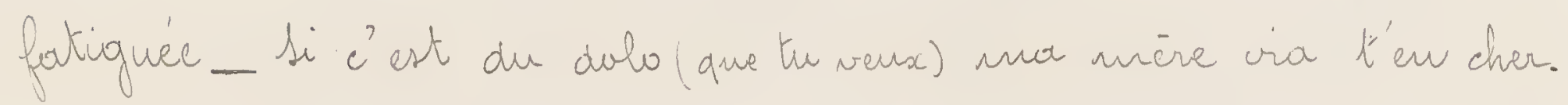
cher_- Ji c'est des toolas, mon peire ira t'an acheter à

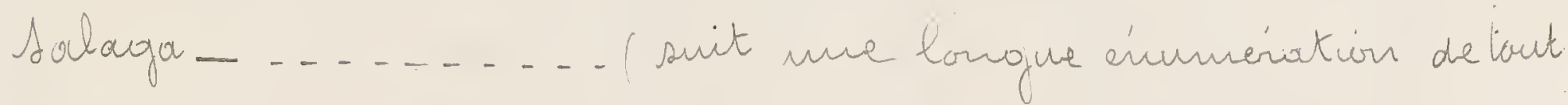
ee que fraurrait désirer Bila has l'étreinite amaurause) Ct be

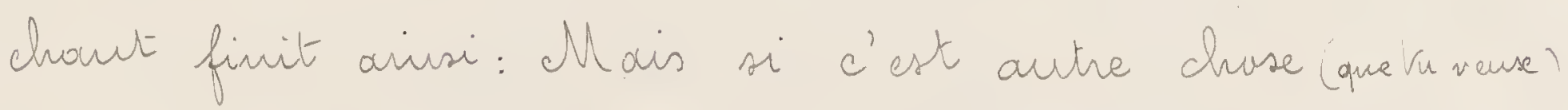
Mon, laisse noi, ge suis fatiguée.

\section{Mingida yidre"}

Ba Rinugidor to Ran iradenr fívoris Panis a sémodeldé gie.

Ba Rimajudar to ten far dorifo den a man doipla-apparifla biga xara, Oúmura sitrta banné. Melura fan Grúbgor cooghén brogaré 


\section{a Ringit goubjo}

Ba Rinngida fí bé Birgui

Mad pa ña a kou dé Mé

Ba dabali a danié ya

Ba Ringiat kon yal ye'.

\section{Rimgida l'hétaire __ Ringioda a au bras}

le frarrier des hétaires - It s'en va à l'averature - Rinigida est enceinte - qui saura farmais si e'est d'un garcons - On d'une fille? - Car taut le monde va chey le surcier - Paur avair des enfants - Ringida ma (aussi chey he sorcier wais cat)

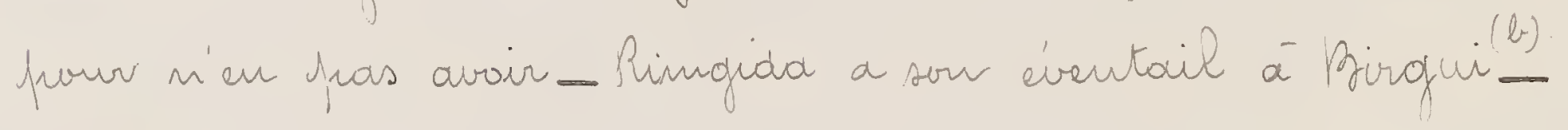

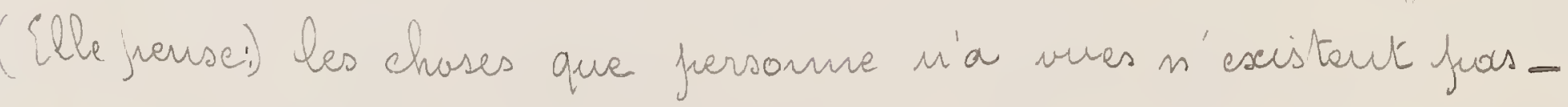
Daboli s'ennuie dours sa case _ Sagerf tranguille, cela n'ar. rivera famais à Rinugida.

a of bs Copressions figurées qui n'out qui mure signification lacale.

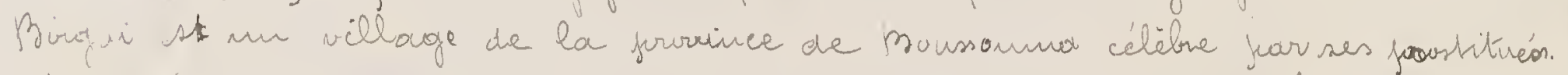

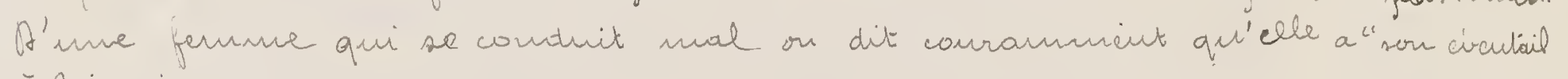
à Bringui.

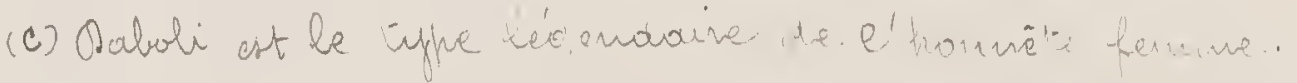

Ses chants querriers n'out fas la miense siojinalite?;

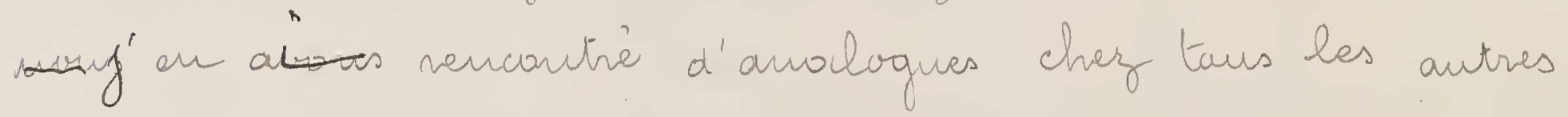
pengles de la beucle du Aaiger. Le sont en gérienal des brousdotes ot frovocations a'autant plus fieires qu'elles 


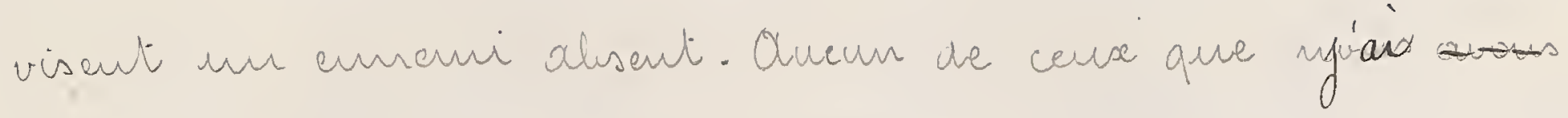
recucilli re mérite d'étive veteru. Les joueurs de hemderé nous l'avous wu chantent an som de leur instinumant des devises des nabas ot buodent sur ce thèrre des lacionopes

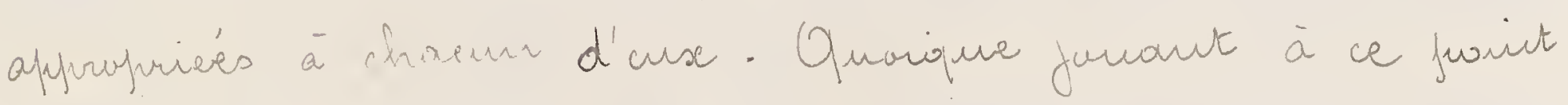
de vere un vile analogue à celui tu dicali des juays Mandés, is ne dorivent fous être assimilés à ee demier; lar fornetion ne les déconsidére juas aux yeuse des combat.

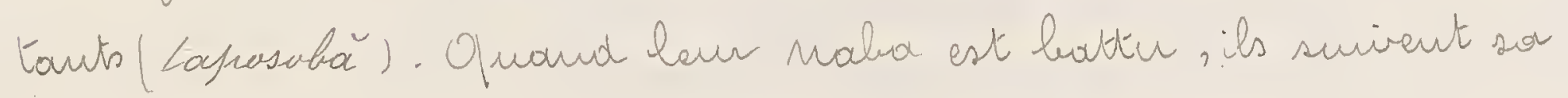

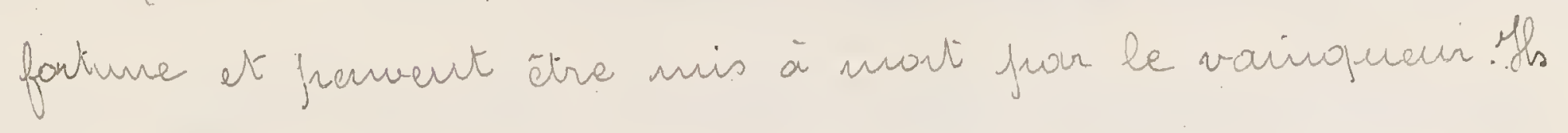
n'injurient ni ne mendient. In un mot le griot clas. sique n'existe fras an Massi.

Orts owivers _. Les arts graphiques sout four airsi dive ricomus an Massi. Les vores dess-ains que

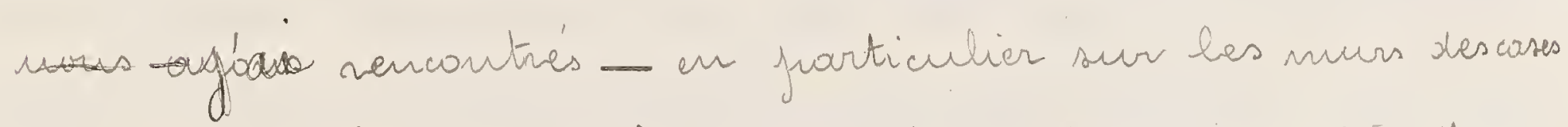

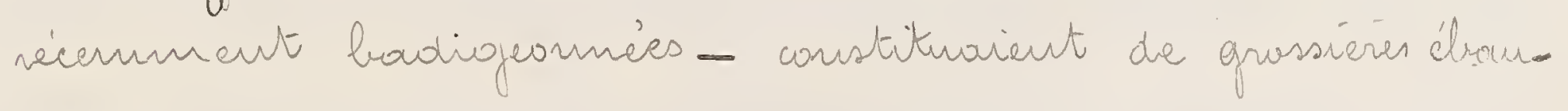
ches à peine digure de ce nom.

Il n'en est fros de mierme des arts plastiopues. leux-à sont assez népondus et méritant une mention spéciale. Les nandbas et les grótses en fout leur monofuble. Les pre. miers utilisent particulierencut le bis at la terre, les se. conds les miétaux. Ceux ei south les phus intéressants et de. beaucoup les plus nombreux. Sn gírieral il commen. cout fuar excicuter une maquatke en cire aierge qu'ils 
curtourent de glaise nécemmeut fuétrie. Dès que celle -i, laissie au saleil a frir de la cunsistonce on coule le métal en fusions foor mue omverture qui a èté ménagée.

Mans avons mu que des statuctties némandi veprésen. tout taus les Mabas apant négrié, sant dèpasées à Lum.

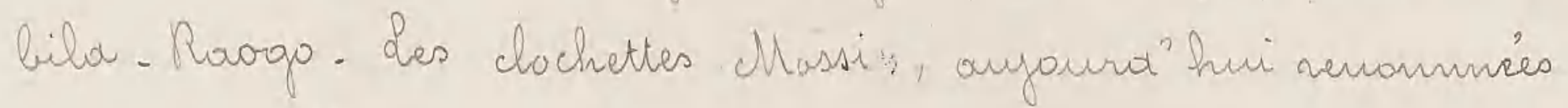
dours trut le soudan, représenteut un type de création recente. Acount motre arrivél dours le juays, les vérita. bles clochettes Massi étaient conyeletement déjaururs des sujets et des accessoives qui en fout aujourd'hui le plus bel onament. On distrigaait trois catéganies: la graude cochette lámbre?. la pretite yoédrtue et le grebot bougoulli. Sarni les imonubrables productions des grótsés

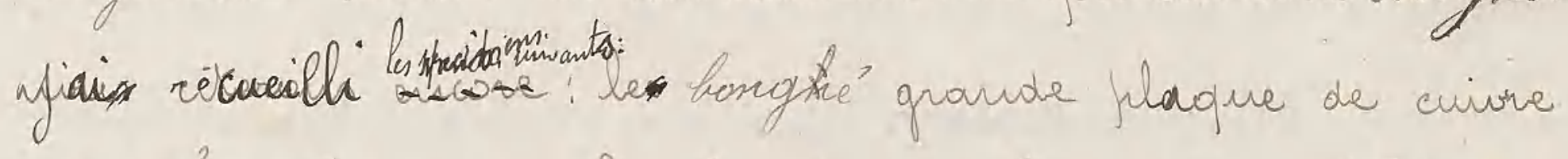
auragé qui onnent le frout des chevause; d'élégants átriers alábici les variétés de bracelets dites Kan-banga (petit, portés aux biceps, spéciause ause soithonés) zocúcoif porties aux furignetis) Kalemblanga (fer eh crive unis ew torsade)

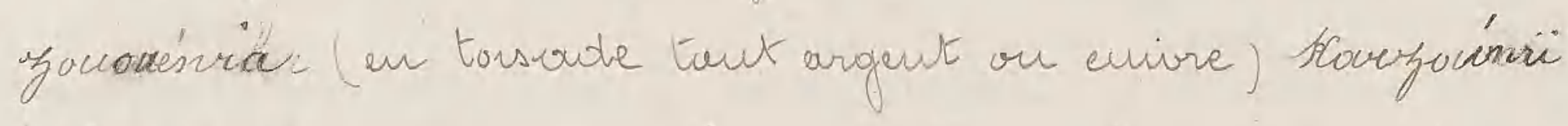
(bies gros en cuivere massif, se met en filace aw martion

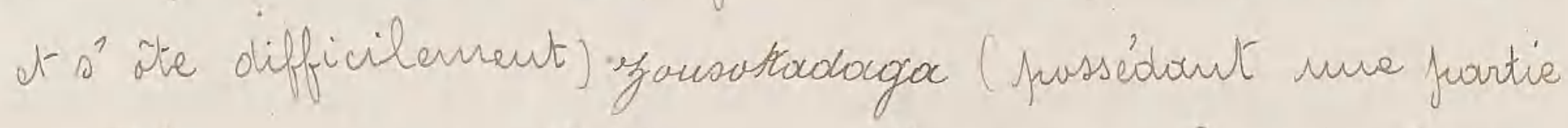
mobile qui singilifie ces operations) Les ranbre's gus. ses boules de cuivere creuse séservées aux fermmes des nabos at aux soyponies, de meine que les fodo fambieieres an cuive houte de 2,5 à 30 centinietres. Ohes baucles d'orélles 
160

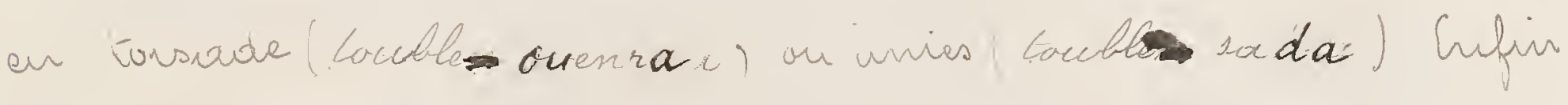
des bagues ( noungon binga siruples ou onrés de sujet's divers refuresentaut un grand nombere de variètés.

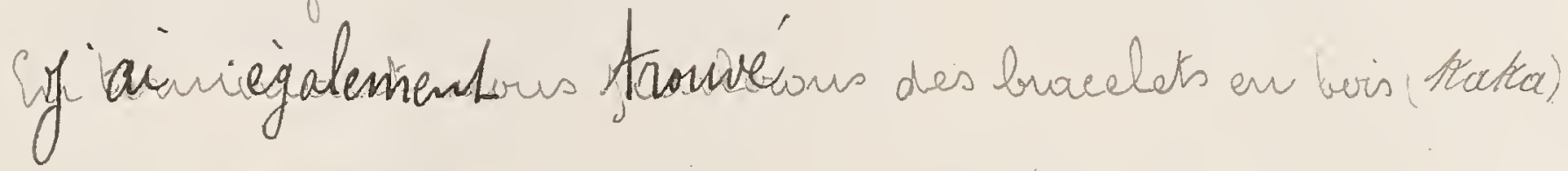

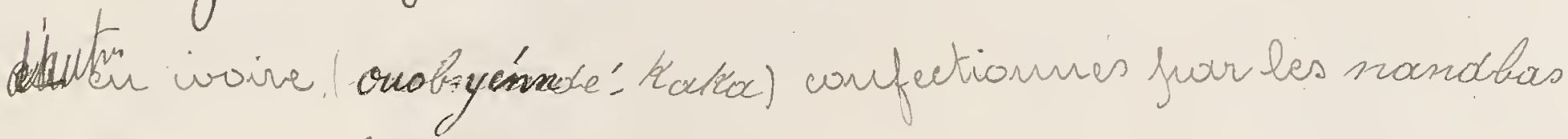
four être frortés aur dessus du conde.

III. Vie affective

A. Sensibilité marabe. Sentiment affectueux

Caractère. Maralité - Les Massi. nes deux leses sont en acrieral exycansip et assez versatiles. If est

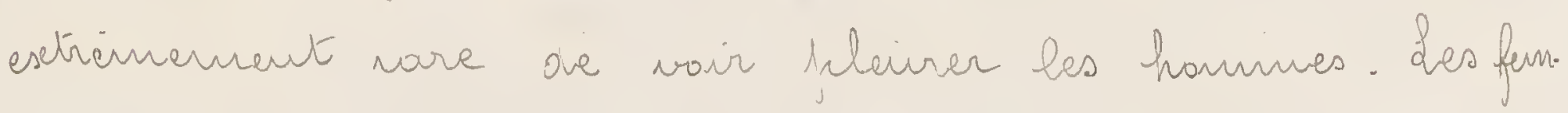
mes ont our contraire les larmes faciles: la minivare controvieté frersomelle suffit soment à les prowo. quer. Clles sont tautefois ris soumises ot accpatent sars se foloindre les durs cravaus que a'me focon gárerale la contime a deirblu à laur sexe.

Le caractere Gouraunsi est grave et enfurerint de

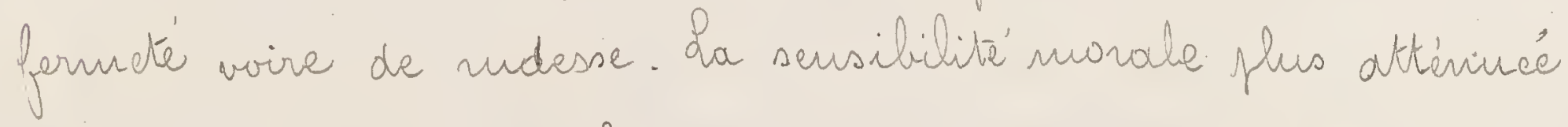
que chez les Mssi. Les qualités de bravoure trés appréciées. egalement de ces demiers sont plus developpées chezenx.

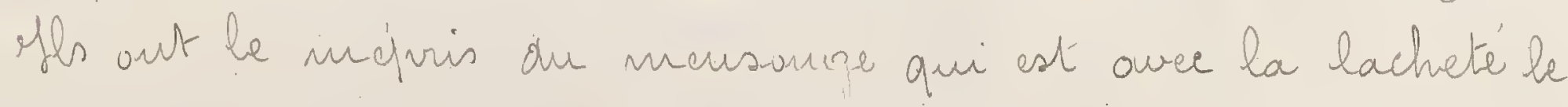
défout qu'is haissent le phis. 
Masti.et Gaurounsi. sout géréralement de berme foi t ves contestations an suget d'engagements foris sout irès vares sauf lonsqu'il s'agit de liquidation de dot aprés divonce; sours ce aemion cas be sentinum, t'hosti. litè qui arime joresque taufaurs les éfionse l'in contre l'autre, bes farte à enufloyer taus bes noyers pour se nuire.

Les Peulhs sont phes conceutres et la diosinulation ost nue de laurs carracteristiguves. Ils mentent avec la phes grounde facilité lorsagu'ils of ant un avantage in.

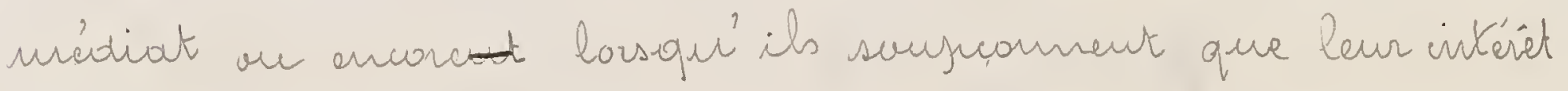
nẽue lointair est en jen. Leurs femmes suirt légères et infpré. vayantes. Clles surfpartent avec infratience les alseruations de leurs moris, s'iritant lorson'elles sout infustifiées, en cas contraire, pretestent en versant d'abondantes lar. mes et out l'air de se croire juersécutées; elles boudent aur moinare prietesete. Clles ne sant habituellencent fixées an donicile confugal qu' aperè la moisoarree d'un enfant et encove y a.t.il des execpations.

L'anitié, en Massi yádó, st un sentiment trés né.

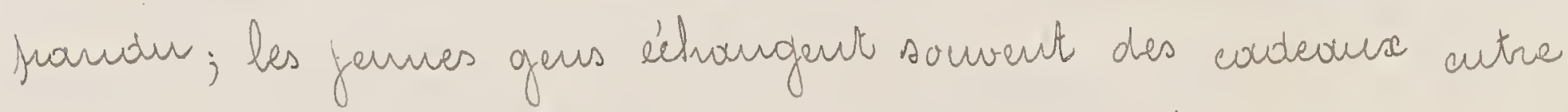

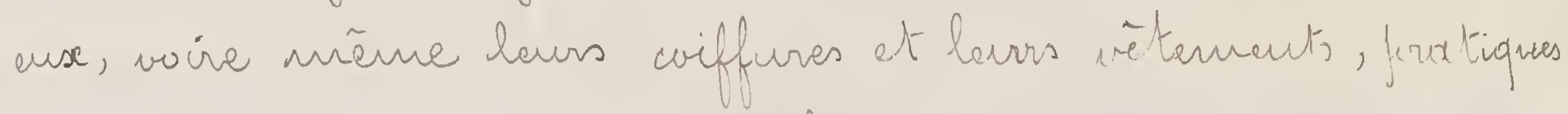
qui caractèrisent l'irtinite'. Ils me want cefrendant for fusqui à faire de grands sacrifices les mis puur les autres et ces liaisons dureut peu en géréral.

Le sentimat de l'amaur, nong-lóm, ast frarfois triés 
vik. In le nomme dasse' torsap' il s'acconyraugne de rela. tions sexuclles. Chez les cemes il rever savent un caractione platonique surturt à l'égard des fanmes aifficilement accessibles comme celbs des makas, qui sout falmse. inent gardées. Saus ce cas lonque l'riffidielité atait ve. comne les deuse smoureuse ristavient leur vie. Ses eseécu. tions de ce genre étaient avout notre arrivée dours le wapp extrênement fréquentes, d'autaut pilus que les moindres irdices suffisaieut à iveiller la suseegrtibilité du mari. Mfes raconteraps iesi un fait récent qui est caractéristi. que à divers furits de vere et éclaire curieusemeut les moeurs locales antour du suset qui nous occufre.

de Malar de dalero avait appris qu'une de ses fermmes, fort tolie. était courtisée frour trois jeurres gens. \&a fermme dut périr onssitiot poor le froison et les amoureuse arrẽ. tés fureut reconduits à laurs fomilles par des gens du Maba jorteurs du message suivont "Si cenx. gui out oublie' le respect qu'ils me devaieut ew nchereinask une des mes fenmes re se domment fias la anodt, fe m'en preridrai à laus parents, Oterxe des femes gens se tirirent aussitiot ave des fliches enquaisomées. Ae troisière qui habitait un wil. lage voisin me dut la vie quíà l'irtervention du foro. fore fiels du Malma de salero chef de cette localité. Celui.ei après avoir pressé le malheurause de dire la virité et s'étre nevitu confite qu'il we frouvout suroir commis vien de grave, déclara que la senterce de sour pére niétait 
fols basee sur dés prenves auffisaintes at défenolit de lesè. cuter. C'est à la suite de cette corrongeuse intervention que les faits ont eté comus et sanctionnis. Salero Maba est' encove en frisons à Onagadangar.

Les suicides volantaires sant aussi brés frë̈üents soit comme dans le cas pirécédent juar jiquires de flechesem. joisonnces, soit jair strarqulations. Les nutios les phis coun.

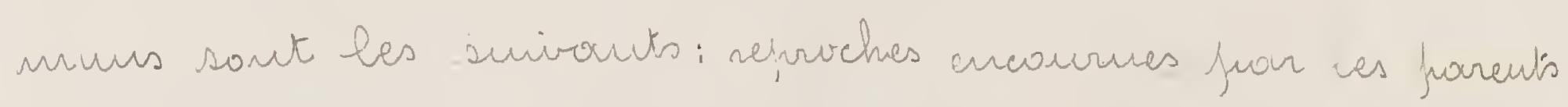
à la suite a'mue action malhonmer" le fait a'itre frivé fear l'arbitraire d'un chef d'une terrme aimes, la nerte d'inn enfant. Daus ce dernier cas un a wu le jière se domver la moat en mène terups que la mire.

Le suricide n'est yourais bläné; it est aur controure considèré comme alsalmment légitime en taut que cons. séqiesace d'une action qui aurait attiré sur son outsur la véprobations inublique. In paralle circansotance éest surs vent une sonte de conseil de famille qui conclut à sor meses. sité. Nul me saurait se soustraire à la décision prise mi de. mander un délai. Coutefois lasopi'un hamme a dé de'cidé à se domer la mort an s’y est vesolu spoutoninacut, il arrive que des amis perueut l'erriféetrer d'exéeuter La détermination. Shars ce but is freiviement le forgerom $\therefore$ saja de la localité qui se acna la mit pris de la juste du dèsesfièré et if fette san martean avec frowas an pramansant des paroles sacrameutelles. Ois ce moment le suiciole ve pent se provduire car son auteur se verrait 
priver de la séfueture et les vites furrèbres ne pourravent etre accomplis an sou hommeur. Son äme sirifa seraiten conséquence condannee à errer misirablemeut autour des vivarts quil a comu et chéris et à les nulester sours trieve. Thi Massi reculera Caujours devant cette persiseeticis, redoutable. D'autre part ceux qui firandraieut l'initia. tive des córinanies dout sa désobéissance aux infanctions du sáija a juour effet de priver le suicidé el qui sentes assureraiaut la tranquillité de ce dorrier dours l'autre mande, s'exijuseraicut selas la crafance fofurlaire à nourir subitament.

Il n'y a pas d'exemple de desespióre ayaut frassé outre à l'avertissement du salfa.

- Les frostituées professionnelles (-ijadike”) saut nom. bereuses. Clles se vecuteut en gériéral farmi les fermos mariées affant alomolome' le donnicile caryugal foun me axison quelconque. Clles vivert tayjours avec laur meive ou ine matrome de leurs jareuties, s'etablis. sent de frefference dous les grands centres commerciaux préquentís puar les étrangers. E'endroit an elles se tièn. nent d'ondinaire sur les marche's, endroit qui finite frar

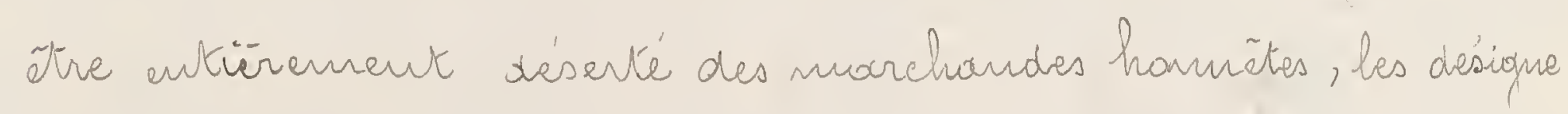
ause clieutis. Clles se fout en outre remaropuer fuar le soin spécial qu'dles apponteut à leur coiffure ct à leur vête. ment airsi que juar l'aisance avec laquelle elles afuastruf hisent 
les fossouts mérue incomus et qui cantraste alsolument

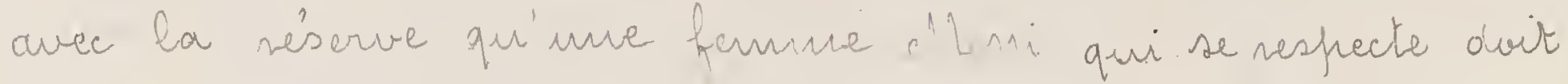
Kunfaurs gorder vis.ä-vis des houmes.

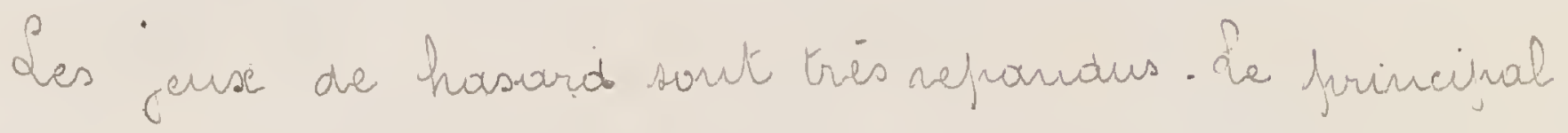
d'entre ense, le tétérika consiste darrs le jet de quatre su sise cauris qui am... le qair lorsque ces coquillages vetombent en nombre fair sur la frartie bomber ot néei. proguement. On a me greopuenment des gens qui arovient risqué tart leur avoir, yuer teur femme et l'afout per. duesse jurer firalement eux.urines. lettie mise acéses. Trérée Jomout si le sant leur itoit contraire les

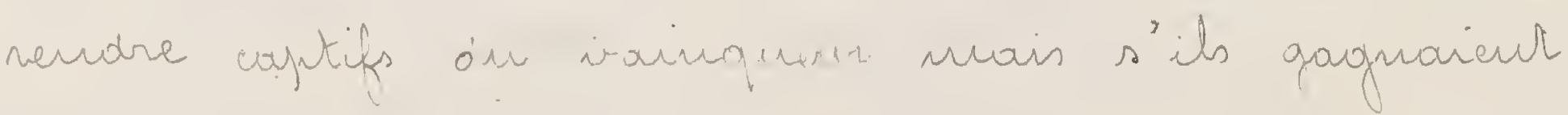
its neutroieut en possessions de taut ce qir' is avorient perdir; aussi arrivait.il sanvent que l'adversaire Peureux n'aceprait fas le coupr.

L'action decionalisatrice our jen et les growes in.

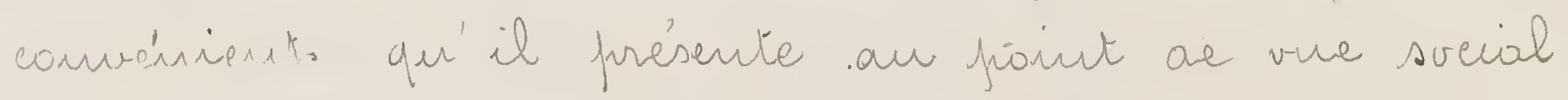
ont anené defuris longtenys eartains chefs, tels canx de Bèbussa, à l'interdire nigaurensernout dars turte l'èteridue de leur jovince.

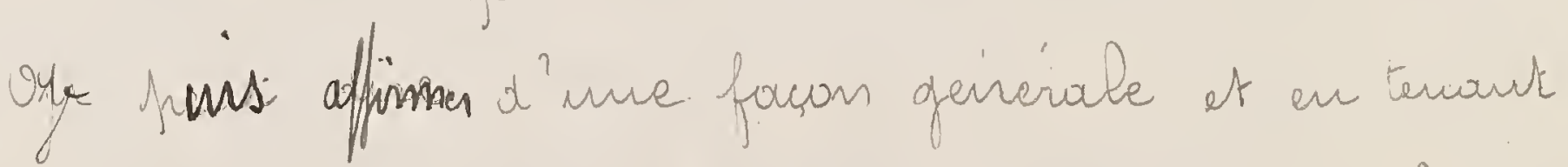
compete de la dersité de la jugnulation que la dilin. quance st assez nare. le n'en est f was de mõure de la criminalité; les mentres sont selativencut fréqueuts et commis presque turfaurs surs le coup de la coline et de l'ivesse 
protuite" fuar le dame. L'alus de cette boisson constitue évi. demmant l'un des alstacles ques les itiées civilisatrices sout appuelées à nencoutrer dours de juays.

La conviction profondémart enrocinée cher le preuple ape

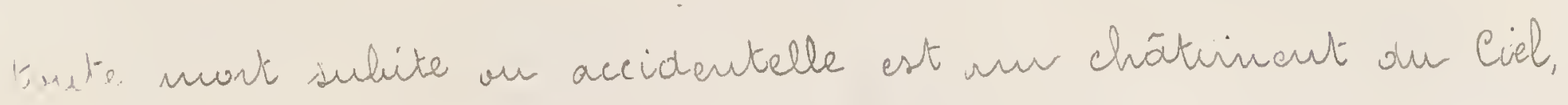
connue aussi saus doute les lixis-lations draconiennes ausopuelles il a dé soumis, sout frourvenues peur à freur à le doter d'une Wracience monale qui hii est en marite circonstance un frein salutaire. Noir à ce suget la pratique si csrisuse de.

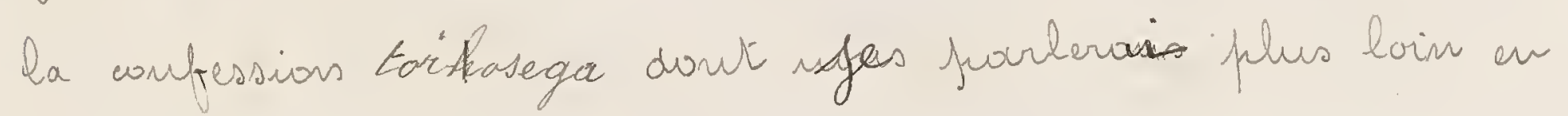
trantant du sentinent veligiense.

\section{Salitesse _ Les régles de la fulitesse sout strie.} tement dablis an cllassi et nul me s'en jeut écouter sors peime a'encourir la réfrobation fubliopue. Baus la cauversa. tion, l'usage de la deusirime persome du pluriel. lors. qu'on ne s'adresse fuas à un égal est de nigueur. Au licur de "fo, toi, on dirxx. "I" constractions de "'jamba " vous. C'est arisi qui on fuarle à un naba, à un étranger, à un un vieillard; une fermue se sert de la mière expression à l'égourd de son mouri; les enfourts disent"fon à leurfière

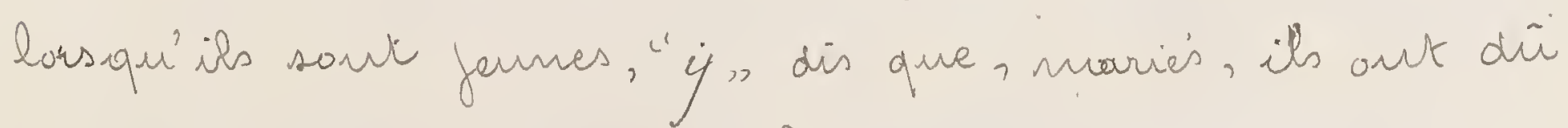
quitier le domicile patemel.

$$
\text { quand deuse persomnes qui se comaissent seven. }
$$
coutrent, la plus femre dit sous s'arrieter 


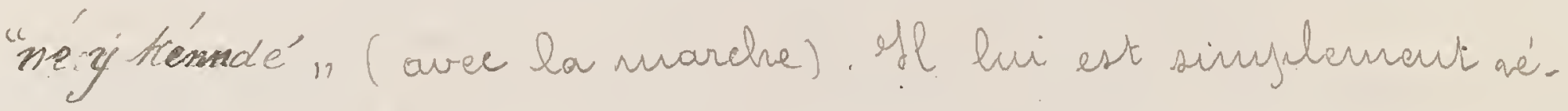

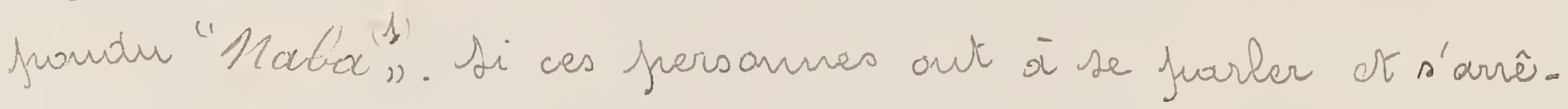
teur, elles préludent à la conversation fiar le dialoojue suivaut qui nappelle les litaries habituclles des Mandis. 12. Mé. ga:" I de vepus avee vour) R. Ga be'láfi:" ( Le nefors a.t. il été en jaixe). R. Ráflibe' (il fut en hais). D.

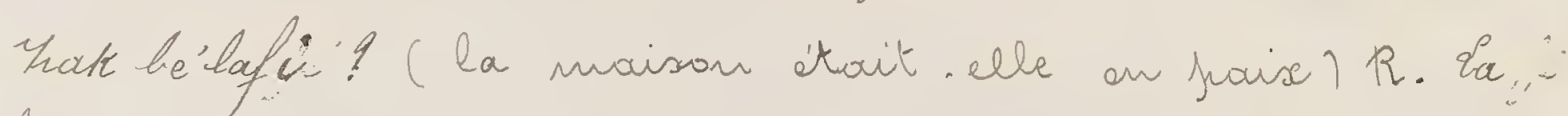

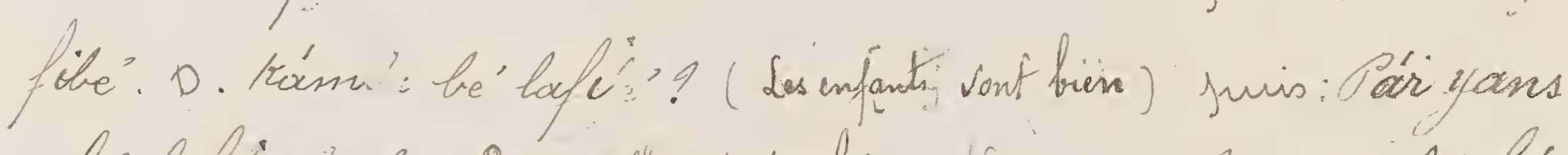

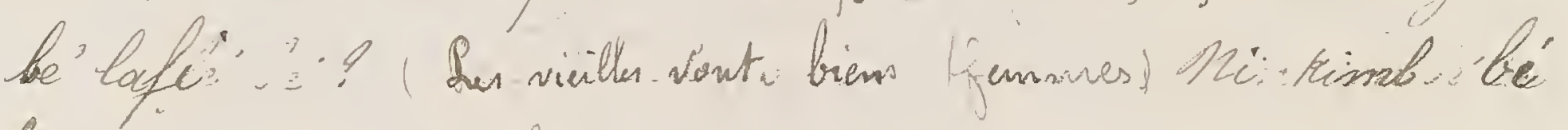

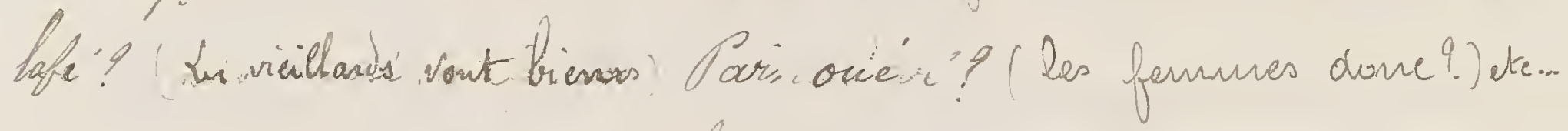
La veponse est taufours Laf '" be'.

Si ces salutation ont lien à midi an licu de Niéga.

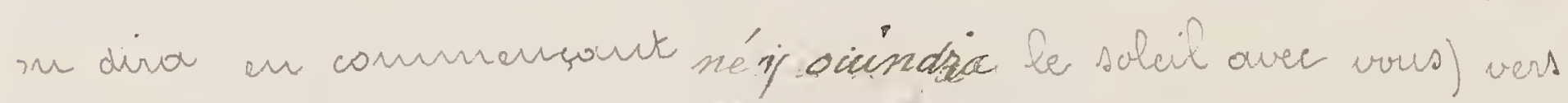

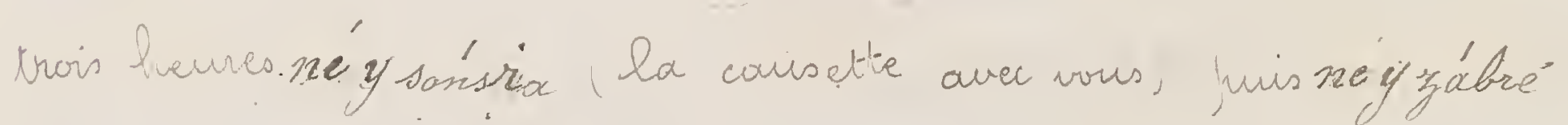
(le soir aver vous). Sifuir aprè le caucher du soleilnezzyónágo la mit avee vous fot on se quitte en disant Ociére: na Kón

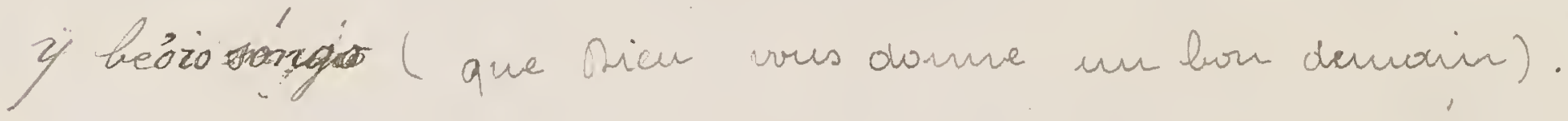
Quount on se verid en visite on s'arrête à la poite

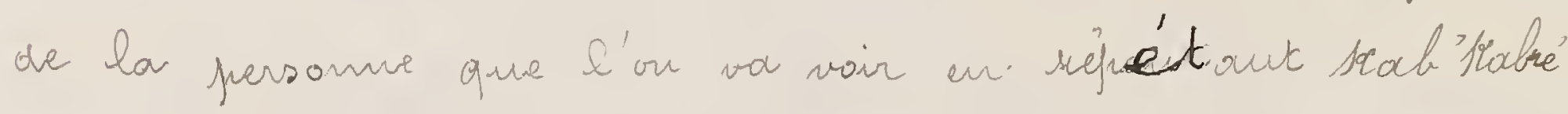
(pardon fariton) fusqu' à ce qu'il soit répanau kab. da Resr que celui qui demande frondoni entre.

(1) Sei Mabor ast intraduisible. Cette cepression est l'égui. valeut de "mésont, dours la biuche d'une frersonne que l'on vient d'appicler. 


$\times 175$

suik

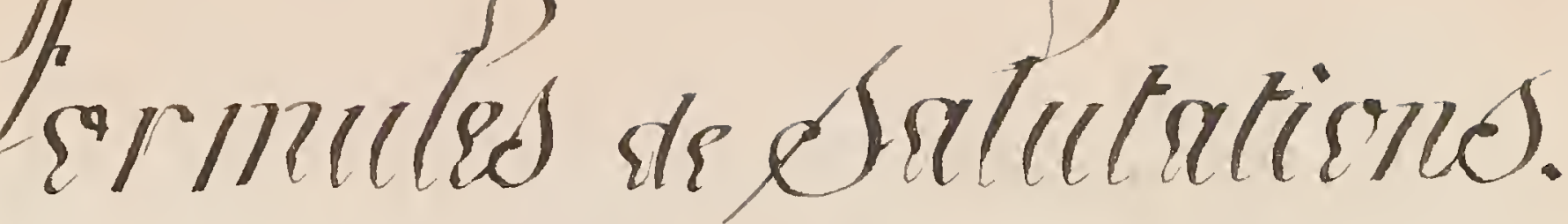

$x$

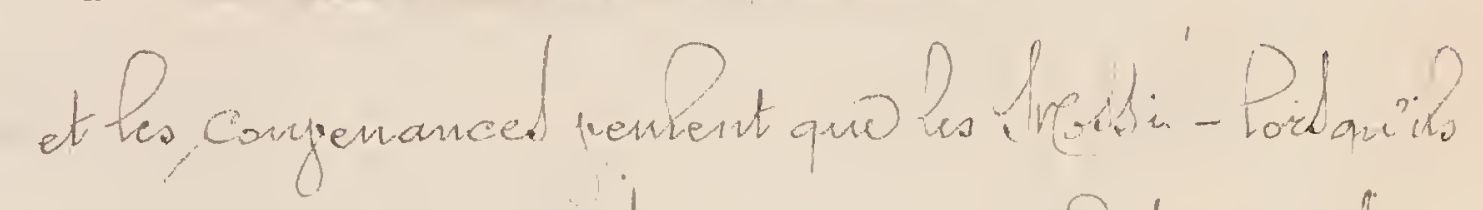

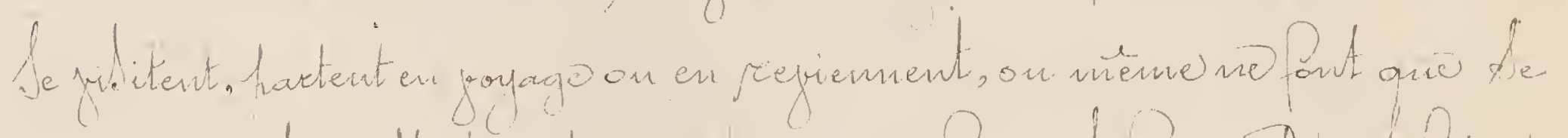

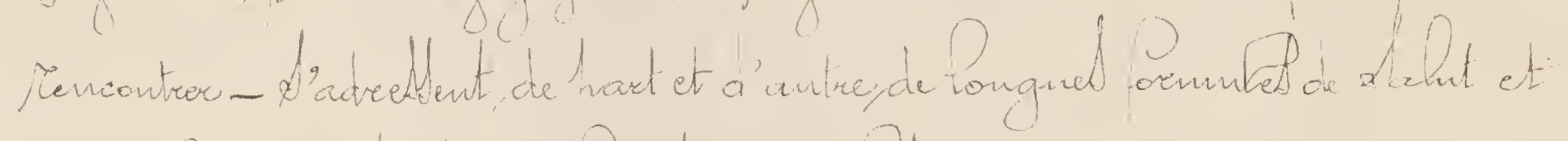

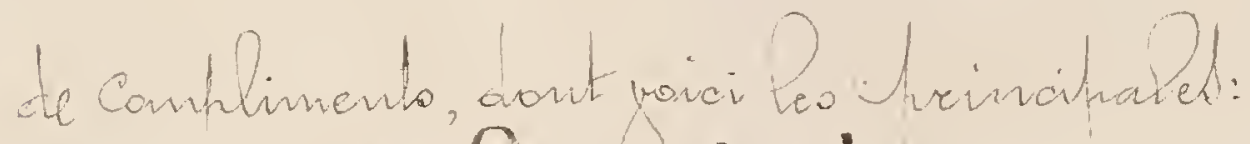

A. Sn visite.

Celivain rend sidite

Celin qui réegait

Im Se mation.

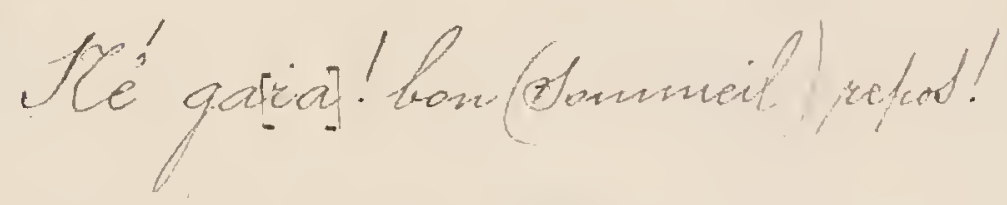

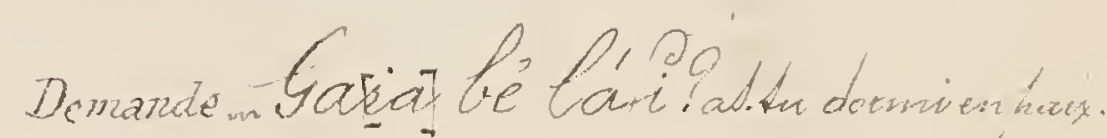

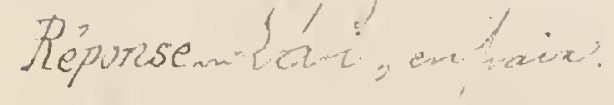

R- faili.

D-yakratie lan?

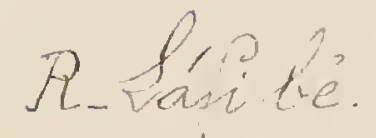

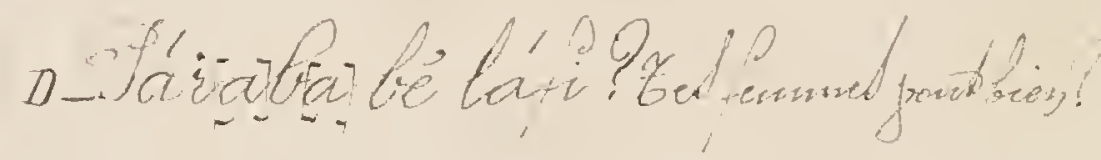

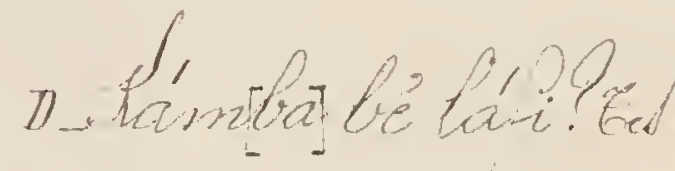

R-Siatib.

R. Lafifiè

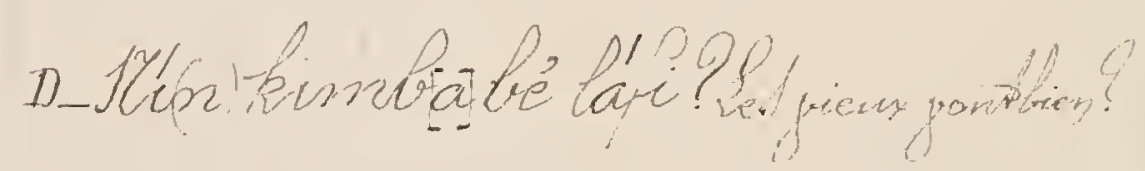

A. Laible

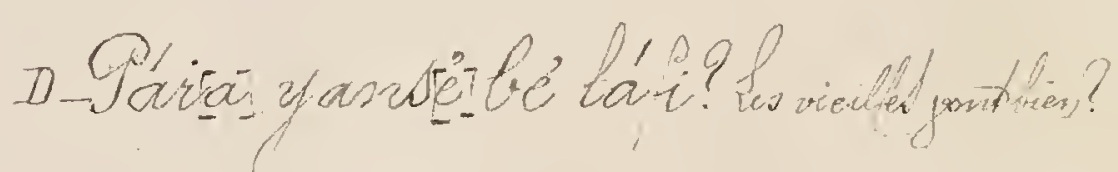

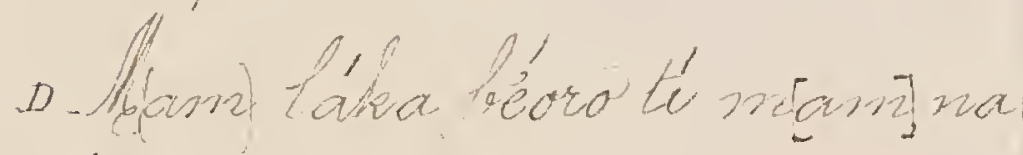

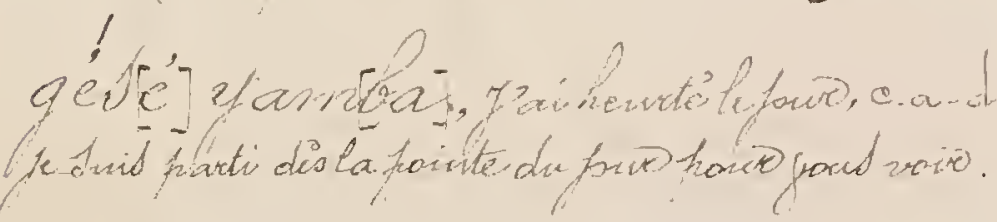

Yốba!

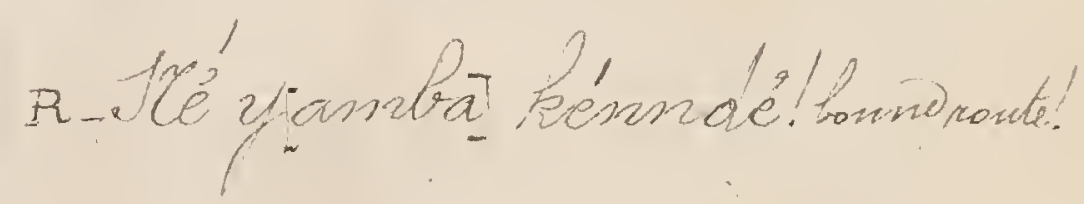


126

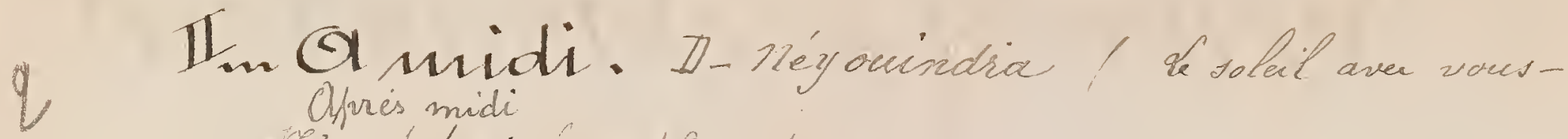

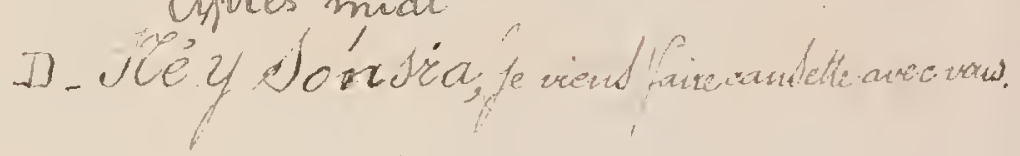

R. Ketaba!

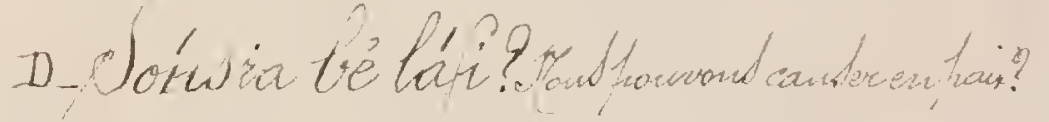

R. Barbe

Difondêt lamété á na qédéy.

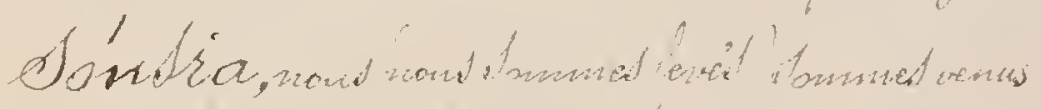

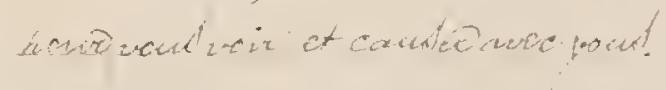

R. Fúla!

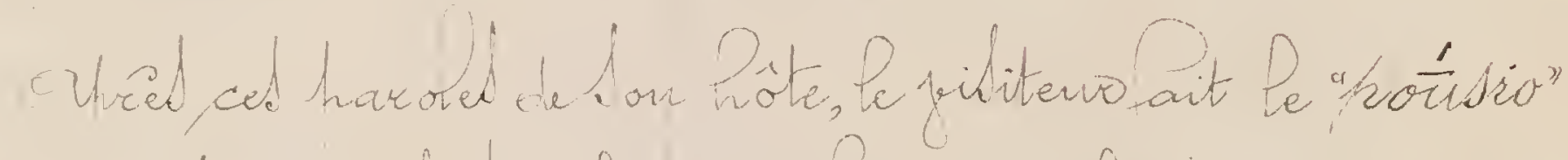

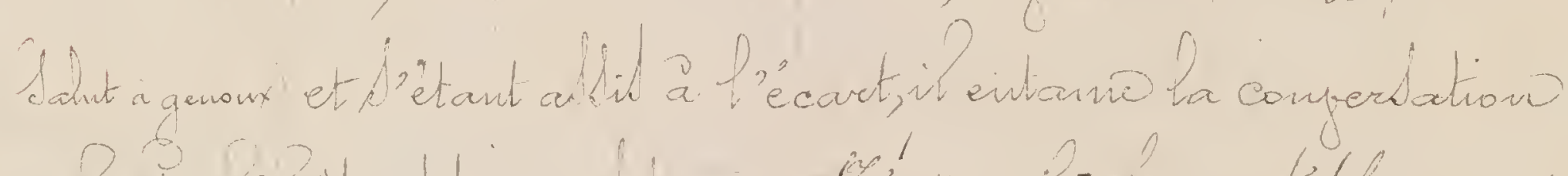

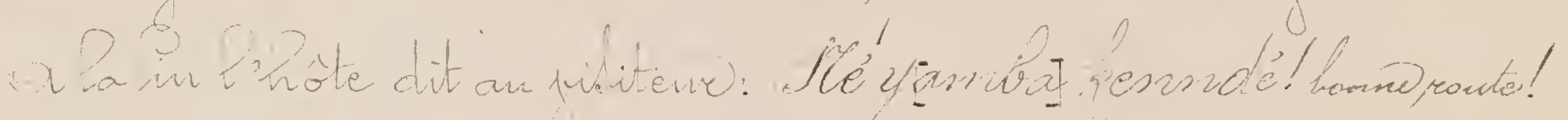

IIr. Se poir.

té, yambaryábré! lomodoriee!

R. Thál

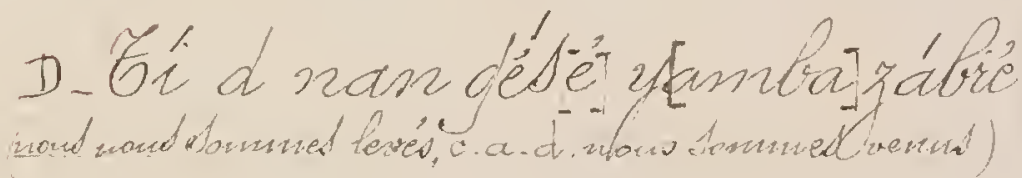

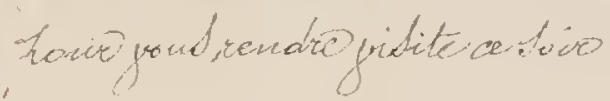

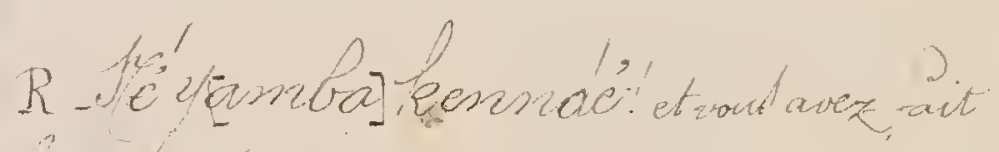

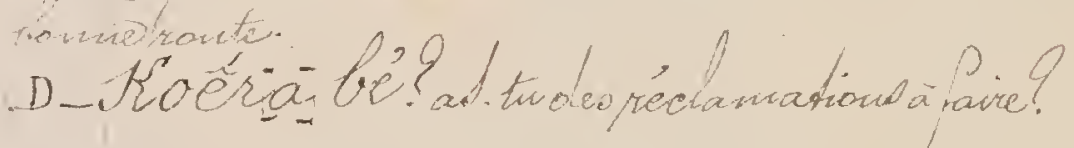

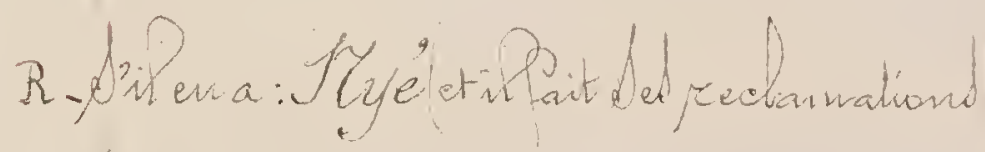

Linow: Ai et in be retire

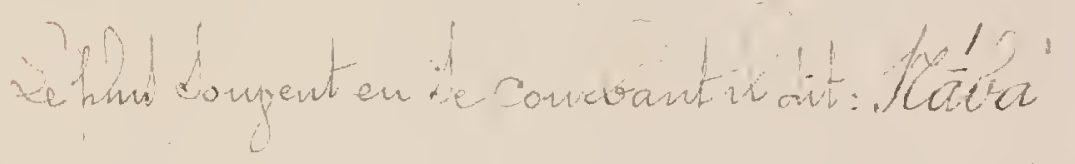

IV Samite.

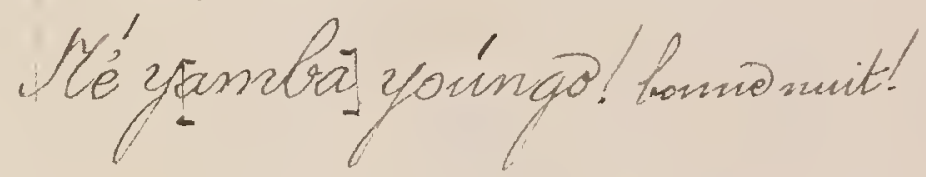


biand

3

R. Sain be

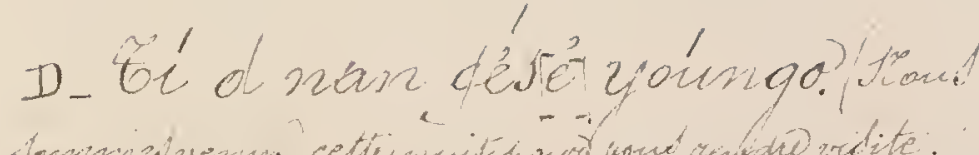

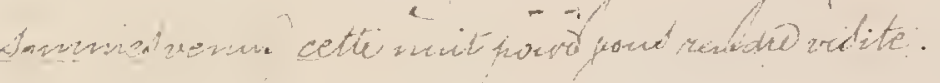

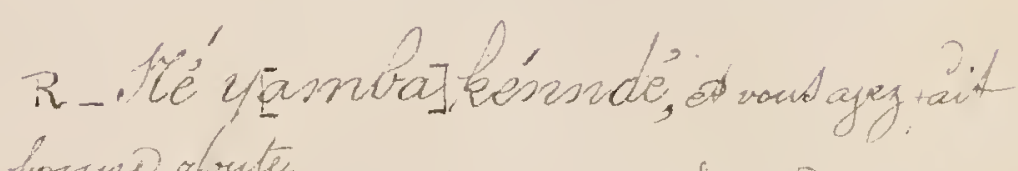
An nioment an déchaté de Lon frote, if fuidat:

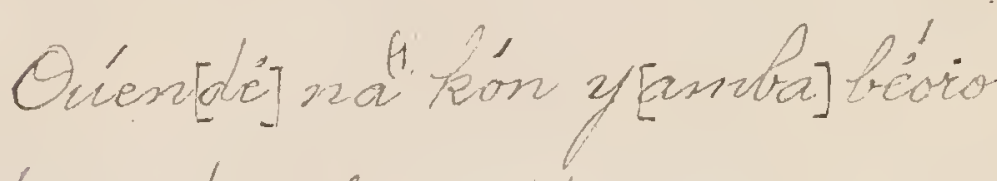

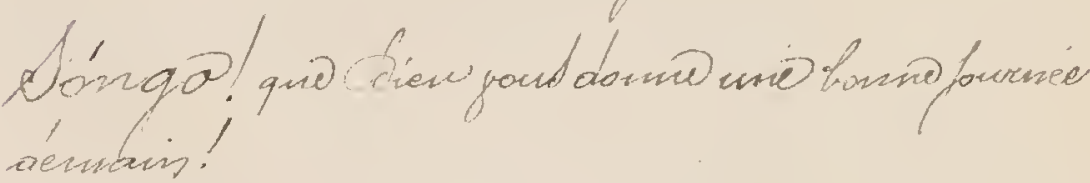

B. Oouhrite de voryage.

Im Au defract.

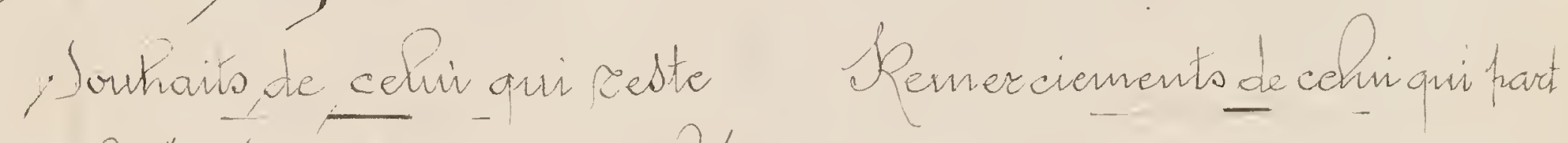
Orénidé nan ouatar né-o!

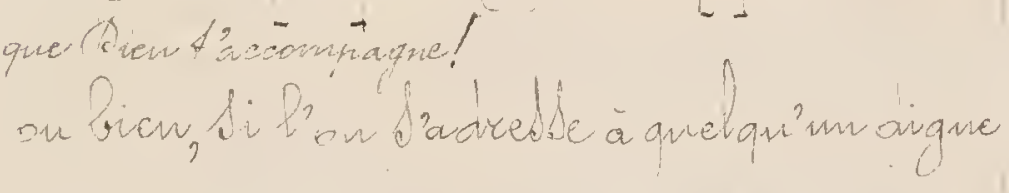

d'un trawa pethect, su ac creana àge, on à

im êtranger

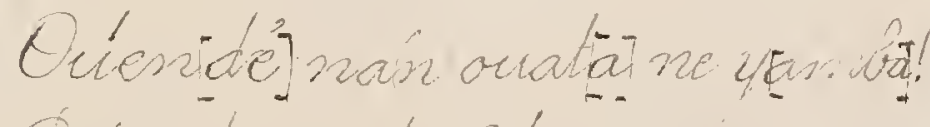

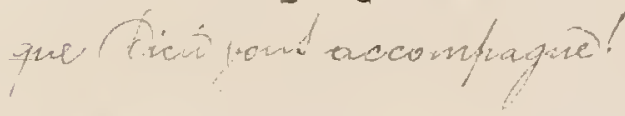

R. Thấa

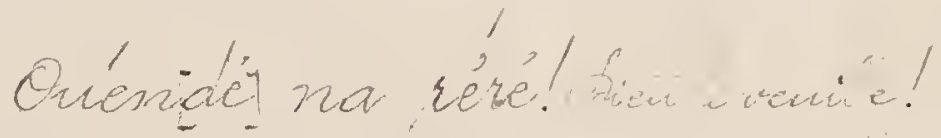

tomina! Pu:

Im a Parcivée.

Cehingir arrige Yếla!

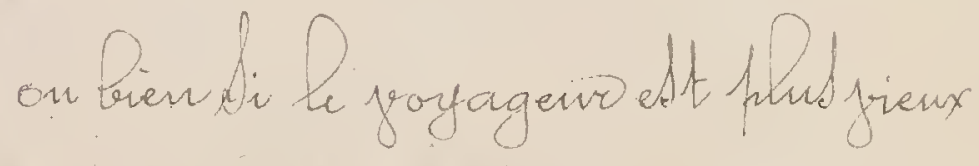

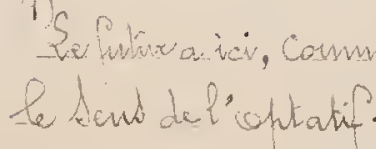




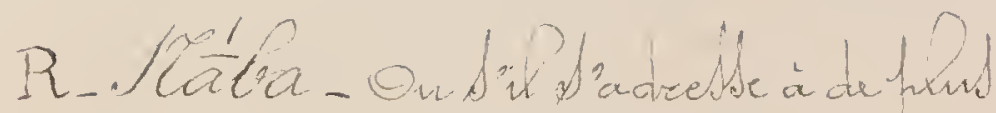

rieux que hin, i dit:

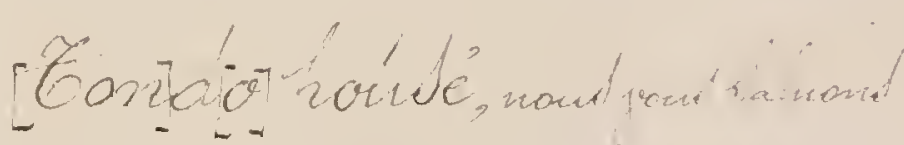

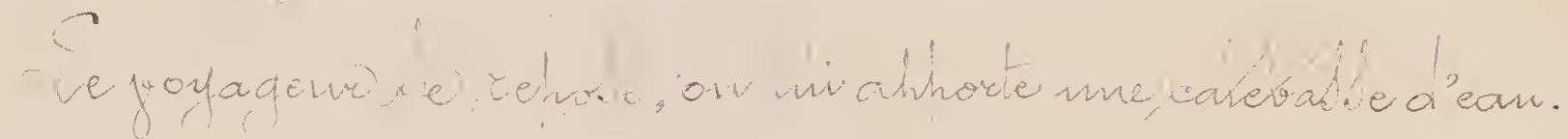
té qaisiat! tom retuot!

\section{R. fitra}

D.Ýsanbaidébr) táépr dásuba?

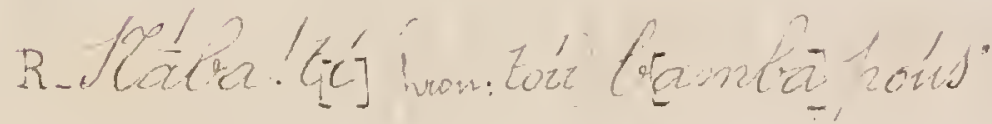

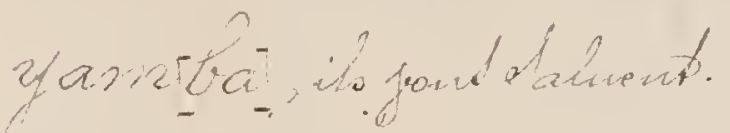

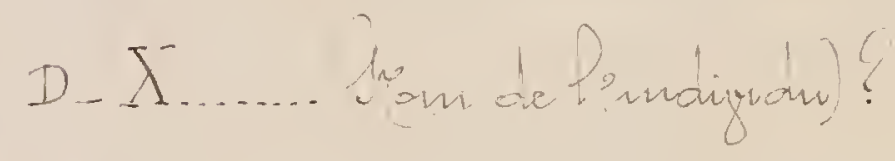

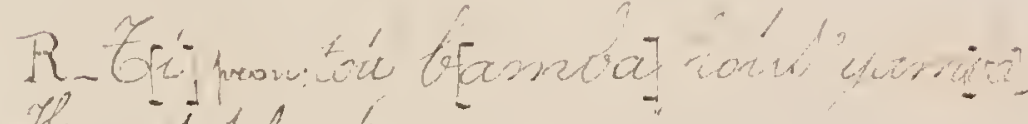
Ho wais salwent.

$$
\begin{aligned}
& \text { Inid iro haldent en regue tonted teo } \\
& \text { herbonneb qui ont regule yoyageno. }
\end{aligned}
$$

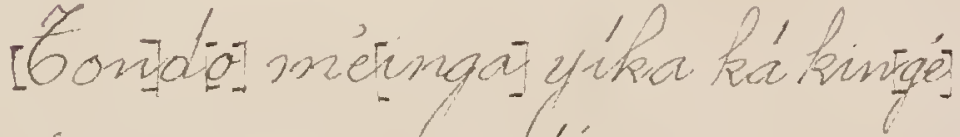
Ra inum du gilage n'aédé tamtas hám. - ba n'léfienn - yére tej itoniáas

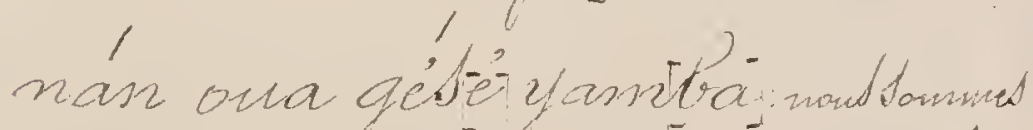

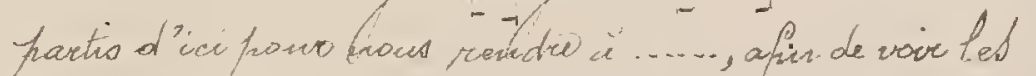

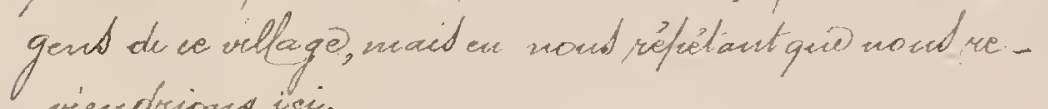

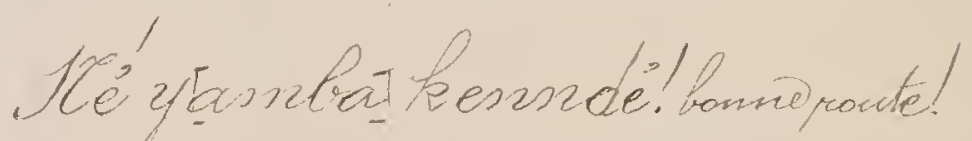

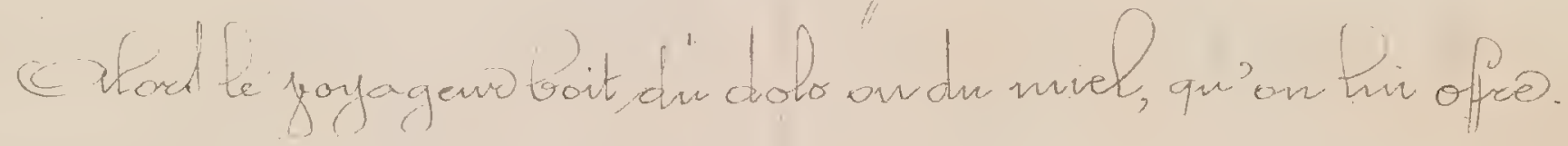

\section{Cur soute.}

Celin ain vervant de la brouble va verd le yiftage 


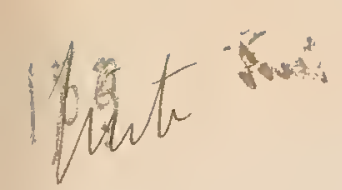

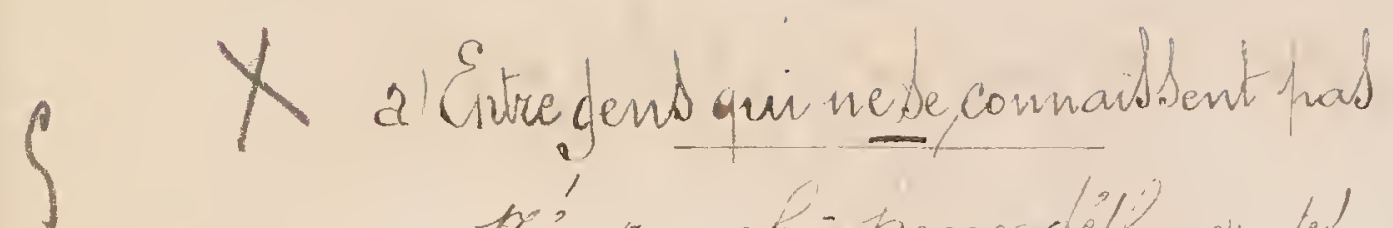

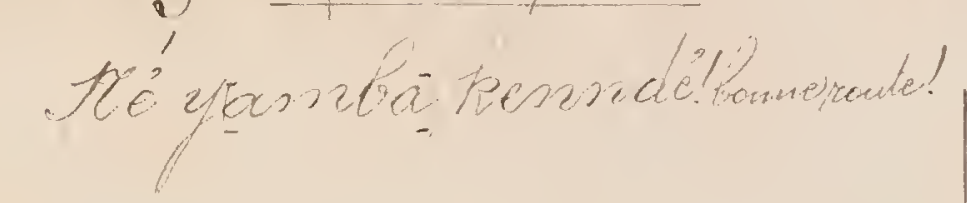

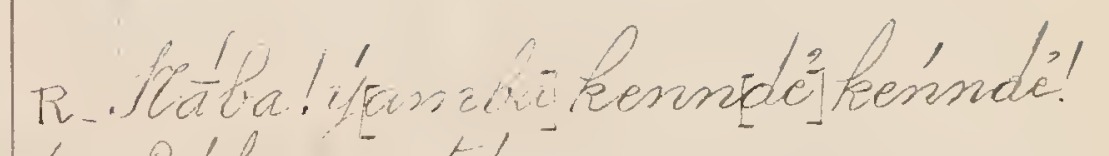

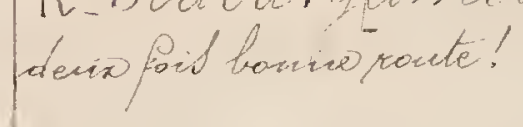

b) intre connaiblanced m.

R. Letaba

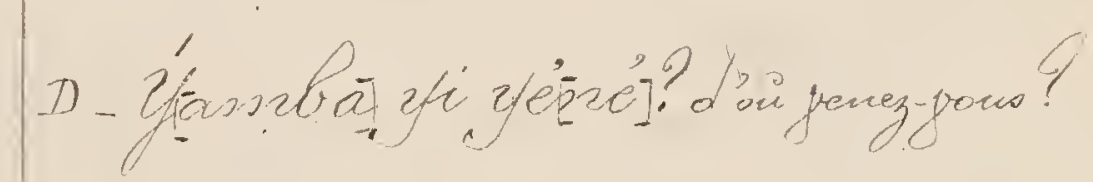

R.X... (heandn in illage)

Lehir la hiu demande alord des nougel.

- Reb de toutel les hertomed qui it a gued.

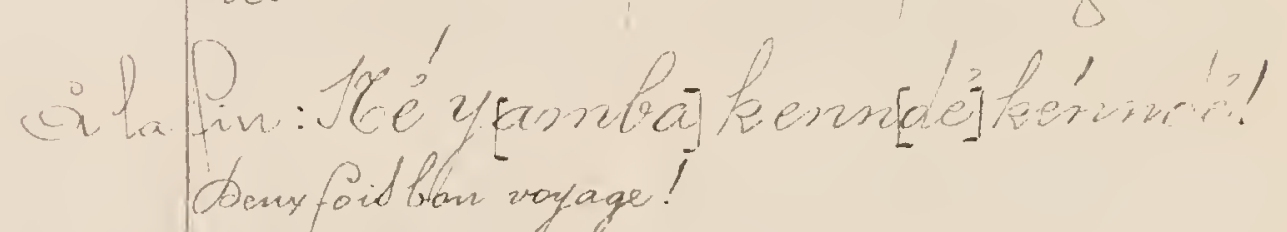

R. Kratra! domy foid bran voryage!

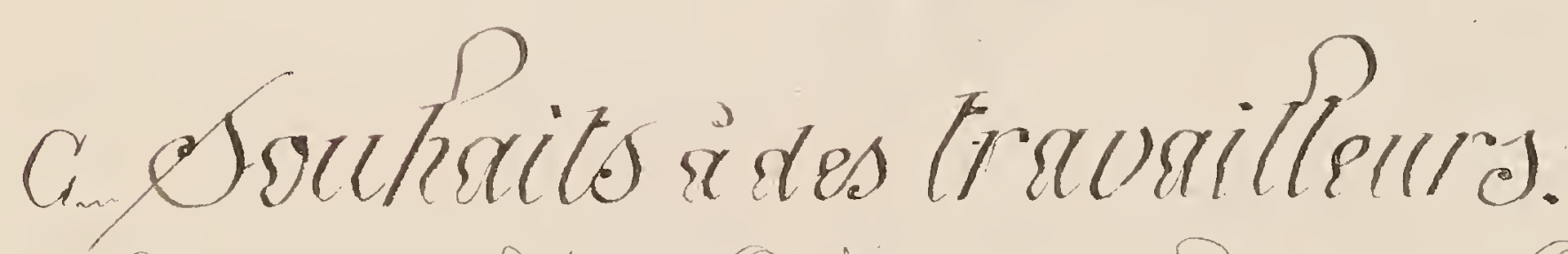

Célim qui pa yoir les beagarlened

Ses traysailfond

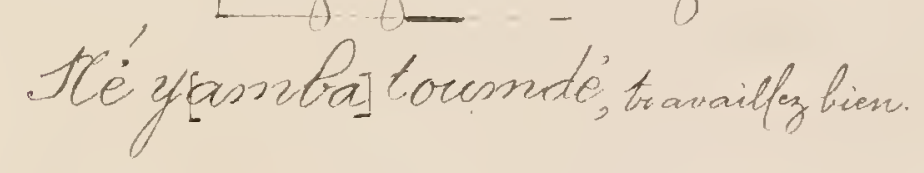

R. Jéba

Colim qui ne fait que fabser

Boum toúmdé! bon hrovail!

R. Jestra

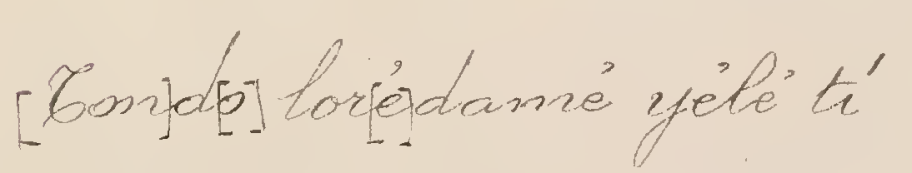

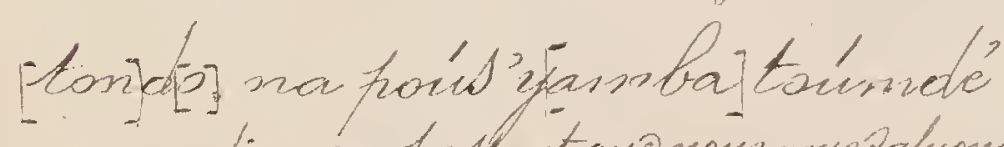

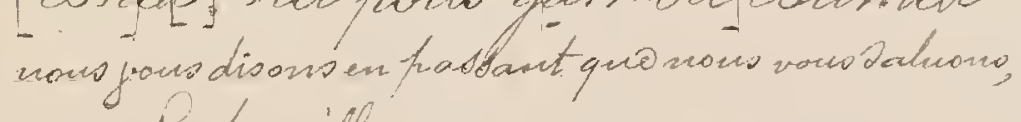
mecus, ter havoillenso.

R_. Hézfanabaténndé! bou gayage! 
的

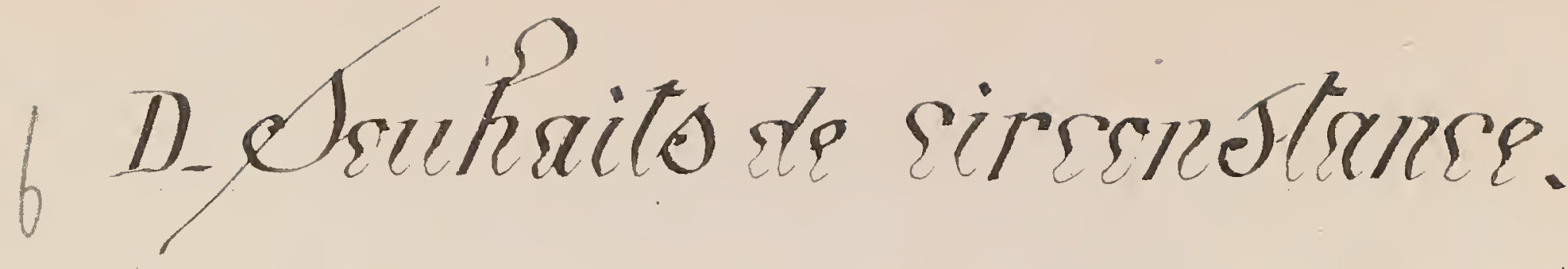

a) Se bornc année Oúenidéin nan ovilg ýanbas.

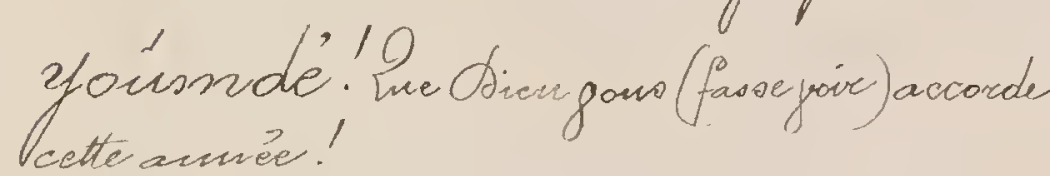

R. Seaboa

b) Sendant une matadie.

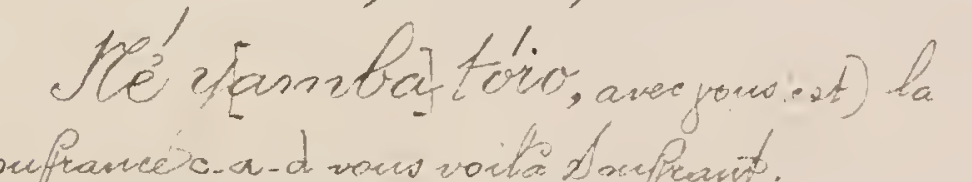

souf haver

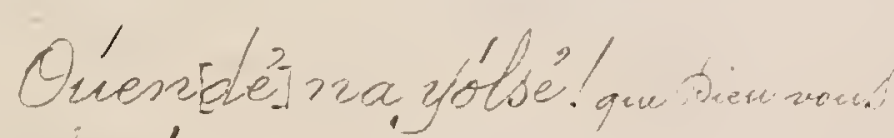

On bien

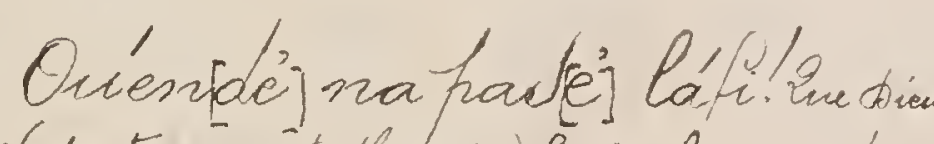

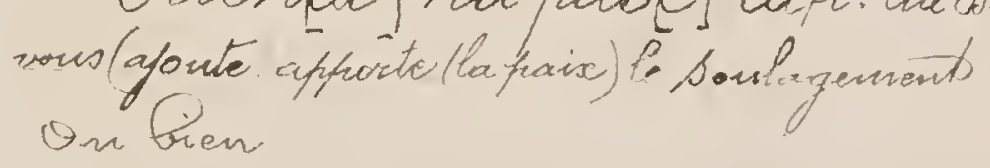

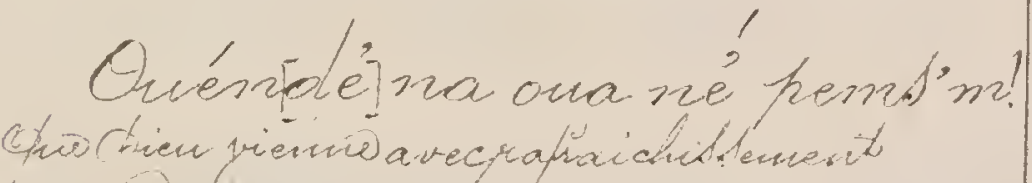

Ons neon

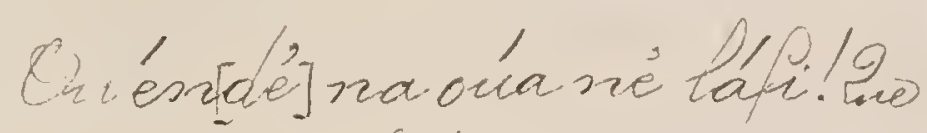

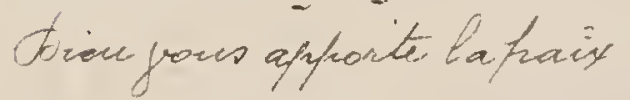

R. Kâba

c) à la mairsance d'un enfant.

Hé zourgout nório! hamo chance!

d) a la moot d'un enfants.

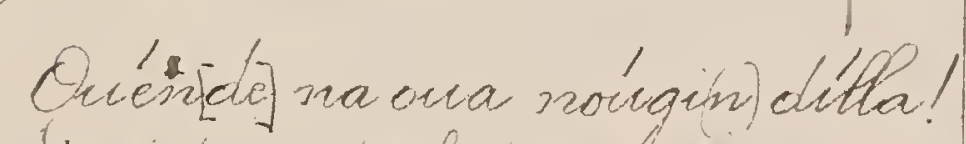

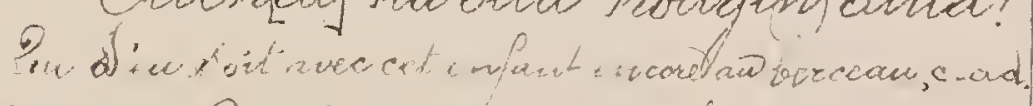

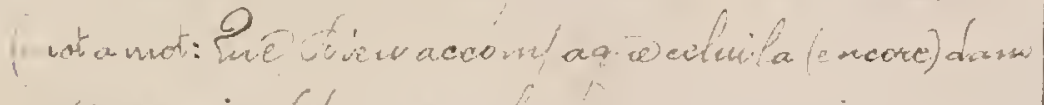

c) á la mort de quelquim

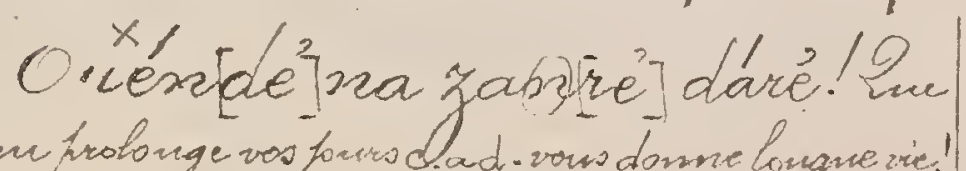

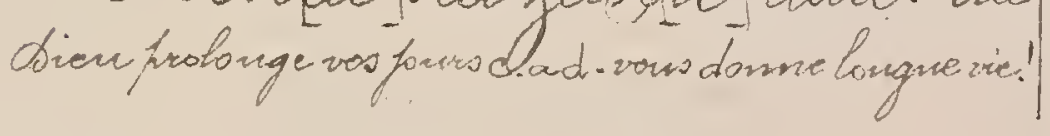




Se ivisiteur enleive aussitiot ses souliers et ro coiffure

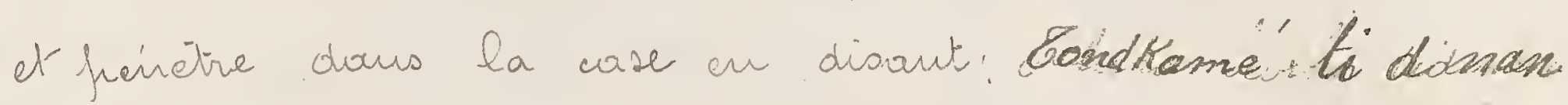
gese'y (mous avous affranté le juur funr vous voir) d'ex. pressión consacrée fuur une visite de devil est la siri.

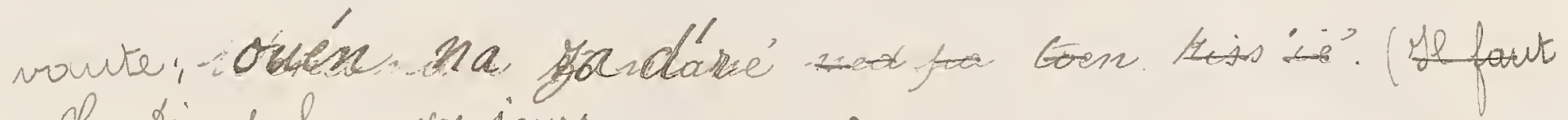

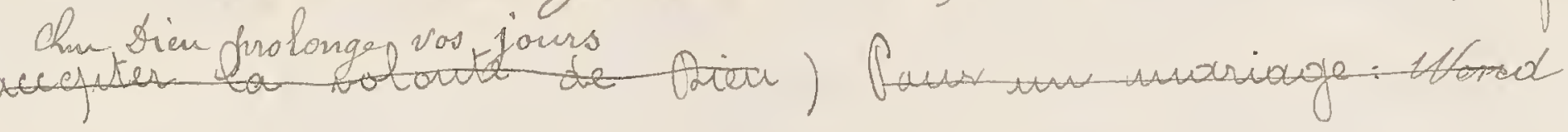

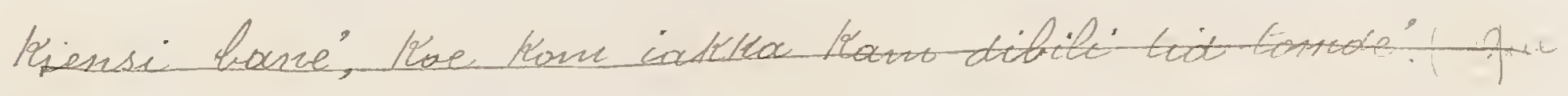

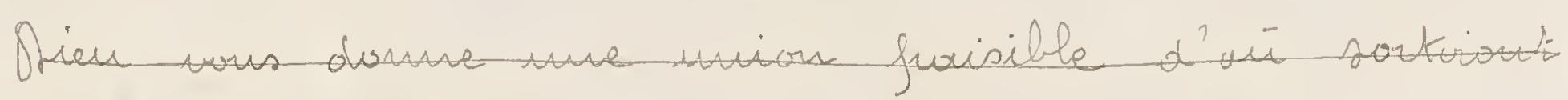

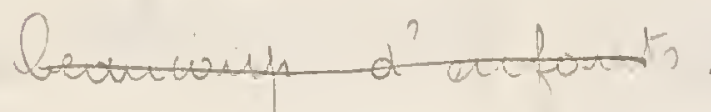

Lorsqui on vertire chez un naba apries le Toab.' Mabre' prononcé sur le senil de la porte, le visiteur taut en suivaut le sonthané qui l'introduit we cesse de claquer des dorigts en rejpétaul Ma............... Jusqu' à ce qu'il soit arrimé prés du Maba. Ie se prostarne alors $x$ salue en frappant trois fois la tarre du coude les fraings fermès.

L'hosjitalite' gairita est bres en honneur au cllosis a queloue frate qu'il frapple l'itranger est taufours sir d'être recur ane cordialité.

Les Gourounsi: out feur de fommules de fulitesse. Il n'existe chey ens aucur geste de solutation; tsme manifestent leur déférence que frar lour enyressement à venir. 'th sout assez hosfutaliers; eette vertur n'ast cefrendant fuas chey ans très ancienne. Seurs voisins serap. pelleut qu'autrefiris au Gourounsi la persome de l'höte n'avout nien de sacré et les ètrangers ne se risopuaient 
fus nolontions dours ce joufs.

If n'y a sur ce chafitive rien de frorticulior à dire au suget des Ceulhs car ils out conservé au Mussi les salutations traditionnellus (fofo, da idiams, a ova lidians) des qu' ow Reur. comait dours taut le Saudon. Il fassent jueur être movis hosititaliers que tes Mossi..

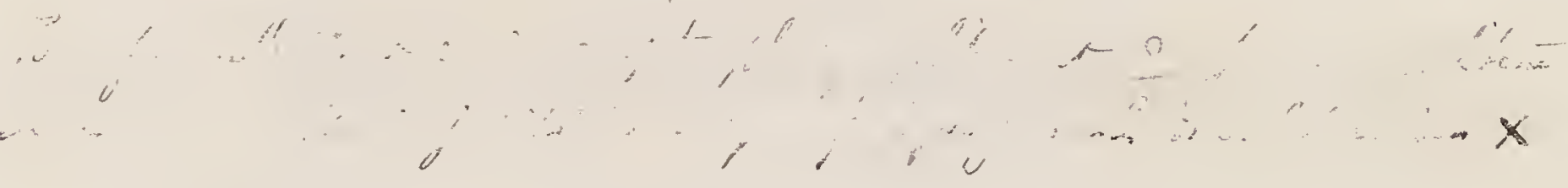

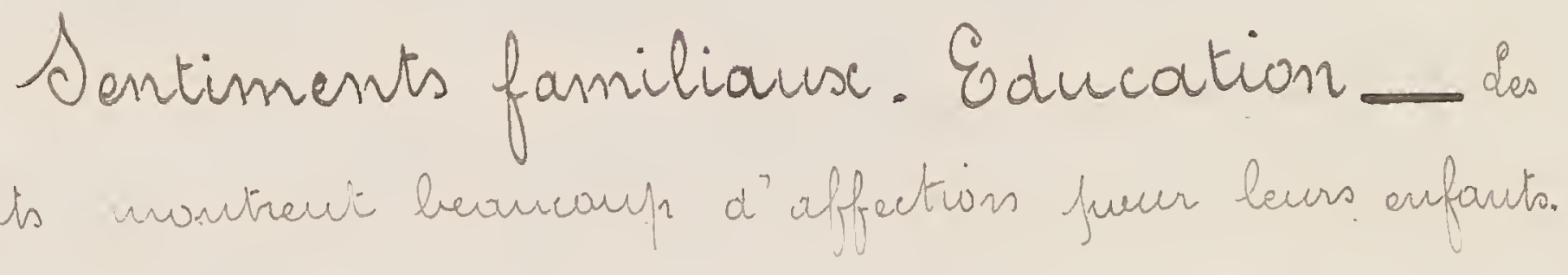
Ils les corressent et foumt ovee ense.

Dies que les garsous aut six an sept ans, en gèré. ral dès l'époque de la circoncision, ils surivent leurs jère arse chanuss ot acquièreut airsi fss quelques notions aqui. wles qu'its utiliserant dous la vie. If n'y ar fras d'éducation progremant dite. Certairs chefs confient ce.

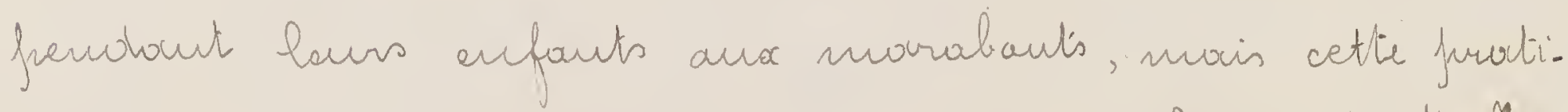
que récertie est demenvé esceptionielle. Ee seul chorho. Maba qui ait sin ac et écrire, Maba Routou, owait èté instruct par mu noûtre d'écale d'origine Mondé. Yarsé dabli à Sarifabartenga. In genéral l'unigue enseignement que acsonicut les fils de chefo furte siur bes trois vertus qui sont considérés comme l'afonage du commanowementsavair: la bravoure, zacido; la larfauté, sarnde; la générasitè, Hasta.

A l'äge an les garcous commencent à suiove leur jère 
ause champs les filles dorivent aussi jrendre leur jiart des traveuse véservés ause femmes.

Rien ve narque que l'ciducation ast terminée: il nify a fias nou filus de cénémonie d'initiatión ni fuour lés garcuss ni nour les filles.

Q'iffluence du jeire sur les enfourts oure autaut que la vie. La mère st soment l'alyet d'une affec. tion tres vive. Mien que la constitution niterdise ause Alupho. Mabas de s'alsenter de la lafitale nus avousm que Maba sighiri sollicitait tars les trois ans du adridi l'autonisation d'aller voir sa vicille mère vetirie au village de Kansunadivie?

Les enfauts sout temus de trourailder juenr leurs fuarcent's in. finues et leur domer euseneines les soirs que nécessite leur ćtat. lorsquils out atkeirt un ãge ourancé.

Les fermues sont tayjaurs maintencos dars ne situa.

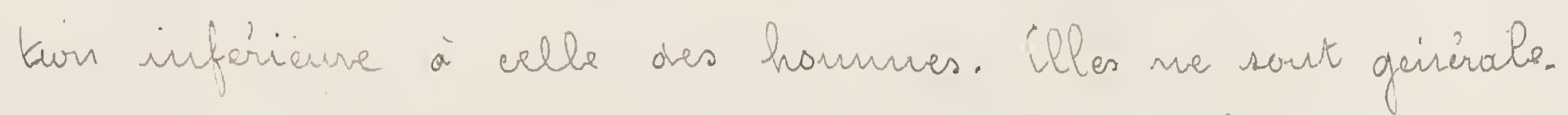
ment fas maltraités et si le fait arriue il ast suffisant

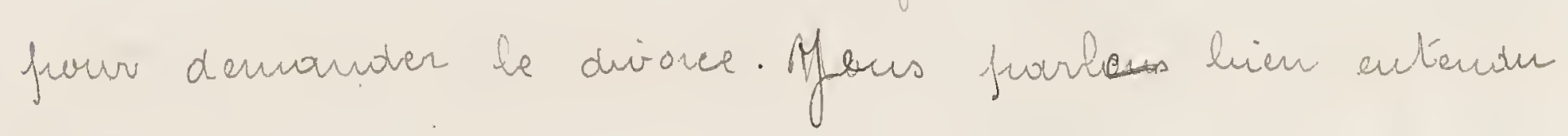
des seivices growes.

In principue les forrarts n'out jas le aroit de verwhe leurs enfouts, mais en foit le mariage des felles n'est qu'une verte déguisèe. Ohès leur flus femre agestis sant en effet joromises fuar le jère qui disfuse d'ellesen faveur de tel individus tenu de hi affrir for cela méne des coudearae périodiques; des quimr gendre phus géréreuse 
est entrear, la destinaturn de la fille pect ètre nuvaifiée à son avartouge. A la mozt du frère it arrime très somvent que le fils âné ue se cousiovere 'jas comme obligè

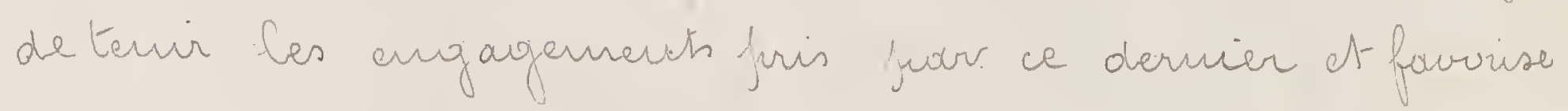
un autre preetendant. Nhre fermue jeut âtre culcuée à son moni par be fuère un le frère aìré aprèr quélle a en dos enfourts, mais daus ce cas cenx.y demenrent la prapriètá du mari.

Al arrive soment que des cheps de case out à laur service des jemes gers qui arfinient à la manir de leurs filles. On les nomme ctastasrrés, its sout tomus de trourdil. cer puur leur futur beau. jère et le mariage n'est sonvent obtenu frar eux qu aprés un stowe asseg long qui jeent duver fusqu à sefat ou huit arriées.

Les enfouts contrefoits sant turfaus mis à mart faor sulfocation, an we voit gamais t'infinmes de maissance. L'avontement (froce-yíri est pratique' cacuramment war les porustituées at les filles mieres vers le troisieine nois de la grussesse. Clles utilisent dous ce but une racine

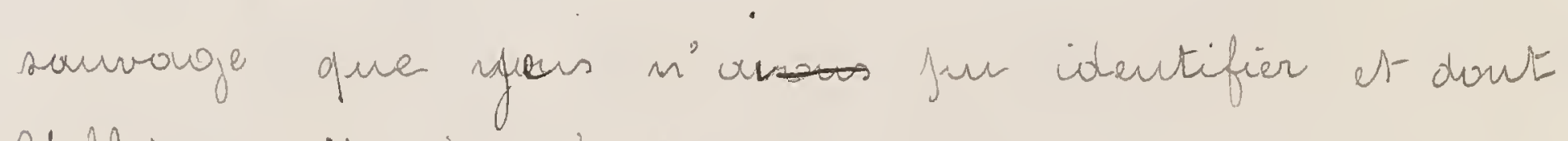
l'effet scrait immédiat.

Les nualades ne sout jamais abomdomies ot bien que la nont des veillards soit considérée comme ure délivance

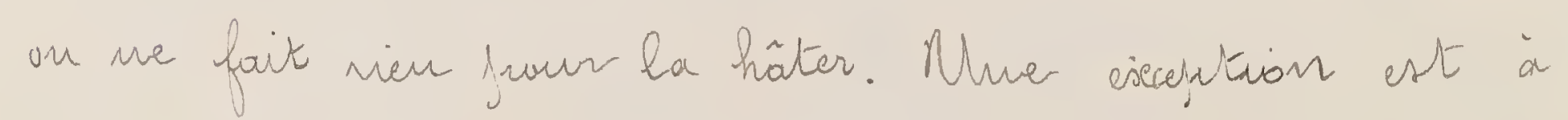


wever chey les Teulhs de la négion de kunjuéla un l'on a curturne de mairtenir la tête des moribonds aous me bas. sive enterre (singa) rermplie d'ear prendaut qu'our leur de. mande d'aller saluer les nembres de la famille au des amis qu'ils sowt cerse's devoir rebrumer daus l'autre non. de. Ses hopucts provtuits par la suffocation sout cunsidérés comme des sigues a'acquiescement.

\section{Pites funeraires_ Un Massi les moits sout} en geniéral ensevelis pries dubavillage an its ont vécu ou dans la cour qui se trouve habituellement an coutre des cases

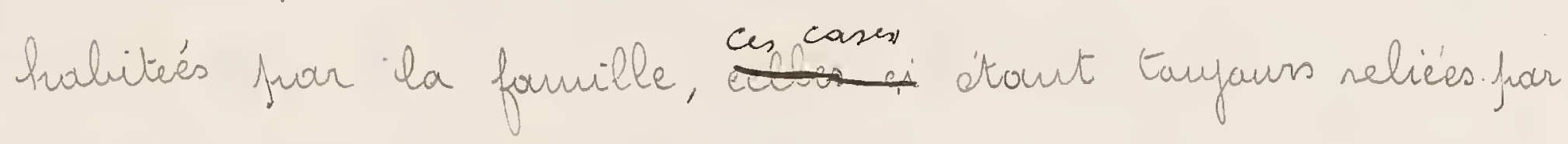
un mur an confprives dous une enceivite de grossefraille tressée. Les Mabus fout construire de leur vivart, à l'Sst de l'habitation qu'ils vecupreut, une petite case nommiée yongo destincis à laur servir de touble. viur tracíder à lessenelissoment in commence faor creuser une onvertire

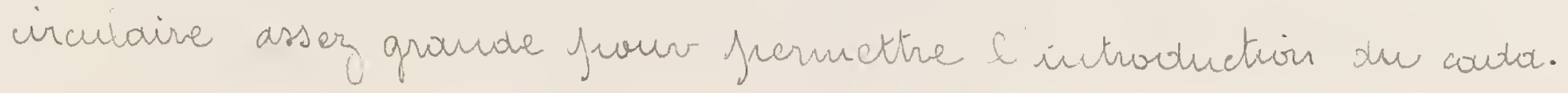
vre; le Conblean froprenvent dit s'élargit en voite an dessous. Le sol est somvent ondré et frassé aur néré comine celui des cases habities. Le local étant airsi arréiagé le mout y est introohit après avoir été lavé et vevètu de vẽtaneuts blauss j on le jlace sur le côté aroit, la face tournée vers l'bst. Cris de hi sant dispusés des aliments, des teulas, souvent mäne des cauris et des vêtements de vecharige. 
La tonule ane bois secller, un wase en terne nerversé ext Macé à l'enaroit de l'ouvertire.

On sacrifie tarymers en cetise circoustance des voenfs

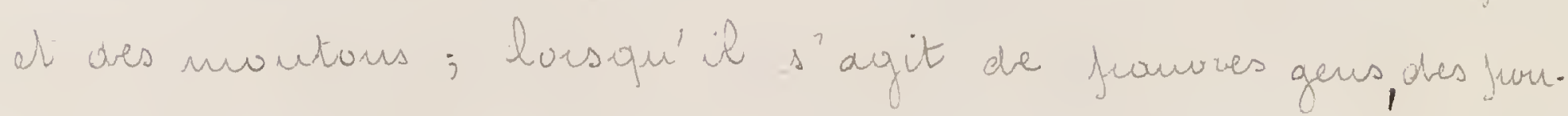
lest senlement. A la navt d'un naba son cheral pré. fère est eqalanut mis à mont arisi que les amimanx familiers qu'il jaurait jasséder. Lu racante qu'aubrefors

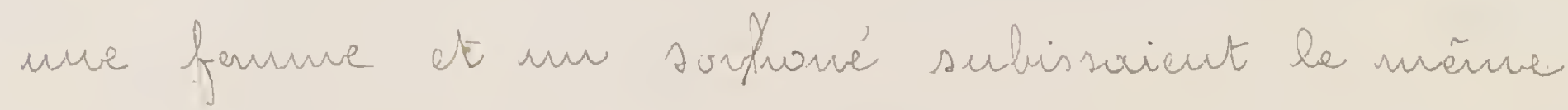
sot ar farcille circonstance.

C'est Turugurs le fils airié qui présiove à ces cuernomies.

Darrs la vigion de Qukadogo les choses se fuassent sensiblement de mêrue mais on fiarait s’y préoccuper darantage de la pussibilité qu'aurait le mort de vevenir unlester les vivarts. Paur l'en enprècher l'ou Mend la frécoution de compre les noubres du condoure surout la nise an tombani; la jorte juar laquelle on l'a sorti de sex case si l'ensevelissencut a lian

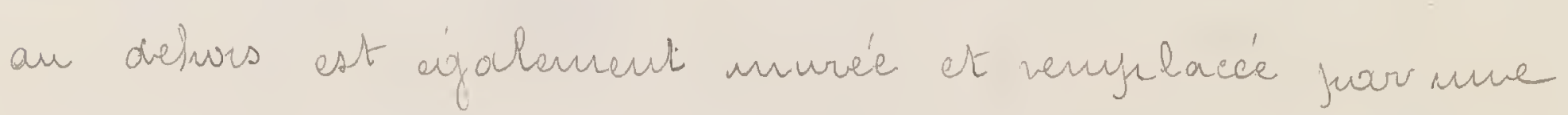
autre onverture que le mait n'esera fronchir ne l'ajant fuas comne de son mivant.

Cher les Boussanga it existe une couturne bigarre qui consiste à juramener le cowtoure à trowers les nues du village araut son ensevelissencert. On cours de cette

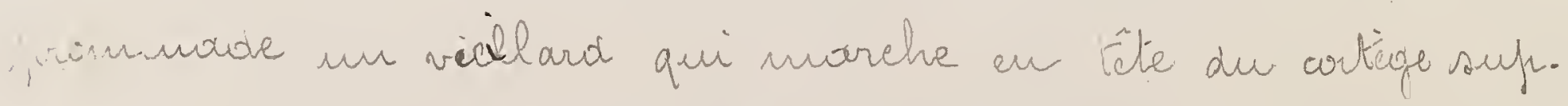
plie à haute woise le mont de foire camaître foar opi

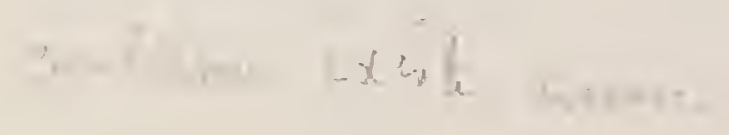


il a èté tué et il arrive quelquchors que bes jurteurs s'arrêtent devart une case comme si he caram afusait d'ar. vancer; la juersome qui habite la case airsi irwiqueé serat aussitat accuse' d'auoin fout jeérir le mort fuar des ma.

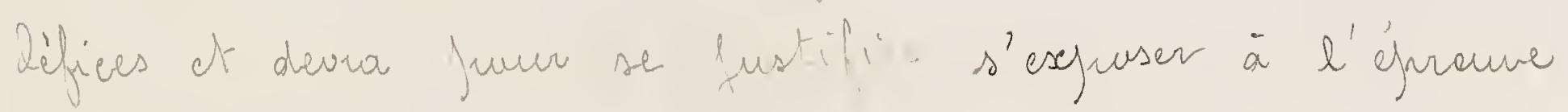
fublique quicosnsiste à se friquer la crisse avec muefle.

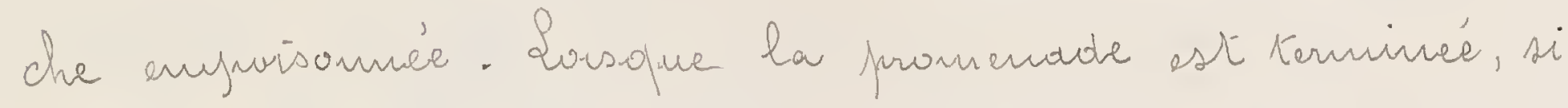
le mart we sest arrête' unlle juart le vieilourd directeur de la córenonie uni demande cos. tu été tuéfuar

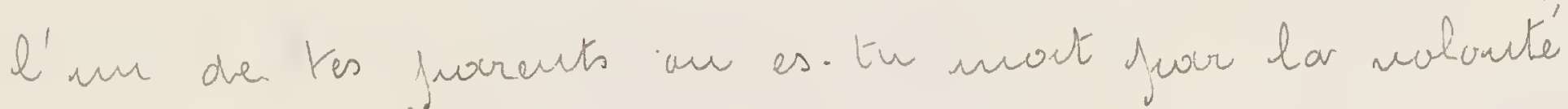
de Bien '."I; si les aseillatious qu'il provorope chez les portens noubent que le mat népand affinmatives ment à la prenicie question on duit provecider à des wiba.

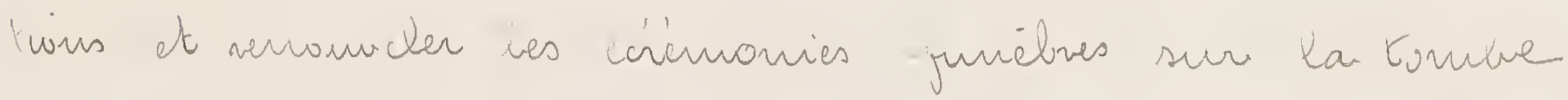
de $x$ ancêtre irrité. Whe lonque matique de cette contir.

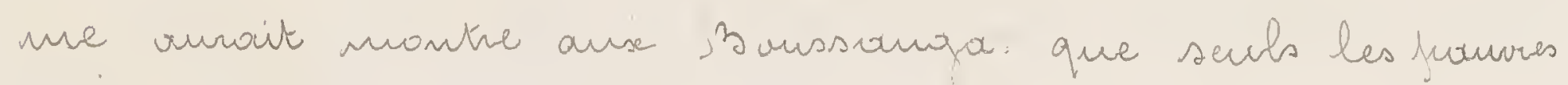
gens mencut jar la voloute de Dien.

the Gouraunsi les nites funibves out me grande rifiatance dt les cereinonies junibues constituaient

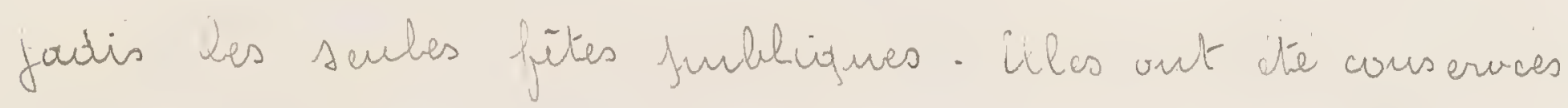
Jusap'à mo jours at leur ulservation ast rifourause. Vir. ci an quivi cles constitirent.

Anméaciaternat apriès le deciès la formille du viéfunt envir chercher un homme qui nerugulit dours la localité les fouctions de Cavecer de morts. Cchir, or serrive, savonme

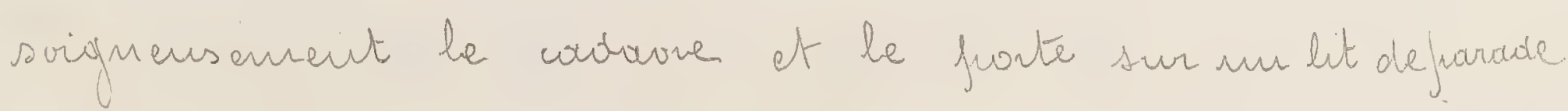


en bois api ser trome ar nilier de la place fubblique. Il n'y a pas de village Gouranusi qui ne soit Jourou a'un lit de ce gave; toilté dours un seul blve de bois ce mauble a gérieralencut deuse mítres de long et est sup. Jwaté Juar des Jieats quessièrement éperourris. Par dessus, on dépase le novt necouvert de vietements que détient en tempis or. dinaise le chef de village ot qui ne sout utilisès que durs cette circon.tance. Dans les villoges riches ces vêtements sont nombreux; it y en a dix à Léo du nudeíle wurant aue vevitent les habitants ais is. Dausles villages jaurve un simple frougue est consaceré à cet usage. On. Waire sur la tète du nour une sorte de chéchia nange éga.

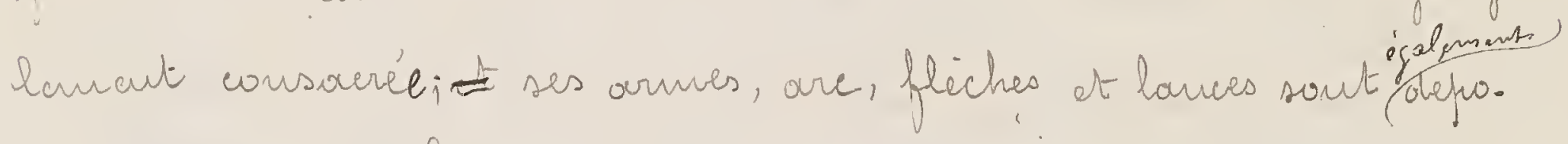
sées juès de hri. La formille commence alors à vociférer ses lamen. tatious. Qurt le village s'assenble et foit chorus. Le caucur

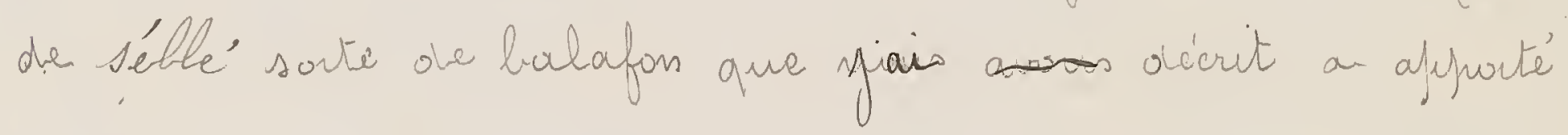
son instrument et excecutic un sir furicbre. On attorche an jried du lik un bouf obstine' a âtre sacrifié of on tue des

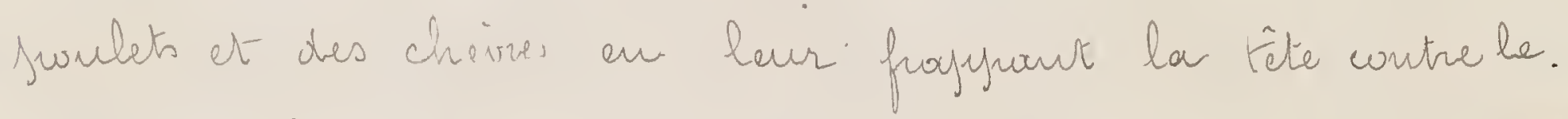
sol car il me ouit fras âtre répandu de sang avour que le nout soit inkumé. On contimue à se lannauter fusquian urcher du soliel, et là s'il s'agit d'un viellard, bes cris cessent jwor forive felace anx réfouissances. On donsera

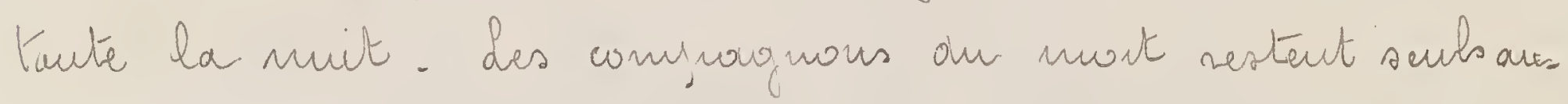
près du lit et taus les jemses gens s'en wout sur la fala. ce our monché aver les tam. taurs et les séblés. 


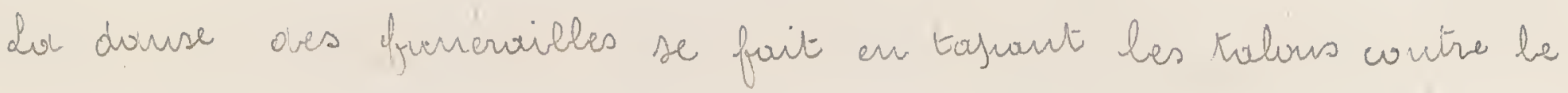
sol en aifant soin que les friess we se rewntrent fras.

Ou juetit gaur on cuterre le noat. On chvisit fune cela un enyelacenent dours l'nitérieur de far cose ot on creuse unce fosse owimaire de 80 centimétres envious. On y défuse le cowiarre anquel on a aliré ses effets de farowde et que l'on a cuvelopgé d'un javine neuf. Il est couché sur be cöté troit. La fusse est ensuita referniée et l su dome le sol avee soin. Athe patite calchasse marquera seule cet enflacenaut. Pate operation teminée, on procéceira aux

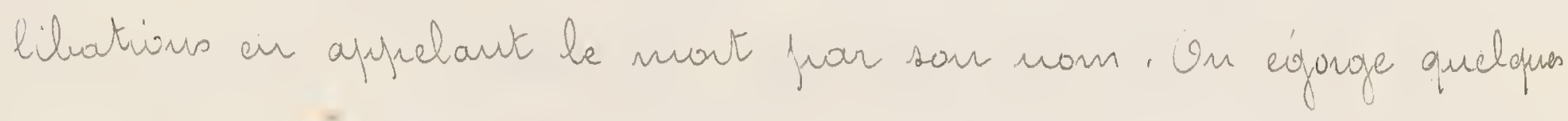
faulets 'sant an fait couler le soung sur la tombe, des aliments saut refouctus à l'eutour et l'on immole en. fir le bueuf jrépraré la veille jaur en distriduer la viim. de entre cerx qui out assisté aux olsiques.

Our Mssi les cerimonies furiekes en hoimeur des naris revètent un caractère obligataire et jarenneutle nom de

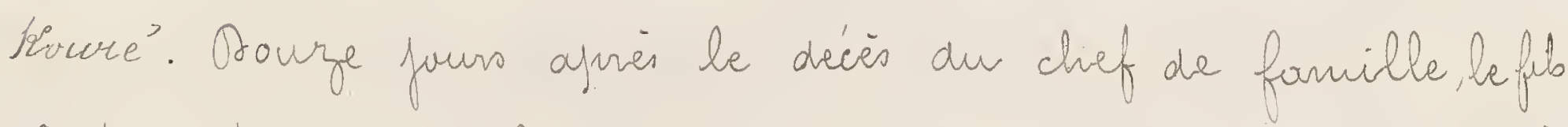
airré est tén de célebres le fremier Kowre", il sacrifie selon des ites consacrés un ou phesieurs foulets dout il vépand le soung sur la tombe en minue, timps que du dom ot du saiba...... La farmille veimi consomme ensulte une farkic de ces alimants et des dourses ont lieu affrartie.

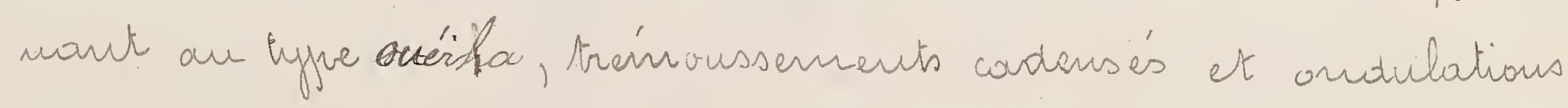


195

In cons an son des violous: le secont Koure' st célebvé kren.

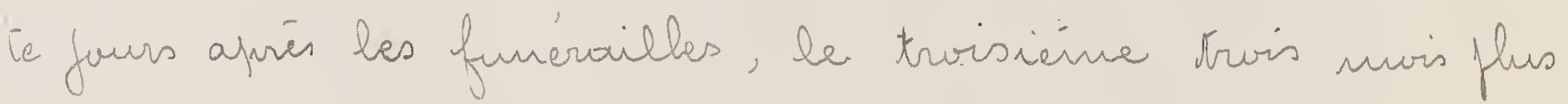
tard, le quatriéne an bout de six mins, le dernier eufin lossque l'année est terminèe. e'cst alors que vieut le cour de la coreinonie dite bossonga dout fiouis frarlé en haitour des atributions om Morpo. Maba et qui est celébré Kars les ons à la méne date dars Vautes les familles. Offort plus spécialement ause mânes du chef de farmille, le hassagor constitue en méne verups une commeimonation de taus les arcêtres défunts. Les person. nes qui menreut sans justerité en soir exclues car les vivouts les carisidirent comme des étrangers qui M ont fait que" juasser. At que nier ne rattache pilus à la terre.

The corfance minersellement accegtée seut que les mouts conservent la faculté de se nauriir de la fartie la plus subtile des alinats véfwirdus sur lour tomber à l'occosion des cérémonies que feas vicans de décrise, maisce joint intiresse of hus joorticulièremant l'ibée de la vie future dowt il est traité du farougraphe suivont.

B. Sentrinent rebigierse

Ome. Vie future Les Assis. ervient à l'esis. Kance et à la survivance de deuse àmes distincties; l'âne 


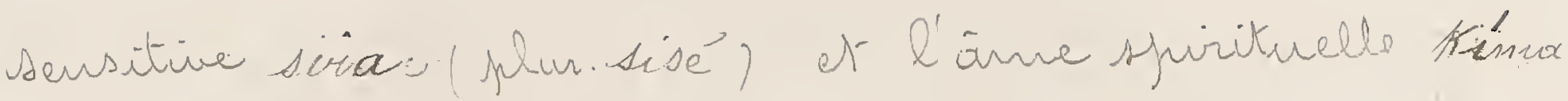
(phir Kinsó)

La premiére est consideré comine le purinciprevilial et identifié geirérabunent auer le soufbe; elle geut Naute. fois quitter l' homme en certonives circonstances fernowout le sommail for exerufle et les syjucapes. Le rêre (zannsolo) n'est

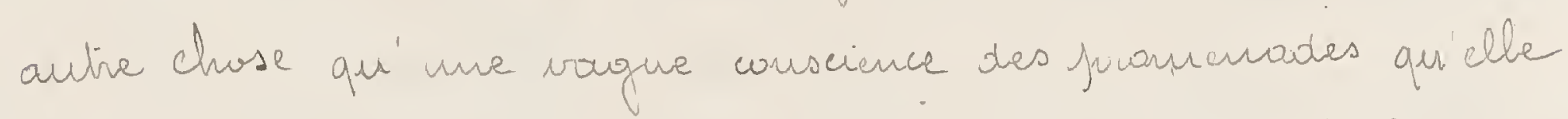
accomplit toin aur conp, aussi les Mossi. attachent. ils à ce fait une grande infrovtance

L'interpretation d'un sonoge bizarre les preivecupre beaucoup. Clle ost confièe genièralement à une atégoive

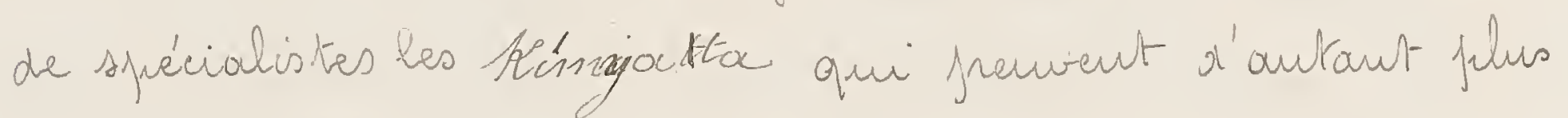

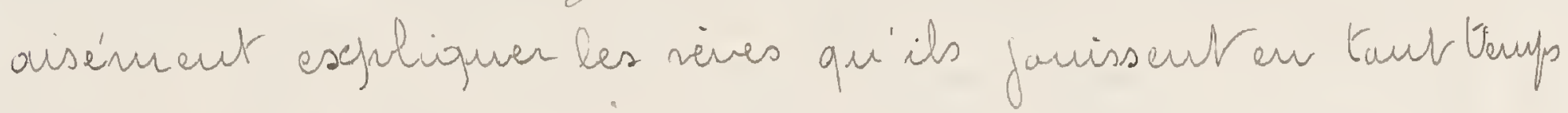
de la focultie d'apercevoir les sisé arrarites sufaut quitté Kenforairenaut les coups quielles habiteut. Ils bes sur. prement someut in bain de boire le soung des fusulets socerifiés pour un defunt of lossqu' is ow fur les recomair. the, en forrille circans vance amansent infailliblemat la modt jorchairie de leur pussesseur our mieux de leur double

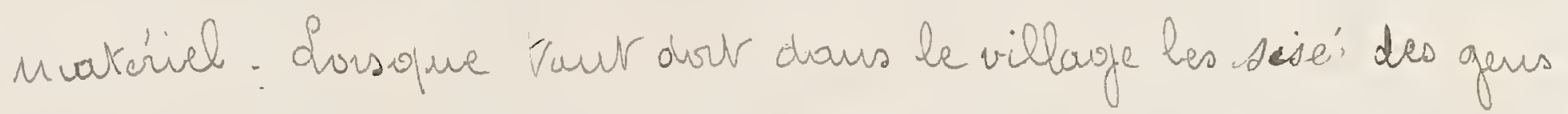

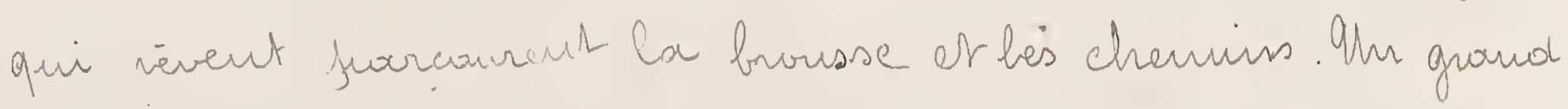

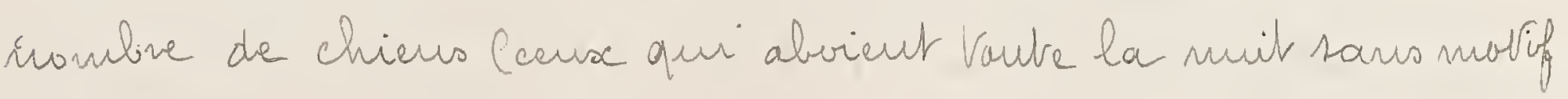
afforats) frartagent anse Yeux des Mossiv le privili. ge des Aisnyoutta; ils penveut voir les sise., ot mêne les mictre en fuite.

Dans beancaup de localités ont croit que la sirifox 
de chaque homme est nitumenant lié avee celle d'un ani-

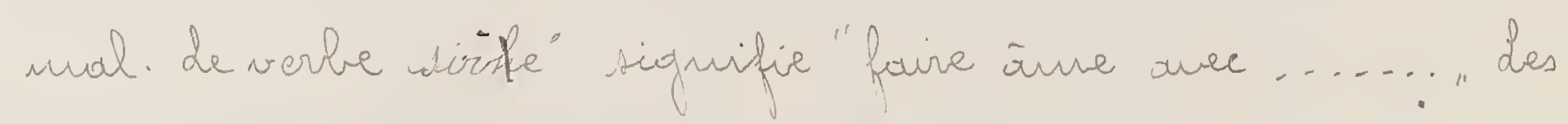

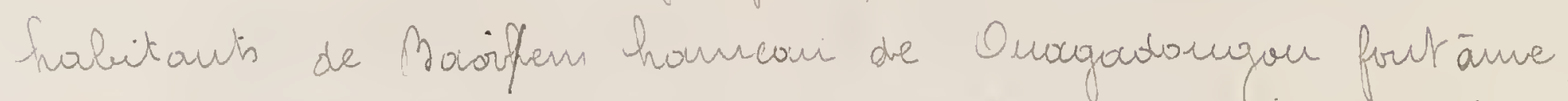
aree des gazelles cenx de Thetranuga, Barhogo th thavigo aver des cainmaus. Orés de ces dermières localités quicanque lécroit un caiman s'expraserait s'il était un, à ètremis a modt par la pufurbation, car un caimion ne prent nourir saus arim homme du villowge subisse par con. the curp le mêne sort. Si un not était avenofle, arufurté

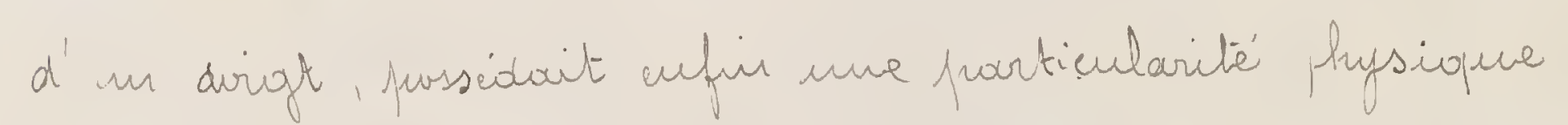
quelconque on paurrait si l'ou cherchait bien netrouver daus les envirous le cowiove d'un cainan vềetur du

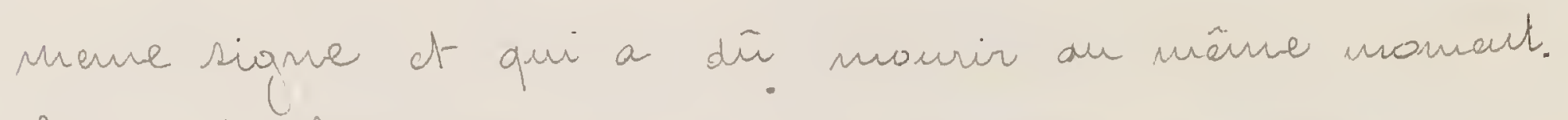
La an les habitouts fout anre avee des cainmans cesari. manx sont mor seulement respectés mais encore nourris fuar la pupuclation qui and de leasps en voups seter de la viande aux antervits qu'its frépucutent. A Kundongion (Kipquisi) in l'ow fout âne avee des iguea nes, ces reptiles out firi par publuber a'me foxcon extrowdinaire. Its se tiement dous un gus fourré voisin ou 'village qu' ils quittent somout le funr frour circuler dous les mes en quête de nouriture; ils peirètrent librement daus les cases on nul we s'aviserait de les nolester. Gies ai awit un cervarirnombo pacter des bracelets ot des aundettes que les habitants 
beur ont ploce' aux pattes ot an cen!"

Al est une cotégone de sorcières les sókéla Lau sing. sónufas qui preubut dit.on s'enfparer de la sïrta des vianouts, la nuetre four un terups déterminé dous un ustensibe de torre ot firalement la nonger, assenbbés ar des sortes de sabbat dout on me joorle qu'ave terreur. Mu graund nombre de décès d'enfourts et de feumes geus sout attribués à cette pratique. des sókéha sout tries vedoutées.

L'ärve sensitive soïta envelopure l'àme sfirituelle kinna. Leur séfuaration n'a lieu qui aprè la mout.

Vivi quelles sout sur ce ponit les idéés les plus véparnotues.

Il existe darrs le canton de Jondéré (pravince dellam. sono) un petit villowge is alé du nan de Yabtawé dent le

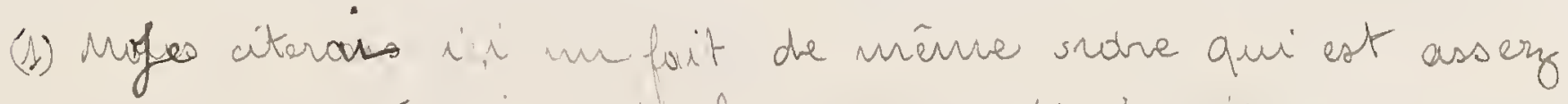

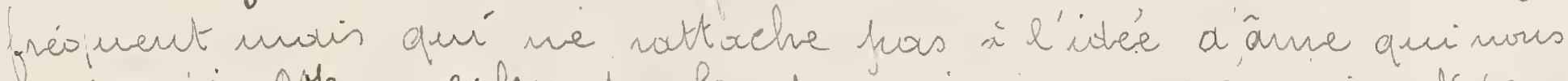

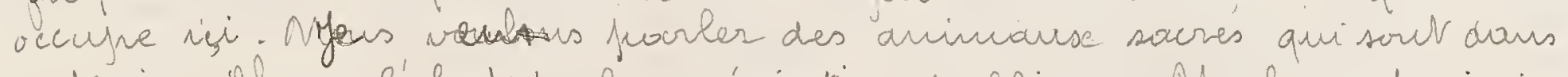
certarin villowes l'obet de la vericiakions fublique. An boa est airsi eurreteme fuar les habitaus de Garrpíla nor bin de Oragadoungur.

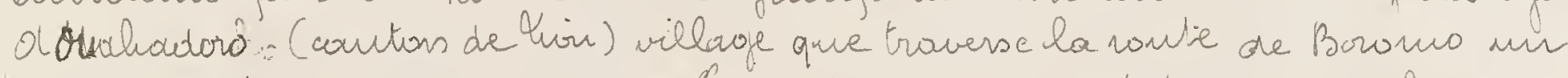

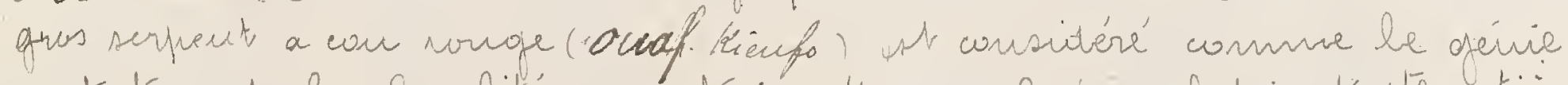

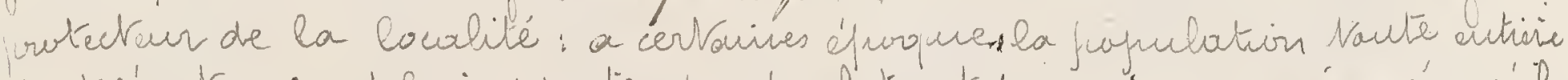

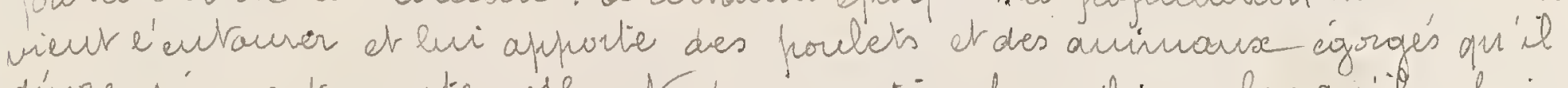

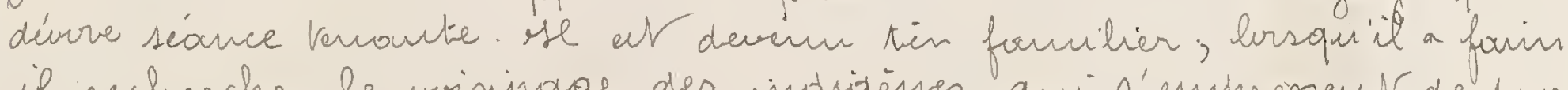
il recherche le voisinage des indigères qui s'empresseut de forr. vir à sol nourriture. 
chef doit the owengle. S'it we l'st was an momentr de sor elcction il le devientra fatalement aussitôt appèi, car wur bien voir les ámes - At cela opt rindis prensabbe à thab. towré. Maba - il we fout pas être krouble' par la grassière affuarence des olgets matériels: Les ârves sise' de tous les

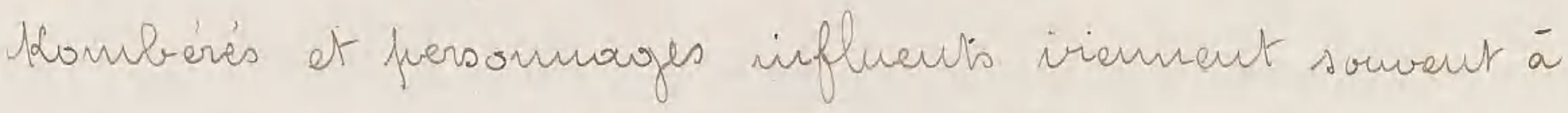
l'insu de ces aemiers et en genirial besourils sout mala. des, renobre visite à. Mabtaure'. Maha. Mles hi denarident à boire, si Tabthave'. Maba aefuse la Sï̈ka verthe en häte ot le nualoude se nétablit, s'il laur tand une collebosse d'eour c'est que la mont doit se foroduire. Oours ce

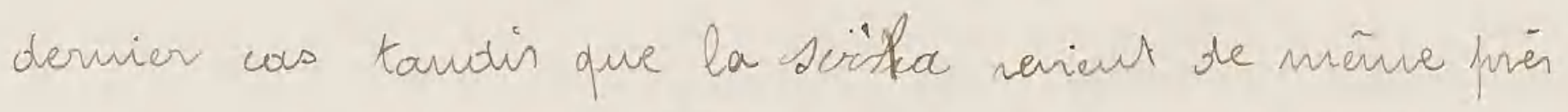
du cups qui a cesse' de vivive et qu'elle ne doit phos quit. Ner (cor c'est elle qui se nourriora des offroundes faites aux

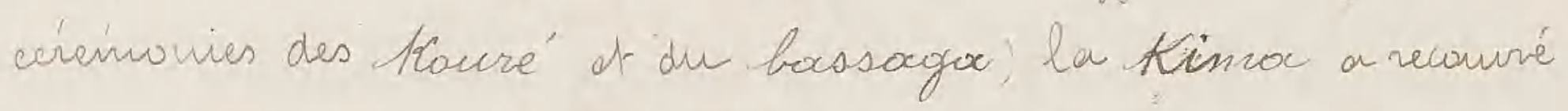

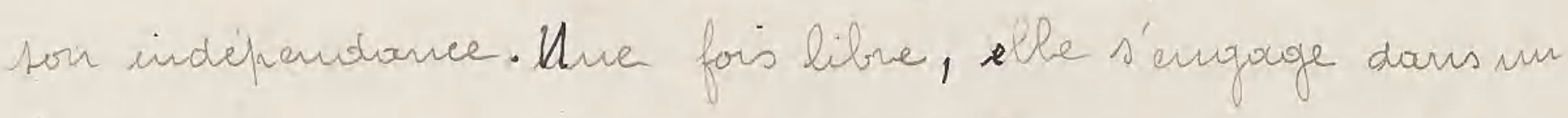
Chemin qui passe aux environs de Manga se dirige en. suite entre Moberé At Mongo et de là, lougeaut la Volta Blanche aboutit à Slimfiran an se trause la fox. mense cowerne que les teimsé ourveut définiti. veneut habiter.

Ce chanin des änes, bearicoup déclareut l'aviniver, il ast large conme ceuse que les blanes out l' habi.

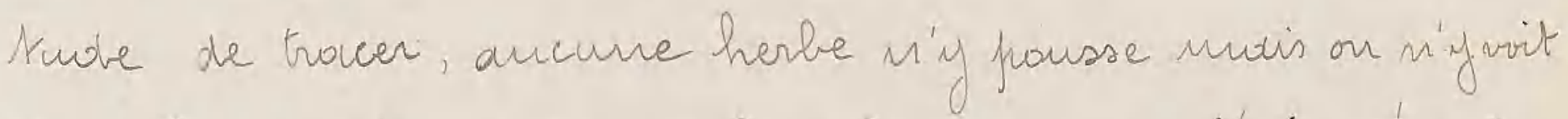
Jas d'empraintes de fas et le sol a Vaufaurs l'air d'avoir été frachernout vemué-

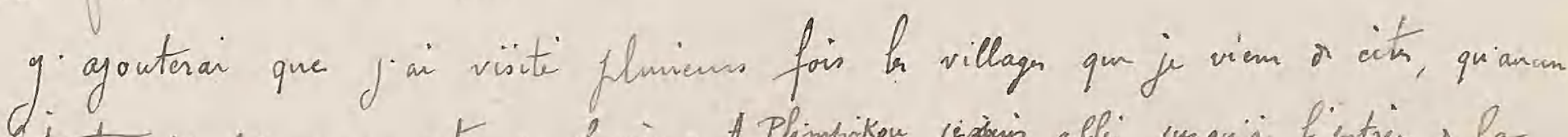

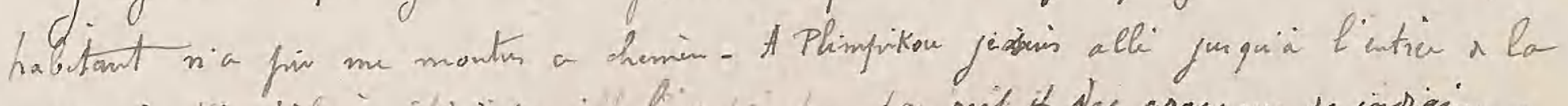

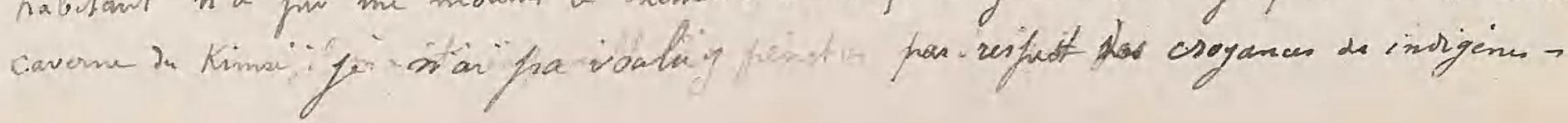




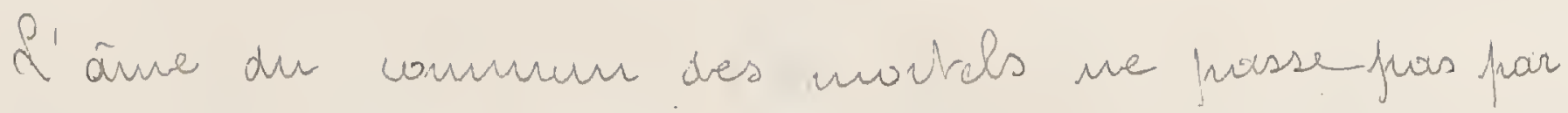
Thabtousé, elle se rend directemant à Aibzongan dours les ar. virous immédiats de la coverne. La les esprits qui prési. dent an fugement, la metteut aux fers, l'interrogent et de'. libiraut sir son sort. Li elle peut se disculfver de sesfoutes son coups maríriel lorsqu elbe revient recouvie la sarté.

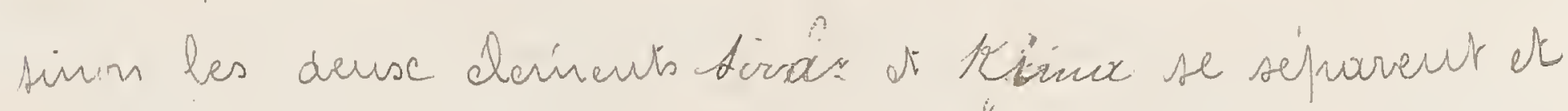
comme nus l avous we la noat s'en suit. La mise aux fers nevectaut un caractere infamant est prargucé à l'ãme des chefs of le jergement four ens se réduit an geste fartidique de Yabkowie'. Mabot qui dome on vefuse à lovire.

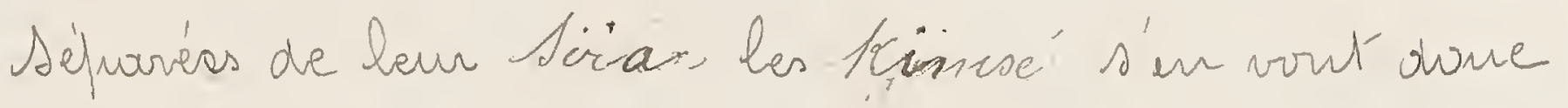
àla coverne qui se traure owar la montagne de

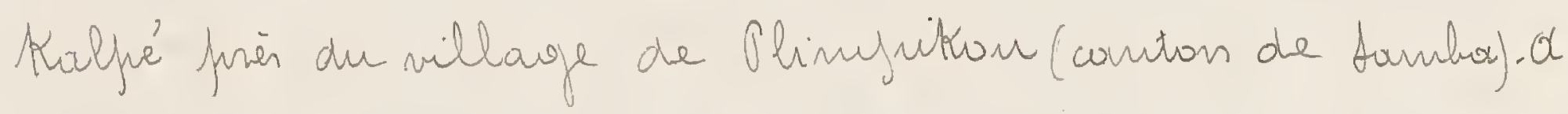
l'autrée de cette coverue se aresse un granit arbue soubre nommé raporä: que le vofougeur attoude' ne argarde qu'avec effrii; it est cependourt queleques hommes qui ont la hardiesse de se risoprer à l'nitèriour four vir l'äme d'ancétres défunts, moin mul d'entre arxe, - n'est veverum. On ignove harconscoqueut ce qui s'y passe ot la aie qu'y mirrant les kimse'. On we peut dire avee certetude que cette ire ne firivia fuss. La Jira se nuurrit bien des affrandes forites aux

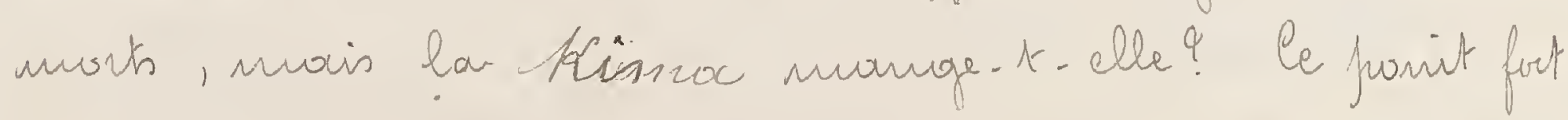
discuté ve parait has aisé à éclairier. Quelques uni 
14)

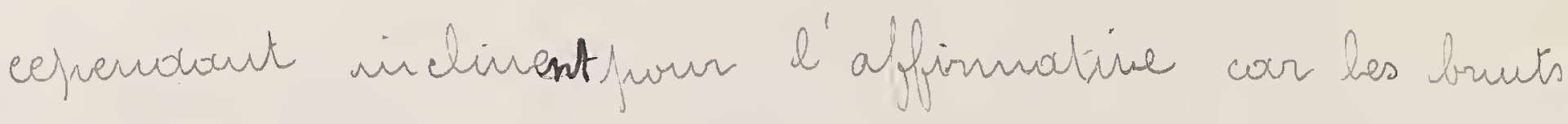
lointouis an'cutendeut de Taups à autre les habitowts de

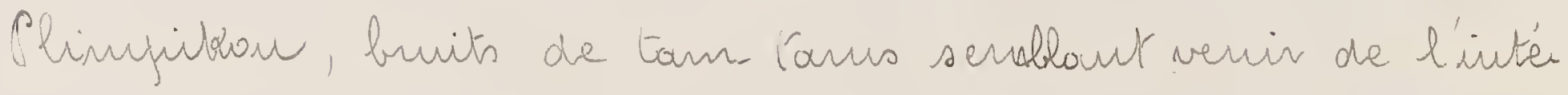
rieur de la caverne permettrovieut de souffusoer qu il s'in Kieut des morchés périvohiques.

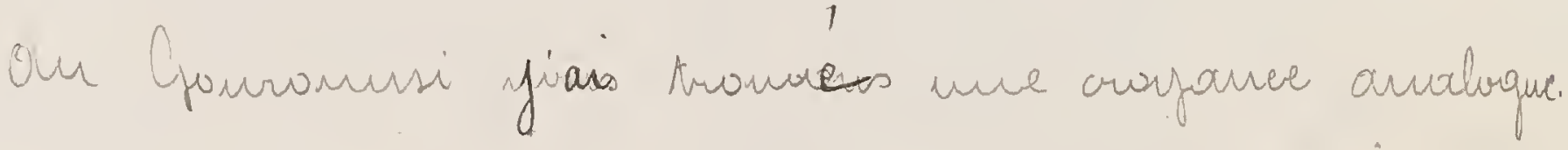

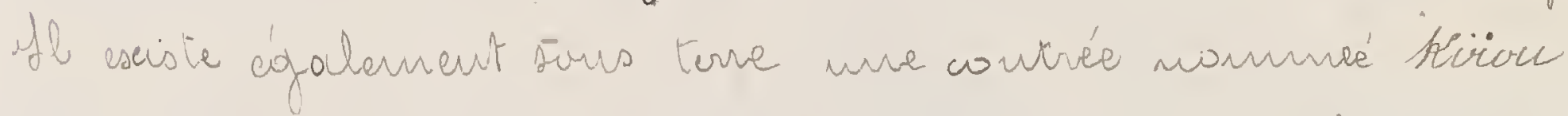
sir vout haliter les annes des noonts. Ce qu' il y a de

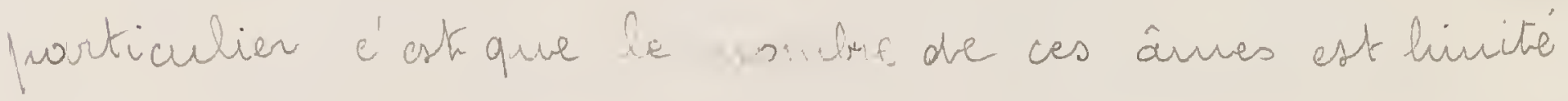
ot qui clles prewart se séircarmer. A choque décès sun

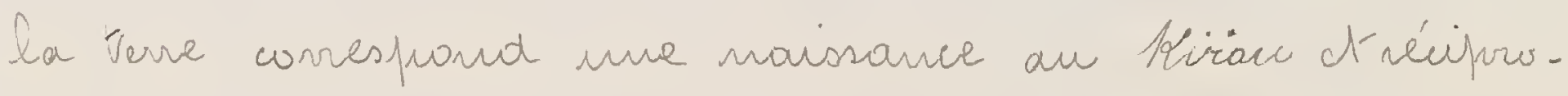

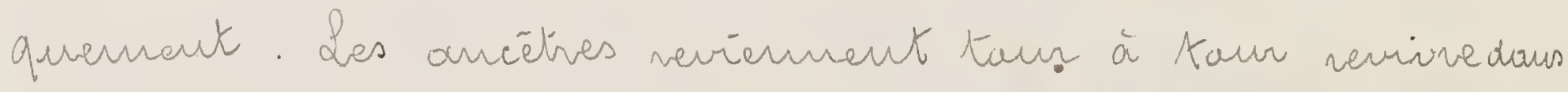
leurs descendants el bosaqu' on présente un nouveour-né à unsoneier celvi-èi pent dire à quel parrent défunt appar. ténait l'ane dout il a herité.

Relugion _ Les Massi. croieut in un Dieururi.

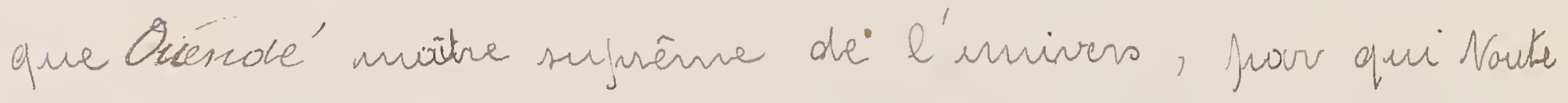
chase sulusiste. Ils l'identifient le phlus somentaver le solail (oninderx). Lorsopue cet astre se live on dit

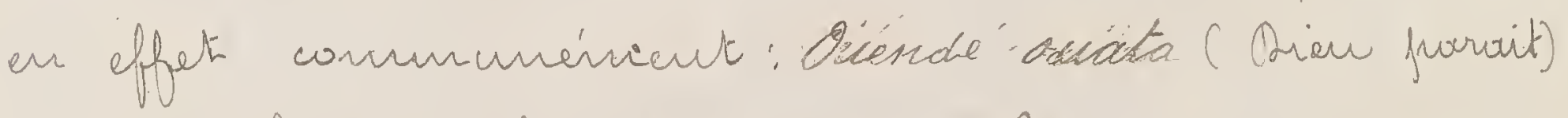
at lorsapuiel se couche: Ociénde'tion libiga (Ohen eutre dius les teiribves). On dit oussi que la tare (Genga, est la

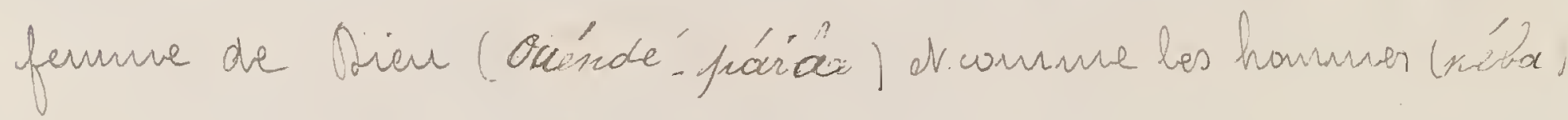

Wot 
Aout les enfauts de la terre Eenga kianba) il est facile de dégager de ces expressions le sustéme que ianoint envelupNer la crajance mossi, systeine d'mue incontestable affarrence logique qui se peut formuler ainsi qu' il suit: "La Perre fécondée fuar le Soleil a domì naissonce à la Uien.

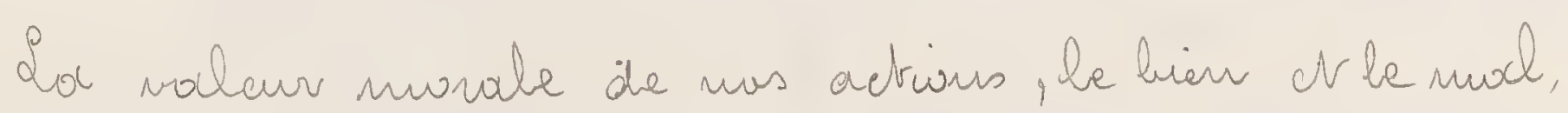
l'épurtable of l'ánfusté out été décrétés frar Dieu qui an a sistrivit les hommes dis l'oriofine et commerien ve hir échappe, mi les chases les plus cachées mi les persées les phus secréties il diotrilure avee nue fustice infaillible, punitions et réconfpesses: la noor exr Voufaus le chathineut d'une namouse action. L'cscistence de l'horn. me ast Pleino a'épranes oifficiles dout il we sera sir de rriompher que s'il a la conscience netre. deubleusant l'ail at pur verrout clairament avec be dienger le majen de s'en garoutir d' jusront marcher avec assurvance dous cettevie. de respect dout on wise les viellarits est surtant fustifié en ceci que leur langévité constitue hour tous une présonption a'inrocence conservé à kruvers les omiés.

Mais la confession (toikaséga) qu'inn cunjuable fout de sa faute peut atoriter be châtiment encouru si elle ne lui peaner fas de l'éviter axupliterient.

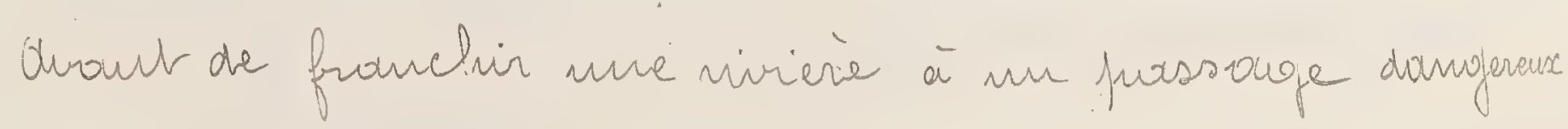


le voyageur s'arvête taufours hrè d une pierre nummée téstacogri... qui a éte disfusie en cet endruit, yfoit l'aveu de ses fourtes, 'varfoir mêne, s'it se secit très wuprable, un sacrifice exprataine. Il sait que si à ce moment ses péchés re lui sout fas semis la rivière ve peut manquer de l'engloutir.

La pratique an torikasega nevet pour les fermues en. ccintés un caracterie obligatoire lassque la délivrance est priche. Bour éviter mue issue foutale elles sout en effet Kenves dès que les premières douleurs fout présagier l'as.. unchement d'avourer aux matromes qui bes assiskent but ce qui peut charger leur conserience.

Lorsqu'une ferume a Norminei sa confessions, le mari est pris a faurt et mis ari couraut far be détoul de taut ce qu'elle vieut de dire th re peut refuser sour juordon encette circonstonice. Cour moutrer qu'il an st anisi il doit prendre devout tars une gowgée a'cour à la calebasse qu'ou hir tend et qu'il passe ensurite à so foume. Celle-gi fait comme hir, quir kaus deuse en mérue temps refettent le liopuide ingurgité. Dès ce nomartil vereste plus vien au curr de la femme de la souillure qu'auraieut fur y laisser les fartes commises, it ve vesteplus nien éfalorraut dores la mémaire du mari de ce qu'il a euteridu. Opprès que ce dernier a domé l'exerupule, d'aur. tres persomes sant introoluites; avisines qui out fur avoir. a se fubinobre de celle qui doit accoucher, ete. Chacume 


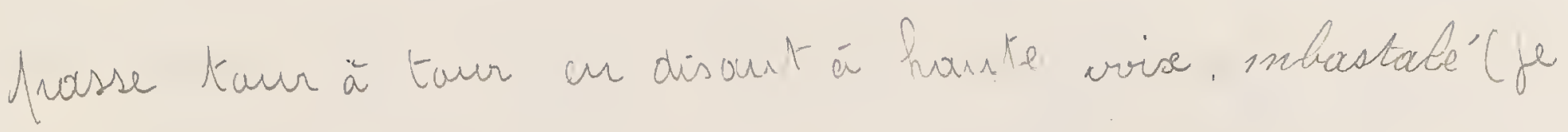
pardanne). Eufir les pelits eufauts quiclbe a dî paorfors inaltraiter sous waison s'apperochent de leurs mire crele la mair, hir afflenraut la jare.

Li la délinance s’apère saus aifficulte' c'ats que les fautes de l'accouchée out été parfaitement effacées; s'il est falus laboricux e'est que quelque passonve a conserve'vis.à. is Selle un ressentiment secrer; enfin, sila mast sumint celos signifie que la corfession a dî être infourfoute. un pecehé grave onis.

Rien ve se passe darue ohaus le mande qui ne sait l'effet an hasard; le maindre ivervenvent y trahtit les in.

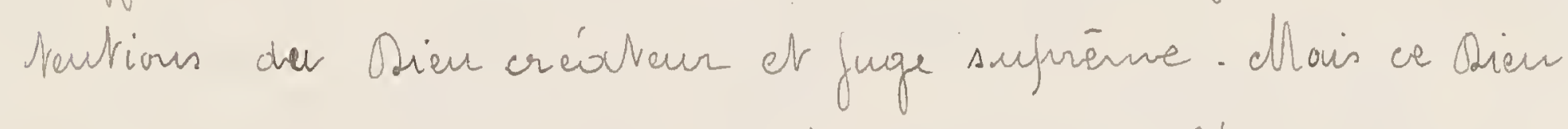
n'est fros inmoment à sa creation, on we l'a famais an sur la Cerre; juour se nomifester dours l'oritre des chases et of faire aégner sa wolonté sonveraine il a hesoin d'inter. mídiaives. Ces intermédiavires sout les Génies et les phé. nouriva shisiques.

Les Geiries sout de deuse sortes, les mus out unve exis. Nonce indéprendaute les autres adhèreut aux objeth natu.

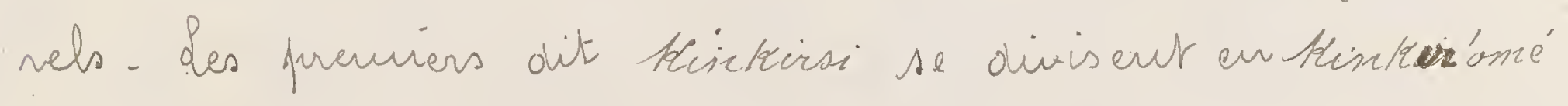

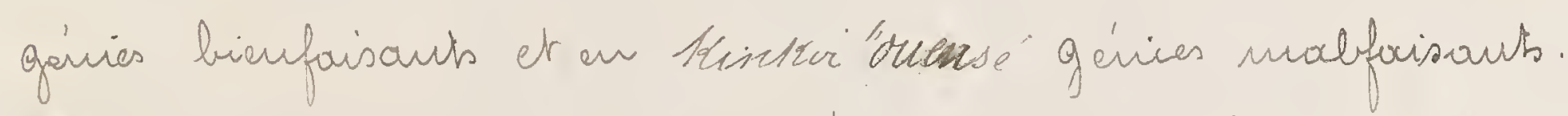

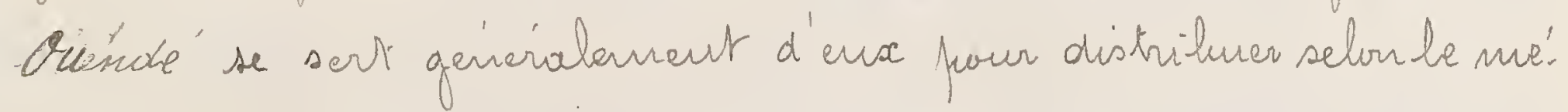

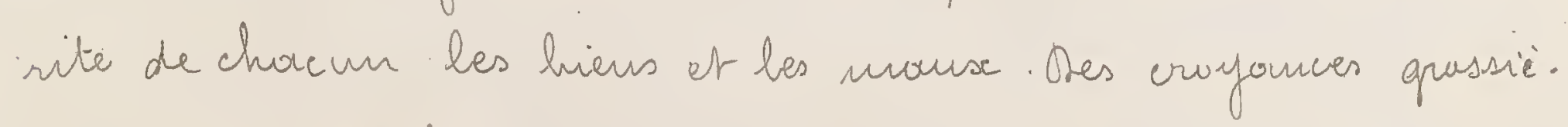
ves sout attachies à la notion de leur existence. Las 


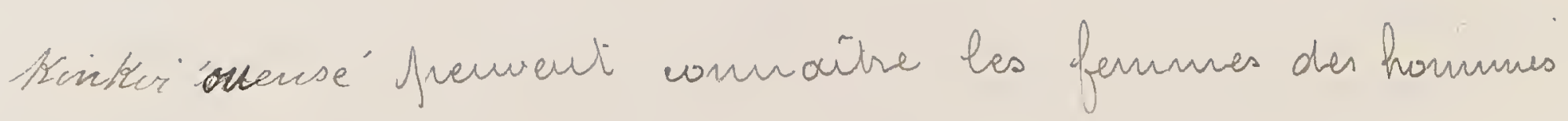
lósqu'elles sout folies et out l'infrudence de se nisiguer

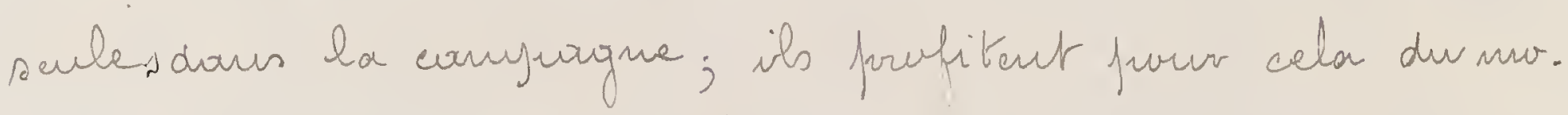

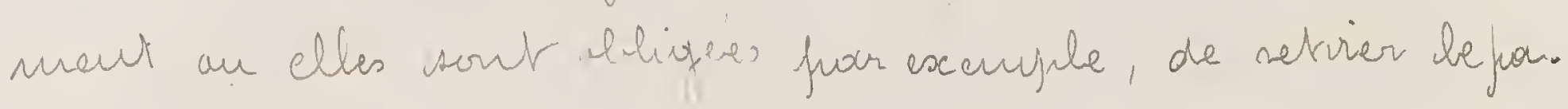

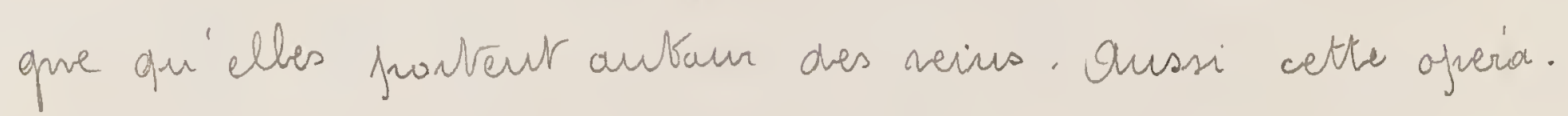
then obit. elle se foire tries napidement, surt aut lossague les ferrmes sout déjá anceintes des ouvves de leur mari.

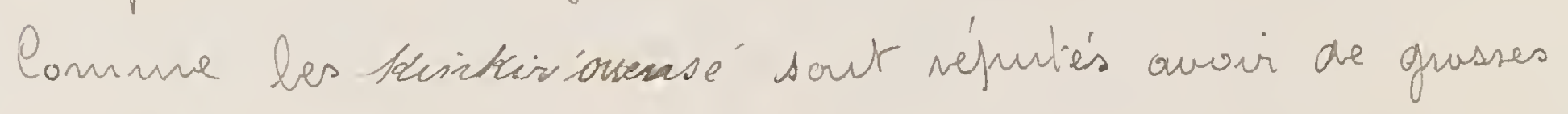
Hèter sur de quetits cosprs, on distingue disément les aufauts dout la venue au monde doit. ctre attribuée à leur intowention. les enfouts sout mis à mout aussität ofprier leur maissance.

Ses chieus qui nfees l'a dit, peuveut vecomaître les sise'errautes, viieur aussi les kintric ouenss'; ils défen. dent la maison contre leur afproche et jeuveut, en aboyant, les metre en fuite. Mais les hammas ne sau. rovieut en gevieral vesister efficacencut à beurseubeprises malfaisantes si le concours des Akictici asse' ne leurctait assurré. Ces Genies se livent aubaur d'euse à des conchats incessouth; là ar bes Kinteir auersé out appouté la maladie,

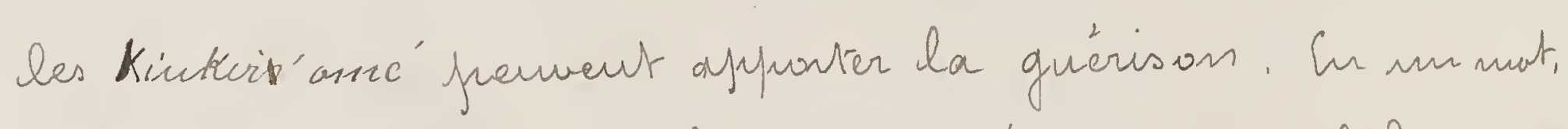
selan que les auns au les autives l'empouteut, l'homme est ou non la provie du malheur.

On woit frar l'esprose' de ces sufuerstitions les altiration qu'à oni subir la croyance à laquelle alb se aattacheut Sent. etre néme les thireteirse me sout. ils que 
les disnes des Manthingues, ount la notion into.

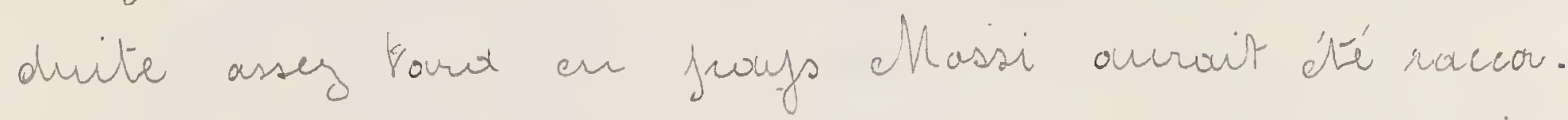
dée Mur hien que mal avee les idées frimivives quielle y a trouvies.

Les forces waturelles, bes introumènes fhysioures dout aussi func la voloutié de Diar le royen de s'exercer.

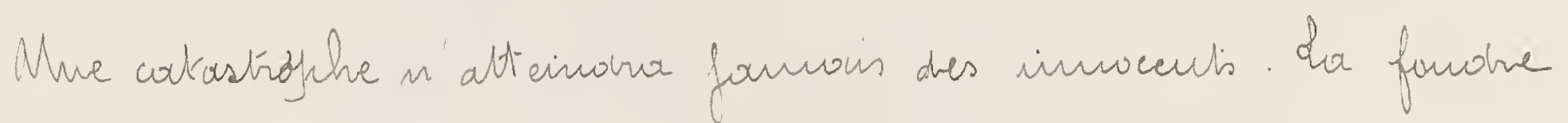

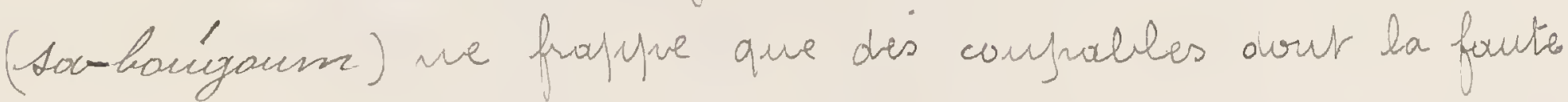
ignuvée des hommes n'a fur e'chapper à la claveroyance di. vine. Il in est de mèrre des ourougans, an cours infuetuenx des nivières gowflées fuar les pluies.

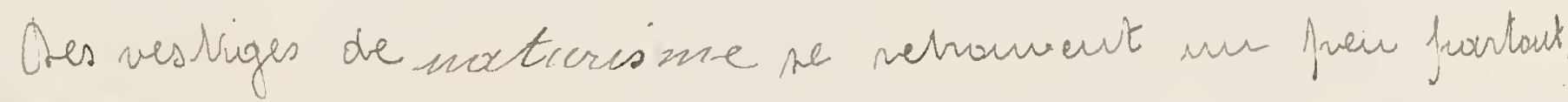

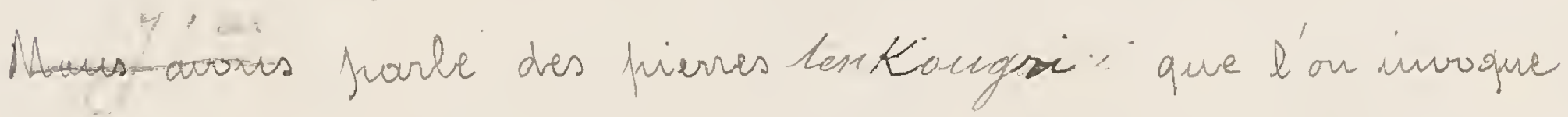
et prier desquelles on fait des sacrifices; il existe dues arbves, Lise' (survaut owus la région de l'Orest) et des foune's sacrés; des noutragues aut l'alyet d'mn culte et recoinieut des prieries prufitiatones. On

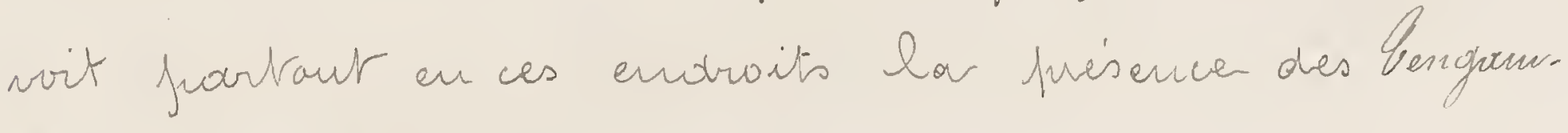
ba Géries de la verre.

La plus célibue des nowragnes tarnes ot celle de Qoutilu pries de afantanga à \& kil de Onagadougan. On s'adresse à elle four oltenir diverses faveurs, particu. lièrement pour avoir des aufourts. Pelui qui a déé escouce' est

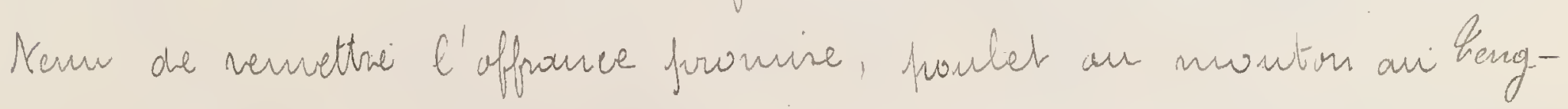
- Sabar maitre des sacrifiles. 
Les. Ceng-solax remilisseut an Massi des fonctions assez amalragues à celles du frêtre. Ils descendeut dit-on des plus arreiens habitours du fouss ot à ce vithe javissent

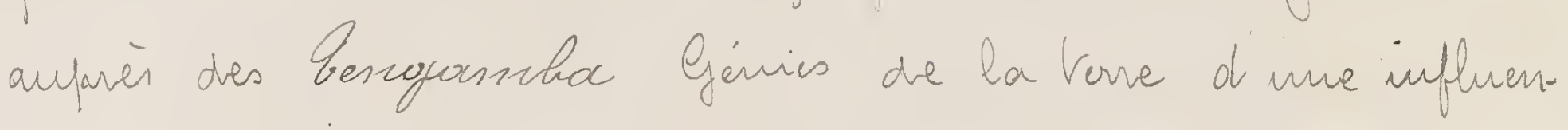
ce particulière. Leur vétemaut hahituel est la grusse

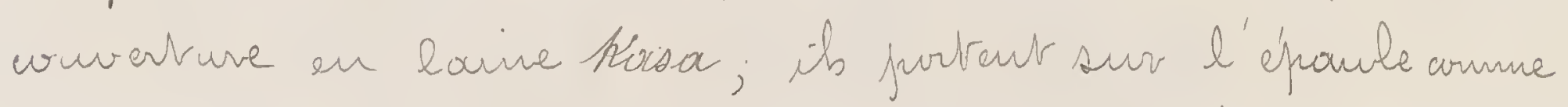
signe distinctif la háche aituelle des sacrifices

deur chet habite au village de Gazani; il vicut pe'. nodiquement cheig le Motho-Maba acconflir des sacrifices privés et adars les gramoves circoustonces vinmule des victimes

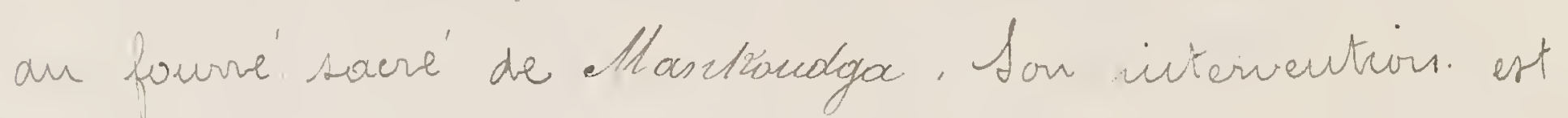
niohifarsable fuur obterio la cessation d'miné épidé. mie, lavenue des fluies en termps de sécheresse, l'heuren. se ssive d'une expédition militaire. Il ne se charge fros a'vitérets particuliess mair cenx qui afreis avoir demande' une faveur à la nour ouge Gonstibo out abterm ce qu'its desirovient hi seirrettect leur affromide et hi corfieut be soin de nemercier en leur nomble Genies qui les a secouruos.

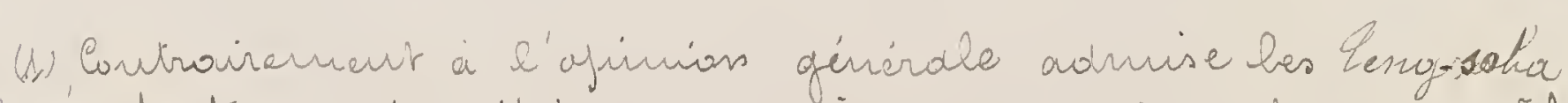
quirefrésentout une trodition tries ancienne ne funcur aucun söle dours la circoncision. Cette frodtique est dì en effet à l'ivilucune

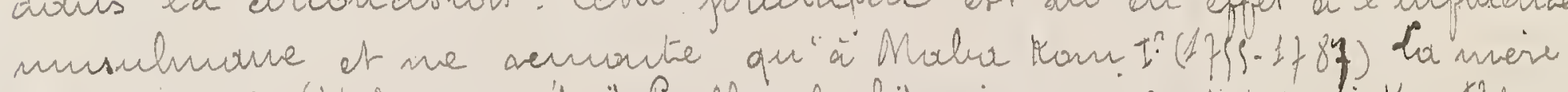
dece dernier (Haho) qui ctait Peulhe le fit circoncine et deprins kourt? tous

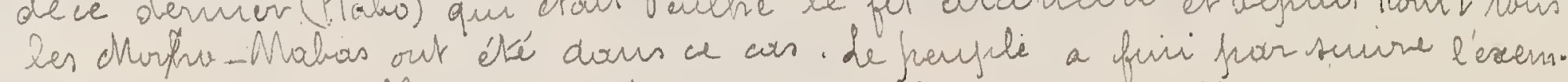

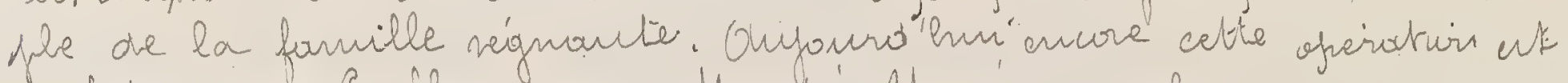

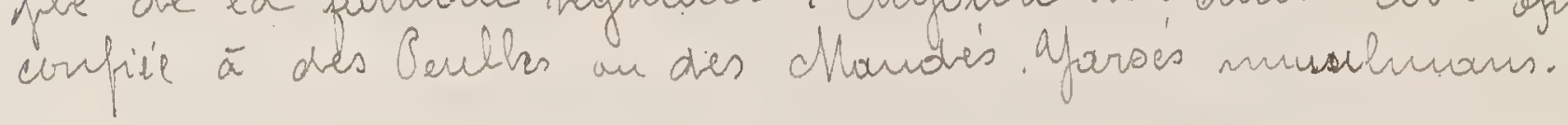


Les Lerry-soba sunt en outre considérés cormme détew. Parrs de preciense provéóvés trerapreabiques. On les consulte

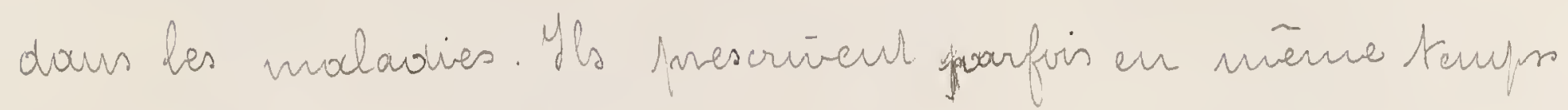
que des nemides cectarives alstinanes frouticulières. Ifs recommonderit aussi les nontifications et la confession cortasega; le nualoude n'est assure' de giverir que lovinue sa conscience we hi reproche rien.

Les Earay-sohor constitueut whe caste spéciale, its

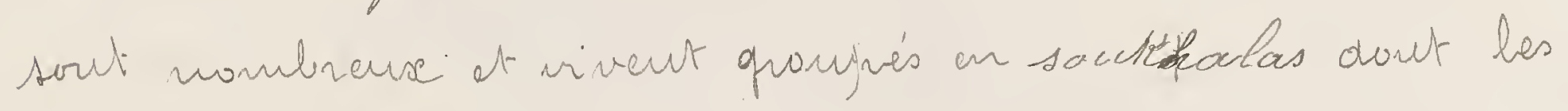
plus conmies sowt celbs de Sazani (pies Onagowongur) Doundarndé et Doundanlauna (Arumince de Gaunga)

Hl pares veste à fourber des soveiers profremout dits. Les phes comus parmi cenx. is se nomment bócitka: ils ont la spécialité de prédive l'avenir et d'nidiquar les procédies fuar lesquebs out paent atteindire in hut difficile, satisfaise ure andition, éviter des ennuis préus.

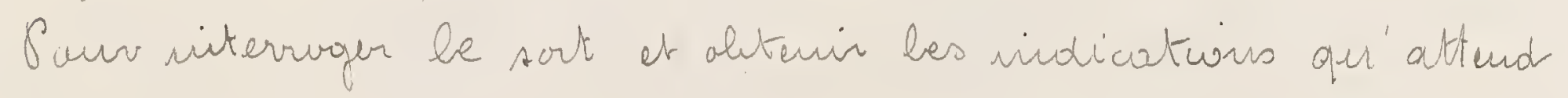
le visiteur ils out coutume de tracer davout hi des si. grves mystérieux dars le sabre, de aisfueser des figures symátriques de petites firerses ranges ou des couris suit en. core des grain végétause appelís Miou. Oprès s'être liviés à des combinaisons variées ausopuelbes be client arocieuse ne frent rieur conforadre, ils hi amoncent gravenent ce qu'il owit forive paur wir son surhait se ríalises: ce sera la

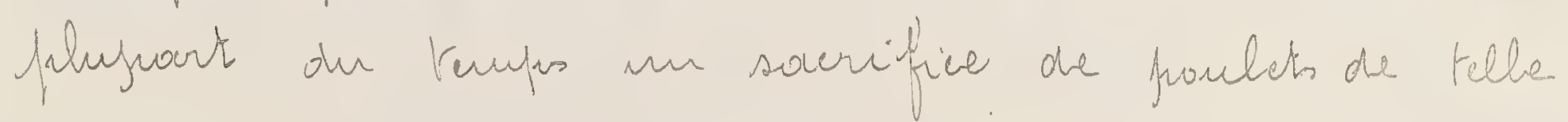


un relbe wileur, an le déput d'un alyet doins la brousse àm endrivit détemine'; d'autre furs (surtrout s'il s'owjt de faire cesser la stérilité d'une fenme) il conscillera d'acouérir un aminal domestiane jussédant telle far. ticularité et awoul on deuva mettre des braceletict des anulettes. La consultation sera taujaurs grassement frajfée. le sunt en somme des explaiteurs cyniques dout le procé. de' est presque identioure à celui des Kéesiciélala si num-

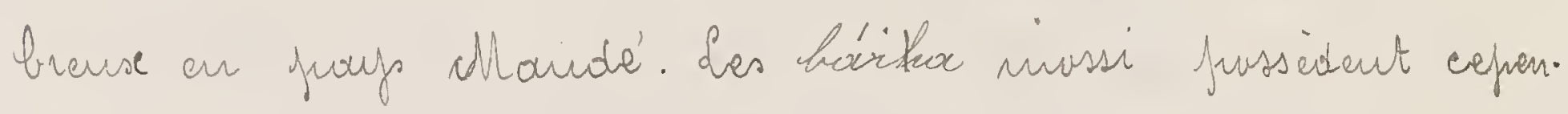
dant me attribution ignonée de ces dermiers: on his apprelle souvent aufuris des nouvcour. nés furur déciober du nom de founille (Sandéré), qui laur vevieut. Onfitant dur monnent ar l'erfour fleure, be sorcierné. cite beutencut la série des Sonchéré qu'il est susceptible de prendre, sil'cufaut se tout à l'nisioust au l'un d'euse vieut d'étre foromancé, e'est edmi-ci qui sera chisisi.

\section{Hess gfiraw dit un mot des sakéba noungerses d'ämes.}

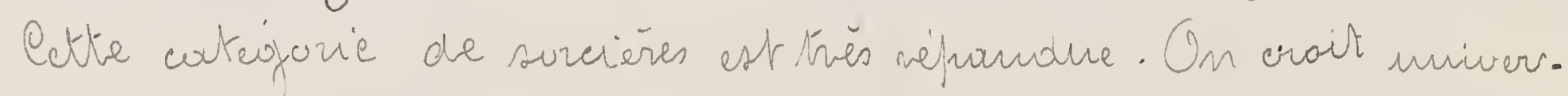
sellement à leur jawair et elles jaraissent elles-mèmes

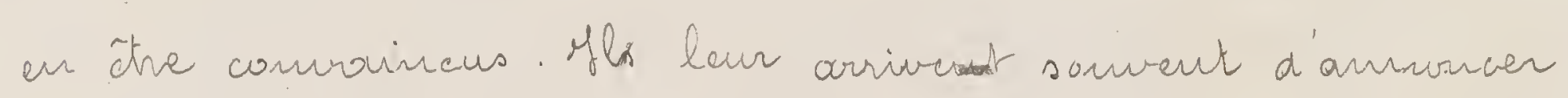
foar onticiguation la mort de leur vichime; si celle. ir à comaissouce de la chase, il devieut riugassible

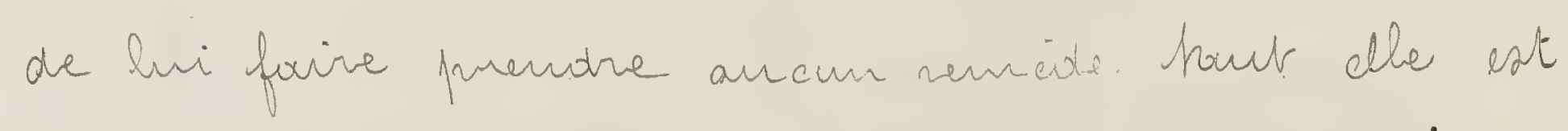

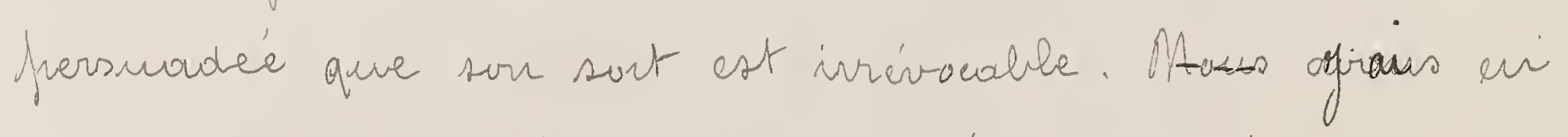
récemment sous les yeure un cas d'autrasuggestroon de ve 
geure: dis que le malade eut afparis que la mongeuse a'ámes avoit amoncé sa mont il acpoussamon senb.

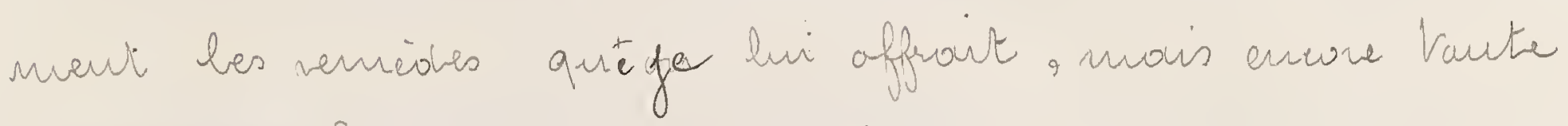
mouriture. Sa mont s'est fourolement prodrite au lunt de quelopies fours.

On croit qu'arout a'ctre monogées, les ámes sise' sout enfermées prè des sotiéla dours un wase en torre

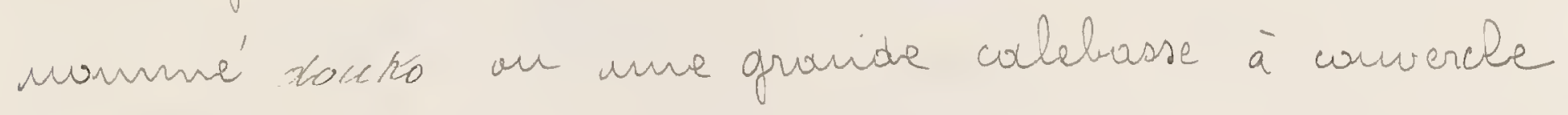
(fralensfora), qui we servent qu' à cet usage, elles y de.

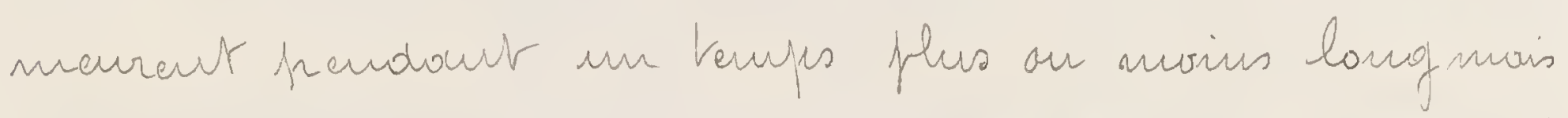

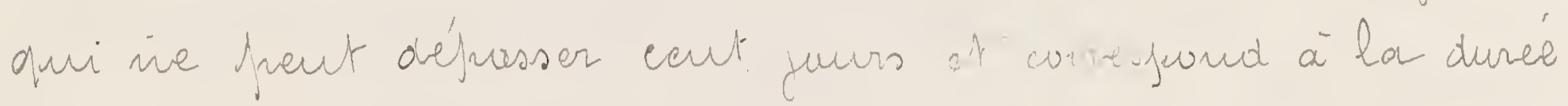
de la maladie. E'est arfir farmi l'hovreur d'un sabbat auquel tioutes les sorcièves des emirous sout comorgués, qu'elle wir être mangít.

On considiere aussi conne surciers les grognasé: qui seraieut de méme que les Geno-sola les descendants

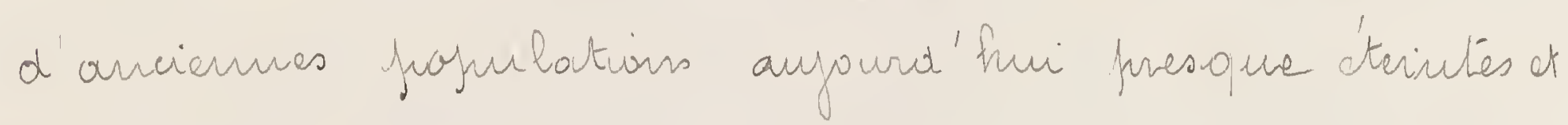
qui auraieut domine' dours le fuafs avout l'arrivée de Maba Gubir. Hls se recomaissent à teurs nous dé fa. mille Jébio', vent) it da-cíadro (mouge) its prement obt. on commander aux pheiromeines raturels of se venger ohe mal qui its recoivent en dechaimant leselé. ments contre leurn amonis, an en attirart des calamitiés sur les localités qu'ils habitant. Ils prowoopueur à leur gié l'ouragour of la se'cheresne. 


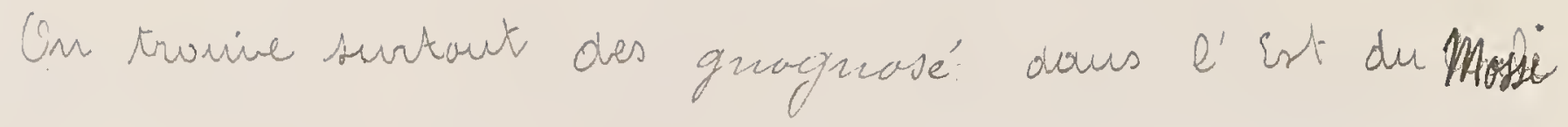
vir il constituent des villouges entiors.

Cae ménve que les omagnasé sout aftuarenté suee be

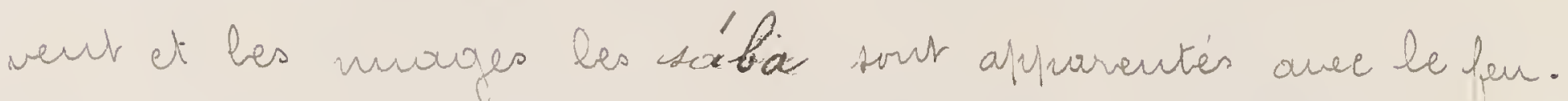
Leurs ancéctres étoicut sussi étahhis dours la vegions auaut

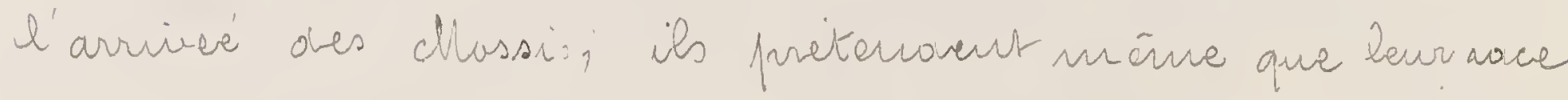

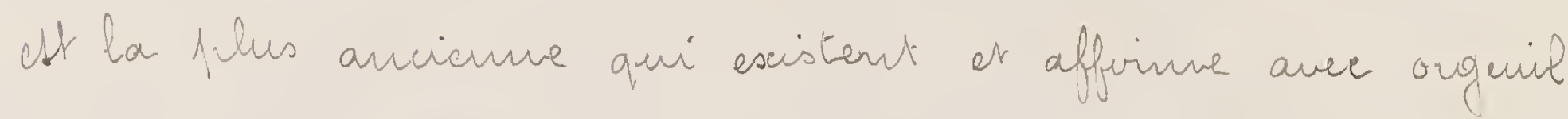
que "créés rimméoliaterneut après la pluie ih out narroés bes prenciars les nécultes de la terre...

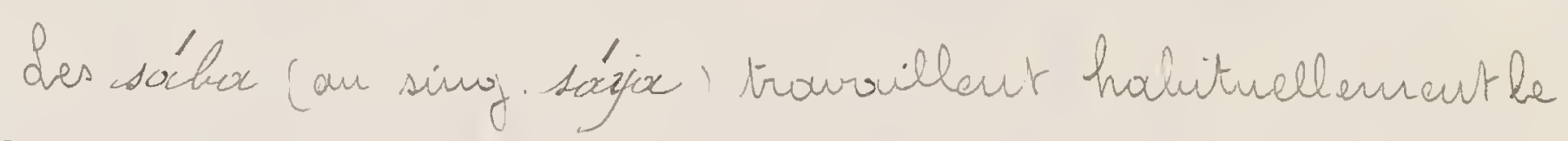
Per. Leur vỉe en ce qui tunche à la soricellerie est sachusi. vement benefique. On avit eirter l'endroit air est toun.

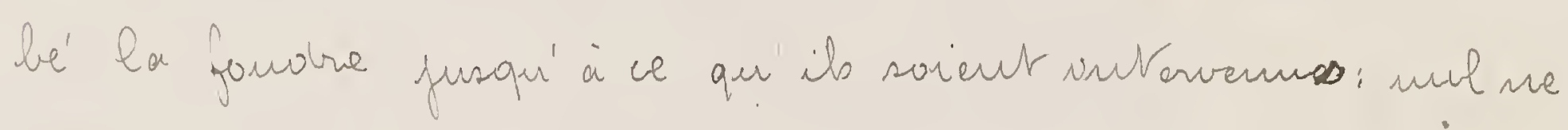
peut rours s'coepuser à la mort foorter la main sur l'olyet

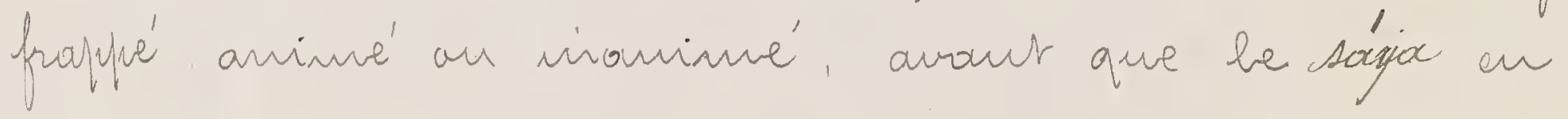
ait foit le taur on l'asprergeaut an moyour d'mu lifus. de dout hi sucl à be secret et pronongourt des founules sacronnentelles. Sur ses indicatioins on creuse an Four daus

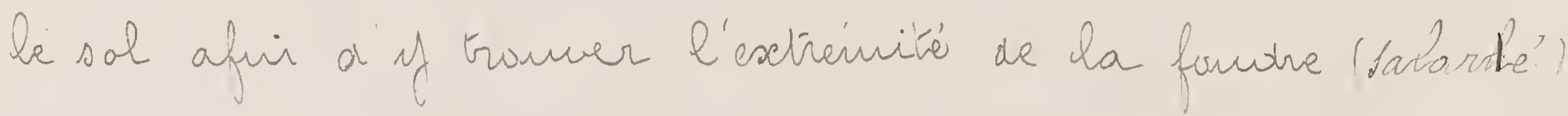
fín ans dongeneuse Kout que son extraction na pas en line. Le coyps de la personne forrobrapie seste en place at me peut ctive enseveli fusqu' à ce que l'intervention aur saja ait weruth sou contact insoffersif.

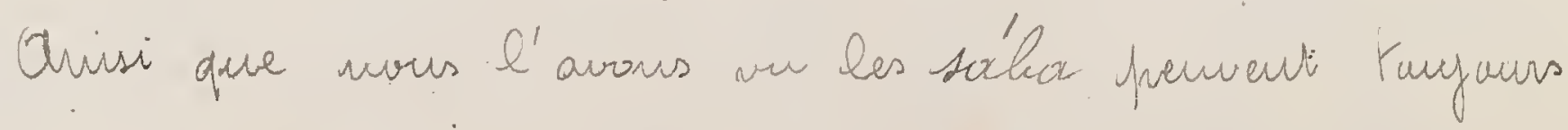

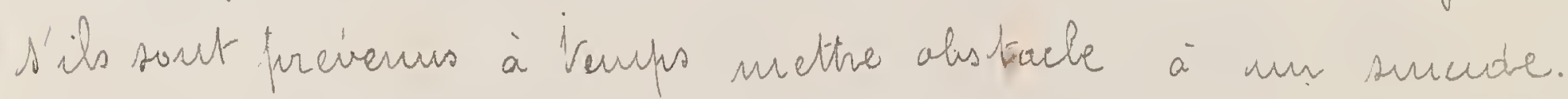




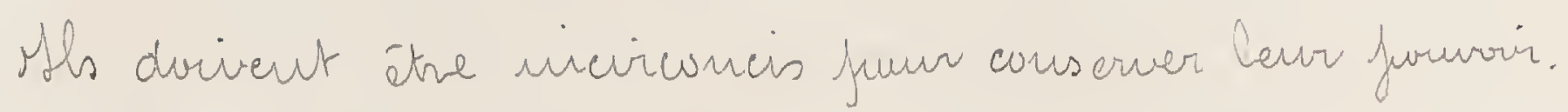

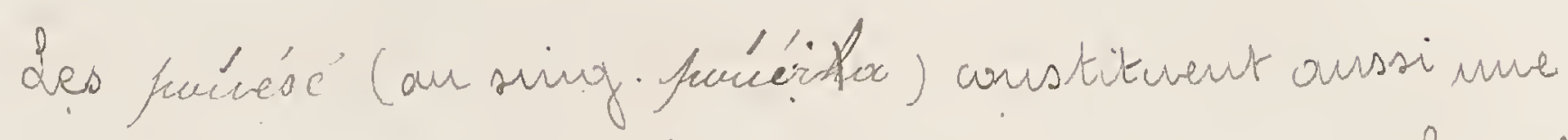
catégarie de surciers cunt la vertu eat heriéditoure Leur cheff

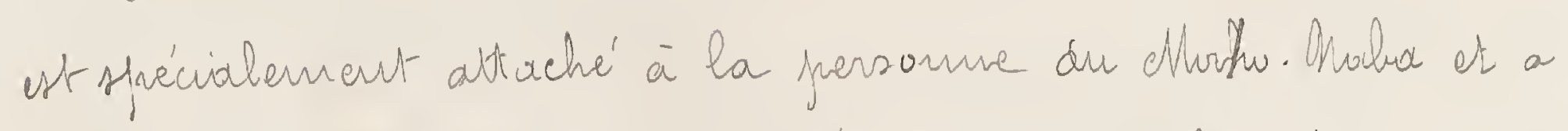
le proude de dignivaire de l'enfire, sa fonctions est an. jurrs hivi purement hanorifique. Anout nothe corrivée dours le paup il opeérait en général tors les Krois aus. Ossis de. iout un vase de verre (forié. laxiffox) reuypli d'ean dars lequel il jetait préabablencur la pondre mystoriarse de

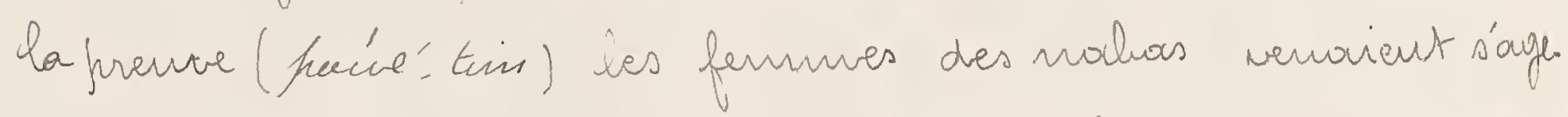

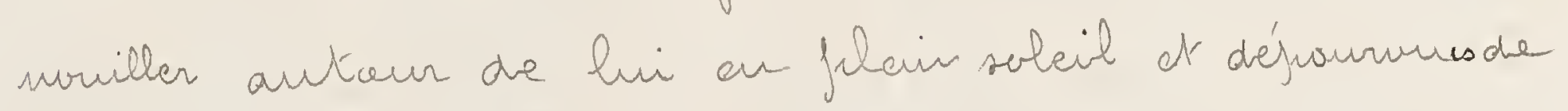

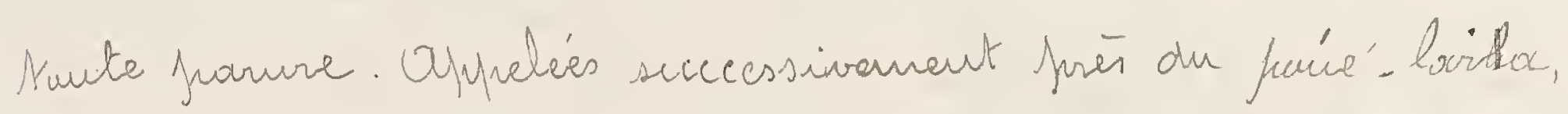
le surcier considerait aver attention le hiquide con'? is dours ce vase of decrétait si elles avaieur quelque in. fidelité à se reprocher. Oaus ce cos la comprable átait a'abord. fructtée fusqui à ce qu'elle ait fait l'aver irconstancié dre sa foute ot révelé le nom de son confilice; fuis mise à mort en mience temps que ce dernier.

\section{In Kenps oudinaine lossop' on avoit des frerues meime} évidentes de la culpabilité d'une funme, celles-si devaiaut être confirmées far l'éfrume du frocié. tisse. Lavecherche et le chätiment des sabíba mangarses a'ãnes dxait également du sessant des fraciésé. Lasquie la muatralité nifoutile revêtait un caractère de fréquence uisolite une éfreme gérèrale était déciotée faor le Moofo. 
Malua, épreme à laquelle tautes les vieilles fermues du village '́rareut termes de se sounctre. Là aussi la mort conskituait la seule souction.

des gris. gis on anulettes an usage an chosis sont nimonbrables. Leur confections nest jas farticulièrenent devolue aux sonciers; taut le mande s'y comait flus on moirs mais on consulte de préférence des visilords expé. rimentés déteuteurs de frécieuses recettes qu'its hivent volon. tiers à leurs fuareuts et curis. Les cendres, le charbur juché de plauties vorves fiogureut presapue Énjours daus leur conjusition.

Les gis-gris servent à Vitre proventif on curatis contre Vautre sortie de nuaux; its serut utilisés dours le lut de mever à baume fir des entreforises dout l'issuve est inceataine dre. Anidiquess ci-apuès les plus réparious.

Le overara-tiga petit sachek en cuir contantur be charkon d'une plautre particuliòre of qui perwi à un bracelet furté à lachaille. Coutre la moasure des serpreuts.

Le sonfo-liga hetit sochet verformant un mélange de divers charbans fibés, susipendu à une harobe de air

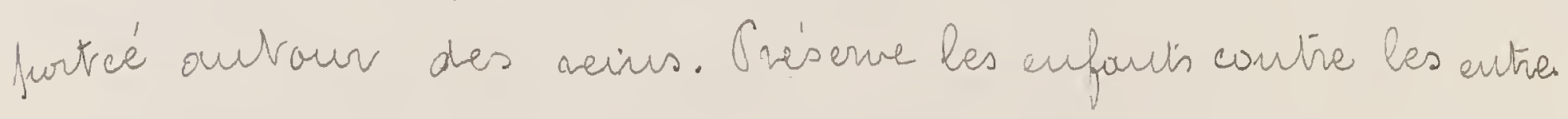
Prises des sonciēres.

Le fouson-tiga jivetit bac habituclternartsuspendu an unur de la case qui contrieut un häte d'éconce de fi.

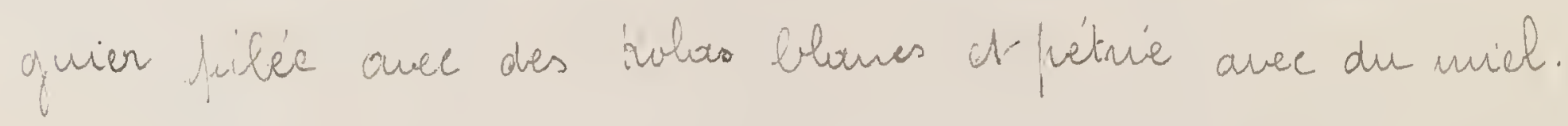


On quite de cette niacture avout de Ceuter une dérrabrehe dour l'issue est nicertaive.

Le fwiklo-tion. Onène genre. An fiel de bouf ot du

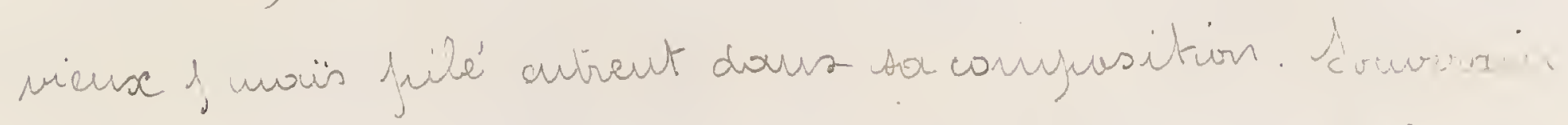
wutre les moux de veutre. Oren irauve un sochet de neserve dous Varte les wases.

Le youga - tin pelit sachel de cuir suspendu aux cheveux. Srévient les courp à la tête. Xle puruvir de sendive four

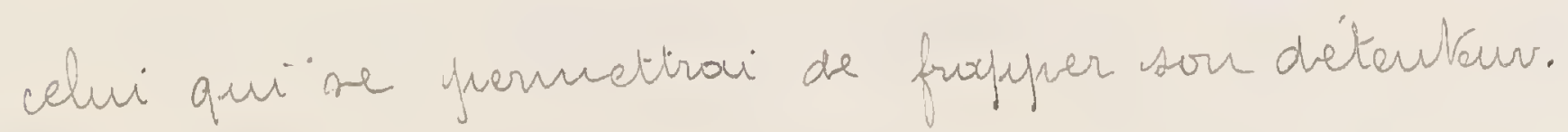

de borle'-turs, opeinati, qui consiste a cuire ensenble certaines acines en promancout le nour d'une persomme fens.

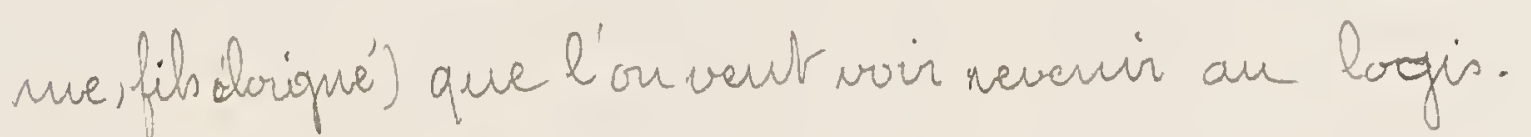

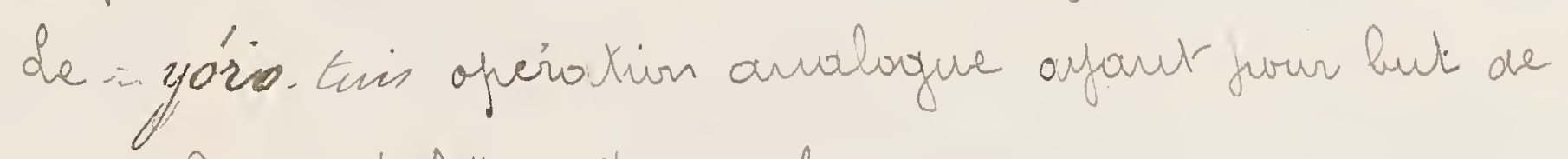
faire esser la stérilité o'une fermue.

de Kowse'-ynaga bracelet de uir ani confere la bruge'. vité à celur qui le forte.

Le niri. tirir. Sovte de cullier en ecorce de sousautio. Préserve des maladies d'yaux.

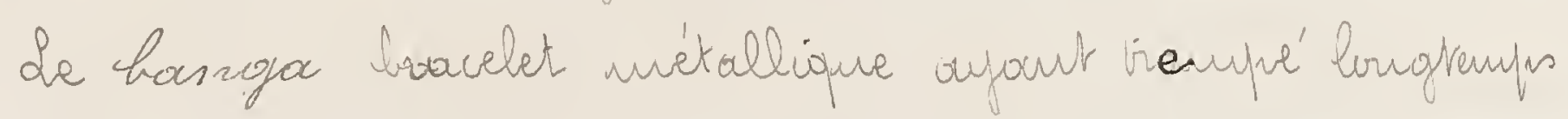
darrs un hiquide spécial. Onde porte habitiellement un furiguet mais il etr scusiblement plus laroge, de torte que pour le faire achierer il four be funsser vers le cande. e'art dours cette position qu'il posside vaute son efficacité. di juar exenule son déteubeur fosssait en fustice, il rerait assuré aufout frocédé airsi, de s'en tiver aver un mi. nimum de freive. 
Le rérité-tigo miscture compliginée contenure daus une corms que l'on susperid owdinairement à l'interieur de

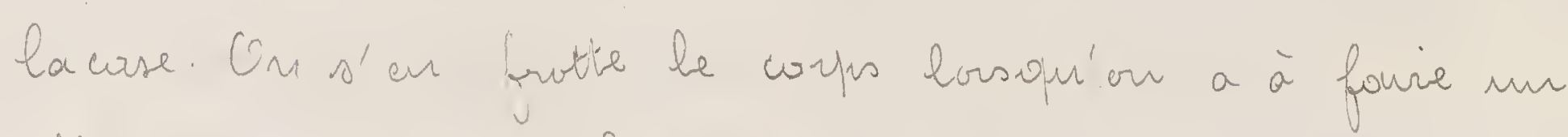
effout exceptrioncel. Se rérike-Aga pent dut-on domer nomentarréneut è un cufart la force d'un homme; it vend les charoux plus napides, active le retiur de la santé cher les consina

Le zouré, quare d'aninal (le jhos stoment vache, cheive, gazelle an hyine, susfrenduce à une condelelts que l'on nave jaorfois jour abterin un thes haut dergréd'ef ficacite. Premunit contre les maunaises rencoutres, pernuct de farcourir sous danger une acojion peur sirre, de. Sorsopict

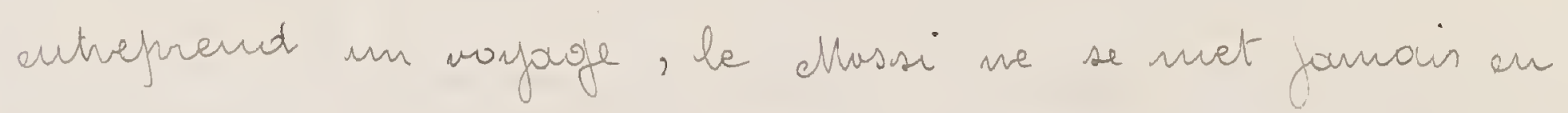
wite sours sons youre?'

In ce a concome le Boussangsé ijers signaleracer

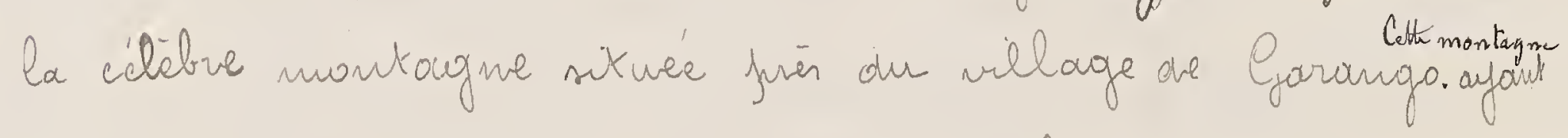
a diverses vefrises servi de refuge aux habitants contre les envahisseurs, devrait à cette circonstance le cultie dantelle est l'abjet. On obrieut fuar clle taut ce qu'on désire. Yl n'y a qu'à se voubre à l'enarait consacré juur les offrandes et à y ígarger selass les régles établis, un foulat que l'on jette aussitit à quielques fras: s'il netombe sur le dus la nontowgue agpée la frière ct le voen sora exacé. An cas an le froulat tombe differanment on doit recanmencer 
le sacrifice avee un sccond ct airsi de surite fusapi'à ce que l'animal inmalé conserve ar tombant l'attitude vou. lue. In temps a'épidernic, de séè esse, ete, on fout á la montougre de Parango de grands socerifices qui sout ardon. més fuar le fróéré de l'ariorit.

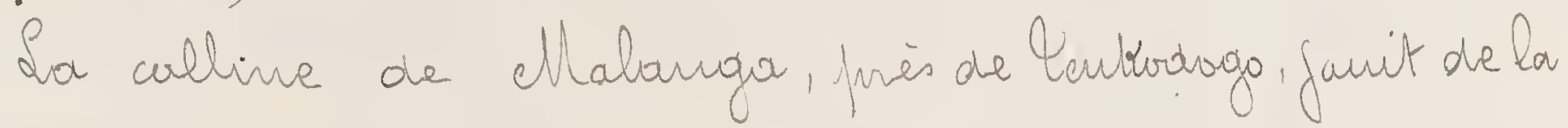
mêrne réputiation et est l'alyet d'un culte arralogue.

Ou Gauranusi on ervit connme au Mossi à l'eseis.

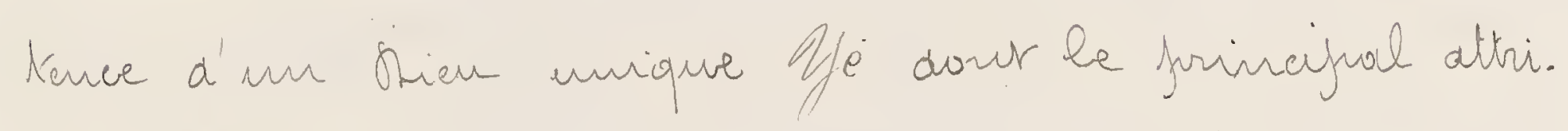
but ast le tomerre. Les rivières sour l'alyet d'un culte Apécial et recaivent fréoummant des sacriticas an joulets et en hérail.

Cour ce qui est des Ceulh ugess n'a rien de hiess interessaut à relater. Its se disent taus nubuhnous moir se montrent en foits de veligion d'une ignorance crasse. On sein a'agglomérations relativencut considérables telles

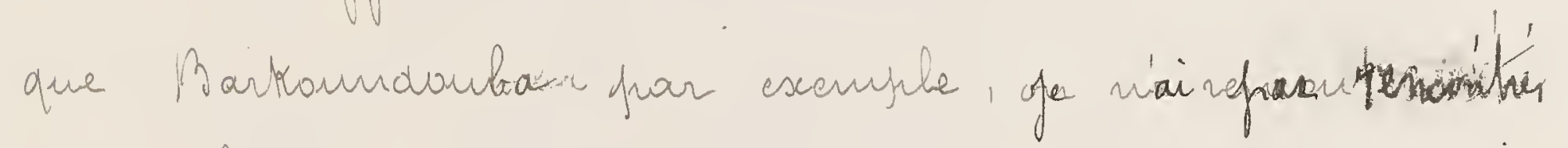
un seul hamme qui comonsse l'arabe, sain to to rwaver dous taute la localité un sent axenfulaire du Haran. Darrs certaines régions vers Qeutavovgo farticulièrement, les Peulhs out firi joor adufeter un guound nambive de sufrers. titions Mossi; la plugart d'entre ense boiveutle done sous aucun scrujiule.

Les Mar dés. Marsés praraisseut aussi ignonouts que les 
Reulhs ales chuses de l'islarn; ils viveut Yaureforis monin isolies, vecoivent avee cajarols les marublauts étroungers os dours les nombicuse voyages qu'its entrepremant axe-ménes dous un but mercoutile, finissent sauveut foor acopuerir sur la aeligion du Propthiete des notions assez précises. Partaines farmilles ou la dignité a iman est héréditaire comaissent

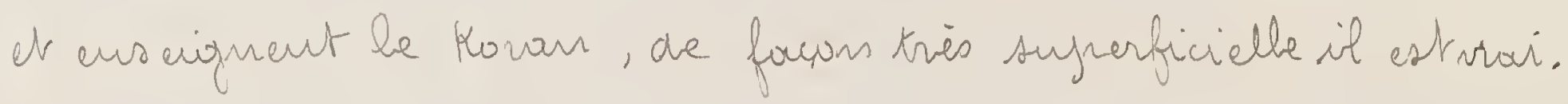
Leur coutact a nềre fini pour déternimer la conversion - de quelopies Cllasi parmi les chefs notamment; mais le ques de la pufuclations se montre netterment séfractaine à Yurtie propraganove islamique.

IV. Vie dociale

Tamille La fomille est la lase de l'angani. satkin suciale. de chef de formille yir-sohas est vespousa. Ple vis-à-vis du villouge de la conduite de vaus cense qui vivent saus son toit et en veranche il a chey fui Eaus les furi.

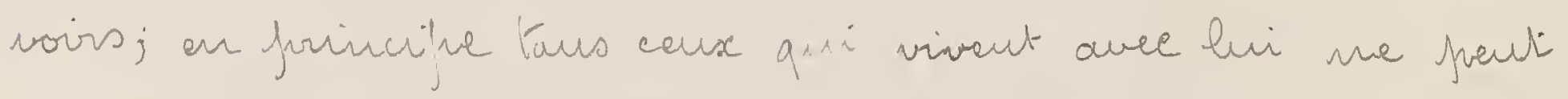
possider autre chuse que quelques effets an abyets nuali. liers. C'est hir qui décide des fuurs au l'on doit trourailler ause champs, qui gardie bes nagasins et fait chaque matior la discrilution des graius of des cauris néces. saires puur la journée. 
Co promoir due cheit de amille, airsi que e hiritage:

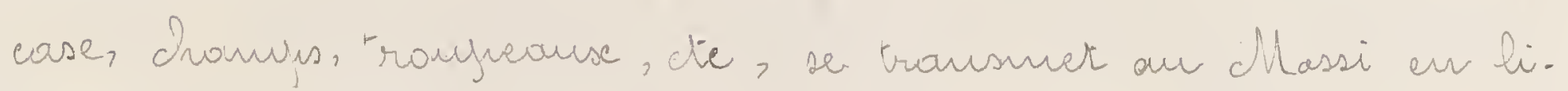
gne oirecte. A difaut d'enfautis kancka) garçous use filles, ce sout les frères utèrin (nox-bisi) qui héritent, à défout de ces derriers les frères cansanguins (ba-bisi).

Iu Gaurounsi au coutraire l'héritage se trousmet exclusivement de nuäle en míale en lique callatérale. A l'ainé wurcecida sou coutet et twus les autres frères frar rang d'äge. S'il n'y en a tas, la succession vevient an plus àgé des enfarts de la seconde gérieration quel qu'ait étá le wang de sun peere juarni les náles de sa profore géné. nation.

a la mast du Chef de famille, les fermues de ce

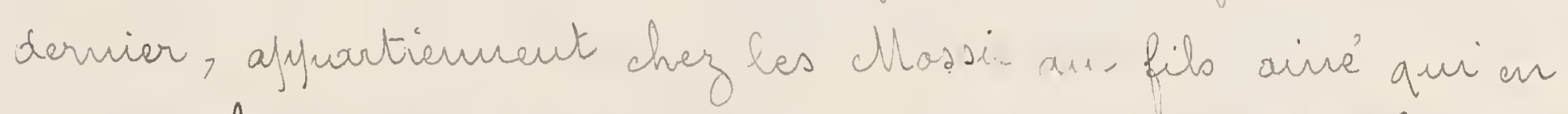

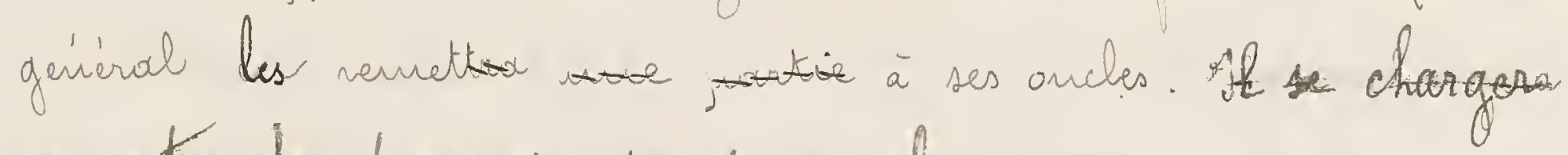

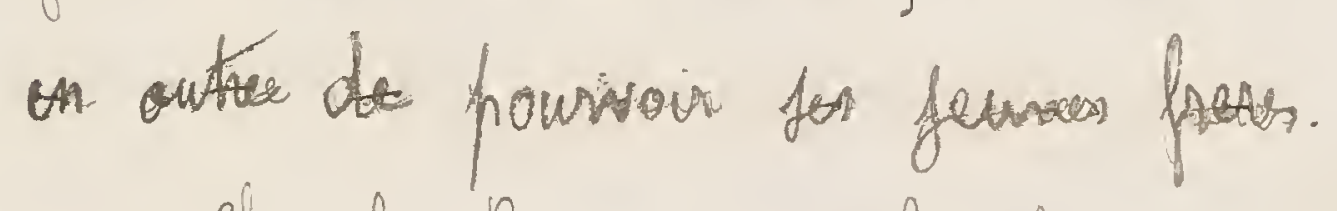

Chey les Boussariga. les fermmes du défunt de meẽre que ses enfouts ei bas äge sout la profirièté exclusine de l'airié de ses frères. S'it n'y a fuas de frère, le fils ârié devient chef de fanrille unais dans aucun cas il n'ar be droit dédisproser des femmes de sour jeere comme celar se Jratioue chez les Mossi.

Ses Coulhs hiritent des bieus de lours fromatits comme les Massi et les Baussanga:; quant ouse femmes du difunt elles se vetient en geiveral chez leurs enfourts ou premuent de nonvearse maris si cla leur plait. 
On renourque dome cher ces divers jeugiles ence qui concerne la condition des fommes ar fuivt de vue de la succession, les differences ci, après: Les fermues Mossi

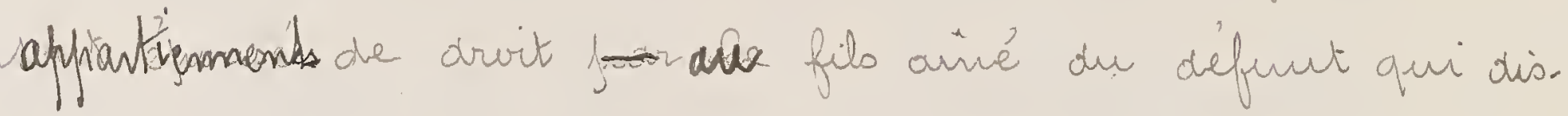
fuse d'un cortoin nombre d'entre elles ot faarticulierianaut de sa moire au frerfit de ses oucles at frères cadets.

Les femmes Baussariga of Gouramiga devieumant

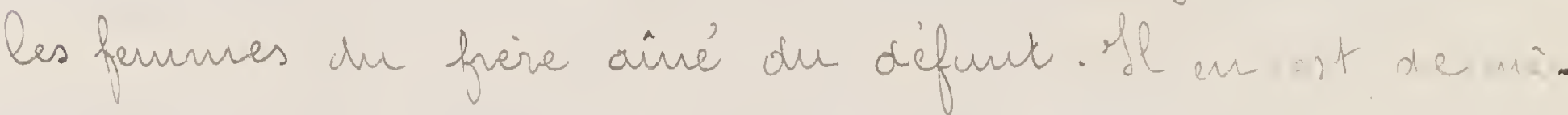
we cher les cllardés. Marsès.

Les femmes foulhes à la nort de leur mari, recour. went lour antière liberté.

chey les clossi les termes qui serveut à déterminer les degrés de fuarenté sout les suivants: Arrière ground.

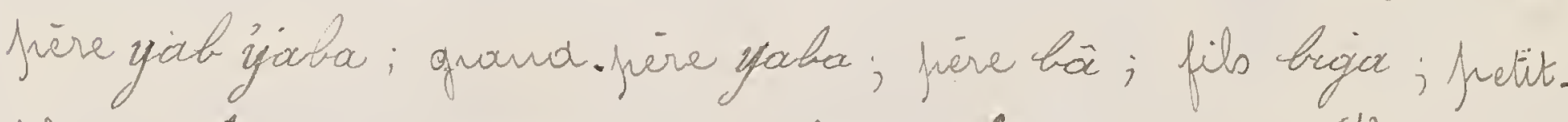

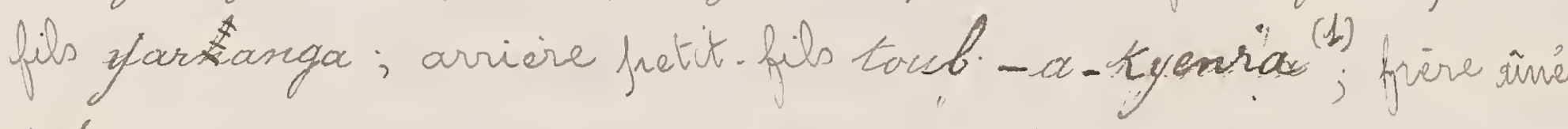

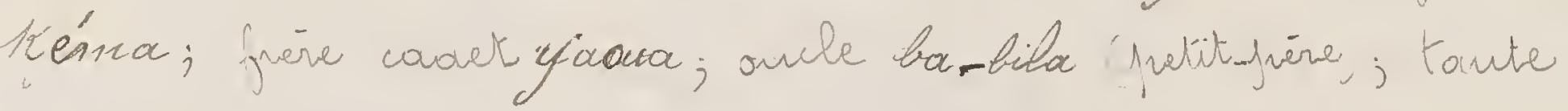

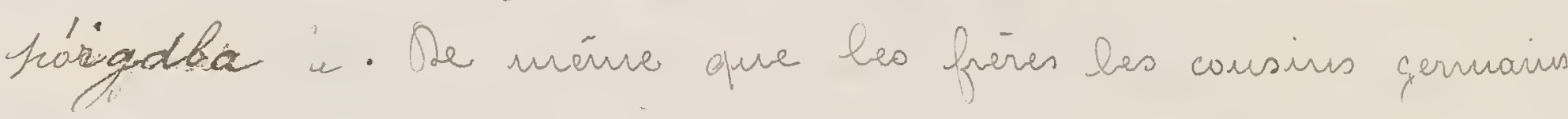
our issus de gemmains pusopu' à cing ur six geninénations se servent du mot Kéma furr se désigner réciproquernout.

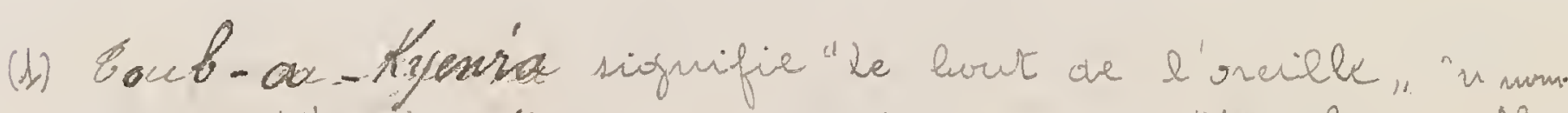

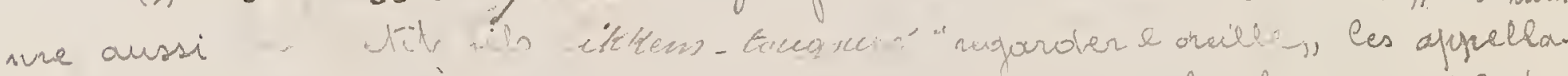
tious bizarres triewt leur origine de la suferstition lvcale que vorigi: $l^{\prime}$ ar. rière palit fils ne sromait frarler à l'uncille de son aicul sours nut.

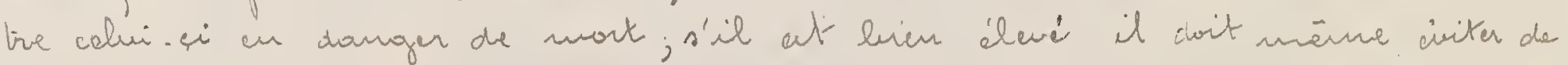
reparter les acielles de ce demier. 
Les peulls se sevent des expressions sumantes: arricie

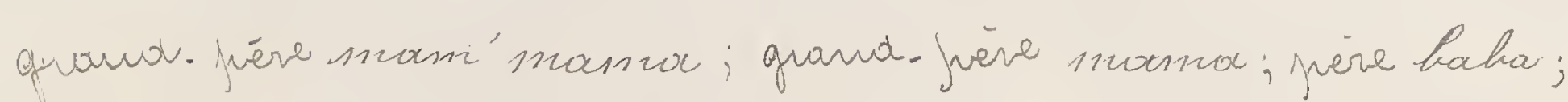

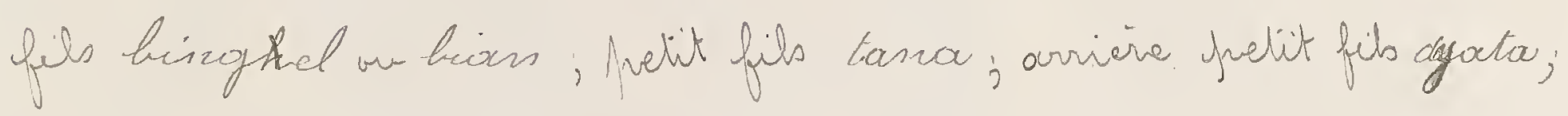

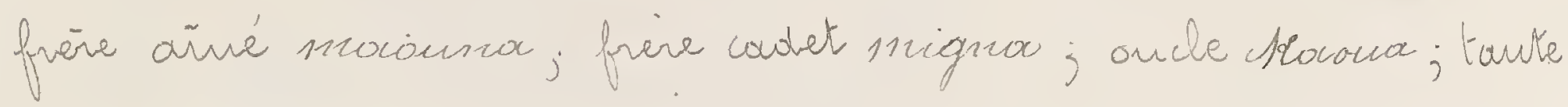
gogyo.

L'aduption est proutiquée fwartaut souf aur Gourounsi or coufeire les mèrues aroits que la filiation naturelle à la condition qu'dle iert de notorièté fubliojue cr que cehi qui adopte i'ait frois a cuffunts.

Le chef de fomille a seul qualité jurur décider du mariouge de sa fille, it n'est yamais tenw conyje de l'apainion de celle. çi qui d'ailleurs ne sourait se nuon. nifes ver saus inconvenancel. O' autive part, il arrier le flus sorvent que les fernmes sout fiancés dès laur plus jeur

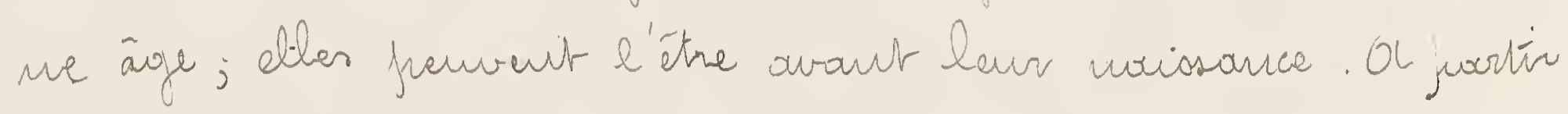
dece nomeut l'homme à qui elles out été promisesect Soun de venir se methe taus les ous à la disfusition de son fut'ur bean-pieve pusur le travaib des champs, il doit

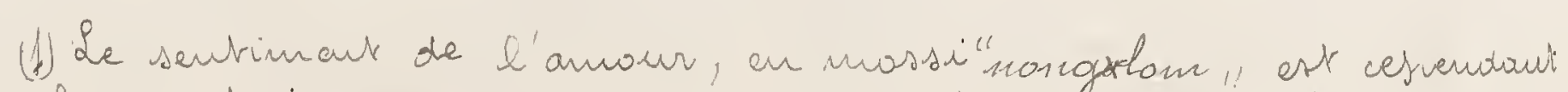
asser vif en certaris cas, movies cefuendaut cher les ferres filles que cher les ferrmes a'un certrain äge (Lo à zo aus). Maus avors ver qu'il est

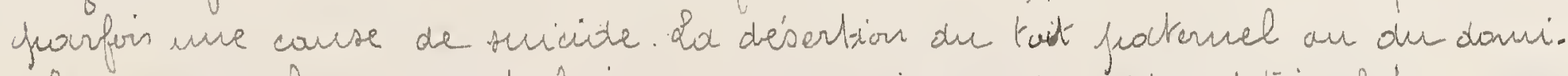
cile conjugal furer satisfaire une frossion contrarie'e ast thès frépupuls.

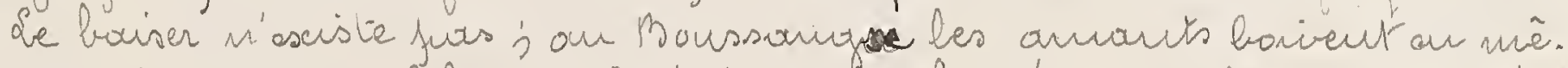

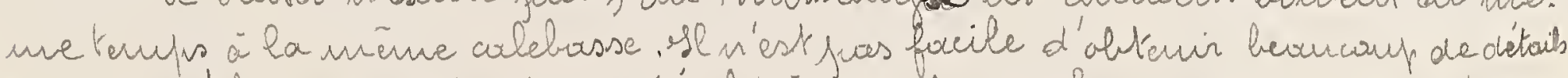
surce sufet les nidigeires dtaut en geiréral très funtibonds. Les fratiques contre vature si

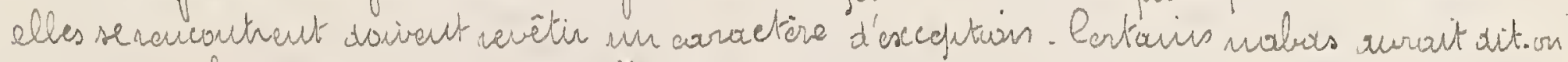

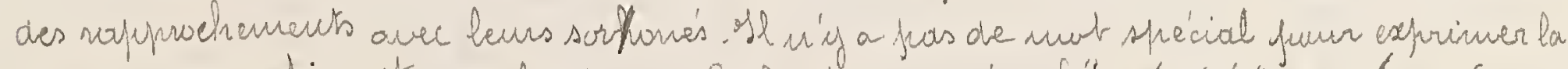

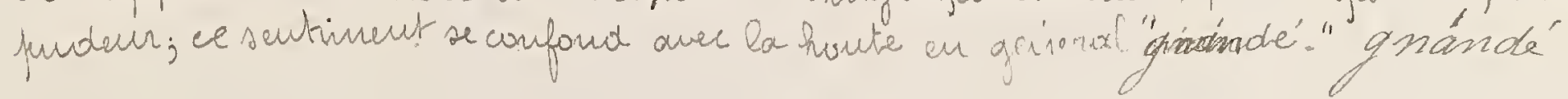


eni faire des cordecaux à certorives épuques et il y moulie d'aur.

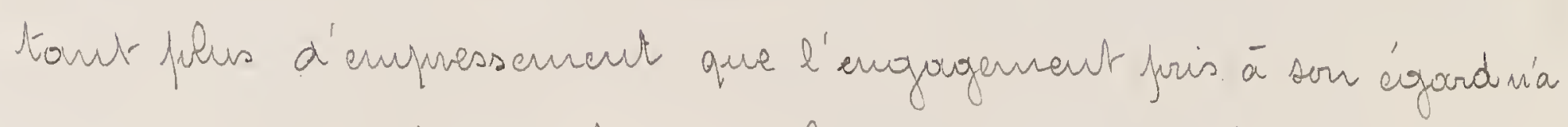
rien a'inreiveable. Quelquefors il couscut à vive chez le pēre de Ax fioncée en qualité de datroné ot y venjelit en quelque soute les forction de asmestique.

A l'áge de sept an huit aus la jenne fille commence à aller de Kunpo à autre dars la farrille de son fiancé.

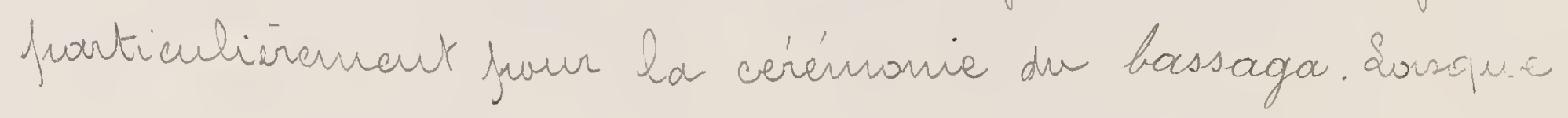
son fiène fuge qui elle est nubile il priviewt le nari d'avoir à verir chercher so fermue ot fisce la dut qui sera paufe'e. Il n y a jas de contrat projerenrent dit muxis be numtout de la dot stepulée, n'est pas cxigible d'avance; taut' qu' il u'a pas 'ké necu jar de jeere il est tautefois admin

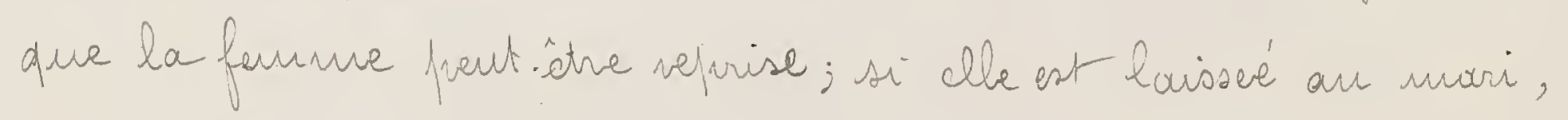
tes filles aui naîtrout du nariage alvivarticnatrant serr. vent an bear-jerve qui paicra an disfuser à son api. En gèneral la dut varie eutie 5.000 et 401.100

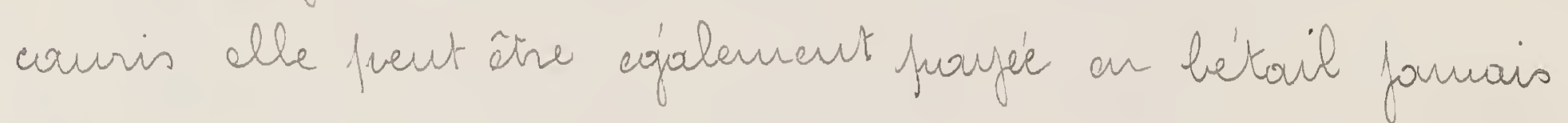
en chevaux; ce serait expuser l'union à être inféconde.

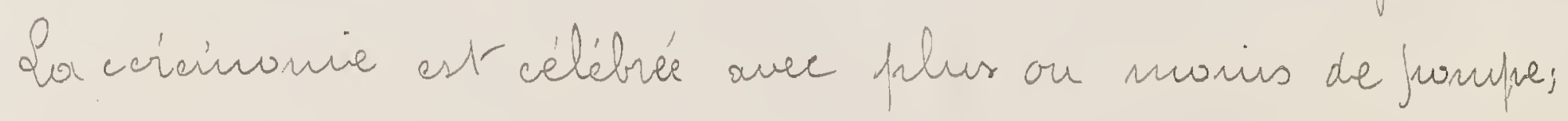
elle dome taufaurs lien à des onjies de dom, des darses des véfourissances; sulle, la nouvelle nariée est Kenve de verser d'abomatautes lamies of de noutior un wif regret de quitter le tait jatemel.

Il est adris geírichement que deux sceurs funclles der. vent appartenir an mîne mari; cla est de iegle larsqu'il 


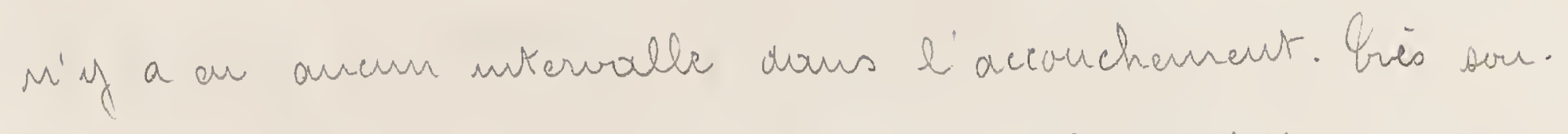
went les Baussonga: doment turtes leurs filles aurnếme nori qui quarse arisi à la fois deus au tris seeurs. Cette contanne a dé la régle antrefois; anjoura'l hi encore

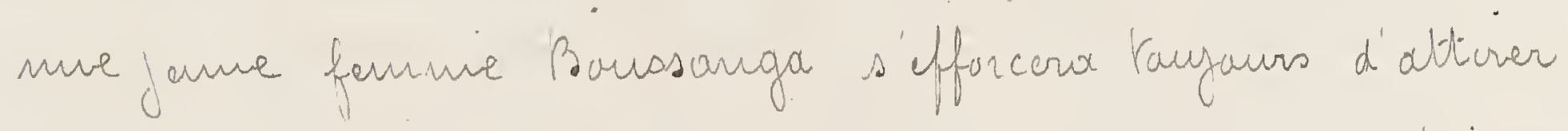
aupriè d'elle sa fenne sour surs le pretesete de l'aider dours les trovaux domostiques, et ferix ensuite de celle. ei la secoside fenme de son mari.

Crez les Massi, de la province de kiowjibla, an fuarti. culier daus le cantou de Qialgo, la jenne fiancee est en.

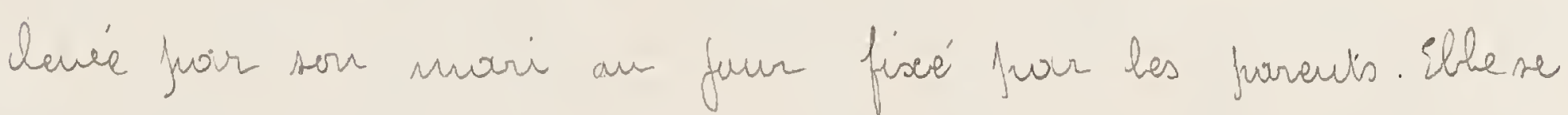
rend comme a'habitude sort an frits, soit dous la buruse

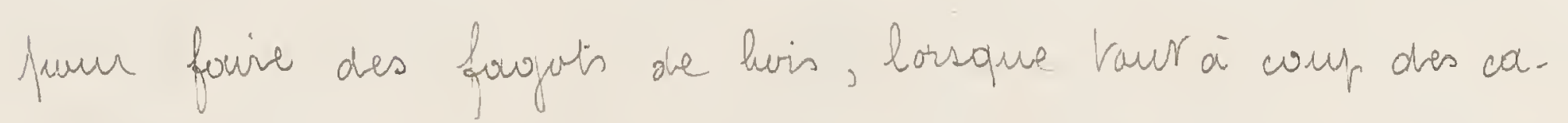

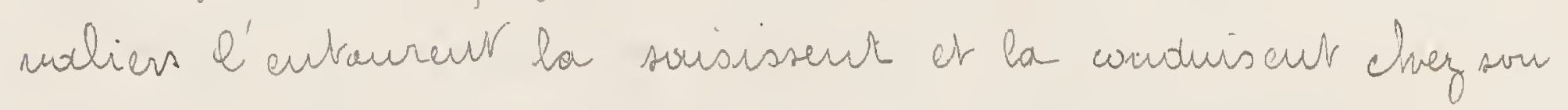
mari. E est le seul vestioge que yiais nercoutré an fiaup chassi du marioge fat capture.

La vinginité ar nomeut du morriage n'est fias con. tritée, mais le mari fait beancour de cas de la fidélité

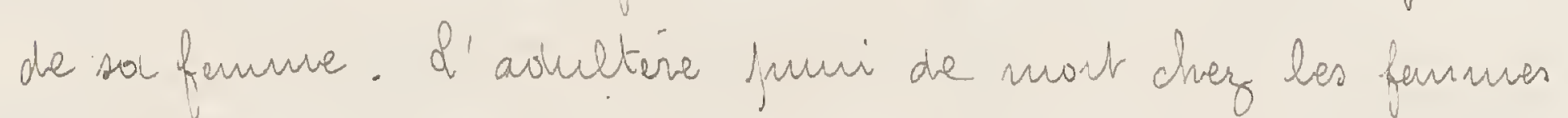
de Maba, n'entrairve qu'ure amende darro les autres cas.

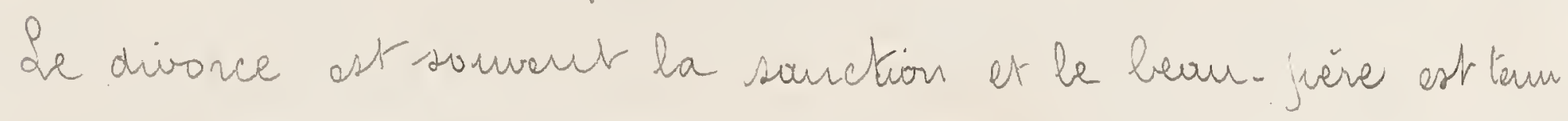
de remburser la dot.

Our Banssangá les fames filles out be furs sument des arrants ourout beur marionge; elles déserteut facilement le domicile conjugal si elles ne s'y plaiseut fors, danogéét. Coat elles se véfugient dours un villoge emeni avee 
laur complice. So farrille de celui-ei ast alors mis ar demene de veryplacer la femme du'un de ses nerubues a

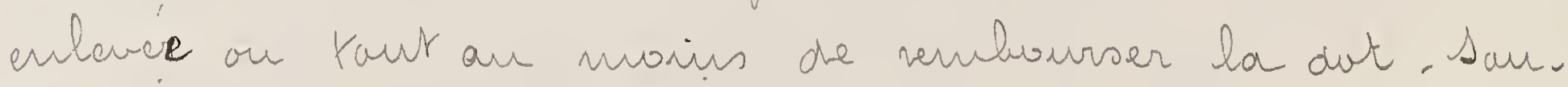
veut le moiri frustiré se sendera avee des amis à un manche' voisin et dés qui il verria une deme fermuc oir. ginaire du village an la sieme a 'wouve'un vefuge, it s'ar enguarera jour, la vialence. Ce genre de aajts ost extrômencut fréqueut.

dur Gouranusi le mari k ranjue' ausait aurvefors be arvit de vie or te movt sur bes coujuables surfaris en fla. grout délit; actucllemavr il se contente de aivorcen. Le divere est d' ailleurs tis fréquent dous la végion; outio que bes fenr. mes Gouranusi sout assez leigères, elles out somvent mauvain

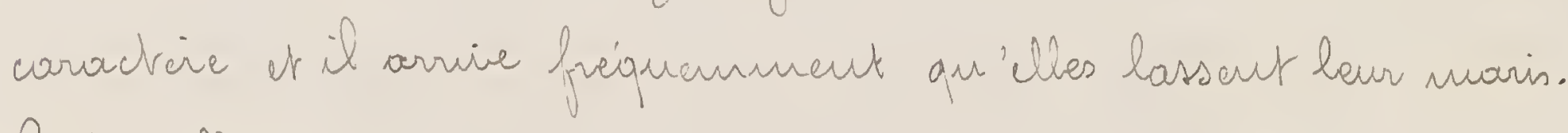
Carfors elles se plaignents de manques d'égards four s'en. fuir chez elles. Les frarcutis renvoject alors la dot que le mari a parfée. Comme au dossi douns tars les cas de diverue les enfouth verienneut au jères.

Les feurmes divorcées trouvent triès foulenrent à se re. nariar; actuelleneut frar nite des nourouges des Siabersnas les fennues sout envivon quatine fois moins nombreusesque les hommes. E'est frour cetle naison probablement qu'il n'y a has an Gauraunsi de prostitueis porofessionnclescomme an Massi ot an Banssongsé. If carsie cejpendant dours ce froups une forme a'aduetère dégal si g'ear peist 
- esclorimer airni, asser sinqulior.

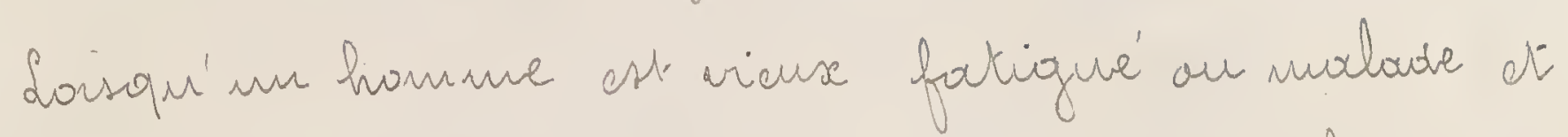

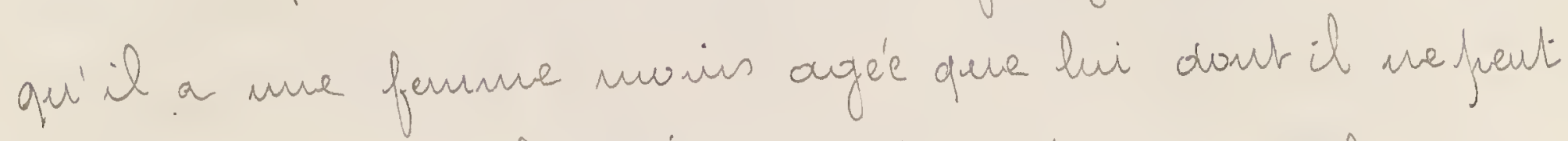
obtenir d'enfanis il arrioe souvent qu'un zenne hamme de son choise devianne l'anour afficiel de sa fanme. de jenne hamme en question dvit trourailler aux chanps. à la ploce on mari, qui le traite comme un domeatigumisis hi abanitame compeleternar ses droits coujugauce. Les emfauts qui maissont de ces unious appartienmout taufours aur motri ct mon au frère.

Chase curieuse, malogé la situation niférieures qu'elles fout à la fernme, les mocurs Gouronusi me laissurtpos au mari la liberté de cromper celle-ci. Sour le forire il est oblige' de se cucher et a'agir à l'insu de sa fermue qui jeat four ce nuvif excriger le divoree.

La constitution de la fanille at trowp ancieme cher

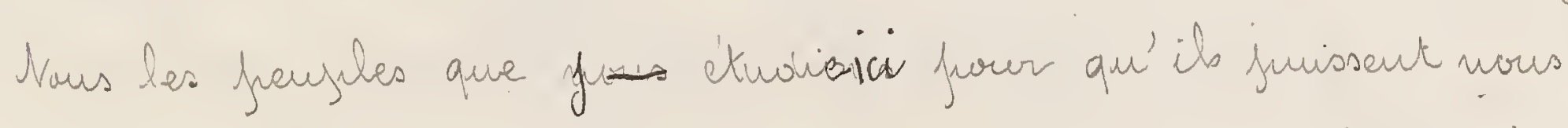

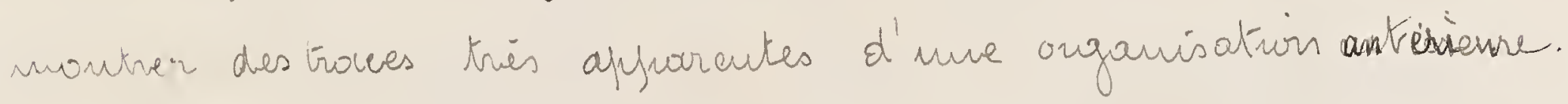
Il n'y a mille part des vestiges d'endorganie un d'exerga. mie; seube l'umion avec les lowbés (sattas) est nigourause-

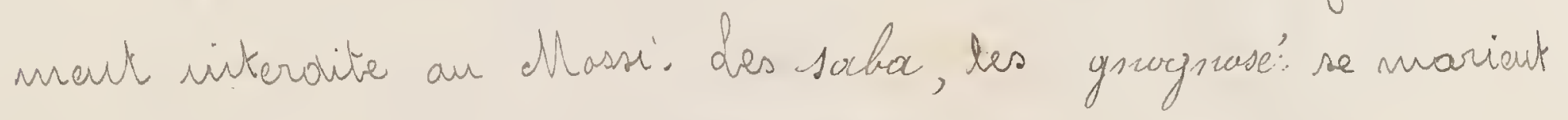
de Areiference entre ase mais les exceptions ne sout pas nares.

Cela, m'ancive à traiter de l'onomostiopue qui présente ici quelques fuarticulorités vraiment oniginales. 
On a cru fusquià ces dermieres amues qu' il n'excistout pas our Massi de noun de famible. e'est nue erreur; an

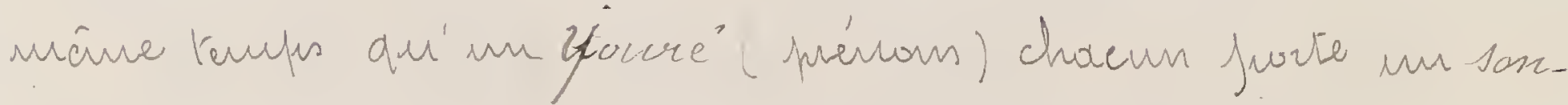
dère'. Le sondéré il est wuil me se transmet farmais de

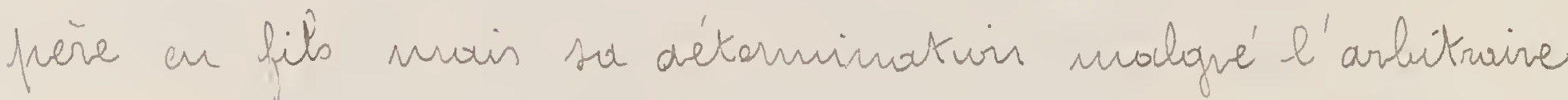
qu' elle porait présenter me preut sontir jour chouque fanille d'ure série unomastione domée. Oans cer. tarives localutés le sonodéré est attribué au nouvear-né sous ouroir vecours au soncier boxita mais ve demiver intemient en genéral. Il owit choisin de Mréférence le no. ment oir l'enfout fleure furer prononcer l'un après l'autine les soncdére' qu'il est susceptible de frendre. Celui qui est supfuse' l'avair fait toire, hi aestera. Dours cette émune'. vation be sorcier commence taufaurs juar le sanderié du guand. Fère et de la grand-mère qui sant chisis le fhus sou. vert mais en supprasout qive l'enfout t reeu le frenior

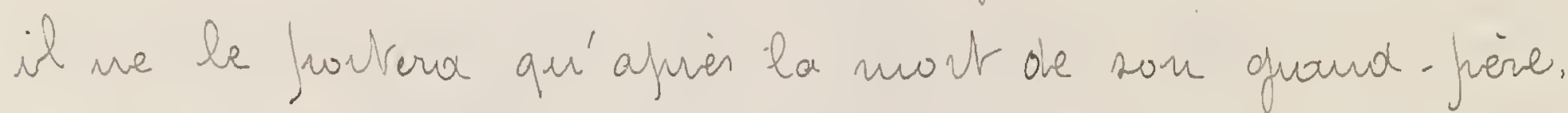
mort qu'il jourrait hâter s'il n'agissout airsi. He

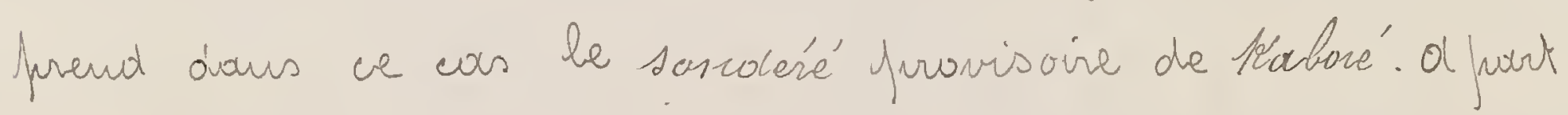
Trabone' qui est arisi le sublstitut de Vous les sonderé

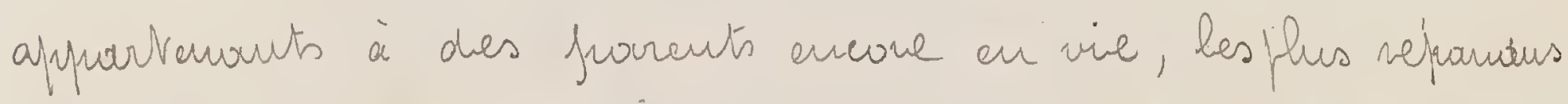

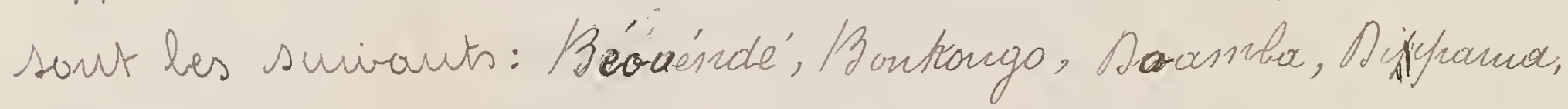

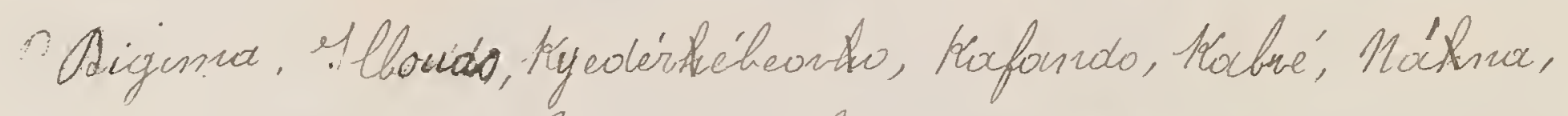

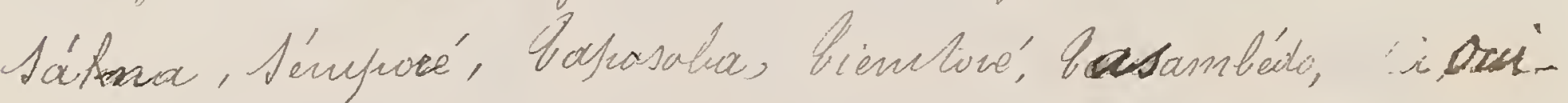

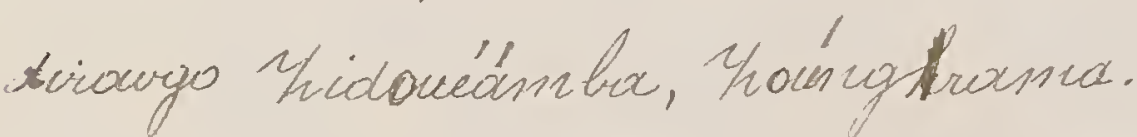

Chacun de ces sanderé doit corresigondre àun totem 


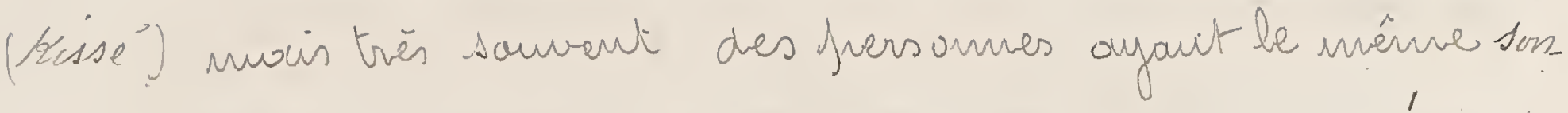
déré accusent des totens différent's. Yae nia nercontire de concoswance alsolu que là on un nom a'aninal ou

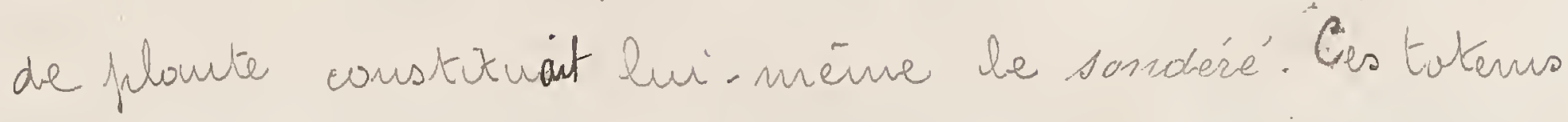

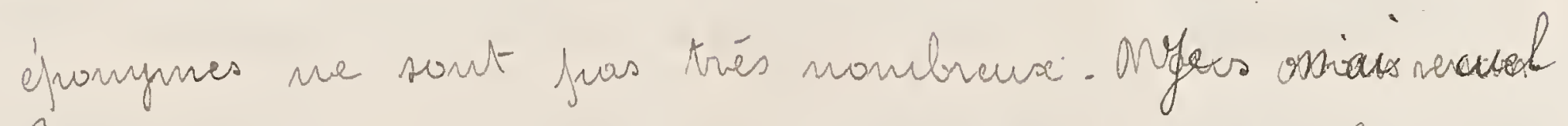

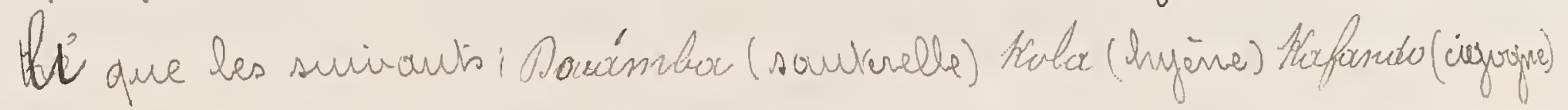

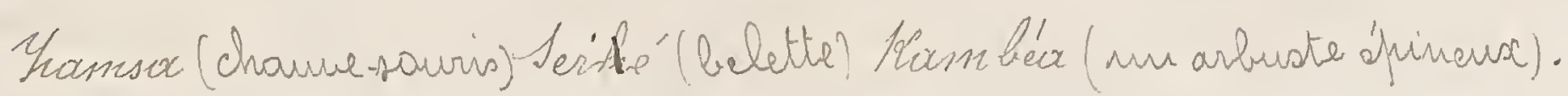

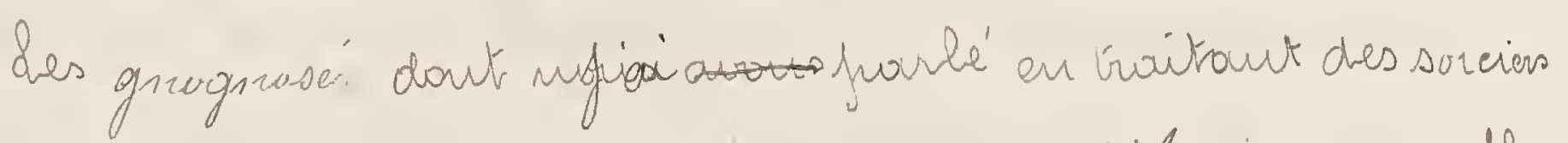

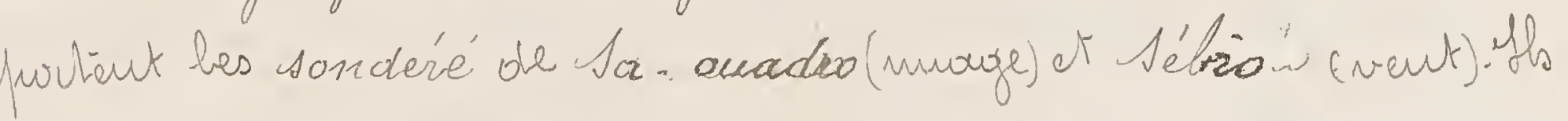
se disent applarentes avee ces pheinomiencs.

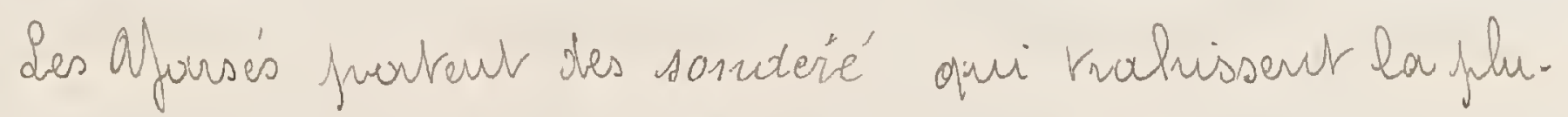
pout du Kenfs beur onigive Momolingue of sout trous. missibles a' ailleurs de jeère en fils: Fon 'da (Roné, Rande')

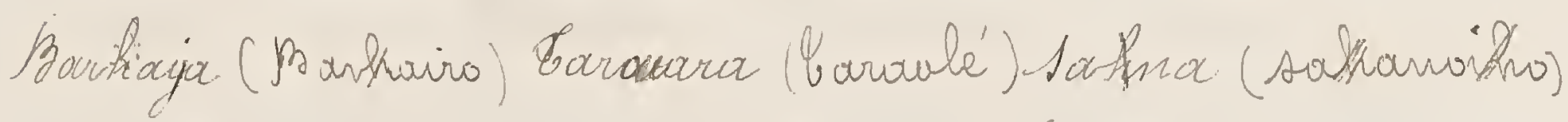

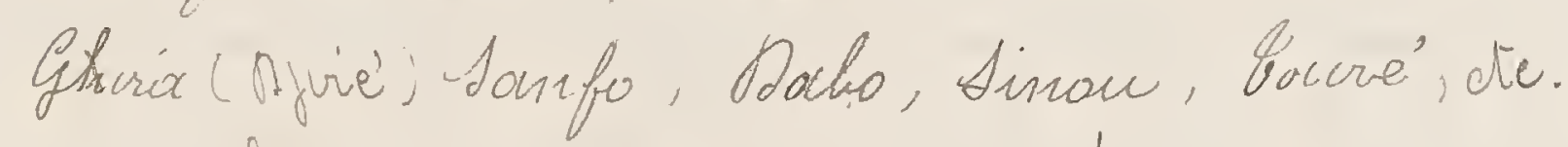

chez les Ceulhs fai croune surturt des Diallo, Bấ Bou et 1o.

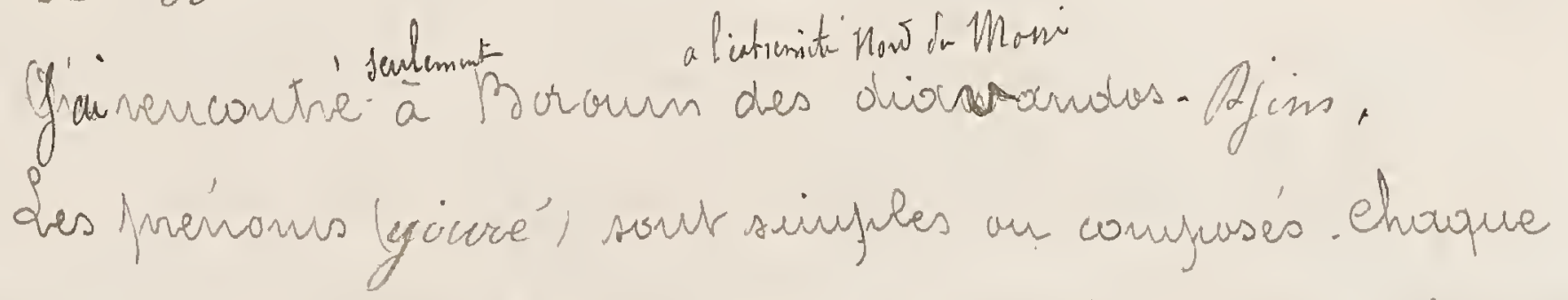
prenour siniple coustitue le funirt de defrart d'ume foule

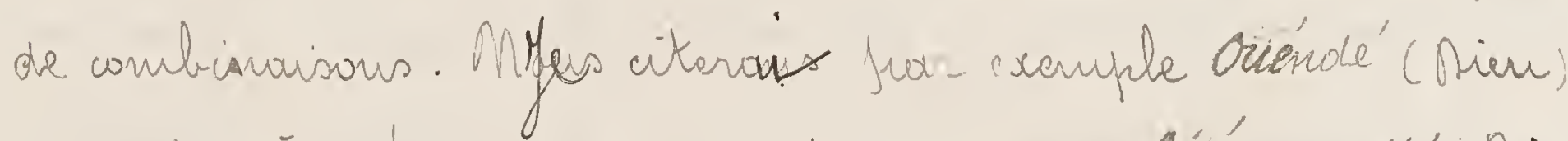

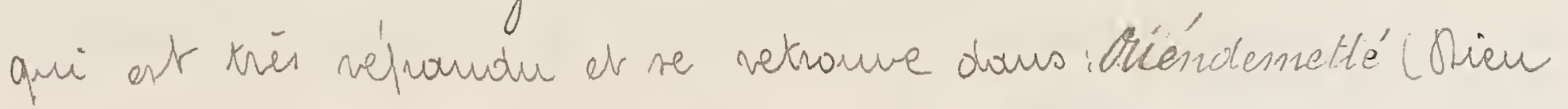

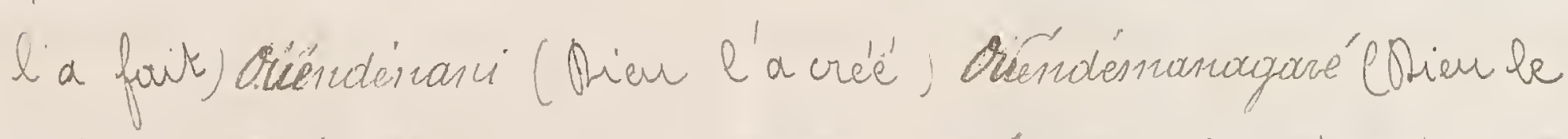

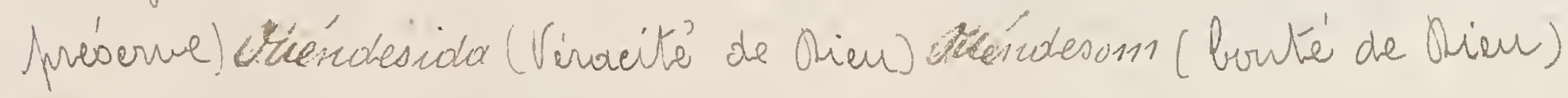

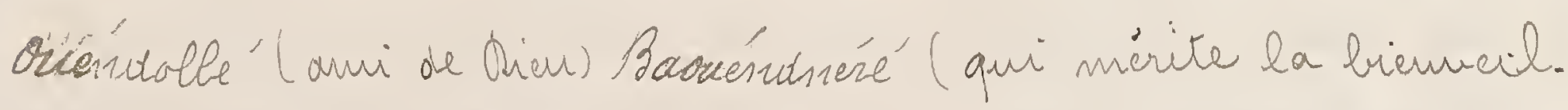

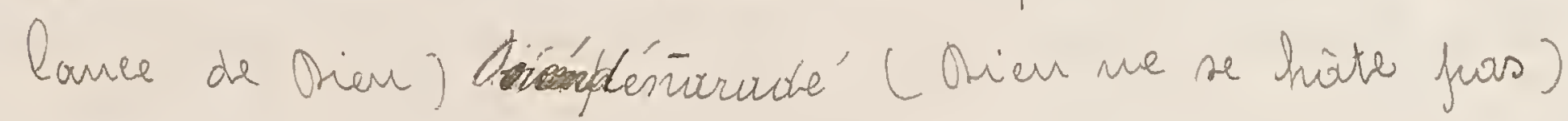




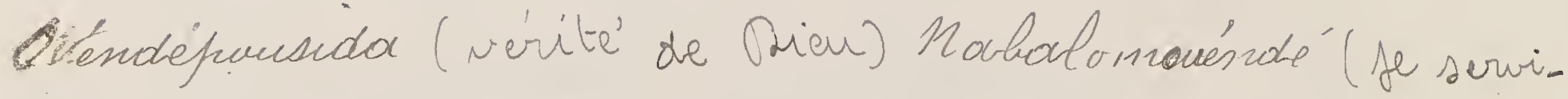

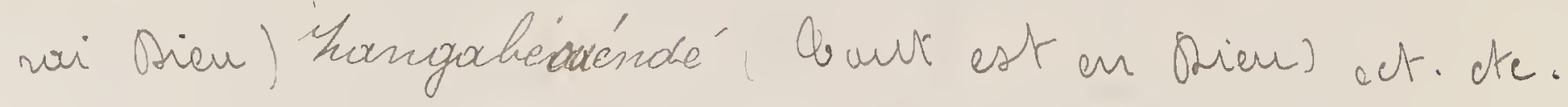

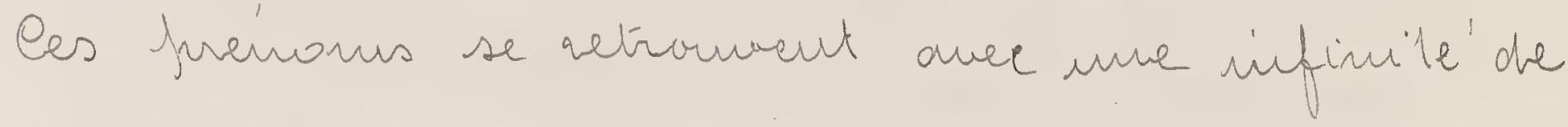
inariontes.

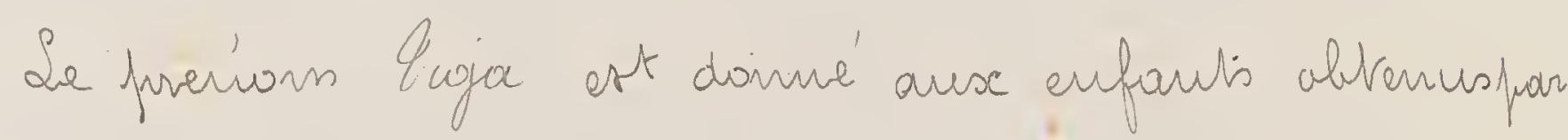

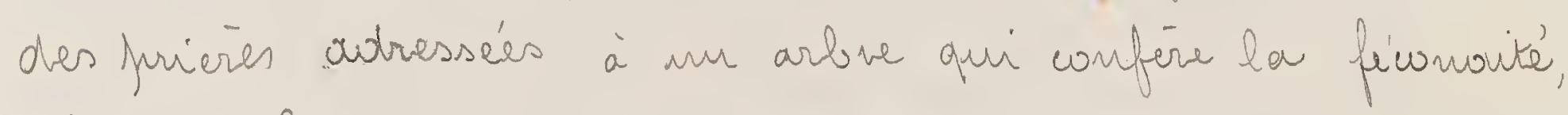
celvi de Geriga ause enfouts obtenus fuar l'intersosian

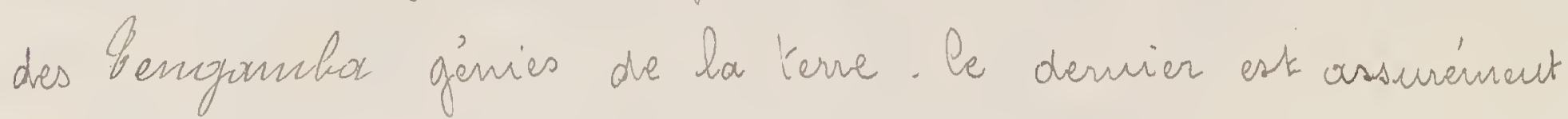
ke flus séponam.

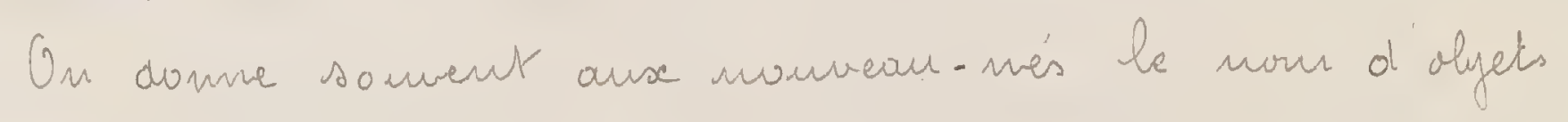

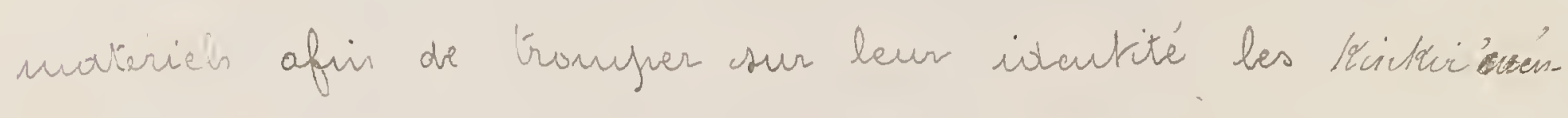

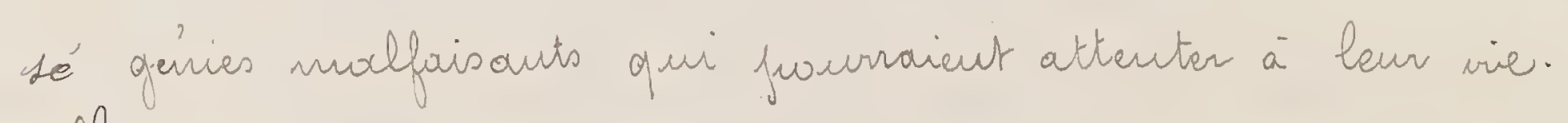

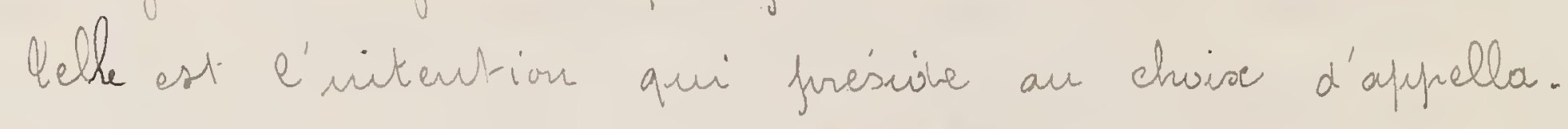

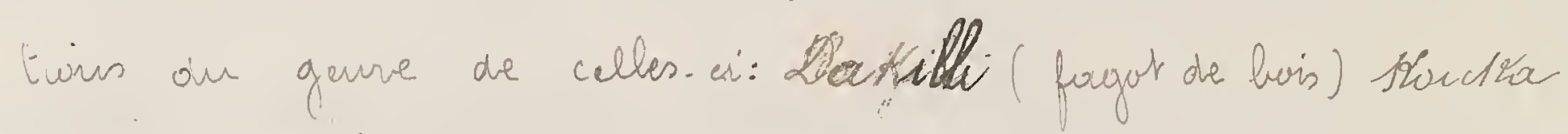

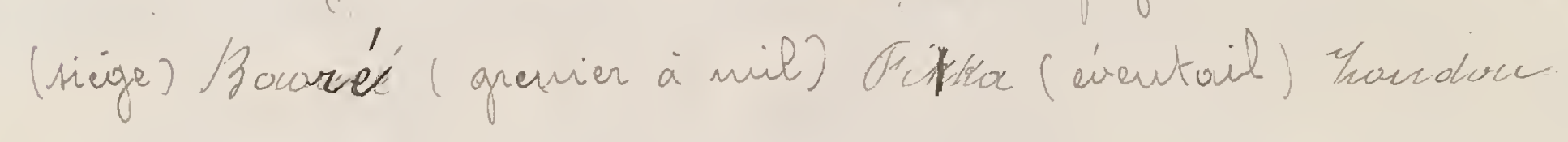
(dopet un cuivere) Thomé' (quave).

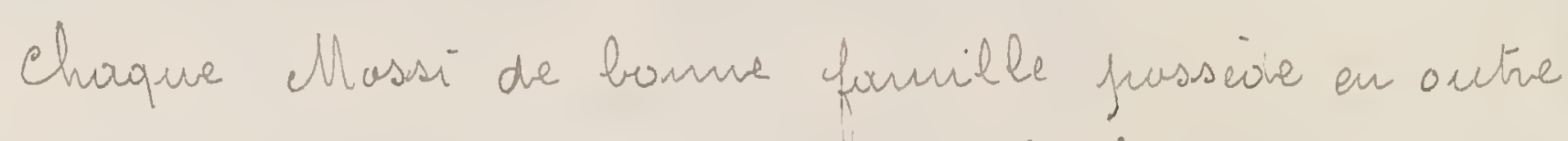

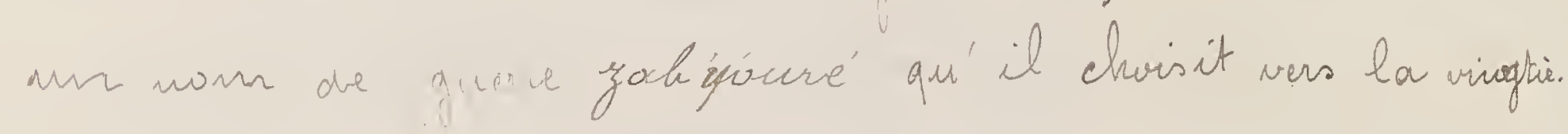

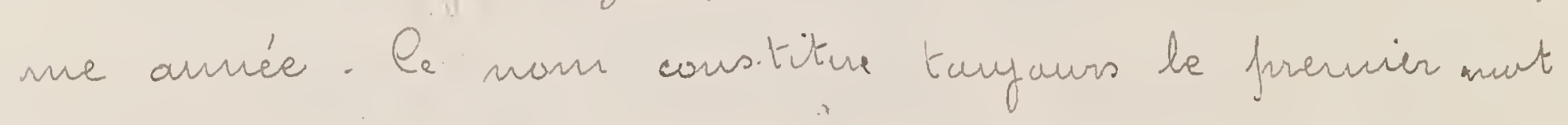

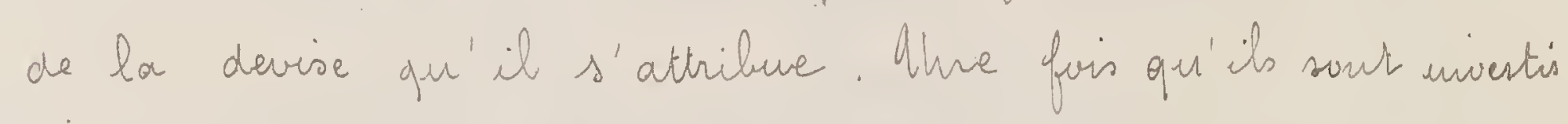

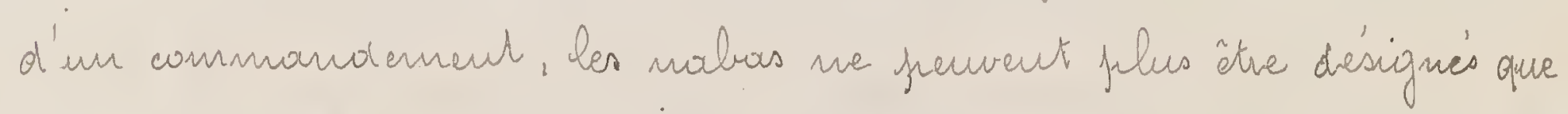

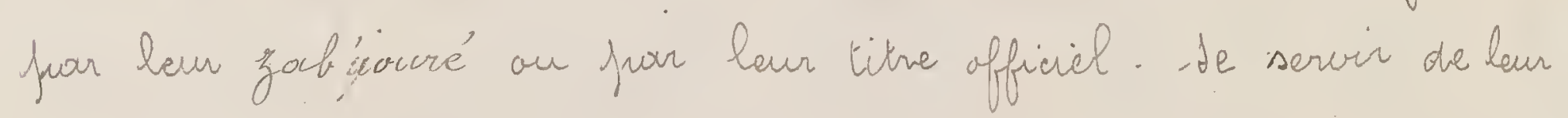

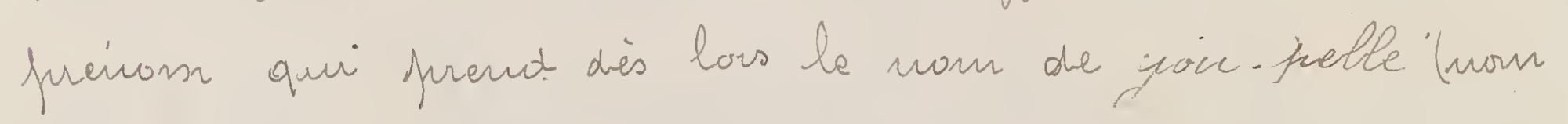

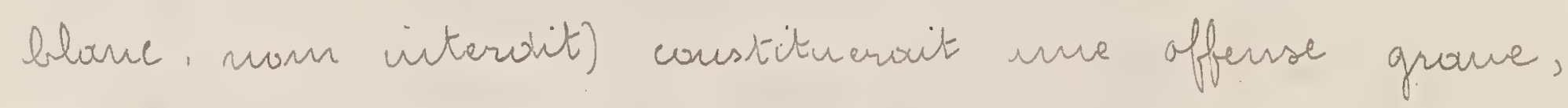

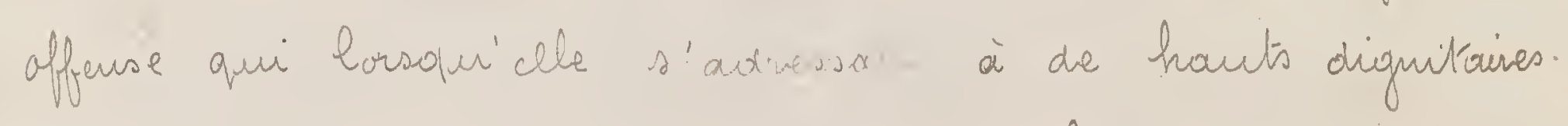

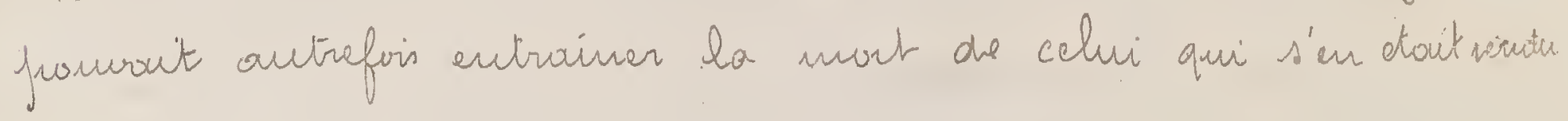


confrable. Surr avair des escemples de zab-zjouré se vepouter ause nutices concomont les diofho. Mabus.

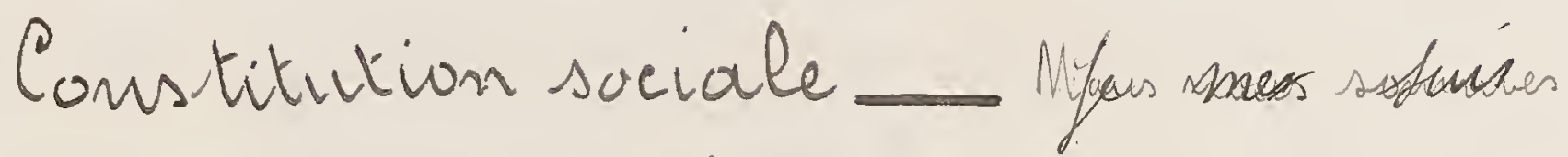
oceugnes phis hout de la hiéarchie qui coustitivait le gou.

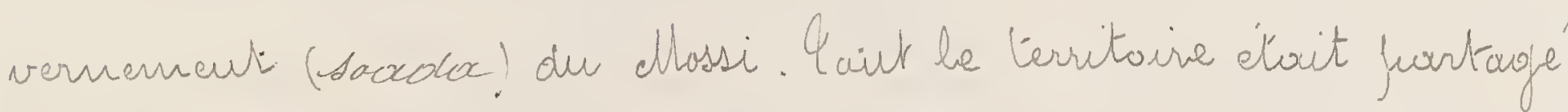
eutre bwis cents orignitaires qui l'administrarieut eur vertur d'une délégation spéciable au Morkw. Maba. Ms étaient Terur de hi

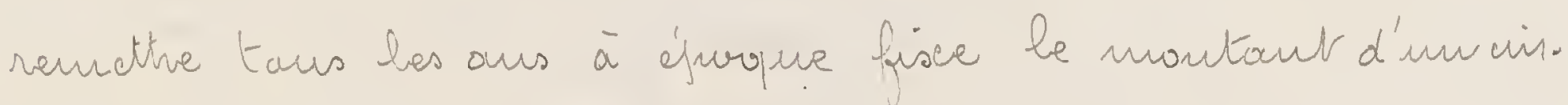

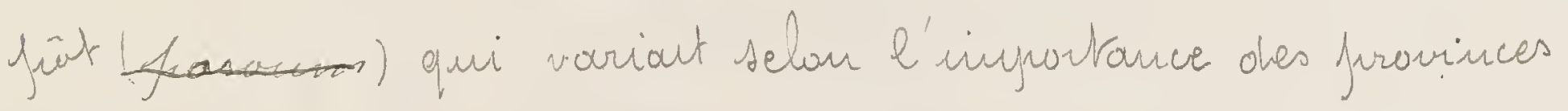
at ansistait surtaut en auris et en cheurause.

- Sa fustive étoit fuarfaitement ongarisée et si elle laissait soment à désirer daus son afplications cela froverrait de la facilité aver las, w. certaris chefs se baissaient corrampre. Saur ceux- it la concussions étoit de régle et les riches n'aurrient jamais quana chase à redouter de leur juart.

Le cheff de case (zuiri-sobor! kranchait les aifferents qui Gouvaieut s'elever entre gens de la case; le chef de Souktha.

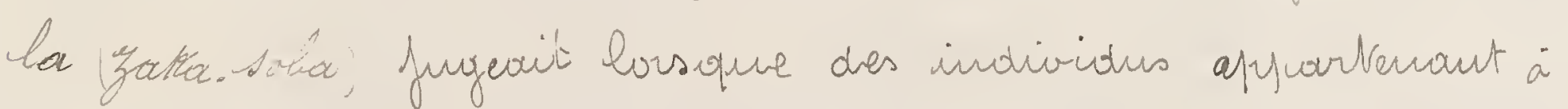
des cases differenties dtaicir ur jen. Le chef de village (tan. ga-sobor) camaissait des afforives an bes parties habitovient des Soutrhochas différentes in mêrue village. Eo déeision ourchef de Jrovince (Mombéré) nitervenait lorsqave les nitéressés itorient somiciliés dours des villouges différents dégrenctour 
Ut?

che sa furidiction. Wifir les affaives ar se trouraient in.

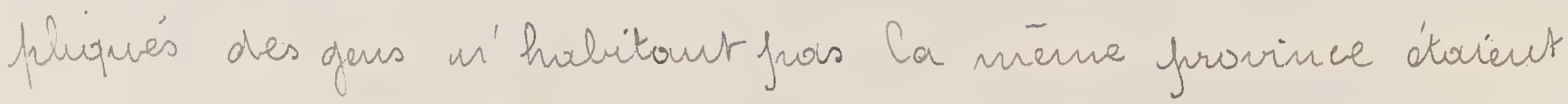
du vessout due clutho- Maba.

If existrout une sovite de ade troutitioncl (aliffeto)

La ferire de nuat etorit furevue fruur les cas smivonts:

Prine de lise. mayesté (na bivithe')

deutre voloutaire (rous).

aduetère kicodon; avee la fermme de sun frére an d'in naba.

Vol (ouardeum) lorsqu'il ét oit commis jar un étromojer.

(actes de surcecllerie'tins) ayourt entrainé la movt are entrepris dours ce lut.

Dours be was de lese. majesté le Somandé. habo ésécu.

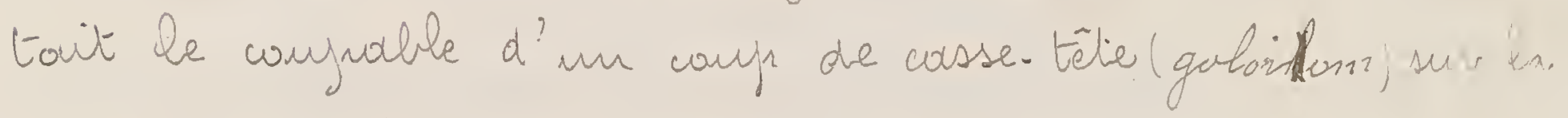
nuque. Orans les autres cas on enfflonait la strangulations (scinko) à l'aide d'une bounde de coton que deuse acolytes trionieur de chaque euté.

Les nahars s'enfpoisonnaient fiar ondre ower de l'arsenic

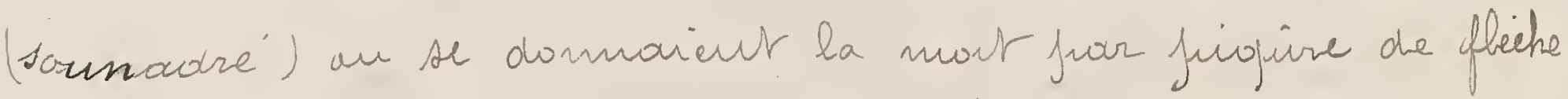
enfuisomée ( fi-giscara).

In delwas de la mise à nout malheurausennent trop fré. quente, les prinalités en vigueur dovieut les suivartes:

La barre de tustice (dabbaszigo) a laquelle le condanné Kait fisé fuur un délai mascimum de trois ass, chatiment barbare qui entrainait presque taujaurs la mouk. 
Les fers ause frieds (hanga) ani jounait confworter la mie. me durée; fe condommé conservait la faculté de se frome. ner à l'interieur de la sackfforla an il dónit détemi en réalite' il se trañuait páriblement, mais be Jeen S'exer. cice qu'il jomait prendre hi joemettait de durer fusagu'à l'espiration de sa jevine.

Eufir la fustrigation (fabre') qui constituait la preire la plus légère et était sonvent administrie preiventive. mat juevr el tari l'aven des criminels.

Les temoris apprelés en fustice, les accusés ens.mémes

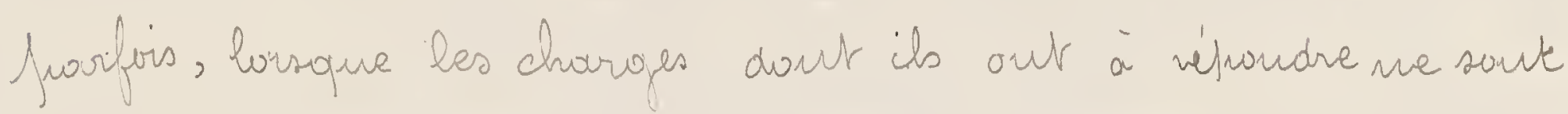
fras cerlóines, dovieut Jures sur le tengandé génie pro. tecteur an villonge.

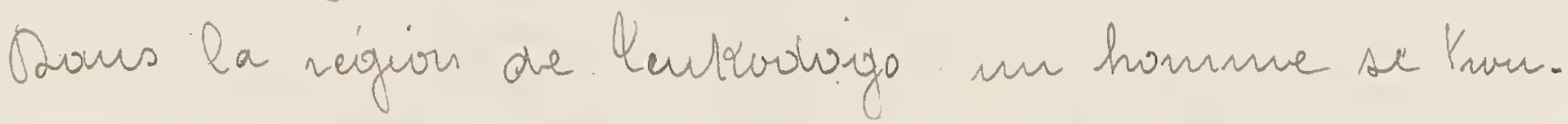
mant suns be coup d'me accusation grane fromoit frour

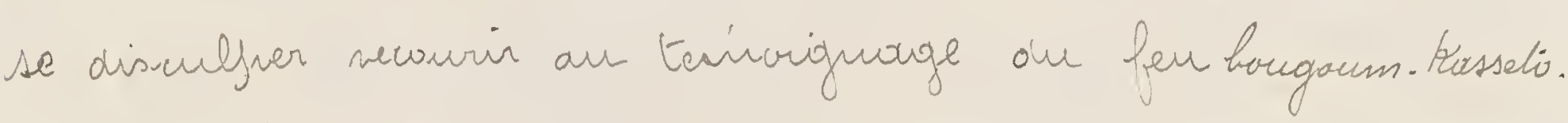
Cn général it e hassait un fer nonge sur la langue ou leterwait dours les moirs fusqu'à conyaler sefroidisse. ment. So frigüre an noyer d'une lonse on d'une fleche enfroisamée était ágalement adrise. L'accuse' devait Naufasars succomber à l'éprenue s'il ćtoit coupable. Oaus

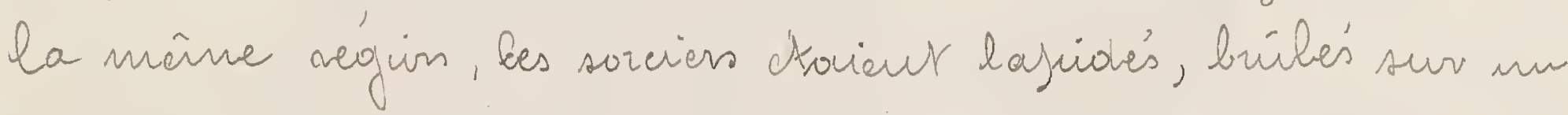
biviher, an encove cuiffe's d'un ustansile de Kerre chauf. fe' à blane.

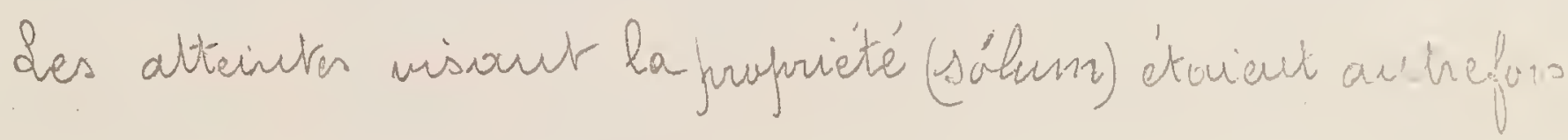


4

tries nares mais elles n'out cesset de se Imultiplier ctoout de nov faurs asser communs.

Sa joupprieté nohiriduelle existe senle an Massi. Cle Krie son onijuive, en ce qui concerne la terre, de l'autroisations de cultiver denandée au chef. lotte autorisohion étoit quatuite fuur les gars de la communauté, mais les étrongers demonieut taufaurstan en la sollicitant un cadeour de foible infortonce, tribut jas legiel ils se recommoissaieut vassoux du chef sur be territoi. ve arguel its s'ćablissaient. L'usouge consacerait la

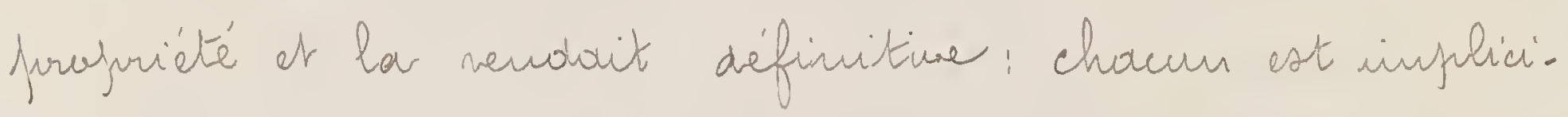
temant vecomm propriétaire du sol qu'il a rendu féiond fear son travail. Eelui-ai est transmis ause heritiers de móne quie bes bins nubles.

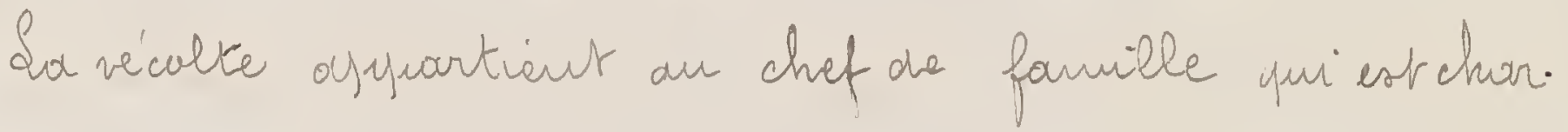
ge' de frourvoir à la sulsistance de tuus coux quilhabitant chez lui. Quis somvent aur Massi les femmes qui particifrent aur Krourail commun des cultures en four clles nuẽ. mes isuleninewt, dous ce cas be froduit abtern leur appoartient autiei ement at n'est fras verse'a la communouté?.

Aars ourous an an joorougrophle précédect les sé. gles qui priésideut à la kansmission de la propriétié de chef de village on de pronince n'herite à ce titre que des biens Vombés en déshèrence et le cos ne se présente que horsquid. s'agit a'étraungers décétés. 
Le drvit de fropricité ne samble jas avair évolué.

d'institution de l'esclauonge n'a juénétré que fout tarow an Massi de y ast demenré le nonujube d'une caste pri. vilecis. Les derniers Mopho. Onatuas farisonicut habituellement au début de laur végue une axpéditions nilvtoire dars la rigirs de Garoungo destine' à leur procures des axpliff its consenvieut à laur service la magarre frortie de cenx-ei qui Rabitaient, arisi que des prisomiers de querre de diverses/prove.

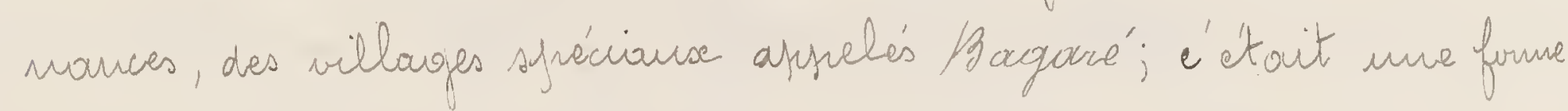

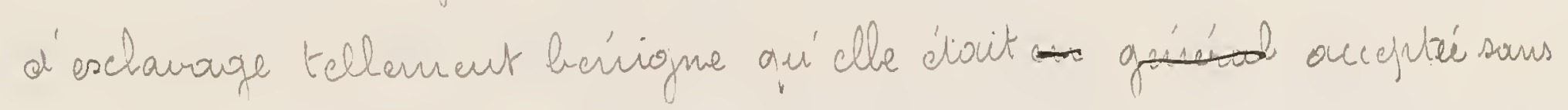
coutraintie faor cense quil s'y bromolient sounnis.

Caute persomne riche au influente a Taufours aufner d'elle des gars qui viemeut libsement hi affir beurs services un échan.

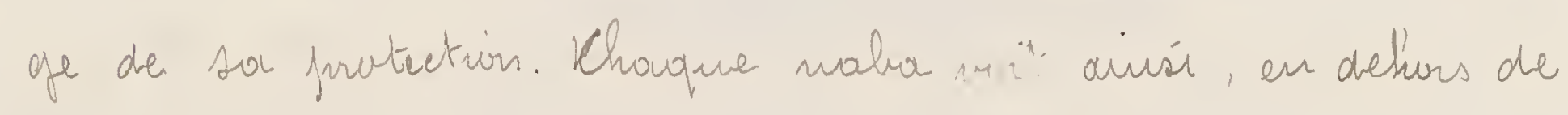
cos awministives habituels, se grouper autrour de bi une clieutele dout le chiffre varie suivact sa fmissomere et qui

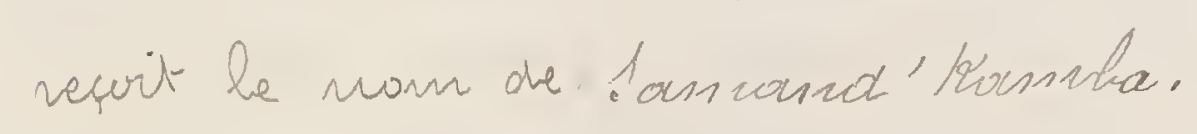

Le visinange du Massi a excercé une influence incoutéstio. ble sur l'onganisation sociale que le Barssangace' à véalisée, mais cette ongarisation est flus maimentoine et surtaut re nevêt fras be caractire de ceubralisation sifstimatiojus qui distringue C'enfive des Marfo-Mabas. Les chels aut

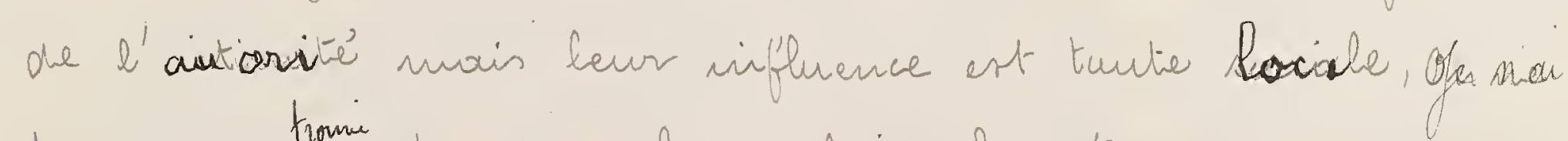

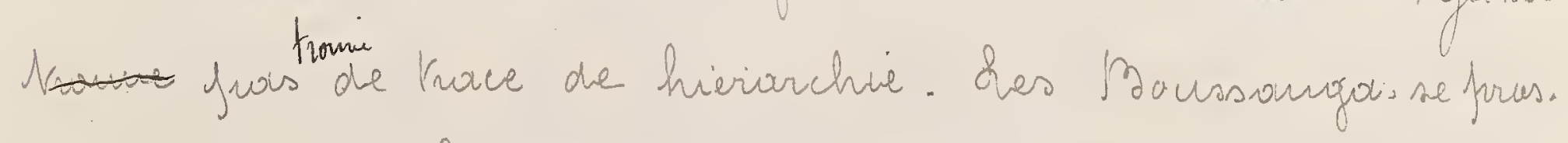
Nomeut comme les Massi devaut leur nabas man sans 
faapper la rerve du coude et se recouvivi la rête de durs. siòre arisi que le fout ces dermiers.

Les Baussangai n'avarient fras d'esclaves; ib en four.

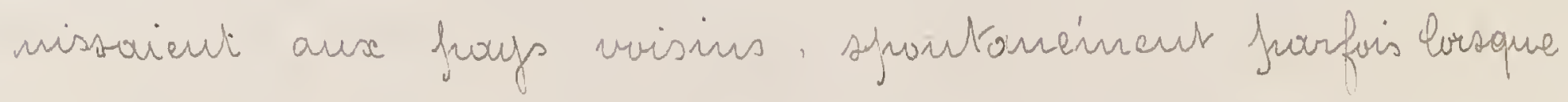
la oisdte les abligexient a in leurs kemmes an enfouts furr se frocurer de quir vive.

Che Gounounsi l'onganisation pulitique est encove murius aromcé. Le viole des chets qui sout taufaurs

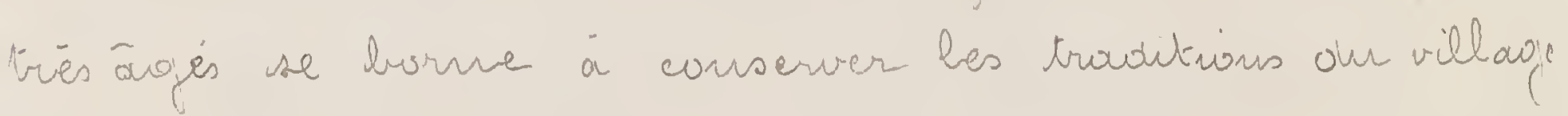
on ce quielles out d'essentiel et à frésider aux cerimo. mies des funéroilles.

Il n'y a mi castes, ari aristocratie, mi hierorchie quelwuame; on respecte surtaut les viches et les milents.

d'esclavowig excistrait avaut motre arrive'e mais il était fuarticulieiement doux. Maitres ef caphifs havoillaieut ct

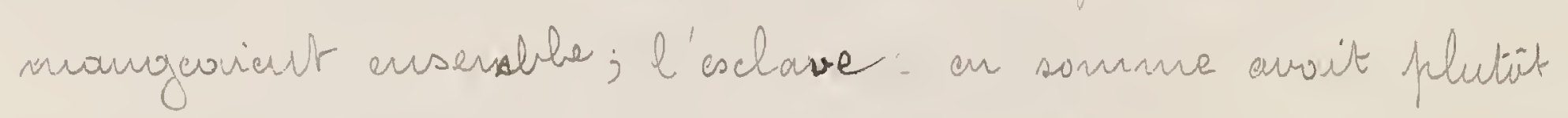
Chanai te fo nu. que de situation saciale. On ne distringuait twas entre les captits de cases of cence de frise ou d'acquir. sition véente; Kans étaient indistincterment bies traités et me cherchovicut pas à s'enfuir.

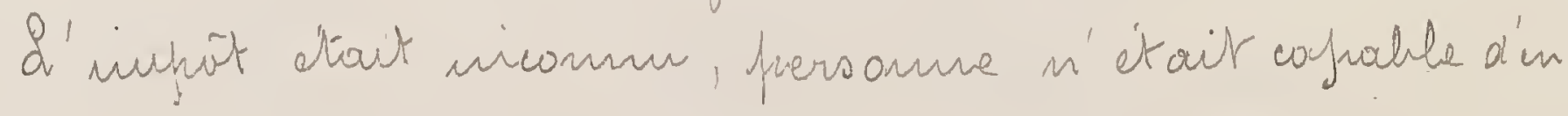
assurer la perception of la vejurtition dours un but quelconque.

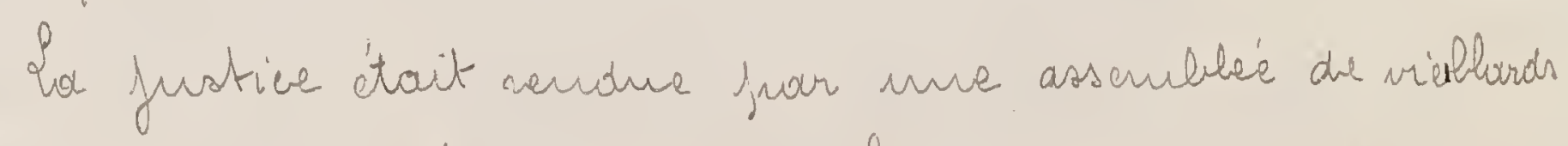
que présidait be chef de villouge. Les crirves currannaient 
habituellement la mont. On appliopnoit suit la jnentaison soit nue coutume siriogulière: le coufuable étoit attoché

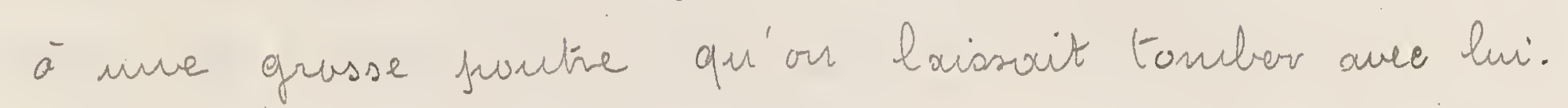

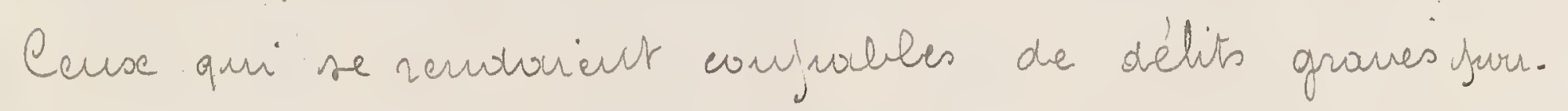

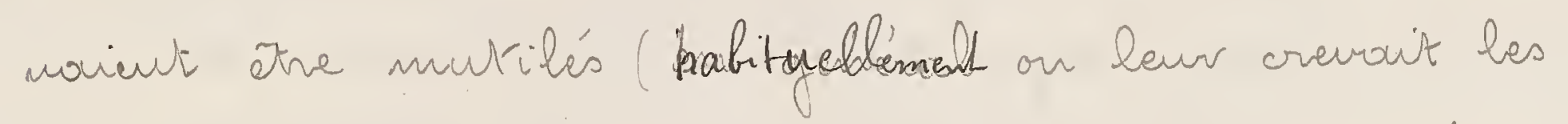
Henx an confout la moni) an otwe veridus conme esclaves. bours un ground nombre de localitis, chacen se foisait s'il le jourait, fustive sui-mérme fuur cette nousour qu'il

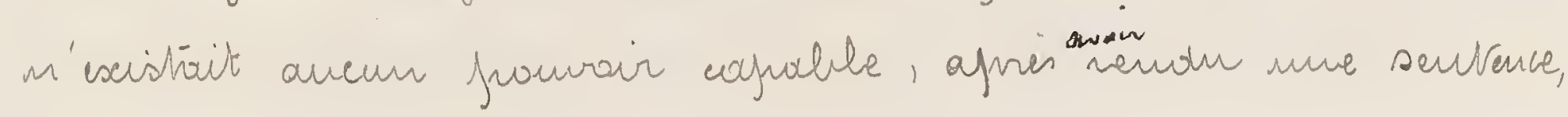
de la faire appliaver.

\section{Vie intellectuelle}

Les nuivifestativis de la wie nitellectuclle drez les dhssi'

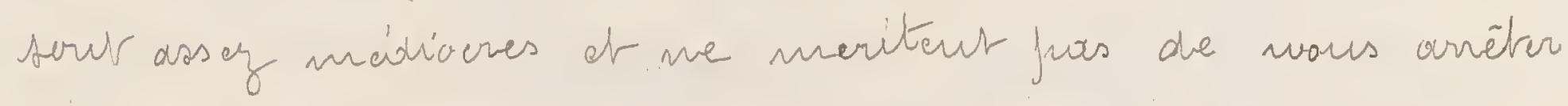

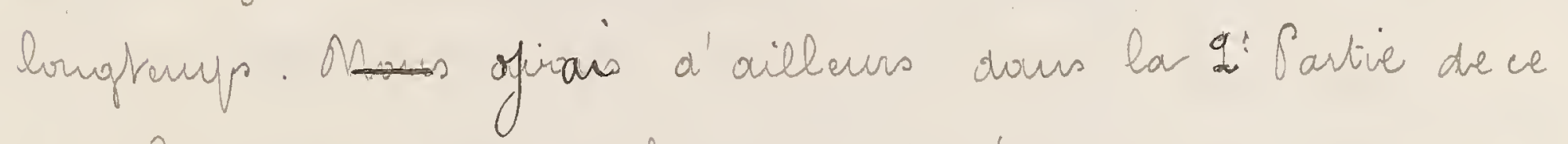

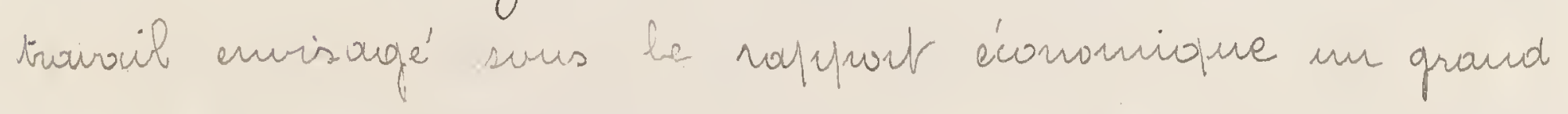
nombre de questions qui out leur flace ici: arts awiers, in. ohstivie, commerce, ete; soucrèus d'éviter les redites mintiles

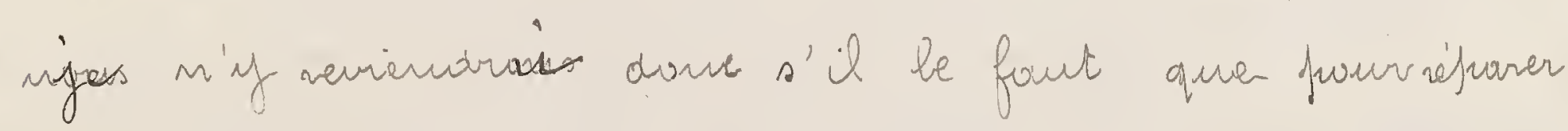
des onvissions et conyuliter fuar quelques détails Nechniques les apercus défà furumis.

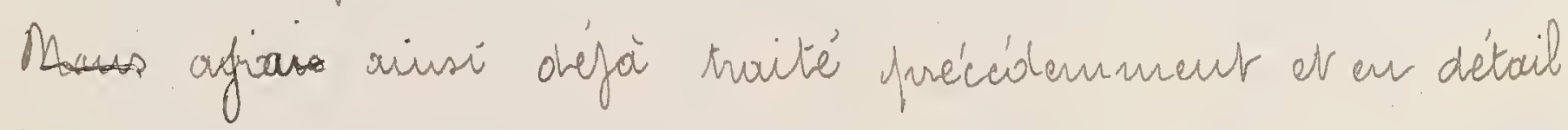
de l'elendig proprement dit. Les dlassis nous l'aurus un 
me cessent de s'appliquer de filus en flus à cette notrstrie

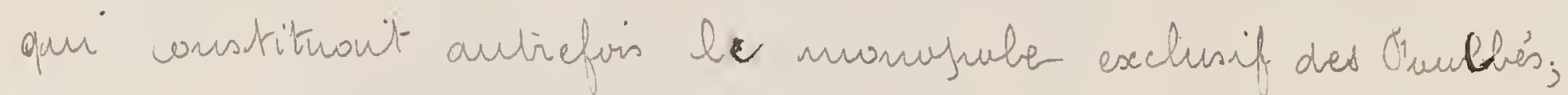
mettout à jrufit l'exerufle de ces derriers ils y réalisent

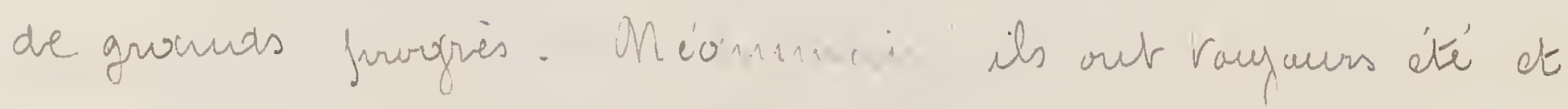
dencurcur agricultewis.

Cour bes Massi. (et il en est de nuéme de la jelufuint des preuples primutilg) les ápurques air il comieur a'eurrepren. dre les trourause des chomps sout riglées faor be vetour

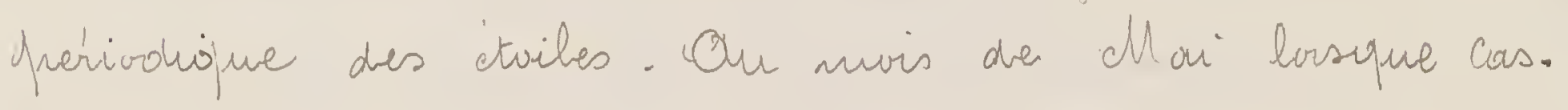
-iajnée (en Massi boudoula. Kouktouri le nounche à senver) se montre an 11.E'. ourout be lever du soleil, be dé. frichage owit ètretenmiré. 'e'est en effet le noment a'ensemencer avec be naserimum de chanees de sueceis

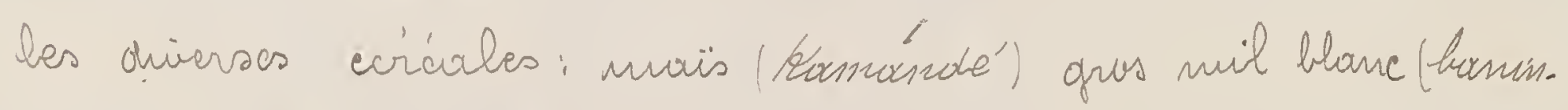

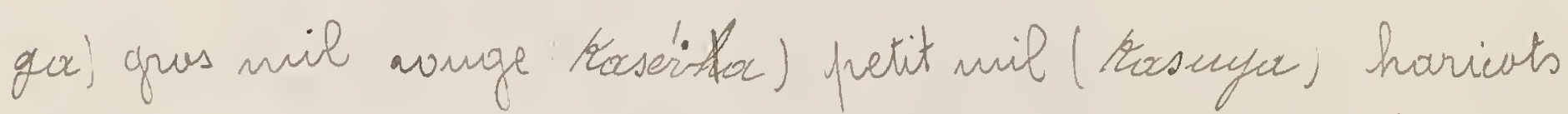

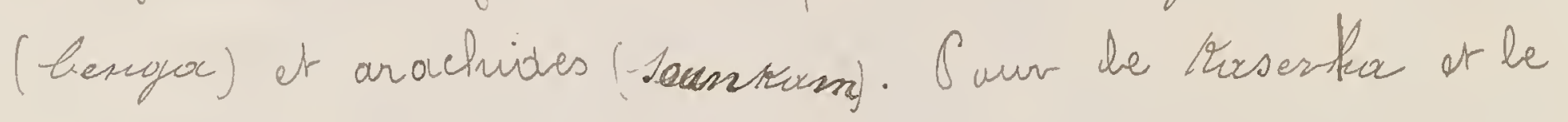
Laninga les semailles doincut être terminaés lors. que les Plïioudes (momnis Sacsi, les crins du cheval) se montrent à l' Lt. Las outres graines preuvent encove etre mises en torre avec la cortitude d'aleteriit un resultat; four détaminer cette perióde de refit d'une forons preicise les chassir useut o'un proceide' cur. rieux. A l'aubre lorsque les étoiles sout sur be firit

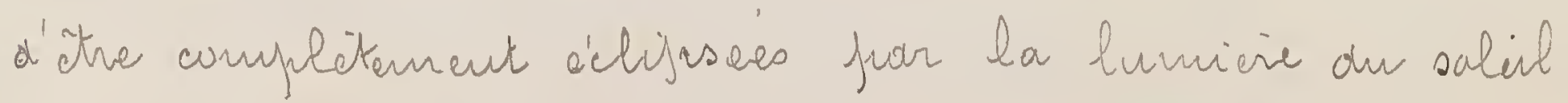

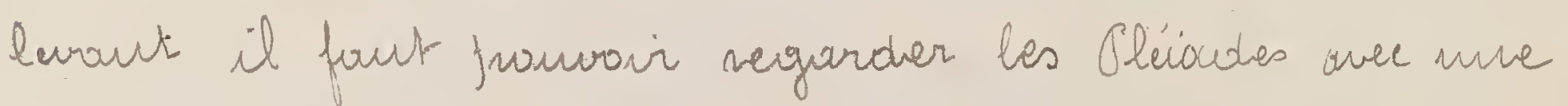


pierve sur la tëte; si eet asterismo astencure assez hos sur l'horizon fuur que l'alservateur l'a prersoive sours - que la juirre setumbe, il sera jussible de semer enco. ve ave frivit.

Le sésarme (sim) neowit àtre servé qu'apreis l'appua. nition des Sleioutes.

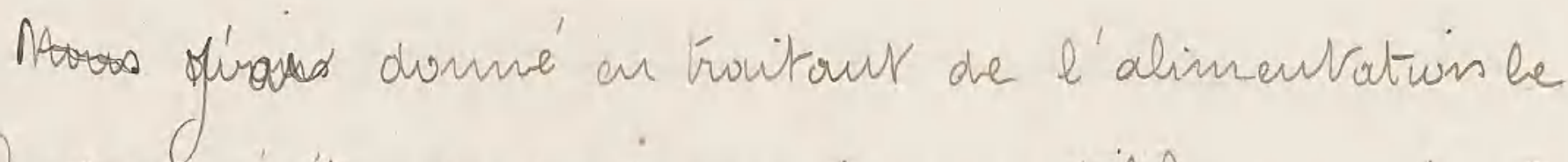
dékail des vagitaux projnernent connestiblis au entraut dours la fréprarations des sames indrigernes; il est abue inutile a'y zaverior

des vrouraux des chomus detuis be défrichouge er les

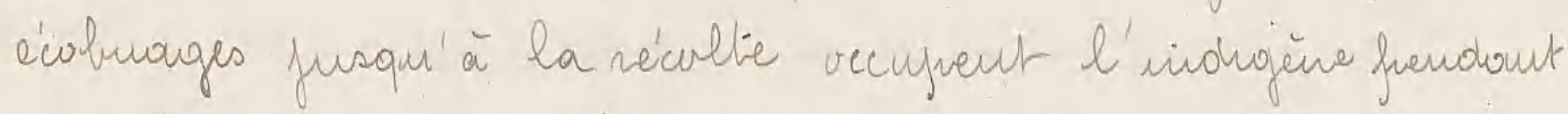
runti la poiviobe quir s'eterrod d'arib à Gerobme. Saur le muxis (Fumánde') que l'ou sème le premier et qui mient triés vite, de nuère que four la tomate (toímbiré) et la

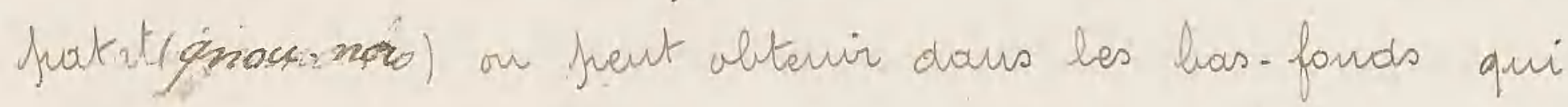

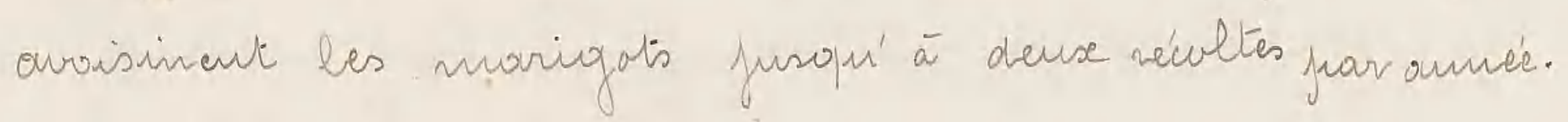

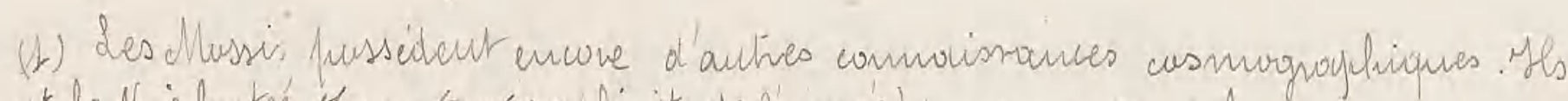

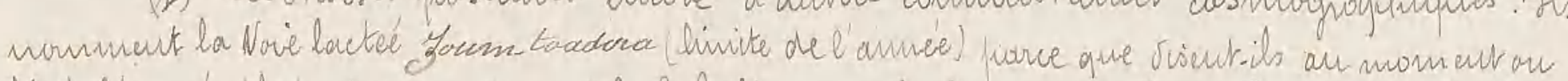

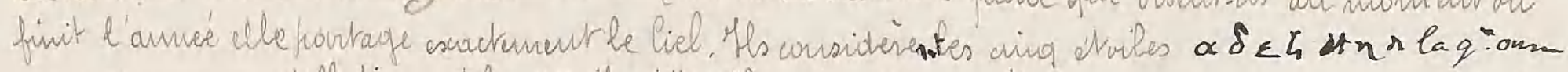

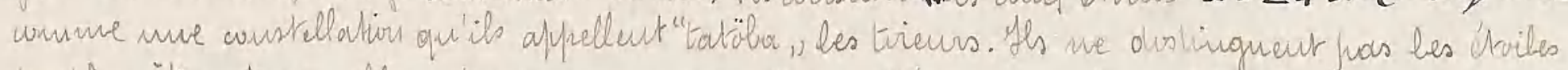

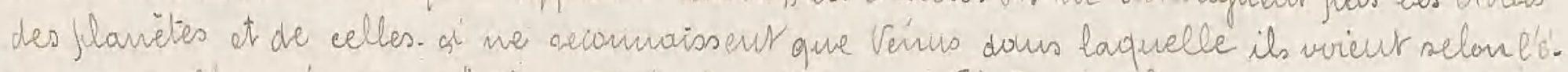

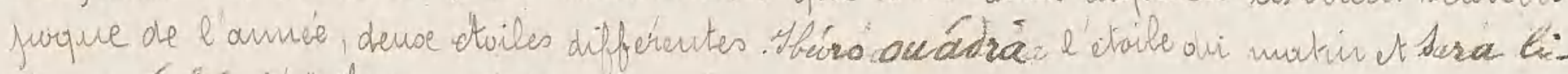

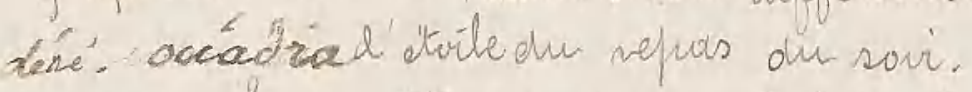

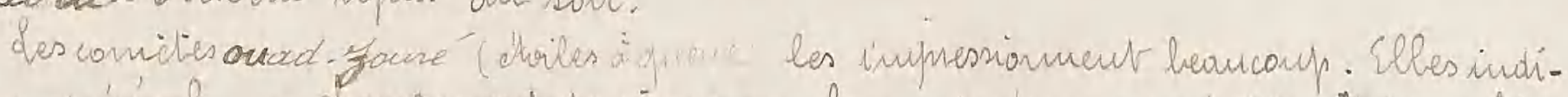

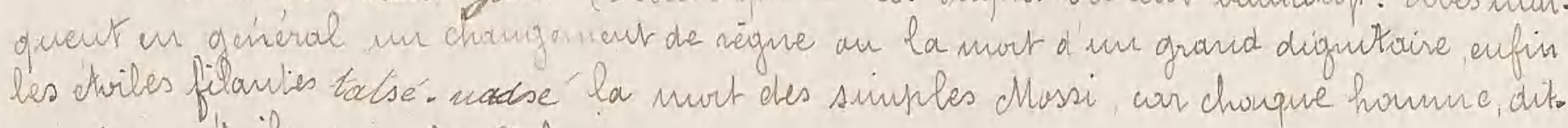
on à son cturite dours de liel.

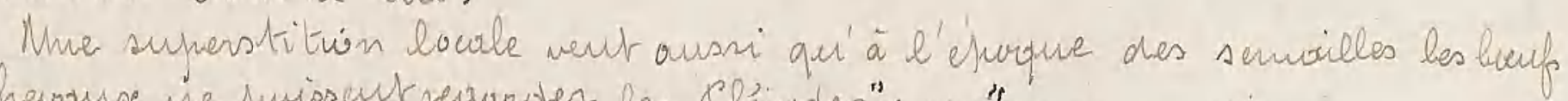

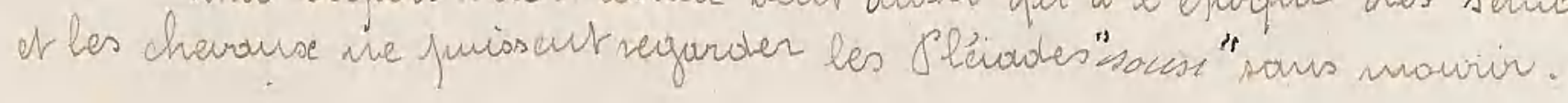


Le funier (nebdo) de bouf et de chèrre ost surtaut utilsé dares la culture du miis.

Les nistimmants enfloyés sout assey nombreuse nais me frisoutcut fras une grande variété dhe types. Le scan. gex surte de linette serr ò defficher; les trous destinésá necevair la semence sout erensés soit à l'avide au Fontorga instrument du meine geure mais de noindre di.

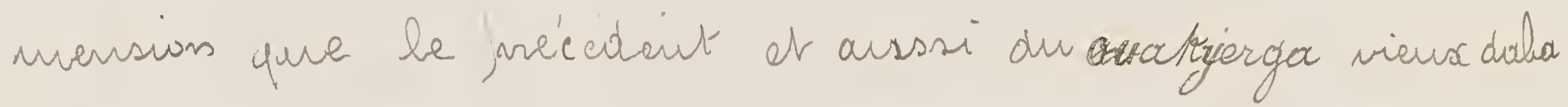

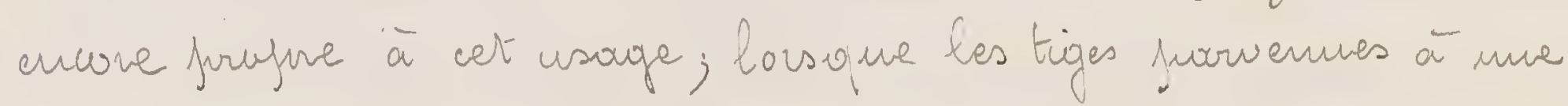
corvaire hauteur commencent ôtre geviées dours leur cruissan. ce Juar les nuavaises herbes qui les eurourent, on les dajage à l'aide du cuaka dalux des Mondingues. Enfir lossque le grain a atteint sa maturité la tige est confuei an as on sol avec le soanga et les dpis de'tachés quelques jaurs afirès an noujar du Jace. "ra, coutuxe à grounde lame.

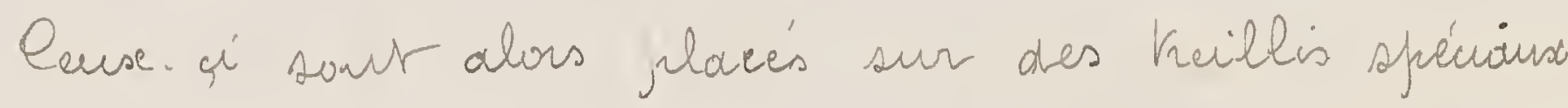

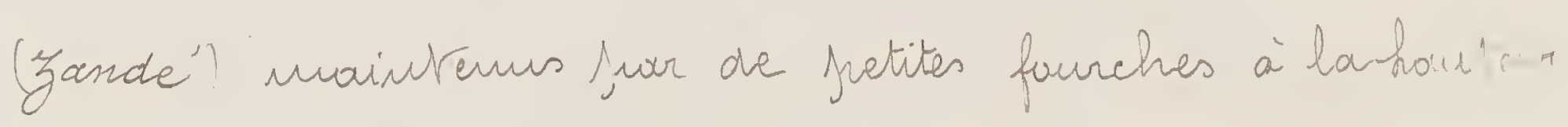

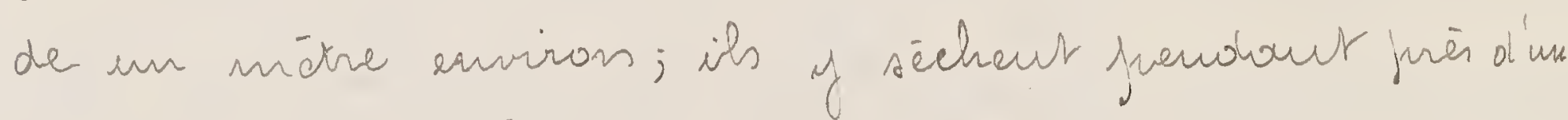
urois es l'on prend soin de les frotíger foor des sétis an. tre l'ardar excossive dur sobil.

Daus cortoines localités des gavirs sout couserwe's ar épis

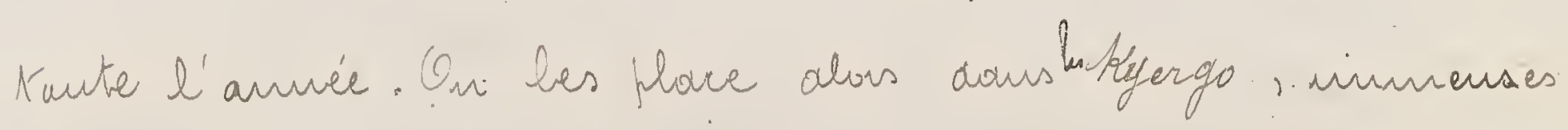
jomiers en gros séko, isulés du sol fear des fieuse or newavers d'un chapreau de faille. On enlive ce chaprear sour introduine an vetiver bes épis. Gousque 
Grain a été séparé ar mayen du battage il cat enfermé dars les haouré genriers à nil fuoframeut dis, cojalement munis a'un chafreau de paille mais cors.

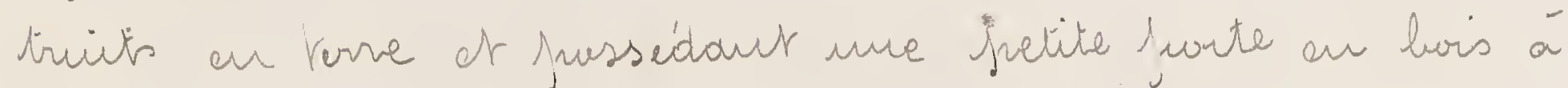
leur juartie niférieure.

our Massi, hammes et fermmes prement leur fourt des travaux agricules, l n'en est fas de méne aur. Gour. nounsi aris la culture est l'apanouge des hammes seubs. Caus cette région, it arrive fuarfors que les habitants o'un quartier du villoge conviement de bavailter ensemble et l'on fait de cette facon tars les champs on commun.

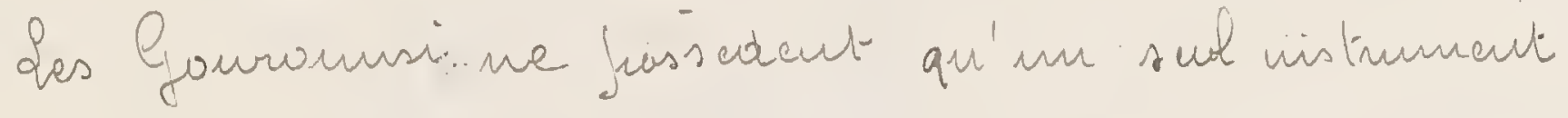
soute de daba dout le fer rionugulaive de dix à quinze centinietires de longuaur ot autour de lorgeur à la hase. Ifls se vautrout jourtaut d'être milleurs agrieulteurs que

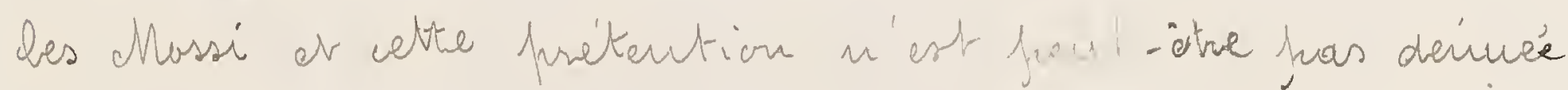
de fordement, deur ingeiriasitie se monifeste surtrout dous le movde d'anneinougenent du sol sebu les bescoirs de la plaute. E'est anisi qu' an fur or a nuesure que ce divelappent an hicol des tiges de mil les nocimes adventives, ils buttent la kerre fruur les recouvirir otrecom. menceut be kavail chaque fois que la jhive a entroti.

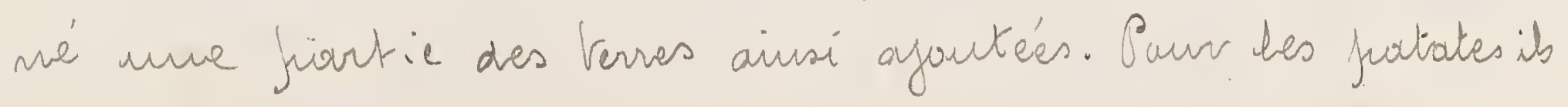
Pert de viritables plauches en Verve appuartée ce funve'c de

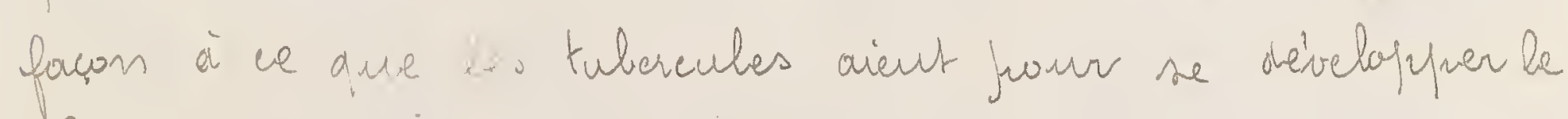
sol nuenble qui lour comient. 
La chasse (tonsorito) we constitue fuas me des nesserer. ces inolispensable de l'alimautatión.

Il y a cejendaur des chasseurs preffessiommels (toms.

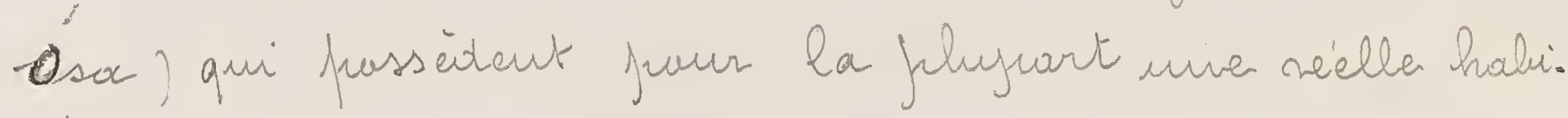
lete', mais is...terut jas érés noubreuse.

Les vaus jennes gens se vémisseut serueut an bandes fuur foire lachasse ause liènes qu'ils treur à couf de

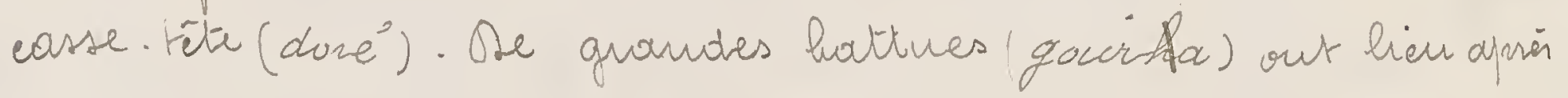
l'hivernaige of sout owownées le palus souvent far les nabas. Qaut be village y prend paort. On allurve daus la burusse des incendies circulaires ne laissaut qu'mue seule issue an gilier qui s'enfuit affolé et est troqué juar les chasseurs

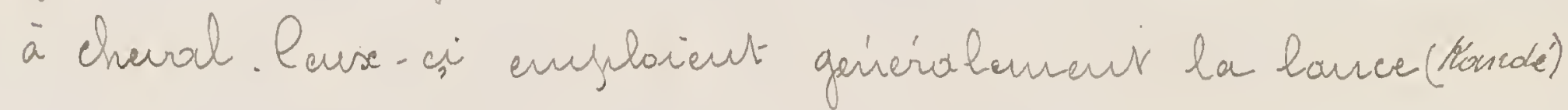
ou le javelot (tanbure').

Les fliches (finis) et le fusil (houg - ravgo) sout les armos des piètons. Ses risclews, an Mossi senlement, se sevvent de pièges (Houssese) en criss de cheval.

Ohes trous (lovisiogo) pratioués dours le sal et recouverts de branchanges sout enfoloyés frour la capture des fauves. Les chasseurs creusent enfir an bond des marigats des abis adroitement masopues qui leur permettert de suypren. dre le gilier au moment on il vient boire.

La pieche (zin Gmabo) ost usitée sur les nives des twis Vobtas airsi que des innombrabbes affluents qui sillonneut le hays en hivenoage. Les engiis les flus communs sout 


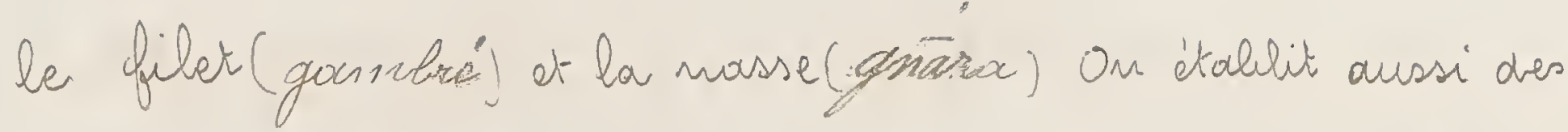
barringes (majaix) dous le lut disaler le jwisson ct de sen. orre sa capture plus aisée. Les indrigères me fabriquent fars de havrecons (léd:"); ceuse qu' is utilisent vienneut de la lote juar l'internuédriaire de marchands havussas.

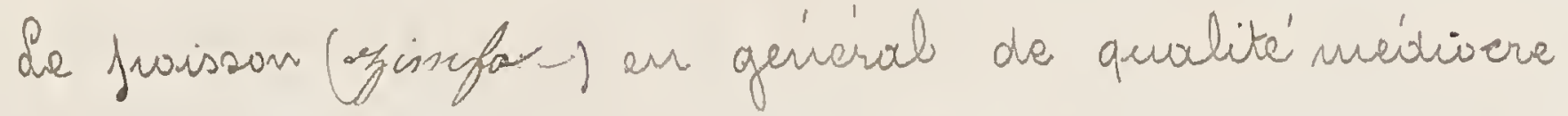
est desséché frour servir d'alinent de réserve. Dans aueloues Cocolites on se hive aussi à la fueche des huitreo(yersole') dout le contenu apries dessications eutre daus la confection de cerkarives sances indrigènes.

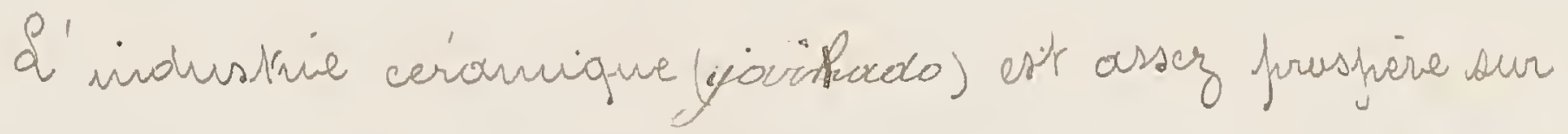
Koute l'átare du fuafs mais ne dome hieu qu'à des kronsactions furancit locales.

Les fennmes an out be nonafuble. Clles utilisent on genieral une argile quisätre (yaito) qui, soigneusement tricé et foitrie est cuite dous des fours en terre (goanga) ot four. nit d'excellentes foteries. Les usterisiles arisi confectionnés se trouvent sur turs les nuarchis à un farise modiopue. Les tifpes sout exetrinement nombreus; ngers me didotesas que les flus comrus: le singax grande cure évasée ayout souveut

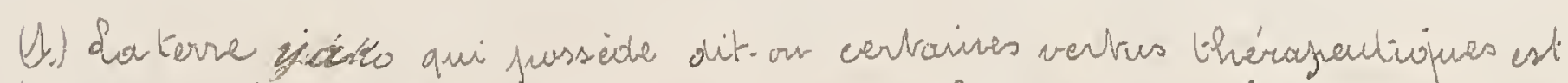

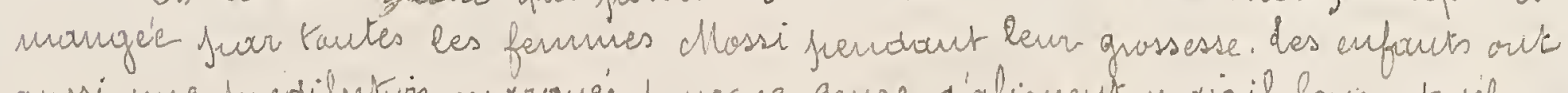

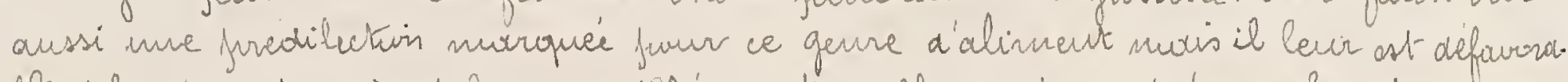

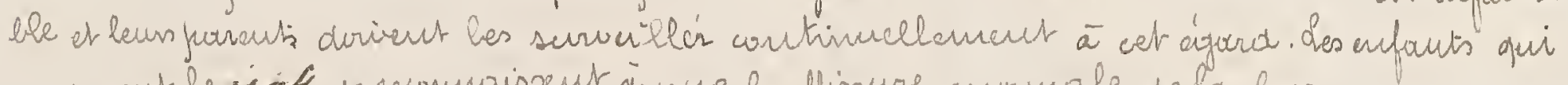

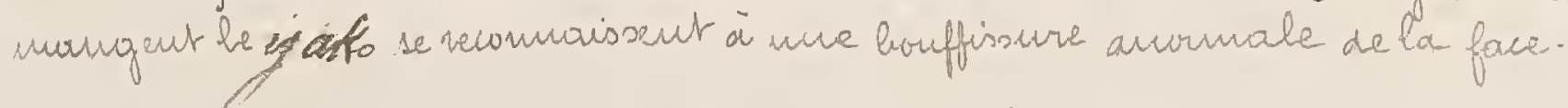




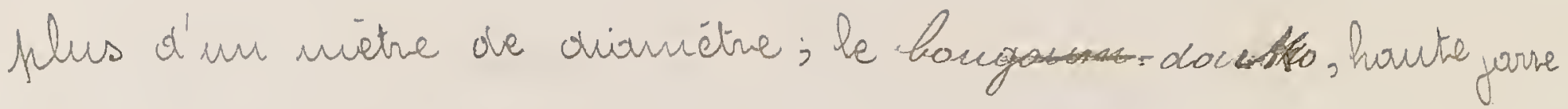
étranglée dours laquelle mu enfout pourrait se terir dellour.; lesin-bich, nièrve toille mais plus évasé; on s'en sert fuur laver le linge. le dernier uskensile we doit Juas aller an

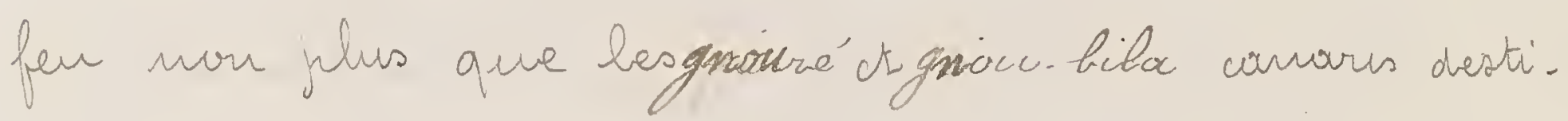
vis à coutenir le doun aprèr sa fréfuaration. Il existe aussi une varièté nifinir de pretits to laiffa réservés arse sances of recouverts d'ure sorte de verni soborffo qui leur òte tautie fourasité.

On Gourounsi les afpes sant noins nombreuse; the dureisserneut des patéries est obtemus à l'oir libre.

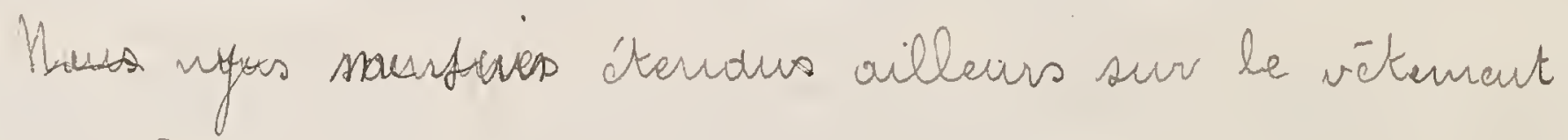
(fongon). le sout surssi les fennmes qui à leurs noments de Coisois fileut le wto. (On troune du fil gisga ) en éche.

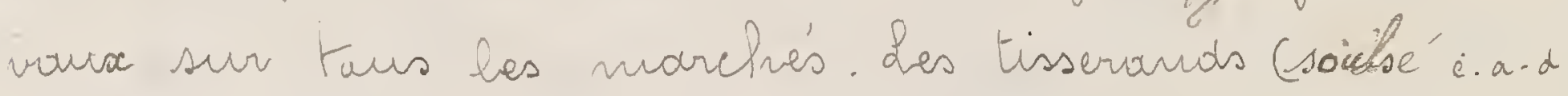
araignécs) l'utilisent juer la frabricatit des étoffes. Celbes-ei sont aébitées an bandves de dixe centinuétres de

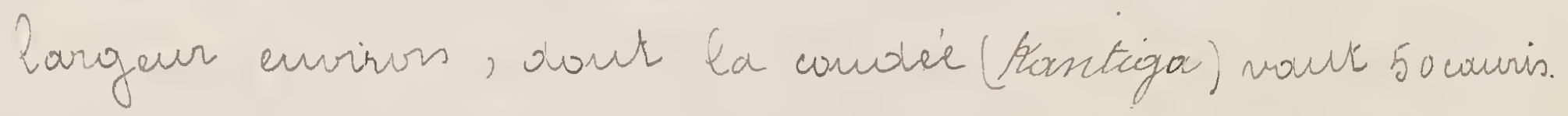
Les vêtemewis sout coufectionnco fur les vailleurs (fun-sistate). Le frincifial accessovie de cette industrie at la

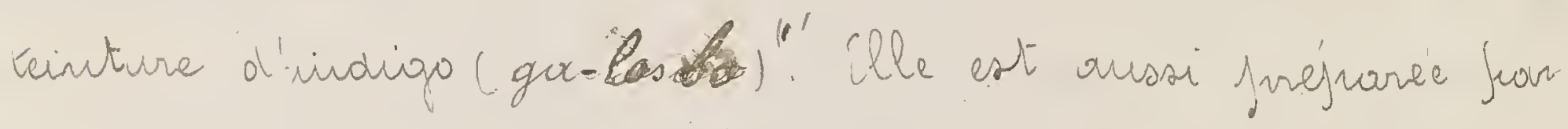
les fannes ot foit surs forme de baules l'ohet d'mue aseoutation lucrative.

$$
\text { (1) ga }
$$


114 


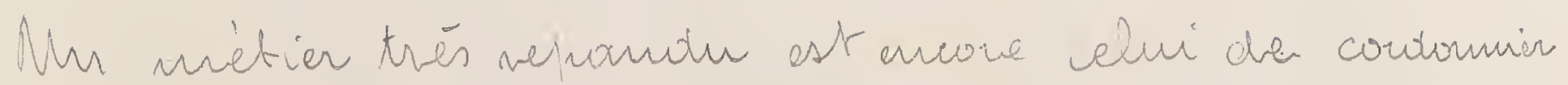
(Yáffux) confection de tars olyets de cuir joincialement

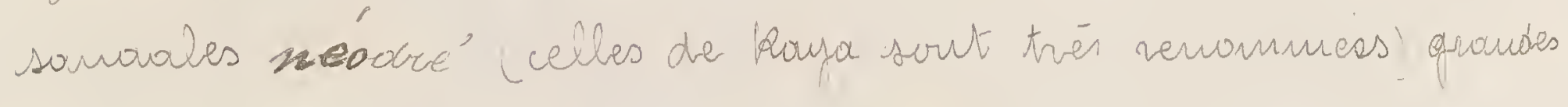

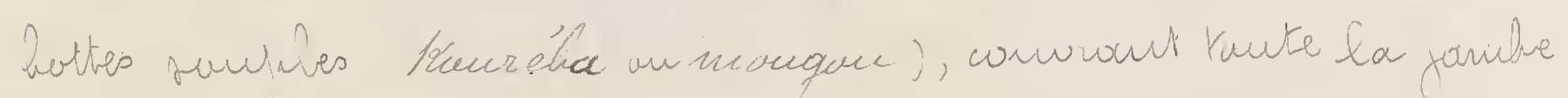

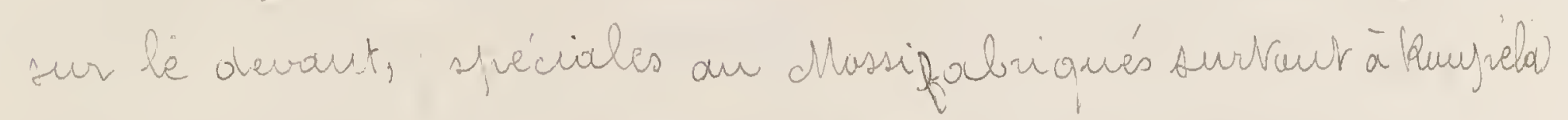

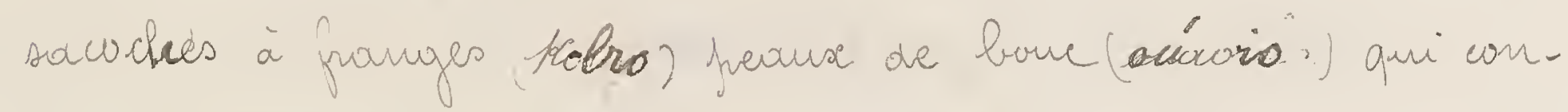
tiemeut les alimients ot les véterneuts de sechange que raut.

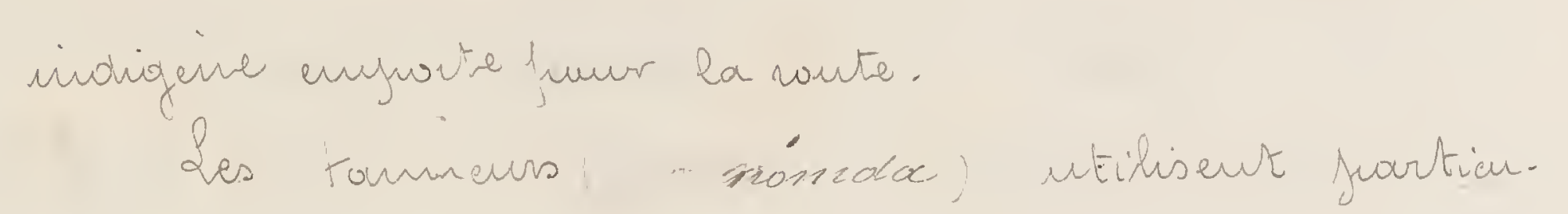

Sur le nafyout de la metallurgie on 'iarraile sur raut le fer at le cuive. de promier se vencoutre un

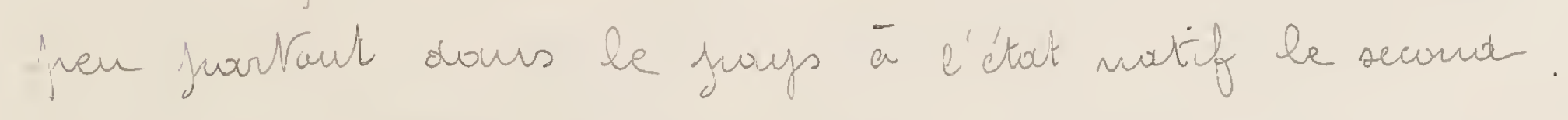
ats inguorté de Cjold. Coast.

Se fer est extrait du nimerai jar fusion obtenu

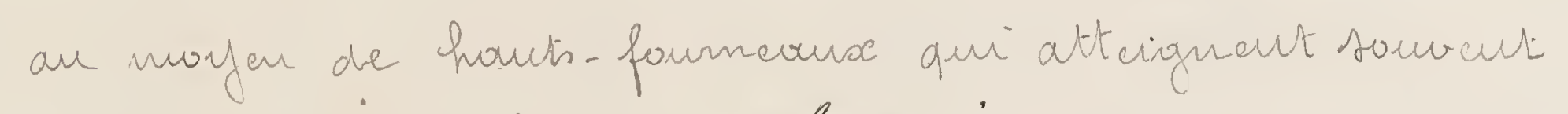
quatine unctres de houbleur (Bongansi.) an bien dous des sortes de cylindres (forlac) haut de un wétre avirons. Paur ces domiors qui sout fearticuliess au Mossi la forte est achivéc à l'a obe de suufflet yaígdga ;) en peour de chère.

Mar gious décrit sommairement en traitaut des arts plastiques les driers elyets de chive en usage an Massi (varì f. 158 Je fer sert surtraut à confectionner 
les mistimnats ougriables dout il est questain filus hout.

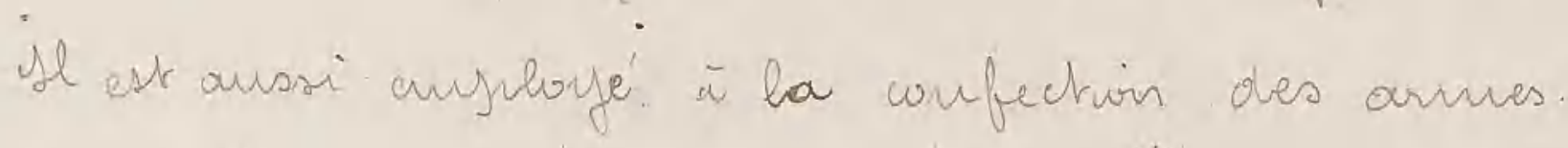

Les princijuales armes Gabtedo (1) dhassi, sout bes suivanVes: la lance (toonde? qui se présentie surs la forme din. nombrables varieties; le favclot count (toubre'); le grand sabire à lanne droite (Song-bangandya) Apécialité de la végiors de Hi-

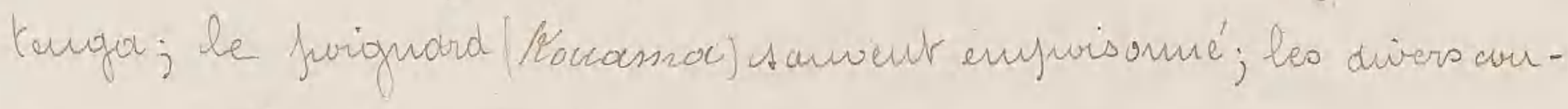

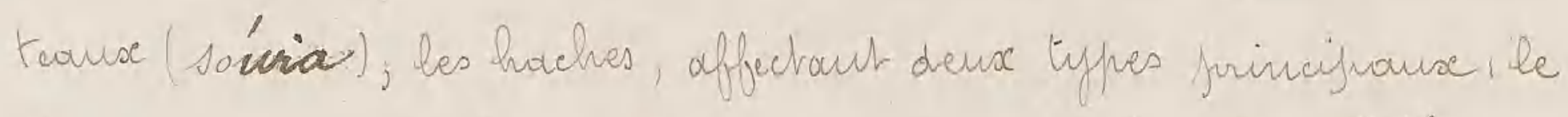

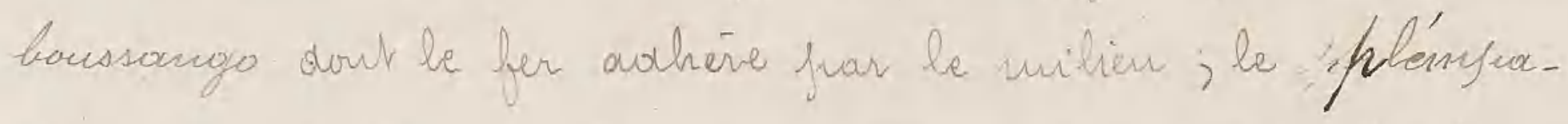
To qui jusséde wre lanne en furme de croissaut fiscée an somnct der manche par ses deuxe extrémitiós.

Mges citerais encove farmi les anmes, les fliches (jum)

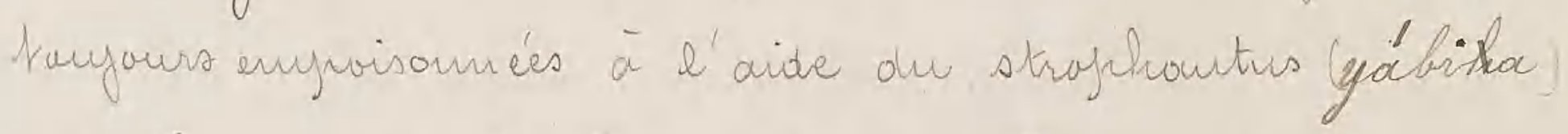
que t'on cultivait beancaur autrafois à l'entaur des villowes. Clles compuntent comme accessoires l'are (támpios)

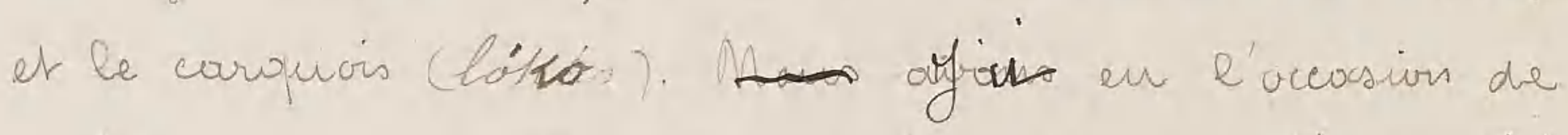
farrler our casse. Eẽte, arme foworite des Massi. Giai

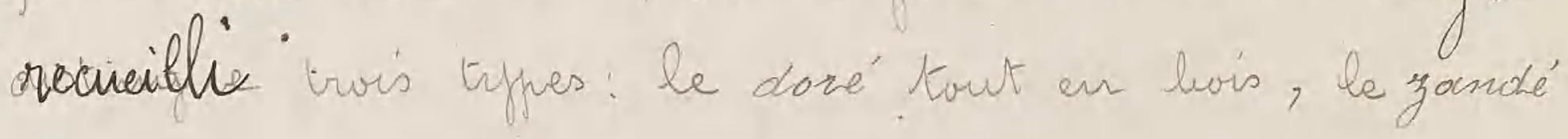

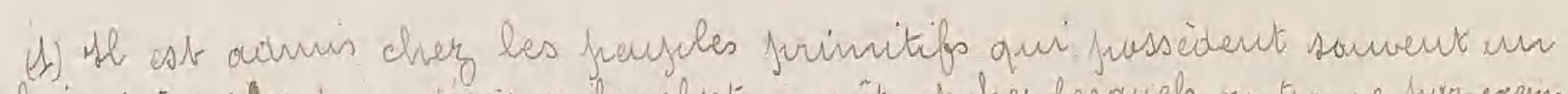

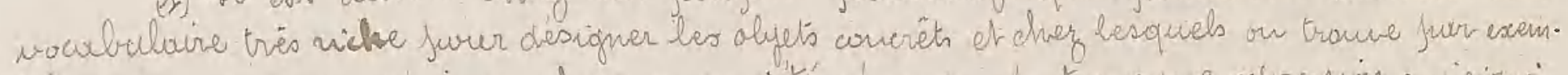

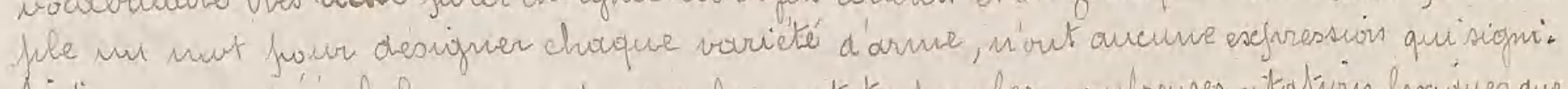

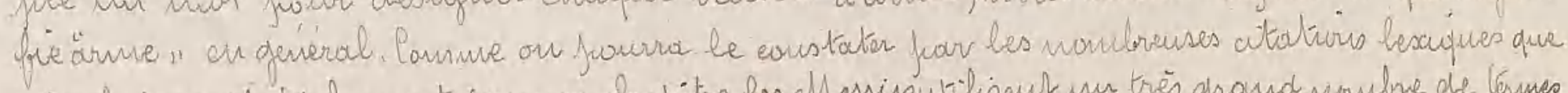

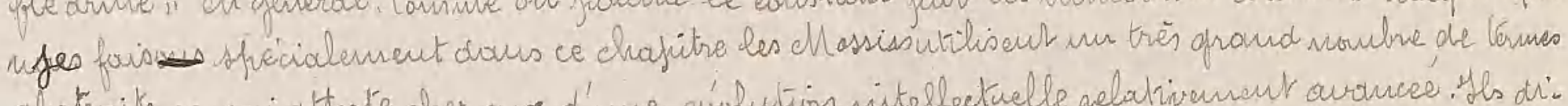

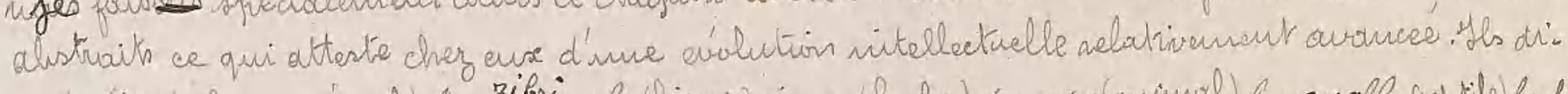

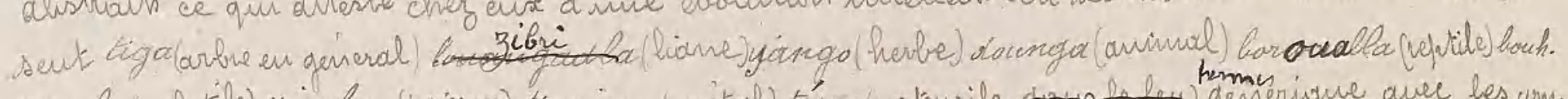

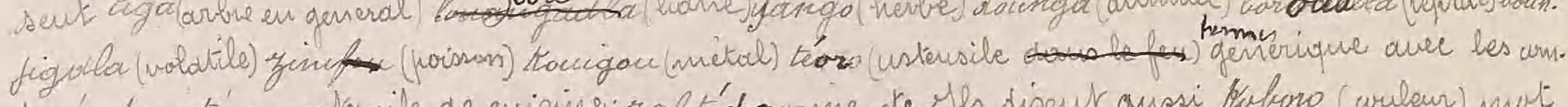

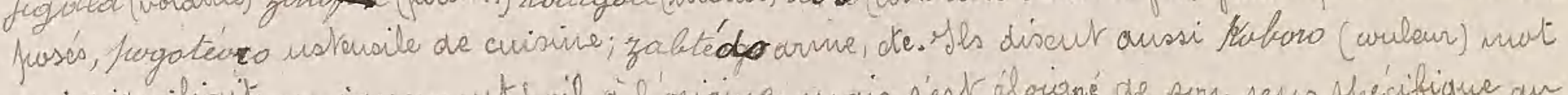

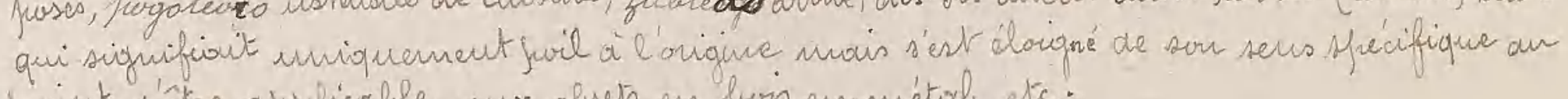
fisint d'écre affelicable ause abests en livis, en niétal, etc. 


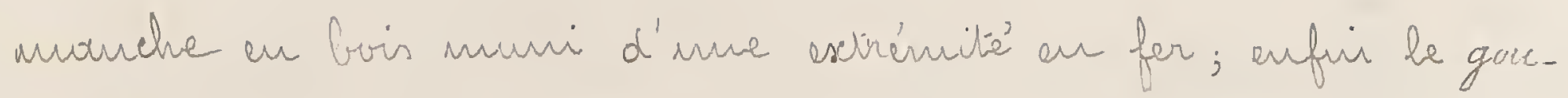

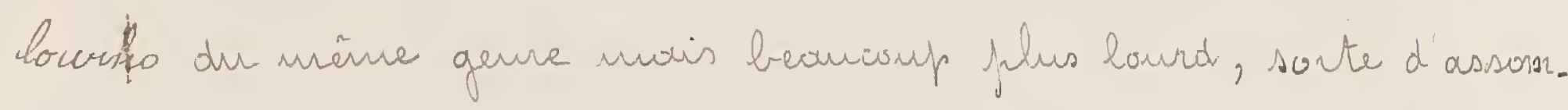
moir, atribut diskirctif du Samarrde'. Maba, escécutair des haus. tes. ounves." On se sert áqalement de la frante (láxbiaci). Les armes defensives sont uniquenrent vepresentées foor le bouclier (ganrgo) en cuir de babuf que l'ou vencoutre surtaut

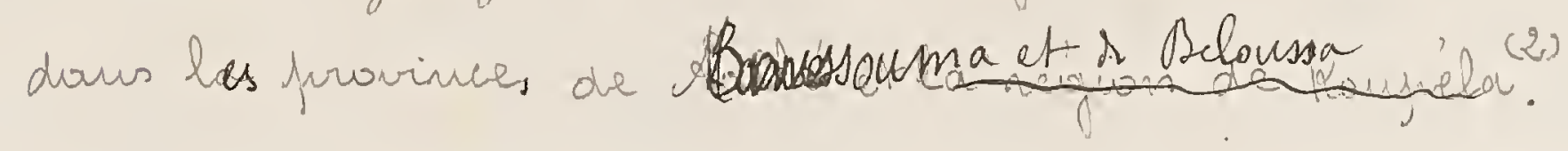

S'habitation fomiliale type Girc') ast twyaurs coustiture an Mojsi frar un graupre de cases disfoséés en cercle et conn. prises dours la minue encerinte (loelojocebo-cega).

Ces cases (dório) dout la prenvière à gauche ferès de l'en. thée est toujours séservéc à la ferrume de fremier rang at

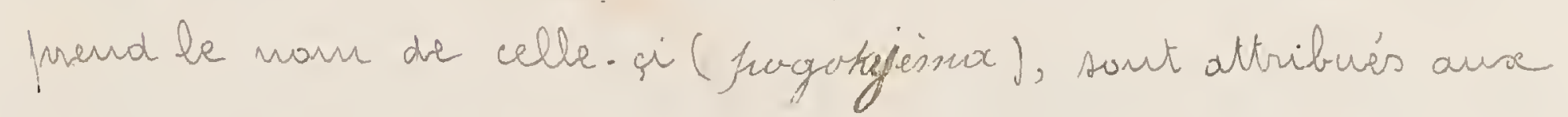

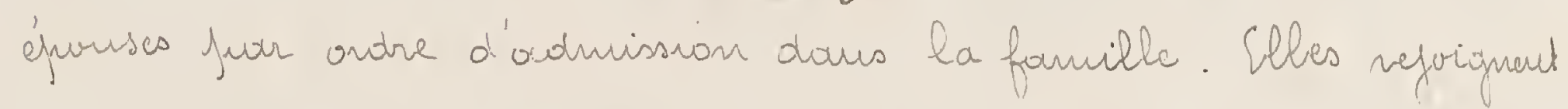
vers la gourche un groupe de conskructions qui conufeste le cercle ot qu'on karue forrcoursépuent à drvite en entraut:

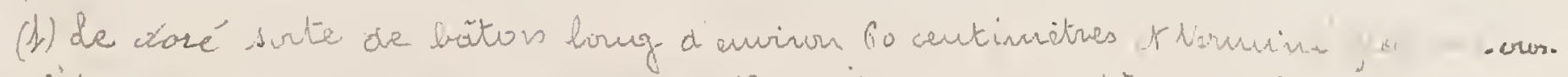

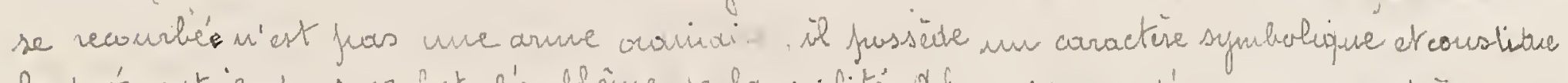

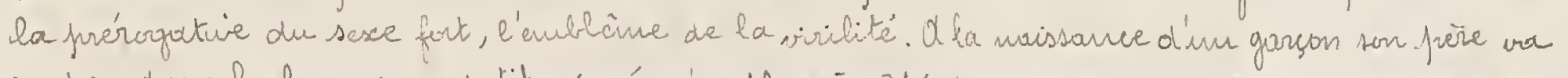

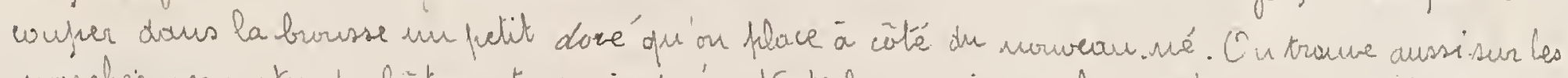

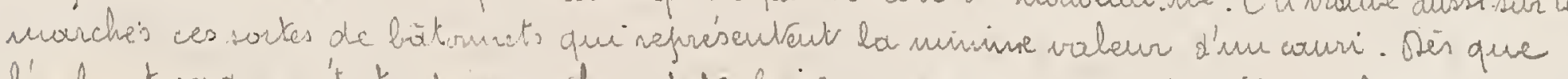
l'enfaut sera en état de warcher ct de faire usage de ses nuairs il nemplaccra ce doré

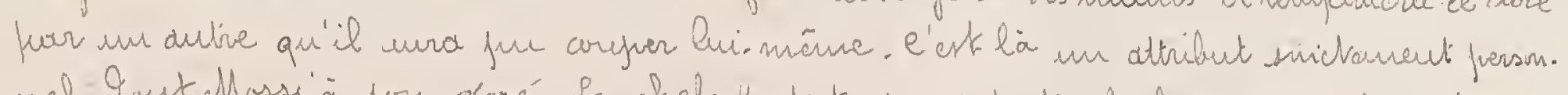

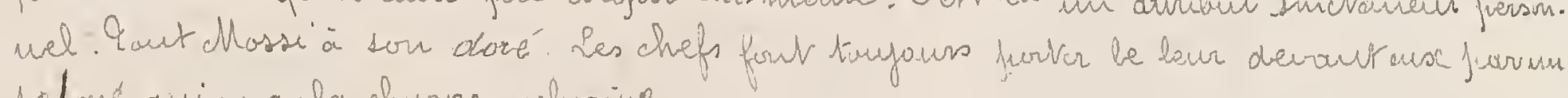
so / and quien a la charge axchrosive.

(2) On utilise anssi un frocédé frarticulier ayour furur but de endre la tumigu

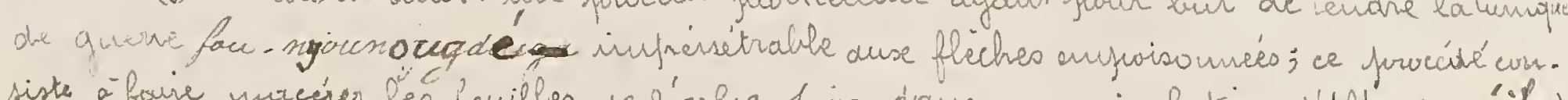

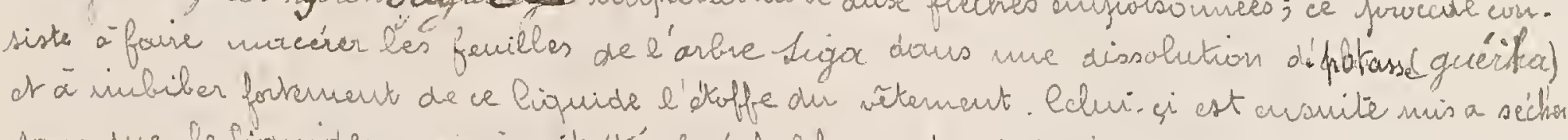
saus que be Ciquide en excés ait dé' foréalablancent exprinié. 


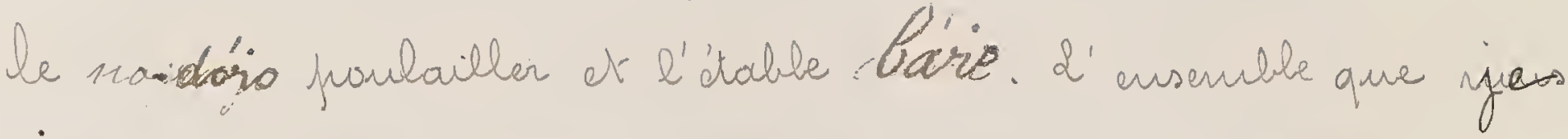
viens de décrire circonscrit la cour sirtérieure yatio du milien de laquelle se détache un grougue de trois cases veliées entre elles frar un petit mur: wase du chef de bamille izin-soba),

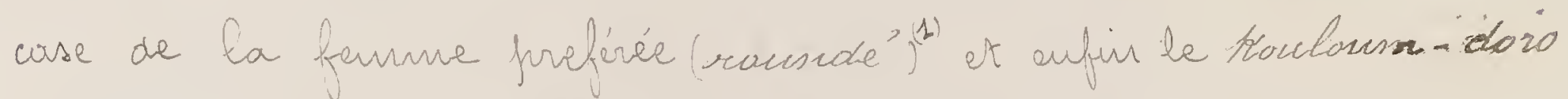

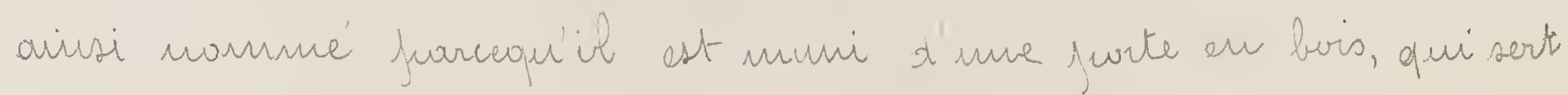
à enforruer ce que le chef de funille a de phus précieus.

C'est le yasgo case à deuse futtes, arrabogue au bantous.

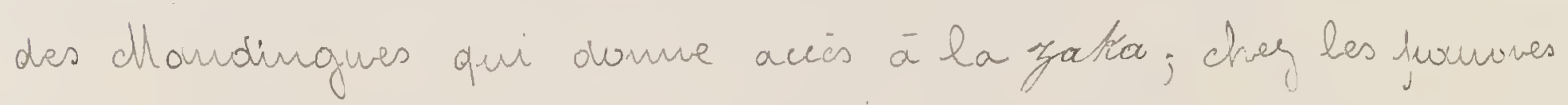
gens il est venulacé Jar une simple brieche dours le mur b. voune.

Les vésidences des chelp fussèiart deux fuotes: la grande, dommant sur la flowe (gassande?) un se tienneut les ar.

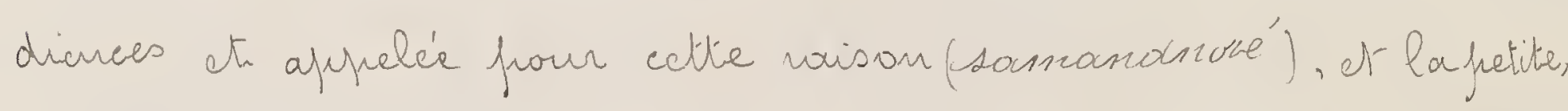

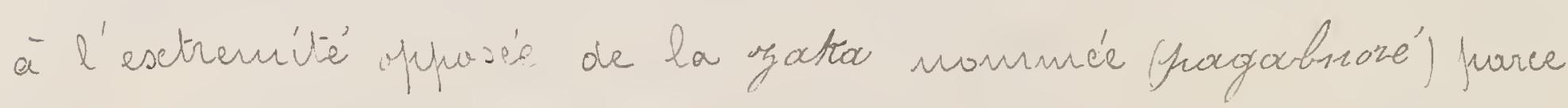
qu'elle conduit ause appartement's des fermues un dovi-nobérifor (entrée prerfiove) car elle ast tris surveillée et trahit ceux qui cherdent à s’y introanive. Chez les nabas ćgalenreut la

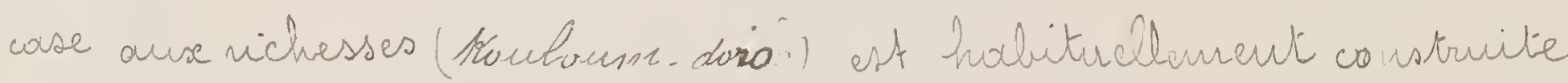
à argannasse et oniée sur le devant de fisintes corractèristiques

1) Les ferrmes our chef de familbe riennent à tún de wobe farser la mit sur

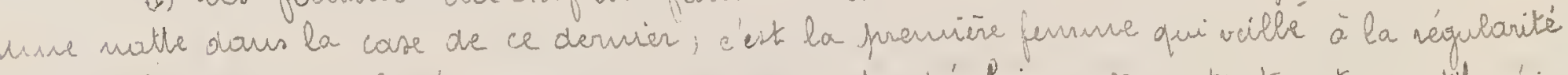

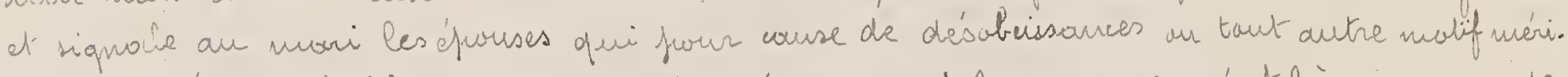

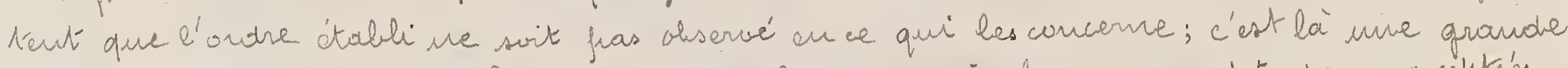

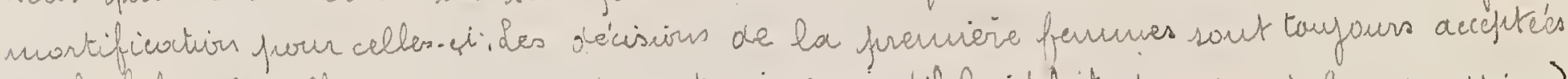

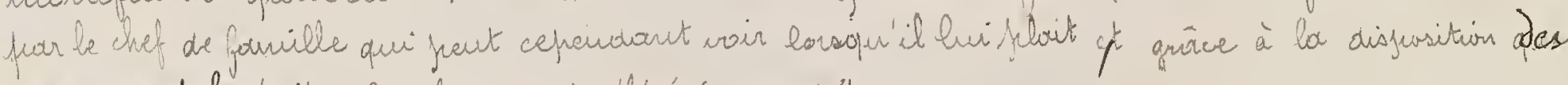

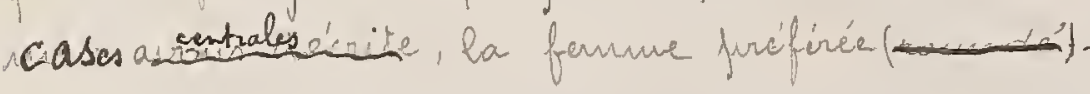




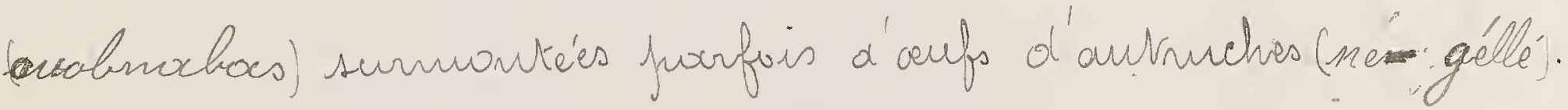
Les nabas conchent d'owhiovie dous be touko case Vaute en paille possédaut à l'irtérieur un verre.flair hour de quorwarte centimietres at large de un miètre cinquaute qui lnisert de bit.

Hors de la grande encente (laloganbangor) se tranent a'habitude un certrain nombue de casses isolées les soithado, destinées aux sourestiques et aux doctasorés.

Le néré córre. plair garmi de pierres plates serwait à

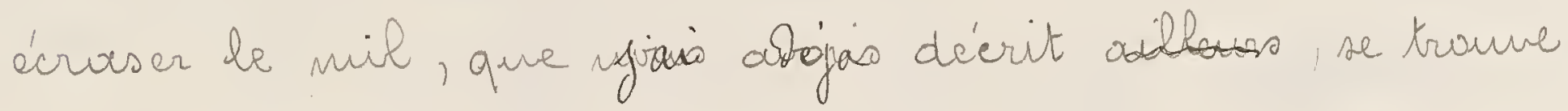

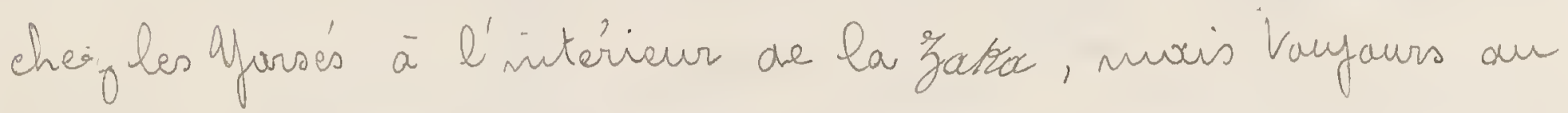
dehois chez les Massi.

Darrs cartaires régions notammert an II. ctà l'O. de Onougadougan se vencontre un type fuarticulier de cases qui a vegu le nam de doblilga. Dours un but de défeuse et de protection coutre les tomodes, comme aussi frour atcinver la lumière verout in dehors l'entrée qui n'a jus filus de soiscante quinize centinictres de houteur est encore barrée à l'intérieur foor mu pelit nur en terre, de sovte que four pénétrer dours ces cosses il fout sébaisseri confletement.

Les cases sout en géréral surmontées d'un toit en failve Songaceri) du type owhiraire.

Quoique primitifs, les clements du nablibier sorte. lativement assez momistanx.

Daur une case d'hamme on Vrouve tanjaurs deymattes 
(débré') on de sinuples sékus frire') servaut an couchage. ilit an verre ost particulier aux Mabas. Des défrié curssins en difffes an ar cuir garris de bourre de formager. On wit souvent hisiés our mur dis fevite. noublemix en

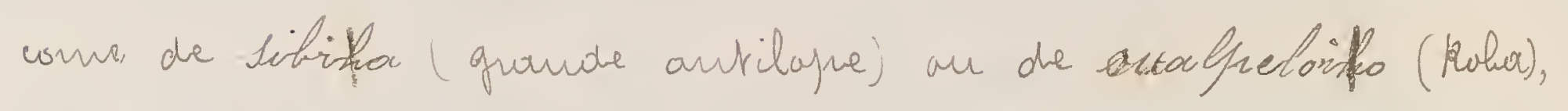

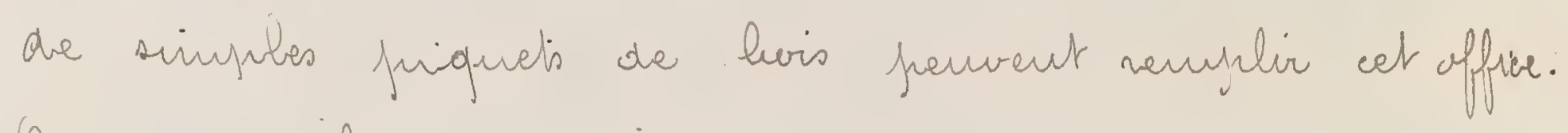
On y accorche des mistimments de culture et des armes voriess; la joour de bour gabóxiaq et la gourde (limiga) accessovies de voyage. Mh panier special filorion susprenin au flafond contieut des vêtemants de rechange of de menus alyets.

On vort anssi dours les cases d'hourmes ct farticu. lieienneut de fenres geus des nistrunculs de musique secondaires que dédorionent un puer les professiomels: le

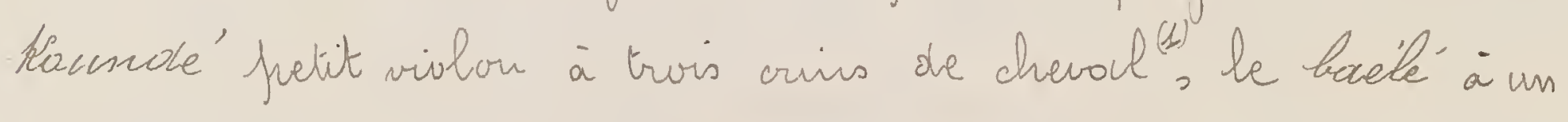
seul crin. Enfin le aunzbila, petite caldebasse seruourt de coupre à dasse, fuse' sur un comori verufeli de ce higui. de, et le doré Koufaurs placé en évidence.

de nubbilier des cases de femmes caryrarte essentielle. ment les tris priesses (docrana qui constituent le foyer

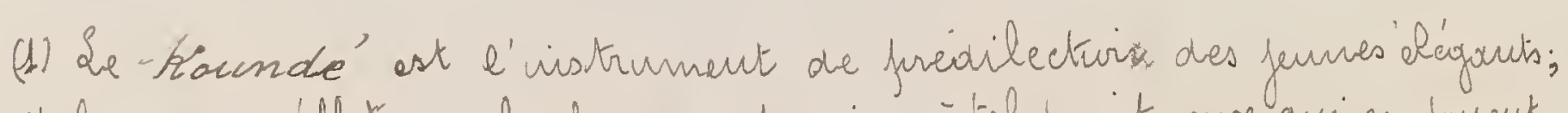

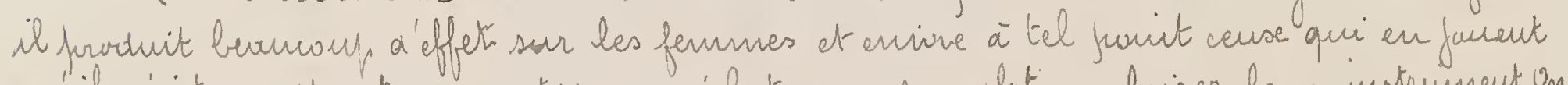

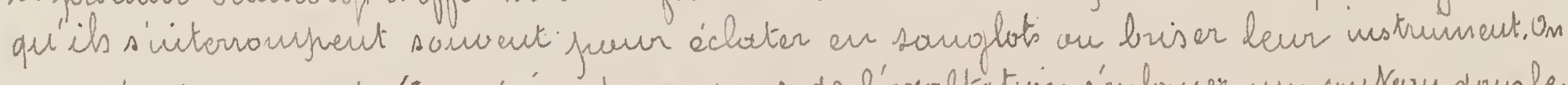

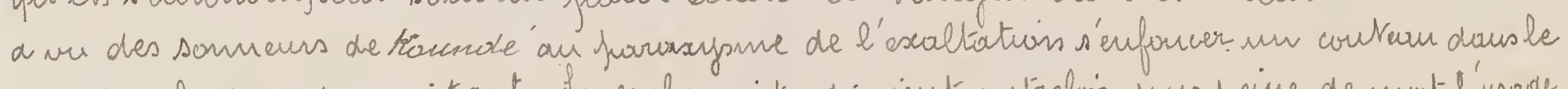

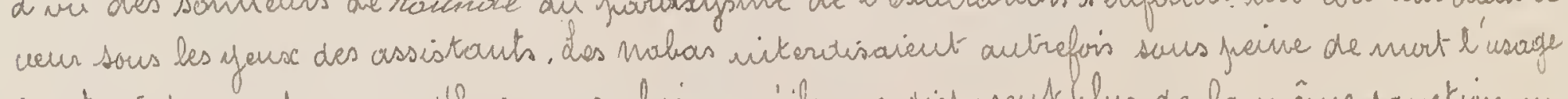

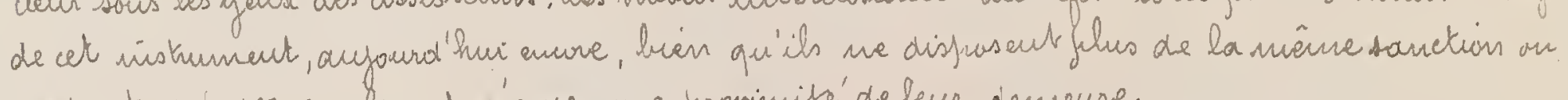

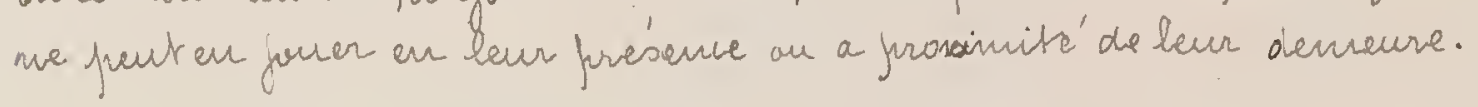




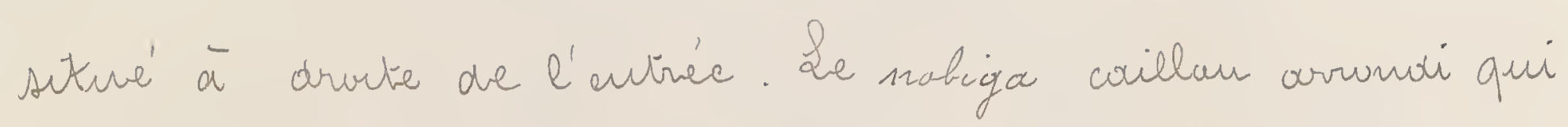
sert à noudre le grain sur le réré; le cóftra sorte d'us. tarsile à l'nitérieur onquel on fait áganter l'ear qui apries avoir troversi une wuche de cerrobres est utilisée daus $l$ alimentation en guise de sel. Ohes futeries variées

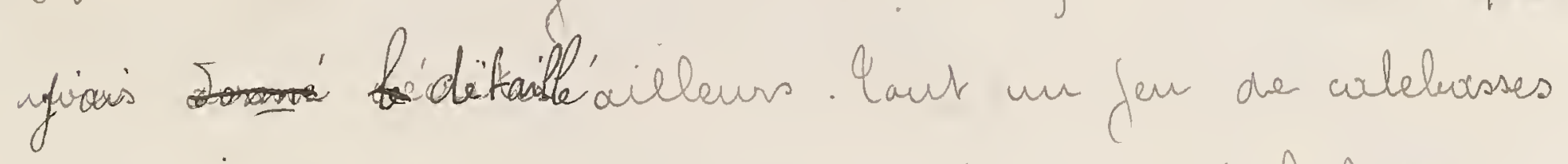
de arisine qui congorte comme types essentiels le doam-

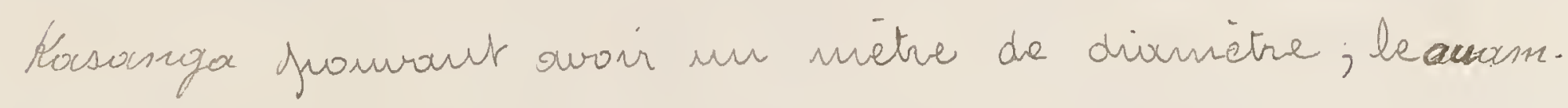
youga grainbe calchasse à manche; le soutrouga petite du même gense en fame de cuiller; enfirle acimovorom. dé qui mérite une mention sfréciale. Cotte caldebasse dont la cafracite' st définie, contient aviran trois kiloys de mil et chouque fanille en fussède un asemplaire. Clle a exacte.

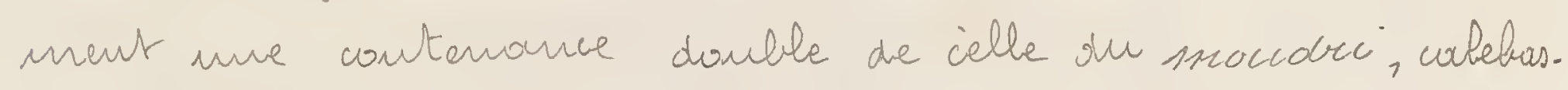
se étalon que détient dours chaque marché le Raga.naba. baus les villages on Massi pusséobent un ssondri absalu. ment semblable qui mesure la voleur de firc cauris de mil en tenups andinmine. In cas de disetile les variations de frixe sont jrachanies pror le Raga. Maba.

On voit. encore dous les cases de fermmes tes framiers (néorio) me des spécialités les plus originale de l'industrie Massi, des fuassovies en froille (joíatia."), divers accessov. nes velatif an filowge ou cuton (lowasos) et enfin des

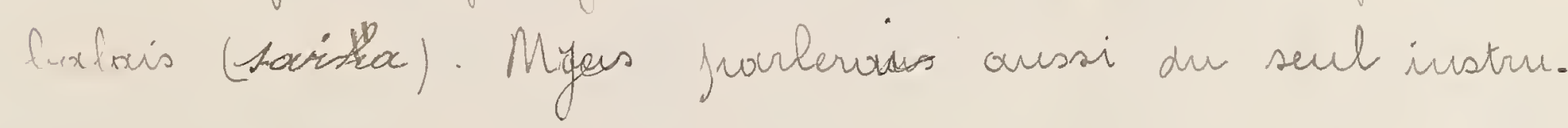
meut de musique qui leur afprartiemment en fropure si tout est que efoar furisse domer ce nour à une sortede 
gourde nenfilie de caillouse, la silsartra, qu'elle fout souter a'une marir à l'autre feour se divertir.

On trome également dours las cases Massi: des siéges ronds (gonéfle') creusis dours ine fieice de bis tendre sur lesquels les femmes s'assoieut foun vemwer le saibo "i. A defout de govélle' alle utilisent le doutroucka trone d'arbre pleir. Les fismmes s'asseyeut sur des sélas (ficic) ou bien si ce sout des nabas sur mue sorte de gouéfle' de forme blongue assez élégart, polus sanient encove sur le socofaforve' coussin sond en cuir brodé an sur me pean de mouton (gando).

Les cases Gourunusi: aifferent complètement des cases Mossi. Clles sout de forme carrée ot fussiovent une taiture à argannasse que suffportent des forurches situées daus l'in. terieur de la case; sur ces fourches on flace deuse lits on heris en sers alterne' et l'on newoure le taut d'une éansar canche de terre darmée avee soin. Es cases sont farfaitement étanches et l'our y est twut à fait à l'abrix

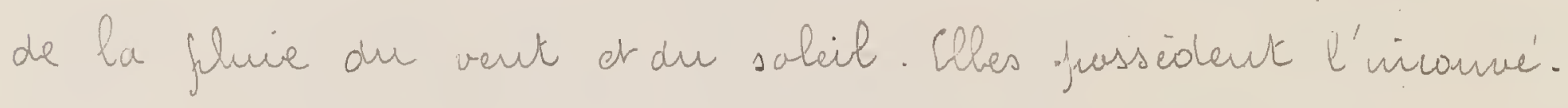
mieut d'être extrèmement chaudes car l'air ne jueut eir. culer que fuor les frortes, hasses et étraities Les Gouranusi vivent beancauf sur leurs. terrasses, souvent its construisent foar dessus d'autres cases qui consti. twent un prenier 'to ac. Le rez-de. Chanssée est alors réservé auxe Lemmes. Les cases sout Shabitudlement groupris foor ing 
an sise, tartes orientés vers un esfocee libme formont ainsi cour intérieure. lette cour ast entourée joor un pretit mur prence' d'une joorte que l'ou ferme chouque soir avee de grus madriers.

Les villages Gourounsi. présentent en géréral four les diverses soutrtalos, sinur jour l'ensemble, une forma. tion confracte; our Massi ar controine, et frimeipalement dours la région du N. et de l'O. Chaque gruugue de cases peut être isolè au milien des terres cultiviés dout il tive sa sulsistance, de sorte que les villoges s'étendent tur des suprerfieies consideriables. 


\section{Yndex alphabétique}

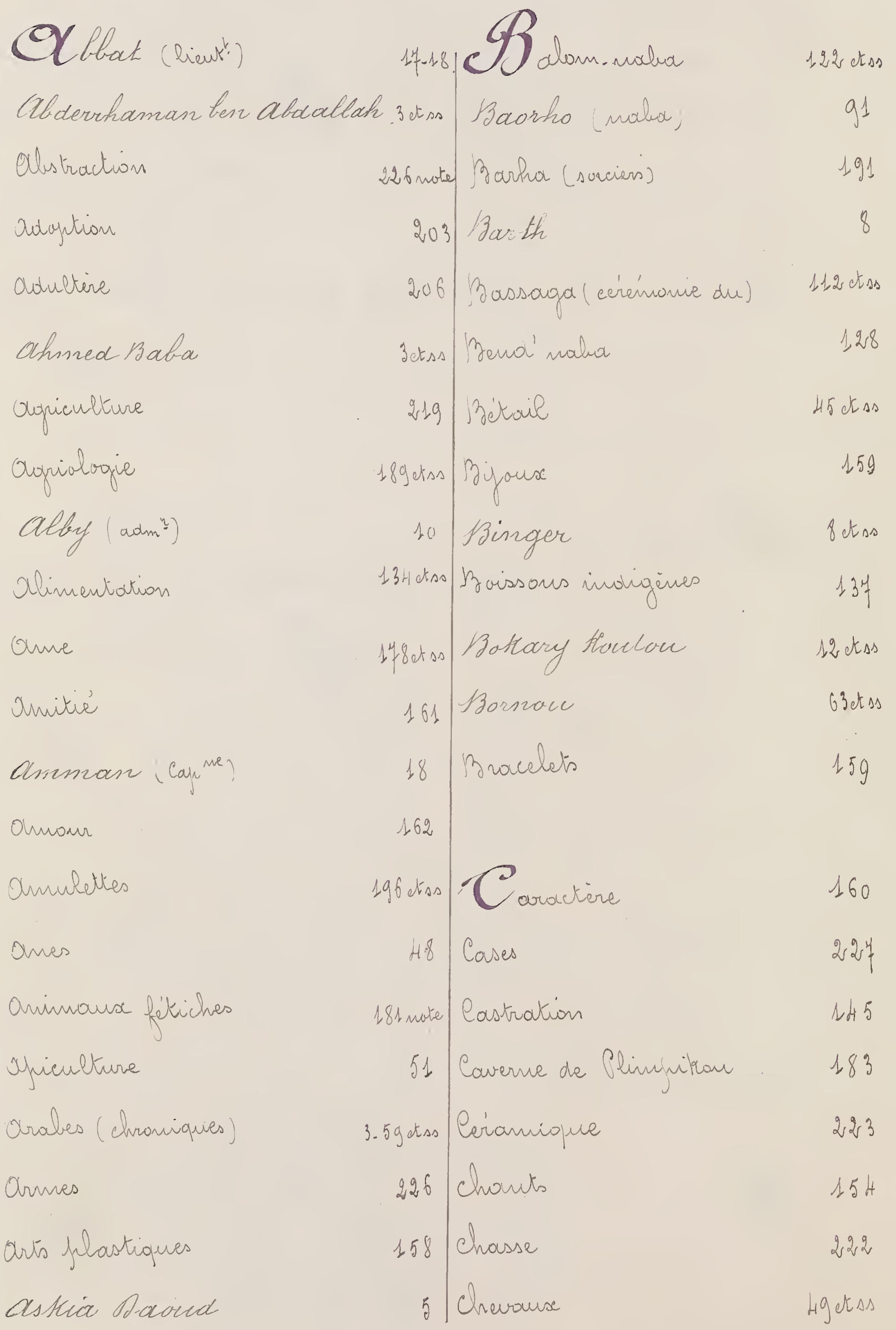




\begin{tabular}{|c|c|c|c|}
\hline Cirioncision & 145 & Sufouts & $1,41 d x$ \\
\hline Clochettes & 159 & Esthétiouere & 1) fots etss \\
\hline code & 212 & Emmopues & 145 \\
\hline Coiffure & 148 & Etoiles & 218 \\
\hline munerce & $38 c t s s$ & & \\
\hline unfession & 185 & amille & 2000 tos \\
\hline Condomiers & 225 & Gaune & 3Hetas \\
\hline Casmogranhlie & 218 & unes & $20130 t s s-222 f \operatorname{cts} s$ \\
\hline Courtisanes & 164 & Tergusson & $1 \ell$ \\
\hline Crave & 18 & Gilature & 224 \\
\hline brogat & 20 & Glove & 33 \\
\hline & & Stourrages & 51 \\
\hline agonsba & 66 & Grurérailles & $1 y_{3} t_{\Delta s}$ \\
\hline alionrés & 142 & & \\
\hline Danses & 151 & andé. maba & 130 \\
\hline Dafoe".naba & 1,26 & Ganiza. Maba & 129 \\
\hline Danvina & yg & Genies & 187 \\
\hline Dreformations diniques & LHLetss & Géologie & 32 etsas \\
\hline Destesiave (comm") & 11 ctss & Groognasé (soreiers) & 193 \\
\hline Orivinité & 18thetsis. & Gounga-naba & 120 \\
\hline Donlangan (maba) & 88 & gris.gris & 196 cotss \\
\hline Docbreciel (capre) & 23 & Grivort (lisut.) & 21 \\
\hline changes & 53 & If abitation & $22 \%$ crss \\
\hline Concation & 169 & Heeritage & 201 \\
\hline action du Morho. Mabre & $1050 t a s$ & Hosfuitalité & \\
\hline
\end{tabular}


Hbaubie' nalua)

Hanobrognaphive

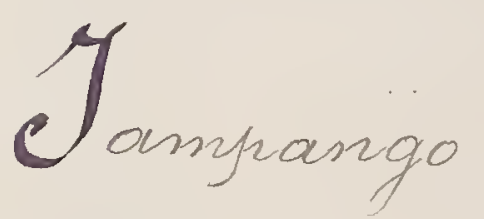

'Jandse' (naba)

Inmiot

Sndustries

Iyfherrendo (maba)

Yslancisme

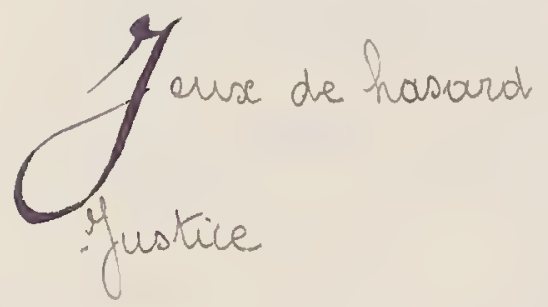

Uadiogo naba

Mamibo. naba

Mansoho. Maba

kiarfo (Mabou)

Biba (naba)

Himba (nala)

Kan 1". (maba)

Hom It (nabd)

Marcolar nalua

Ronga.malua

Haunde: Sembre' (nalua)

Plouritas

Houlare (maba)
8H 2 artrallé. natua

120

2. Lu - nalua

y 12 ampocisi

66

80 Marchés

3 getso

212 Mariages

2rictins Al attöba (naba)

zoltetsos

85

78 Métallurgie

Igg) Mobilier.

225

230

5

Mohanmmed-Asticia

165 Montagues sacrées 189 otss-198

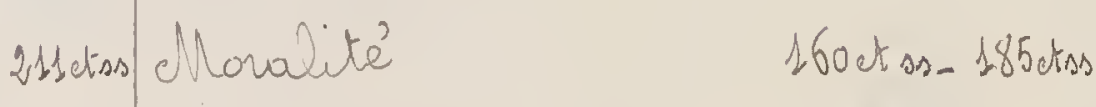

Morno. Matua rolutron

130 Classochs $\quad 62$

1,21 Massi bes 64

1,2 Cllosi: Tés $\quad 68$

g1 dlulets $\quad 48$

82. Chisione stgetss

82 Mnsulnavo $\quad 199$

87erso Mutilations atrmiques 1,43etss

102 Chvendé. naba 130

48

130 M akieroha (maba) 81

98 Mathansess 132

1 1H Mamerho (nala 81

92 Mananus is 


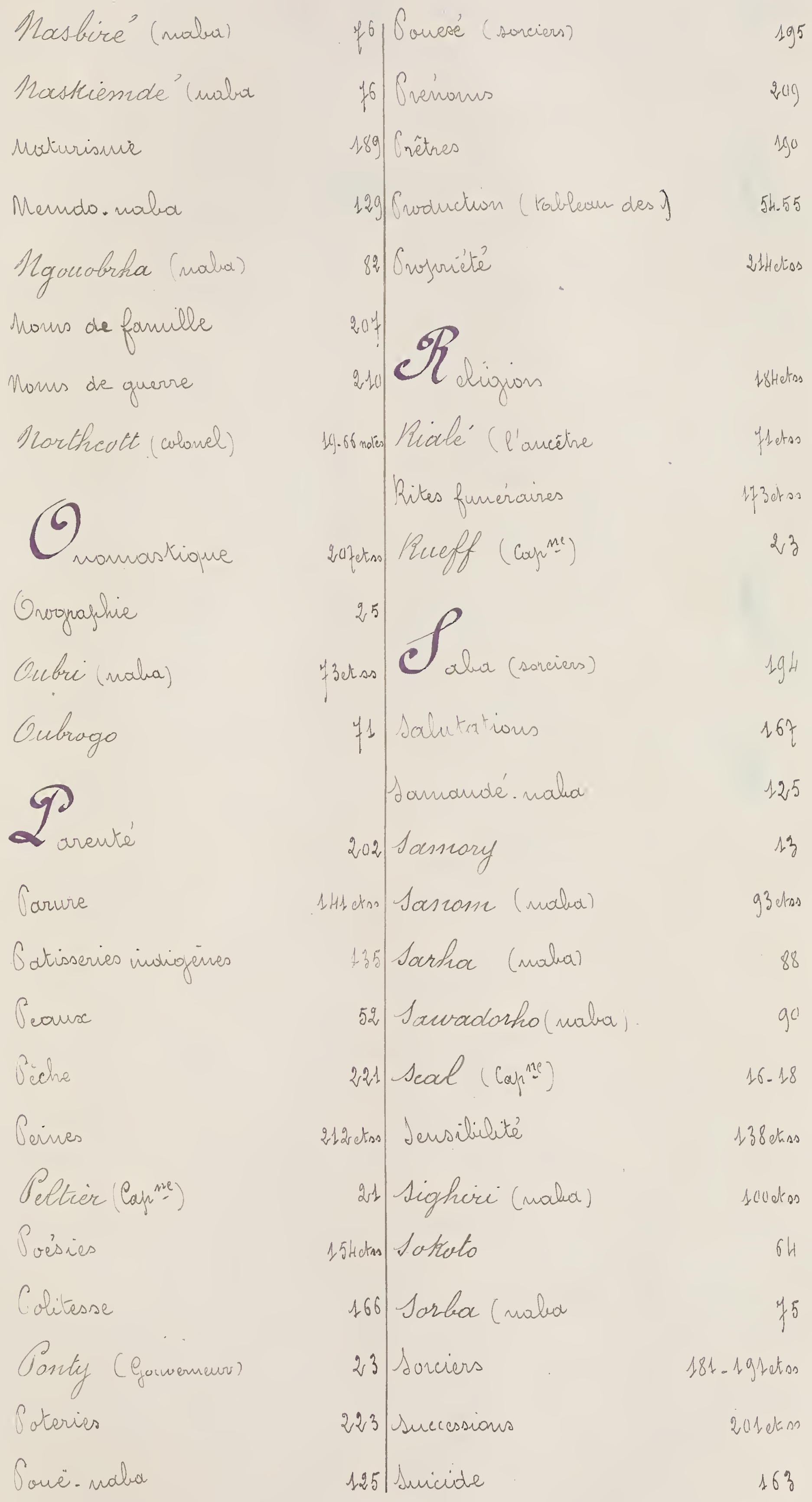




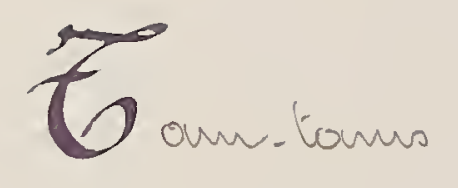

Gameurs

Gansaba. naba

Garithe es Joudân

Qotanoves

Qense' Rêtedus

of to fietiche)

Gomibouctan prise de

Qotémisme

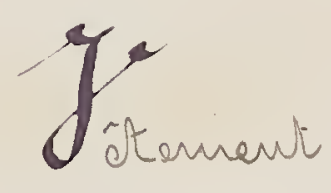

Nie future

Voltas (nivieres)

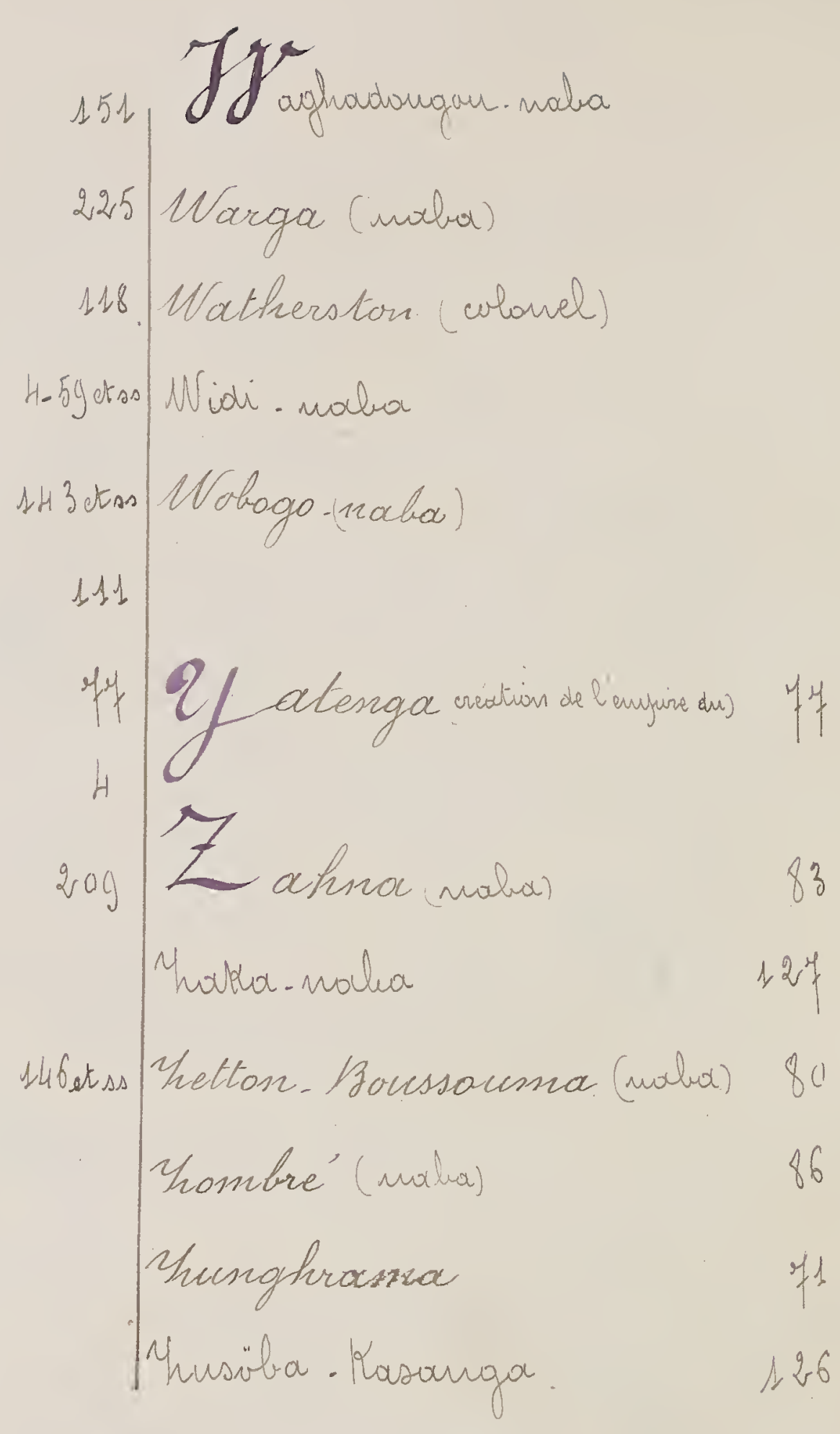




$$
\text { . }
$$
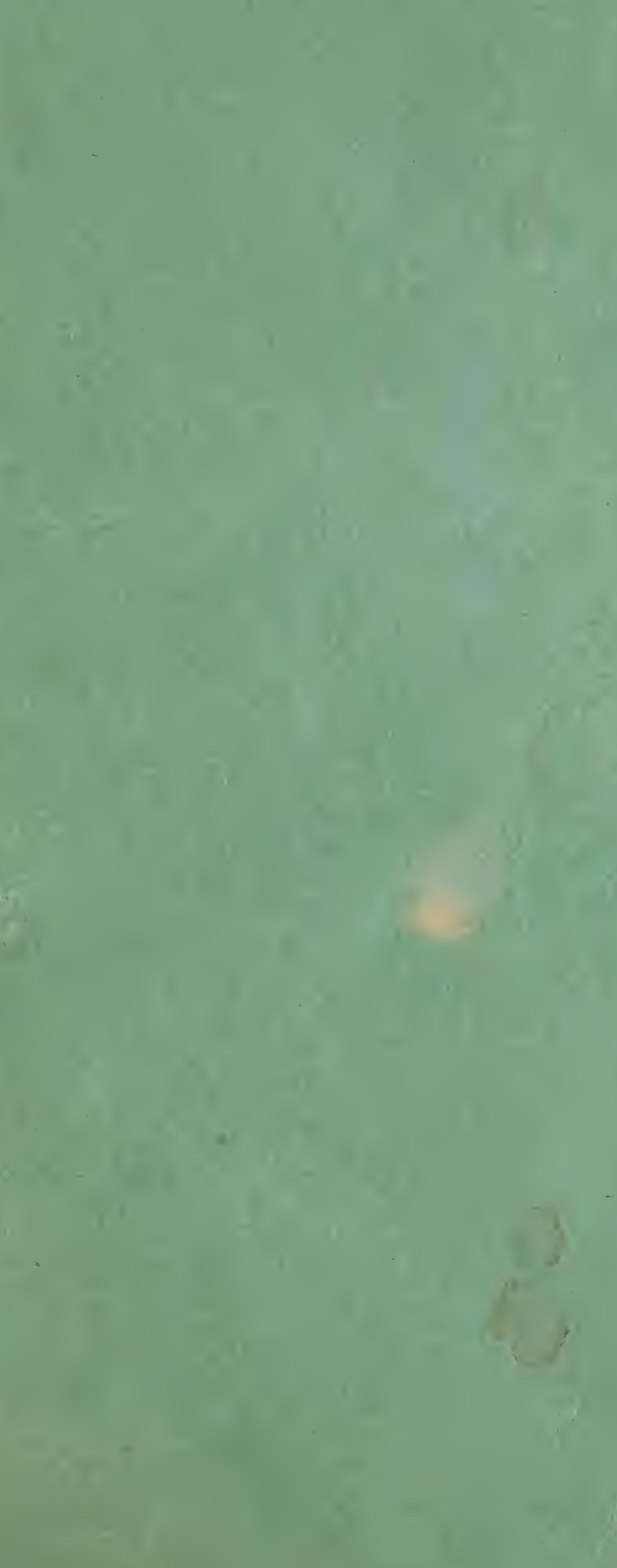


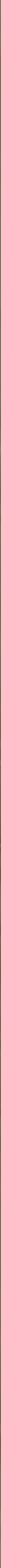

